Schneider, Klaus

\title{
Der Berufseinstieg von Lehrpersonen. Übergang und erste Berufsjahre im Kontext lebenslanger Professionalisierung
}

Bad Heilbrunn : Verlag Julius Klinkhardt 2021, 216 S. - (klinkhardt forschung) - (Dissertation, Universität Innsbruck, 2020)

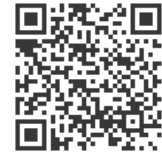

Quellenangabe/ Reference:

Schneider, Klaus: Der Berufseinstieg von Lehrpersonen. Übergang und erste Berufsjahre im Kontext lebenslanger Professionalisierung. Bad Heilbrunn : Verlag Julius Klinkhardt 2021, 216 S. - (klinkhardt forschung) - (Dissertation, Universität Innsbruck, 2020) - URN: urn:nbn:de:0111-pedocs-213717 - DOI: 10.25656/01:21371

https://nbn-resolving.org/urn:nbn:de:0111-pedocs-213717

https://doi.org/10.25656/01:21371

in Kooperation mit / in cooperation with:

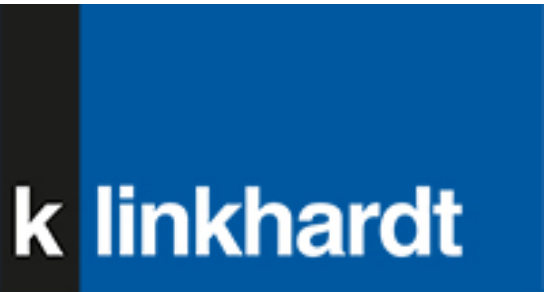

http://www.klinkhardt.de

\section{Nutzungsbedingungen}

Dieses Dokument steht unter folgender Creative Commons-Lizenz: http://creativecommons.org/licenses/by-nc-sa/4.0/deed.de - Sie dürfen das Werk bzw. den Inhalt unter folgenden Bedingungen vervielfältigen, verbreiten und öffentlich zugänglich machen sowie Abwandlungen und Bearbeitungen des Werkes bzw. Inhaltes anfertigen: Sie müssen den Namen des Autors/Rechteinhabers in der von ihm festgelegten Weise nennen. Dieses Werk bzw. der Inhalt darf nicht für kommerzielle Zwecke verwendet werden. Die neu entstandenen Werke bzw. Inhalte dürfen nur unter Verwendung von Dizenzbedingungen weitergegeben werden, die mit denen dieses Lizenzbedingungen weitergegeben werden,

Mit der Verwendung dieses Dokuments erkennen Sie die Nutzungsbedingungen an.

\section{Terms of use}

This document is published under following Creative Commons-License: http://creativecommons.org/licenses/by-nc-sa/4.0/deed.en - You may copy, distribute and transmit, adapt or exhibit the work in the public and alter, transform or change this work as long as you attribute the work in the manner specified by the author or licensor. You are not allowed to make commercia may distribute the resulting work only under this or a comparable license.

By using this particular document, you accept the above-stated conditions of

\section{Kontakt / Contact:}

\section{peDOCS}

DIPF | Leibniz-Institut für Bildungsforschung und Bildungsinformation Informationszentrum (IZ) Bildung

E-Mail: pedocs@dipf.de

Internet: www.pedocs.de

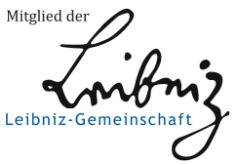




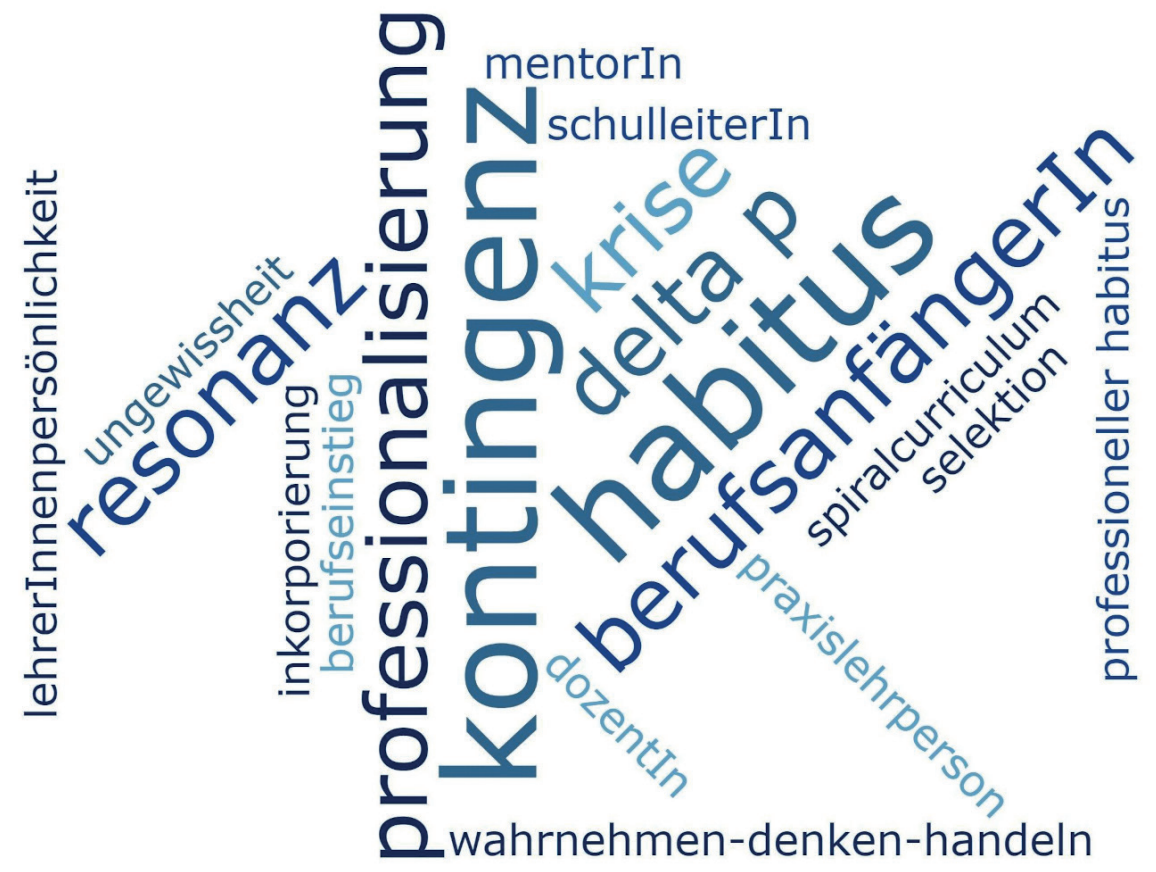

Klaus Schneider

\section{Der Berufseinstieg von Lehrpersonen}

Übergang und erste Berufsjahre im Kontext lebenslanger Professionalisierung 
Schneider

Der Berufseinstieg von Lehrpersonen 
Für Barbara, David und Jacob. 


\section{Klaus Schneider}

\section{Der Berufseinstieg von Lehrpersonen}

Übergang und erste Berufsjahre im Kontext lebenslanger Professionalisierung 
Die Open Access-Publikation dieses Titels wurde von der

Pädagogischen Hochschule Tirol finanziell gefördert.

Die vorliegende Arbeit wurde vom Institut für LehrerInnenbildung und Schulforschung der Fakultät für LehrerInnenbildung an der Universität Innsbruck unter dem Titel „Der Berufseinstieg von Lehrpersonen als Übergang. Die ersten Berufsjahre im Kontext lebenslanger Professionalisierung“ als Dissertation angenommen. Gutachter: Univ.-Prof. Mag. Dr. Christian Kraler, Univ.-Prof. Mag. Dr. Hans Karl Peterlini.

Tag der Disputation: 30.04.2020.

Dieser Titel wurde in das Programm des Verlages mittels eines Peer-Review-Verfahrens aufgenommen. Für weitere Informationen siehe www.klinkhardt.de.

Bibliografische Information der Deutschen Nationalbibliothek Die Deutsche Nationalbibliothek verzeichnet diese Publikation in der Deutschen Nationalbibliografie; detaillierte bibliografische Daten sind im Internet abrufbar über http://dnb.d-nb.de.

2021.kg. (C) by Julius Klinkhardt.

Satz: Friedrich Wall, Spreeau.

tag cloud Umschlagseite 1: ㄷ Klaus Schneider.

Druck und Bindung: Bookstation GmbH, Anzing.

Printed in Germany 2021.

Gedruckt auf chlorfrei gebleichtem alterungsbeständigem Papier.

Die Publikation (mit Ausnahme aller Fotos, Grafiken und Abbildungen) ist veröffentlicht unter der Creative Commons-Lizenz: CC BY-NC-SA 4.0 International

https://creativecommons.org/licenses/by-nc-sa/4.0/ 


\section{Zusammenfassung}

In der vorliegenden Arbeit werden die Phasen der Ausbildung, des unmittelbaren Berufseinstiegs (Induktionsphase mit Mentoring) und der weiteren ersten Berufspraxis von Lehrpersonen primär aus der Perspektive des berufsbiografischen Ansatzes (Keller-Schneider \& Hericks 2014) untersucht. Das Forschungsziel lautet, Gelingensbedingungen für den Berufseinstieg und die weitere berufsbiografische Entwicklung zu identifizieren. Nach der Grounded Theory (Strauss \& Corbin 1996), Clusteranalyse (Buber \& Kraler 2000) und qualitativen Inhaltsanalyse (Kuckartz 2016) werden Interviews mit SchulleiterInnen und BerufsanfängerInnen mittels multimethodologischen Zugangs analysiert und interpretiert. Das empirisch gewonnene Konzept konstituiert sich als Professionalisierung durch Kompetenzgewinn, die professionelle Habitualisierung etabliert sich als Weg zum Erfolg. Das Habituskonzept nach Bourdieu (2015b) mit der Triade des Wahrnehmens, Denkens und Handelns seht im Zentrum dieser Arbeit und begegnet den Phänomenen Ungewissheit und Kontingenz (Paseka et al. 2018) mit der schöpferischen Dimension des Generierens von beliebig vielen Handlungsschemata für beliebig viele Situationen. Mit dem Ausblick auf konkrete Umsetzungsmöglichkeiten der gewonnenen Erkenntnisse in den Ausbildungs- und Fortbildungscurricula schließt die Arbeit.

Schlagworte (alphabetisch gereiht)

Curriculum, Habitualisierung, Induktionsphase, Kontingenz, Lehramtsstudium, LehrerInnenbildung, Mentoring, Professionalisierung, Professioneller Habitus, Ungewissheit 


\begin{abstract}
In this dissertation, the phases of training, the immediate entry into the profession (induction phase with mentoring) and the further first professional practice of teachers are examined primarily from the perspective of the professional biographical approach (Keller-Schneider \& Hericks 2014). The research goal is to identify conditions necessary for both a successful career start and further career development. After grounded theory (Strauss \& Corbin 1996), cluster analysis (Buber \& Kraler 2000) and qualitative content analysis (Kuckartz 2016), interviews with school leaders and young professionals are analyzed and interpreted using a multimethodological approach. The empirically gained concept is constituted as professionalization through gaining competence, the professional habitualization is established as a way to success. The Habitus concept according to Bourdieu (2015b) with the triad of perception, thinking and acting is at the center of this work and meets the phenomena of uncertainty and contingency (Paseka et al. 2018) with the creative dimension of generating any number of action schemes for any number of situations. The work concludes with the prospect of concrete implementation options for the knowledge gained in the training and further education curricula.
\end{abstract}

Keywords (alphabetically sorted)

Contingency, continued professional development, curriculum, habitualization, induction phase, mentoring, professional habitus, professionalization, teacher training, uncertainty 


\section{Inhalt}

Vorwort

\section{Einleitung}

1 Forschungsinteresse und Fragestellungen $\ldots \ldots \ldots \ldots \ldots \ldots \ldots \ldots \ldots \ldots \ldots \ldots$

2 Stand der Forschung und Konzeption der Arbeit ................... 15

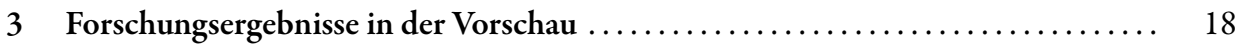

\section{Grundlagen}

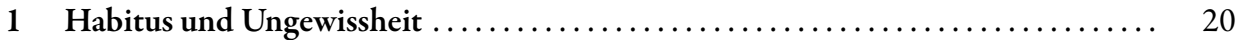

1.1 Ungewissheit als Teil der pädagogischen Profession .................. 20

1.1.1 Konstitution der Ungewissheit im pädagogischen Kontext ......... 20

1.1.2 Umgang mit Ungewissheit und Krisenerfahrung ............... 24

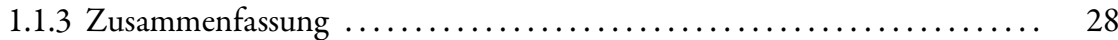

1.2 LehrerInnenpersönlichkeit und Habitus im Kontext von

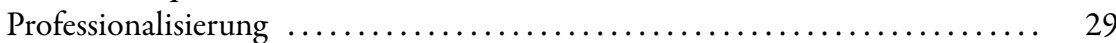

1.2.1 Habituskonzept nach Bourdieu als „Theorie der Praxis“ ............ 29

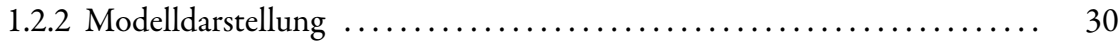

1.2.3 Übertragung des Habituskonzepts auf LehrerInnenprofessionalisierung . . . 37

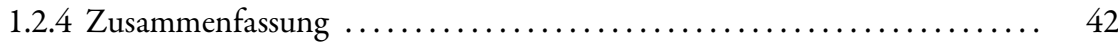

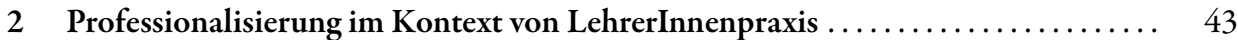

2.1 Pädagogische und allgemeine Professionalisierung $\ldots \ldots \ldots \ldots \ldots \ldots \ldots \ldots .43$

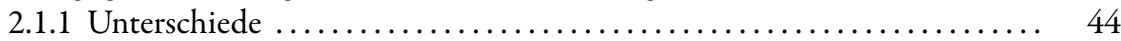

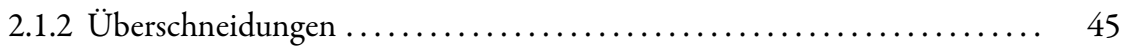

2.2 Professionalisierung in den Praxisphasen: Pädagogisch-praktische Studien .... 49

2.2.1 Professionalisierung durch Forschendes Lernen ................. 51

2.2.2 Professionalisierung durch Reflexion über Unterricht ............ 55

2.2.3 Professionalisierung durch Kooperation der AkteurInnen ........... 59

2.3 Professionalisierung beim Berufseinstieg: Induktionsphase und Mentoring .... 63

2.3.1 Einführung und Begriffsbestimmungen ................... 63

2.3.2 MentorInnen und Mentees: die ProtagonistInnen des

Mentoring-Programms ................................. 66

2.3.3 SchulleiterInnen im Professionalisierungsprozess von Lehrpersonen .... . 69

2.3.4 Mentoring in Theorie und Praxis ......................... 71

2.3.5 Status quo an österreichischen Schulen $\ldots \ldots \ldots \ldots \ldots \ldots \ldots \ldots \ldots . \quad 80$ 


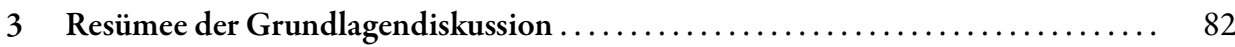

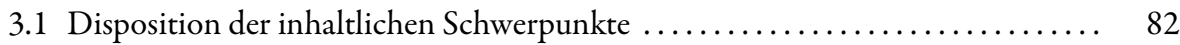

3.2 Ungewissheit, Kontingenz und doppelte Kontingenz $\ldots \ldots \ldots \ldots \ldots \ldots \ldots . \quad 83$

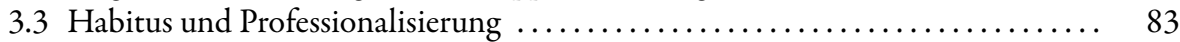

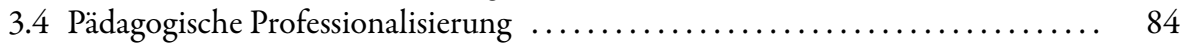

\section{Empirischer Zugang}

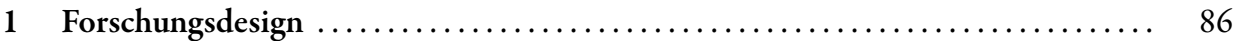

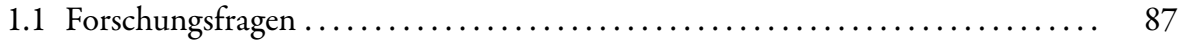

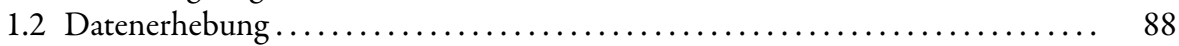

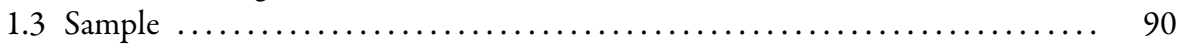

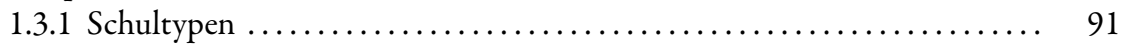

1.3.2 SchulleiterInnen .................................... 91

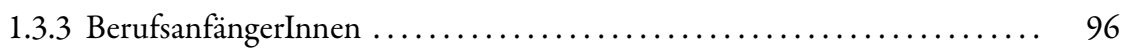

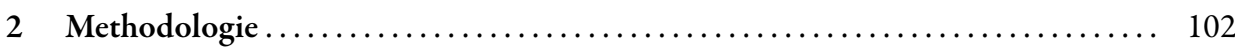

2.1 Datenerhebung: Leitfadeninterview, ExpertInneninterview, Postscript . . . . . 103

2.2 Datenanalyse: Grounded Theory und Clusteranalyse ................ 104

2.3 Dateninterpretation: Strukturierende qualitative Inhaltsanalyse,

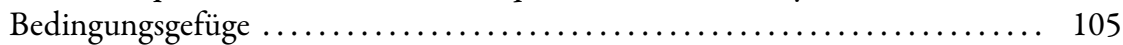

2.4 Methodologische Grenzen der Untersuchung .................. 106

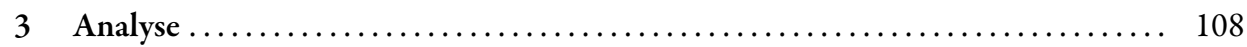

3.1 Haupt-, Sub-, Subsub- und Subsubsubkategorienbildung ................ 108

3.1.1 Darstellungsformen der Kategoriensysteme .................. 108

3.1.2 Kategoriensystem: SchulleiterInnen ....................... 109

3.1.3 Kategoriensystem: BerufsanfängerInnen .................. 110

3.1.4 Interviews mit SchulleiterInnen ........................ 112

3.1.5 Interviews mit BerufsanfängerInnen ...................... 125

3.2 Zusammenfassende Darstellung der Hauptkategorien .................. 144

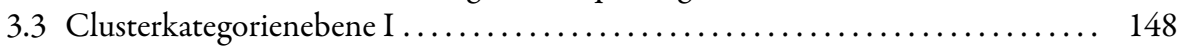

3.3.1 Schlüsselbegriffe und Clusterbildung: Clusterkategorie 1 (CK_I_1) .... 149

3.3.2 Schlüsselbegriffe und Clusterbildung: Clusterkategorie 2 (CK_I_2) .... 149

3.4 Konzeptualisierung: Clusterkategorie 3 (CK_II_3) .................. 150

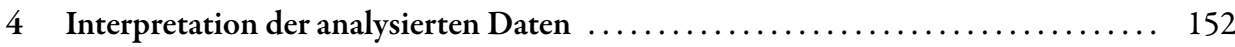

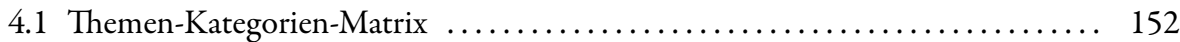

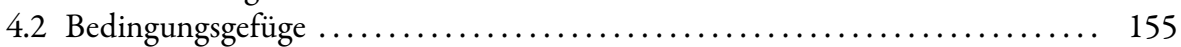

4.2.1 Vorbemerkungen zu Abbildungen und Tabellen der Bedingungsgefüge .. 156

4.2.2 Bedingungsgefüge Administration ..................... 156

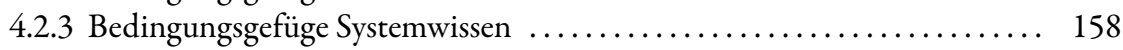

4.2.4 Bedingungsgefüge Reflexion \& Feedback .................. 160

4.2.5 Bedingungsgefüge Autorität in der Klasse ................... 162

4.2.6 Bedingungsgefüge Kooperation mit SchülerInnen .............. 163

4.2.7 Bedingungsgefüge Habitus: Metabedingungsgefüge ............. 167 


\section{Zusammenführung und Integration}

1 Vereinigung theoretischer und empirischer Erkenntnisse $\ldots \ldots \ldots \ldots \ldots \ldots \ldots 172$

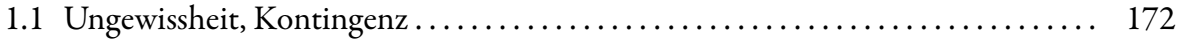

1.2 LehrerInnenpersönlichkeit \& Habitus ........................ 176

2 Erkenntnisse zu den Forschungsfragen: Conclusiones ................ 181

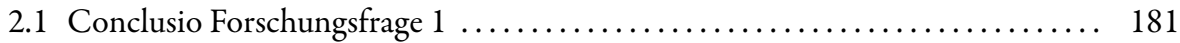

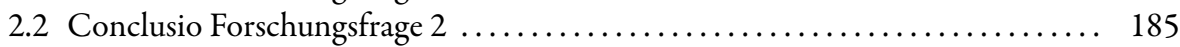

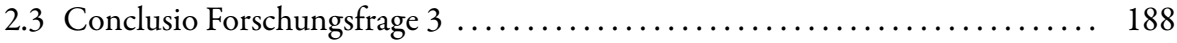

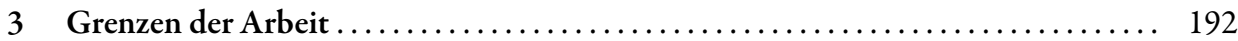

V Ansätze zur gelingenden Professionalisierung - Ausblick

1 Spiralförmig in kleinen Schritten zur Professionalität: Delta P . . . . . . . . . 195

2 Ganzheitliche Professionalisierung in der Ausbildung . ................ 197

\section{Verzeichnisse}

Literaturverzeichnis .......................................... 201

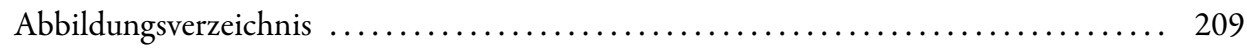

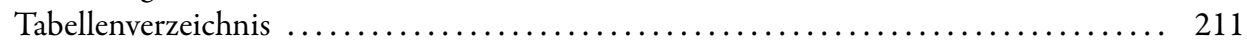

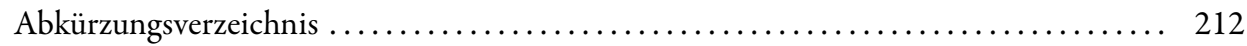

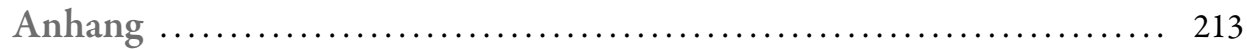





\section{Vorwort}

In 23 Jahren eigener Unterrichtstätigkeit entwickelte sich mein Forschungsinteresse, den Berufseinstieg von Lehrpersonen zu untersuchen. Dieses Interesse entstand einerseits aufgrund eigener Erfahrungen beim Einstieg in die berufliche Praxis, andererseits durch Beobachtungen nachfolgender, im berufsbiografischen Sinne jüngerer KollegInnen, durch Gespräche und letztlich durch informelles Mentoring, das ich leisten durfte. Die Wahrnehmung unterschiedlicher Berufseinstiegsszenarien, diese reichten vom optimalen Start über Fehlstart mit Restart im nächsten Schuljahr bis zur Disqualifizierung (durch sich selbst, andere AkteurInnen oder das System) mit Ausscheidung, um in der Terminologie des Sportes zu sprechen, weckten mein Interesse, Gelingensbedingungen für den Übergang vom Studium in den Beruf zu identifizieren. In meiner nunmehrigen Funktion als Hochschuldozent des Ausbildungsverbundes West ${ }^{1}$ im Lehramtsstudium Sekundarstufe-Allgemeinbildung boten sich für mich durch das Praxis- bzw. Forscherwissen, die Arbeit mit Studierenden und den Fachdiskurs mit AkteurInnen im Kontext der Ausbildungsinstitutionen Pädagogische Hochschule und Universität ideale Rahmenbedingungen, diesem Forschungsinteresse nachzukommen. Eingebettet in die von Univ.-Prof. Mag. Dr. Christian Kraler gegründete Forschungsgruppe Teacher Education Research Group (TERG) am Institut für LehrerInnenbildung und Schulforschung (ILS) konnte ich den Übergang in den Beruf als einen durch viele Faktoren determinierten Prozess untersuchen. Die Zeit vorher (LehrerInnenausbildung) und nachher (Induktionsjahr und weitere Berufspraxis) wurden in die Forschung im Sinne von Rahmungsphasen des Überganges integriert.

Als Ergebnis der vorliegenden empirischen Forschungsarbeit liegt das Konzept Professionalisierung durch Kompetenzentwicklung vor, es wird in der nachfolgenden Arbeit als Gelingensbedingung für einen erfolgreichen Berufseinstieg und als Perspektive für die weitere berufliche Professionalisierung diskutiert.

An dieser Stelle gebührt vielen Personen ein großer Dank, ohne deren Unterstützung die Entstehung dieses Buches nicht möglich gewesen wäre.

Allen voran danke ich den hilfsbereiten und in ihren Erzählungen sehr offenen GesprächspartnerInnen. 15 SchulleiterInnnen und 25 BerufsanfängerInnen mit maximal drei Unterrichtsjahren Berufserfahrung teilten mir in Interviews ihre Erkenntnisse, Anliegen und Ideen mit, die nach qualitativer Analyse zur Generierung des bereits genannten Konzepts der Arbeit führten. Ein besonderer Dank gilt meinem Betreuer und Doktorvater Christian Kraler an der LeopoldFranzens-Universität Innsbruck. In unzähligen Treffen forcierte er meine bildungswissenschaftliche Professionalisierung maßgeblich, sein klares und direktes Feedback war stets wertschätzend und konstruktiv. Vielen Dank für die Offenheit, die Betreuung und den großen Beitrag an meinem wissenschaftlichen Erkenntnisgewinn.

Frau Irmgard Plattner, Vizerektorin für Forschung und Entwicklungsangelegenheiten an der Pädagogischen Hochschule Tirol, danke ich ganz herzlich für die Genehmigung von Zeitres-

1 Ausbildungsinstitutionen im Ausbildungsverbund WEST/Österreich: Universität Innsbruck, Pädagogische Hochschule Tirol, Pädagogische Hochschule Vorarlberg, Kirchliche Pädagogische Hochschule Edith Stein, Mozarteum. 
sourcen im Rahmen meiner Dozententätigkeit. Ohne diese Zeitgefäße läge dieses Buch jetzt nicht vor.

Zahlreiche KollegInnen in meinem beruflichen Kontext bekundeten in vielen Begegnungen ihr Interesse an meiner Forschung. Die Fachdiskurse wirkten als Impulse im Forschungs- und Schreibprozess, verschonten mich vor manchen Irrwegen und brachten neue, wertvolle Ideen. Ich danke euch dafür.

Ein großes Dankeschön fehlt noch, es gilt meiner Familie. Barbara, David und Jacob zeigten viel Verständnis, wenn ich in den vergangenen Jahren bei familiären Unternehmungen nicht dabei war. Zudem haben mich eure aufmunternden Worte über manches Motivationstief gerettet. Vielen Dank euch dreien.

Hall in Tirol, im Jänner 2021

Klaus Schneider 


\section{Einleitung}

In der vorliegenden Arbeit wird der Berufseinstieg von Lehrpersonen als berufsbiografischer Übergang mit dem Ziel diskutiert, Gelingensbedingungen für einen erfolgreichen Berufseinstieg im Kontext der ersten Berufsjahre sowie der lebenslangen Professionalisierung zu identifizieren. Dem Fokus auf die scheinbar kleinen Details beim Berufseinstieg und der folgenden ersten Berufspraxis wird besondere Bedeutung beigemessen und nachgekommen. In einem ersten Codierdurchgang gehe ich den Aussagen in den Interviews im Sinne der Grounded Theory (Strauss und Corbin 1996) ,auf den Grund“, um später bei der Konzeptentwicklung auf höchsten Kategorienebene wieder darauf zuzugreifen.

In der Einleitung (Kap. I) werden die Fragestellungen bzw. das Forschungsinteresse zur Arbeit dargestellt, um anschließend den wissenschaftlichen Stand der Professionsforschung auszugsweise zu diskutieren. Dabei kristallisieren sich die thematischen Schwerpunkte der Forschungsarbeit heraus: Kontingenzerfahrungen und Ungewissheit, Professionalisierung als Lehrperson durch die Herausbildung eines professionellen Habitus sowie Professionalisierung in den Praxisphasen als LehrerIn (Kap. II). Einblicke in die Konzeption des empirischen Zugangs in Kapitel III sowie Hinweise zu empirischen Ergebnissen (Kap. IV) werden ebenso gegeben wie ein Ausblick auf einen gelingenden Berufsstart (Kap. IV). 


\section{Forschungsinteresse und Fragestellungen}

Wie bereits erwähnt, ist das Ziel und zugleich das Forschungsinteresse der vorliegenden Arbeit, Gelingensbedingungen für einen positiven Berufseintritt als Lehrperson zu identifizieren. Um die Phase des Berufseintritts als Übergang zu positionieren, werden auch die Ausbildungsphase vorher und die erste Praxisphase nachher berücksichtigt. Insofern stellt sich zu Beginn die Frage, welche Inhalte LehramtsstudentInnen in ihrer Ausbildung benötigen, um später als BerufsanfängerInnen einen gelingenden Berufseintritt und als Lehrpersonen einen professionellen Unterricht zu realisieren. Direkt damit hängt die Frage zusammen, welche Anforderungen an die Curricula der Aus- und Fortbildung gestellt werden, um dem Anspruch eines gelingenden Berufsstarts bzw. der LehrerInnenprofession gerecht zu werden. Schließlich wird noch untersucht, wie sich initiale Berufserfahrungen beim Berufseinstieg auf den unmittelbaren weiteren Verlauf in der ersten Berufspraxis auswirken. 


\section{Stand der Forschung und Konzeption der Arbeit}

Im wissenschaftlichen Diskurs wird die Forschung zum Berufseinstieg als Teil der pädagogischen Professionsforschung interpretiert (Helsper und Böhme 2008; Hericks 2006; Herzog 2014; Terhart et al. 2014; Tippelt 2010). Innerhalb dieser lassen sich grob drei theoretische Grundpositionen unterscheiden. Zunächst sind da der strukturtheoretische Ansatz, der auf eine Rekonstruktion der strukturellen Handlungsanforderungen des Lehrerberufs abzielt, und der kompetenzorientierte Ansatz, der nach den zur Bewältigung dieser Anforderungen notwendigen Kompetenzen und Einstellungen der Lehrerinnen und Lehrer sowie deren Entwicklung fragt (Helsper und Böhme 2008; Terhart 2001). Einen Mittelweg stellt aus der Sicht von KellerSchneider und Hericks (2014) der berufsbiografische Ansatz dar, ein spezieller Ansatz der Bildungsgangforschung. Dieser wird in der folgenden Arbeit zum Berufseinstieg von Lehrpersonen favorisiert, wenngleich alle drei genannten Zugänge insbesondere auch deshalb eine Rolle spielen, da in der Befragung der SchulleiterInnen und BerufsanfängerInnen ${ }^{2}$ (Empirischer Zugang, Kap. III) diese Ansätze thematisiert werden. Teilweise explizit, teilweise implizit und durch Interpretation sichtbar gemacht. Aus der berufsbiografischen Perspektive ist Professionalität ein „berufsbiografisches Entwicklungsproblem“ (Terhart 2001) und kann als Herausforderung des lebenslangen Lernens (Schober et al. 2009) gesehen und interpretiert werden. Dabei entwickelt sich die Professionalität im „Prozess des Lehrerwerdens“ (Terhart 2001, S. 56). Insofern startet die Entwicklung der LehrerInnenprofessionalität mit Ausbildungsbeginn und zieht sich durch das gesamte Berufsleben der LehrerInnen mit den weiteren berufsbiografischen Stationen des Berufseinstiegs, der Berufsausübung und der berufsbegleitenden Fort- und Weiterbildung. ${ }^{3}$ Die vorliegende Arbeit konzentriert sich auf den Bereich des Berufseinstiegs als Übergang von der Ausbildung in den Beruf (Abb. 1).

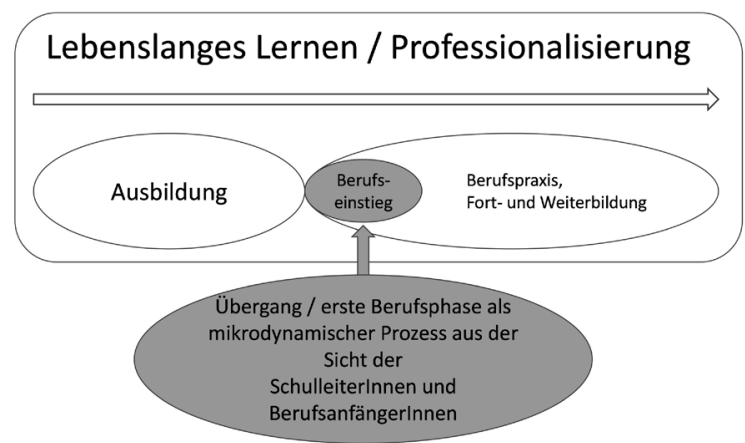

Abb. 1: Berufseinstieg als Übergang

2 Als Datengrundlage dienen Interviews mit SchulleiterInnen und BerufsanfängerInnen.

3 Im Kontext der Pädagogischen Hochschulen und ebenso in der vorliegenden Diskussion liegt der Unterschied zwischen Fort- und Weiterbildung in der Intention der sich fort- oder weiterbildenden Lehrperson. Während eine Fortbildung meist systemimmanent mit dem Ziel der Erweiterung der aktuellen berufsrelevanten Expertise stattfindet, werden Weiterbildungen als Voraussetzungen oder Chancen zum Karrieresprung absolviert, oft an anderen als der eigenen Institution. Der Besuch von ein- bis zweitägigen, in sich abgeschlossenen Seminaren fallen in den Bereich der Fortbildung (Fortbildungsseminare), die Hochschullehrgänge im Umfang von fünf bis 60 ECTS-AP (Pädagogische Hochschule Tirol 2019) sind als Weiterbildung konzipiert. 
In Abbildung 1 wird die Grundannahme der vorliegenden Untersuchung dargestellt. Der Prozess der Professionalisierung als Lehrperson ist ein lebenslanger. Er beginnt in der Ausbildung und setzt sich im Berufseinstieg bis in die spätere Berufspraxis fort (Helsper 2018; Hericks 2006; Hericks et al. 2018; Idel und Kahlau 2018; Kraler 2008b; Paseka et al. 2011; Schratz 2011; Stichweh 1992, 2017). Im Laufe des Berufslebens findet nach der Ausbildungsphase Professionalisierung zudem in der jeweils eigenen LehrerInnenpraxis und im Rahmen von Fortund Weiterbildungsveranstaltungen statt (Schmich und Itzlinger-Bruneforth 2019; Schrittesser 2011; Schumacher 2013). Insofern ist in der wissenschaftlichen Diskussion zur Professionalisierung vom lebenslangen Prozess die Rede (Helmke 2012; Hericks 2006; Hericks et al. 2018; Terhart 2011). Die Professionalisierung während der Ausbildung wird in der folgenden Diskussion ebenso fokussiert wie jene in der Phase des unmittelbaren Berufseinstiegs, der Induktionsphase ${ }^{4}$. Kraler (2008b) sieht in den Bereichen Unterricht, Beurteilung, Kollegium, professionsspezifisches Selbstverständnis und Arbeitsorganisation zentrale zu erfüllende Aufgaben in der Berufseingangsphase und geht infolge der „sukzessiven Weiterentwicklung der Ausbildung [und der] Änderung des Lehr- und Lernverständnisses“ (ebd., S. 6) von einem erweiterten Professionsverständnisses des LehrerInnenberufs aus. Der Thematik der Professionalisierung inkl. des Professionsverständnisses wird in der vorliegenden Arbeit unter besonderer Berücksichtigung der Kompetenzsteigerung im Laufe der LehrerInnenaus- und -weiterbildung bzw. der ersten Berufsjahre diskutiert. Professionalisierung durch Kompetenzsteigerung stellt hier das zentrale Konzept für einen gelingenden Berufseinstieg dar. Das Aufgreifen des Kompetenzbegriffs erweist sich „bei aller Definitionsproblematik insgesamt als fruchtbar“ (Kraler 2008a, S. 153). Insofern sie auch den empirischen Erkenntnissen geschuldet sind, werden Kompetenz bzw. Kompetenzerweiterung im Kontext der Professionalisierung eingehend diskutiert. Den sozialen Eingliederungsprozessen beim Berufseinstieg und der „inhaltlichen Überforderung “von BerufsanfängerInnen (Kraler 2008b, S. 7) werden u.a. beim Berufseinstieg im Rahmen der Induktionsphase Beachtung geschenkt. Die dafür installierten formellen Mentoring-Prozesse, welche auch bereits im Laufe der pädagogisch-praktischen Studien stattfinden, werden als Professionalisierung im Kontext der LehrerInnenpraxis in der vorliegenden Arbeit ebenfalls thematisiert.

Der empirische Zugang (Kap. III) liefert dahingehend eindeutige Ergebnisse, dass der Professionalisierungsprozess mit Kompetenzerweiterung einhergeht und dass dieser Prozess zugleich Verunsicherungen mit sich bringt. Die Präsenz der Phänomene Kontingenzerfahrung und Ungewissheit (Kosinár 2018; Oevermann 2017; Paseka et al. 2018; Scheeler 2008) ist insofern nachvollziehbar, als Kompetenzsteigerung als Veränderung im Denken und Handeln (Kraler und Menges 2007) mit der Konsequenz von auftretenden unsicheren Momenten aufgefasst werden kann. Im Unterricht und in der Ausbildung wissen Lernende und auch Lehrende eventuell auf Fragen keine unmittelbaren Antworten (Kraler 2012b), dies führt zum in der Literatur und ebenso in dieser Arbeit eingehend diskutierten Phänomen der Kontingenz bzw. Unverfügbarkeit mit Ungewissheit als Folge (Combe 2018; Connor et al. 2012; Helsper 2001; Kärtner 2015; Kosinár 2018; Rosa 2018; Schlömerkemper 2010). Der Umgang mit dem KontingenzPhänomen ist auch Teil der Aus- und Weiterbildung von Lehrpersonen und, wie sich in der Diskussion herauskristallisiert, eine Herausforderung für die gesamte LehrerInnenschaft. Kontingenzerfahrungen gehen nicht primär mit dem Versuch deren Lösung einher, sondern mit dem

4 Seit dem Wintersemester 2016 werden in Österreich alle LehrerInnen der Sekundarstufe gemeinsam an Pädagogischen Hochschulen bzw. Universitäten ausgebildet (Lehramt Bachelor- und Masterstudium) und sind bei Diensteintritt dazu verpflichtet, eine einjährige Induktionsphase zu durchlaufen, die durch eine Mentorin bzw. einen Mentor begleitet wird (Höller et al. 2019). 
Stellen neuer Fragen. „What constitutes teacher development? What factors influence teacher development?" (Evans 2002, S. 135).

Besondere Beachtung erfährt in der vorliegenden Arbeit auch die LehrerInnenprofessionalisierung nach dem Habituskonzept Bourdieus (2015a). Bourdieu versteht sein Konzept als Theorie der Praxis (ebd.). Insofern werden die Phänomene und Begriffe Interaktion, Integration, Resonanz, Inkorporierung, Subjekt, Objekt Feld etc. (Bourdieu 2015a; Rosa 2018) theoretisch vorgestellt und in die Praxis im Sinne der konkreten LehrerInnenprofessionalisierung übertragen und diskutiert (Kap. II.1.2.3). Subjekte werden zu BerufsanfängerInnen, Objekte zu SchulleiterInnen oder Schulaufsichtsbehörden und das Feld wird beispielsweise in Ausbildungs-, Praxisoder Fortbildungsfeld gesplittet. Die Habitualisierungstriade aus Wahrnehmen, Denken und Handeln zieht sich durch die gesamte Arbeit und trägt letztlich wesentlich zur Entwicklung des Konzepts in der Interpretation (Kap. III.4) bei. Die Ausbildung eines professionellen Habitus (Bourdieu 2015b; Lenger et al. 2013) erfolgt im Habituskonzept Bourdieus über Inkorporierungsprozesse, die in Feldern (Bourdieu 1996a) über lange Zeiträume ablaufen. Auch dahingehend wird in der vorliegenden Arbeit von Professionalisierung als lebenslangem Prozess im Sinne des lebenslangen Lernens ausgegangen (siehe auch Abb. 1). Der Habitus zeichnet sich gerade dadurch aus, dass er „nicht direkt fassbar ist, sondern sich als stilles und unterschwelliges Prinzip in den Hervorbringungen der Praxis - Spuren gleich - zeigt" (Kramer und Pallesen 2019, S. 14). Kontingenz und Habitualisierung mit ihren jeweiligen Konsequenzen Ungewissheit infolge von Kontingenz (Paseka et al. 2018) und Habitus als Ergebnis der Habitualisierung (Nairz-Wirth 2011) ziehen sich durch die gesamte vorliegende Arbeit und verschränken sich mit dem Thema der Professionalisierung im Kontext von LehrerInnenpraxis. Insofern startet Grundlagen (Kap. II) mit der Diskussion über Ungewissheit und Kontingenz (Gruschka 2018; Oevermann 2017; Paseka et al. 2018; Rosa 2018) und Habitus und LehrerInnenpersönlichkeit (Bourdieu 2015a; Helsper 2018; Lenger et al. 2013), um in der Diskussion zur Professionalisierung im Kontext von LehrerInnenpraxis (Kap. II.2) diesen Themen als Querschnittsthemen durch ständigen Bezug gerecht zu werden.

In Empirischer Zugang (Kap. III) erfolgt die Analyse der qualitativ, durch ExpertInnen- bzw. Leitfadeninterviews erhobenen Daten. Mittels multimethodischen Zugangs durch die Grounded Theory (Strauss und Corbin 1996), Clusteranalyse (Bortz und Döring 2006; Buber et al. 2000) und die strukturelle qualitative Inhaltsanalyse (Kuckartz 2016) wird die Konzeptualisierung eines gelingenden Berufseinstiegs vorgenommen, mithilfe von Bedingungsgefügen erfolgt die Interpretation. Das Konzept der Professionalisierung durch Kompetenzentwicklung wird als Clusterkategorie 3 abgebildet (Abb. 60, Kap. III.3.4) und stellt das empirische Ergebnis dieser Arbeit dar.

Im Anschluss an Zusammenführung und Integration (Kap. IV) der Erkenntnisse aus den Grundlagen und dem empirischen Zugang, wird die Arbeit mit Ansätzen zur gelingenden Professionalisierung als Ausblick (Kap. V) abgeschlossen. 


\section{Forschungsergebnisse in der Vorschau}

In der empirischen Datenanalyse kristallisieren sich sechs Themen ${ }^{5}$ heraus, die mit den diskutierten Phänomenen Kontingenz, Ungewissheit und Unverfügbarkeit sowie mit LehrerInnenpersönlichkeit \& Habitus verknüpft und in Beziehung gebracht werden. Bei den sechs empirisch gewonnenen Themen handelt es sich um Administration, Kooperation, Autorität in der Klasse, Reflexion \& Feedback, Systemwissen und Habitus. Habitus bildet sich in allen hier vorgestellten Themen ab und wird in der vorliegenden Arbeit als Metathema diskutiert.

Ohne an dieser Stelle auf die Forschungsfragen und deren Beantwortung im Detail einzugehen (siehe dazu Kap. IV.2), etabliert sich als Gesamtkonzept der Arbeit Professionalisierung durch Kompetenzentwicklung. Diese Professionalisierung erfolgt unter Berücksichtigung verschiedener Professionalisierungsmodelle in kleinen Schritten (Delta P; Kap. V.1) und erstreckt sich über die Ausbildung bis in die Fortbildung als Lehrperson mit entsprechenden Kompetenzansprüchen. Die Akzeptanz und der Umgang mit Kontingenz- bzw. Krisenerfahrungen bildet sich als wesentliche Kompetenz bzw. Gelingensbedingung im LehrerInnenberuf ab, die im Kontext der Habitualisierung als professionelle Lehrperson zu betrachten ist.

Als zentrale Determinante für eine fundierte Aus- und Fortbildung mit dem Anspruch auf eine ganzheitliche Professionalisierung wird die Vernetzung zwischen Institutionen (allgemein) und Personen (konkret) identifiziert. Die Zusammenarbeit zwischen den Ausbildungsinstitutionen (Universitäten, Pädagogische Hochschulen, Praxisschulen) und den Bildungsdirektionen bzw. dem Bundesministerium ist für einen guten Berufsstart mit optimistischer Berufsbiografie als Lehrperson ebenso relevant wie die Vernetzung einzelner AkteurInnen. Dozierende der jeweiligen Disziplinen (Fachausbildung, fachdidaktische und bildungswissenschaftliche Ausbildung) interagieren und kooperieren idealerweise miteinander, mit Praxislehrpersonen und den HauptakteurInnen: den Studierenden. So können Inhaltsdesidarate vermieden und beabsichtigte thematische Überschneidungen aus verschiedenen Perspektiven initiiert werden. Insofern lautet das Ergebnis der vorliegenden Studie zur LehrerInnenaus- und fortbildung: Professionalisierung durch Kompetenzgewinn kann durch Vernetzung und Kooperation auf ganzheitlicher personaler und institutioneller Ebene zum Erfolgsprogramm werden.

5 Thematisch signifikant abgebildete Gegenstände, die aus den Interviews (BerufsanfängerInnen und SchulleiterInnen) in Bezug auf die Forschungsfragen (Kap. III.1.1) generiert werden. 


\section{Grundlagen}

Im vorliegenden Kapitel wird die Professionalisierung als Lehrperson diskutiert. In der wissenschaftlichen Diskussion kristallisieren sich zwei Querschnittsthemen heraus, welche in Abschnitt II vorweg diskutiert werden, um in späteren Abschnitten im Sinne der Querschnittsthematik darauf verweisen zu können. Es handelt sich dabei um Habitus und Ungewissheit (Kap. II.1). In Ungewissheit als Teil der pädagogischen Profession (Kap. II.1.1) wird auf die Konstitution der Ungewissheit im pädagogischen Kontext (Kap. II.1.1.1) und den Umgang mit Ungewissheit und Krisenerfahrung (Kap. II.1.1.2) eingegangen. Die Erkenntnisse werden in der Zusammenfassung (Kap. II.1.1.3) rekapituliert. LehrerInnenpersönlichkeit und Habitus im Kontext der Professionalisierung (Kap. II.1.2) wird als zweites Querschnittsthema, welches in der gesamten Arbeit zum Ausdruck kommt, durch Habituskonzept nach Bourdieu als „Theorie der Praxis" (Kap. II.1.2.1) und Modelldarstellung (Kap. II.1.2.2) subsumiert. In Übertragung des Habituskonzepts auf LehrerInnenprofessionalisierung (Kap. II.1.2.3) werden die Erkenntnisse der Habitualisierung nach Bourdieu auf die professionelle Habitualisierung als Lehrperson transferiert.

In Professionalisierung im Kontext von LehrerInnenpraxis ${ }^{6}$ (Kap. II.2) liegt der Fokus auf den praktischen Teilen der LehrerInnenausbildung mit permanentem Bezug zu den oben genannten Themen Ungewissheit und Habitus. Nach der Gegenüberstellung von pädagogischer und allgemeiner Professionalisierung (Kap. II.2.1) erfolgt die Diskussion der konkreten Praxisphasen der pädagogisch-praktischen Studien (Kap. II.2.2) unmittelbar vor dem Berufseinstieg und der Professionalisierung beim Berufseinstieg: Induktionsphase und Mentoring (Kap. II.2.3).

In Zusammenfassung der Grundlagendiskussion (Kap. II.3) erfolgt ein Kurzüberblick zur geführten Diskussion des Abschnitts II als thematischer Extrakt.

6 In der vorliegenden Arbeit inkludiert der Begriff LehrerInnenpraxis sowohl Praktika während der Studienzeit im Rahmen der pädagogisch-praktischen Studien als auch die erste berufliche Praxis als bereits ausgebildete Lehrperson (Induktionsphase und zwei folgende Jahre). 


\section{Habitus und Ungewissheit}

Kontingenz, Unverfügbarkeit und Ungewissheit sowie Habitualisierung und Habitus sind Phänomene, die als Querschnittsthematik in der Professionalisierungsdiskussion eine Rolle spielen (Gruschka 2018; Schrittesser 2011). In Kapitel II.1 wird in diesen Diskurs Einblick gegeben. In Ungewissheit als Teil der pädagogischen Profession (Kap. II.1.1) wird zuerst die Konstitution der Ungewissheit im pädagogischen Kontext diskutiert (Kap. II.1.1.1), um anschließend auf den Zusammenhang von Ungewissheit und Krisen bzw. Krisenerfahrungen (Kap. II.1.1.2) einzugehen. Schließlich folgt eine Zusammenfassung (Kap. II.1.1.3) der bisherigen Erkenntnisse. In LehrerInnenpersönlichkeit und Habitus im Kontext der Professionalisierung (Kap. II.1.2) wird einleitend die theoretische Einordnung des Habitusbegriffs nach Bourdieu vorgenommen (Kap. II.1.2.1). Seine Erkenntnisse über Feld, Macht, Kapital, Subjektivismus, Objektivismus und Inkorporierung, die letztlich zur Habitualisierung überleitet, werden in der Modelldarstellung (Kap. II.1.2.2) diskutiert. In Übertragung des Habituskonzepts auf LehrerInnenprofessionalisierung (Kap. II.1.2.3) werden die Begriffe des Bourdieu'schen Habituskonzepts auf die Professionalisierung von Lehrpersonen umgelegt, um schließlich zur Kernidee der vorliegenden Rekonstruktion zu gelangen: Habitus als Konzept der Professionalität.

\subsection{Ungewissheit als Teil der pädagogischen Profession}

In der wissenschaftlichen Diskussion zur pädagogischen Professionalisierung wird das Phänomen der Ungewissheit ausführlich behandelt (Gruschka 2018; Helsper 2001, 2005; Oevermann 2017; Paseka et al. 2011; Paseka et al. 2018; Schrittesser 2011).

Im vorliegenden Kapitel wird das Phänomen Ungewissheit im pädagogischen Feld thematisch eingeordnet und eingegrenzt. Es erfolgt die Diskussion über Ungewissheit bzw. Umgang mit Ungewisheit als Krise. Ebenso werden der Umgang mit Kontingenz und die daraus resultierende Ungewissheit im Unterricht bzw. im schulischen Umfeld konkretisiert und zur Diskussion gestellt.

\subsubsection{Konstitution der Ungewissheit im pädagogischen Kontext}

Weite Übereinstimmung herrscht darüber, dass Ungewissheit im pädagogischen Feld in der „Nicht-Standardisierbarkeit des pädagogischen Handelns“ (Helsper 2001) begründet ist. Combe (2018) identifiziert Ungewissheit als „eine Erfahrung, ein Widerfahrnis“, weil die handelnden Personen insofern in einer Entscheidungskrise stecken, als sie eine Selektion von Handlungsoptionen angesichts des „immer anders Möglichen, Denkbaren und Interpretierbaren“ vornehmen müssen. Der Autor spricht die beiden Phänomene Ungewissheit als Krisen- bzw. als Kontingenzerfahrung an und identifiziert Ungewissheit als eine Bedingung, unter der die AkteurInnen kommunizieren, handeln und letztlich, trotz der im pädagogischen Feld allgegenwärtigen Kontingenz, entscheiden.

Connor et al. (2012) unterstreichen dieses Kommunikationspotenzial aufgrund von Ungewissheit: „[...] the word [contingency] descends etymologically from the prefix con-, which indicates the proximate positions of with or together" und sehen es als Chance, über Kontingenzerfahrungen Resonanzen zu erzeugen: „[...] a unique opportunity to reflect on our close contacts and resonant connections" (ebd.).

Kosinár (2018) lenkt den Blick bei der Erfassung des Begriffs der Ungewissheit auf die Person der Lehrerin bzw. des Lehrers, konkret auf deren Handlungsweisen. Die Autorin sieht Unge- 
wissheit im Unterricht bzw. im schulischen Feld u.a. darin begründet, dass LehrerInnenhandeln „kein technisches, standardisiertes und regelgeleitetes Handeln ist“ (ebd., S. 258). Dafür sind die Handlungen der Lehrpersonen interaktiv, da Arbeitsbündnisse sowohl mit einzelnen Schülerinnen bzw. Schülern als auch einer ganzen Gruppe geschnürt werden. Die Komplexität im Sinne der Ungewissheit erhöht sich, da sich verschiedene Gruppenkonstellationen (Klassengröße, Heterogenität etc.) in ihrem Verhalten unterscheiden (Kontingenz) und die angesprochenen Arbeitsbündnisse im Gegensatz zu anderen Professionen (Oevermann 2017) keine freiwilligen sind. Ungewissheit entsteht laut Kosinár (2018) auch, weil die Lehrperson Bildungsprozesse aufgrund des individuellen Lernens der SchülerInnen „nur kommunikativ anregen, aber nicht direkt steuern kann“. Nachdem das Resultat des genannten Bildungsprozesses auch von der „Motivation und den Verstehensprozessen“ (ebd.) der SchülerInnen abhängt, ist es unsicher, was und wie viel vom Unterricht aufgenommen und später relevant sein wird.

Der Begriff der Kontingenz spielt in der Diskussion über die Ungewissheit eine entscheidende Rolle, da durch die schon beschriebene Nicht-Standardisierbarkeit von Handlungen und Reaktionen im schulischen Feld diese gemäß der Begriffsdefinition möglich und gleichzeitig nicht notwendig (Duden 2017) sind. Im Unterricht, wo ständig mit dem direkten Gegenüber (einzelne SchülerInnen oder eine Gruppe) interagiert wird, spielt „die Häufigkeit des gemeinsamen Auftretens zweier Sachverhalte“ (ebd.) eine bedeutsame Rolle hinsichtlich der Quantität an Entscheidungsoptionen der Lehrperson und/oder der SchülerInnen.

Dieser Ansatz führt unmittelbar zum Fachterminus der doppelten Kontingenz, welcher in der soziologischen Systemtheorie von Talcott Parsons eingeführt (Kärtner 2015) und von Luhmann (2018) übernommen und abgeändert wurde. Die doppelte Kontingenz ist in ihrer reinen Form als eine soziale Situation, ,in der [sich] mindestens zwei Teilnehmende gegenseitig wahrnehmen und in der noch völlig offen unbestimmt ist, was als Nächstes geschehen soll“ (Luhmann 2018, S. 152), zu verstehen. In der Ausschließung von Notwendigkeit und Unmöglichkeit besteht die Kontingenz. Aufgrund der Tatsache, dass auch nach dem Muster des didaktischen Dreiecks (Bönsch 2006) in schulischen Situationen häufig mindestens zwei Person interagieren, wird daraus die doppelte Kontingenz. Der Grad der Ungewissheit im pädagogischen Feld steigt und wird zugleich nachvollziehbar. Aus systemtheoretischer Perspektive ist Kontingenz ein in allen Systemen auftretendes konstitutives Element (Luhmann 2018), aus bildungstheoretischer Perspektive ergibt sich die Kontingenz bzw. die doppelte Kontingenz aus der „Fallorientierung“ (Combe et al. 2018). Damit ist die Zuwendung der Lehrperson zu den Lernenden bzw. die Interaktion mit den jeweils individuell am Lern- und Bildungsprozess teilnehmenden Schülerinnen und Schülern angesprochen (ebd.).

Für Combe et al. (2018) wird Kontingenz in verschiedenen Erscheinungsformen in Bezug auf Ungewisheitsmomente im unterrichtlichen Handlungszusammenhang sichtbar (Abb. 2).

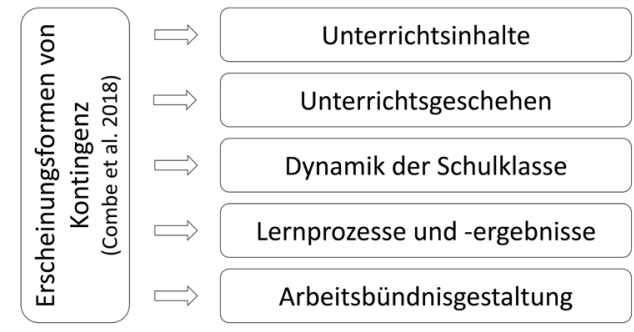

Abb. 2: Erscheinungsformen von Kontingenz; eigene Abbildung nach Combe et al. (2018, S. 54) 
Die AutorInnen identifizieren Kontingenz bzw. Unsicherheitsmomente im Unterricht u.a. abhängig von den Unterrichtsinhalten. Die curricularen Vorgaben spielen in diesem Zusammenhang eine ebenso große Rolle wie deren Umsetzung durch die Lehrperson. Bereits in der Planungsphase der zu vermittelnden Inhalte orientiert sich die Lehrperson in eine offene, ungewisse Zukunft.

Die Ungewissheit im Unterrichtsgeschehen entsteht durch die „eigensinnigen und unterschiedlich motivierten Zugänge der Lernenden“ (ebd., S. 54) zu Unterrichtsinhalten. Die wechselseitigen Beziehungen der einzelnen Individuen bewirken eine offene Dynamik der Schulklasse mit unsicherer innerer Strukturiertheit. Hinsichtlich der Lernprozesse und -ergebnisse gibt es keine Garantie, dass Lernen zum Erfolg führt. Die individuelle Passung der schulischen Anforderungen an die Lernbereitschaft der SchülerInnen fordert von den Lehrpersonen eine hohe Adaptionsfähigkeit und die Bereitschaft, „sich auf Kontingenz als Herausforderung einzulassen“" (ebd.). Schließlich müssen sich Lehrpersonen, insbesondere BerufsanfängerInnen, in die unterschiedlichen Möglichkeiten der Arbeitsbündnisgestaltung erst hineinfinden, zeigen sich in den Schulklassen doch spannungsreiche Überlagerungen von „Unterrichts-, Peer- und Familienkulturen“ (ebd., S. 55). Die Unsicherheitsvariable der Unterrichts-, Peer- und Familienkultur hängt auch von der jeweiligen standortbezogenen Schulkultur ab.

Einen ähnlichen Befund über die Präsenz von Ungewissheit im Unterricht liefert Keller-Schneider (2018) und betont zusätzlich die Notwendigkeit der SchülerInnenzentrierung im Unterricht. Konkret spricht die Autorin davon, dass die Durchführung von Unterricht kein Vollzug von Unterrichtsplänen, sondern die „Wahrnehmung von situativen Handlungen“ bedeutet und sie beleuchtet in ihrer Studie die „Inkongruenz von Planung und Durchführung [von Unterricht]“. Damit wird aufgezeigt, dass neben der unklaren Wirkung und der nicht vorhersehbaren Bedeutung von Erkenntnissen auch die begrenzte Planbarkeit von Unterricht Ungewissheit zur Folge hat. Keller-Schneider (2018) legt in Bezug auf die Planbarkeit des Unterrichts drei Facetten der Ungewissheit dar. So ist innerhalb der Bildungslandschaft erstens kein geteiltes Verständnis von Planungskompetenz erkennbar, auch wenn Studien belegen, dass der Erwerb von Planungskompetenz im Rahmen der Ausbildung bis zu einem gewissen Ausmaß erreicht werden kann und sich auch erste Routinen einstellen (Keller-Schneider 2018).

Im Rahmen der LehrerInnenbildung NEU wird den Studierenden zur Erlangung von Planungskompetenz viel Raum geboten. Einerseits in den universitären Seminaren, insbesondere vorbereitend auf die Praxisphasen, andererseits in den pädagogisch-praktischen Studien, also zur unmittelbaren Anwendung (Kompetenz) des Gelernten, Erforschten etc. (Leopold-Franzens-Universität Innsbruck 2016). Der Hinweis von Keller-Schneider kann dahingehend interpretiert werden, dass einheitliche Vorgaben seitens der Ausbildungsinstitutionen in Bezug auf Planungskompetenz der Studierenden die Ungewissheit der Planbarkeit reduzieren und umso mehr zur Sicherheit der Studierenden beitragen.

Zweitens ist für Keller-Schneider (2018) ungeklärt und insofern ungewiss, „was sich [bei der Entwicklung der Planungskompetenz] genau tut " und wie sich diese Kompetenz im unterrichtlichen Handeln zeigt. Außerdem bleibt die Frage offen, ob die erstellte Planung als Grundlage und nicht als Leitlinie benutzt und bei Bedarf in professioneller Weise davon abgewichen wird. Schließlich zeigt die Autorin drittens die Ungewissheit in der Planbarkeit und Durchführung von Unterrichtssituationen aufgrund der Interaktionen von AkteurInnen in ihren je unterschiedlichen Rollen auf. In diesen Situationen wird die Dringlichkeit einer „Adaption des Handelns der Lehrperson an die Resonanz des Unterrichts bei den Lernenden ersichtlich“. Als Schlussfolgerung bzw. Lösungsansatz nennt Keller-Schneider einmal mehr, Ungewissheit als Herausforderung anzunehmen und mithilfe einer adaptiven Lehrkompetenz eine hohe Unter- 
richtsqualität zu erreichen. Der Ansatz, Ungewissheit als Herausforderung zu sehen, wird im Kapitel Umgang mit Ungewissheit und Krisenerfahrung (Kap. II.1.1.2) näher beleuchtet.

Die Thematik der Vermittlung eines offenen, ganzheitlichen und möglichst wenig subjektiven Zugangs zu schulischen bzw. interaktiven Herausforderungen in der LehrerInnenausbildung spricht Scheeler (2008) an: „Generalization training continues to be a missing link between preservice teacher preparation and inservice application of skills." Hierbei liegt eine große Verantwortung bei den Dozierenden: „Teacher educators [because they have to] generalize effective teaching." (ebd.) Sie tragen letztlich neben dem erfolgreichen Berufseinstieg auch zum besseren Lernen der SchülerInnen bei.

Gruschka (2018) artikuliert eine Reihe von Fragen aus der Sicht von BerufsanfängerInnen und der LehrerInnenschaft insgesamt. Die formulierten Fragen drücken Ungewissheit aus und betreffen am Ende, wie von Scheeler (2008) bereits angesprochen, auch oder gerade die SchülerInnen (Abb. 3):

\begin{tabular}{l|c|}
$\begin{array}{c}\text { Welche Umwege, Sackgassen } \\
\text { und Fehlkonzepte tun sich auf? }\end{array}$ & $\begin{array}{c}\text { Fällt mir zum richtigen Zeitpunkt } \\
\text { der richtige Impuls ein? }\end{array}$ \\
\hline $\begin{array}{c}\text { Wer bin ich als } \\
\text { Erzieher und Lehrer? }\end{array}$ & $\begin{array}{c}\text { Was geschieht in den } \\
\text { Köpfen der Schülerlnnen? }\end{array}$ \\
\hline $\begin{array}{l}\text { Werden die SchülerInnen } \\
\text { die Aufgaben bearbeiten? }\end{array}$ & Wer kommt wann ans \\
\hline $\begin{array}{l}\text { Werde ich die SchülerInnen vom } \\
\text { Lohn des Lernens überzeugen? }\end{array}$ & $\begin{array}{c}\text { Werde ich mein Programm } \\
\text { sinnvoll bearbeiten können? }\end{array}$
\end{tabular}

Abb. 3: Fragen zur Ungewissheit; eigene Abbildung nach Gruschka (2018, S. 18)

Bereits mit dem Titel seines Kapitels „Offenheit und Ungewissheit“ gibt Gruschka (2018, S. 18) seine Antwort auf die Frage der Ungewissheit und geht davon aus, dass PädagogInnen zum „Grundtatbestand der Ungewissheit der Praxis“ (ebd.) mit der Zeit ein breites Erfahrungswissen entwickeln und trotzdem im Verlaufe des Unterrichts jeweils nur wenige Möglichkeiten haben, auf die Fragen (Abb. 3) konkrete Antworten zu finden. Gruschka geht davon aus, dass der Großteil der Lehrpersonen in ihrer Praxis die belastende und befreiende Ungewissheit ambivalent erleben. Belastend wird es dann, wenn es um den Bildungsertrag der SchülerInnen geht. Befreiend kann Ungewissheit sein, wenn diese als „produktive[r] Eigensinn der pädagogischen Kommunikation“ (ebd., S. 19) wahrgenommen wird.

Kosinár (2018) nimmt sich in ihrer deutschen Studie „Konstruktionen von Professionalität und Ungewissheitserfahrungen im Referendariat“ der Frage an, welchen Formen der Ungewissheit BerufsanfängerInnen in der zweiten Phase der Berufsausbildung zur Lehrperson in Deutschland ${ }^{7}$ begegnen und wie sie Ungewissheitserfahrungen vor dem Hintergrund ihres Professionsverständnisses deuten. Die aufgetretenen Ungewissheiten zeigen sich vor allem durch die nicht

7 Das Referendariat ist in der zeitlichen Positionierung mit der neuen Induktionsphase in Österreich vergleichbar, wenngleich das Referendariat als zweite Ausbildungsphase gilt und die Induktionsphase der Ausbildung zeitlich nachgestellt ist. Aufgrund der Tatsache, dass die Induktionsphase eine durch den Mentoring-Prozess betreute Berufseinstiegsphase darstellt, gehe ich in der vorliegenden Arbeit von parallelen, durchaus vergleichbaren Berufseinstiegserfahrungen von Referendarinnen bzw. Referendaren in Deutschland und Vertragslehrpersonen in der Induktionsphase in Österreich aus. 
kontrollierbare Interaktionsdynamik und die damit verbundene Notwendigkeit einer „Arbeitsbündnisgestaltung “ (ebd.). Die Autorin weist in ihrem Beitrag explizit darauf hin, dass die vielfach angestrebte „Schließung von Ungewissheit“ (ebd., S. 272) im Sinne einer Auflösung bzw. Behebung oft nicht gelingt. Diese Phase des Berufseinstiegs wird häufig als „berufsbiografisch hoch brisante Phase“ erlebt und ist „nicht selten von Krisen bewegt“ (ebd., S. 256). Auf das Thema der Krise durch Ungewissheit gehe ich im folgenden Kapitel näher ein.

\subsubsection{Umgang mit Ungewissheit und Krisenerfahrung}

In der bildungswissenschaftlichen Diskussion haben Kontingenz und Ungewissheit als zentrale Merkmale des LehrerInnenberufs Anerkennung gefunden (Kosinár 2018). Rosa (2018) bereichert den Diskurs mit dem Phänomen der Unverfügbarkeit (siehe auch Exkurs Kap. II.1.2.2.3). Kosinár (2018) stellt fest, dass gerade Ungewissheitserfahrungen bei angehenden Lehrpersonen zu Krisen führen können. Ausschlaggebend dafür sind Differenzerlebnisse zwischen den bereits thematisierten Unterrichtsplanungen, den beruflichen Selbstbildern und Vorstellungen der eigenen Wirksamkeit einerseits und der realen, im Unterricht vorgefundenen Situation andererseits. Ein Ansatz zum Umgang mit Ungewissheit und Krisenerfahrungen im Unterrichtsalltag stellt die Institutionalisierung der Berufseinstiegsphase ${ }^{8}$ dar (Dietrich 2018). Hier werden BerufsanfängerInnen von erfahrenen Lehrpersonen bzw. MentorInnen begleitet und diese Phase kann als Professionalisierungsmodell, welches über die Aneignung kanonisierten Wissens hinausgeht, gesehen werden. Im Zentrum dieser Professionalisierungsphase steht der kreative Prozess der Krisenbearbeitung.

Die durch die handlungspraktische Auseinandersetzung mit Ungewissheit hervorgerufene spezifische Krisenhaftigkeit ist für die Professionalisierung angehender Lehrpersonen „von konstitutiver Bedeutung“ (ebd., S. 277) und lässt sich insofern auch als Prozess der Krisenbewältigung verstehen, als dass berufsbezogene „Überzeugungen und Glaubenssätze“ (ebd.) der BerufsanfängerInnen nicht unangetastet bleiben. Allfällige Spannungsfelder zwischen den Ausbildungsinstitutionen (Universität bzw. Pädagogische Hochschule und deren Lehrende) und den am Berufseinstieg für BerufsanfängerInnen beteiligten Institutionen (Schule, Lehrerkollegium, MentorInnen etc.) hinsichtlich der durchaus belastenden Krisensituationen für die Beteiligten scheinen vorprogrammiert. Jedoch gilt die Krisenhaftigkeit laut Dietrich (2018) ,als strukturell unhintergehbar, also auch nicht organisational etwa durch bessere Ausbildungsbedingungen vermeidbar".

Das Erleben einer Krise bezieht sich folglich auf den handlungspraktischen Teil des Berufseinstiegs und wird durch eine „unbekannte, eine neue oder neu zu entdeckende Begebenheit“ (ebd., S. 283), die sich einer routinierten Deutung und Bearbeitung entzieht, ausgelöst. Tradierte oder ausprobierte Reaktionsweisen der BerufsanfängerInnen scheitern. Hier liegt der Bezugspunkt zum handlungspraktischen Teil des Berufseinstiegs im Gegensatz zur theoretischen Ausbildung. Auf das Unbekannte bzw. Ungewisse muss die neue Lehrperson („Subjekt“ in Dietrich 2018) reagieren. Dies kann letztlich eine krisenhafte Erfahrung darstellen. Scheeler (2008, S. 146) hebt in diesem Zusammenhang folgende Unstimmigkeit hervor: „[...] skills and techniques that teachers learn and practice in college classrooms are not always maintained over time." Ebenso vermisst die Autorin den Erkenntnistransfer der Studierenden aus den Praktika bzw. Übungsklassen in die eigene Unterrichtstätigkeit: „[There is not necessarily a] transfer to actual classrooms with children." (ebd.)

8 Referendariat bzw. Induktionsphase mit formellem Mentoring-Prozess 
Als Variante für einen offensiven Umgang mit Kontingenz kann der Vorschlag von Combe et al. (2018) angesehen werden, wonach die BerufsanfängerInnen nicht versuchen, die Krise zu umgehen, sondern sich als „Kriseninduzierer“ sehen. Dabei konfrontiert die Lehrperson die SchülerInnen mit Inkonsistenzen ihrer Konzepte und Denkweisen und gibt ihnen damit die Möglichkeit, „am Widerstand der Sache“ (ebd.) neue Perspektiven zu gewinnen. Die Chance in diesem durchaus „wagnisreichen Vorgehen [liegt darin, dass die] Irritation durch das Fremde“ (ebd.) - in diesem Fall die Lehrperson - oftmals krisenhaft-offene Bewegungen in Gang setzt. Empirisch zeigt sich diese Entwicklung dadurch, dass sich Lehrpersonen und SchülerInnen „experimentell-deutend und sprachsuchend der noch unbegriffenen Vieldeutigkeit einer Sache nähern“" (ebd.).

Die hier beschriebene Vorgehensweise ist Teil der paradoxen Techniken (Molnar et al. 2013) und kann eingesetzt werden, um Verhaltensweisen und Ereignisse zu verändern, die scheinbar ohne Bezug zum Problem sind. Auf diese Weise beeinflussen paradoxe Techniken bzw. paradoxe Interventionen, im vorliegenden Fall das absichtliche Herbeiführen einer Krise bzw. einer Krisensituation, die problematische Situation aufgrund des progressiv initiierten Perspektivenwechsels oftmals positiv.

Für Dietrich (2018) steht außer Zweifel, dass Krisenbewältigung keineswegs von selbst und aus dem Nichts erfolgt. Sie basiert auf der Erfahrung anderer, vorheriger Krisenbewältigungen inklusive derer Routinen. Letztlich greift die in die Krise geratene Lebenspraxis „auf die Krisenbearbeitung strukturierende Struktur" zurück.

Trotz passender Unterrichtssettings und dem Wissen über Krisenbewältigung mit positiven Ergebnissen bzw. Auswirkungen kann es vorkommen, dass Irritationen und Krisen nicht angenommen werden (Keller-Schneider 2018). Diesbezüglich können die individuellen Überzeugungen und Vorannahmen der Studierenden Grenzen in der Bereitschaft zur Krisenannahme setzen. Insofern gilt es auch für die Lehrenden im Lehramtsstudium, als Vorbilder zu wirken und „Ungewissheit auszuhalten [bzw.] Kontingenz als Herausforderung anzunehmen“ (ebd. S. 251), zumal Kontingenz ohnehin per se präsent ist.

Keller-Schneider (2018) erörtert eine weitere Dimension der Ungewissheit im LehrerInnenberuf und konstatiert, dass für den Umgang mit Ungewissheit die Positionierung zwischen dem Phänomen der Ungewissheit und den BerufsanfängerInnen eine Determinante darstellt. Für die Autorin sind BerufsanfängerInnen bzw. in den LehrerInnenberuf einsteigende Personen fortgeschrittene AnfängerInnen, die vorerst über grundlegende Fähigkeiten verfügen, um sich in der Praxis weiteres Wissen und Können anzueignen. Der Status der fortgeschrittenen Anfängerin bzw. des fortgeschrittenen Anfängers folgt zum Teil daraus, dass die BerufsanfängerInnen aus den bisherigen Praxisphasen ${ }^{9}$ der LehrerInnenausbildung erste Unterrichtserfahrungen mitbringen. Zugleich wird die Diskrepanz betont, die „aus den Erfahrungen während der Ausbildung bekannten Anforderungen und den im Berufseinstieg [tatsächlich] erlebten“ (ebd., S. 246) entsteht. Beim unmittelbaren Berufseinstieg erhöht sich u.a. aufgrund der plötzlichen Verantwortungsübernahme der Komplexitätsgrad für die BerufsanfängerInnen und in Verbindung mit den zu meisternden Anforderungen werden bei den angehenden Lehrpersonen Irritationen ausgelöst. Die Erwartungshaltung, diese Aufgaben entsprechend „zu meistern“ (ebd.), erhöht den Irritationsgrad noch weiter. Neben der Fähigkeit der Wahrnehmung dieser komplexen Anforderungen ist es gerade auch das „Anerkennen von Ungewissheit“ (ebd., S. 233), welches die einsteigende Lehrperson als Kompetenz mitbringt. Die Bereitschaft, sich mit Ungewissheit aus-

9 Pädagogisch-praktische Studien 
einanderzusetzen und dadurch weiter zu professionalisieren, ist ebenfalls Teil der grundlegenden Fähigkeiten von Berufsanfängerinnen und Berufsanfängern. Diese Anforderungen sind im Vorfeld des Berufseinstiegs ,in der gesamten Dynamik strukturbedingt nur begrenzt erfahrbar“ (ebd.). Insofern sind nicht nur konkrete Prozesse und Handlungen beim späteren Berufseinstieg im Unterricht Auslöser von Ungewissheit, sondern der Übergang als solcher stellt eine zusätzliche Unsicherheit dar. Der Wechsel von Studierenden zu BerufsanfängerInnen ist kein reibungsloser. Weiterlernen ist erforderlich und die Herausforderung anzunehmen, sich durch Erfahrungen weiter zu professionalisieren, ist Voraussetzung.

Eine Aufgabe der LehrerInnenausbildung liegt in der Vorbereitung der Studierenden auf die Ungewissheit im späteren Berufseinstieg, auf den Umgang mit dieser und mit den dadurch auftretenden Irritationen. Die Intention kann sein, den angehenden Lehrpersonen durch eine „vertiefte Auseinandersetzung mit dem Lerngegenstand und der Ungewissheit (ebd., S. 247) die Wirkungserfahrung des zukünftigen Handelns als Lehrperson zu ermöglichen. Dietrich (2018) zeigt die in der wissenschaftlichen Diskussion thematisierten Zugänge zu Ungewissheit bzw. Kontingenz auf, strukturtheoretisch argumentiert als Ungewissheit, kompetenztheoretisch diskutiert als doppelte Kontingenz. Während ,in Bezug auf den Gedanken“ (ebd., S. 279) Einigkeit über die Faktizität von Ungewissheit herrscht, verläuft die Diskussion über den Umgang mit Ungewissheit teilweise konträr. So ist von „nicht technologisierbar[em Umgang]“, von „ingenieurale[r] Handlungslogik“ und ebenfalls von „technologische[m Handlungs]Repertoire" (ebd.) die Rede.

Kosinár (2018) unterscheidet drei Formen des Umgangs mit Ungewisheit, wobei sich diese gegenseitig nicht ausschließen (Abb. 4).

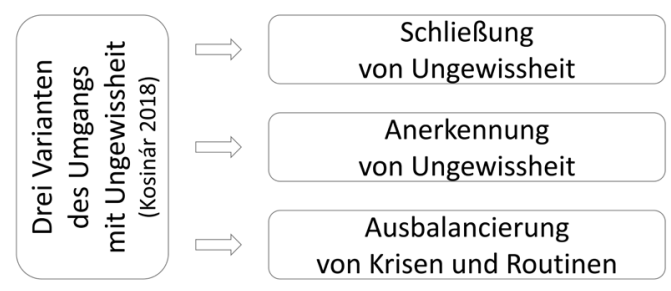

Abb. 4: Umgang mit Ungewissheit; eigene Abbildung nach Kosinár (2018)

Die Möglichkeit, Kontingenz bzw. Ungewissheit aufzulösen ${ }^{10}$ und damit angehenden Lehrpersonen Gewiss- und Planbarkeit anzubieten, entspricht dem Paradigma des kompetenztheoretischen Ansatzes (Kosinár 2018). Von erfahrenen Lehrpersonen kann nach diesem Muster ein breites Erfahrungswissen im Unterricht abgerufen werden. Dies betrifft sowohl Wissensbestände als auch Planungshandlungen. Kosinár diskutiert die „Definition lehrerberuflicher Standards [und die] Darstellung von Kompetenzmodellen“ (ebd., S. 259) als Gelingensbedingungen für den Berufseinstieg und als Voraussetzungen für erfolgreiches LehrerInnenhandeln generell. 
Die theoretischen Ansätze der Anerkennung und Ausbalancierung von Ungewissheit setzen stärker beim professionellen Selbstverständnis der Lehrpersonen an, wobei in der Anerkennung die Routine, mit Unsicherheitspotenzial umzugehen, in den Vordergrund rückt. Erfahrungswissen wird mit „wissenschaftlichem Erklärungswissen“ (ebd.) in Beziehung gesetzt und dadurch kann mit vorhandener Handlungsroutine die Reflexion unterrichtlicher Geschehnisse aus der Retrospektive erfolgen. Die Lehrperson kommt auf diese Weise in die Beobachtung zweiter Ordnung, indem nicht isolierte Einzelhandlungen, sondern Zusammenhänge zwischen Handlungen bzw. Beziehungen zwischen Handlungen und Personen beobachtet und interpretiert werden. Es kommt zu einer „Strukturdeutung des Unterrichts“ (ebd.), Personen und Sozialsystem werden getrennt wahrgenommen und in Beziehung gesetzt. Die Beobachtung zweiter Ordnung mit der Deutung und Interpretation von Handlungen erfordert schließlich das situative Ausbalancieren von Ungewissheit. Es „bedingt ein spontanes [und] flexibles Handeln“ (ebd.). Charakteristisch dafür ist eine Pendelbewegung zwischen Krisen und Routinen. „Situierte Kreativität und schöpferische Intuition“ (Combe und Paseka 2012) sind zentrale Bestandteile der pädagogischen Handlung. Professionstheoretisch liegen in der Pendelbewegung des Ausbalancierens Gemeinsamkeiten des struktur- und kompetenztheoretischen Ansatzes (Kosinár 2018).

Combe et al. (2018) stellen in Anspielung an den kompetenztheoretischen Professionalisierungsansatz fest, dass es einer Auseinandersetzung mit Unterricht bedarf, die „über Rezeptologien“ (ebd., S. 72) hinausgeht. In Bezug auf die Variante des Ausbalancierens von Ungewissheit (Abb. 4) schreiben sie diesem Ansatz zwar eine Bedeutung zu, im Sinne des Bildungsanspruchs von Schule ist dieser Zugang jedoch nicht ausreichend. Ungewissheiten sollten aktiv provoziert und die damit einhergehenden Krisen aufgegriffen werden. Aus dieser Perspektive sind Gewissheitsverluste keine Defizite von Unterricht, sondern „initiale Momente von Erfahrungs- und Lernprozessen“ (ebd.). Die daraus gewonnenen Erkenntnisse schlagen sich nachhaltig im jeweils individuellen Bildungsgang nieder. Schlömerkemper (2010, S. 9) bemerkt, dass zur pädagogischen Reflexion neben dem Blick aufZielsetzungen und normative Vorgaben „trotz allem immer verbleibende Ungewissheit pädagogischen Handelns" hinzukomme (ebd. S. 9). Diese Aussage ist insofern interessant und merkwürdig, als der Autor selbst All-Aussagen und ob „etwas immer so ist" grundsätzlich infrage stellt (ebd. S. 29). Im Falle der Ungewissheit scheint sich die Frage nach deren Existenz also tatsächlich nicht zu stellen und der proaktive Umgang auch oder gerade im Sinne der bereits diskutierten Kriseninitiierung (Combe 2018) wird insofern bestätigt.

Als Voraussetzung dafür, Diskrepanzerfahrungen zuzulassen und folglich sogar zu initiieren, ist eine Ungewissheit anerkennende Grundhaltung der Lehrperson von Bedeutung. Die doppelte Kontingenz von Unterricht und der Umgang mit Ungewissheit müssen in diesem Sinne als „konstitutives Element von Professionalität“ (Keller-Schneider 2018) anerkannt werden. Combe et al. (2018) nehmen allerdings in Bezug auf die Kontingenzbelastung im Lehrberuf die Vorstellung einzelner AkteurInnen wahr, dass Kontingenz und Unsicherheit kontrolliert und aufgehoben ${ }^{11}$ werden müssten. Das Gegenteil soll aber in den Blick genommen werden. Ganz im Sinne der schon diskutierten Kriseninduktion (ebd., S. 69) sollen Kontingenzentfaltung und Kontingenzbejahung als Chance gesehen werden. Als Chance für die Nutzung von „Möglichkeitsräumen des Denkens“ (ebd., S. 61) und als Chance für die produktive „Öffnung zur Vieldeutigkeit einer Sache“ (ebd.).

Connor et al. (2012) konnotieren das Phänomen der Kontingenz positiv: „I would like to approach contingency through its more attractive sister term, creativity, and the idea of possibility." 
Die AutorInnen verweisen damit ebenfalls auf die Chance und den positiv herausfordernden Charakter des Kontinuums Kontingenz. Sie gehen noch einen Schritt weiter und beschreiben ästhetische Züge von Kontingenz: „[...] contingency has been viewed as markedly aesthetic.“ (ebd., S. 348) Andererseits beschreiben Connor et al. (2012) ganz pragmatisch auch das Gegenteil: „[...] creative thinking [im Sinne von Suchen nach Lösungen aufgrund von Kontingenz] is less often bestowed by the muse than it is brought forth by frustration and the need to find alternative solutions.“ (ebd., S. 345) Die Strategie lautet: „[...] most creativity comes about by hitting one wall after another, and breaking through them." (ebd.)

Gruschka (2018) spricht zwar nicht von einer aktiven Herbeiführung von Ungewissheitsmomenten im Sinne einer Kriseninitiierung, sie sollte als zentraler Kern von Bildungsprozessen jedoch anerkannt und keinesfalls abgebaut werden. Eine „Entdidaktisierung“ (ebd., S. 15) kann, so der Autor, gehaltvolle Bildungsprozesse fördern. Er spricht damit die bereits diskutierte „schöpferische Intuition“ (Combe und Paseka 2012) an. Aus der Perspektive der Berufsbiografie führt die Einbeziehung eines „kontingenzgewärtigen Verständnisses von Unterricht“ (Combe 2018) zu neuen Aktzentsetzungen in der Konzeptualisierung berufsbiografischer Entwicklung von Berufsanfängerinnen und Berufsanfängern.

Die „lebendige produktive Kommunikation mit Schülern und Kindern“ nennt Gruschka (2018) als die Voraussetzung, um eine positive Einstellung zur Ungewissheit aufbauen zu können. Damit kann „auf einen positiven Ausgang“ (ebd.) der Situationen in Ungewissheit vertraut werden, auch wenn die Auseinandersetzung einen anderen als den antizipierten Verlauf nimmt. Das ungewisse Überraschungsmoment verunsichert nicht, sondern belebt und das pädagogische Interesse an den Ergebnissen steigt im Sinne der Ungewissheit. Wesentlich in diesem Zusammenhang ist die Wirkung der Fragen bzw. die Art der Fragenstellung. Der Satz „Fragen heißt ins Offene zu stellen“ (Gadamer 1990) drückt zum einen die Ungewissheit, andererseits auch die Chance für Erkenntnisgewinnung aus. Die Kunst des Fragens und des Weiterfragens gewährleistet das Vorwärtsgehen der Entwicklung der Sache im Gespräch (ebd.).

Diese Fragen sind es, die „weiterführende interessante Impulse“ (Gruschka 2018) bringen und die Themen bereichern. Dass es für manches nicht die richtigen Antworten, sondern „problematisierende Rückfragen“ (ebd.) gibt, eröffnet das Prinzip des Weiterfragens (Gadamer 1990) und stiftet Neugier, Spannung und Erkenntnisinteresse. Darin liegt die Chance in der Ungewissheit (Gruschka 2018). Diese Chancen und die bereits diskutierten Krisen verweisen in empirisch untersuchten Verläufen darauf, dass die Konfrontation mit Ungewissheit bzw. mit begrenzter Verfügbarkeit der schulischen Handlungssituationen „grundlegende Überzeugungen und Glaubenssätze der angehenden Lehrpersonen“ (Dietrich 2018) berührt. „Dies“, so Dietrich, „spricht dafür, die sich vollziehende Professionalisierung als Habitualisierung zu fassen.” (ebd., S. 294) Der Autor nimmt insofern Bezug auf das Kapitel LehrerInnenpersönlichkeit und Habitus im Kontext der Professionalisierung (Kap. II.1.2) und weist bereits auf die Relevanz des Zusammenhangs von Professionalisierung und Habitualisierung hin. Die Erkenntnisse in Kapitel II (Empirischer Zugang) bestätigen diesen Ansatz.

\subsubsection{Zusammenfassung}

In Ungewissheit als Teil der pädagogischen Profession (Kap. II.1.1) wird der Zusammenhang zwischen Kontingenz, Unverfügbarkeit und Ungewissheit diskutiert. Combe (2018) und Kärtner (2015) stellen Erscheinungsformen von Kontingenz und doppelter Kontingenz dar, während Rosa (2018) den Begriff der Unverfügbarkeit einführt. Es wird betont, dass letztlich Ungewissheit (Gruschka 2018; Helsper 2005; Paseka et al. 2018) im Allgemeinen und Nicht- 
Standardisierbarkeit von pädagogischen Handlungen (Helsper 2005) im Speziellen permanent vorliegen und dass daraus resultierende Krisen unvermeidbar sind (Dietrich 2018). Der Umgang mit Ungewissheit und Krisen reicht von Anerkennung über Ausbalancierung bis zum Versuch der Auflösung bzw. Schließung (Kosinár 2018). Die progressive Form der absichtlichen Krisenherbeiführung (Kriseninduzierung) vertritt Combe (2018). Beobachtungen auf der Metaebene bzw. der Ebene zweiter Ordnung (Kosinár 2018) erlauben den Lehrpersonen den nötigen Abstand, in erster Linie Fragen zu stellen, anstatt nach Antworten zu suchen (Gruschka 2018). Neugier, Erkenntnisinteresse und - metaphorisch schließt sich hier der Kreis: Offenheit im Umgang mit Krisenerfahrung - sind die anzustrebenden Zugänge.

\subsection{LehrerInnenpersönlichkeit und Habitus im Kontext von Professionalisierung}

Bromme und Haag (2008) konstatieren in ihrer Studie zur LehrerInnenpersönlichkeit, dass Unterrichten als komplexer personaler Prozess zwischen LehrerInnen und SchülerInnen angesehen werden kann. Die pädagogische Wirksamkeit von Lehrpersonen wird dem „Black-box-Faktor Persönlichkeit“ (ebd., S. 804) zugeschrieben. Eng damit hängen die beruflichen Erscheinungsformen Erfolg, Misserfolg, Zufriedenheit und Resignation zusammen. Lehrkräfte beziehen ihre Berufsbefähigung nicht nur durch ihre Unterrichtsexpertise, sondern auch über fachliche und/ oder personale Kompetenzen. Berufserfolg und -zufriedenheit werden damit sowohl der zunehmenden Professionalisierung als auch dem Faktor Persönlichkeit zugeschrieben. Obwohl für LehrerInnen offensichtlich ist, dass „ihre Persönlichkeit den Zugang zu den Schülern eröffnet“ (ebd., S. 803) und damit über beruflichen Erfolg oder Misserfolg mit entscheidet, kann trotz der Berücksichtigung „qualitativ neuartiger Variablen“ (ebd., S. 804) mithilfe empirischer Methoden der „universell gute Lehrer“ (ebd.) nicht identifiziert werden. Gerade diese Annahme mag ein Ansporn für die wissenschaftliche Betrachtung dieser Frage sein, denn die Suche nach der idealen Lehrerpersönlichkeit ist der Klassiker unter den empirischen Ansätzen zur Ermittlung guter Lehrer (Terhart 2007).

Der Terminus LehrerInnenpersönlichkeit legt nahe, sich mit dem Begriff des Habitus eingehend zu befassen, denn dieser bedeutet in seiner allgemeinen Form „Aussehen, Erscheinungsbild; Anlage; Körperbau“ (Riecke 2014) und kann als eine erste Umschreibung der Persönlichkeit herangezogen werden. Im Duden-Fremdwörterbuch (Duden 2015) wird Habitus neben „Aussehen, Erscheinungsbild“ auch durch „Erscheinung, Haltung, Gehaben“ beschrieben, was die Handlungsebene des Individuums stärker betont. Außerdem wird auch noch die Unterscheidung "guter Habitus (Tugend), böser Habitus (Laster)“ angegeben. Im vorliegenden Kapitel wird das Habituskonzept Bourdieus (Bourdieu 1982, 1996a, 1996b, 1998a, 1998b, 2011, 2015a, 2015b; Bourdieu und Wacquant 1996; Lenger et al. 2013) rekonstruiert.

Zunächst erfolgt die Begründung der Modellwahl (Kap. II.1.2.1), anschließend die Darstellung und Beschreibung der zentralen Begriffe (Kap. II.1.2.2). Schließlich wird das Habituskonzept nach Bourdieu auf die LehrerInnenbildung respektive auf die Professionalisierung von Lehrpersonen übertragen. Dazu werden Bezüge zwischen dem theoretischen Modell und praktischen Vorgängen hergestellt (Kap. II.1.2.3).

\subsubsection{Habituskonzept nach Bourdieu als „Theorie der Praxis“}

Die Theorie der Praxis macht insofern die Profession des LehrerInnenberufs aus, als der Fokus auf das Handeln gerichtet ist. Wissenschaftlich generiertes (theoretisches) Wissen wird in diesem Sinne mit (praktischem) Handlungswissen in konkreten schulischen Situationen 
verknüpft bzw. angewandt (Keller-Schneider und Hericks 2014). Ebenso stellt der TheoriePraxis-Bezug ein Spannungsfeld in der Pädagogik dar. Die Beziehung zwischen Theorie und Praxis wird oft als Kluft oder gar als Problem bezeichnet (Patry Jean-Luc 2014). Reay (2004, S. 442) meint: „I would argue a real need, for putting habitus into practice“ und attestiert dem Bourdieu'schen Habituskonzept „potential strength“ (ebd., S. 441). Das Habituskonzept als Basis der „Theorie der Praxis“ (Lenger et al. 2013) ist als das Schlüsselkonzept in Bourdieus bildungssoziologischer Forschung anzusehen (Nairz-Wirth 2011). Das Habituskonzept Bourdieus zeigt die Chance und Möglichkeiten auf, die Theorie-Praxis-Schere (Terhart 2001) weitgehend zu schließen.

Im Folgenden wird das Habituskonzept nach Bourdieu mit den wesentlichen Begriffen vorgestellt. Anschließend erfolgt die konkrete Anwendung in der LehrerInnenbildung bzw. im Übergang in den LehrerInnenberuf im Sinne einer Rekonstruktion.

\subsubsection{Modelldarstellung}

Bourdieu (1982, 1996b, 1998a) definiert den Habitusbegriff als „Wahrnehmungs-, Denk- und Handlungsschemata" (Lenger et al. 2013) eines Menschen, in denen sämtliche inkorporierten, früheren sozialen Erfahrungen zum Ausdruck kommen. Er legt damit das Hauptaugenmerk auf die inneren, wirksamen Strukturen der Person. Für die vorliegende Untersuchung und Diskussion stellt der Fokus auf eben diese inneren Vorgänge der Inkorporierung der betreffenden Person, konkret der Berufsanfängerin bzw. des Berufsanfängers und im Sprachgebrauch Bourdieus des Subjekts, den wesentlichen Kerngedanken dar.

Zur Rekonstruktion des Bourdieu'schen Habituskonzepts bedarf es der Darstellung der bereits verwendeten Begriffe des Habitus, der Habitualisierung, der Inkorporierung sowie jenes des Subjekts. Weitere relevante und von Bourdieu eingeführte Begriffe werden ebenfalls beschrieben: das Feld inklusive der Phänomene Macht und Kapital, das mit dem Subjekt resonierende (Rosa 2018) Objekt und der für den Übergang charakteristische Akt der Inkorporierung.

\subsubsection{Feld, Macht und Kapital}

Der Begriff des Feldes bzw. die Feldtheorie Bourdieus beschreibt ein gesellschaftliches System (Diaz-Bone 2010). In einer Gesellschaft existieren mehrere autonome Felder, wobei jedes Feld eine abgrenzbare Funktion in der Gesellschaft einnimmt. Eigenständige Felder sind etwa Justiz, Gesundheit, Wirtschaft (Bourdieu und Wacquant 1996). Für die vorliegende Arbeit sind u.a. die Felder LehrerInnenbildung und formale Bildung (siehe auch Abb. 11: Wechselwirkung zwischen den Feldern im schulischen Kontext) von Bedeutung. Die grundlegenden Merkmale des jeweiligen Feldes sind nach Bourdieu die klaren Regeln und die feldinternen Gemeinsamkeiten (Barlösius 2011). Ein bestimmtes Feldinteresse konstituiert somit ein eigenständiges und autonomes Feld, indem es einen Schwerpunkt bzw. Hauptgegenstand festlegt (ebd.). Dadurch wird inhaltlich bestimmt, was sich im Feld ereignet. Atkinson (2011) drückt den Zusammenhang zwischen Feld und Habitus folgendermaßen aus:" [...] each organisation [...] has its own habitus."

In Bezug auf das jeweilige inhaltliche Feldinteresse führt Bourdieu den Begriff der Illusio (Bourdieu 1998a) ein und gibt zu bedenken, dass es neben dem eigentlichen Feldinteresse auch andere Interessen gibt: Erlangung von Macht, Geld, Ansehen etc. Im Feld kann z.B. ein gutes Image aufrechterhalten werden, indem die Illusion hierarchische Auseinandersetzungen etc. verschleiert (Barlösius 2011). Aus der Sicht der im Feld Beteiligten (Subjekt, Objekt) entsteht die Illusion durch die jeweils feste Überzeugung, dass es sich trotz des Wissens um die beschriebenen Nebeninteressen lohnt, im Feld teilzunehmen (Fuchs-Heinritz und König 2014). Das Ergebnis 
ist ein „heimliches Einverständnis“ (Barlösius 2011, S. 100) mit den Spielregeln des Feldes, die stillschweigend übernommen werden.

Das Konzept der Feldstruktur (Bourdieu 1996a) liefert neben den wichtigsten Eigenschaften und Metakonzepten zur Analyse des existierendes Feldes auch zwei weitere wesentliche Erkenntnisse: den Einblick in die „Machtstruktur [und in die] Distributionsstruktur von Kapitalsorten“ (ebd., S. 139). Bourdieu spricht in diesem Zusammenhang von „Herrschenden und Beherrschten“ (ebd., S. 137). Die Herrschenden sind stets in der Minderheit, haben im Feld jedoch den größeren Einfluss als die Beherrschten, die Feldstrukturen zu bestimmen. Die Beherrschten mit weniger Kapital und Machtpositionen kämpfen für Veränderungen in der Feldstruktur. Insofern sind Feldstrukturen bzw. die charakteristischen Eigenschaften eines Feldes stets im Wandel (Bourdieu 1996a; Fuchs-Heinritz und König 2014).

Unter Kapital oder Kapitalsorte versteht Bourdieu (1996a, S. 128) eine „Waffe“ oder ein „umkämpftes Objekt“, was der/dem BesitzerIn (Subjekte und Objekte) Macht und Einfluss gibt. Bourdieu unterscheidet vier Kapitalsorten: soziales, ökonomisches, kulturelles und symbolisches Kapital. Je nach ihrer „Kapitalausstattung“ (Nairz-Wirth 2011, S. 168) positionieren sich die AkteurInnen im Feld. Soziales Kapital umfasst Beziehungen, Freundschaften, Kooperationen etc. Unter ökonomischem Kapital sind Geld und Besitz gemeint. Das kulturelle Kapital bildet den großen Bereich des Wissens, der Bildung, der Kunst etc. ab, während zum symbolischen Kapital Anerkennung, Ehre etc. gehören (Barlösius 2011; Bourdieu 1996a). Nach der Darstellung des Feldes als Wirkungsbereich bzw. -raum der Habitualisierung, mit den Determinanten Macht und Kapital, folgt im Anschluss der Blick auf die zentralen Elemente im Feld im Sinne des Habitualisierungskonzepts nach Bourdieu: auf Subjekt und Objekt.

\subsubsection{Subjekt und Objekt}

Im Habitusmodell von Bourdieu (Bourdieu 2015a; Lenger et al. 2013; Schumacher 2013) interagieren innerhalb des Feldes Subjekt und Objekt, jeweils ausgestattet mit Macht und Kapital (Abb. 5).

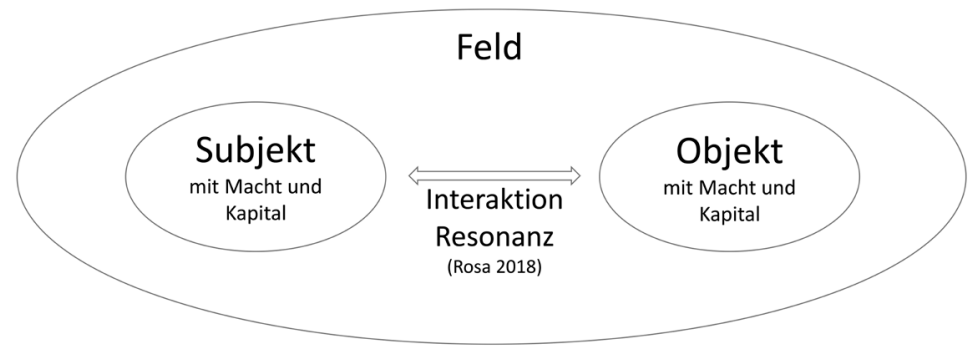

Abb. 5: Subjekt und Objekt interagieren und resonieren im Feld

In Abbildung 5 wird die Positionierung von Subjekt und Objekt innerhalb des Feldes dargestellt. Subjekt und Objekt stehen sich dynamisch interagierend gegenüber und verfügen ihrerseits jeweils über Macht und Kapital. Die in Kapitel II.1.2.2.1 beschriebenen Phänomene Macht und Kapital determinieren die Interaktion ${ }^{12}$, welche auf einer Überwindung der Differenz zwischen der äuße-

12 In Exkurs: Unverfügbarkeit und Resonanz (Kap. II.1.2.2.3) wird auf Resonanz (Rosa 2018) als besondere Form der Interaktion eingegangen. 
ren physischen Welt, dem Objekt, und einer inneren psychischen Sphäre des Subjekts basiert. Das bedeutet, dass sich die soziale Welt auch im Subjekt spiegelt und nicht rein äußerlich aufzufassen ist (Schumacher 2013). Bourdieu (1996a) verwendet für Subjekt und Objekt den Begriff AkteurIn. Er vermeidet damit, das Verständnis auf rationale Einzelpersonen zu reduzieren. Die kollektiven AkteurInnen wie Organisationen, Institutionen werden damit berücksichtigt, wenngleich in einem Feld die meisten AkteurInnen (Subjekte und Objekte) tatsächlich Einzelpersonen sind.

Durch das „Eindringen der Gesellschaft in die einzelnen Menschen“13 (Schumacher 2013, S. 142) erscheinen auch Einzelsubjekte (neben den Objekten) letztlich nicht als individuell handelnde Personen, vielmehr als soziale AkteurInnen. Für Bourdieu (2015a) ist der Akt der Interaktion zwischen Subjekt und Objekt auch insofern ein zentrales Moment seines Habituskonzepts, als er die Gegensätze zwischen Subjektivismus und Objektivismus als die grundlegendsten und verderblichsten in den Sozialwissenschaften ansieht.

Rosa (2018) stellt mit seinem Zugang zur Resonanz als eine Form der Interaktion (Abb. 5) eine Verbindung zur bereits diskutierten Kontingenz bzw. Unverfügbarkeit (Kap. II.1) und letztlich zur Habitualisierung her. Mit der Darstellung des Resonanzbegriffs nach Rosa im anschließenden Exkurs wird diese Brücke sichtbar gemacht, um sich darauf folgend der Rekonstruktion seines Resonanzbegriffs in Bezug auf die Habitualisierung anzunehmen.

\subsubsection{Exkurs: Unverfügbarkeit und Resonanz}

Rosa (2018) diskutiert das Phänomen der Resonanz aus dem Blickwinkel der Unverfügbarkeit. Aufgrund inhaltlicher Parallelitäten zum Bourdieu'schen Habituskonzept im Allgemeinen und dem Akt der Inkorporierung im Speziellen wird der Resonanzbegriff im Verständnis Rosas skizziert. Im ersten Schritt wird Bezug auf den Begriff der Unverfügbarkeit genommen, damit im zweiten das Resonanzverständnis daraus abgeleitet werden kann. Schließlich erfolgt die Darstellung der erwähnten Parallelität und relevanter Zusammenhänge mit dem Habituskonzept Bourdieus. Für Rosa (2018) liegt der Verdacht nahe, dass „das Verschwinden der Erfahrung des Angerufenwerdens eine Folge der grenzenlosen Verfügbarkeit [ist]“, wobei mit „Angerufenwerden“ der Beginn eines Resonanzprozesses gemeint ist. Unter der grenzenlosen Verfügbarkeit versteht der Autor das zentrale Bestreben der Moderne, die Welt ökonomisch und technisch verfügbar, wissenschaftlich erkennbar und beherrschbar, rechtlich berechenbar, politisch steuerbar und zugleich alltagspraktisch kontrollierbar und erfahrbar zu machen. Die „Verfügbarmachung“ (ebd., S. 21) besteht aus vier unterschiedlichen Elementen bzw. kann in vier Dimensionen unterteilt werden: Zunächst werden Dinge, Phänomene etc. (1) „sichtbar“ (ebd.) gemacht, damit sie in Folge (2) „erreichbar bzw. zugänglich“ (ebd.) sind. Damit „unauflöslich verbunden“, so Rosa (2018, S. 21-22), ist die Dimension des (3) „Unter-Kontrolle-Bringen[s]“ (ebd., S. 22) bzw. des Bestrebens nach dem „beherrschbar [M] achen“ (ebd., S. 21f). Schließlich erfolgt das (4) „Nutzbarbachen bzw. [die] Indienstnahme“ (ebd., S. 22), die Instrumentalisierung durch Transformation im Sinne der eigenen Projektionen und Wünsche ist das Ergebnis. Die Diskussion über Unverfügbarkeit lässt sich auf Ungewissheit als Teil der pädagogischen Profession (Kap. I.1.1) übertragen. Hier wie dort herrscht Ambivalenz zwischen dem Wunsch nach Kontrolle bzw. Beherrschbarkeit einerseits und dem offenen Umgang mit Kontingenz bzw. Krisen andererseits. Die Kontingenzerfahrung zieht Ungewissheit und mögliche Krisen nach sich, daraus werden im Sinne der pädagogischen Professionalisierung Lösungen generiert. Analog dazu bereitet Unverfügbarkeit den Nährboden für Resonanz und Interaktion, wodurch Professionalisierung bzw. Habitualisierung initiiert werden. 
Rosa (2018) hebt hervor, dass gerade die Unverfügbarkeit im Sinne der fehlenden Voraussagbarkeit von Reaktionen von Menschen, Organisationen etc. eine Voraussetzung dafür ist, „in Resonanz treten zu können“. Insofern, und hier zeigt sich eine erste Parallelität zu Bourdieu, sind Feldstrukturen mit ihren je eigenen Macht- und Kapitalausstattungen der Motor für die Interaktion, die Resonanz und die im nächsten Kapitel thematisierte Inkorporierung. Der Autor identifiziert außerdem Resonanz als „Beziehungsmodus“ mit den Momenten der „Berührung“ (Affizierung), der "Selbstwirksamkeit“ (Antwort) und der „Anverwandlung“" (Transformation), was als eine weitere inhaltliche Überschneidung mit dem Bourdieu’schen Habitusmodell gesehen werden kann (Abb. 6).

In Abbildung 6 werden die jeweiligen Triaden der Resonanz nach Rosa (2018) und der Habitualisierung nach Bourdieu (1996a, 2015a) dargestellt und in Beziehung gesetzt.

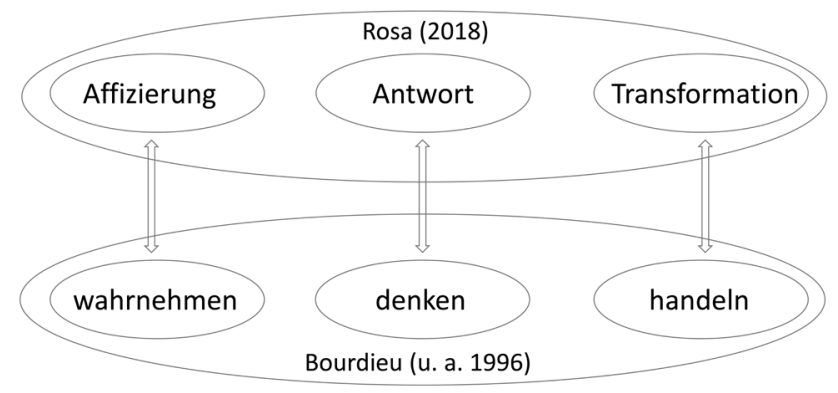

Abb. 6: Trias von Resonanz (Rosa 2018) und Habitualisierung (Bourdieu 1996)

Die Triaden „Affizierung - Antwort - Transformation“ (Rosa 2018) und „wahrnehmen denken - handeln“ (Bourdieu 1982, 1996a, 2015a) lassen sich in inhaltlich verwandte Paare gruppieren: Affizierung - wahrnehmen, Antwort - denken und Transformation - handeln. Folglich deckt sich die Grundidee Bourdieus, wonach Habitus (siehe auch Kap. II.1.2.2.5) als „Wahrnehmungs-, Denk- und Handlungsschemata" und Habitualisierung als Inkorporierung sämtlicher früher sozialer Erfahrungen (Bourdieu 1982, 1996a; Lenger et al. 2013) gesehen werden kann, weitgehend mit dem Konzept Rosas (2018) zum Phänomen der Resonanz. Mit einer Idee in Resonanz zu treten, setzt der Autor mit „erreichen, berühren“ (ebd., S. 39) bzw. Affizierung gleich, was im Sinne Bourdieus mit „wahrnehmen“ (Bourdieu 1982) übersetzt werden kann. Rosa (2018) spricht erst dann von Resonanz, wenn anschließend eine „eigene, aktive Antwort erfolgt“ (ebd., S. 39) und wenn die "Selbstwirksamkeit“ (ebd., S. 40) von der Person selbst ausgeht: Das Subjekt denkt bzw. reflektiert im Habituskonzept (Bourdieu 1982). Schließlich folgert Rosa (2018), dass die Veränderung der Weltbeziehung durch Transformation ein konstitutives Element der Resonanz darstellt. Durch Handlungen verändert das Subjekt bei Bourdieu (1982) die Weltbeziehung. Die Trias aus Affizierung, selbstwirksamer Antwort und Transformation (Rosa 2018) weist zur Triade wahrnehmen - denken - handeln (Bourdieu 1982) eine weitere Parallele auf. Rosa sieht das Moment der Unverfügbarkeit als notwendige Voraussetzung für Resonanz (ebd., S. 43). In der Habitualisierung ist das Moment der Kontingenz (Paseka 2011; Schrittesser 2011) ein ständiger Begleiter. 
Die Triaden nach Rosa (2018) und Bourdieu (1982) greifen mit der bereits diskutierten Ungewissheit als Teil der pädagogischen Profession (Paseka et al. 2018; Schrittesser 2011) in Kapitel I.1.1 thematisch ineinander und sind konstitutiver Teil der LehrerInnenaus- und -weiterbildung, in letzter Konsequenz auch der LehrerInnenprofessionalisierung. Dieses Konzept der Professionalisierung wird in Anwendung des Habituskonzepts nach Bourdieu auf die LehrerInnenprofessionalisierung (Kap. II.1.2.3) näher beleuchtet.

Im Habituskonzept nach Bourdieu ist die Inkorporierung eine bzw. die entscheidende Phase. Im Folgenden wird dieses Phänomen genauer betrachtet.

\subsubsection{Inkorporierung}

Die Inkorporierung stellt in der vorliegenden Rekonstruktion des Habituskonzepts nach Bourdieu (Bourdieu 2015a; 2015b; Lenger et al. 2013; Schumacher 2013) den wesentlichen Prozess im Laufe der Habitualisierung, der letztlich zum Habitus führt, dar (Abb. 7). Unter Inkorporierung versteht Bourdieu (2015a) die Einverleibung von Erkenntnissen, die über Interaktion und Resonanz, letztlich basierend auf den Triaden wahrnehmen - denken - handeln bzw. Affizierung - Antwort - Transformation, entstehen.

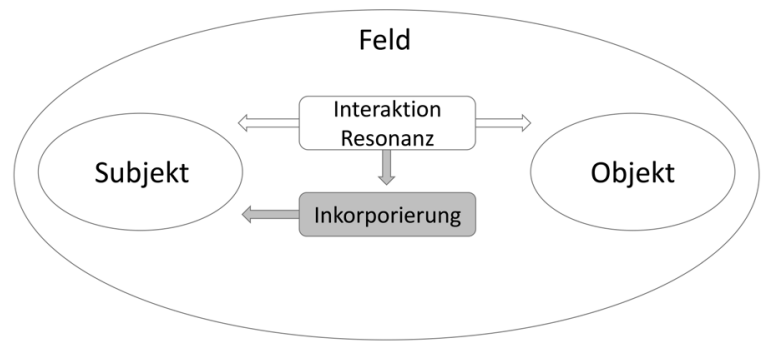

Abb. 7: Inkorporierung im Habitualisierungsprozess

Die Interaktion zwischen Subjekt und Objekt innerhalb des Feldes kann als Resonanz (Rosa 2018) interpretiert werden. Die Resonanz wird von verschiedenen Faktoren, wie Unverfügbarkeit (ebd.), Kontingenz ((Paseka 2011; Schrittesser 2011), determiniert. Im Habituskonzept Bourdieus gestalten sie sich durch Macht- und Kapitalunterschiede innerhalb der Feldstruktur (Bourdieu 1982).

Die „Vermittlung zwischen sozialen Akteuren und gesellschaftlichen Strukturen“ (Schumacher 2013, S. 134), in Abbildung 7 als Interaktion bzw. Resonanz dargestellt, führt über die Inkorporierung schließlich zur Überwindung des Gegensatzes von Subjektivismus und Objektivismus (ebd.). Insofern wird Inkorporierung als „Einverleibung historisch entstandener sozialer Strukturen“ (ebd., S. 135) bzw. als Verinnerlichung früherer sozialer Erfahrungen (Lenger et al. 2013) verstanden. Aufgrund der Einverleibung bzw. Verinnerlichung erfolgt die Umwandlung von Fremdzwängen in Selbstzwänge. Durch die Inkorporierung sozialer Zwänge werden diese schließlich habitualisiert (Schumacher 2013).

Der Vorgang der Einverleibung bzw. der Inkorporierung macht deutlich, dass Habitus keine schicksalhafte, vorbestimmte Determinante darstellt (Nairz-Wirth 2011), sondern dass die Habitualisierung durch integrative, reflexive und in diesem Sinne durch unbewusste sowie bewusste Prozesse determiniert wird. Im anschließenden Abschnitt werden die Begriffe Habitualisierung und Habitus genauer betrachtet. 


\subsubsection{Habitualisierung führt zum Habitus}

Folgt man der Grunddefinition und betrachtet Habitus als „Wahrnehmungs-, Denk- und Handlungsschemata" (Bourdieu 1982), ist damit nicht nur der Habitus als Endprodukt festgelegt, sondern bereits der Weg dahin gekennzeichnet: die Habitualisierung (Abb. 8).

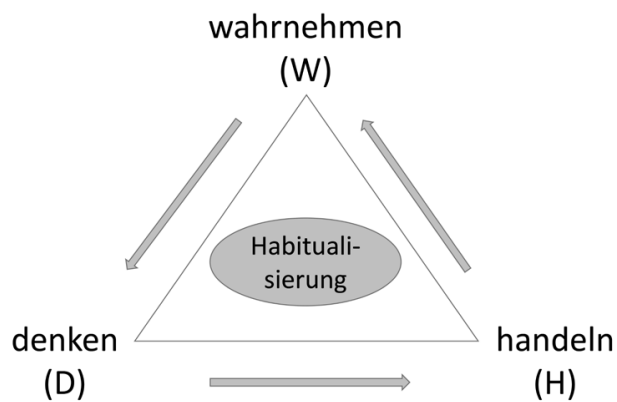

Abb. 8: Habitualisierungsschema nach Bourdieu (1982)

Die Triade wahrnehmen (W), denken (D) und handeln (H) stellt über die Inkorporierung den Prozess der Habitualisierung dar. Bourdieu verortet den Habitus zwischen den sozialen AkteurInnen (Subjekt) und der sozialen Struktur (Objekt). Die Vermittlung (Interkation, Resonanz, Inkorporierung) zwischen Subjekt und Objekt bzw. zwischen Individuum und Gesellschaft im Feld ist ein holistisches Konzept und gilt als die zentrale Idee im Habituskonzept Bourdieus (Bourdieu 2015a; Lenger et al. 2013) (Abb. 9).

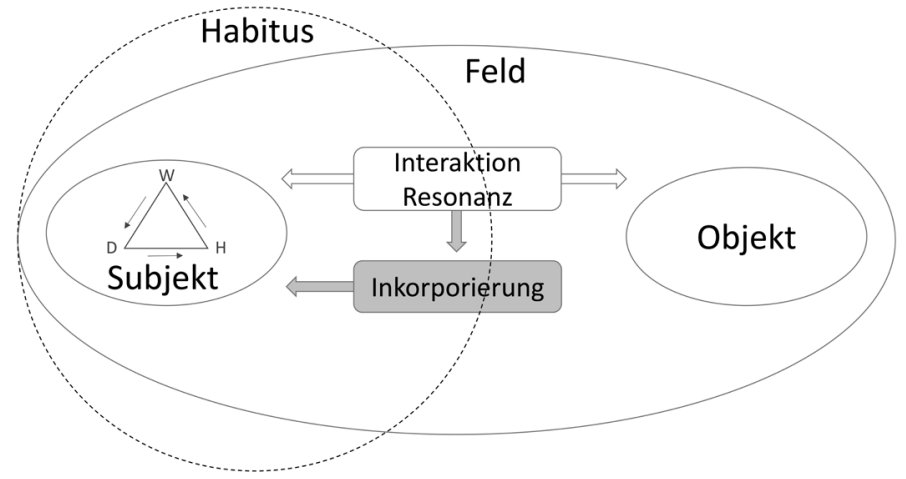

Abb. 9: Habitus als holistisches Konzept (Bourdieu 1982)

Im Feld interagieren und resonieren das mit Macht und Kapital ausgestattete Subjekt und Objekt, wobei sich das Subjekt in der Trias wahrnehmen (W) - denken (D) - handeln (H) bewegt. Interaktion und Resonanz, beides determiniert durch Kontingenz, Ungewissheit und Unverfügbarkeit (Paseka et al. 2018; Rosa 2018; Schrittesser 2011), führen zum Prozess der Inkorporierung, der Verinnerlichung sozialer Erfahrungen (Lenger et al. 2013). Die Kohärenz von Interaktion, Resonanz, Inkorporierung und Triade des Subjekts bildet über Habitualisierung 
den Habitus. Dieser ist in diesem Sinne das Dispositionssystem sozialer AkteurInnen, das die Prozesse der Verinnerlichung der sozialen Praxis repräsentiert. Letztlich wird durch diese Praxis die Inkorporierung der sozialen Laufbahn eines Individuums dargestellt, wobei die Handlungsanweisungen des Habitus unbewusst und eben aufgrund der Inkorporierung automatisch erfolgen. Insofern bleiben Handlungen dem Bewusstsein der Akteurin bzw. des Akteurs meist versagt (Lenger et al. 2013). Damit wird nachvollziehbar, dass die sozialen AkteurInnen nach keinen mechanischen Gesetzen handeln, nicht wie "geregelte Automaten gleich Uhren“ (Bourdieu 2011) funktionieren. Handeln heißt in diesem Zusammenhang nicht Befolgen oder Vollziehen einer Regel, sondern Einsetzen ,inkorporierte[r] Prinzipien eines generativen Habitus“ (ebd.), selbst in den kompliziertesten Handlungsverläufen. Es handelt sich also um „durch Erfahrung erworbene, folglich je nach Ort und Zeit variable Dispositionen“ (ebd., kursiv im Original). Im Kontext der formalen Bildung findet die Habitualisierung des Subjekts (Studierende, Lehrende) durch Inkorporierung von sozialen Erfahrungen (Lenger et al. 2013) aufgrund der Interaktion und Resonanz mit dem Objekt (System, Kollegium etc.) statt. Auf das Phänomen der Triade wahrnehmen - denken - handeln, welches in der vorliegenden Rekonstruktion die Essenz der LehrerInnenprofessionalisierung darstellt, wird im Kapitel Habitus als Konzept der Professionalität: Kernidee der Rekonstruktion (Kap. II.1.2.3.2) gezielt eingegangen.

Als Quintessenz der bisherigen Erkenntnisse zur Habitualisierung im Sinne Bourdieus kann zusammengefasst werden, dass das Subjekt über den erworbenen Habitus je nach Situation beliebig viele Handlungsschemata generieren und danach agieren bzw. reagieren kann (Abb. 10).

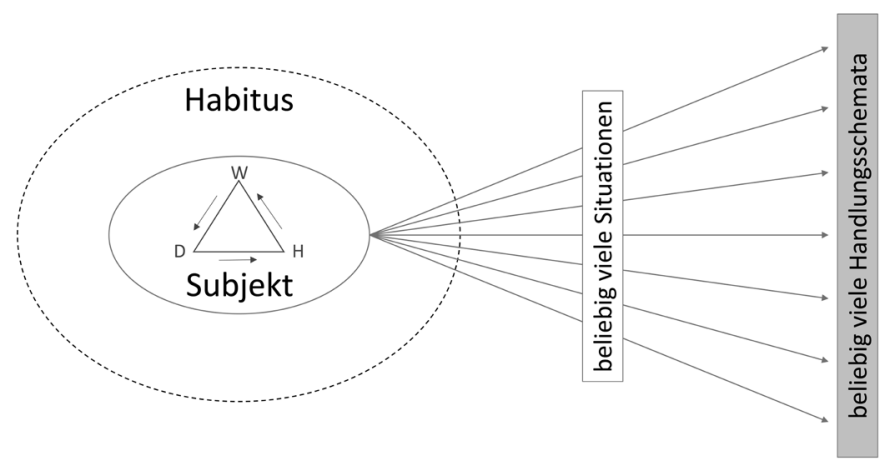

Abb. 10: Generierung beliebig vieler Handlungsschemata

Das Subjekt befindet sich in der Trias wahrnehmen (W) - denken (D) - handeln (H) und bildet über die Habitualisierung als Resultat der Interaktion, Resonanz und letztlich Inkorporierung den Habitus (Abb. 10). Dieser generiert für beliebig viele Situationen beliebig viele Handlungsschemata. Bourdieu (2011) spricht in diesem Zusammenhang von der schöpferischen Dimension des Handelns und der Praxis sowie von den generativen Fähigkeiten des Habitus aus erworbenen und gesellschaftlich konstituierten Dispositionen. „[...] unendlich viele, an stets neue Situationen sich anpassende Praktiken" (Bourdieu 2015a) bzw. Handlungsschemata sind das Resultat eines ausgebildeten Habitus.

Für AkteurInnen im Bildungsbereich bedeutet das generative Prinzip des Habitus eine Chance bzw. Erleichterung: Die beliebig vielen (schulischen) Situationen konstituieren sich als Kontingenz, Un- 
gewissheit und Unverfügbarkeit (Paseka et al. 2018; Rosa 2018; Schrittesser 2011). Anstatt nach dem oftmals artikulierten Werkzeugkoffer zu suchen, kann das habitualisierte Subjekt (Studierende, Lehrende) auf die schöpferische Dimension (Bourdieu 2011) des Habitus hoffen und vertrauen. Handlungsschemata werden im Sinne der Kontingenz, Ungewissheit und Unverfügbarkeit (ebd.) beliebig oft und variabel generiert, aus der Krise (Dietrich 2018; Kosinár 2018) wird eine Chance. Im folgenden Kapitel wird das oben theoretisch dargestellte Habituskonzept nach Bourdieu auf die LehrerInnenprofessionalisierung übertragen und konkretisiert.

\subsection{3 Übertragung des Habituskonzepts auf LehrerInnenprofessionalisierung}

Begriffe und Konzepte des Bourdieu'schen Habitusmodells sind in das Feld der formalen Bildung (Schulpraxis), in die LehrerInnenaus- und -weiterbildung sowie auf die Professionalisierung als Lehrperson übertragbar. In Kapitel I.1.2.3 erfolgt einleitend die Rekonstruktion der Begriffe und Begriffszusammenhänge nach Bourdieu im Kontext der LehrerInnenbildung, um anschließend auf die Kernidee, Habitus als Konzept der Professionalisierung zu verstehen, einzugehen.

\section{2.3.1 Rekonstruktion der Begriffszusammenhänge im Kontext der LehrerInnenbildung}

Der Begriff des Feldes spielt nicht nur in der theoretischen Habitusdiskussion (Bourdieu 1996a; Helsper 2018; Nairz-Wirth 2011) eine zentrale Rolle, auch im Kontext der Professionalisierung als Lehrperson rückt er ins Zentrum. So steht Bourdieu dem „isolierten Gebrauch des Begriffes Profession“ (Nairz-Wirth 2011) ambivalent gegenüber, wenn die „Verwobenheit mit Feld und Habitus" (ebd.) nicht beachtet wird. Dabei geht es letztlich darum, dass sich aufgrund der gegenseitigen Wechselwirkung neben den AkteurInnen der schulischen Felder auch jene der anderen Gesellschaftsfelder habitualisieren (Abb. 11).

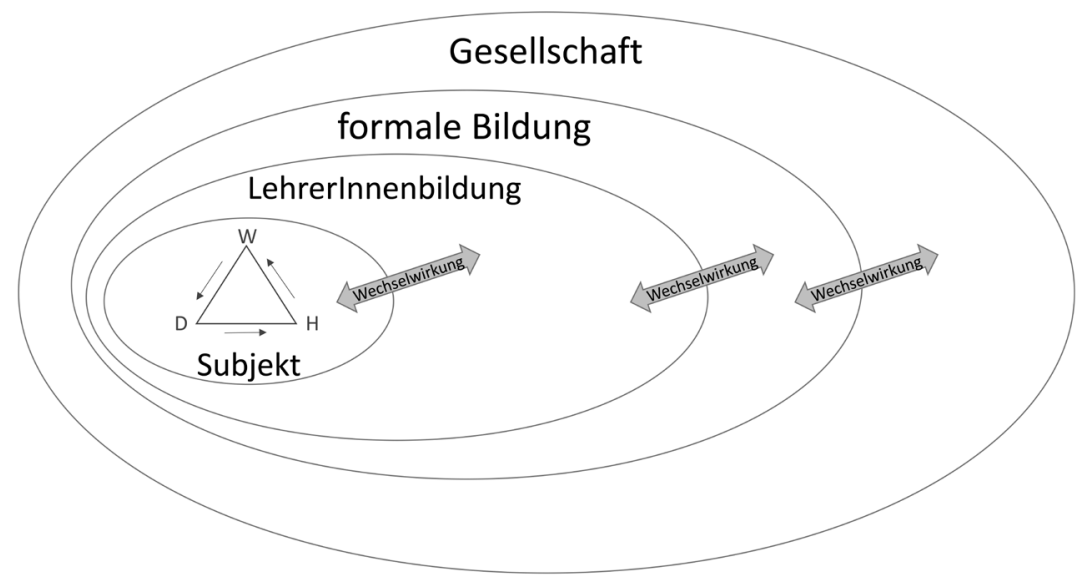

Abb. 11: Wechselwirkung zwischen den Feldern im schulischen Kontext

Dem Subjekt (Abb. 11) entspricht im Kontext der vorliegenden Arbeit ein/e BerufsanfängerIn bzw. ein/e Studierende/r, je nachdem, ob die Phase der LehrerInnenaus- oder -weiterbildung betrachtet wird. Die Felder der individuellen Habitualisierung (Subjekt), der LehrerInnenbildung, der formalen Bildung (Schulpraxis) und der Gesellschaft als Ganzes stehen gegenseitig in 
Wechselwirkung bzw. in „dynamischer Relation“ (Nairz-Wirth 2011, S. 179) zueinander. Der individuelle Habitus wirkt „strukturierend und feldgestaltend“ (ebd.) und insofern beeinflusst er über die Wechselwirkung, Interaktion bzw. Resonanz (Rosa 2018) und letztlich durch Inkorporierung die Habitualisierung der Gesellschaft.

Nairz-Wirth (2011) stellt fest, dass Professionalisierung primär mit Feldgestaltung einhergeht. Erst nach der „Stabilisierung des Feldes“ (ebd., S. 172) kann die Arbeit am „idealen Berufshabitus" (ebd.) erfolgen kann. Dieser wird initial im Studium und in den integriert oder konsekutiv organisierten Praxisphasen erworben, wobei sich der Habitus der Studierenden in der Ausbildung weniger stark verändert als jener der BerufsanfängerInnen in der Berufstätigkeit resp. in der Induktionsphase (ebd., S. 179).

Für die vorliegende Arbeit wird die Konstitution des Feldes „LehrerInnenbildung“ folgendermaßen modelliert (Abb. 12):

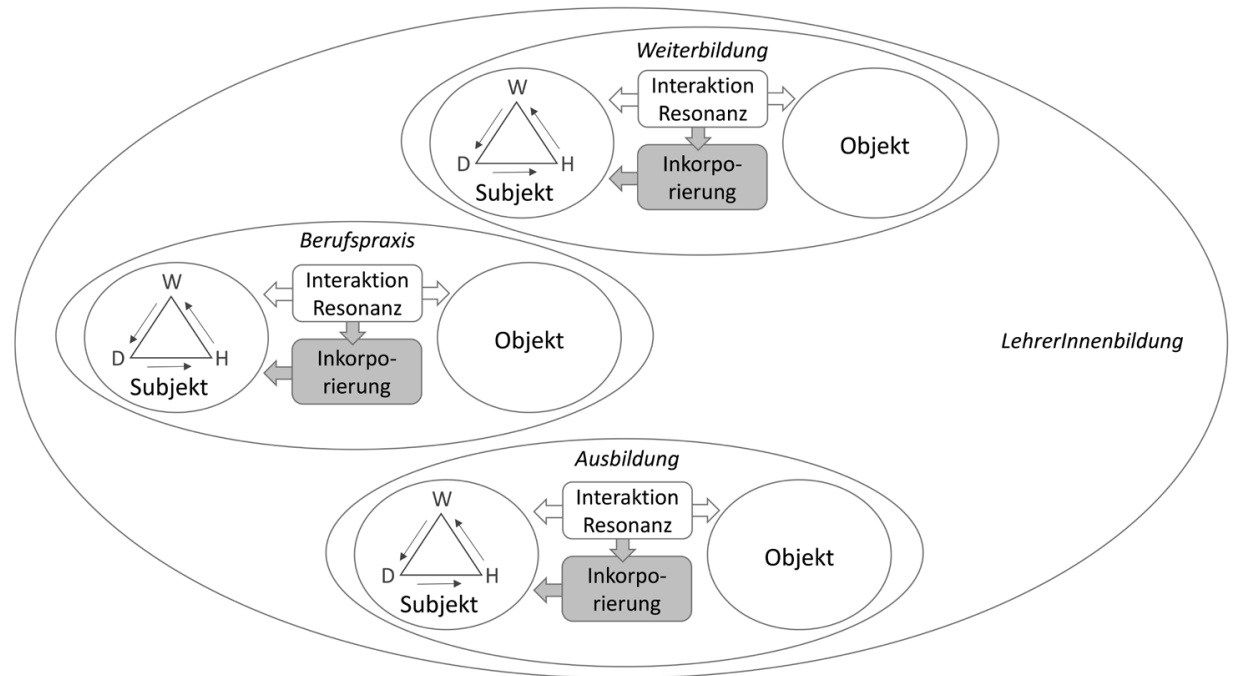

Abb. 12: Konstitution des Feldes LehrerInnenbildung

Die Grundannahme bleibt dieselbe wie oben beschrieben: Das Subjekt befindet sich in der Trias wahrnehmen $(\mathrm{W})$ - denken $(\mathrm{D})$ - handeln $(\mathrm{H})$ und interagiert bzw. resoniert mit dem Objekt. Daraus entsteht Inkorporierung, die wesentlich zur Habitualisierung und letztlich zur Bildung des Habitus beiträgt. Im Gesamtfeld „LehrerInnenbildung“ (Abb. 12) interagiert bzw. resoniert das Subjekt mit dem Objekt und inkorporiert soziale Erfahrungen in den Teilfeldern Ausbildung, Berufspraxis und Weiterbildung. Je nachdem, in welchem sozialen Feld Subjekt und Objekt interagieren bzw. resonieren, finden Handlungen, Lernerfahrungen und letztlich Inkorporierung unterschiedlich statt. Das Modell des Gesamtfeldes LehrerInnenbildung macht deutlich, dass Habitualisierung und letztlich Professionalisierung, wenn Habitus als Konzept der Professionalisierung (Kap. II.1.2.3.2) betrachtet wird, in der Ausbildungs-, Berufspraxis- und Weiterbildungsphase stattfindet. Die Vermutung, dass Professionalisierung einen nie abgeschlossenen Prozess darstellt (Helsper 2018), wird insofern bestätigt. Bourdieu betont in seinem Konzept sozialer Felder, dass Individuen (Subjekt und Objekt) nicht in allen spezifischen Kontexten identisch handeln, son- 
dern dass die jeweilige Handlung letztlich davon abhängt, in welchem Feld sie stattfindet (Lenger et al. 2013). Je nach Feld entsprechen die Studierenden, BerufsanfängerInnen oder in der Praxis stehenden LehrerInnen dem Subjekt, SchülerInnen, KommilitonInnen, MentorInnen, SchulleiterInnen, LehrerkollegInnen, Personen der Schulaufsicht, Dozierende und alle AkteurInnen im Kontext des jeweiligen Feldes dem Objekt (Abb. 13).

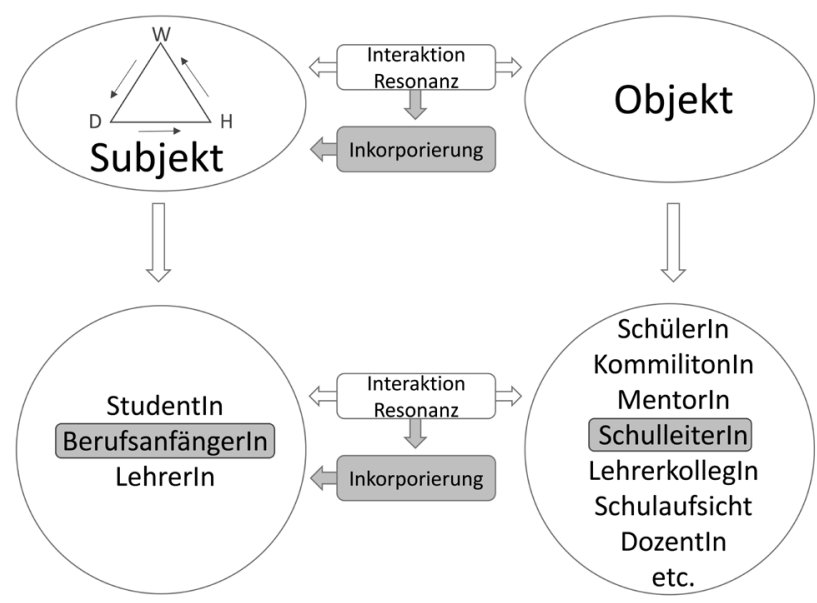

Abb. 13: Subjekt und Objekt in den Feldern Ausbildung, Berufspraxis und Weiterbildung

Die Habitualisierung des Subjekts erfolgt in der Triade wahrnehmen (W) - denken (D) - handeln $(\mathrm{H})$, die vorausgehende Inkorporierung wird von Interaktion und Resonanz determiniert. Kontingenz, Ungewissheit und Unverfügbarkeit (Paseka et al. 2018; Rosa 2018; Schrittesser 2011) beeinflussen die Inkorporierung und die daraus resultierende Habitualisierung ebenso wie die Konstitution von Subjekt und Objekt bzw. im weiteren Sinne des Feldes.

In der vorliegenden Arbeit (Empirischer Zugang, Kap. III) werden die Resonanzphänomene exemplarisch im Feld der ersten Berufspraxis (Übergang) mit BerufsanfängerInnen (Subjekt) und SchulleiterInnen (Objekt) untersucht (Abb. 13).

\section{2.3.2 Habitus als Konzept der Professionalität: Kernidee der Rekonstruktion}

Zwischen Habitus und Professionalisierung entsteht ein Konnex (Helsper 2018; Nairz-Wirth 2011; Paseka et al. 2011; Schrittesser 2011). Helsper (2018) hält fest, dass das Verhältnis von Habitus und Professionalität nicht geklärt ist. Für ihn sind LehrerInnenhabitus und Professionalität „nicht identisch“. Leonhard und Lüthi (2018) verweisen auf den Prozesscharakter in der LehrerInnenausbildung und betonen die "grundlegende Annahme“, dass sich sowohl in den Studiengängen zum Lehrberuf (Ausbildung) als auch in der anschließenden Induktionsphase bei den Studierenden bzw. den BerufsanfängerInnen „etwas tut“. Diese Entwicklungen der BerufsanfängerInnen werden, so die AutorInnen, als „Lernen“ (ebd.) oder als „Habitualisierung“ (Helsper 2018) beschrieben. Hericks et al. (2018) sehen die reflexive Haltung in Bezug auf die Biografie, das Fach und den antizipierten Beruf als einen Grundstein zur Professionalisierung angehender Lehrpersonen. „Längerfristig“, so die AutorInnen, „wird dadurch eine Professionalisierung vorbereitet und in einen professionellen Habitus übergeleitet“ (ebd., S. 267). Die Anbahnung eines „wissenschaft- 
lich-reflexiven Habitus“ (ebd., S. 268) steckt den Rahmen der ersten Phase der LehrerInnenausbildung ab und ist zugleich „notwendige Bedingung für die Ausbildung von Professionalität in den folgenden Phasen“ (ebd.).

Auch Helsper (2018) proklamiert die Herausbildung eines wissenschaftlich-reflexiven Habitus und ergänzt, dass zusätzlich ein forschender Habitus die Studierenden und angehenden Lehrpersonen dazu ermächtigt, „auch eigene ideale Ziele, normative Entwürfe und Orientierungen einer Relativierung und Geltungsprüfung zu unterziehen“" (ebd., S. 129). Diese reflexive selbstbezügliche Haltung der Auseinandersetzung mit „dem Eigenen und dem eigenen Fremden“ (ebd.) ist eine Kernaufgabe des hier thematisierten professionellen LehrerInnenhandelns und stellt nicht zuletzt auch die bereits diskutierte Basis für eine ,angemessene Kriseninitiation und Krisenlösung“ (ebd.) dar. ${ }^{14}$ Unterbleiben derartige Anstöße zu einem reflexiven und letztlich professionellen Bildungsprozess, verläuft die Herausbildung der „Habitussensibilität individualisiert“ (ebd., S. 134) und gebunden an die Vorerfahrungen von Lehramtsstudierenden und bereits im Dienst stehenden Lehrpersonen.

Die Triade wahrnehmen (W), denken (D) und handeln (H) ${ }^{15}$ stellt unter Berücksichtigung des wissenschaftlich-reflexiven Zugangs zur Habitualisierung (Helsper 2018; Hericks 2006; Hericks et al. 2018) die Kernidee der hier dargelegten Rekonstruktion des Habituskonzepts nach Bourdieu dar. Wie in Abbildung 14 dargestellt, habitualisiert sich das Subjekt (BerufsanfängerIn) durch sukzessives Wahrnehmen, Denken und Handeln über Inkorporierung und Lernprozesse und bildet dabei einen individuellen Habitus aus.

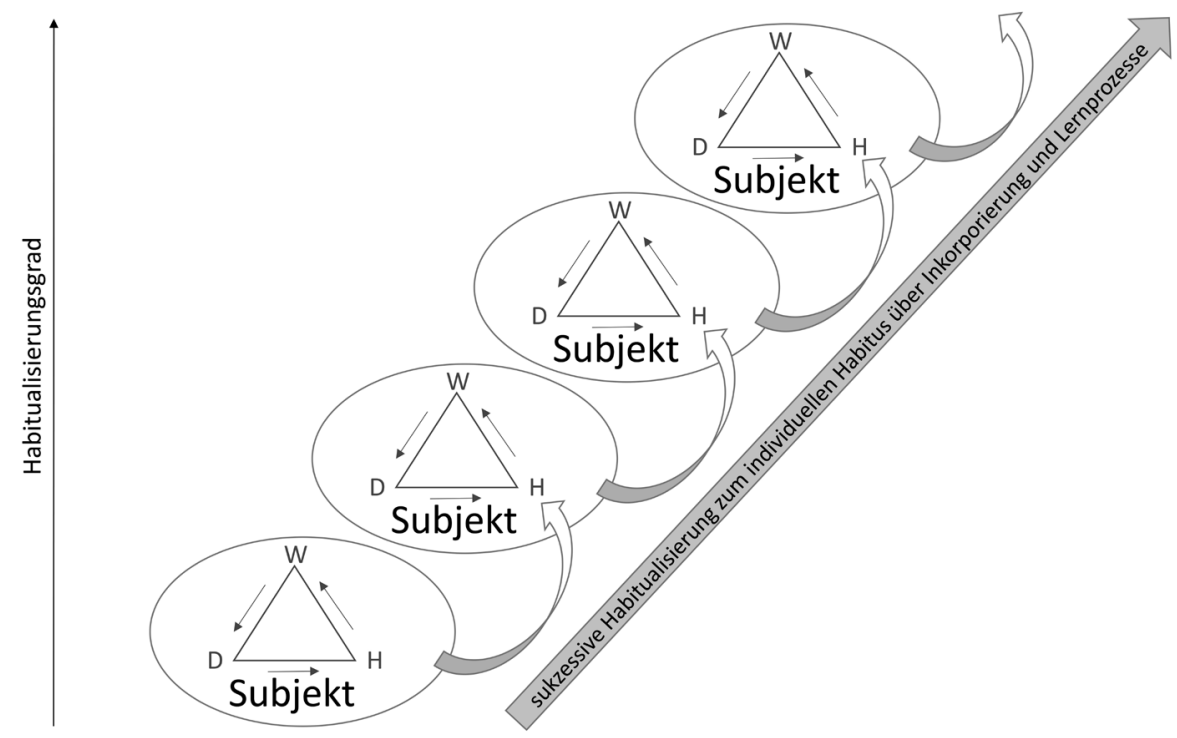

Abb. 14: Sukzessive Habitualisierung über Inkorporierung und Lernprozesse

14 In Kapitel I.2.2 wird auf den Aspekt der Professionalisierung durch forschendes Lernen näher eingegangen. 15 Habitus als Wahrnehmungs-, Denk- und Handlungsschemata (Bourdieu 1982). 
Im Unterschied zum individuellen Habitus (Abb. 14) wird in der vorliegenden Arbeit der LehrerInnenhabitus (in Relation zum individuellen Habitus) aufgrund der Wechselwirkungen der Felder (Abb. 11) mit den vielen sich konstituierenden Habitualisierungsdeterminanten als relativ stabil dargestellt. Er ist gewissermaßen träge, da er sich über die Zeit nicht so schnell verändert wie der individuelle Habitus. „Träge“ ist in diesem Kontext eine positive Eigenschaft, da sich der LehrerInnenhabitus dadurch als repräsentativer Vergleichswert zum jeweiligen individuellen Habitualisierungsgrad heranziehen lässt. Das Ziel der sich habitualisierenden Lehrperson ist ein hohes Maß an Deckung zwischen dem eigenen, individuellen Habitus und dem LehrerInnenhabitus. Idealerweise repräsentiert der individuelle Habitus den LehrerInnenhabitus.

Die Kernidee der hier dargelegten Rekonstruktion bildet die Darstellung des professionellen LehrerInnenhabitus. In Anlehnung an Helsper (2018), Hericks et al. (2018) und Kraler (2008b) sind Lehrpersonen im Allgemeinen und BerufsanfängerInnen im Speziellen durch wissenschaftlich-reflexive Kompetenzen in der Lage, die Triade wahrnehmen - denken - handeln auf ein professionelles Niveau zu heben: durch professionelles Wahrnehmen (PW), professionelles Denken (PD) und professionelles Handeln (PH) (Abb. 15).

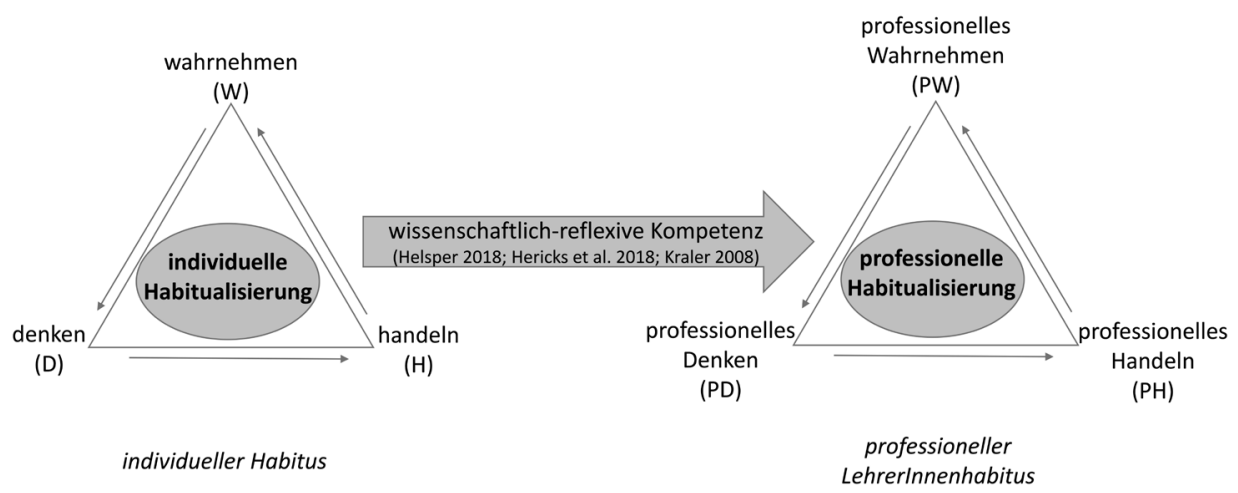

Abb. 15: Professionelle Habitualisierung

In Abbildung 15 wird die Weiterentwicklung des individuellen Habitus zum professionellen Habitus dargestellt. Die individuelle Habitualisierung erzeugt den individuellen Habitus. Dieser wird durch wissenschaftlich-reflexive Kompetenzen (Helsper 2018; Hericks et al. 2018; Kraler 2008b) über das professionelle Wahrnehmen (PW), das professionelle Denken (PD) und das professionelle Handeln $(\mathrm{PH})$ letztlich durch die professionelle Habitualisierung zum professionellen LehrerInnenhabitus weiterentwickelt (Tab. 1).

Tab. 1: Professionelles Wahrnehmen, Denken und Handeln

\begin{tabular}{|c|c|c|}
\hline $\begin{array}{c}\text { professionelles Wahr } \\
\text { (PW) }\end{array}$ & $\begin{array}{l}\text { professionelles Denken } \\
\text { (PD) }\end{array}$ & $\begin{array}{l}\text { professionelles Handeln } \\
\text { (PH) }\end{array}$ \\
\hline $\begin{array}{l}\text { - Art der Wahrnehmung } \\
\text { - jeweiliger individueller } \\
\text { Habitualisierungsgrad ist } \\
\text { ausschlaggebend }\end{array}$ & $\begin{array}{l}\text { - Verarbeitung des Wahrgenom- } \\
\text { menen: wissensbasiert, profes- } \\
\text { sionsspezifisch, reflektiert }\end{array}$ & $\begin{array}{l}\text { - agieren und reagieren in } \\
\text { verschiedenen Situationen: } \\
\text { theoriegeleitet (Ausbildung), } \\
\text { reflektiert }\end{array}$ \\
\hline
\end{tabular}


In Tabelle 1 sind die Besonderheiten des professionellen Wahrnehmens, Denkens und Handelns dargestellt. Die professionelle Wahrnehmung unterscheidet sich in diesem Modell (Abb. 15) vom Wahrnehmen durch die Art der Wahrnehmung aufgrund des jeweiligen Habitualisierungsgrads infolge der Ausbildung (siehe auch Abb. 14: Sukzessive Habitualisierung über Inkorporierung und Lernprozesse). Professionelles Denken betont das wissensbasierte, professionsspezifisch reflektierte Verarbeiten des Wahrgenommenen und mit professionellem Handeln ist das reflektierte, theoriegeleitete, in der Ausbildung erarbeitete Agieren und Reagieren in verschiedenen Situationen gemeint (siehe auch Abb. 10: Generierung beliebig vieler Handlungsschemata). Ich greife Helsper (2018) auf, wonach LehrerInnenhabitus und Professionalität nicht identisch sind und das Verhältnis zwischen Habitus und Professionalität nicht geklärt ist, und komme zum Schluss, dass sich die LehrerInnenprofession über den professionellen Habitus durch die Triade professionelles Wahrnehmen - professionelles Denken - professionelles Handeln abbildet.

\subsubsection{Zusammenfassung}

Im vorliegenden Kapitel LehrerInnenpersönlichkeit und Habitus im Kontext der Professionalisierung (Kap. II.1.2) wurde eingangs das Habituskonzept nach Bourdieu (2015a) als „Theorie der Praxis“vorgestellt. Anschließend erfolgte die Modelldarstellung mit den zentralen Begriffen Feld, Macht, Kapital, Subjekt, Objekt, Inkorporierung und Habitualisierung. Im Exkurs Unverfügbarkeit und Resonanz (Rosa 2018) konnten Parallelitäten und inhaltliche Ergänzungen zur Theorie Bourdieus (Bourdieu 1982, 2015a), insbesondere in Bezug auf die Phänomene der Interaktion und Inkorporierung, dargestellt werden. Schließlich wurde das Habituskonzept Bourdieus auf die LehrerInnenprofessionalisierung angewandt (Kap. II.1.2.3), um als Conclusio die Kernidee der Rekonstruktion zu positionieren: Habitus als Konzept der Professionalität. Im Folgenden werden die Erkenntnisse über Kontingenz, Ungewissheit und Unverfügbarkeit (Paseka et al. 2018; Rosa 2018; Schrittesser 2011) des Kapitels II.1.1 sowie jene über Habitus im Allgemeinen und Habitus als Konzept der Professionalität im Speziellen (Kap. II.1.2) auf die spezifischen Phasen der LehrerInnenpraxis übertragen. Die Phasen der LehrerInnenpraxis finden sowohl während der Ausbildung im Rahmen der pädagogisch-praktischen Studien statt als auch nach Abschluss des Lehramtsstudiums im Rahmen des Induktionsjahrs, welches durch einen formellen Mentoring-Prozess begleitet wird. 


\section{Professionalisierung im Kontext von LehrerInnenpraxis}

In den folgenden Abschnitten wird die Professionalisierung von LehrerInnen im Kontext zur Praxis diskutiert. Nach der Darstellung von Unterschieden und Überschneidungen der pädagogischen und allgemeinen Professionalisierung (Kap. II.2.1) wird der Diskussionsfokus auf die Praxisphasen der Studierenden bzw. BerufsanfängerInnen gerichtet, um die berufliche und zeitlich kurze Phase des Übergangs im Sinne des mikrodynamischen ${ }^{16}$ Prozesses zu beleuchten (siehe auch Abb. 1). Im Kapitel II.2.2 Professionalisierung in den Praxisphasen der LehrerInnenbildung: Pädagogischpraktische Studien wird die Professionalisierung durch Forschendes Lernen (Kap. II.2.2.1), Reflexion über Unterricht (Kap. II.2.2.2) und Kooperation der AkteurInnen (Kap. II.2.2.3) dargestellt. Die unmittelbare Phase des Übergangs bzw. des Berufseinstiegs steht in II.2.3 Professionalisierung beim Berufseinstieg: Induktionsphase und Mentoring im Zentrum.

Nach einführenden Begriffsbestimmungen (Kap. II.2.3.1) erfolgt die Diskussion zu den HauptakteurInnen im Mentoring, MentorInnen und Mentees (Kap. II.2.3.2) sowie SchulleiterInnen (Kap. II.2.3.3), die zum Erfolg oder Misserfolg des Mentoring-Konzepts u.a. in administrativer Hinsicht wesentlich beitragen. In Kapitel II.2.3.4 werden theoretische Modelle, Wirksamkeit, Determinanten und Ziele des Mentorings vorgestellt und diskutiert, um schließlich den Status quo des Mentorings an österreichischen Schulen (Kap. II.2.3.5) darzustellen.

\subsection{Pädagogische und allgemeine Professionalisierung}

Um den Bereich des Berufseinstiegs zu erfassen und darzustellen, werden in der vorliegenden Arbeit drei Phasen berücksichtigt. Neben dem unmittelbaren Berufseinstieg (Induktionsphase bzw. erstes Dienstjahr) werden auch die Ausbildungszeit vorher und die Berufspraxis unmittelbar nach der Induktionsphase untersucht. Die Ausbildungsphase unmittelbar vorher ist für einen erfolgreichen Berufseinstieg ${ }^{17}$ ebenso relevant wie die Zeit während des Berufseinstiegs bzw. wie die unmittelbar nachher. Aufgrund der Erkenntnis, Habitus als Konzept der Professionalität zu verstehen (Kap. II.1.2.3.2), und der daraus abgeleiteten mehrstufigen, sukzessiven Habitualisierung, die letztlich vom individuellen Habitus zum professionellen Habitus führt, nimmt Professionalisierung viel Zeit in Anspruch. Die Dauer des Berufseinstiegs wird hier mit einem Jahr angenommen, was der Induktionsphase bzw. im alten LehrerInnenausbildungssystem im höheren Schulbereich dem Unterrichtspraktikumsjahr entspricht. In dieser Zeit findet das Mentoring statt, welches als Modell zur berufsbegleitenden Professionalisierung eingeführt wird. Das Mentoring ist ein formeller Begleitungsprozess von BerufsanfängerInnen durch MentorInnen während des ersten Dienstjahrs, dem Induktionsjahr. Diese Phase der Professionalisierung wird im Abschnitt Professionalisierung beim Berufseinstieg: Induktionsphase und Mentoring (Kap. II.2.3) diskutiert. Der Zeit unmittelbar nach dem Berufseinstieg entsprechen im Kontext dieser Arbeit das zweite und dritte Berufsjahr ${ }^{18}$.

16 Mikrodynamisch wird hier als Entwicklung, Habitualisierung, Professionalisierung etc. auf persönlicher Ebene der Berufsanfängerin bzw. des Berufsanfängers verstanden.

17 Der Fokus dieser Arbeit ist auf den erfolgreichen Berufseinstieg und die daraus abzuleitenden Gelingensbedingungen gerichtet.

18 Als Sample für die Untersuchung werden LehrerInnen mit bis zu drei Dienstjahren befragt. In den Interviews wird die Phase der Ausbildung retrospektiv analysiert, BerufsanfängerInnen im ersten Dienstjahr decken die Phase des unmittelbaren Berufseinstiegs ab, Lehrpersonen im zweiten und dritten Dienstjahr die Phase der ersten Praxis unmittelbar nach dem Berufseinstieg. 
Brandhorst et al. betonen die Notwendigkeit von Theoriebezügen, „damit eine kritische Distanz zum eigenen und fremden Handeln aufgebaut werden kann und beobachtete Praktiken nicht unreflektiert übernommen werden" (Brandhorst et al. 2018). Schulische Praktika, explizit auch Langzeitpraktika, tragen insofern nicht automatisch zur Professionalisierung angehender Lehrkräfte bei (ebd.). Die Annahme, Habitus als Konzept der Professionalität zu verstehen (Kap. II.1.2.3), und die dieser Arbeit zugrunde liegende Habituskonzeption nach Bourdieu (2015a) unterstreichen die Meinung von Brandhorst et al. (2018). Es wird bestätigt, dass in die Zeit der Professionalisierung neben dem praktischen Handeln vor der Klasse im Rahmen der Praktika und dem eigenen beruflichen Praxishandeln auch die theoriegeleitete Ausbildung im Rahmen der LehrerInnenbildung fällt. Die Triade aus professionellem Wahrnehmen, professionellem Denken und professionellem Handeln (Bourdieu 2015a; Bourdieu und Wacquant 1996; Lenger et al. 2013), siehe auch Kap. II.1.2.3, rückt in diesem Zusammenhang die theoriegeleitete Ausbildung mit reflexiven Inhalten ins Zentrum. Leonhard und Lüthi (2018, S. 183) weisen darauf hin, dass die Idee der Professionalisierung deskriptiv auf berufsbezogene Veränderungen „bei Individuen bzw. Studierenden des Lehrberufs" abzielt. Aufgrund der unterschiedlichen theoretischen Rahmungen sehen sie „gerade Professionalisierungsprozesse derzeit empirisch noch nicht vollständig [erfasst]“ (ebd. S. 183). Evans (2002, S. 124) bemerkt dazu: „[...] there remains much within this field of study that is unclear or imprecise [...] and the teacher development process is not clearly identified.“ Und die Autorin ergänzt im Sinne eines positiven Ausblicks: „Teacher development has emerged over the last decade as a recognised area of study." (ebd)

Zur Positionierung des Professionalisierungsdiskurses in der LehrerInnenaus- und -weiterbildung sowie in der LehrerInnenpraxis erscheint es sinnvoll, auf Unterschiede und Überschneidungen zwischen pädagogischer und allgemeiner Professionalität einzugehen.

\subsubsection{Unterschiede}

Zunächst liegt es nahe, die pädagogische Professionalität von der allgemeinen abzugrenzen, da es "grundlegende Differenzen“ (Schrittesser 2011) zwischen pädagogischen Berufen und klassischen Professionen gibt. Aufgrund der bestehenden Schulpflicht besteht zwischen den SchülerInnen und den Lehrpersonen ein völlig anderes Arbeitsverhältnis (ebd.) wie in einem Setting, wo sich der Klient oder die Klientin das professionelle Gegenüber (z.B. der/die PatientIn den/die Arzt/ Ärztin) selbst aussucht bzw. weitgehend aussuchen kann. Nairz-Wirth (2011) stellt in diesem Zusammenhang fest, dass Theorie und Praxis der Professionalisierung im Lehrberuf nach wie vor stärker kontrovers diskutiert werden, als dies bei anderen Professionen, z.B. Ärztinnen und Ärzten, Juristinnen und Juristen, der Fall ist. Einen Grund sieht die Autorin darin, dass im Vergleich zu den Feldern Medizin und Recht im Feld Schule international theoretisch und praktisch „höchst heterogen[e]" (ebd., S. 167) Strukturen vorliegen und es daher keinen wissenschaftlichen Konsens darüber gibt, „wie professionelle Autonomie von Lehrpersonen definiert werden soll“ (ebd.).

Ein wesentlicher Unterschied zu den genannten ,anderen“ Professionen besteht zudem in der Tatsache, dass es sich bei Schülerinnen und Schülern um „keine kontraktfähigen Klientinnen und Klienten “ (ebd.) handelt - zumindest nicht in der Sekundarstufe 1 und in den unteren Jahrgängen der Sekundarstufe 2. Die hierarchische, durch Macht ausgestattete Positionierung der Lehrperson gegenüber den Schülerinnen und Schülern betont im Arbeitsverhältnis zwischen Lehrperson und SchülerIn die Komponente der Abhängigkeit.

Dass die Lehrperson meist einem Kollektiv gegenübersteht und nur in Ausnahmefällen, wie Einzelgesprächen, einem einzelnen Schüler oder einer einzelnen Schülerin als Individuum gegenübersteht und unterschiedlich agiert, stellt einen weiteren Unterschied zu anderen, klassi- 
schen Professionen dar (ebd.). Schließlich ist pädagogisches Handeln nach vorne gerichtet, gewissermaßen prophylaktisch (ebd.), um stets situationsgerechte Lernbedingungen zu schaffen. Oevermann (2017) spricht in diesem Zusammenhang von der dritten Funktion pädagogischen Handelns, vom implizit Therapeutischen. Diese dritte Funktion ergibt sich daraus, dass sich neben der Wissens- und Normenvermittlung in der Schule durch den sozialen Kontext zwangsläufig eine Interaktionspraxis mit den Schülern einstellt. Diese ist angesichts des noch offenen Bildungsprozesses zumindest bis zur Adoleszenzreife für die spätere personale Integrität „objektiv folgenreich" (ebd., S. 146).

In Bezug auf das Alter bzw. infolge der großteils Nicht-Volljährigkeit der SchülerInnen ${ }^{19}$ eröffnet sich ein Querschnittsthema, welches ebenso den Unterschied der pädagogischen Professionalität zu anderen Professionen herausstellt, nämlich die Stellung und Relevanz der Eltern im pädagogischen Umfeld der Schule. Aufgrund der bereits thematisierten fehlenden Kontraktfähigkeit der SchülerInnen und der Schulpflicht, welche auch durch die Eltern gewährleistet wird, kommt diesen im Wirkungsfeld Schule im Vergleich zu anderen Professionen eine besondere, nicht von vornherein auf der Hand liegende, Aufgabe zu. Oevermann (2017) präzisiert, dass Kinder, sofern „nicht mündig im Sinne von prinzipiell autonomiefähiger Lebenspraxis“, auch nicht „vollgültig autonome Partner eines Arbeitsbündnisses [seien]“. Die Eltern übernehmen einerseits für ihre Kinder das Mandat eines „stellvertretenden Partner[s]“ in den schulischen Arbeitsbündnissen, andererseits sind sie für die Einhaltung der Schulpflicht ihrer Kinder verantwortlich (ebd., S. 175). Um diesem Spannungsverhältnis standzuhalten, dürfen die Eltern weder eine Koalition mit ihren Kindern gegen die Lehrpersonen (Mandat für das Kind), noch eine Koalition mit den Lehrpersonen gegen die Kinder (Verantwortung der Schulpflicht) eingehen. Das professionelle Handeln der Lehrperson besteht nun darin, „die Eltern in der Schwierigkeit dieser Aufgaben an[zu]erkennen und an[zu]nehmen“ (ebd., S. 175). Die Vertrauensbildung zwischen den Parteien SchülerInnen, LehrerInnen und Eltern ist eine entscheidende Aufgabe der professionellen Lehrperson, ebenfalls ein Unterscheidungsmerkmal zu herkömmlichen Professionen.

Nach der Diskussion von Unterschieden zwischen pädagogischer und allgemeiner Profession wird im Folgenden der Fokus auf Überschneidungen bzw. Gemeinsamkeiten gelenkt.

\subsection{2 Überschneidungen}

Schrittesser (2011, S. 104f) stellt Überschneidungen zwischen professionalisiertem Handeln allgemeiner Professionen und dem professionellen pädagogischen Handeln fest. Die inhaltlichen Überschneidungen identifiziert die Autorin darin, dass sowohl die pädagogischen als auch die allgemeinen Professionen für gesellschaftlich besonders relevante Themen zuständig sind. Eine weitere Analogie besteht darin, dass diese, für beide Seiten relevanten Themen krisenhaften Charakter haben und Standardlösungen nicht vorliegen. Die in diesem Zusammenhang auftretenden Kontingenzerfahrungen wurden im Abschnitt Ungewissheit als Teil der pädagogischen Profession (Kap. II.1.1) ausführlich diskutiert.

Den Aspekt der gesellschaftlichen Relevanz thematisiert auch Evans (2008): „Many interpretations [...] seem to focus on professionalism's being an externally imposed, articulated perception of what lies within the parameters of a profession's collective remit and responsibilities." Eine weitere, in der vorliegenden Arbeit noch eingehend zu diskutierende Analogie bzw. Über-

19 In Österreich ist das Alter der Volljährigkeit auf 18 Jahre festgesetzt ( $\$ 21 \mathrm{ABGB}$ ). Vorher ist die natürliche Person nicht voll geschäftsfähig und auf die Unterstützung der Eltern bzw. Erziehungsberechtigten angewiesen. Aufgrund dieser Altersgrenze sind SchülerInnen im Regelfall (ohne Klassenwiederholung) bis zur achten Klasse der Sekundarstufe auf die Unterstützung der Eltern rechtlich angewiesen. 
schneidung zwischen allgemeinen und pädagogischen Professionen ist das Konzept des Habitus als Professionalität im Abschnitt II.3. (Bourdieu 2015a; Lenger et al. 2013). Die Trias wahrnehmen, denken und handeln als Habitualisierung und Professionalisierung gilt im jeweiligen Feld. Die konkrete Interaktion, Resonanz und schließlich Inkorporierung (Bourdieu 1982, 2015a; Rosa 2018) erfolgt zwar feldspezifisch (hier pädagogisch oder allgemein), das Wesen des Habitus als Konzept der Professionalität mit dem Muster vom Wahrnehmen, Denken und Handeln bleibt jedoch identisch.

Schrittesser (2011) identifiziert fünf Strukturanalogien von pädagogischem und allgemeinem professionellen Handeln (Abb. 16). Diese Schnittmenge stellt „eine Art Selbstähnlichkeitsbezug“ (ebd.) des allgemeinen und pädagogischen Professionszugangs dar und dient als Rahmen für die in diesem Abschnitt weiteregeführte Diskussion über professionsspezifische Gemeinsamkeiten.

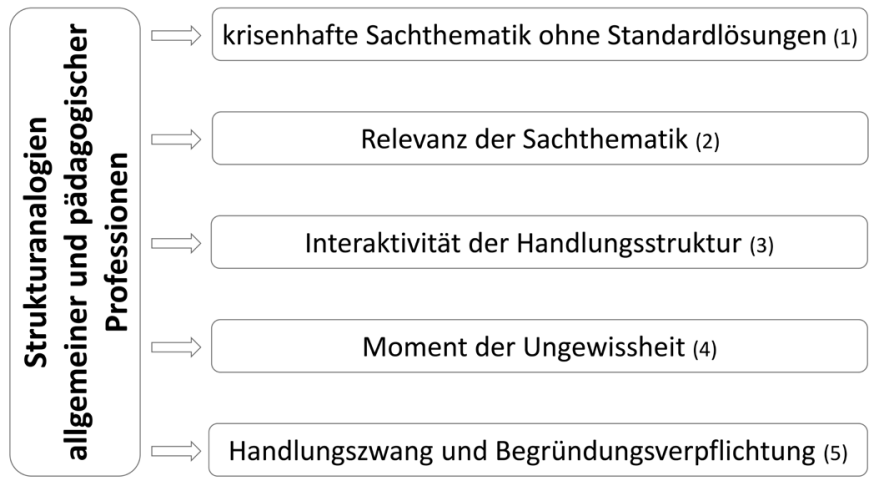

Abb. 16: Strukturanalogien allgemeiner und pädagogischer Professionen; eigene Abbildung nach Schrittesser (2011)

Schrittesser (2011) unterscheidet zwischen allgemeiner und pädagogischer Profession (Abb. 16). Die Autorin verzichtet auf eine detaillierte Unterscheidung innerhalb der „allgemeinen“ Professionen, zumal der Hauptfokus ohnehin auf die pädagogische gerichtet ist und sich der Erkenntnisgewinn im Gegensatz zur Komplexität bei weiterer Analyse (z.B. der juristischen oder medizinischen Profession) nicht erhöhen würde. Die Begründung kann in der Feldtheorie Bourdieus (Barlösius 2011; Bourdieu 1996a; Nairz-Wirth 2011) nachvollzogen werden, wonach sich zwar Subjekt und Objekt je nach Feld spezifisch habitualisieren, der Kern des Habitus als Konzept der Professionalität aber gleich bleibt: die Triade wahrnehmen, denken und handeln.

Die fünf Strukturanalogien nach Schrittesser (2011) setzen sich aus (1) krisenhafte Sachthematik ohne Standardlösungen, (2) Relevanz der Sachthematik, (3) Interaktivität und Handlungsstruktur, (4) Moment der Ungewissheit und (5) Handlungszwang und Begründungspflicht zusammen (Abb. 16). Einzelne Analogien bzw. Phänomene wurden bereits an anderer Stelle diskutiert (z.B. krisenhafte Sachthematik, keine Standardlösungen, Moment der Ungewissheit im Abschnitt Ungewissheit als Teil der pädagogischen Profession in Abschnitt I.1.1). Nachfolgend gehe ich auf jede einzelne Strukturanalogie nach Schrittesser (2011) ein. Bei Bedarf werden Bezüge zur bereits erwähnten Diskussion (Kap. II.1) hergestellt.

Zur Strukturanalogie (1) ist anzumerken, dass auch professionelle PädagogInnen (neben den anderen Professionen) für gesellschaftlich besonders relevante, krisenanfällige Sachthematiken zuständig sind, für die es keine Standardlösung gibt (ebd. S. 105). Die Thematik der Nicht-Standardisierbar- 
keit im pädagogischen Feld wird in der facheinschlägigen Literatur diskutiert. Unter anderen widmen sich Paseka (2011), Gruschka (2018) und Combe (2018) diesem Thema mit der gemeinsamen Erkenntnis, die Ungewissheit als Chance bzw. Herausforderung und nicht etwa als Hindernis oder Negativum in der pädagogischen Arbeit zu sehen. So schreibt Paseka (2011, S. 154) in Bezug auf die EPIK-Domäne Personal Mastery ${ }^{20}$, dass Professionelle im Allgemeinen und professionelle Lehrpersonen im Speziellen „Ungewissheitsstrukturen, die das Feld und die eigene Profession bestimmen, nicht nur aushalten, sondern aktiv balancieren", und bringt damit den bewussten Umgang mit Ungewissheit bzw. das Sich-zunutze-Machen der Ungewissheit im professionellen Feld ins Spiel.

Gruschka (2018) rückt die strukturelle Ungewissheit als Kern gehaltvoller Erziehungs- und Bildungsprozesse ins Zentrum dieses Diskurses. „Ohne Ungewissheit keine Bildungsprozesse“ (ebd., S. 15), lautet seine knappe Formel und er lenkt den Blick in Richtung Offenheit im System Schule. Deshalb spricht er explizit von der strukturellen Ungewissheit. Ungewissheit ist insofern positiv konnotiert und inspirierend und die Offenheit, welche die Unsicherheit ermöglicht - ich entscheide mich bewusst für das Ermöglichen, um die positive Konnotation zu unterstreichen -, belebt die Auseinandersetzung mit Sachverhalten, stiftet Neugier, Spannung und Erkenntnisinteresse (ebd., S. 15f). Rosa (2018) wählt in diesem Zusammenhang den Begriff der Unverfügbarkeit. Unverfügbarkeit liegt dann vor, wenn das Sichtbar- und/oder Erreichbar-, Beherrschbar- und Nutzbarmachen „von Welt“ (ebd., S. 23) nicht erreicht werden kann. Vordergründig löst dies Krisen (Combe 2018) bzw. krisenhafte Situationen ohne Standardlösungen (Schrittesser 2011) aus, auf längere Sicht besteht in dieser Kontingenzerfahrung, Ungewissheit oder Unverfügbarkeit (Paseka et al. 2018; Rosa 2018; Schrittesser 2011) die Chance zur individuellen Lösung, indem die schöpferische Dimension des Habitus (Bourdieu 2015a; Lenger et al. 2013) die jeweils passenden Lösung generiert. Außerdem stellt Unverfügbarkeit eine notwendige Voraussetzung für Interaktion und Resonanz dar (Rosa 2018). Beide Phänomene determinieren über die Inkorporierung (Bourdieu 2015a) den Habitus, der letztlich das Agieren und Reagieren ohne Standardlösungen gewährleisten kann. Aus dem Blickwinkel der Hermeneutik argumentiert Combe (2018, S. 81), dass man im „Vorhinein [...] nie ganz genau weiß, welcher Sinn bei den jeweils anderen ankommt“, und deshalb ortet er im Unterricht „den Zug zur schnellen Überwindung solcher Zonen der Unbestimmtheit“. Die Bedeutung des Gesprächs als hermeneutisches Werkzeug ist in diesem Zusammenhang neu zu justieren und dessen Kontingenz im Unterricht zu berücksichtigen. Der „Sinn für die Produktivkraft des Unverständlichen" muss entwickelt werden, so lautet das Resümee Combes.

Als zweite Strukturanalogie nennt Schrittesser (2011) die Relevanz der Sachthematik. Daraus leitet die Autorin das Erfordernis einer besonderen Expertise und einer erweiterten, wissenschaftlich fundierten Wissensbasis ab. Die Professionalisierungsbedürftigkeit ist für Oevermann (2017) allerdings nicht klar zu beantworten. Die kategoriale Differenz von Wissenschaft und Praxis, die sich in den Erfahrungswissenschaften deutlich ausprägt, verlangt eine idealisierte Abstraktion von konkreten Interessen im wissenschaftlichen Diskurs. Insofern konstituiert sich der wissenschaftliche Diskurs als „paradoxale unpraktische Praxis“ (ebd., S. 98f). Diese Differenz zwischen Wissenschaft und Praxis wird in der Untersuchung mehrfach diskutiert und stellt, um noch einmal auf die Unsicherheit im LehrerInnenberuf zurückzukommen, die BerufsanfängerInnen, aber auch die erfahrenen Lehrpersonen, vor große Herausforderungen. In vielen schulischen Situationen zeigt sich die methodologische Schwierigkeit, die Praxis als Forschungsge-

20 EPIK steht für Entwicklung von Professionalität im internationalen Kontext. Eine vom Bundesministerium für Unterricht, Kunst und Kultur (BMUKK) eingerichtete Arbeitsgruppe identifiziert fünf EPIK-Domänen zu Professionalität, welche als Grundlage für weitere Überlegungen und Empfehlungen in der Professionalisierungsdebatte im LehrerInnenberuf dienen (Schratz et al. 2008). 
genstand in die abstrahierte Wissenschaft aufzunehmen, sind diese doch ,untilgbar in der Praxis fundiert“ (ebd., S. 99). Diese Überschneidung zwischen Wissenschaft und Praxis bzw. zwischen Theorie und Praxis führt unmittelbar zur nächsten Strukturanalogie nach Schrittesser.

Der Anspruch der (praktischen) Fallorientierung setzt die Interaktivität der Handlungsstruktur (3) voraus. Sie ist typisch für pädagogische Professionalität, so Schrittesser (2011). Neben der fachlichen Expertise der Lehrperson, die für die Interaktivität auf der Handlungsebene in Bezug auf das jeweilige Unterrichtsfach als unbestrittene Voraussetzung angesehen werden kann, spielt im Kontext der Bildungswissenschaften die Empathiefähigkeit bzw. die Bereitschaft zur Empathie eine wesentliche Rolle. Die Beziehungsfähigkeit, ich sehe sie ebenfalls als Voraussetzung zur Interaktion an, kann aus der Perspektive der Lehrperson auf zwei Ebenen konkret sichtbar gemacht werden. Einerseits in der Beziehung zwischen LehrerIn und SchülerIn, andererseits zwischen LehrerIn und StudentIn. Poel und Heinrich (2018) sehen die LehrerIn-SchülerIn-Beziehung als für den Bildungserfolg essenziell. Eine Erkenntnis, die auch in der vorliegenden Untersuchung mehrfach bestätigt wird. Bemerkenswert in der Professionalisierungsdiskussion ist auch, dass die Autoren als Gelingensbedingung für die Beziehungsfähigkeit zwischen LehrerIn und Schülerin auf der LehrerInnenseite „eine stetige kritisch-reflektive Auseinandersetzung mit dem eigenen Handeln, den eigenen Gedankenmustern [...] kurzum mit der eigenen Persönlichkeit“ (ebd., S. 223) verdeutlichen. Ich sehe in diesem Sinne nicht nur die Beziehungsfähigkeit und die daraus resultierende Interaktionsfähigkeit als Maß des Professionalitätsgrads, sondern schon den Weg dahin, die Bereitschaft, sich zu verändern, sich weiterzuentwickeln, sich auf Unsicherheiten einzulassen.

Stichweh (1992) thematisiert die Interaktionsabhängigkeit der Beziehung zwischen Professionellen und Klienten aus systemtheoretischer Sicht und stellt dies als Kernproblem der pädagogischen Tätigkeit dar. Die bereits diskutierte Tatsache, dass sich SchülerInnen im Normalfall ihre Lehrpersonen nicht aussuchen können, und umgekehrt, ist nur ein Argument für die Belastung der LehrerIn-SchülerIn-Beziehung. In Bezug auf die Habitusdiskussion (Bourdieu 1982, 2015a; Bourdieu und Wacquant 1996) in Abschnitt I.1.2 mit dem großen Einfluss des jeweiligen Feldes wird der Gedanke Stichwehs noch transparenter. Interaktion und Resonanz (Bourdieu 2015b; Rosa 2018) finden zwischen Subjekt und Objekt statt, die jeweils mit Macht und Kapital ausgestattet sind. Treffen Subjekte und Objekte mit unausgewogenen Macht- und Kapitalressourcen aufeinander, was z.B. bei professionellen Lehrpersonen und schulpflichtigen Kindern zutrifft, können Interaktion und Resonanz ungünstig beeinflusst werden. Auch auf der Ebene zwischen LehrerIn (Praxislehrperson) und StudentIn bzw. zwischen MentorIn und VertragslehrerIn in der Induktionsphase spielt die Beziehung resp. die Beziehungsfähigkeit eine zentrale Rolle. In der schulpraktischen Ausbildung wird diese initiiert, in der Induktionsphase intensiviert und gepflegt. Allerdings sind die Macht- und Kapitalverhältnisse (Bourdieu 1982) auch hier nicht ausgewogen, zumal ein natürliches Macht- und Kapitalgefälle aufgrund des Dozierenden- bzw. Studierendenstatus besteht. Der entscheidende Faktor ist die Interaktivität der Handlungsstruktur bzw. die Beziehung. Sie dient zur „Überwindung der Kluft“ (Patry Jean-Luc 2014) zwischen Praxislehrperson und StudentIn bzw. MentorIn und VertragslehrerIn in der Induktionsphase. ${ }^{21}$

Als vierte Strukturanalogie zu anderen Professionen nennt Schrittesser (2011) das Moment der Ungewissheit aufgrund der nicht verallgemeinerbaren Besonderheit jedes Einzelfalls, expliziert für pädagogische Situationen. Es herrscht Singularität und unüberbrückbare Differenz zum jeweils anderen. Auf die wiederholte Querschnittsthematik der Ungewissheit wurde im Abschnitt Ungewissheit als Teil der pädagogischen Profession (Kap. II.1.1) detailliert eingegangen. Trotz-

21 Die (angehenden) LehrerInnen befinden sich aus berufsbiografischer Sicht kurz vor dem Übergang in den Beruf (Schulpraktika) oder im unmittelbaren Übergang im Rahmen der Induktionsphase (erstes Unterrichtsjahr) (Abb. 1). 
dem sei an dieser Stelle noch einmal die Chance der Kontingenz bzw. Ungewissheit erwähnt. Im Sinne des professionellen Habitus können für „nicht verallgemeinerbare Besonderheiten“ (Schrittesser 2011) besondere, individuelle Lösungen als beliebig variable Handlungsschemata (Bourdieu 2015a; Bourdieu und Wacquant 1996) generiert werden (siehe auch Abb. 10: Generierung beliebig vieler Handlungsschemata).

Schließlich stehen PädagogInnen in der jeweiligen Situation unter Handlungszwang und unterliegen einer Begründungsverpflichtung (5) zur Legitimation der gesetzten Handlungen (ebd.). Keller-Schneider und Hericks (2014) halten in diesem Zusammenhang fest, dass das berufliche Handeln von Lehrpersonen für die SchülerInnen „in der Ganzheit ihrer Personen als potentiell krisenhaft und objektiv folgenreich anzusehen ist“. Selbst bezogen auf den Kernauftrag von Unterricht, denn auch hier greifen die Lehrpersonen im Zuge ihrer beruflichen Tätigkeit immer wieder in den Bereich der persönlichen Integrität der ihnen anvertrauten SchülerInnen ein. Aufgrund der vorhandenen Macht- und Kapitaldifferenzen im Feld Schulklasse (Barlösius 2011; Bourdieu und Wacquant 1996; Diaz-Bone 2010) stehen Lehrpersonen unter Beobachtung des Objekts (Bourdieu 1996a), konkret sind das SchülerInnen, KollegInnen, SchulleiterInnen, Schulaufsicht, Eltern. Die professionelle Lehrperson hat die Aufgabe, im Sinne von Handlungszwang und Begründungsverpflichtung, innerhalb der Klasse auf ausgewogene Interaktion und Resonanz (Bourdieu 1982; Rosa 2018) zu achten. Ein Ansatz, diese Herausforderung professionell zu lösen, liegt in der Kernidee der vorliegenden Arbeit, Habitus als Konzept der Professionalität zu verstehen. Professionelles Wahrnehmen (PW), professionelles Denken (PD) und professionelles Handeln (PH) mit den jeweiligen Merkmalen Art der Wahrnehmung je nach individuellem Habitualisierungsgrad (PW), wissensbasierte, professionsspezifisch reflektierte Verarbeitung des Wahrgenommenen (PD) und theoriegeleitetes, reflektiertes Agieren und Reagieren (PH) (siehe auch Abb. 15: Professionelle Habitualisierung) sind nach den Erkenntnissen der vorliegenden Arbeit ein Schlüssel für professionelles LehrerInnenhandeln, auch in Bezug auf die Strukturanalogien Handlungszwang und Begründungsverpflichtung.

Nach der Einführung in die Professionalisierung von Lehrpersonen im Kontext von LehrerInnenpraxis und der Gegenüberstellung von Unterschieden und Überschneidungen zwischen allgemeiner und pädagogischer Profession in den vorangegangenen Abschnitten (Kap. II.2 und II.2.1) wird im Folgenden der Fokus auf zwei konkrete Praxisphasen gerichtet. Zum einen auf die Professionalisierung Studierender bzw. angehender Lehrpersonen im Rahmen der pädagogisch-praktischen Studien während der LehrerInnenausbildung (Kap. II.2.2), zum anderen auf die Professionalisierung von LehrerInnen nach dem Lehramtsstudium beim unmittelbaren Berufseinstieg im Rahmen der Induktionsphase mit begleitendem Mentoring-Prozess (Kap. II.2.3).

\subsection{Professionalisierung in den Praxisphasen: Pädagogisch-praktische Studien}

Wie bereits eingangs festgehalten, bildet die Übergangsphase zum Berufseinstieg das Zentrum des Interesses der vorliegenden Arbeit. Die Ausbildung unmittelbar vorher mit den enthaltenen Praxisphasen dient bereits zur Professionalitätsentwicklung und wird in diesem Kapitel genauer in den Blick genommen. Allein die Vermutung, dass eine „kompetenzorientierte Ausbildung [...] ohne die frühe Einbindung der Praxis wohl nur schwer möglich [ist]“ (Kraler 2008a, S. 175) und Praktika oftmals im Sinne von Orientierungsphasen entscheidend für den weiteren Studienverlauf sind (Schneider \& Haas 2019), begründet die Diskussion dieser Phase der LehrerInnenprofessionalisierung. Der Umstand, dass gerade frühe schulpraktische Erfahrungen Einsichten liefern, „die individuelle Entwicklungsprozesse wesentlich beeinflussen“ (Kraler 2008a, S. 175), unterstreicht die Relevanz dieses Diskurses. Die Vermutung, dass sich 
die angesprochenen Entwicklungsprozesse über einen längeren Zeitraum ziehen und insofern berufsbiografisch wirken, macht die Dringlichkeit des Diskussionsfokus noch deutlicher.

Leonhard und Lüthi (2018, S. 183) behaupten ebenso, dass Praktika weit verbreitete Elemente des Studiums zum Lehrberuf sind und einen Beitrag zur Professionalisierung von Studierenden leisten. Wie Professionalisierung als Prozess theoretisch modelliert wird und wie solche Prozesse empirisch zu erfassen sind, „wird keineswegs einheitlich diskutiert“, so die Autoren. An der Leopold-Franzens-Universität Innsbruck fand und findet eine wissenschaftliche Diskussion zum Thema der Modellierung des Professionalisierungsprozesses im Rahmen der Schulprakti$\mathrm{ka}^{22}$ im Rahmen der Konzipierung des Curriculums (Leopold-Franzens-Universität Innsbruck 2016) und dessen Weiterentwicklung statt.

Poel und Heinrich (2018, S. 221) sehen in den pädagogisch-praktischen Studien neue Möglichkeiten, den Lernort Hochschule professionalisierungsförderlich mit der beruflichen schulischen Praxis angehender Lehrkräfte zu verknüpfen. So können bereits während der Ausbildungsphase die Professionalisierungsprozesse nicht nur bereits praxisreflexiv unterstützt und erforscht werden, sondern die Studierenden können auch bereits auf die im Kontext Schule über das fachliche Können hinausgehende, erforderliche pädagogische Professionalität vorbereitet werden. Die Praxisphasen im Lehramtsstudium zielen laut Brennecke et al. (2018, S. 39) darauf ab und bieten gewissermaßen auch die Chance, die schulpraktische und universitäre Ausbildung stärker miteinander zu verzahnen. Den Perspektiven Entwicklung von Reflexivität, Handlungsfähigkeit und forschungsbezogene Professionalisierung (ebd., S. 39) stellt sich die Herausforderung der dafür vorauszusetzenden Kooperation zwischen den Studierenden, den Praxis- und Hochschullehrpersonen und, nicht zu vergessen, den Schülerinnen und Schülern.

Liegmann et al. (2018) stellen einen Ansatz zur Professionalisierung in den Praxisphasen der LehrerInnenausbildung vor und sprechen von „drei Arten von Herausforderungen“ (ebd., S. 11) für alle beteiligten AkteurInnen, d.h. Studierende, Lehrende, MentorInnen, SchulleiterInnen, Hochschullehrende etc. Diese Herausforderungen sind die Professionalisierung durch (1) Forschendes Lernen, (2) Reflexion über Unterricht und (3) Kooperation der Akteure im Rahmen der pädagogisch-praktischen Studien (Abb. 17).

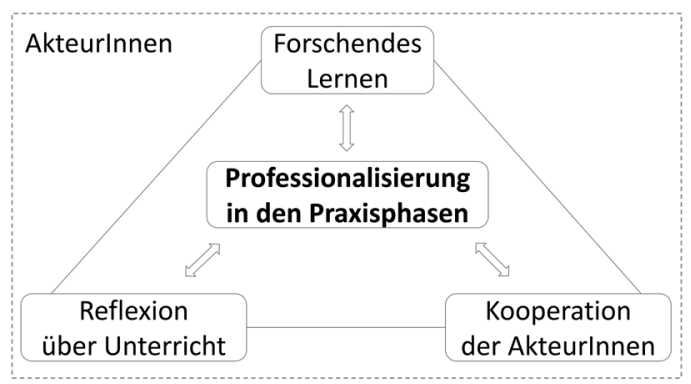

Abb. 17: Professionalisierung in den Praxisphasen; eigene Abbildung nach Liegmann et al. (2018)

22 Im Curriculum zur LehrerInnenausbildung NEU des Ausbildungsverbundes WEST Leopold-Franzens-Universität Innsbruck (2016) werden die Schulpraktika 1 bis 3 als pädagogisch-praktische Studien bezeichnet. Das ehemalige einjährige Unterrichtspraktikum im Curriculum der „alten“ LehrerInnenausbildung für die Sekundarstufe wird durch die an die Ausbildung anschließende Induktionsphase, begleitet durch MentorInnen, ersetzt. In der vorliegenden Arbeit werden die Begriffe Schulpraktika, pädagogisch-praktische Studien, Praktika, schulpraktische Studien synonym verwendet. 
Die AkteurInnen bilden den Rahmen, über sie findet Professionalisierung in den Praxisphasen statt. Studierende, Lehrende, MentorInnen, Schulleiterinnen und SchülerInnen schaffen zwischenmenschliche, kooperative etc. und letztlich inhaltliche Knotenpunkte. Insofern fokussieren die einzelnen Themen Forschendes Lernen, Reflexion über Unterricht und Kooperation der AkteurInnen zwar jeweilige Spezifika, sie greifen aber auch kontinuierlich ineinander und vernetzen sich. Das Ziel ist die Professionalisierung in den Praxisphasen, die Verbindung zwischen den einzelnen Bereichen erfolgt über die AkteurInnen. Mein Beweggrund, mich in der vorliegenden Arbeit am Ansatz Liegmann et al. (2018) zu orientieren, liegt in der Übertragbarkeit der „Herausforderungen“ (ebd.) Forschendes Lernen, Reflexion über Unterricht und Kooperation der AkteurInnen auf die Triade wahrnehmen, denken und handeln (Bourdieu 1982, 2015a) (Abb. 18).

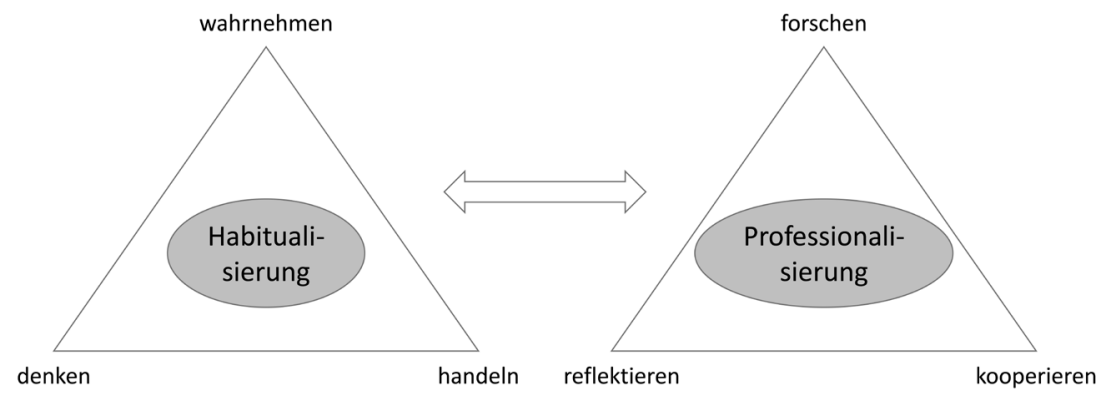

(Bourdieu 1982, 1996, 1998)

(Liegmann et al. 2018)

Abb. 18: Triaden der Habitualisierung und Professionalisierung in den Praxisphasen

Ausgehend von der im Abschnitt LehrerInnenpersönlichkeit und Habitus im Kontext der Professionalisierung (Kap. II.1.2) diskutierten Triade wahrnehmen - denken - handeln (Bourdieu 1982, 2015a), die den Motor der Habitualisierung darstellt, kann nach Liegmann et al. (2018) die Trias forschen - reflektieren - kooperieren als die zu bestehende „Herausforderung“ (ebd.) der Professionalisierung in den Praxisphasen betrachtet werden. Die Begriffspaare wahrnehmen forschen, denken - reflektieren und handeln - kooperieren decken jeweils gleiche bzw. ähnliche Handlungsfelder ab. Insofern kann die Trias forschen - reflektieren - kooperieren (Liegmann et al. 2018) dem Kerngedanken der vorliegenden Arbeit, wonach sich Habitualisierung durch wahrnehmen - denken - handeln konstituiert (Bourdieu 1982, 2015a) und letztlich Habitus als Konzept der Professionalität (Kap. II.1.2.3.2) dargestellt wird, untergeordnet werden.

Im Anschluss folgt die Diskussion der Bestandteile der Trias Forschendes Lernen, Reflexion über Unterricht und Kooperation der AkteurInnen (Liegmann et al. 2018) im Einzelnen, wobei Bezüge zur Triade wahrnehmen, denken und handeln (Bourdieu 2015a) hergestellt werden.

\subsubsection{Professionalisierung durch Forschendes $\operatorname{Lernen}^{23}$}

Pollmanns et al. (2018, S. 21) gehen der Grundfrage nach, ob und wie sich Professionalisierungsprozesse innerhalb schulpraktischer Studien „forschungsbezogenen Typ[s]“ vollziehen.

23 Durch die Etablierung und spezifische Verwendung dieses Terminus im Rahmen der LehrerInnenausbildung ist dieser mittlerweile zum Eigennamen avanciert. In dieser Arbeit und zum größten Teil auch im wissenschaftlichen Diskurs wird die Großschreibung des vorangestellten Partizips präferiert. 
Der forschungsbezogene Typ im Rahmen der schulpraktischen Studien hat sich als Forschendes Lernen herauskristallisiert und beschreibt eine forschungsbezogene Variante der bzw. innerhalb der pädagogisch-praktischen Studien. Das Forschende Lernen wird laut Pollmanns et al. (2018, S. 22) dadurch charakterisiert, dass die Studierenden in rekonstruktiven Methoden zur Erschließung der schulischen Wirklichkeit eingeführt werden und angehalten sind, Unterricht derart zu dokumentieren, dass sie ihn entsprechend der verwendeten Analysemethode rekonstruieren können. Dieses Fallverstehen in Bezug auf Unterrichtssequenzen, Unterrichtsgeschehnisse oder Interaktionen im Unterricht wird mit dem Ziel praktiziert, die Professionalisierung der Studierenden zu befördern.

An die Ausbildung bzw. an das Curriculum der LehrerInnenausbildung stellt sich die Herausforderung, wie das Forschende Lernen in das Studium konkret integriert wird, um die angestrebte Professionalisierung zu erreichen.

Nach Artmann und Herzmann (2018, S. 65) gehen die Konzeptionen Forschenden Lernens insofern von einer berufspraktischen Relevanz aus, als durch die Erforschung schul- und unterrichtsnaher Problemstellungen professionelles LehrerInnenhandeln angebahnt werden soll. In Anlehnung an das Habitualisierungskonzept Bourdieus (2015a) mit der Triade wahrnehmen - denken - handeln entspricht das Konzept des Forschenden Lernens dem Wahrnehmen im Sinne Bourdieus. Studierende der Universität Innsbruck führen im Rahmen ihrer pädagogisch-praktischen Studien und der begleitenden Lehrveranstaltungen an den Ausbildungsinstitutionen Projekte Forschenden Lernens durch, wo das Wahrnehmen im Zentrum steht, die Beobachtung des Unterrichts im Rahmen von Hospitationen. Der Beobachtung folgt eine systematische Protokollierung, womit in einem nächsten Schritt zur Analyse bzw. Reflexion übergegangen wird. Herzmann und Liegmann (2018, S. 75) identifizieren in ihren Untersuchungen mehrdeutige Zielbestimmungen des Forschenden Lernens.

Sowohl die Entwicklung der Reflexionskompetenz angehender Lehrpersonen als auch die Ausbildung berufsrelevanter forschungsmethodischer Fähigkeiten werden dabei angestrebt und je nach Stellung im Curriculum verändert sich der Schwerpunkt des Erkenntnisinteresses (Abb. 19).

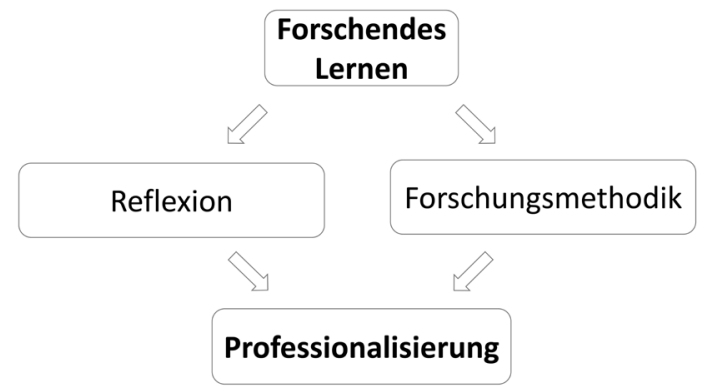

Abb. 19: Professionalisierung durch Forschendes Lernen; eigene Abbildung nach Herzmann und Liegmann (2018)

Forschendes Lernen setzt Kenntnisse über Forschungsmethodik voraus, um über die Wahrnehmung zur Reflexion zu gelangen. Die Kombination aus der Beherrschung der Forschungsmethodik und der Reflexion im Bereich des Forschenden Lernens (Abb. 19) trägt zur Professionalisierung der (angehenden) Lehrperson während der Praxisphasen bei. Boer (2012a) betont die Bedeutung der Beobachtung für Professionalisierungsprozesse. Damit etabliert die Autorin 
Beobachtung als Forschungsmethodik für Professionalisierungsprozesse und attestiert dem „geschulte[n] beobachtende[n] Blick“ (ebd. S. 309) das Durchbrechen von Automatismen und Routinen. Das „aufmerksame Wahrnehmen“ (Reh 2012) rückt ins Zentrum, um „Erkenntnisse über die Wirklichkeit“ (ebd., S. 21) zu erlangen und schlägt gewissermaßen die Brücke zum professionellen Wahrnehmen (siehe auch Abb. 15: Professionelle Habitualisierung) in der Kernidee des Habitus als Konzept der Professionalität (Bourdieu 2015a). Mit dem Blick auf LehrerInnen (Bennewitz 2012) und auf sich selbst (Boer 2012b) werden Reflexionsprozesse ausgelöst und insbesondere die Frage nach der „eigene[n] Perspektive“ (ebd., S. 223) und deren Veränderung machen StudentInnen nachdenklich, stellt die Autorin fest. Dies kann ein Hinweis auf die Initiierung des Denkens bzw. des professionellen Denkens in Bezug auf das Habituskonzept (Bourdieu 2015a) sein, vorausgesetzt die Verarbeitung des Wahrgenommenen erfolgt wissensbasiert, professionsspezifisch und reflektiert (siehe auch Tab. 1: Professionelles Wahrnehmen, Denken und Handeln). Reflexion (siehe auch Kap. II.2.2.2 Professionalisierung durch Reflexion über Unterricht) ist in diesem Sinne eine Metakompetenz, die in vielen Phasen der Professionalisierung eine entscheidende Rolle spielt. Die Kernidee der vorliegenden Arbeit, Habitus als Konzept der Professionalität, deutet darauf hin. Sowohl beim professionellen Denken als auch beim professionellen Handeln (siehe auch Abb. 15: Professionelle Habitualisierung und Tab. 1: Professionelles Wahrnehmen, Denken und Handeln) ist Reflexion ein wesentliches Element, das über den weiteren Verlauf im Professionalisierungsprozess mitentscheidet.

Boelhauve (2005) bemerkt, dass Studierende „bewusst in Distanz zu sich und [der] Umgebung“ (ebd., S. 105) treten, um Neues zu entdecken. Die Autorin spricht das professionelle Wahrnehmen (Bourdieu 2015a) der Kernidee Habitus als Konzept der Professionalität an und konkretisiert mit fünf Kriterien das Format des Forschenden Lernens, die sich mit der Triade der Habitualisierung bzw. der Professionalisierung (ebd.) in Einklang bringen lassen. Boelhauve (2005) stellt fest, dass (1) Aufbau einer bestimmten Haltung, (2) unterschiedliches Anspruchsniveau, (3) theoriegeleitetes Lernen, (4) berufsbiografische Gesichtspunkte und (5) Kontakt zwischen Lernenden und Lehrenden das Format und die Voraussetzung für das Gelingen des Forschenden Lernens charakterisieren (Abb. 20).

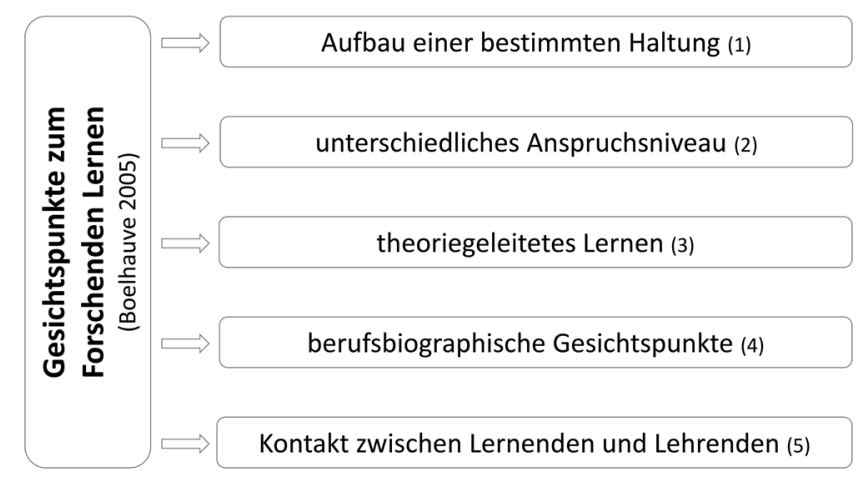

Abb. 20: Gesichtspunkte zum Forschenden Lernen; eigene Abbildung nach Boelhauve (2005)

Im Folgenden werden die einzelnen Kriterien bzw. Gesichtspunkte (Boelhauve 2005) diskutiert und mit dem Habitus- bzw. Professionalisierungskonzept nach Bourdieu (2015a) in Beziehung gesetzt. 
Grundsätzlich sieht Boelhauve den (1) „Aufbau einer bestimmten Haltung“ (ebd.) der Studierenden als zentrales Charakteristikum bzw. als Voraussetzung, sich mit den Feldern Schule und Unterricht wissenschaftlich auseinanderzusetzen. Die Felder Schule und Unterricht sind mit Macht- und Kapitalressourcen ausgestattet (Bourdieu 1996a), Interaktionen, Resonanz und schließlich die Inkorporierung (Bourdieu 1996a; Rosa 2018) werden durch die Positionierung von Subjekt (Studierende/r) und Objekt (Umfeld) determiniert. Die Position, die Initialisierung der Triade wahrnehmen - denken - handeln (Bourdieu 2015a), letztlich die Haltung der betroffenen Person wirkt sich auf die Professionalisierung der angehenden Lehrperson im Kontext des Forschenden Lernens aus.

Forschendes Lernen wird mit (2) unterschiedlichen Anspruchsniveaus verknüpft. Dies hebt den Prozesscharakter des Studienformats des Forschenden Lernens hervor und bestätigt die Untersuchungsergebnisse von Herzmann und Liegmann (2018) in Bezug auf die mehrdeutigen Zielbestimmungen je nach persönlichen Voraussetzungen und Zielen (siehe auch Abb. 19: Professionalisierung durch Forschendes Lernen). Studierende entwickeln, habitualisieren und professionalisieren sich individuell. Die sukzessive Habitualisierung zum individuellen Habitus (siehe auch Abb. 14: Sukzessive Habitualisierung über Inkorporierung und Lernprozesse) erfolgt in vielen individuellen Schritten. Der jeweils persönliche Lernprozess impliziert persönliche, unterschiedliche Anspruchsniveaus.

Forschendes Lernen ist (3) „theoriegeleitetes Lernen“ (Boelhauve 2005, S. 106). Ein differenziertes Verständnis von Theorie und Praxis wird etabliert und vor dem Hintergrund geeigneter Theorieansätze werden neue Sichtweisen auf Phänomene der Schul- und Unterrichtswirklichkeit erworben. Die wissensbasierte, theoriegeleitete Ausbildung ist eine wesentliche Voraussetzung für die professionelle Habitualisierung, konkret für das professionelle Denken und professionelle Handeln in der Triade professionelles Wahrnehmen - professionelles Denken - professionelles Handeln (Bourdieu 1982, 1996b, 2015a) (siehe auch Abb. 15: Professionelle Habitualisierung). Ebenso wird die wissenschaftlich-reflexive Kompetenz (Helsper 2018; Hericks et al. 2018; Kraler 2008b) theoriegeleitet erarbeitet und als Gelingensbedingung für die Entwicklung eines professionellen LehrerInnenhabitus angesehen (siehe ebenso Abb. 15: Professionelle Habitualisierung). Forschendes Lernen, so Boelhauve (2005), soll sich unter (4) berufsbiografischen Gesichtspunkten als persönlich bedeutungsvolles Lernen erweisen. Das bedeutet, im Kontext eigentätig durchgeführter Erkundungen bisher für wahr gehaltene Sichtweisen über Schule und Unterricht aufzugeben, zu modifizieren oder, mehr noch, durch etwas anderes zu ersetzen. Artmann und Herzmann (2018) halten in diesem Zusammenhang fest, dass die persönlichen Vorerfahrungen der Studierenden bei der Bearbeitung der Vorgaben eine wesentliche Rolle spielen. Auch bei der wissenschaftlich orientierten Bearbeitung von Aufgabenstellungen im Forschenden Lernen zeigt sich, dass die „Erfahrungsbasierung nicht verschwindet und dass keine Verwissenschaftlichung im Sinne eines Ablöseprozesses der Vorerfahrungen durch wissenschaftliche Zugänge stattfindet" (ebd., S. 72). In Bezug auf den laut Boelhauve (2005) zu berücksichtigenden berufsbiografischen Gesichtspunkt stellen Herzig und Grafe (2005) fest, dass sich Lehramtsstudierende in ihren Voraussetzungen von anderen Studierenden u.a. dadurch unterscheiden, dass sie als „ehemalige Schülerinnen und Schüler bereits über vielfältige Erfahrungen in ihrem späteren Berufsfeld verfügen“ (S. 151). Die AutorInnen geben allerdings zu bedenken, dass diese „vermeintlich gute Kenntnis“ (ebd.) mitunter auch dazu führe, Annahmen über Lehr- bzw. Praxislehrpersonen und deren berufliches Handeln „nur allzu schnell“ (ebd.) aus der eigenen, ehemaligen SchülerInnenperspektive zu verallgemeinern. An dieser Stelle wird deutlich, dass sich die Habitualisierung vom individuellen zum professionellen LehrerInnenhabitus entwi- 
ckeln muss. Je nach Feld (Bourdieu 1996a), konkret Ausbildung und Berufspraxis (siehe auch Abb. 12: Konstitution des Feldes LehrerInnenbildung), erfolgt die bereits diskutierte sukzessive Habitualisierung vom individuellen zum professionellen LehrerInnenhabitus.

Schließlich stellt Boelhauve (2005, S. 107) fest, dass sich Forschendes Lernen (5) in direktem Kontakt mit Lernenden und Lehrenden ereignet und deshalb mit ethischen Leitlinien verknüpft werden muss, um die Wahrung der persönlichen Integrität aller Beteiligten zu garantieren. Der Professionalisierungsprozess der Studierenden im Rahmen des Forschenden Lernens startet bereits mit der Themenwahl für das Projekt. Herzmann und Liegmann (2018) zeigen in ihren Studien diesbezüglich auf, welche Forschungsgegenstände die Studierenden bearbeiten und diskutieren davon ausgehend, welches Verständnis von Forschung im Kontext Forschenden Lernens darin zum Ausdruck kommt. An dieser Stelle kommt den Hochschullehrenden ${ }^{24}$ eine besondere Bedeutung zu, die im Abschnitt Professionalisierung durch Kooperation der AkteurInnen (Kap. II.2.2.3) thematisiert wird. Die Annahme, die „forschende Grundhaltung [sei] ein Teil der Professionalisierung" (Brandhorst et al. 2018) erfordert die Vermittlung von Kompetenzen im direkten Kontakt zwischen Lehrenden und Lernenden. Dabei handelt es sich um Forschungsmethoden und deren regelgerechte Anwendung, die Beachtung wissenschaftlicher Gütekriterien und die Einhaltung methodologischer und forschungsethischer Regeln (ebd., S. 95). Neben der sorgfältigen Themenwahl im Forschenden Lernen, diese beeinflusst Forschungsinteresse und -methodik unmittelbar, sind Interaktionen und Resonanzen (Bourdieu 2015b; Rosa 2018) im Feld wesentliche Gelingensfaktoren für ein erfolgreiches Forschungsprojekt im Sinne der praxisorientierten Professionalisierung als Lehrperson.

Zorn (2018) erwähnt in Bezug auf den Kontakt zwischen Lernenden und Lehrenden, dass das Forschende Lernen als ein mögliches vernetzendes Element innerhalb der LehrerInnenausbildungsorganisationen bisher "noch keine verbindliche Funktion übernommen hat" (ebd., S. 286). Die Autorin ortet Vorbehalte seitens der Studierenden und Unsicherheiten in der Institution Schule. Mangelnde Transparenz und Kommunikation auf Einzelebene und zwischen den Institutionen Universität und Schule sind für Zorn dafür ausschlaggebend und weisen auf einen diesbezüglichen „Entwicklungsbedarf“ (ebd.) hin. In diesem Zusammenhang werden die Relevanz der Feldtheorie (Bourdieu 1996a) sowie die Wechselwirkung der schulischen Felder (siehe auch Abb. 11: Wechselwirkung zwischen den Feldern im schulischen Kontext) deutlich. Einerseits ist auf Macht- und Kapitalverhältnisse im Feld (Barlösius 2011; Bourdieu und Wacquant 1996; Diaz-Bone 2010), andererseits auf Interaktionen und Resonanz (Bourdieu 2015a; Rosa 2018) zu achten, um die notwendige Transparenz und Kommunikation zu gewährleisten. Nach dem Forschen im Forschenden Lernen als Pendant zum Wahrnehmen in der Triade wahrnehmen, denken und handeln (Bourdieu 2015a) wird im nächsten Abschnitt die Reflexion im Sinne von denken (ebd.) diskutiert.

\subsubsection{Professionalisierung durch Reflexion über Unterricht}

In diesem Abschnitt wird der Zusammenhang zwischen Reflexion und Professionalisierung diskutiert. Es wird der Frage nachgegangen, inwiefern Professionalisierung durch Reflexion über Unterricht in Praxisphasen der LehrerInnenbildung initiiert bzw. gefördert werden kann. Eingangs stehen allgemeine Überlegungen zur Reflexion im Mittelpunkt, anschließend wird auf die Theorie-Praxis-Verknüpfung eigegangen, um schließlich konkret auf die Reflexion in Unter-

24 Im Lehramtsstudium der PädagogInnenbildung NEU (Ausbildungsverbund West) sind DozentInnen aus den Ausbildungsinstitutionen Universität und Pädagogische Hochschule tätig. Mit der Bezeichnung „Hochschullehrende“ sind Dozierende beider Ausbildungsinstitutionen angesprochen. 
richtsbesprechungen, die einen Teil der Ausbildung im Rahmen der pädagogisch-praktischen Studien ausmachen, einzugehen.

Führer und Heller (2018) geben zu bedenken, dass empirisch noch weitgehend ungeklärt ist, was das Reflektieren im Gespräch ausmacht, und stellen zugleich fest, dass in Bezug auf den Reflexionsakt ein Perspektivenwechsel notwendig ist: vom Reflektieren als individuelle Denkanstrengung hin zu einer gemeinsam im Gespräch vollzogenen Aktivität. Dafür müssen aber „nicht nur die kognitiven, sondern auch die interaktiven Strukturen des Reflektierens“ (ebd., S. 115) in den Blick genommen werden. Es stellen sich die Fragen, wie es in den Gesprächen überhaupt zum Reflektieren kommt, wie sich die Beteiligten gegenseitig anzeigen, was und worüber reflektiert werden soll und mittels welcher kommunikativen Mittel schließlich das Reflektieren vollzogen wird.

Die Analysen von Führer und Heller zeigen, dass im Rahmen der Reflexionsgespräche über Unterricht in zwei Varianten reflektiert wird, in einer „Elementar- oder einer Ausbauvariante“ (ebd., S. 119). Während die elementaren Reflexionsgespräche auf Ebene der „rekonstruierende[n] und bewertende[n] Problemdarstellung " (ebd.) erfolgen, werden in der Ausbauvariante die dargestellten Probleme im Hinblick auf das Zustandekommen bzw. zur Identifikation möglicher Lösungen ergründet. Die Minimalbedingung für eine reflexive Auseinandersetzung, das Formulieren von Begründungen, wird laut Führer und Heller in der genannten Elementarvariante nicht oder nur selten erfüllt. Das „häufige Vorkommen der Elementarvariante“ (ebd., S. 127) deutet darauf hin, dass die Reflexion aus Sicht der Beteiligten auch aus einem Bewerten von Unterrichtsgeschehnissen bestehen kann. Dieses „Teilnehmer-Konzept“ (ebd.) steht allerdings in Diskrepanz zu „präskriptiven Reflexionsbegriffen“ (ebd.), wie sie von der Lehrerprofessionsforschung an Unterrichtsnachbesprechungen herangetragen werden. Dieses Teilnehmer-Konzept wird am Ende dieses Kapitels noch einmal diskutiert.

Haas und Schneider (2018) stellen in ihrer Studie über die Erkenntnisse von Studierenden der Sekundarstufe Allgemeinbildung in den pädagogisch-praktischen Studien fest, dass diese im Rahmen der Reflexionsgespräche „ihren Blick jeweils auch auf die eigene Person und das im Schulkontext persönlich vollzogene pädagogische Handeln “werfen. Dies gilt insbesondere für Fragen zum Ge- und Misslingen der jeweils eigenen Handlungstätigkeit. Dieser Umstand fordert einen bewertungs- und beurteilungsfreien Reflexionsraum ein, damit sich die Gespräche offen und von gegenseitigem Vertrauen geprägt gestalten.

Rosemann und Bonnet (2018) betonen in diesem Zusammenhang das vorliegende Hierarchieverhältnis zwischen MentorIn ${ }^{25}$ und Mentee und machen auf die Relevanz der bewertungsfreien Reflexionszonen aufmerksam, um die Hierarchie im Sinne offener Reflexionsgespräche nicht weiter zu begünstigen. Weiters geben die AutorInnen zu bedenken, dass MentorIn und Mentee „zumindest primär zwei unterschiedlichen Institutionen angehören“ (ebd., S. 132). Ob MentorIn und Mentee nun aus ihren jeweils institutionellen Logiken heraus „dieselben oder zumindest kompatible Ziele“ (ebd., S. 132) in den Gesprächen verfolgen und „gemeinsame Perspektiven auf Unterricht entfalten können“ (ebd., S. 133), bleibt offen. Wesentlich in Bezug auf den bewertungsfreien Raum und die kompatiblen Ziele ist ein professionelles MentoringSetting, das eine professionsspezifische Resonanz zulässt bzw. unterstützt. Die Trias der Resonanz aus Affizierung, Antwort und Transformation (Rosa 2018) führt letztlich zur Triade des

25 Der Begriff Mentoring wird in der vorliegenden Arbeit für Begleitung bzw. Begleitprozess verwendet, unabhängig davon, ob es sich um eine Praxisphase im Rahmen der pädagogisch-praktischen Studien oder den Mentoring-Prozess im Rahmen der Induktionsphase handelt. Insofern werden die Begriffe MentorIn und Praxislehrperson sowie Mentee und PraktikantIn synonym verwendet. 
Wahrnehmens, Denkens und Handelns (Bourdieu 1996b; Lenger et al. 2013), idealerweise zu professionellem Wahrnehmen, Denken und Handeln (siehe auch Abb. 15: Professionelle Habitualisierung und Tab. 1: Professionelles Wahrnehmen, Denken und Handeln). Ausschlaggebend für eine erhöhte Resonanzerfahrung ist die Interaktion zwischen Subjekt und Objekt im Feld (Bourdieu 1996a), was ebenso das Verhältnis zwischen MentorIn und Mentee in den Vordergrund stellt. Auch die Schulleitungen bzw. übergeordnete Organisationen, wie z.B. die Bildungsdirektion, beeinflussen die Feldgestaltung. Darauf wird in SchulleiterInnen im Professionalisierungsprozess von Lehrpersonen (Kap. II.2.3.3) näher eingegangen.

Zur Theorie-Praxis-Verknüpfung in Bezug auf die Professionalisierung in der LehrerInnenausbildung gehen Rosemann und Bonnet (2018) auf die Lösungsformel „Professionalisierung durch Praxisbezug" (ebd., S. 132) ein. So werden in der Professions- und Lehrerbildungsforschung die Fragen nach einer „sinnvollen Theorie-Praxis-Verknüpfung“ (ebd.) differenziert und durchaus kontrovers diskutiert. Für Führer und Heller (2018) steht außer Zweifel, dass zwischen den Phasen der universitären Theorievermittlung und der schulischen Unterrichtserfahrung in den Praktika der Reflexion eine „besondere Bedeutung“ (ebd., S. 114) zukommt, und unterscheiden in diesem Zusammenhang zwischen deskriptiven und präskriptiven Ansätzen der Reflexionsgespräche.

Auch für Herzig und Grafe (2005) führt der Weg von der Theorie zur Praxis „weder über einen naiven Transfer" (ebd., S. 157) noch über spezifische Transformationsregeln. Es kommt darauf an, Theorie und Praxis bzw. Wissenschaftswissen und Handlungssituationen in Relation zueinander zu setzen. Die eigentliche Reflexionsleistung der Studierenden besteht laut Herzig und Grafe (2005) darin, Wissenschaftswissen zu spezifischen Lehr- und Lernsituationen in Beziehung zu setzen (Abb. 21).

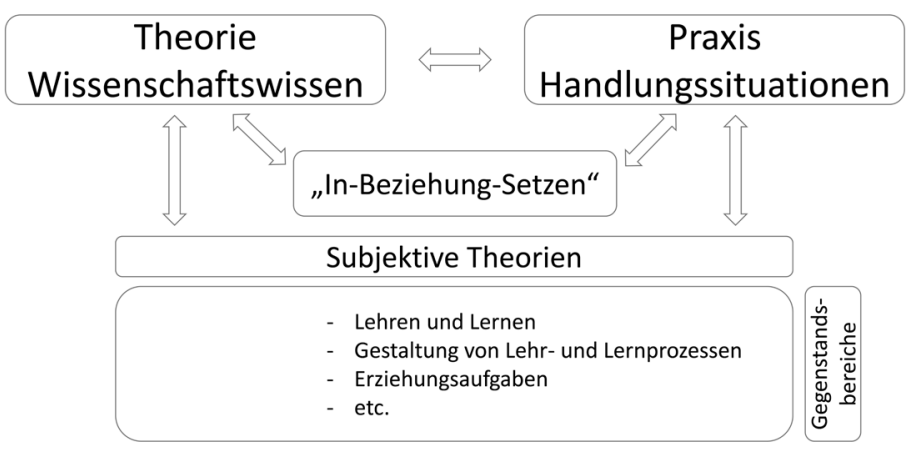

Abb. 21: Professionalisierung durch reflexives Lernen; eigene Abbildung nach Herzig und Grafe (2005, S. 158)

Ebenso werden Theorie und Praxis mit subjektiven Theorien verglichen und in ersten berufspraktischen Erfahrungen verifiziert oder falsifiziert. Dieses reflexive Lernen wird in verschiedenen Gegenstandsbereichen realisiert (Abb. 21).

Theorie als Wissenschaftswissen und Praxis in Form von Handlungssituationen wird in Beziehung gesetzt und in der Reflexion können subjektive Theorien abgeleitet werden. Diese subjektiven Theorien konstituieren sich in konkreten Gegenstandsbereichen, wie Lehren und Lernen, Gestaltung von Lehr- und Lernprozessen, Erziehungsaufgaben etc. Die Verknüpfung von Theorie und Praxis entspricht dann weder einem einseitigen Erwerb von Können mithilfe von Expertinnen und Experten (Mentorinnen und Mentoren) im Sinne einer Meisterlehre 
noch einem „isolierten Erwerb“ (ebd. S. 157) expliziten Wissens. Professionalisierung durch reflexives Lernen ist die Idee dieses Konzepts. An dieser Stelle wird der Bereich „denken“ der Triade wahrnehmen, denken, handeln (Bourdieu 2015a) sichtbar. Über das Wahrnehmen und das anschließende Denken in Form der Reflexion entwickelt sich idealerweise das professionelle Denken (siehe auch Abb. 15: Professionelle Habitualisierung), die Ausbildung des professionellen LehrerInnenhabitus bzw. der Professionalität im weiteren Sinne wird eingeleitet.

Ein weiteres Thema in Bezug auf die Theorie-Praxis-Verknüpfung im Rahmen der Professionalisierung durch Reflexion ist die Habitualisierung (Helsper 2018) bzw. die Chance der „Herausbildung eines wissenschaftlich-reflexiven Habitus" (Rosemann \& Bonnet 2018) durch Mentoring-Gespräche, wenn diese als Experimentierfeld ohne die bereits oben diskutierte hierarchische Gesprächskultur gesehen werden. Auch Kraler (2008b), Helsper (2018) und Hericks et al. (2018) verweisen auf die wissenschaftlich-reflexive Kompetenz, mithilfe derer ein professioneller LehrerInnenhabitus durch professionelles Wahrnehmen, Denken und Handeln erreicht wird.

Aufgrund der unterschiedlichen Institutionszugehörigkeiten der Akteurinnen und Akteure (Studierende, Mentorinnen und Mentoren, universitär Lehrende) läge es für Rosemann und Bonnet (2018) nahe, die Unterscheidung zwischen einem routinierten Habitus der Praxis und einem wissenschaftlichen Habitus der Reflexion in Mentoring-Gesprächen zu verwenden. Aus ihren Untersuchungsdaten geht allerdings hervor, dass es vielmehr „das Verhältnis zwischen Habitus und Normen“" (ebd., S. 134) ist, das in Mentoring-Gesprächen verhandelt wird. Damit wird die Diskussion um die Facette der Normen reicher, die beispielsweise institutionalisierte Verhaltenserwartungen, mit denen sich Akteure konfrontiert sehen“ (ebd., S. 135), betreffen. Bohnsack (2017) sowie Rosemann und Bonnet (2018) unterscheiden in diesem Zusammenhang Identitäts- und Institutionsnormen. Unter Identitätsnormen wird die Auseinandersetzung mit von außen herangetragenen Fremdbildern und Normalitätsvorstellungen verstanden (Rosemann und Bonnet 2018), Institutionsnormen definiert Bohnsack als „Erwartungen der Lehrpersonen an das, was von ihnen innerhalb der Institution Schule erwartet wird“ (ebd., S. 161). Er spricht deshalb vom Begriff der „Erwartungserwartungen“ (ebd.).

Leonhard und Lüthi (2018) stellen fest, dass Studierende in den Praktika mit einer „Vielzahl und Vielfalt berufsbezogener Normen“ (ebd., S. 197) in Kontakt kommen. Die „sprachliche Mühe“ (Rosemann und Bonnet 2018), diese argumentativ zu begründen, ist für Rosemann und Bonnet ein Indiz für den nicht bzw. noch nicht vollständig ausgebildeten Habitus der Praxis und dem der wissenschaftlichen Reflexivität. „Das“, konkretisieren die AutorInnen weiter, „zeigt mögliche Grenzen der [...] in der Lehrerbildung angestrebten Förderung eines wissenschaftlich-reflexiven Habitus auf“ (ebd., S. 145), wenn dieser einer Konfrontation mit der Praxis nicht standhält. Zum Verhältnis von Norm und Habitus im Kontext der Professionalisierung durch reflexives Lernen wird festgehalten, dass mit der Fokussierung der Analyse eben dieser Begriffe ein zentraler Aspekt der Mentoring-Gespräche erfasst wird. Das Professionalisierungspotenzial von Mentoring liegt laut Rosemann und Bonnet (2018) in der Auseinandersetzung mit von außen herangetragenen Verhaltenserwartungen und der eigenen Handlungspraxis als angehende Lehrperson. Im Sinne Bourdieus (1996a) handelt es sich hier um eine feldspezifische Habitualisierung (Barlösius 2011), wo Unverfügbarkeit (Rosa 2018) aufgrund von Macht- und Kapitaldifferenzen (Bourdieu und Wacquant 1996) Interaktion und Resonanz sowohl als Herausforderung als auch als Potenzial in Erscheinung treten lassen. Nach der Diskussion der Theorie-Praxis-Überschneidungen im reflexiven Lernen richtet sich im folgenden Abschnitt der Fokus auf die konkrete Situation der Unterrichtsbesprechung. Diese stellt einen wesentlichen Aspekt in den pädagogisch-praktischen Studien im Rahmen der LehrerInnenausbildung dar. 
Führer und Heller (2018) betrachten die sequenzielle Organisation des Reflektierens in Unterrichtsbesprechungen ausgehend vom Verständnis des Reflektierens als interaktive Praktik zwischen den AkteurInnen, den Studierenden und Lehrenden. Insgesamt weisen die empirischen Befunde der Autoren darauf hin, dass es in Unterrichtsnachbesprechungen „nicht von selbst zum Reflektieren kommt“ (ebd., S. 117). Dafür bedarf es einer interaktiven Vorbereitung und Unterstützung der Mentorinnen und Mentoren. Für Leonhard und Lüthi (2018) scheinen die Studierenden bei den Praktikumsnachbesprechungen „eher Teilnehmende als Akteure“ (ebd., S. 197) zu sein, ähnlich dem oben bereits diskutierten „Teilnehmer-Konzept“ (Führer \& Heller 2018). Diese Erkenntnisse unterstreichen die Notwendigkeit der Initiierung eines interaktiven Reflexionsprozesses durch die Professionellen, die MentorInnen, und generieren gewissermaßen eine Verantwortung seitens dieser, die angesprochene Interaktivität in den Unterrichtsnachbesprechungen einzufordern, um letztlich Gespräche mit Reflexionscharakter zu erhalten. Rosemann und Bonnet (2018) sprechen von ,an den Leistungsstand [des betreffenden Mentees] angepasste[n] Hilfestellungen“ (ebd., S. 132) durch die Mentorin oder den Mentor, die es dem Studierenden ermöglicht, die „Zone der nächsten Entwicklung" (ebd.) zu durchschreiten und in der Folge die Kompetenz zu erweitern. Insofern kommt den MentorInnen nicht nur die Rolle zu, ihr Erfahrungswissen an die Mentees weiterzugeben, sondern auch und vor allem, „adaptiv auf deren Bedürfnisse einzugehen“ (ebd.). Zusammenfassend halten Führer und Heller (2018) fest, dass die Reflexion im Rahmen von Unterrichtsbesprechungen „keine individuell zuschreibbare Handlung“ (ebd., S. 127) entweder der Studierenden oder der Mentorinnen und Mentoren ist, sondern eine „gemeinsam hervorgebrachte [...] und organisierte Aktivität“" (ebd.).

Als Ausblick bzw. Anregung für die Mentorinnen und Mentoren legt Helsper (2001) großen Wert auf die „Nicht-Standardisierbarkeit des pädagogsichen Handelns“ (ebd., S. 10) und will diese als Kernelement des Lehrerhandelns begriffen sowie Irritationen und Ungewissheit als Normalfall empfunden wissen. „Nur so“, erläutern Rosemann und Bonnet (2018, S. 145), „kann sich eine Praxis des Hinterfragens und Begründens des eigenen Handelns etablieren, ohne dass dies als persönliches Versagen gedeutet würde." Der Auftrag an die MentorInnen, Irritationen und Ungewissheit zuzulassen, wird damit ebenso formuliert wie jener, auf die Defizitzuschreibung des oben erwähnten, teilweise unter Studierenden vorzufindenden „TeilnehmerKonzeptes" (Führer \& Heller 2018) zu verzichten, ja mehr noch, dies sogar anzuerkennen und als Ausgangspunkt für die gezielte Weiterentwicklung der Studierenden, die sich auf dem Weg zur Professionaliserung befinden, zu nutzen. Die Verantwortung zur Schaffung dieser Voraussetzungen liegt im Aufgabenbereich der MentorInnen. Konkrete kommunikative Mittel und Reflexionsgelegenheiten müssen initiiert und den Studierenden nachhaltig zugänglich gemacht werden (ebd.).

Nach der Diskussion zur Reflexion bzw. des professionellen Denkens der Professionalisierungstriade (siehe auch Abb. 15: Professionelle Habitualisierung) folgt im Anschluss der nächste logische Schritt, das professionelle Handeln im Sinne der Kooperation.

\subsubsection{Professionalisierung durch Kooperation der AkteurInnen}

Im folgenden Kapitel werden die Positionen der AkteurInnen und die Professionalisierung durch deren Kooperation dargestellt. Im Laufe des Professionalisierungsprozesses angehender Lehrpersonen sind die Studierenden die HauptakteurInnen im Rahmen der pädagogisch-praktischen Studien. Im Habitusmodell (Bourdieu 2015a; Lenger et al. 2013; Schumacher 2013) sind es die Subjekte, die ihrerseits mit Macht und Kapital ausgestattet sind. Die Lehrenden an den Ausbildungsinstitutionen Universität und Pädagogische Hochschule, die MentorInnen 
(Praxislehrpersonen an den Schulen oder auch Lehrende der Ausbildungsinstitutionen), SchulleiterInnen und die SchülerInnen sind ebenfalls AkteurInnen im Sinne des dieser Diskussion zugrunde liegenden Schemas nach Liegmann et al. (2018) (siehe auch Abb. 17: Professionalisierung in den Praxisphasen). Nach Lenger et al. (2013), Schumacher (2013) und Bourdieu (2015a) entsprechen diese AkteurInnen im Habitusmodell dem Objekt, der sozialen Umwelt. Über Interaktion und Resonanz (Rosa 2018) findet Kooperation statt.

In Abbildung 22 werden die am Professionalisierungsprozess während der Praxisphasen der LehrerInnenausbildung beteiligten AkteurInnen und die betreffenden Institutionen mit möglichen Kooperationsvarianten dargestellt. Es wird veranschaulicht, dass sich zwischen den Institutionen (Universität bzw. Pädagogische Hochschule und Schule) viele Kooperationsvarianten anbieten. Die institutionsinternen Möglichkeiten (z.B. Hochschullehrende - Studierende oder SchulleiterInnen - Praxislehrpersonen sind hier nicht berücksichtigt, aber ebenso möglich und wichtig). Auf institutioneller Ebene können die Ausbildungsinstitutionen der Studierenden (Universität und Pädagogische Hochschule) und die Schulen kooperieren (Abb. 22).

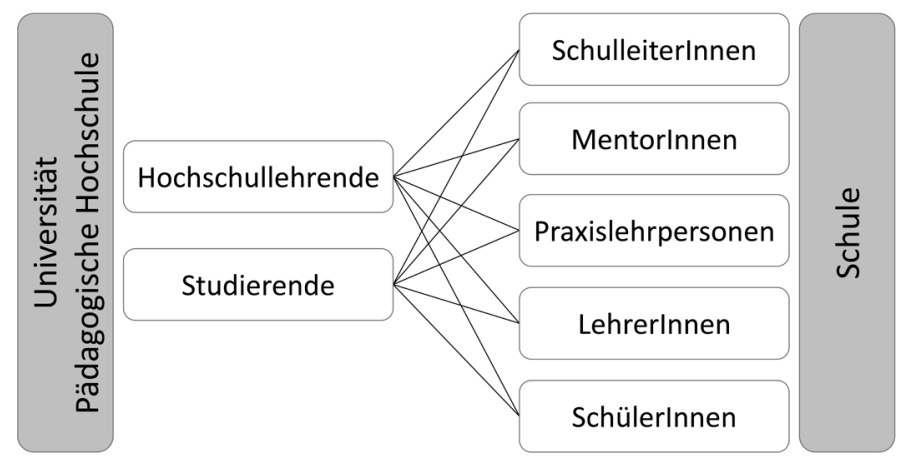

Abb. 22: Mögliche Kooperationsvarianten der Akteure zwischen den Ausbildungsinstitutionen

Auf personeller Ebene bieten sich Kooperationen sowohl von Hochschullehrenden als auch von Studierenden mit SchulleiterInnen, MentorInnen, Praxislehrpersonen, LehrerInnen und SchülerInnen an. Diese Darstellung erhebt nicht den Anspruch auf Vollständigkeit, es sollen lediglich die häufigsten und am nächsten liegenden Varianten gezeigt werden (Abb. 22).

Im Folgenden werden die Akteurinnen und Akteure in den verschiedenen Settings und den Phasen der Kooperation nicht immer alle explizit genannt, wenngleich sie im Sinne eines umfassenden Systems, das alle anderen mit einschließt, immer mit dabei sind (Luhmann 2015).

In den Phasen der LehrerInnenausbildung und explizit auch in den Phasen der pädagogischpraktischen Studien vollziehen die angehenden LehrerInnen wesentliche Entwicklungsschritte, die nach Hericks et al. (2018) gedanklich als Professionalisierung rekonstruiert werden können. Für eben diese Professionalisierungsprozesse in den Praxisphasen wird die Begleitung der angehenden LehrerInnen „als eine zentrale Gelingensbedingung“ (Zorn 2018) angesehen. Diese Begleitung erfolgt in erster Linie durch die MentorInnen und, wie die empirischen Ergebnisse dieser Arbeit zeigen, auch durch LehrerkollegInnen und die SchulleiterInnen.

Mit den pädagogisch-praktischen Studien wird laut Brennecke et al. (2018, S. 38) eine in das universitäre Studium eingelagerte Phase etabliert, die Professionalisierungsanforderungen von 
Universität, Pädagogischen Hochschulen und Schule u.a. durch das hochschuldidaktische Konzept der Forschenden Lernens verbindet. Allerdings „wird bei den Orientierungen bzgl. der Professionsverständnisse auch die Verschiedenheit der Institutionen deutlich“, so Zorn (2018). Dieses, für die Studierenden durchaus herausfordernde Spannungsfeld, das sich ,in der Gesprächsgestaltung sowie bei den Professionalisierungsverständnissen“ (ebd.) konstituiert, ist nicht lediglich durch die subjektiven Sichtweisen der Einzelpersonen begründet. Vielmehr sind die Unterschiede auf das ,jeweilige institutionelle Selbstverständnis und [die] entsprechenden Strukturmerkmale“ (ebd.) zurückzuführen. So basiert die Existenz der Ausbildungsinstitution Universität auf der Grundannahme, dass Professionalisierungsprozesse „gestaltet, begleitet und befördert" (ebd.) werden können, während sich laut Zorn bei schulischen Lehrkräften tendenziell eine Orientierung finden lässt, die Professionalisierung als einen automatisch ablaufenden Prozess durch Erfahrung im Handlungsfeld Schule versteht. Insofern bedarf es zur Kooperation der Ausbildungsinstitutionen „genauer Abstimmungsprozesse über Ziele und Selbstverständnisse“ (ebd., S. 288). Diese, so die Autorin weiter, sind „nicht nur in Steuergruppen auf einer übergeordneten Ebene, sondern auch auf Einzelebene bei der gemeinsamen Begleitung Studierender" (ebd.) notwendig.

Als möglicher Lösungsweg, gewissermaßen zur Professionalisierung der Professionalisierung, gilt es künftig, „Kooperationsprozesse sowohl auf der benannten übergeordneten als auch auf der Einzelebene in den Blick zu nehmen" (ebd.), um die Entwicklung des kooperativen Netzwerks verfolgen zu können. Dafür bieten sich allgemeine Gesprächssettings ebenso an wie in künftigen Forschungsfeldern auszuwertende Interviews mit den Beteiligten. Durch Letztgenanntes kann man der Erforschung der Kooperation in Bezug auf die „Multiperspektivität aller Beteiligten und der Eigenlogik der Institutionen“ (ebd., S. 287) gerecht werden.

Auf der Einzelebene zwischen den Studierenden und Lehrenden, der Mikroebene im Gesamtkomplex der universitären LehrerInnenbildung, ist es das programmatische Ziel, insbesondere in den Studien-Praxis-Projekten (pädagogisch-praktische Studien, Projekt Forschendes Lernen) „symmetrische Formen des Zusammenhandelns“ (Idel \& Kahlau 2018) zu ermöglichen, um bei den Studierenden die bereits diskutierte reflexive Haltung zu fördern. Mit dem Ziel der Professionalisierung und dem Setting, diese Projekte im Team mit einer symmetrischen, weitgehend gleichberechtigten und der bereits diskutierten auf Hierarchie und Beurteilungszwang verzichtenden Arbeitsbeziehung durchzuführen, wird nicht nur der wissenschaftlich-reflexive Habitus (Helsper 2018) gefördert, sondern die Studierenden gelangen in Zugzwang. Sie müssen sich aktiv gegenüber der Lehrkraft positionieren, um ihre Erwartungserhaltung an diese und an sich selbst zu reflektieren (Idel \& Kahlau 2018).

Poel und Heinrich (2018) identifizieren eine weitere Facette der Kooperation zwischen Studierenden und Lehrenden. Die Hochschullehrenden, seien es Lehrende in den universitären Kursen oder Lehrende bzw. Mentorinnen oder Mentoren in den pädagogisch-praktischen Studien, fungieren als „stellvertretende Krisenbewältiger für ihre Studierenden“ (ebd., S. 234), wenn es darum geht, den Studierenden Empathie und vor allem Persönlichkeitsbildung als Professionalisierungserfordernis bewusst zu machen. Diese kooperative Funktion als stellvertretender Krisenbewältiger nehmen Hochschullehrende gegenüber Studierenden in ähnlicher Form ein wie Lehrerinnen oder Lehrer gegenüber ihren Schülerinnen bzw. Schülern (ebd.). Die Hochschullehrenden haben die Chance, durch die jeweils eigene Beziehungsgestaltung zu ihren Studierenden auf deren Entwicklungsprozesse in Bezug auf Empathie und Persönlichkeitsbildung positiven Einfluss zu nehmen. „Dies stellt“, so Poel und Heinrich (2018, S. 235), „eine für jede in diesem Kontext tätige Person zugängliche und wenig aufwändige Möglichkeit dar." Hattie (2015) stellt einen besonders hohen 
positiven Einfluss der LehrerIn-SchülerIn-Beziehung in Bezug auf die Lernerfolge fest und identifiziert innerhalb der großen Kategorie Beziehung die stärksten positiven Zusammenhänge zwischen „personenzentrierten Lehrervariablen und Leistungsoutcomes“ (ebd., S. 142) im Bereich des kritischen und kreativen Denkens. Dieser Zusammenhang auf der Ebene LehrerInnen-SchülerInnen kann aus den empirischen Erkenntnissen der vorliegenden Untersuchung (Auswertungskategorie Institutionelle Ausbildung aus der Sicht der BerufsanfängerInnen/Curriculum/DozentInnen, BA_C_3_1, Kap. III.3.1: Haupt-, Sub-, Subsub- und Subsubsubkategorienbildung) vorsichtig auf die Ebene Hochschullehrende-Studierende übertragen werden, wobei das kritische und kreative Denken auf SchülerInnenebene dem bereits ausführlich diskutierten und für die Ausbildung des wissenschaftlich-reflexiven Habitus zugrunde liegenden Reflektieren (Liegmann et al. 2018) bzw. dem professionelle Denken (Bourdieu 2015a) entspricht.

Zur Beziehung und Empathie zwischen den Akteurinnen und Akteuren kann auch „Enthusiasmus“ (Helmke 2012) beitragen und der Autor hält fest, dass das Fehlen von jeglichem Enthusiasmus auf der Seite der Lernenden auf Dauer zu „Langeweile und Monotonie“ (ebd.) führen dürfte. Aber, und das kann auch als Hinweis für Hochschullehrende bezüglich der kooperativen Hingabe verstanden werden, „[es geht] nicht darum, ein Maximum sondern ein Opium von Enthusiasmus zu verbreiten" (ebd.).

Da sich die Studierenden während der pädagogisch-praktischen Studien weitgehend bewertungsfrei im Unterricht bewegen, haben sie damit laut Poel und Heinrich (2018) die Möglichkeit zur „bewussten Auseinandersetzung mit der eigenen Persönlichkeit und deren spezifischen Bedeutung für die Interaktion mit Schülerinnen und Schülern“ (ebd., S. 223). Diese gezielte Professionalisierung angehender Lehrpersonen mit Blick auf die Aufgabe der Beziehungsgestaltung auf der Kooperationsebene StudentIn-SchülerIn kann von den Lehrenden bzw. Mentorinnen und Mentoren durch die Hinführung zur Reflexion und die Unterstützung bei „beginnenden Transformationen von Handlungsmustern“ (ebd.) auf der nächsten Kooperationsebene Studierende bzw. Studierender-Lehrende bzw. Lehrender begleitet werden.

In Bezug auf Studierende als Akteurinnen bzw. Akteure bringen Wilfert und Thünemann (2018) deren „Voreinstellung“ (ebd., S. 216) ins Spiel und verweisen darauf, dass sich eben diese sowie auch das Vorwissen auf die Kompetenzentwicklung von Lehramtsstudierenden auswirkt. Die Autoren stellen die Praxisphasen als komplexe Lern- und Ausbildungsangebote dar. Letztlich kann die erwähnte Voreinstellung als Ausgangsbasis für das spätere Kooperationsverhalten herangezogen und die Kompetenzentwicklung als Teil der Professionalisierungsphase gesehen werden.

Hericks et al. (2018) identifizieren bei Studierenden während der universitären Praxisformen bzw. während der pädagogisch-praktischen Studien parallel laufende Professionalisierungsprozesse. Gemeint ist die „mehrfache Reflexivität“ (ebd., S. 257) im Umgang mit fachlichen, fachdidaktischen und bildungswissenschaftlichen Wissensbeständen sowie mit dem für das spätere Kooperationsverhalten essenziellen eigenen Selbst. Zugleich zielen die Praktika „auf eine Habitualisierung ab“ (ebd.), wenngleich die AutorInnen feststellen, dass die Frage, „wie sich diese mehrfache Reflexivität erforschen lässt [...], ob die Befunde dann als Habitualisierung rekonstruiert und [...] als Professionalisierungsprozess“ (ebd.) bewertet werden können, bislang methodologisch weitgehend ungeklärt ist. Erwähnenswert in der Diskussion des Kooperationsverständnisses bzw. -vermögens der Studierenden erscheint auch die Erkenntnis, dass sich der LehrerInnenhabitus in einem komplexen Bezug zu vorgängigen Habitusformen, dem familiär erworbenen Herkunftshabitus, dem Schülerhabitus und dem in der Gesamtbiografie eigen erworbenen Habitus bildet (ebd., S. 256). Die sukzessive Habitualisierung über Inkorporierung und Lernprozesse (Bourdieu 2015a; Hericks et al. 2018; Nairz-Wirth 2011) und schließlich 
die Entwicklung des professionellen LehrerInnenhabitus aufgrund wissenschaftlich-reflexiver Kompetenzen (Helsper 2018; Hericks et al. 2018; Kraler 2008b) (siehe auch Abb. 15: Professionelle Habitualisierung) wurden im Abschnitt Übertragung des Habituskonzepts auf LehrerInnenprofessionalisierung (Kap. II.1.2.3) ausführlich diskutiert.

Als Resümee wird festgehalten, dass das Professionalisierungsschema in den Praxisphasen nach Liegmann et al. (2018) mit den Bereichen Forschendes Lernen, Reflexion über Unterricht und Kooperation der AkteurInnen auf die Habitualisierungstheorie nach Bourdieu (2015a) mit der Triade wahrnehmen, denken und handeln übertragen werden kann. Die Diskussion führt über fünf Gesichtspunkte zum Forschenden Lernen (Boelhauve 2005) zur Professionalisierung durch reflexives Lernen, indem Wissenschaftswissen in Beziehung zu Handlungssituationen gebracht wird (Herzig \& Grafe 2005). Die Kooperation der AkteurInnen ist durch Interaktion und Resonanz (Rosa 2018) determiniert und schließlich sind, wie auch in der späteren Berufspraxis, bereits in den Praxisphasen im Rahmen der Ausbildung wissenschaftlich-reflexive Kompetenzen (Helsper 2018; Hericks et al. 2018; Kraler 2008b) notwendig, um aus der individuellen in die professionelle Habitualisierung zu gelangen.

Nach der Diskussion zur Professionalisierung in den Praxisphasen der Ausbildung liegt im nächsten Abschnitt Professionalisierung beim Berufseinstieg: Induktionsphase und Mentoring, der inhaltliche Schwerpunkt auf der Professionalisierung bereits ausgebildeter Lehrpersonen im unmittelbaren Übergang in den Beruf.

\subsection{Professionalisierung beim Berufseinstieg: Induktionsphase ${ }^{26}$ und Mentoring}

Der Übergang von der Ausbildungsphase in den Berufbzw. in die Berufseinstiegsphase ist national und auch international nicht einheitlich definiert (Greiner 2019), weshalb sich Schwierigkeiten hinsichtlich dessen empirischer Erfassung ergeben. In der vorliegenden Arbeit wird der unmittelbare Übergang von der Ausbildung in den Beruf bzw. die Berufseinstiegsphase mit dem ersten Dienstjahr, der Induktionsphase, definiert (siehe auch Abb. 1: Berufseinstieg als Übergang). Die Berufseinführung bzw. die Berufseinführungsphase und das dazugehörige formelle MentoringProgramm für Lehrpersonen sind der Professionsforschung mit Fokus auf den Übergang vom Studium in den Beruf zugeordnet (ebd.). Die ersten Berufsjahre stellen eine „besonders sensible Phase dar" (Kraler et al. 2017, S. 202), in der BerufsanfängerInnen über Einführungsprogramme „nachhaltig und wirkungsvoll unterstützt werden [können]“ (ebd.).

In diesem Kapitel wird das Professionalisierungskapital der Berufseinstiegsphase in Verbindung mit dem formellen Mentoring-Programm untersucht.

\subsubsection{Einführung und Begriffsbestimmungen}

Aus historischer Sicht geht das Mentoring bis in das zwölfte vorchristliche Jahrhundert zurück, als Odysseus seinem Freund und Gefährten namens Mentor die Erziehung seines Sohnes Telemach übertrug (Ziegler 2009). Der Ausflug in die Geschichte ist aufschlussreich. Mentor war ein väterlicher Freund, kluger Ratgeber und aufmerksamer Beschützer. Diese Eigenschaften finden sich zumindest teilweise im heutigen Verständnis der MentorInnenrolle und es gibt unzählige

26 Seit dem Wintersemester 2016 werden alle LehrerInnen der Sekundarstufe gemeinsam an Pädagogischen Hochschulen bzw. Universitäten im Rahmen der PädagogInnenbildung Neu (Leopold-Franzens-Universität Innsbruck 2016) im Bachelor- und Masterstudium ausgebildet. Sie sind dazu verpflichtet, bei Diensteintritt eine einjährige Induktionsphase zu durchlaufen, die durch eine Mentorin bzw. einen Mentor begleitet wird. Diese verpflichtende Induktionsphase mit formellem Mentoring-Begleitprozess gilt auch für neu eintretende LehrerInnen im Bereich der Primarstufe (Bundeskanzleramt 2013). 
Definitionen von Mentoring, die alle eine unübersehbare Ähnlichkeit zum historischen Mentor aufweisen, etwa in der Betonung einer persönlichen, dyadischen, hierarchischen Beziehung, die auf die Förderung des Lernens, der Entwicklung und das Vorankommen der Mentee ${ }^{27}$ bzw. des Mentees durch die Mentorin bzw. den Mentor ausgerichtet ist (Ziegler 2009). Dass aus dem Telemach die bzw. der Mentee wurde, könnte als historische Inkonsequenz gewertet werden und ist insofern an dieser Stelle auch erwähnt. Der „Mythos des Mentors“(ebd.) wurde in bekannten Beispielen realisiert. So war Aristoteles für Alexander den Großen ebenso Mentor wie Johann Christian Bach für Wolfgang Amadeus Mozart (ebd.). Diese prominenten Vorreiter mit ehemals erfolgreichem Mentoring-Programm können ein gutes Omen für die Implementierung der Induktionsphase mit dem begleitenden Mentoring-Programm im Hier und Jetzt sein.

In Bezug auf den LehrerInnenberuf kann der Berufseinstieg als eigene Phase identifiziert werden, da sich in ihr das Professionswissen der BerufsanfängerInnen von einem „regelgeleiteten, wenig vernetztem Wissen hin zu einem Richtlinien und Prinzipien folgenden Wissen, welches das Handeln steuert“" (Keller-Schneider und Hericks 2014, 390) entwickelt. Die Herausforderung für die beteiligten AkteurInnen ${ }^{28}$ besteht darin, eine derartige Phase zu etablieren, die die Professionalisierung der BerufsanfängerInnen fördert. Der Prozess des Lehrerwerdens ist ein komplexer, krisenhafter und individueller, der sich aus dem Zusammenspiel von person- und situationsspezifischen Faktoren ergibt (ebd.). In diesem Zusammenhang ist das zu implementierende Induktionsjahr mit dem formellen Einführungsprogramm Mentoring für BerufsanfängerInnen zu einem drängenden Umsetzungsthema geworden. Induktion und Mentoring sind derzeit in Österreich viel diskutierte Begriffe und Fragen der konkreten Umsetzung der neuen Berufseinführungsphase, der Induktionsphase, hängen mit der Gesamtreform der LehrerInnenbildung zusammen. Diese wird seit 2016 als PädagogInnenbildung NEU im Verbundstudium zwischen den Universitäten und den Pädagogischen Hochschulen durchgeführt (Kraler et al. 2017; Kraler \& Reich 2019). Bei der Umsetzung der genannten ersten Berufsphase, dem Berufseinstieg für BerufsanfängerInnen, geht es auch um schulorganisatorische und rechtliche Klärungen, in welcher Beziehung Schulpraktika während der Ausbildung und das neu eingeführte Induktionsjahr zueinander stehen (Greiner 2019). Im Zuge der Implementierung der Reform der PädagogInnenbildung NEU in Österreich werden strukturelle Veränderungen in Bezug auf die konkrete Gestaltung des Berufseinstiegs für BerufsanfängerInnen initiiert, die den AkteurInnen im Feld teilweise weder begrifflich noch inhaltlich ausreichend bekannt sind. Schulleitungen und angehende MentorInnen sind mit einer Tradition der formellen Berufseinführung, welche sich nicht mehr im Kontext von Ausbildung und Praktikum darstellt, noch nicht vertraut. Insofern ist es schwierig, die Themen rund um Induktionsphase und begleitendes Mentoring bei der vorhandenen Skepsis positiv zu besetzen (ebd., S. 82), wenngleich, wie weiter unten ausgeführt wird, diesem Berufseinstiegsprogramm ${ }^{29}$ ein großes Potenzial in Bezug auf die weitere Professionalisierung der BerufsanfängerInnen zugesprochen wird. Hier wird insofern von der „weiteren“ Professionalisierung gesprochen, als Professionalisierung im Gesamtfeld LehrerInnenbildung stattfindet (siehe auch Abb. 12: Konstitution des Feldes LehrerInnenbildung; Kap. II.1.2.3.1). Der Professionalisierungsprozess beginnt über Interaktion, Resonanz und Inkorporierung (Bourdieu 1996a, 2015a; Rosa 2018). Die Triade wahrnehmen, denken und handeln (Bourdieu 1996a, 2015a; Lenger et al. 2013) beginnt bereits im Teilfeld Ausbildung und wird in den Teilfeldern Berufspraxis bzw. Weiterbildung fortgesetzt.

27 Betreute im Mentoring-Prozess (Duden 2017).

28 BerufsanfängerInnen, Mentorinnen und Mentoren, LehrerInnen, SchulleiterInnen, Ausbildungsinstitutionen, Abteilungen und Ministerien auf Landes- bzw. Bundesebene etc.

29 Induktionsphase mit formellem Mentoring-Programm 
Mit der Frage, inwieweit es sich beim Induktionsjahr mit begleitetem Mentoring-Programm um einen Professionalisierungsprozess im Sinne des Erreichens eines LehrerInnenprofessionsstatus oder einen individuell gestalteten Berufseinstieg handelt, befasst sich die internationale TALIS-Studie ${ }^{30}$ (Schmich \& Itzlinger-Bruneforth 2019). Sie wurde von der Organisation für wirtschaftliche Zusammenarbeit und Entwicklung (OECD) initiiert und untersucht konkrete Rahmenbedingungen des schulischen Lehrens und Lernens aus der Sicht von Lehrerinnen und Lehrern sowie Schulleiterinnen und Schulleitern. Die empirischen Daten der Studie zeigen, dass der sichtbare Gestaltwandel der Einführungsphase von einer individualisierten Interaktionspraxis zwischen der neuen Lehrperson und deren MentorIn zu einem strukturell-systemisch verankerten Programm international zwar mehrheitlich, auf nationaler Ebene jedoch noch nicht vollzogen ist (Greiner 2019; Schmich \& Itzlinger-Bruneforth 2019). Zur genaueren Fassung des Begriffs der Einführungsphase verständigt sich der TALIS-Fragebogen, der an LehrerInnen und SchulleiterInnen ausgegeben wurde, auf „die Einführung neuer Lehrer[Innen] in den Lehrberuf sowie erfahrener Lehrer[Innen] an einer neuen Schule“ (Höller et al. 2019). Es kann festgehalten werden, dass sich die Einführungsphase für BerufsanfängerInnen, konkret die Induktionsphase mit begleitetem Mentoring-Programm, an österreichischen Schulen erst etablieren muss (Greiner 2019; Schmich \& Itzlinger-Bruneforth 2019). Dafür wird es auch nötig sein, dieses Bewusstsein bei SchulleiterInnen und (angehenden) MentorInnen zu schärfen. Die Pädagogischen Hochschulen als Anbieter der Hochschullehrgänge für die Ausbildung von MentorInnen sind in dieser Hinsicht besonders gefordert.

International variieren die Berufseinstiegsphasen und die begleitenden Mentoring-Programme stark (Greiner 2019). Die Einführungsphasen können in strukturierten Programmen ${ }^{31}$ informell arrangiert oder formell organisiert sein (ebd.). Der Hauptunterschied zwischen dem informellen und formellen Mentoring besteht in der Art und Weise der Bildung des MentorInMentee-Tandems, dem Matching. Beim informellen Mentoring kümmert sich ein Mentee in Eigeninitiative darum, eine passende Mentorin bzw. einen passenden Mentor zu finden. Die Initiative kann auch von der Seite der Mentorin bzw. des Mentors ausgehen. In formellen Mentoring-Programmen ${ }^{32}$ wird die Mentoring-Beziehung von einer Institution ${ }^{33}$, einem Netzwerk oder einem Unternehmen vermittelt (Schmid \& Haasen 2011). Ein weiterer wesentlicher Unterschied zwischen informellem und formellem Mentoring besteht darin, dass bei der formellen Variante regelmäßige und standardisierte Rückmeldungen bzw. Feedbackschleifen zwischen MentorIn und Mentee vorgesehen sind. So ist der Unterricht „zu analysieren und zu reflektieren" (Bundeskanzleramt 2013) sowie ein Entwicklungsprofil der Vertragslehrperson und ein Gutachten bzgl. des weiteren Verwendungserfolgs (ebd.) zu erstellen. Raufelder und Ittel (2012) stellen zur Existenz informellen und formellen Mentorings fest, dass in der Schule vielerorts verstecktes bzw. informelles Mentoring stattfindet, während das standardisierte und strukturierte informelle Mentoring noch keinen Platz gefunden, geschweige denn sich etabliert hat. Mit der neu eingeführten Induktionsphase für BerufsanfängerInnen wird die Richtung des formellen, standardisierten Mentorings eingeschlagen. In Bezug auf das Spannungsfeld zwischen der beschriebenen Standardisierung der Berufseinstiegsphase und durch Resonanz, Inkorporierung, letztlich Unverfügbarkeit (Bourdieu 2015b; Rosa 2018) charakterisierten Professionalisierung

30 TALIS steht für „Teaching and Learning International Survey“.

31 Mentoring

32 Die Induktionsphase für BerufsanfängerInnen ist ein formelles Mentoring-Programm.

33 Im Falle der Induktionsphase sind die jeweiligen Bildungsdirektionen für das Matching verantwortlich (LDG 1984, 01.09.2018). 
stellt Ziegler (2009) fest, dass Mentoring zu den spannendsten, aber auch verwirrendsten Themen der Pädagogik gehört und damit ambivalentes Potenzial hat.

Die Ambivalenz entsteht auch dadurch, dass Mentoring einerseits „die mit großem Abstand effektivste Methode sein kann“. Andererseits sorgen Metaanalysen, die Mentoring lediglich bescheidene Ergebnisse attestieren, für Verwirrung. „Insofern ist“, so der Autor, „keine verbindliche Leitdefinition in Sicht." (ebd.) Auch aufgrund der großen Bandbreite an angebotenen Mentoring-Programmen mit unterschiedlichen Zielsetzungen gibt es keine einheitliche Definition des Mentoring-Begriffs. Ziegler (2009) beschreibt Mentoring als „eine zeitlich relativ stabile dyadische Beziehung zwischen einem/einer erfahrenen Mentor/in und seinem/r/ihrem/r weniger erfahrenen Mentee“ (ebd., S. 11). In der TALIS-Studie (Schmich \& Itzlinger-Bruneforth 2019) wird Mentoring als „eine Unterstützungsstruktur an Schulen, bei der erfahrene Lehrpersonen weniger erfahrene Lehrpersonen unterstützen“, beschrieben.

Schmid und Haasen (2011) bezeichnen Mentoring als einen

„individuellen Lernprozess, in dem eine erfahrene Person (Mentor oder Mentorin) eine weniger erfahrene Person (Mentee) über einen längeren Zeitraum in ,Vier-Augen-Gesprächen' berät. Das können punktuelle Begegnungen sein, bis hin zu systematischen, in regelmäßigen Abständen geführten Gesprächen. Ziel ist es, für persönliche und berufliche Themen und Fragestellungen individuelle Lösungen zu finden und den Mentee in seiner persönlichen Entwicklung zu unterstützen.“

Die Betonung des Individuellen stellt die am Mentoring-Prozess beteiligten Personen und deren jeweilige Partizipation in den Mittelpunkt. Insofern gilt es nun, in den nächsten Abschnitten die beteiligten Personen im Mentoring-Prozess näher zu betrachten. Das sind in erster Linie die Betroffenen (Mentees) mit deren MentorInnen (Kap. II.2.3.2). Aber auch den SchulleiterInnen kommt für das Gelingen der Berufseinstiegsphase mit begleitetem Mentoring eine große Bedeutung zu (Kap. II.2.3.3).

\subsubsection{MentorInnen und Mentees: die ProtagonistInnen des Mentoring-Programms}

Im folgenden Abschnitt werden die Rollen der Mentees und MentorInnen und vor allem ihre Beziehung zueinander diskutiert. Keller-Schneider und Hericks (2014) sehen in der Phase des Berufseinstiegs eine aktiv handelnde und lernende Person (Mentee) mit ihrer aktiven sozialen Umwelt (MentorInnen und andere AkteurInnen) im Austausch. Diese zwischenmenschliche Beziehung von MentorIn und Mentee wird in neueren Definitionen z.B. als „Wechselseitigkeit und Komplexität der Beziehung" (Raufelder \& Ittel 2012) dargestellt. Zugleich werden die ursprünglichen hierarchischen Strukturen mehr und mehr aufgehoben (ebd.). Höller et al. (2019) stellen fest, dass in neueren Zugängen im Gegensatz zum Ausbildungsansatz die Beziehung zwischen MentorIn und Mentee nicht mehr als eine hierarchische, sondern als eine wechselseitige gesehen wird. MentoringProgramme, die diesen Zugang verfolgen, tragen zur „erfolgreichen persönlichen, sozialen und sozioemotionalen Entwicklung der Mentorinnen bzw. Mentoren und Mentees bei“ (ebd., S. 72). Ebenso dienen sie dem Professionalisierungsprozess und erfolgreichen Qualitätsmanagement im gesamten Bereich der Schule (ebd.). Ein wesentliches Merkmal des „neuen“ Mentoring-Verständnisses ist der Ansatz, dass beide, MentorIn und Mentee, von der „Beziehung als Kooperation“ profitieren (Raufelder \& Ittel 2012). Steinke und Putnam (2011, S. 43) drücken den Benefit für Mentees und MentorInnen im Mentoring folgendermaßen aus: „While assisting beginning teachers is the primary goal [...], experienced teachers who participated as mentors can also benefit from such programs." Insofern verstehen sich MentorIn und Mentee in der LehrerInnenbildung mehr und mehr als gleichberechtigt (Raufelder \& Ittel 2012). Schmid und Haasen (2011) erwähnen in diesem Zusam- 
menhang, dass „es meist nicht [stört]“ (ebd., S. 31), wenn die Mentorin bzw. der Mentor fachlich mit dem Gebiet des Mentees wenig vertraut ist. „Fachliche Nähe“, so Schmid und Haasen weiter, „kann sogar eher dazu führen, sich darin zu verlieren [...] und persönliche Themen nicht anzugehen." (ebd.) Die Begründung für diese Annahme liegt darin, dass die relevanten Themen im Mentoring Persönlichkeitsbildung, Kooperation und Besprechung von Strategien sind (ebd.). Auf den Mentoring-Prozess in der Induktionsphase übertragen, kann daraus abgeleitet werden, dass es für das Matching nicht relevant ist, ob das Unterrichtsfach des Mentees dem Fach der Mentorin bzw. des Mentors entspricht. Wesentlicher ist es laut Raufelder und Ittel (2012), gegenseitige Abhängigkeiten zwischen MentorIn und Mentee zu vermeiden, um die hierarchische Struktur zugunsten von Reflexionsgelegenheiten auf Augenhöhe zu gewährleisten. Die Bereitstellung der dafür konkret notwendigen kommunikativen Mittel, um den Weiterentwicklungsprozess der Mentees zu gewährleisten, liegt im Aufgabenbereich der Mentorin bzw. des Mentors, so Führer und Heller (2018). Es ist leicht nachvollziehbar, dass sich auch in Bezug auf die hierarchische Struktur das gleiche Unterrichtsfach von MentorIn und Mentee nachteilig auswirken kann. Sobald eine Mentorin bzw. ein Mentor das Amt der Fachvorständin bzw. des Fachvorstands oder der Fachkoordinatorin bzw. des Fachkoordinators ausübt, ist die beschriebene Hierarchie automatisch gegeben.

Neben den gesetzlichen Voraussetzungen ${ }^{34}$, als MentorIn eingesetzt zu werden, identifizieren Raufelder und Ittel (2012) weitere „pädagogische“ Merkmale, die eine Mentorin bzw. ein Mentor haben muss, um den Professionalisierungsprozess der BerufsanfängerInnen positiv zu beeinflussen. Diese Merkmale beziehen sich stark auf die Rollenausübung als MentorIn. Insofern verstehen sich MentorInnen selbst als LehrerausbildnerInnen und reflektieren ihr eigenes Handlungswissen. Sie teilen diese Reflexionsergebnisse den Mentees mit und tragen damit zur Entwicklung der beruflichen Werte und Einstellungen für die Mentees und für sich selber bei. MentorInnen müssen laut Raufelder und Ittel auch die Kompetenz entwickeln, Unterricht und LehrerInnenhandeln $\mathrm{zu}$ analysieren und sich auf akademische Ziele und Inhalte der LehrerInnenbildung zu beziehen.

Schließlich sind MentorInnen mittels guter Unterrichtspraxis für die Mentees ein Vorbild bezüglich der Integration von Theorie und Praxis.

Mit der Erfüllung der genannten Rollenanforderungen kann es unmittelbar zu „Fehldeutungen der MentorInnenrolle“ (Schmid \& Haasen 2011) kommen. So ist es nicht zielführend, wenn die Mentorin eine mütterliche bzw. der Mentor eine väterliche Rolle zur bzw. zum Mentee einnimmt und diese bzw. diesen als „erwachsenes Kind“ (ebd., S. 51) behandelt. In dieser Eltern-Kind-ähnlichen Konstellation fühlen sich zwar beide Seiten wohl, sie ist jedoch für das angestrebte persönliche Wachstum der Mentee/des Mentees hinderlich. Ebenso wenig hilfreich ist es, wenn Mentorinnen bzw. Mentoren versuchen, in den Mentees eigene Kopien zu erzeugen, gewissermaßen um sich selbst zu verwirklichen. Hier kann bereits im Matching darauf achtgegeben werden, nicht „in ihren Grundüberzeugungen sehr ähnliche“ (ebd.) Paarungen zusammenzuführen. Ebenso können Mentorinnen bzw. Mentoren in die Retter-Rolle verfallen und versuchen, Schwierigkeiten für die bzw. den Mentee zu lösen. Mit diesem Vorgehen wird die bzw. der Mentee abgewertet und die Chance, etwas zu lernen oder Neues auszuprobieren, geht verloren. Eine weitere Verkennung der Mentorinnen- bzw. Mentorenrolle liegt laut Schmid und Haasen auch dann vor, wenn die Men-

34 Mindestens fünfährige Berufserfahrung und Absolvierung des Hochschullehrgangs (HLG) „Mentoring, Berufseinstieg professionell begleiten“ (LDG 1984, 01.09.2018). Bis zum Jahr 2029/30 gilt eine Übergangsregelung: MentorInnen in den pädagogisch-praktischen Studien (Praxislehrpersonen) müssen einen 15-ECTS-HLG absolviert haben, MentorInnen in der Induktionsphase benötigen einen weiteren 15-ECTS-HLG, müssen insgesamt also eine 30-ECTS-Ausbildung vorweisen können. Ab 2030/31 ist für MentorInnen in der Induktionsphase eine 60-ECTS-Ausbildung im Rahmen eines Mentoring-Hochschullehrgangs vorgesehen (Bundeskanzleramt 2013). 
torin bzw. der Mentor als Karrierevermittler auftritt. Selbst erarbeitete Karriereschritte geben dem Mentee die notwendige Zeit für die persönliche Entwicklung.

Schmid und Haasen (2011) identifizieren drei Bereiche, die im Sinne eines professionellen Mentorings in der Verantwortung der Mentees liegen. So zeichnen sich Mentees durch (1) Aktivität und Initiative dahingehend aus, dass sie sich maßgeblich für die Richtung und Intensität des Mentoring-Prozesses verantwortlich fühlen. Aufgrund der bereits diskutierten, weitgehend aufgelösten Hierarchie bestimmen sie Themen und Fragen im Prozess aktiv mit. Der Bereich (2) Selbstverantwortung bedeutet für die Mentees die ständige Überprüfung des MentoringProzesses auf „gefundene Wege und Lösungen“ (ebd., S. 54) in Bezug auf Authentizität und Umsetzbarkeit für die bzw. den Mentee. Offene Gespräche mit der Mentorin bzw. dem Mentor gehören ebenfalls dazu wie, im Falle eines anhaltend nicht zufriedenstellenden Mentoring-Prozesses, Gespräche mit der Schulleitung oder der für den Prozess verantwortlichen Institution, der Bildungsdirektion. Schließlich, ausgehend von einem positiven Verlauf des Mentorings, liegt die (3) Umsetzung der gefundenen Erkenntnisse und Lösungen auch im Verantwortungsbereich der Mentees. In der Reflexion der Handlungsstrategien kann „das Tandem ${ }^{35}$ lernen, was [die bzw.] der Mentee wirklich braucht“" (ebd., S. 55).

Ausgehend von diesen Erkenntnissen, leiten Schmid und Haasen (2011) Voraussetzungen von Mentees im Sinne von Gelingensbedingungen für einen erfolgreichen Mentoring-Prozess ab. So müssen sich Mentees entwickeln wollen und den Willen und die Fähigkeit besitzen, eigenständig Initiative zu ergreifen. Die Aussage, Mentees „[... ] develop a vision of the kind of teacher they wanted to be" (Hanuscin und Lee 2008, S. 56), bringt sowohl die Entwicklung als auch die Eigenständigkeit der Mentees zum Ausdruck. Den Idealfall der MentorIn-Mentee-Kooperation formulieren die AutorInnen folgendermaßen: „Effective Mentoring works best when mentors and mentees share a vision of good teaching." (ebd., S. 57) Schmid und Haasen (2011) stellen zur Kooperation fest, dass MentorInnen einerseits die Verantwortung im gesamten Mentoring-Prozess übernehmen und andererseits das Entwicklungspotenzial der Mentees und deren Aktivität nutzen sollen.

Zusammenfassend wird festgehalten, dass sich im Mentoring-Prozess sowohl die Mentees als auch die MentorInnen im Sinne des Habituskonzepts (Bourdieu 2015a; Lenger et al. 2013) professionalisieren (Abb. 23).

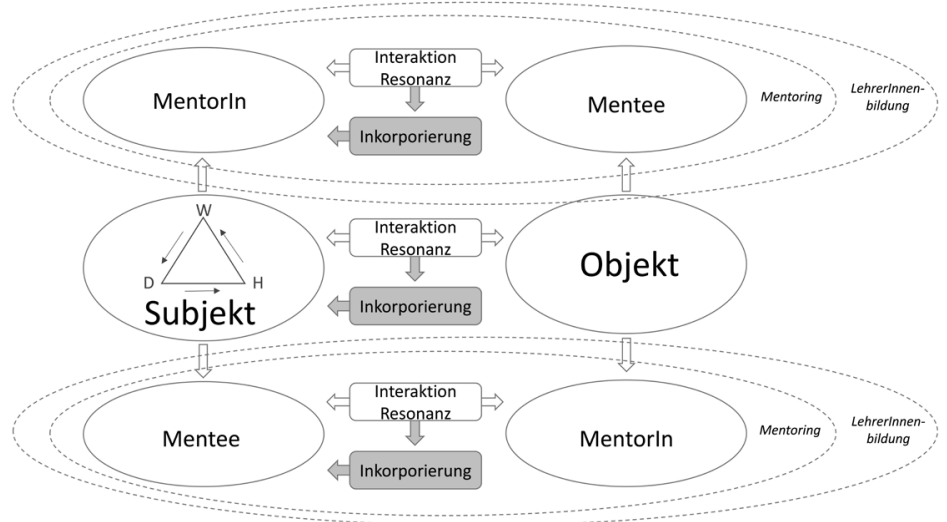

Abb. 23: Professionalisierung der Mentees und MentorInnen 
Mentees und MentorInnen sind mit Macht- und Kapitalressourcen ausgestattet (siehe neben Abb. 23 auch Abb. 5: Subjekt und Objekt interagieren und resonieren im Feld). Sie interagieren und resonieren (Rosa 2018) aufgrund von Unverfügbarkeit (ebd.) und Kontingenz (Paseka et al. 2018; Schrittesser 2011). Dies findet im Teilfeld Mentoring statt, welches in das Feld LehrerInnenbildung (siehe auch Abb. 12: Konstitution des Feldes LehrerInnenbildung) eingebettet ist. Durch differenzierte Macht- und Kapitalressourcen übernehmen Mentees und MentorInnen je unterschiedliche Aufgaben und Verantwortungen (Raufelder \& Ittel 2012; Schmid \& Haasen 2011). Trotzdem agieren Mentees und MentorInnen idealerweise weitgehend in hierarchisch aufgelösten Strukturen (Raufelder \& Ittel 2012) und gehen wechselseitige Beziehungen ein (Höller et al. 2019; Keller-Schneider \& Hericks 2014; Schmid \&Haasen 2011).

Das Phänomen der Inkorporierung (Bourdieu 1996b) betrifft Mentees und MentorInnen gleichermaßen, da beide Parteien zugleich Subjekt und Objekt sind. Insofern unterstützen als vordergründiges Ziel im Mentoring-Prozess die MentorInnen als Objekte die Mentees als Subjekte in der professionellen Habitualisierung im professionellen Wahrnehmen, Denken und Handeln. Gleichzeitig, und das kann als Benefit für die MentorInnen, als Win-win-Situation oder positiver Nebeneffekt gesehen werden, tragen die Mentees als Objekte zur weiteren Professionalisierung der MentorInnen (als Subjekte) bei.

Nach der Rollendiskussion von Mentee und MentorIn wird im folgenden Abschnitt der Einfluss der Schulleitung im Mentoring-Prozess näher betrachtet.

\subsubsection{SchulleiterInnen im Professionalisierungsprozess von Lehrpersonen}

SchulleiterInnen tragen als Führungspersonen an ihren Schulen u.a. die Verantwortung für den großen Bereich Schulentwicklung und Mentoring wird als ein Instrument dafür angesehen (Ittel \& Raufelder 2009). Im folgenden Abschnitt richtet sich der Fokus auf die Begleitung von BerufsanfängerInnen beim Berufseinstieg (Buhren \& Rolff 2011; Horster 2001), auf einen Teilbereich der Personalentwicklung.

Im Schulunterrichtsgesetz ist festgelegt, dass die Schulleitung die Lehrpersonen „in ihrer Unterrichts- und Erziehungsarbeit" (Bundeskanzleramt 1986) zu beraten hat. Diese Aufgabe gilt für SchulleiterInnen gegenüber allen Lehrpersonen im Kollegium. Darüber hinaus können sie gezielt für BerufsanfängerInnen durch administrative und organisatorische Maßnahmen eine gelingende Begleitung durch erfahrene KollegInnen bzw. MentorInnen in der Berufseinstiegsphase begünstigen (Buhren \& Rolff 2011). Dies geschieht z.B. durch einen strategisch geplanten Unterrichtseinsatz von BerufsanfängerInnen, indem sie nicht „als willkommene Verfügungsmasse betrachtet [werden], um Löcher im Stundenplan zu stopfen“ (Horster 2001, S. 142). Ebenso sind SchulleiterInnen bzw. AdministratorInnen ${ }^{36}$ dazu angehalten, die Stundenpläne der MentorInnen so zu konzipieren, dass Hospitationen, Feedback, formelle Nachbesprechungen etc. adäquat und für Mentees gewinnbringend umgesetzt werden können. Allerdings stellen Ittel und Raufelder (2009) fest, dass dieser zusätzliche bürokratische Aufwand seitens der Behörden durch Bereitstellung von Zeitressourcen mitgetragen werden muss, um die Implementierung einer formellen Berufseinstiegsphase mit Mentoring-Programm in das Schulsystem gewährleisten zu können. Weiters betonen Buhren und Rolff (2011), dass die Förderung von Kooperationen im LehrerInnenteam wesentlich zum gelingenden Berufseinstieg von Berufsan-

36 An großen Schulen, weitgehend sind dies Bundesschulen (AHS, BMHS), übernehmen AdministratorInnen die Stundenplanungen. 
fängerInnen beiträgt, indem ein Fachdiskurs z.B. über Unterrichtsgestaltung, Beurteilung von SchülerInnenleistungen sowie über organisatorische und erzieherische Herausforderungen geführt werden kann.

In diesem Zusammenhang kommt dem Matching, der Zusammenführung des Tandems MentorIn - Mentee, eine wichtige Bedeutung zu. Die Paarbildung im schulischen MentoringProzess folgt dem Muster eines externen Matchings (Schmid \& Haasen 2011), wo die Organisation das Tandem zusammenstellt und sich nicht Mentees oder MentorInnen selbst das Gegenüber aussuchen. Die Schulleitung resp. der Dienstgeber bildet das Tandempaar MentorIn - Mentee und muss im Sinne eines effektiven, weitgehend hierarchiefreien Mentorings auf Augenhöhe (Höller et al. 2019) einige Determinanten für einen positiven MentoringProzess berücksichtigen. Schmid und Haasen (2011) stellen fest, dass (1) MentorInnen nicht Vorgesetzte der Mentees „sein [dürfen]“ (ebd., S. 87) und die MentorInnen (2) hierarchisch nicht zu weit von den Mentees entfernt sein sollten. Insofern ist ein/e FachkoordinatorIn keine gute Wahl als MentorIn für eine/n BerufsanfängerIn desselben Fachs, noch weniger die Schulleitung selbst. Dass idealerweise MentorInnen (3) mit Erfahrungen und Zielen zu den Mentees passen und (4) MentorInnen Mentees ergänzen, kann mit persönlichen Entwicklungspotenzialen, aber auch mit dem professionellen Habitualisierungsprozess erklärt werden. Wenn das triadische Habitualisierungsmuster des professionellen Wahrnehmens, Denkens und Handels (Bourdieu 2015a; Lenger et al. 2013) der MentorInnen jenem der Mentees nahekommt, wird der Identifikationsprozess der Mentees möglicherweise begünstigt. Insofern nehmen die Schulleitung bzw. der Dienstgeber in Bezug auf den Professionalisierungsprozess von BerufsanfängerInnen bereits beim Zusammenstellen der Tandempaare MentorIn - Mentee einen großen Einfluss und ihr kommt im Qualitätsmanagement eine zentrale Position zu (Schratz et al. 2015). Bereits der erste Auswahlprozess von angehenden MentorInnen findet durch die SchulleiterInnen statt, indem diese ein Empfehlungsschreiben an die Ausbildungsinstitutionen ${ }^{37}$ richten, sofern eine Lehrperson aus dem Kollegium Interesse an der Ausbildung zur Mentorin bzw. zum Mentor bekundet. Dieses Empfehlungsschreiben ist eines der Aufnahmekriterium für den Hochschullehrgang (Bundesministerium für Bildung, Wissenschaft und Forschung 2014).

Als KoordinatorIn der Entwicklung (Schratz et al. 2015) installiert die Schulleitung Arbeitsstrukturen (z.B. Tandembildung durch Matching) und sorgt für Informationsflüsse, indem die Rahmenbedingungen dafür geschaffen werden. Letztlich tragen Führungskräfte über die Planung, Administration etc. von innerschulischen Strukturen und Prozessen, wie dem MentoringProzess in der Berufseinstiegsphase, auch zur Steigerung von SchülerInnenleistungen bei (ebd.). In Bezug auf das Bewusstsein über die Relevanz von Mentoring an Schulen zeigt die SchulleiterInnenbefragung im Rahmen der TALIS-Studie (Schmich \& Itzlinger-Bruneforth 2019) die in Tabelle 2 dargestellten Ergebnisse (Tab. 2). An dieser Befragung nahmen ausschließlich SchulleiterInnen teil, deren LehrerInnen zum Zeitpunkt der Befragung Zugang zu einem Mentoring-Programm hatten. Die Prozentwerte beziehen sich auf Österreich, da in der vorliegenden Untersuchung (Empirischer Zugang, Kap. III) SchulleiterInnen und BerufsanfängerInnen österreichischer Schulen befragt wurden und ein internationaler Vergleich nicht angestrebt wird.

37 Angehende MentorInnen werden an Pädagogischen Hochschulen im Hochschullehrgang „Mentoring: Berufseinstieg professionell begleiten" ausgebildet. 
Tab. 2: Relevanz von Mentoring; Quelle: Schmich und Itzlinger-Bruneforth (2019, S. 79)

\begin{tabular}{lc}
\hline Kriterium & Angabe in Prozent \\
\hline Für die Unterstützung von weniger erfahrenen LehrerInnen im Unterricht & $77 \%$ \\
Für die Verbesserung der pädagogischen Kompetenzen von LehrerInnen & $64 \%$ \\
Für die Stärkung des beruflichen Selbstverständnisses von LehrerInnen & $62 \%$ \\
Für die Verbesserung der Zusammenarbeit von LehrerInnen mit KollegInnen & $58 \%$ \\
Für die Verbesserung der Leistung von SchülerInnen allgemein & $42 \%$ \\
Für die Erweiterung des Wissens der LehrerInnen in deren Hauptgegenständen & $31 \%$ \\
\hline
\end{tabular}

Wie in Tabelle 2 dargestellt, sehen 77 Prozent der befragten SchulleiterInnen die Relevanz des Mentorings in der Unterstützung von weniger erfahrenen Lehrpersonen. 64 Prozent geben an, Mentoring sei für die Verbesserung der pädagogischen Kompetenzen von LehrerInnen geeignet. Für 62 Prozent der SchulleiterInnen ist Mentoring zur Stärkung des beruflichen Selbstverständnisses von Lehrpersonen wichtig, während 42 Prozent eine allgemeine Leistungssteigerung von SchülerInnen sehen. 31 Prozent der Befragten nennen Mentoring als relevantes Instrument zur Erweiterung des Wissens der LehrerInnen in deren Hauptgegenständen.

Die Analyse der Daten zeigt, dass sich die theoretischen Annahmen über Mentoring-Programme weitgehend mit dem Bewusstsein der SchulleiterInnen decken. So kann die allgemeine Weiterentwicklung von BerufsanfängerInnen (Führer \& Heller 2018) in der Unterstützung von weniger erfahrenen LehrerInnen und in der Verbesserung pädagogischer Kompetenzen gesehen werden, während sich individuelle Lernprozesse (Schmid \& Haasen 2011) und die Beziehungsebene der Kooperation (Raufelder \& Ittel 2012) in der Stärkung des beruflichen Selbstverständnisses bzw. in der Verbesserung der Zusammenarbeit im Kollegium als Wahrnehmung der SchulleiterInnen darstellt. Die Leistungssteigerung von SchülerInnen wird in den theoretischen Konzepten des Mentorings nicht explizit erwähnt, und die relativ niedrige Bewertung der Wissenserweiterung der Lehrpersonen in den eigenen Fächern mit 31 Prozent in der SchulleiterInnenbefragung unterstreicht die Annahme, Mentoring nicht auf das Unterrichtsfach zu reduzieren (Führer \& Heller 2018; Raufelder \& Ittel 2012). Die Analyse der empirischen Daten der vorliegenden Arbeit zeigt weitgehende inhaltliche Überschneidungen zur Darstellung im vorliegenden Abschnitt (siehe Hauptkategorie Unterstützung \& Begleitung der BerufsanfängerInnen im Schulalltag (SL_D), Kap. III.3.1.2). Die ausführliche Diskussion der empirischen Daten findet im Abschnitt Analyse (Kap. III.3) statt.

Im folgenden Abschnitt wird Mentoring in Bezug auf Wirksamkeiten, Ziele und Determinanten betrachtet. Die Diskussion findet auf Basis theoretischer, im Anschluss vorgestellter Mentoring-Modelle statt.

\subsubsection{Mentoring in Theorie und Praxis}

Je nachdem, welches Ziel bzw. welcher Wirkungsbereich mit dem Mentoring-Programm verfolgt wird, welche konkrete Situation vorliegt und welche Personen am Prozess beteiligt sind, wird entweder der Ansatz des personenzentrierten oder des strukturellen Mentorings gewählt (Ittel \& Raufelder 2009). Beim personenzentrierten Mentoring steht die persönliche Entwicklung des Individuums durch eigene Kräfte und Impulse im Zentrum, das strukturelle Mentoring hingegen fokussiert die Gemeinschaft. Im Sinne des Schulmanagements sollen alle an Schulen 
am „organisatorischen und mentalen Wandlungsprozess“ (ebd., S. 201) beteiligten Akteurinnen und Akteure Unterstützung erhalten.

Es ist davon auszugehen, dass in der Induktionsphase implementierte Mentoring-Prozesse weder rein personenbezogener noch ausschließlich struktureller realisiert werden. Vielmehr ist es im Sinne der Akteurinnen und Akteure sowie der Schulorganisation insgesamt, für eine ganzheitliche Induktion sowohl personenzentriertes als auch strukturelles Mentoring anzuwenden. Unabhängig vom theoretischen Ansatz eines Mentoring-Prozesses sehen Koballa, JR. et al. (2008) die Handlungswirksamkeit zwischen MentorIn und Mentee im Vordergrund. „Conceptions of mentoring help shape interactions [...]“ (ebd., S. 43), so lautet die zentrale Formel zur Wirksamkeit des Mentorings. Ziel von Einführungsprogrammen ist, unabhängig von den dahinter liegenden theoretischen Ansätzen, „die fachliche und soziale Integration neuer Mitarbeiter am Arbeitsplatz“ (Kraler 2008b, S. 7). So müssen sich BerufsanfängerInnen u.a. „auf das neue Umfeld einstellen, eine Arbeitsrolle lernen, [...] soziale Beziehungen aufbauen und mehr oder minder stark die neue Schulkultur übernehmen“ (ebd.).

Im Folgenden werden theoretische Modelle zum Mentoring vorgestellt, um anschließend Wirksamkeit, Determinanten und Ziele zu diskutieren.

\subsubsection{Theoretische Modelle}

In diesem Abschnitt stelle ich Modelle zum Mentoring vor und unterscheide einerseits nach personenzentriertem und strukturellem Ansatz (Ittel \& Raufelder 2009), andererseits weise ich auf das lehr- bzw. lernseitige Paradigma des Wissens- und Kompetenzenerwerbs (Christof \& Schwarz 2013; Schratz et al. 2012) hin. Ich beziehe mich in der folgenden Diskussion auf die in Tab. 3 angeführten theoretischen Modelle zum Mentoring.

Tab. 3: Mentoring-Modelle

\begin{tabular}{lll}
\hline Modell & $\begin{array}{l}\text { personenzentriert/ } \\
\text { strukturell } \\
\text { (Ittel \& Raufelder 2009) }\end{array}$ & $\begin{array}{l}\text { lehrseits/lernseits } \\
\text { (Christof \& Schwarz 2013; } \\
\text { Schratz et al. 2012) }\end{array}$ \\
\hline $\begin{array}{l}\text { Mentoring-Ansätze } \\
\text { (Wang \& Odell 2007) }\end{array}$ & personenzentriert, strukturell lehrseits, lernseits \\
\hline $\begin{array}{l}\text { Drei-Wege-Modell } \\
\text { (Niggli 2005) }\end{array}$ & personenzentriert & lernseits \\
\hline $\begin{array}{l}\text { Lerntriade beim Mentoring } \\
\text { (Ziegler 2009) }\end{array}$ & personenzentriert & lehrseits \\
\hline $\begin{array}{l}\text { Effektives Lernen beim Mentoring - } \\
\text { „Big Four“ (Ziegler 2009) }\end{array}$ & personenzentriert & lernseits \\
\hline
\end{tabular}

Nachfolgend werden die personenzentrierten und strukturellen Mentoring-Ansätze (Wang \& Odell 2007) mit lehr- und lernseitigem Paradigma diskutiert. Anschließend stelle ich drei jeweils personenzentrierte und lernseitig orientierte Modelle vor, das Drei-Wege-Modell nach Niggli (2005), die Lerntriade beim Mentoring (Ziegler 2009) und effektives Lernen beim Mentoring durch die „Big Four“ (ebd.).

Wang und Odell (2007) teilen Mentoring in den humanistischen Ansatz, den Ausbildungsansatz und den kritisch- konstruktivistischen Ansatz (Abb. 24). Diese drei Ansätze beziehen sich 
auf Mentoring innerhalb der LehrerInnenausbildung (Höller et al. 2019) und können u.U. nicht direkt auf die Induktionsphase für BerufsanfängerInnen übertragen werden. Jedoch sind einerseits Essenzen des personenzentrierten Mentorings (humanistischer Ansatz) und des strukturellen Ansatzes (kritisch-konstruktivistischer Ansatz) erkennbar (Ittel \& Raufelder 2009), andererseits befinden sich auch BerufsanfängerInnen während der Induktionsphase noch in Ausbildung, sofern sie in dieser Zeit das Masterstudium absolvieren. Der humanistische Ansatz (1) umfasst die persönliche und zwischenmenschliche Unterstützung durch die Mentorin bzw. den Mentor. In der Rolle der Beraterin bzw. des Beraters fördern sie u.a. das Selbstvertrauen des Mentees (Wang \& Odell 2007). Warme Atmosphäre, positives Feedback und kollegiale Solidarität kennzeichnen den humanistischen Ansatz ebenfalls (Raufelder \& Ittel 2012) und es liegt ein lernseitiger Schwerpunkt vor (Abb. 24).

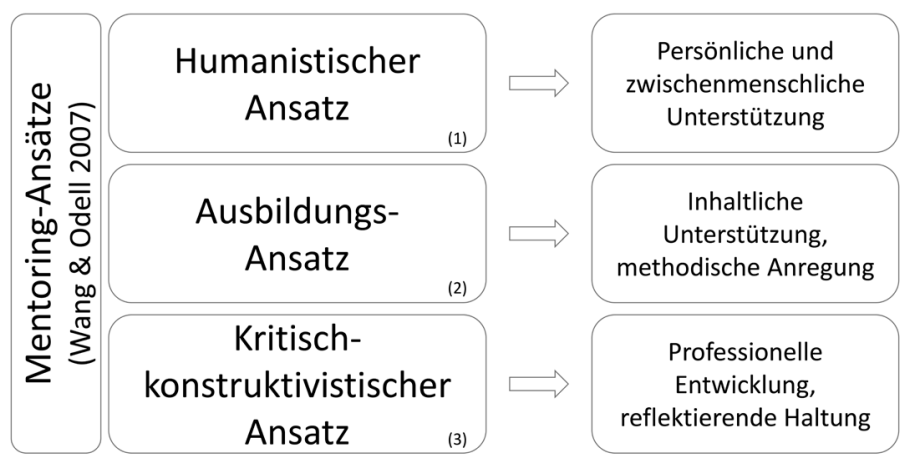

Abb. 24: Mentoring-Ansätze; eigene Abbildung nach Wang und Odell (2007)

Als inhaltliche Unterstützer geben die Mentorinnen und Mentoren ihren Mentees im Ausbildungsansatz (2) methodische Anregungen in Bezug auf Unterricht und Klassenführung. Steinke und Putnam (2011, S. 45) betonen: „The reality ist that knowledge and skill set to be an effective teacher is different than the knowledge and skill set to effectively mentor a colleague" und heben damit die unterschiedlichen Zugänge zwischen Lehrpersonen im Klassenzimmer und MentorInnen bei der Betreuung von Mentees hervor. Das lehrseitige Paradigma dominiert.

Im Mittelpunkt des kritisch-konstruktivistischen Ansatzes (3) steht die professionelle Entwicklung des Mentees, die reflektierende Haltung des Mentees wird dabei fokussiert (Höller et al. 2019). Die Mentorin bzw. der Mentor fungiert in diesem Ansatz als Motivator und Partner für Veränderungen im schulischen Kontext und hat die gesamte professionelle Entwicklung des Lehrerberufs im Blick (Raufelder \& Ittel 2012). Der Zugang ist lernseitig dominiert.

Im Drei-Wege-Modell nach Niggli (2005) steht die Interaktion durch das Gespräch zwischen MentorIn und Mentee im Mittelpunkt und es ist der Kategorie der personenzentrierten Ansätze zuzurechnen. Hier werden drei Gesprächsebenen unterschieden, wobei von Ebene 1 über Ebene 2 zu Ebene 3 eine Erhöhung des Professionalisierungsgrades erkennbar ist (Abb. 25).

Der vertikale, nach oben gerichtete Pfeil signalisiert die Steigerung des Professionalisierungsgrades. 


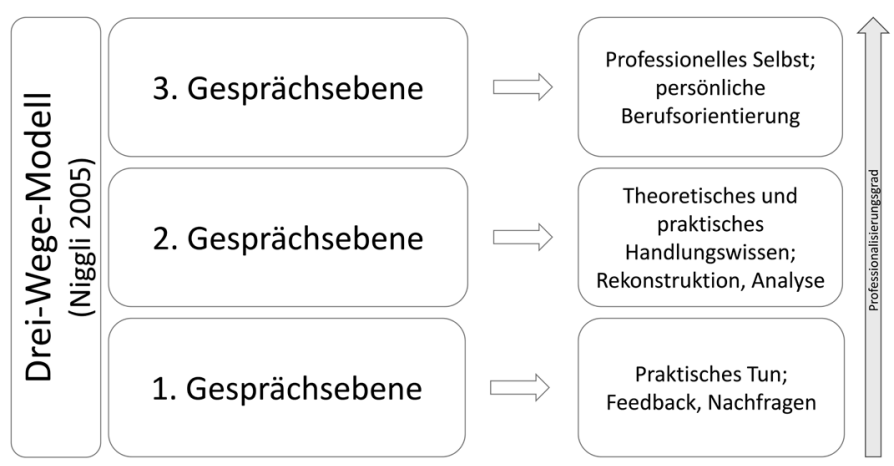

Abb. 25: Drei-Wege-Modell; eigene Abbildung nach Niggli (2005)

Auf der ersten Gesprächsebene steht das praktische Tun im Unterricht im Vordergrund. Feedback und „vorsichtiges Nachfragen“ (Ittel \& Raufelder 2009) der MentorInnen optimiert das Unterrichtsverhalten bezüglich der praktischen Fertigkeiten. In Gesprächsebene zwei wird das theoretische und praktische Handlungswissen thematisiert und reflektiert. Mithilfe des theoretischen Wissens der Mentorin bzw. des Mentors und auch durch die parallelen Studien des Mentees werden Unterrichtssituationen rekonstruiert und analysiert (Ittel \& Raufelder 2009; Niggli 2005). In der dritten Gesprächsebene liegt der Fokus auf Zielen, Werten und Motivationen des professionellen Selbst der Mentees. Es geht um eine Art der persönlichen Berufsorientierung, indem die Mentees das Wissen über sich selbst fokussieren (Ittel \& Raufelder 2009) und damit lernseitig und reflektierend agieren.

Ziegler (2009) weist auf pädagogische Modellbezüge des Mentorings hin und stellt fest, dass in den Mentoring-Programmen alle drei Formen einer „Lerntriade“ (ebd., S. 13) zum Einsatz kommen können (Abb. 26). Die Lerntriade nach Ziegler ist ein personenbezogenes Modell und beschreibt im Unterschied zu den anderen hier vorgestellten Modellen Handlungsweisen der MentorInnen, nicht jene der Mentees.

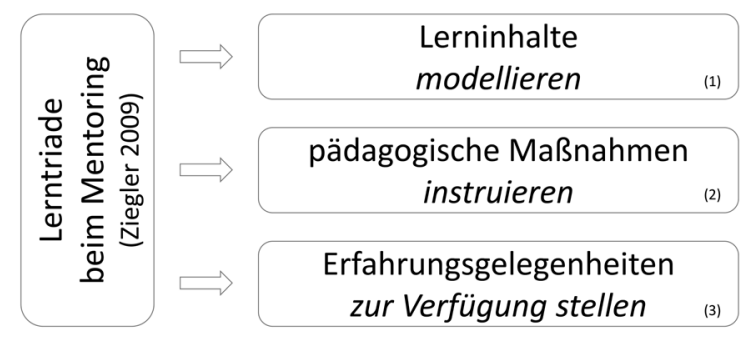

Abb. 26: Lerntriade; eigene Abbildung nach Ziegler (2009)

Aufgrund ihrer Erfahrung können MentorInnen Lerninhalte modellieren (1) (Abb. 26). Sie können dadurch bestimmte Handlungen, Einstellungen, Werte, Charaktereigenschaften etc. (Ziegler 2009) abstrahieren und den Mentees die komplexe Wirklichkeit besser verständlich machen (Schlömerkemper 2010). Modelle können aus didaktischer Perspektive das Wichtige herausstellen, leichter vorstellbar machen und insofern den Entwicklungsprozess der Lernen- 
den fördern (ebd.). Weiters können MentorInnen pädagogische Maßnahmen instruieren (2). Sie geben an die Mentees praktikables Handlungswissen und Informationen zu pädagogischen Maßnahmen weiter. Schließlich, und damit wird dieser Ansatz zur Triade, können MentorInnen ihre Erfahrungsgelegenheiten zur Verfügung stellen (3), indem sie z.B. Buchtipps geben oder Führungen, Exkursionen etc. anbieten (Ziegler 2009). An dieser Stelle weise ich darauf hin, dass dieser Ansatz der Lerntriade sehr stark das Lehren fokussiert und weniger das Entwickeln professioneller Kompetenzen, die u.a. aus den selbstregulativen Fähigkeiten der Mentees hervorgehen (Keller-Schneider \& Hericks 2014) und im Mittelpunkt des personenorientierten, kritisch-konstruktivistischen Ansatzes (Ittel \& Raufelder 2009; Wang \& Odell 2007) stehen.

Ziegler (2009) beleuchtet den Mentoring-Prozess noch von einer anderen personenorientierten Seite und betont, dass Mentoring „wie keine andere pädagogische Maßnahme optimale Rahmenbedingungen für die Big Four effektiver Lernprozesse“ (ebd., S. 14) schafft (Abb. 27).

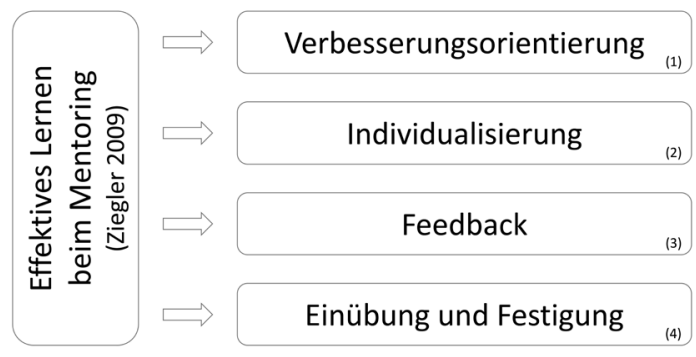

Abb. 27: Effektives Lernen beim Mentoring; eigenen Abbildung nach Ziegler (2009)

Die Big Four des effektiven Lernen bestehen nach Ziegler (2009) aus (1) Verbesserungsorientierung, (2) Individualisierung, (3) Feedback und (4) Einübung und Festigung und fokussieren wieder die Kompetenzen der Lernenden, der Mentees.

Die Verbesserungsorientierung (1) zeichnet sich dadurch aus, dass der Blick der Mentees nach vorne gerichtet ist. Die gesteckten Verbesserungsziele werden in der Regel nicht durch die einfache Beschäftigung mit Sachverhalten erreicht und es liegt auch im Verantwortungsbereich der Mentorin bzw. des Mentors, auf die Entwicklung der bzw. des Mentees zu achten und diese nicht auf „suboptimalem Niveau“ (ebd.) zu beenden. Das Prinzip der Individualisierung (2) kann im Rahmen des Mentorings „nahezu perfekt“ (ebd.) eingelöst werden, da sich die Mentorin bzw. der Mentor auf eine einzige bzw. einen einzigen Mentee konzentriert. Jeder Lernschritt kann durch individuelle Planung und individuelles Feedback (3) auf die bzw. den Mentee zugeschnitten sein. Damit ist bereits das nächste Charakteristikum der Big Four erwähnt. Eine der wichtigsten Funktionen der Mentorin bzw. des Mentors besteht laut Ziegler (2009) darin, der bzw. dem Mentee häufiges und qualitativ hochwertiges Feedback zur Verfügung zu stellen, da sich bei unangeleitetem Lernen sehr schnell Routinen einschleichen. Außerdem übersehen die Mentees durch die oftmals raschen Anfangserfolge den doch noch bestehenden Lernbedarf. Unter Einübung und Festigung (4) versteht Ziegler die Anwendung von Gelerntem mit minimalen Transferleistungen und spricht damit eine Art Automatisierung der Handlungen an. Als Beispiel nennt der Autor das Erlernen eines Musikinstruments und konstatiert zugleich, dass beim Mentoring im schulischen Kontext die große Auswahl der Übungsaufgaben und deren sequenzielle Anordnung zur Überforderung der Mentees führen. Die MentorInnen haben auch 
die Aufgabe, die Erfahrungsschritte der Mentees „zu leiten“ (ebd.). Der lernseitige Erwerb von Mentoring-Kompetenzen steht bei allen vier Phasen des effektiven Lernens im Zentrum.

Zusammenfassend kann festgehalten werden, dass die hier vorgestellten Mentoring-Modelle personenzentrierte und strukturelle Ansätze (Ittel \& Raufelder 2009) mit lehr- und lernseitigem Paradigma (Schratz et al. 2012) darstellen. Die in den vorigen Abschnitten diskutierten und anzustrebenden Prinzipien, wonach die kooperierende Beziehungsebene zwischen MentorIn und Mentee im Gegensatz zu hierarchischen Strukturen im Zentrum steht (Höller et al. 2019) und wo die zwischenmenschliche Beziehung mit hohem Reflexionscharakter (Raufelder \& Ittel 2012) den Mentoring-Prozess maßgeblich definiert, spiegelt sich in den MentoringModellen wider. Der Schwerpunkt liegt auf Personenzentrierung (Ittel \& Raufelder 2009) mit lernseitigem Erkenntnisparadigma (Schratz et al. 2012).

\subsubsection{Wirksamkeit, Determinanten und Ziele}

Zur Wirksamkeit von Mentoring haben Ingersol und Strong (2011) in einem Review von 15 empirischen Studien festgestellt, dass schulische Unterstützungsstrukturen, wie etwa Einführungsphasen in die Unterrichtstätigkeit, positiven Einfluss auf die Einsatzbereitschaft und die Unterrichtsgestaltung von Lehrpersonen sowie auch auf den Lernerfolg von SchülerInnen haben. Dieser positive Einfluss auf die Unterrichtsgestaltung wirkt sich konkret, bezogen auf die Bedürfnisse der SchülerInnen, auf die Abstimmung der Unterrichtsaktivitäten aus. Aus der TALIS-Studie (Schmich \& Itzlinger-Bruneforth 2019) geht zudem hervor, dass Lehrpersonen, die an formellen und informellen Einführungsphasen teilnehmen, im Durchschnitt der OECD-Länder „tendenziell eine höhere Selbstwirksamkeit und Arbeitszufriedenheit“ (Höller et al. 2019) erleben. Aufschlussreich ist in diesem Zusammenhang der Befund von Raufelder und Ittel (2012), wonach die AkteurInnen, also MentorIn und Mentee, in ihrer persönlichen Weiterentwicklung unterstützt werden. Dies ist im Übrigen einer der wesentlichen Unterschiede zu anderen Kooperationsformen, wie dem Coachen oder der Supervision, wo die Entwicklung des Klienten, im Falle des Berufseinstiegs als Lehrperson der bzw. des Mentees, im Vordergrund steht. Aus dieser Perspektive wird Mentoring in der pädagogischen und schulischen Praxis, explizit in der LehrerInnenausbildung, als ein Instrument der persönlichen und professionellen Weiterentwicklung des Lehrpersonals verstanden (ebd.). In diesem Sinne kann laut Ittel und Raufelder (2009) mit Blick auf das gesamte Lehrpersonal die Etablierung eines MentorInnen-systems helfen, alle AkteurInnen zur Teilnahme an der Schul- und Unterrichtsentwicklung zu motivieren. Der Benefit geht dann über den persönlichen Nutzen für die MentorInnen und die Mentees hinaus. Zudem tragen regelmäBige Berichte von MentorInnen und Mentees, etwa im Rahmen von LehrerInnenkonferenzen, und Evaluationen der MentorIn-Mentee-Beziehung zu einem offenen und dynamischen System des Austauschs innerhalb des gesamten LehrerInnenkollegiums bei. Ein weiteres, eher pragmatisches Argument für das implementierte Mentoring im Rahmen der Induktionsphase zielt auf die finanziellen Gegebenheiten ab. „Anstatt nur auf externe und häufig sehr kostspielige Angebote zurückzugreifen“ (Ittel \& Raufelder 2009, S. 205), können sich Schulen mit dem Aufbau einer Mentoringkultur ${ }^{38}$ mit internen, gewissermaßen , hauseigenen“ BeraterInnen, „von innen heraus stärken“ (ebd.) und nebenbei die Kosten gering halten.

Derzeit, so Höller et al. (2019), wird Mentoring vielfach während der LehrerInnenausbildungsphase angeboten. Dieses Kooperationssetting kann aber durchaus auch als eine Art der beruflichen

38 Voraussetzung ist das Angebot an genügend MentorInnen. Die Ausbildung dieser erfolgt im Rahmen des Hochschullehrgangs „Mentoring: Berufseinstieg professionell begleiten“. Die Thematisierung dieser Ausbildung ist jedoch nicht Teil der vorliegenden Arbeit. 
Fortbildung gesehen werden. Als Instrument zur persönlichen und professionellen Entwicklung eignet sich Mentoring in allen Phasen des LehrerInnenberufs und kann in diesem Sinne zur Weiterbildung genutzt werden (Raufelder \& Ittel 2012). Das Konzept „Induktion als fortlaufende kollegiale Supervision“ (Greiner 2019) könnte demzufolge für alle Phasen der LehrerInnenbiografie wichtig sein, nicht nur in der Zeit des Berufseinstiegs. Allerdings zeigen die Befunde aus der Praxis (Keller-Schneider \& Hericks 2014), dass sich diese Idee noch nicht etabliert hat.

Auf internationaler Ebene liegen diverse empirische Studien zu Mentoring-Programmen vor (DuBois et al. 2002). Auf nationaler Ebene wurden Mentoring-Programme mit formellem Charakter erst im Schuljahr 2019/20 installiert und es werden zukünftig vermehrt Möglichkeiten und Chancen bestehen, diese begleiteten Einführungsphasen empirisch zu evaluieren (Raufelder \& Ittel 2012). Odell und Huling (2000) zeigen in ihrer Studie, dass angehende Lehrpersonen von Mentoring-Prozessen profitieren. Ebenso gewinnbringend gestalten sich die begleiteten Berufseinstiegsphasen aber auch für die betreffenden MentorInnen (David 2000; Holloway 2001; Resta et al. 1997). Insgesamt wird in den Studien dargelegt, dass sich Mentoring nicht nur auf die MentorInnen und Mentees, sondern im Sinne der Langzeitwirkung auf berufsbiografischer Ebene auf die gesamte LehrerInnenschaft positiv auswirkt: „Mentoring [...] not only can enhance the abilities of teachers, but it also can have a significant impact on overall retention of teachers", stellen Steinke und Putnam (2011, S. 47) dazu fest. Auch Schmid und Haasen (2011) identifizieren im begleitenden Mentoring-Prozess der Induktionsphase Chancen sowohl aufseiten der Mentees als auch auf jener der MentorInnen. Während sich die Chancen der Mentees zum Großteil mit schon Genanntem in den bereits zitierten Theorien und Modellen decken, bringen die AutorInnen mit den Chancen für die MentorInnen durchaus neue Gedanken ins Spiel. Als Chancen für Mentees werden die Entwicklung der Persönlichkeit, die Erweiterung der Problemlösestrategien, das Lernen anhand von Beispielen, der Blick für das Umfeld und das „große Ganze“ (ebd., S. 22), die Stärkung des Selbstbewusstseins und Diskussionen über den Karriereweg bis hin zur Bildung von Netzwerken genannt. Die Chancen für die MentorInnen betonen die bereits erwähnte beidseitige Partizipation an persönlicher Entwicklung. Durch den reflexiven Charakter des MentoringSettings üben sich neben den Mentees auch die Mentorin bzw. der Mentor in Selbstreflexion, was neben der persönlichen Entwicklung auch eine Chance für die gesamte Organisation im Sinne der Weiterentwicklung darstellt. Die MentorInnen kennen Abläufe im Schulsystem und haben die Chance, im Mentoring einen Beitrag zur „Kulturentwicklung“ (ebd., S. 29) zu leisten, auch aufgrund der gemeinsamen Verantwortung z.B. mit der Schulleitung. Sayer (2006, S. 69) drückt es folgendermaßen aus: „It is frequently asserted that future role of schools requires innovation, adaptability and new forms of open professionality.“ Der Autor apostrophiert weiter: „In much of Europe, teachers are civil servants, employed and controlled by the state." (ebd.) Schließlich spricht Sayer (2006) sogar von „pedagogical command economy“. Für die oben erwähnte und anzustrebende Kulturentwicklung bedeutet das einen hinderlichen, mindestens einen nicht förderlichen Zustand. Ostinelli (2009, S. 304) bringt die notwendige Freiheit zur Professionalisierung generell und im Bereich des Mentorings speziell zum Ausdruck: „[...] it should be stressed that even the better idea, if proposed in a top-down fashion, risks leading to failure."

Die im Feedback und in der Reflexion mit den Mentees gewonnenen Erkenntnisse sind einerseits eine Chance für die Mentees, ebenso bietet sich aber auch deren Transfer für die Mentorin bzw. den Mentor in den eigenen Schulalltag an. Positive Rückmeldungen seitens der Mentees, der Schulleitung und anderer AkteurInnen erhöhen die berufliche und persönliche Zufriedenheit und Wertschätzung, was schließlich auch eine emotionale Bindung an die Schule bewirken kann - ebenfalls eine Chance für die MentorInnen und ein Sinn des Mentorings, so Schmid und 
Haasen (2011). Raufelder und Ittel (2012) identifizieren im Rahmen ihrer Studie Gelingensbedingungen für ein erfolgreiches und nachhaltiges Mentoring und teilen dies im Wesentlichen in die beiden Bereiche Persönliches und Institutionelles.

Als Grundvoraussetzung für ein gelingendes Mentoring im schulischen Bereich sehen die Autorinnen die gute persönliche Beziehung zwischen MentorIn und Mentee. Erst dadurch kann die notwendige emotionale Unterstützung seitens der Mentorin bzw. des Mentors erfolgen. Auch die erforderliche Offenheit zwischen MentorIn und Mentee wird dadurch begünstigt und ist für die bzw. den Mentee der „Rollenfindung als Lehrkraft“ (ebd., S. 150) zuträglich. Die Umsetzung handlungsorientierter Anleitungen und das Einlassen auf die methodische Unterstützung fallen den betreuten Lehrpersonen leichter, wenn die persönliche Basis durch Vertrauen und Sicherheit gebildet wird. „Handlungsroutinen und Entwicklung von Veränderungen“ (ebd.) sind dann Teil eines gemeinsamen Prozesses. Auch wenn in der Induktionsphase „der Erfolg des Mentorings zwar in erster Linie auf der Entdeckung und Nutzung von individuellen Ressourcen" (ebd., S. 151) beruht, ist es aber auch die Struktur des Umfelds, die für eine solide Grundlage und dauerhafte Implementierung dieses Betreuungskonzepts beitragen kann. Raufelder und Ittel (2012) unterscheiden drei verschiedene institutionelle Bedingungsfaktoren, die zum Gelingen des Mentoring-Prozesses in der Schule beitragen können (Tab. 4).

Tab. 4: Institutionelle Rahmenbedingungen für Mentoring in der LehrerInnenbildung (Raufelder \& Ittel 2012)

\begin{tabular}{ll}
\hline Institutionelle Rahmenbedingungen für Mentoring in der LehrerInnenbildung \\
\hline 1. Entscheidungsfaktor & entscheiden und gemeinsam durchführen \\
2. Zeit- und Geldfaktor & Institutionalisierte Ressourcen \\
3. Entwicklungsfaktor & Mentoring als Schulentwicklung \\
\hline
\end{tabular}

Raufelder und Ittel (2012) identifizieren Rahmenbedingungen in Form von Determinanten und Indikatoren für funktionierendes, gewinnbringendes und letztlich sinnvolles Mentoring an Schulen (Tab. 4). Primär nennen sie den Entscheidungsfaktor (1). Wenn sich die Institution Schule und die LehrerInnen für die Integration von Mentoring in ihrer Schulkultur entschließen, müssen alle Beteiligten die Bereitschaft und das Ziel verfolgen, pädagogische und persönliche Prozesse im pädagogischen Alltag offenzulegen. Ferner müssen Ressourcen in Form von Zeit und Geld (2) von institutioneller Seite sichergestellt sein. Zeit muss von den Beteiligten investiert und der Institution bzw. Verwaltung zur Verfügung gestellt werden und spielt eine bedeutende Rolle. Die notwendigen Ressourcen müssen von den zuständigen AkteurInnen (Bildungsdirektionen, SchulleiterInnen etc.) freigegeben werden, um Mentoring als Professionalisierungsprozess mit speziellen Rahmen- und Prozessbedingungen zu etablieren. Besonders den SchulleiterInnen fällt eine große Verantwortung zu (siehe auch Kap. II.2.3.3). Sie stellen den konkreten schulinternen Rahmen zur Verfügung, damit aus dem Mentoring ein „Instrument zur Qualitätsverbesserung und -sicherung“ (ebd.) für Lehrende und damit für die ganze Schule werden kann. Schließlich hat Mentoring das Potenzial eines Entwicklungsfaktors (3). Durch die Implementierung und Weiterentwicklung von Mentoring-Prozessen werden „althergebrachte Denkmuster aller Beteiligten“ (ebd.) modifiziert, eine nachhaltige Befähigung zu kooperativer und verantwortungsbewusster Gestaltung von Schulkultur wird erreicht.

Aus der Umkehrung der oben diskutierten Gelingensbedingungen für erfolgreiches Mentoring identifiziert Ziegler (2009) die „Nichtrealisierung allgemein bekannter Erfolgsbedingungen er- 
folgreichen Mentorings“ (ebd., S. 12) als ein Problem, welches die Effektivität und letztlich den Erfolg des Mentoring-Prozesses negativ beeinflusst. Die häufigsten Defizite liegen erwartungsgemäß im persönlichen und institutionellen Bereich. Laut Ziegler wird die Relevanz der sorgfältigen Zusammenstellung der MentorIn-Mentee-Paare, das Matching, zu wenig berücksichtigt. Die oben erwähnte persönliche Beziehung als vorausgesetzte Gelingensbedingung für erfolgreiches Mentoring macht deutlich, dass dieser Begleitprozess bereits mit dem richtigen Matching beginnt. Steinke und Putnam (2011, S. 45) stellen fest: „Selecting the right mentors and matching those mentors with the proper protégés can be crucial in any mentoring relationship." Das Ziel kann sein, den formellen Mentoring-Prozess auf der persönlichen Ebene so informell wie möglich zu gestalten: „The more a formal mentoring program simulates an informal mentoring relationship, the more effective it will be." (ebd.) In engem Zusammenhang damit stehen Maßnahmen zur Förderung der Beziehungsqualität von MentorIn und Mentee. Auch hier sieht Ziegler (2009) ein Defizit und es würde sich ein Blick auf die institutionellen Rahmenbedingungen (Zeitressourcen) und auf die Förderung der Personalentwicklung (in der Verantwortung der Schulleitung) lohnen. Auf der Seite der MentorInnen vermisst Ziegler (2009) ein „angemessenes Training“ (ebd., S. 13) oder zumindest eine Eignungsfeststellung, damit die teilweise sogar „negative[n] Effekte“ (ebd.) von Mentoring-Programmen, wie Vernachlässigung, Täuschung und Inkompetenz, wegfallen. Im Rahmen der neuen MentorInnenausbildung im Rahmen der Hochschullehrgänge „Mentoring: Berufseinstieg professionell begleiten“ (Bundeskanzleramt 2013) sollten diese Sorgen allerdings zerstreut werden. Als abschließendes Defizit in der Umsetzung der Mentoring-Programme sieht Ziegler (2009) die oftmals nicht „angemessene und ausreichende Laufzeit“ (ebd., S. 13) des Betreuungs-Programms. Im schulischen Kontext ist diese Betreuungszeit mit einem Jahr Induktionsphase festgesetzt (Bundeskanzleramt 2013). Es wird sich zeigen, inwieweit sich Chancen, Nutzen und positive Effekte in der Berufseinstiegsphase für BerufsanfängerInnen etablieren.

Als Ausblick formulieren Raufelder und Ittel (2012) Ziele des Mentoring-Prozesses. Die AutorInnen identifizieren ein Hauptziel und mehrere Nebenziele (Abb. 28). Als Hauptziel (HZ) steht die persönliche Entwicklung der Mentees im Vordergrund. Dieses Hauptziel wird zum einen durch die zwischenmenschliche Beziehung zwischen MentorIn und Mentee im Mentoring-Prozess erreicht (ebd.), zum anderen durch die kooperative und wenig hierarchisch gestaltete Arbeitsform im Mentoring (Höller et al. 2019). Auch die Habitualisierung als Lehrperson begünstigt die persönliche Entwicklung der Mentees. Durch die Triade des professionellen Wahrnehmens, Denkens und Handelns (Bourdieu 2015a; Lenger et al. 2013) entwickelt sich der individuelle und später die professionelle LehrerInnenhabitus, die persönliche Entwicklung ist integriert.

\begin{tabular}{|c|c|}
\hline \multicolumn{2}{|l|}{$\begin{array}{l}\text { Haupt- und Nebenziele beim Mentoring } \\
\text { (Raufelder \& Ittel 2012) }\end{array}$} \\
\hline Persönliche Entwicklung der Mentees & $\hat{I}$ \\
\hline Kooperation zwischen Lehrpersonen & \\
\hline Kommunikation und professionelle Interaktion & \\
\hline Sensibilisierung für Lehr- und Lernveränderungen & \\
\hline Positive Entwicklung des Kollegiums und Schulklimas & $\bar{z}$ \\
\hline Profit durch Berufserfahrung der Mentorin / des Mentors & \\
\hline Zugang zu berufspraktischem Wissen & \\
\hline Emotionale und handlungsanleitende Unterstützung & \\
\hline
\end{tabular}

Abb. 28: Hauptziel (HZ) und Nebenziele (NZ) beim Mentoring; eigene Abbildung nach Raufelder und Ittel (2012) 
Basierend auf den oben genannten empirischen Erkenntnissen, dass durch das Mentoring sowohl die Mentees als auch die MentorInnen gewinnbringend partizipieren, ist es naheliegend, dass die durch das Mentoring-Programm begleitete Induktionsphase als ein Nebenziel die Kooperation zwischen Lehrpersonen begünstigt (Abb. 28). Zudem können die Kommunikation und die professionelle Interaktion zwischen den AkteurInnen innerhalb der Schule verbessert werden. Wenn LehrerInnen z.B. durch die schon erwähnten Berichte der MentorInnen an den Mentoring-Programmen teilhaben, auch wenn sie nicht direkt mit dem Mentoring-Prozess in Verbindung stehen, werden diese für Veränderungen des Lebrens und Lernens sensibilisiert, was sich wiederum positiv auf das gesamte Kollegium und mehr noch, auf das Schulklima insgesamt auswirkt. Die weiteren Nebenziele werden weitgehend durch die Erfahrung der MentorInnen in der Berufspraxis erreicht, indem die Mentees davon profitieren, und decken sich zum Teil auch mit den in der von Ziegler (2009) genannten Lerntriade enthaltenen Lernkonzepten. Den Mentees wird berufspraktisches Wissen zugänglich gemacht und sie werden, ebenfalls begünstigt durch die Erfahrungswerte der MentorInnen, in der Umsetzung der gemeinsam erarbeiteten Strategien „emotional und handlungsanleitend“ (Raufelder \& Ittel 2012) unterstützt.

Nach der Diskussion über Modelle, Wirkungen, Determinanten und Ziele des Mentorings im Rahmen der Induktionsphase für BerufsanfängerInnen im vorigen Abschnitt erfolgt im Anschluss eine Bestandsaufnahme des Status quo bezüglich Mentoring an österreichischen Schulen.

\subsubsection{Status quo an österreichischen Schulen}

Mentoring scheint in Österreich noch kein etabliertes und mit Weiterbildungs- und Aufstiegsmöglichkeiten verbundenes, spezialisierendes Berufsprofil für Lehrkräfte zu sein (Greiner 2019). Dieser Befund der TALIS-Studie (Schmich \& Itzlinger-Bruneforth 2019) ist insofern interessant, als das Bewusstsein, dass Mentoring ein geeignetes Instrument zur Professionalisierung für die AkteurInnen im schulischen Kontext ist, sehr wohl vorhanden ist (Ittel \& Raufelder 2009). Den bekannten Vorzügen des Mentorings, dass die Beteiligten in geschützten Räumen ihre Anliegen ansprechen können, der Ansprechpartner (MentorIn) kontextinternes Wissen hat und der Entwicklungsprozess von allen Beteiligten begleitet und institutionell unterstützt wird (ebd.), stehen ernst zu nehmende Argumente gegenüber, nämlich dass das Mentoring für die Mentorin bzw. den Mentor eine Zusatzbelastung einer (gefühlt) freiwilligen ${ }^{39}$ Tätigkeit darstellt, die nicht bedingt als Teil des Berufs wahrgenommen wird, und die vergleichsweise gering eingeschätzte Bezahlung der MentorInnentätigkeit in der Induktionsphase (Greiner 2019).

Im EU-Vergleich zeigt sich, dass in Österreich die Anzahl an MentorInnen und jene von Mentees signifikant niedriger ist. Vier Prozent der LehrerInnen im Berufseinstieg geben an, derzeit eine Mentorin bzw. einen Mentor zur Seite zu haben (EU-Durchschnitt: sechs Prozent) und sieben Prozent der Lehrpersonen sind als MentorIn tätig (EU-Durchschnitt: neun Prozent) (Höller et al. 2019). Die Ergebnisse zur Verfügbarkeit von Einführungsphasen aus SchulleiterInnensicht zeigen, dass formelle Einführungsphasen in Österreich mit 38 Prozent signifikant seltener als im EU-Schnitt angeboten werden, während die österreichischen Schulen bei informellen Aktivitäten in Einführungsphasen mit 76 Prozent im EU-Schnitt liegen (ebd., S. 79). Die auffällige Diskrepanz zwischen den zahlenmäßigen Angaben von SchulleiterInnen und LehrerInnen ${ }^{40}$ lässt zwei

39 Unter Umständen können die Bildungsdirektionen bzw. Schulleitungen die Tandems auch ohne die Zustimmung der Mentorinnen bzw. Mentoren vornehmen (Bundeskanzleramt 2013).

4063 Prozent der AHS-SchulleiterInnen deklarieren Mentoring-Angebote an ihren Schulen, während nur 14 Prozent der betreffenden LehrerInnen diese Angaben machen (Höller et al. (2019). 
Schlüsse zu. Zum einen argumentieren beide Gruppen (SchulleiterInnen und LehrerInnen) aus ihren je eigenen Blickwinkeln (ganze Schule bzw. eigenes Fach) und zum anderen, und das stellt in der vorliegenden Darstellung das interessantere Moment dar, liegen möglicherweise verschiedene Auffassungen von Berufseinführungsphase und Mentoring vor. Nach Auswertung der Fragebögen kann davon ausgegangen werden, dass viele SchulleiterInnen das ehemalige Unterrichtspraktikum an der AHS als Berufseinstiegsphase interpretieren und dass die Betreuung der Studierenden in den pädagogisch-praktischen Studien teilweise als Mentoring verstanden wird (ebd., S. 76f). Beides, sowohl das ehemalige Unterrichtspraktikum der AHS-LehrerInnen als auch die absolvierten Praktika an den AHS, BMHS und NMS im Rahmen der pädagogischpraktischen Studien, ist Teil der Ausbildung und damit laut TALIS-Definition nicht als Einführungsphase bzw. Mentoring-Programm zu verstehen (ebd., S. 72).

Inhaltlich lässt sich zu den bereits stattfindenden formellen und informellen Berufseinführungsphasen feststellen, dass die häufigste Maßnahme geplante Treffen zwischen BerufsanfängerInnen und erfahrenen Lehrpersonen darstellen. Weiters werden Tätigkeiten im gemeinsamen Team-Unterricht und schließlich Maßnahmen zur administrativen Einführung im Schulalltag genannt (ebd., S. 74). Dieser Befund deckt sich mit den Ergebnissen der anschließenden empirischen Arbeit (Empirischer Zugang, Kap. III), wo diese genannten Argumente als Herausforderungen und zu initiierende Hilfestellungen explizit genannt werden.

Für Greiner (2019) steht außer Zweifel, dass sowohl SchulleiterInnen als auch LehrerInnen der formellen Einführungsphase, also der seit dem Schuljahr 2019/20 verbindlichen Induktionsphase mit begleitetem Mentoring-Prozess, eine „hohe Bedeutung zumessen“ (ebd., S. 80). Allerdings wird das in der praktischen Umsetzung noch nicht bestätigt. Insgesamt wird deutlich, dass die Worte in der einzuführenden Induktionsphase in Taten umgesetzt werden müssen. Im Verlgeich zu den AHS und BMHS hat die Begleitung von BerufsanfängerInnen beim Berufseinstieg an der Neuen Mittelschule noch keine Tradition. Es gab bisher zwar von Praxislehrpersonen betreute pädagogisch praktische Studien, jedoch kein Unterrichtspraktikum, welches in Teilen Analogien zur Induktionsphase aufweist (ebd., S. 81).

Abschließend kann festgestellt werden, dass die Attrahierung, Auswahl und Ausbildung der MentorInnen, verbunden mit den ihnen zur Verfügung gestellten Ressourcen, von zentraler Bedeutung sein wird, wenn es gilt, die Herausforderung der Umsetzung der Induktionsphase mit dem begleitenden Mentoring-Programm zu meistern. Diese Einsicht wird von vielen AkteurInnen des österreichischen Bildungssystems geteilt, die Etablierung im Gesamtsystem der LehrerInnenbildung steht jedoch noch aus (Greiner 2010). 


\section{Resümee der Grundlagendiskussion}

In Kapitel II Grundlagen wird die Professionalisierung von Lehrpersonen diskutiert, da diese einerseits Teil der Aus- und Fortbildung ist, andererseits als Gelingensbedingung für einen positiven Berufsstart gesehen wird. An dieser Stelle werden die theoretischen Erkenntnisse rekapituliert.

\subsection{Disposition der inhaltlichen Schwerpunkte}

Der Grundlagenteil der vorliegenden Arbeit ist so konzipiert, dass ausgehend von den allgemeinen Phänomenen Ungewissheit bzw. Kontingenz (Kap. II.1.1) und LehrerInnenpersönlichkeit \& Habitus (Kap. II.1.2), die sich als Querschnittsthemen durch die gesamte Arbeit ziehen, in weiterer Folge die Aufmerksamkeit auf die Praxisphasen im Rahmen der LehrerInnenausbildung und des Berufseinstiegs gerichtet wird. Pädagogisch-praktische Studien während der LehrerInnenausbildung und die Induktionsphase mit dem begleitenden Mentoring-Prozess werden diskutiert, um die pädagogische Professionalisierung rund um den Übergang vom Studium in den Beruf auf mikrodynamischer Ebene darzustellen (Abb. 29).

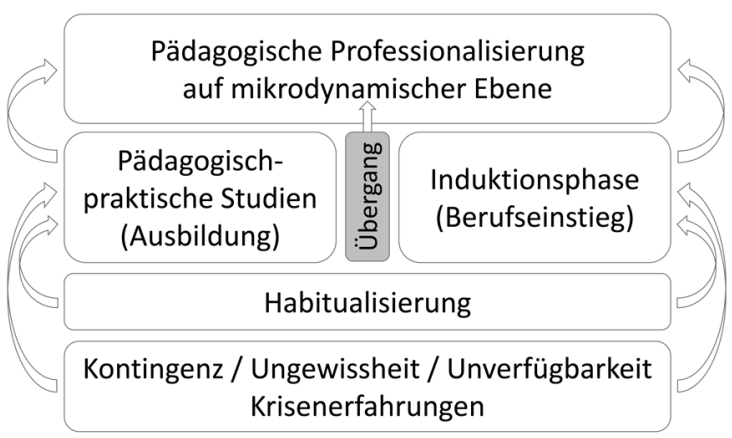

Abb. 29: Pädagogische Professionalisierung auf mikrodynamischer Ebene

Wie in Abbildung 29 visualisiert, stellen die Phänomene Kontingenz, Ungewissheit und Unverfügbarkeit (Gruschka 2018; Helsper 2001, 2005; Oevermann 2017; Paseka et al. 2018; Rosa 2018; Schrittesser 2011) mit den einhergehenden Krisenerfahrungen (Dietrich 2018; Kosinár 2018; Scheeler 2008) und der Habitualisierung nach dem Bourdieu'schen Habituskonzept (Bourdieu 1996a, 2015a; Lenger et al. 2013; Nairz-Wirth 2011; Schumacher 2013) die Diskussionsgrundlage der vorliegenden Arbeit dar. Der Interessensschwerpunkt liegt im Grundlagenteil auf der Professionalisierung in den Praxisphasen als (angehende) Lehrperson. Diese werden zum einen durch die pädagogisch-praktischen Studien im Rahmen der LehrerInnenausbildung und zum anderen durch die Induktionsphase mit formellem Mentoring abgebildet. Zwischen den pädagogisch-praktischen Studien in der Ausbildungsphase und der Induktionsphase im ersten Berufsjahr liegt der Übergang, der in der vorliegenden Arbeit fokussierte berufsbiografische Lebensabschnitt. Als Ziel wird die Rekonstruktion der pädagogischen Professionalisierung auf mikrodynamischer Ebene unter besonderer Berücksichtigung der Phase des Übergangs im Kontext der Prä- und Postprofessionalisierungsphasen verfolgt. 


\subsection{Ungewissheit, Kontingenz und doppelte Kontingenz}

Als Querschnittsthemen innerhalb der Professionalisierungsdebatte kristallisieren sich Ungewissheit, Kontingenz, Unverfügbarkeit und als häufige Konsequenz eine damit einhergehende Krisenerfahrung heraus. Es herrscht diesbezüglich Einigkeit darüber, dass die Ungewissheit im pädagogischen Feld in der Nicht-Standardisierbarkeit des pädagogischen Handelns begründet ist (Helsper 2005; Nairz-Wirth 2011; Schrittesser 2011). Neben den verschiedenen Erscheinungsformen von Kontingenz, z.B. hinsichtlich der Unterrichtsinhalte, des Unterrichtsgeschehens oder der Lernprozesse und -ergebnisse, wird dieses Phänomen insofern noch komplexer, als es sich durch das Zusammentreffen mindestens zweier Parteien um doppelte Kontingenz handelt. Viele Fragen zur Ungewissheit, z.B. „Wer bin ich als LehrerIn und ErzieherIn?“, „Was geschieht in den Köpfen der SchülerInnen?" (Gruschka 2018), tragen u.a. zur unvermeidbaren Krisenerfahrung und -bewältigung im LehrerInnenberuf, insbesondere beim Berufseinstieg, bei. Einen Lösungsweg stellt für die Lehrperson die Rollenübernahme des Kriseninduzierers (Combe et al. 2018) dar, ganz im Gegensatz zum Versuch der Krisenvermeidung im Sinne der Schließung von Ungewissheit (Kosinár 2018).

In Bezug auf den Umgang mit Krisensituationen besteht für die LehrerInnenausbildung die Herausforderung darin, die Studierenden und späteren BerufsanfängerInnen auf die Ungewissheit, deren Komplexität beim Berufseinstieg etwa im Vergleich zu den Praktika sprunghaft ansteigt, vorzubereiten. Die in der Theorie diskutierten Varianten zum Umgang mit Ungewissheit reichen vom Versuch der Schließung bis zu ihrer Anerkennung. Einen Mittelweg stellt die Ausbalancierung von Krisen und Routinen dar (ebd.).

\subsection{Habitus und Professionalisierung}

Die pädagogische Wirksamkeit einer Lehrperson hängt eng mit der jeweiligen LehrerInnenpersönlichkeit zusammen (Helsper 2018), die sich aufgrund der Habitualisierung konstituiert, sofern Wahrnehmungs-, Denk- und Handlungsschemata als Ausprägungsmerkmale für die LehrerInnenpersönlichkeit angenommen werden (Bourdieu 1996a, 2015a).

Der von Bourdieu diskutierte Habitusbegriff weist auf die Vermittlung zwischen Subjekt und Objekt, zwischen Individuum und Gesellschaft durch den Vorgang der Inkorporierung sozialer Zwänge beim Individuum hin. Der Begriff des Feldes spielt in der Habitusdiskussion eine zentrale Rolle. Das Individuum (Subjekt) wirkt im jeweiligen Feld, ausgestattet mit Macht- und Kapitalressourcen. Ebenso wie sich das Individuum im Feld ausrichtet, wirkt es seinerseits strukturierend und feldgestaltend und interagiert bzw. resoniert (Rosa 2018) mit dem Objekt, der sozialen Umwelt. Als Grundbedingung für Interaktion und Resonanz wird die Konstitution der oben genannten Ungewissheit und Unverfügbarkeit angenommen (Paseka et al. 2018; Rosa 2018; Schrittesser 2011).

Die generierende Wirkung des Habitus (Bourdieu 2015a) kommt dadurch zum Ausdruck, dass durch eine kleine Anzahl von Schemata beliebig viele variable Praktiken für neue Situationen erzeugt werden können. Der Handlungsspielraum des Individuums, der Lehrperson, steigt. Insofern verlieren Ungewissheit und Unverfügbarkeit ihren bedrohlichen Charakter und werden zur gängigen pädagogischen Praxis.

Der Zusammenhang zwischen Professionalisierung und Habitualisierung liegt in der Herausbildung eines wissenschaftlich-reflexiven Habitus. Die Habitualisierungstriade des Wahrnehmens, Denkens und Handelns entwickelt sich durch wissenschaftlich-reflexive Kompetenzen (Helsper 
2018; Hericks et al. 2018; Kraler 2008b) zur Triade des professionellen Wahrnehmens, Denkens und Handelns. Über Inkorporierung und durch Wissenschaftslehre sowie praxisgestützte Lernprozesse entwickeln (angehende) Lehrpersonen über sukzessive Habitualisierung einen professionellen LehrerInnenhabitus aus. Dieser gilt als Konzept der Professionalität, das ist die Kernidee der vorliegenden Arbeit.

\subsection{Pädagogische Professionalisierung}

Die größten Unterschiede zwischen allgemeinen und pädagogischen Professionen stellen einerseits die völlig verschiedenen Arbeitsverhältnisse zwischen der Klientel und dem professionellen Gegenüber und andererseits die nicht vorhandene Kontraktfähigkeit ${ }^{41}$ der SchülerInnen dar (Schrittesser 2011). Es gibt aber auch Strukturanalogien allgemeiner und pädagogischer Professionen, wobei die krisenhafte Sachthematik ohne Standardlösungen und der Umgang mit Ungewissheit bzw. Kontingenz zentrale Bedeutung haben und eine inhaltliche Überschneidung zur oben beschriebenen Kontingenz etc. darstellen.

Zur Darstellung des Übergangs von der Ausbildung in den Beruf und des mikrodynamischen Prozesses im Sinne einer kurzen berufsbiografischen Phase im Verhältnis zur gesamten Berufslaufbahn, wird auch die unmittelbare Zeit vor und nach dem Übergang diskutiert. Konkret sind dies die pädagogisch-praktischen Studien und der Berufseinstieg in Form des ersten Berufsjahres, der Induktionsphase mit begleitetem formellen Mentoring. Während der pädagogischpraktischen Studien stehen drei inhaltliche Schwerpunkte, Forschendes Lernen, Reflexion über Unterricht und Kooperation der AkteurInnen, im Zentrum des Interesses (Liegmann et al. 2018; Poel \& Heinrich 2018). Hier zeigt sich, dass über die Reflexion die Herausbildung eines wissenschaftlich-reflexiven Habitus eingeleitet wird (Helsper 2018; Hericks et al. 2018; Kraler 2008b). Der Habitualisierungsprozess ist ein bestimmender Faktor der Professionalisierung als Lehrperson. Dies stellt auch ein Ergebnis des vorliegenden empirischen Teils dar. Insofern wird Habitus als Konzept der Professionalität als die Kernidee in den Grundlagen dargestellt.

Bezüglich der Professionalisierung beim Berufseinstieg wird in der vorliegenden Arbeit zwischen dem ersten Berufsjahr (Induktionsphase) und dem zweiten bzw. dritten Berufsjahr unterschieden. In Kapitel II.2.3 werden der Mentoring-Prozess mit Begriffsbestimmungen, die Rollen der AkteurInnen MentorIn, Mentee und SchulleiterIn, theoretische Modelle mit deren Wirksamkeiten, Determinanten und Zielen sowie der Status quo des Mentorings an österreichischen Schulen diskutiert.

In Empirischer Zugang (Kap. III) werden die in den Grundlagen beschriebenen theoretischen Erkenntnisse aus der Sicht Betroffener (BerufsanfängerInnen) und ExpertInnen (SchulleiterInnen) im Kontext des dieser Arbeit zugrunde liegenden Forschungsinteresses (Einleitung Kap. II) und der Forschungsfragen (Kap. III.1.1) diskutiert. Über die Erhebung, Darstellung, Analyse sowie Interpretation der Daten wird aus evidenzbasierten Erkenntnissen durch einen multimethodischen Zugang ${ }^{42}$ ein Konzept zur Professionalisierung von (angehenden) Lehrpersonen rekonstruiert. Die Diskussion von Bedingungsgefügen schließt den empirischen $\mathrm{Zu}-$ gang ab. Fünf Themen, die als Essenzen aus abstrahierten Aussagen resultieren, werden mit den Hauptkategorien der Analyse in Beziehung gesetzt und interpretiert.

41 Wenn SchülerInnen unmündig im Sinne der noch nicht erreichten Volljährigkeit sind.

42 Begründung und Konzeption des multimethodischen Zugangs werden in Forschungsdesign (Kap. III.1) ausgeführt. 


\section{Empirischer Zugang}

Im zweiten Teil der vorliegenden Arbeit werden die Themen Forschungsdesign (Kap. III.1), Methodologie (Kap. III.2), Analyse (Kap. III.3) und Interpretation der analysierten Daten (Kap. III.4) diskutiert.

In Kapitel III.1 (Forschungsdesign) erfolgt die methodische Darstellung und Begründung der gewählten Erhebungs- und Auswertungsverfahren. In diesem Abschnitt wird der methodologische Weg von der Datenerhebung bis zur Rekonstruktion des Ergebniskonzepts dargestellt. Im Abschnitt III.2 (Methodologie) erfolgt der wissenschaftliche Blick auf die in der Forschungsarbeit gewählten Forschungsmethoden, von der Datenerhebung über die Analyse bis hin zur Auswertung und Darstellung. Begründungen für die Wahl der jeweiligen Verfahren bzw. Methoden werden an dieser Stelle angegeben. Abschnitt III.3 (Analyse) bildet den Prozess der Datenanalyse ab. Das verwendete Kategoriensystem bedient sich des Ansatzes der Grounded Theory (Strauss \& Corbin 1996), die Konzeptentwicklung erfolgt mittels Clusteranalyse (Bortz \& Döring 2006). In Kapitel III.4 (Interpretation) werden nach dem Paradigma der qualitativen Inhaltsanalyse (Kuckartz 2016) kategorienüberschneidende Themen identifiziert und die Vernetzungen bzw. Bedingungen zwischen Themen und Kategorien dargestellt, der Abstraktionsgrad reduziert und der Blick auf Details bzw. auf die empirisch gewonnenen Phänomene gelenkt. Die Interpretationen werden zur Validierung durch Zitate der SchulleiterInnen und BerufsanfängerInnen belegt. 


\section{Forschungsdesign}

Im Folgenden werden die Fragestellungen, die methodischen Überlegungen und die Durchführungspraxis der empirischen Untersuchung dargelegt. Mit der vorliegenden Forschungsarbeit wird die Intention verfolgt, sowohl Gelingensbedingungen für einen erfolgreichen Berufseinstieg von BerufsanfängerInnen zu identifizieren als auch deren Auswirkung auf den weiteren beruflichen Lebenslauf herauszufinden. Das daraus folgende Forschungsziel lautet, Konsequenzen für das Curriculum und die Induktionsphase sowie Möglichkeiten der konkreten Umsetzung aufzuzeigen. Die Darstellung der konkreten Umsetzungsmöglichkeiten ist Inhalt der Zusammenführung und Integration (Kap. IV).

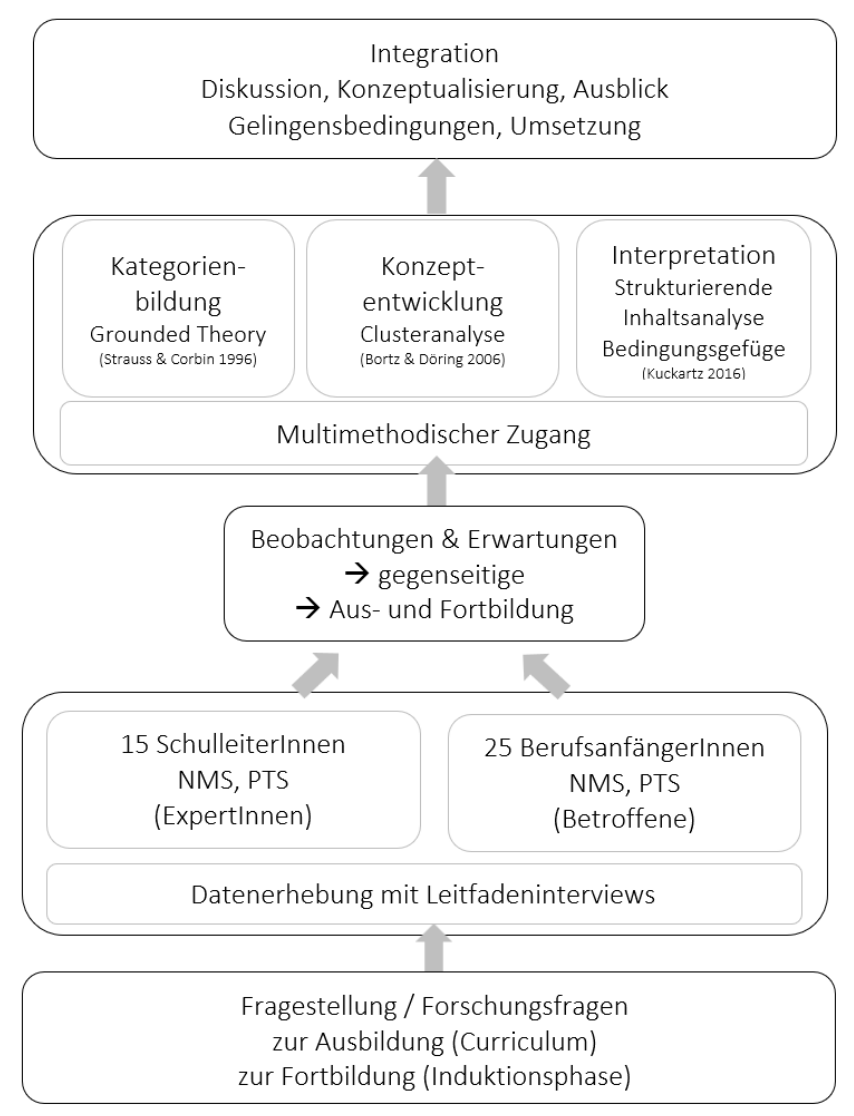

Abb. 30: Forschungsdesign der Untersuchung

In Abbildung 30 ist das Forschungsdesign mit den methodischen Schritten und den zugrunde liegenden Theorien bzw. Absichten dargestellt. Ausgehend von den Forschungsfragen erfolgt mit daraus abgeleiteten Fragestellungen zur Aus- und Fortbildung der (angehenden) Lehrper- 
sonen in Form von Leitfadeninterviews die Datenerhebung zur Untersuchung. Als Sample werden zum einen 15 SchulleiterInnen von Neuen Mittelschulen (NMS) und Polytechnischen Schulen (PTS) (siehe auch Kap. III.1.3.2) und zum anderen 25 BerufsanfängerInnen (siehe auch Kap. III.1.3.3), ebenfalls aus Neuen Mittelschulen (NMS) und Polytechnischen Schulen (PTS), herangezogen. In den Interviews werden Beobachtungen im Sinne von Selbst- und Fremdwahrnehmungen sowie gegenseitige (Schulleitung - BerufsanfängerIn) und an die Ausund Fortbildung gerichtete Erwartungen thematisiert. Mit einem dreiteiligen, multimethodischen Zugang werden die Daten computerunterstützt (Software: MAXQDA) (Kuckartz 2016) analysiert und auf Basis der Grounded Theory (Strauss und Corbin 1996) Kategorien gebildet (Kap. III.3.1), um in Folge mithilfe einer Clusteranalyse (Bortz und Döring 2006) zur Konzeptualisierung (Kap. III.3.4) der analysierten Daten zu gelangen. Schließlich werden im dritten methodischen Schritt nach dem Paradigma der strukturierenden Inhaltsanalyse (Kuckartz 2016) die Daten mithilfe von Bedingungsgefügen interpretiert. Der multimethodische Zugang dient in der vorliegenden Untersuchung nicht der Triangulation, sondern um das Feld hinsichtlich der befragten Personen (SchulleiterInnen und BerufsanfängerInnen) und der gewählten Methoden (Interview, Grounded Theory, Clusteranalyse, Inhaltsanalyse) möglichst breit zu rekonstruieren. Die Erkenntnisse aus den Grundlagen (Kap. II) und der Empirie (Kap. III) werden zusammengeführt und integriert (Kap. IV), um mithilfe der Konzeptualisierung Gelingensbedingungen für den Berufseinstieg zu diskutieren. Schließlich erfolgt ein Ausblick auf konkrete Umsetzungsmöglichkeiten der gewonnenen Erkenntnisse (Kap. V).

In den folgenden Abschnitten steht in Kapitel III.1.1 (Forschungsfragen) die detaillierte Darstellung der Ziele der vorliegenden Arbeit und der daraus resultierenden Forschungsfragen im Mittelpunkt, ebenso wird die Wahl der Leitfadeninterviews als Datenerhebungsinstrument begründet. Im Abschnitt Datenerhebung (Kap. III.1.2) erfolgen die Planungs- und Durchführungsbeschreibung des empirischen Vorgehens sowie die Darstellung des Leitfadeninterviews als wissenschaftliche Datenerhebungsmethode. In III.1.3 (Sample) wird zur Auswahl der InterviewpartnerInnen und zur konkreten Interviewsituation Stellung genommen. Mit bibliografischen Datentabellen zur Sample-Übersicht wird die Darstellung des Forschungsdesigns abgeschlossen.

\subsection{Forschungsfragen}

Im Folgenden werden die dieser Arbeit zugrunde liegenden Forschungsfragen genannt, nachdem einleitend zur Entstehungsgenese dieser Stellung genommen wird. Das Erkenntnisinteresse, welche Determinanten die Berufsbiografie einer Lehrperson im Allgemeinen und jene einer Berufsanfängerin bzw. eines Berufsanfängers im Speziellen in welcher Weise beeinflussen, fokussiert in dieser Arbeit die Gelingensbedingungen für einen erfolgreichen Berufseinstieg bzw. für die weitere positive die Entwicklung der Berufsbiografie. Dies betrifft sowohl die Aus- als auch die Fortbildung von Lehrpersonen. Vor dem Hintergrund, dass nach den Erkenntnissen in den Grundlagen (Kap. II) Kontingenzerfahrungen und Unverfügbarkeit zu Ungewissheit und Krisen führen können (Hericks et al. 2018; Keller-Schneider und Hericks 2014; Kosinár 2018; Oevermann 2017; Paseka et al. 2018; Rosa 2018; Schrittesser 2011) und dass die Professionalisierung durch die Herausbildung eines professionellen Habitus (Bourdieu 1996a, 2015a; Helsper 2018; Nairz-Wirth 2011) im Sinne von Habitus als Konzept von Professionalität (siehe auch Kap. II.1.2.3) ein Lösungsansatz im Umgang mit den Herausforderungen beim Berufseinstieg sein kann, kristallisieren sich die folgenden Forschungsfragen heraus. Die empirische Ar- 
beit baut auf diesen drei Forschungsfragen auf. Sie stehen im inhaltlichen Kontext zueinander und werden stets ganzheitlich betrachtet:

\section{Forschungsfrage 1:}

Welche Inhalte benötigen LehramtsstudentInnen während der Ausbildung und welche Hilfestellungen benötigen BerufsanfängerInnen während der ersten Berufsjahre, um den Berufseinstieg für sich und die Personen ihres unmittelbaren Arbeitsumfelds (SchülerInnen, Eltern, KollegInnen, Vorgesetzte) erfolgreich absolvieren zu können? Hierbei geht es im Kern um Gelingensbedingungen für den Prozess eines erfolgreichen Berufseinstiegs.

\section{Forschungsfrage 2:}

Wie wirkt sich die initiale Berufserfahrung auf den unmittelbaren weiteren beruflichen Verlauf (bis drei Jahre) der BerufsanfängerInnen aus? Mit dieser Frage wird die erste Entwicklungsrichtung der Berufslaufbahnen junger BerufskollegInnen thematisiert.

\section{Forschungsfrage 3:}

Welche Anforderungen ergeben sich aus den gewonnenen Erkenntnissen an ein Curriculum für das Lehramtsstudium und an die Induktionsphase für BerufsanfängerInnen? In der vorliegenden Arbeit sollen konkrete, umsetzbare Erkenntnisse für die Weiterentwicklung der LehrerInnenbildung in der Ausbildungs- und ersten Berufsphase identifiziert werden.

Nachdem mit den Forschungsfragen das Forschungsinteresse konkretisiert ist, wird im Folgenden die Methode der Datenerhebung skizziert.

\subsection{Datenerhebung}

Mit der vorliegenden Untersuchung soll ein Konzept für einen gelingenden Berufseinstieg formuliert werden. Aeppli (2014) stellt fest, dass durch mündliche Befragungen gezielte Informationen in Form von verbalen Antworten eingeholt werden können, um Konstrukte und theoretische Zusammenhänge zu untersuchen bzw. übergeordnete Forschungsfragen zu beantworten. Aus diesem Grunde wird zur Untersuchung der oben formulierten Forschungsfragen das leitfadengestützte Interview als Datenerhebung eingesetzt. In der Arbeit wird Wirklichkeit bzw. soziale Realität in Form einer Rekonstruktion abgebildet. Dafür ist das Interview insofern ein geeignetes Instrument zur Datenerhebung, als im qualitativen Paradigma Konstruktionen von Welt beforscht werden (Friebertshäuser \& Langer 2010) und mithilfe von Interviews Zugang zum Forschungsfeld hergestellt wird. Im direkten Gespräch mit an der Thematik interessierten Personen ${ }^{43}$ können deren Konstruktionen von Sinn und Bedeutungen, die wiederum ihr Handeln steuern, identifiziert werden (ebd.).

\section{Leitfadeninterviews mit Betroffenen und ExpertInnen}

Mit der Methode des Leitfadeninterviews werden subjektive Sichtweisen verschiedener sozialer Gruppen untersucht. Die Zielsetzung kann dabei die Generierung von Hypothesen für spätere quantitative Untersuchungen, aber auch die vertiefende Interpretation experimenteller Ergebnisse sein (Flick 2007, S. 201). Die Leitfadeninterviews werden mit zwei verschiedenen zu untersuchenden Personengruppen durchgeführt, mit LehrerInnen (BerufsanfängerInnen) als Betroffene

43 Die Auswahl des Samples erfolgte über ein Informationsschreiben zu den Untersuchungszielen mit der expliziten Bitte, sich bei Interesse als InterviewpartnerIn zur Verfügung zu stellen. 
und SchulleiterInnen als ExpertInnen. Das Leitfadeninterview eignet sich als Datenerhebungsinstrument für ein anschließendes Kodierungs-Auswertungsverfahren „in ganz besonderem Maße“ (Flick 2007, S. 201) und erfährt in der qualitativen Sozialforschung größte Aufmerksamkeit. Insofern ist der erste Schritt der Datenauswertung in Form einer Kategorienbildung nach der Grounded Theory (Strauss \& Corbin 1996) eine logisch Konsequenz (siehe auch Kap. III.2.2). In der relativ offenen Gestaltung der Interviewsituation kommen die Sichtweisen des befragten Subjekts eher zur Geltung als in standardisierten Interviews oder Fragebögen (ebd., S. 194).

Eine besondere Rolle nimmt in diesem Zusammenhang das ExpertInneninterview ein. Es ist eines der am häufigsten eingesetzten Verfahren in der empirischen Sozialforschung (Meuser \& Nagel 2009, S. 35). Zur Identifizierung interner Abläufe innerhalb einer Institution, in der vorliegenden Untersuchung sind das Vorgänge innerhalb der Schule, im Ausbildungssystem etc., bietet sich nach Friebertshäuser und Langer (2010, S. 438) ein leitfadenorientiertes Vorgehen mit ExpertInneninterviews an. In diesem Sinne wird eine ExpertInnenbefragung mit Betroffenen (BerufsanfängerInnen) als Selbstwahrnehmung und mit ExpertInnen (SchulleiterInnen) als Fremdwahrnehmung durchgeführt.

Die Interviews der BerufsanfängerInnen befassen sich inhaltlich vorrangig mit zwei Themenkomplexen, der LehrerInnenausbildung und dem Berufseinstieg (siehe Anhang). Mit der Auswertung der Interviews dieser Personengruppe werden der Stand der LehrerInnenausbildung und die Phase des Berufseinstiegs aus der Sicht von BerufsanfängerInnen rekonstruiert. Die SchulleiterInneninterviews (siehe Anhang) liefern Erkenntnisse zum Berufseinstieg der BerufsanfängerInnen aus der Perspektive der bzw. des Vorgesetzten. Das Ziel ist die Rekonstruktion der Anforderungen an die BerufsanfängerInnen im LehrerInnenberuf durch die SchulleiterInnen und durch das System Schule. Das betrifft hierarchische Strukturen ebenso wie personelle Ebenen im LehrerInnenberuf. Konkret sind hier Dienstgeber, Eltern, KollegInnenschaft etc. gemeint.

Die vorliegenden Leitfäden zu den Interviews mit BerufsanfängerInnen und SchulleiterInnen dienen als Impulsgeber bzw. Orientierungshilfen für Interviewer und InterviewpartnerIn. Für die narrativen Passagen in den Interviews gilt die Annahme, dass diese eine besonders „dichte" Rekonstruktion des Erlebten liefern. Dies führt zur Darstellung der gegenwärtigen Perspektive und der aktuellen Relevanzsetzungen und Problemdeutungen des Erzählers (Hericks 2006, S. 142). Insofern impliziert die Existenz eines Leitfadens nicht, dass dieser sequenziell abgearbeitet werden muss. So kann man die Abfolge der Leitfragen verändern oder Fragen bzw. Fragebereiche weglassen, wenn diese im Rahmen anderer Ausführungen hinreichend behandelt wurden. Ebenso können Fragen situativ verändert oder ergänzt bzw. durch Nachfragen vertieft werden. Schließlich kann man als Forscher auch auf neue Aspekte, die sich im Gespräch ergeben, spontan eingehen (Weischer 2007, S. 273) und sich die Vorteile der narrativen Gesprächsführung zunutze machen.

\section{Interviewsituation}

Die Interviews wurden als Face-to-Face-Interviews geführt, damit eine gewisse Verbindlichkeit und Vertiefung des Gesprächs erreicht werden konnte (Weischer 2007, S. 276). Durch den persönlichen Kontakt mit den InterviewpartnerInnen fällt die Anfertigung des Postskripts (siehe auch Kap. III.1.2) leichter, welches durch das Face-to-Face-Gespräch auch die Ebene der nonverbalen Kommunikation erfasst.

Alle Interviews fanden an den jeweiligen Dienstorten der InterviewpartnerInnen statt. Diese Entscheidung erfolgte zum einen aus pragmatischen Gründen, da aus eigener Erfahrung InterviewpartnerInnen einem Interview dann eher zustimmen, wenn dieses ohne Wegzeiten durchgeführt werden kann. Zum anderen auch deshalb, um die Gesprächssituationen möglichst au- 
thentisch und angenehm - im Sinne eines bekannten Umfelds - zu gestalten. Darüber hinaus kann davon ausgegangen werden, dass die Erfassung der sozialen Realität (Lamnek \& Krell 2010) im realen beruflichen Umfeld am wahrscheinlichsten zu gewährleisten ist.

\section{Erfassung, Transkription und Auswertung der Daten}

Alle Interviews liegen als Tonaufzeichnungen vor. Das Einverständnis zur Aufnahme wurde von allen InterviewpartnerInnen im Vorhinein eingeholt. Die Transkriptionen sowie die anschlieBende Auswertung wurden mithilfe der Software MAXQDA (Kuckartz 2016) vorgenommen. Als Grundlage für die Transkriptionsregeln wurden jene für eine computerunterstütze Auswertung (ebd.) herangezogen. Insbesondere die Arbeit mit Absatzmarken, unterstützt durch die einfache Umsetzung der QDA ${ }^{44}$-Software (ebd.) stellte sich im späteren Analyseprozess als besonders hilfreich heraus. Jedes Transkript wurde vor der Analyse aus forschungsethischen Gründen allen InterviewpartnerInnen vorgelegt. Erst nach der Freigabe des transkribierten Texts durch die InterviewpartnerInnen wurde damit weitergearbeitet. In der vorliegenden Untersuchung hat nur ein/e InterviewpartnerInnen von insgesamt 40 (15 SchulleiterInnen, 25 BerufsanfängerInnen) die Verwendung des Transkripts untersagt, woraufhin eine Ersatzperson zur Vervollständigung des Samples gefunden wurde. Der Auswertungs- bzw. Analyseprozess erfolgte computerunterstützt. Die Unterstützung durch die QDA-Software begann bereits beim Importieren der AudioDateien, kam jedoch beim Auswertungsprozess durch Kodierung, Kategorienbildung, Memos Schreiben, Sortieren, Visualisieren u.v.m. erst richtig zur Geltung.

Die methodisch-theoretischen Hintergründe zur Analyse im Kontext zur MAXQDA-Software werden in Methodologie (Kap. III.2) ausgeführt.

\section{Postscripts}

Nach jedem durchgeführten Interview wurde ein Postscript verfasst. Dabei wurden Rahmenbedingungen, Kommunikationssituationen, Verhaltensweisen der InterviewpartnerInnen und Interaktionssituationen auf der Metaebene beschrieben. Sachverhalte, die im Interview nicht zum Ausdruck kommen, können für die Interpretation wichtige Aspekte enthalten (Flick 1995, 2007). Ebenso können zusätzliche Metainformationen zu den InterviewpartnerInnen erhoben werden. Nervosität, Gereiztheit, scheinbares Desinteresse oder auch das persönliche Anliegen zum Informationsaustausch können dokumentiert und über Memos (Kuckartz 2016; Strauss \& Corbin 1996) in die Interpretation eingebracht werden (Lamnek \& Krell 2010). Weiters wurden für die Forschungsarbeit inhaltlich relevante Aspekte teilweise erst nach dem offiziellen Interview ausgetauscht. Nach dem Einverständnis der InterviewpartnerInnen wurden auch diese Inhalte transkribiert und in die Analyse mit einbezogen. Somit bilden die Postscripts nicht nur einen interpretativen, sondern auch einen inhaltlichen Wert für die Datenanalyse.

\subsection{Sample}

Für die vorliegende Untersuchung wurden qualitative Daten von SchulleiterInnen $(n=15)$ und BerufsanfängerInnen $(\mathrm{n}=25)$, die an einer Neuen Mittelschule (NMS) oder Polytechnischen Schule (PTS) tätig sind, eingehoben und ausgewertet. Im Folgenden werden nach den beiden Schultypen beide Samplegruppen (SchulleiterInnen und BerufsanfängerInnen) hinsichtlich der soziodemografischen Merkmale bzw. Variablen ${ }^{45}$ (Rädiker \& Kuckartz 2019) beschrieben.

44 Qualitative Datenanalyse

45 Terminologie in der Auswertungssoftware MAXQDA (Kuckartz 2016; Rädiker und Kuckartz 2019). 
Die Diagramme werden ausschließlich aus den Daten der jeweiligen Übersichtstabelle (Tab. 5: Sample der SchulleiterInnen bzw. Tab. 6: Sample der BerufsanfängerInnen) generiert. Stadt NMS (7) bedeutet, dass es sich um sieben Personen einer Neuen Mittelschule im städtischen Bereich handelt. Auf die Angabe der Prozentwerte wird in den Abbildungen verzichtet, da zum einen dieser Größenvergleich durch die Darstellung in Kreisringdiagrammen gewährleistet wird und zum anderen die Größenverhältnisse aufgrund der kleinen Samplegrößen unmittelbar vom absoluten Wert abgelesen werden können. Außerdem können Prozentwerte u.U. eher zur Verwirrung als zur Aufklärung beitragen. Wenn beispielsweise ein/e (1) BerufsanfängerIn von 25 zwei eigene Kinder hat, so ist die absolute Zahlenangabe 1 (bzw. 1/25 beim Gesamtblick) nach Einschätzung des Autors aussagekräftiger als die Prozentangabe von vier Prozent.

\subsubsection{Schultypen}

Alle InterviewpartnerInnen der vorliegenden Untersuchung sind entweder an einer Neuen Mittelschule (NMS) ${ }^{46}$ oder Polytechnischen Schule (PTS) tätig. Die Neue Mittelschule ist eine seit 2012 gesetzlich verankerte Regelschule für zehn- bis 14-jährige Kinder. Alle SchülerInnen mit positivem Abschluss der Volksschule können eine NMS besuchen. Die Aufgaben der NMS bestehen darin, SchülerInnen je nach Interesse, Neigung, Begabung und Fähigkeit für den Übertritt in weiterführende mittlere und höhere Schulen zu befähigen sowie auf das Berufsleben vorzubereiten. Der NMS-Lehrplan orientiert sich an jenem der AHS-Unterstufe, die pädagogischen Merkmale sind u.a. Individualisierung des Unterrichts, differenzierter Unterricht und Unterricht im LehrerInnenteam in Form von Teamteaching (Bundesministerium für Bildung, Wissenschaft und Forschung 1962, 2020a).

Die Polytechnische Schule unterstützt Jugendliche im letzten Jahr der allgemeinen Schulpflicht. Sie hilft den Jugendlichen beim Einstieg ins Berufsleben bzw. in die duale Berufsausbildung (Lehre). Ebenso sind weiterführende Bildungswege möglich. Im Mittelpunkt dieses Überleitungsprozesses stehen individuelle Interessen, Neigungen, Begabungen und Fähigkeiten der SchülerInnen. Die Polytechnische Schule umfasst ein Schuljahr und wird vorrangig von 14- bis 15-jährigen SchülerInnen im neunten Schuljahr besucht. Es sind Ausnahmeregelungen möglich, sodass auch 16- oder 17-jährige Jugendliche im zehnten bzw. elften Schuljahr die PTS zur Erlangung eines positiven Schulpflichtabschlusses besuchen. Die pädagogischen und fachlichen Ziele der PTS konzentrieren sich einerseits auf den Berufsorientierungs- und -findungsprozess, andererseits steht eine solide Berufsgrundbildung in Form von Unterricht in technisch, dienstleistungs- und kaufmännisch orientierten Fachbereichen im Vordergrund (Bundesministerium für Bildung, Wissenschaft und Forschung 1962, 2020b; Jäger 2001).

\subsubsection{SchulleiterInnen}

Die Frage der Auswahl der InterviewpartnerInnen stellt sich im Forschungsprozess an verschiedenen Stellen (Flick 2007). So gestaltete sich die Herangehensweise an das Sampling bei beiden Gruppen ${ }^{47}$ verschieden. Zur Auswahl der SchulleiterInnen als InterviewpartnerInnen spielten verschiedene Kriterien eine Rolle. Ein wesentliches Kriterium stellte in dieser Untersuchung die Geschlechterverteilung dar, um die Wahrnehmungen und Interpretationen der SchulleiterInnen in Bezug auf das Geschlecht repräsentativ abzubilden. Idealerweise entspricht somit das

46 In Österreich wird seit dem Schuljahr 2020/21 die Neue Mittelschule (NMS) als Mittelschule (MS) geführt (Bundesministerium für Bildung, Wissenschaft und Forschung 2020a). In der vorliegenden Arbeit wird die Bezeichnung NMS verwendet, das diese zum Zeitpunkt der Datenerhebung und -auswertung aktuell war.

47 SchulleiterInnen und BerufsanfängerInnen; Sample BerufsanfängerInnen (Kap. III.1.3.3) 
Verhältnis der männlichen und weiblichen InterviewpartnerInnen jenem der SchulleiterInnen an den Schulen, um eine Rekonstruktion des geschlechtsbezogenen Meinungsbilds zu gewährleisten (Abb. 31 und Abb. 32). Weitere Kriterien stellten die Mischung der untersuchten Schultypen, Neue Mittelschule und Polytechnische Schule, sowie die Abbildung der Schulstandorte (ländlicher und städtischer Bereich) dar. Letztlich wurde das Sampling auch von der pragmatischen Überlegung „Wer stellt sich für ein Interview zur Verfügung?" geleitet.

\subsubsection{1 Übersicht}

Das vorliegende Sample erfüllt weitgehend die Kriterien repräsentatives Geschlechterverhältnis, ausgewogenes Verhältnis beider Schultypen sowie Verteilung zwischen städtischem und ländlichem Bereich (Tab. 5). Die einzelnen soziodemografischen Merkmale Geschlecht, LehrerIn seit, Schulleiterin seit, Eigene Unterrichtstätigkeit als SchulleiterIn, Schultyp, Schulstandort, Schulgröße, Kollegium sind in Tabelle 2 dargestellt und werden anschließend beschrieben sowie in Diagrammen visualisiert. Ein zentrales Element stellt die Interviewbezeichnung dar. SL steht für SchulleiterIn, die beigefügte Zahl dient als Nummerierung der Interviews. So wird z.B. mit SL_01 das erste SchulleiterInneninterview gekennzeichnet. Diese Bezeichnungslogik wird in der gesamten Forschungsarbeit angewandt. Insbesondere in der Analyse und datenimmanenten Interpretation werden Belege in Form von Zitaten durch dieses Beschriftungssystem kenntlich gemacht.

Tab. 5: Sample der SchulleiterInnen $(\mathrm{n}=15)$

\begin{tabular}{|c|c|c|c|c|c|c|c|c|}
\hline 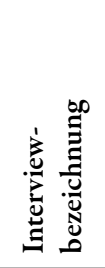 & 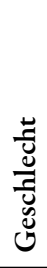 & 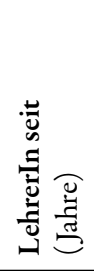 & 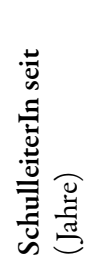 & 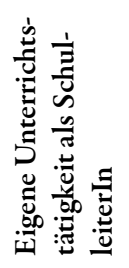 & 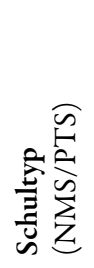 & 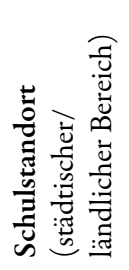 & 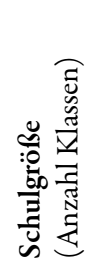 & 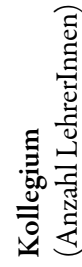 \\
\hline SL_01 & $\mathrm{m}$ & 18 & 4 & $\mathrm{Ja}$ & PTS & städt. B. & 5 & 13 \\
\hline SL_02 & $\mathrm{m}$ & 32 & 6 & Nein & NMS & städt. B. & 14 & 35 \\
\hline SL_03 & $\mathrm{m}$ & 41 & 15 & Nein & NMS & ländl. B. & 8 & 20 \\
\hline SL_04 & $\mathrm{m}$ & 26 & 4 & $\mathrm{Ja}$ & PTS & ländl. B. & 3 & 8 \\
\hline SL_05 & $\mathrm{w}$ & 39 & 10 & Nein & NMS & städt. B. & 8 & 24 \\
\hline SL_06 & $\mathrm{m}$ & 38 & 19 & $\mathrm{Ja}$ & PTS & städt. B. & 6 & 14 \\
\hline SL_07 & $\mathrm{m}$ & 26 & 2 & Nein & NMS & städt. B. & 14 & 35 \\
\hline SL_08 & $\mathrm{w}$ & 27 & 3 & $\mathrm{Ja}$ & PTS & ländl. B. & 4 & 10 \\
\hline SL_09 & $\mathrm{m}$ & 37 & 13 & Nein & NMS & ländl. B. & 11 & 25 \\
\hline SL_10 & $\mathrm{w}$ & 28 & 15 & $\mathrm{Ja}$ & PTS & städt. B. & 5 & 12 \\
\hline SL_11 & $\mathrm{m}$ & 29 & 4 & $\mathrm{Ja}$ & NMS & ländl. B. & 12 & 28 \\
\hline SL_12 & $\mathrm{m}$ & 32 & 5 & Nein & NMS & städt. B. & 9 & 24 \\
\hline SL_13 & $\mathrm{m}$ & 27 & 6 & Nein & PTS & städt. B. & 9 & 23 \\
\hline SL_14 & $\mathrm{m}$ & 34 & 6 & $\mathrm{Ja}$ & PTS & ländl. B. & 2 & 5 \\
\hline SL_15 & $\mathrm{w}$ & 20 & 5 & Nein & NMS & ländl. B. & 12 & 30 \\
\hline
\end{tabular}


Im folgenden Abschnitt werden die Kriterien des SchulleiterInnen-Samples erläutert. Die Darstellungen erfolgen jeweils grafisch und deskriptiv.

\subsubsection{Kriterien}

\section{Geschlecht und Schultyp}

\section{SchulleiterInnen nach Geschlecht und Schultyp - Sample}

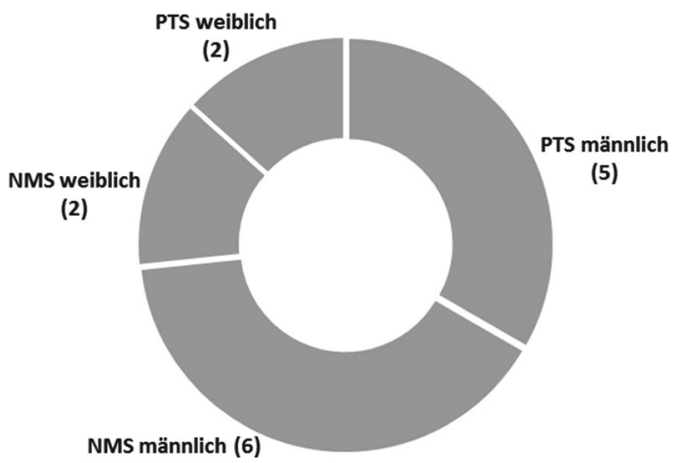

Abb. 31: Kriterien Geschlecht und Schultyp; SL

Insgesamt wurden 15 SchulleiterInnen interviewt, elf Männer und vier Frauen (Abb. 31). Das entspricht einem Männeranteil von rund 73 Prozent, der Frauenanteil liegt bei rund 27 Prozent. Von den elf Schulleitern ( $\mathrm{m}$ ) leiten fünf eine Polytechnische Schule und sechs eine Neue Mittelschule. Von vier Schulleiterinnen (w) sind jeweils zwei an Polytechnischen Schulen und Neuen Mittelschulen tätig.

Der tirolweite Vergleich bzgl. Geschlecht und Schultyp stellt sich wie folgt dar (Abb. 32).

Der Anteil an Schulleitern (m) in Tirol beträgt rund 68 Prozent, die Schulleiterinnen decken rund 32 Prozent ab. Zum Vergleich: Das Sample der SchulleiterInnen besteht aus ca. 73 Prozent (m) und ca. 27 Prozent (w) (Abb. 31). Somit bilden in beiden Gruppen (Sample der Untersuchung und tirolweite Vergleichsgruppe) die Männer einen größeren Anteil zwischen zwei Dritteln und drei Vierteln (Abb. 32).

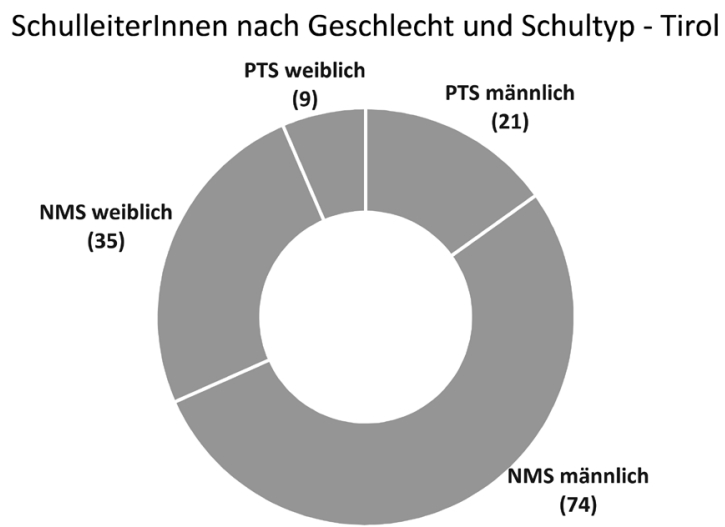

Abb. 32: Vergleichsverteilung nach Geschlecht und Schultyp; SL, Quelle: Bildungsdirektion für Tirol, Stand: 20.02.2019 


\section{Dienstzeit als SchulleiterIn}

\section{Dienstzeit als Schulleiterln}

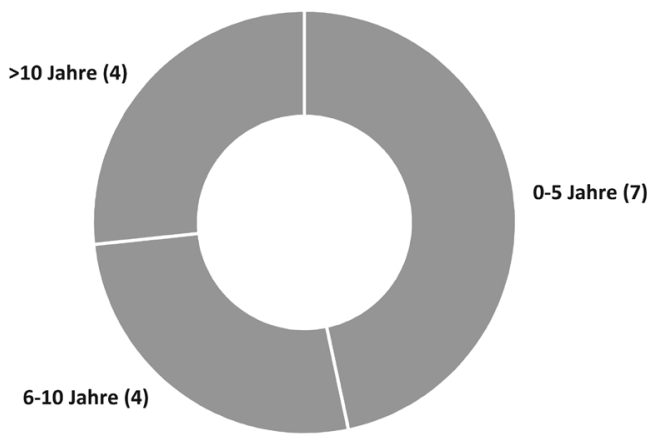

Abb. 33: Kriterium Dienstzeit; SL

Die Dienstzeit als Schulleiterin (Abb. 33) wird in Tabelle 5 unter "SchulleiterIn seit“ abgebildet. Knapp die Hälfte (7 von 15) der befragten SchulleiterInnen waren zum Zeitpunkt der Untersuchung null bis fünf Jahre in der Schulleitung tätig. Jeweils vier SchulleiterInnen hatten sechs bis zehn Jahre bzw. mehr als zehn Jahre Schulleitungserfahrung.

\section{Unterrichtstätigkeit als SchulleiterIn}

\section{Unterrichtstätigkeit der SchulleiterInnen}

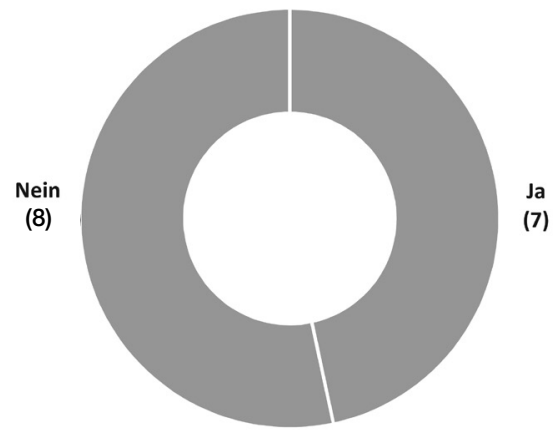

Abb. 34: Kriterium Unterrichtstätigkeit; SL

Das Kriterium Unterrichtstätigkeit der SchulleiterInnen (Abb. 34) wird in Tabelle 5 als „Eigene Unterrichtstätigkeit als SchulleiterIn“ betitelt. Ob SchulleiterInnen ausschließlich für die Schulleitung oder auch im Unterricht eingesetzt sind, hängt von mehreren, in dieser Untersuchung nicht berücksichtigten, Determinanten ab.

Jeweils ca. die Hälfte des SchulleiterInnen-Samples widmet sich ausschließlich der Schulleitung bzw. ist zusätzlich im Unterricht eingesetzt. Exakt sieben von 15 SchulleiterInnen unterrichten neben der Schulleitungsfunktion, acht von 15 SchulleiterInnen absolvieren keinen Unterricht. 


\section{Größe des Kollegiums}

\section{Größe des Kollegiums}

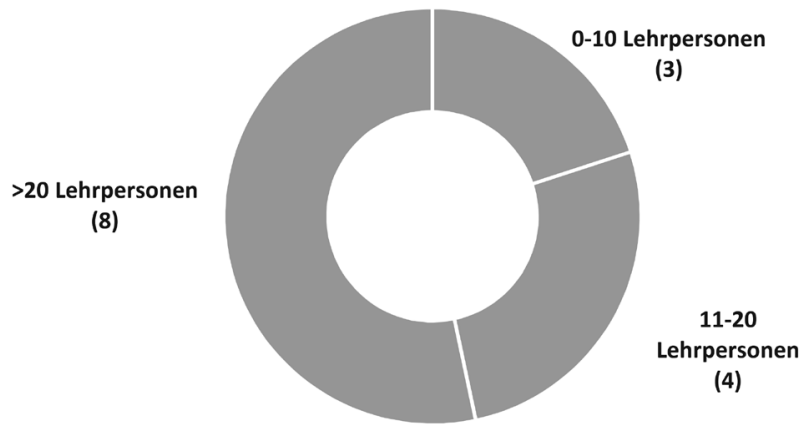

Abb. 35: Kriterium Größe des Kollegiums; SL

Ca. die Hälfte des SchulleiterInnen-Samples (acht von 15) führte eine Schule mit mehr als 20 Lehrpersonen. Vier SchulleiterInnen hatten zwischen elf und 20 LehrerInnen im Kollegium, bei drei SchulleiterInnen unterrichteten zehn oder weniger Lehrpersonen (Abb. 35).

\section{Exkurs: Größe des Kollegiums - Unterrichtstätigkeit als SchulleiterIn}

Zwischen dem Kriterium Größe des Kollegiums und Unterrichtstätigkeit als SchulleiterIn besteht ein Zusammenhang. Die Zahl der Lehrpersonen richtet sich unmittelbar nach der Größe der SchülerInnenzahl, welche wiederum die Anzahl der Klassen beeinflusst (Bundesministerium für Bildung, Wissenschaft und Forschung 1962). Die Anzahl der Klassen ist das Hauptkriterium dafür, ob eine Schulleiterin bzw. ein Schulleiter neben der Schulleitungsfunktion auch unterrichtet.

\section{Lage und Schultyp}

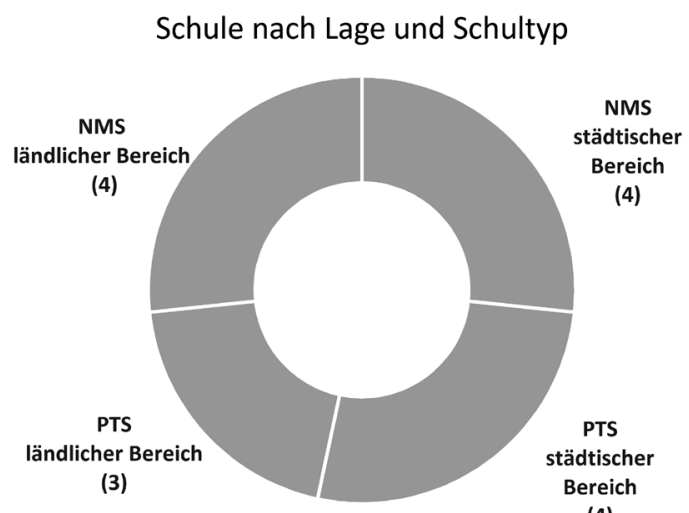

(4)

Abb. 36: Kriterien Lage und Schultyp; SL

Schließlich wurde im Rahmen der Untersuchung das Kriterium ländlicher oder städtischer Bereich erhoben (Abb. 36). Fast die Hälfte der befragten SchulleiterInnen (7 von 15) üben ihre 
Tätigkeit im ländlichen Bereich aus, acht von 15 SchulleiterInnen sind im städtischen Bereich tätig. Die Aufteilung zwischen den beiden Schultypen Neue Mittelschule und Polytechnische Schule war ausgeglichen. Jeweils vier Neue Mittelschulen lagen im städtischen Bereich, drei Polytechnische Schulen waren im ländlichen und vier im städtischen Bereich angesiedelt.

Nach der Darstellung der Kriterien des SchulleiterInnen-Samples erfolgt im nächsten Abschnitt die Beschreibung der Kriterien der interviewten BerufsanfängerInnen.

\subsubsection{BerufsanfängerInnen}

Für die Auswahl der BerufsanfängerInnen als InterviewpartnerInnen wurden spezifische soziodemografische Daten berücksichtigt, damit repräsentative und zur Rekonstruktion eines Konzepts in Bezug auf den Berufseinstieg geeignete empirische Erkenntnisse gewonnen werden konnten. Das primäre Merkmal zur Auswahl stellte die Anzahl der Dienstjahre der interviewten BerufsanfängerInnen dar. Es wurde darauf geachtet, dass BerufsanfängerInnen mit maximal drei Dienstjahren beim Sampling berücksichtigt wurden (Tab. 6, Abb. 37). Ein weiteres Merkmal war das Geschlecht der jungen Lehrperson. Hier wurde darauf geachtet, dass das Verhältnis der Geschlechterverteilung in etwa jenem der im Dienst stehenden Lehrpersonen im Tiroler Pflichtschuldienst entsprach (Abb. 38 und Abb. 39), um im Sinne einer Rekonstruktion repräsentative Daten zu erhalten, was die Wahrnehmung und Interpretation der BerufsanfängerInnen in Abhängigkeit von ihrem Geschlecht angeht. Der Schultyp und die Lokalität der Schule der BerufsanfängerInnen stellten weitere Kriterien dar. Schließlich wurde auch das Alter der BerufsanfängerInnen erhoben.

\subsubsection{1 Übersicht}

Das in Tabelle 6 dargestellte Sample orientierte sich an den oben genannten Kriterien. Die einzelnen soziodemografischen Merkmale Geschlecht, Biologisches Alter, Dienstjahre, Eigene Kinder, Schultyp, Schulstandort werden in Tabelle 6 aufgelistet und anschließend, visualisiert durch Abbildungen, beschrieben. Ein wesentliches Detail bildet die jeweilige Interviewbezeichnung. BA steht für BerufsanfängerIn und kombiniert mit der Nummer beschreibt z. B. BA_01 das erste Interview aus dem Sample der BerufsanfängerInnen. Diese Interviewbezeichnung zieht sich stringent durch die Forschungsarbeit. Insbesondere in der Analyse und datenimmanenten Interpretation werden Belege in Form von Zitaten durch dieses Beschriftungssystem kenntlich gemacht (Tab. 6).

Tab. 6: Sample der BerufsanfängerInnen $(n=25)$

\begin{tabular}{|c|c|c|c|c|c|c|}
\hline 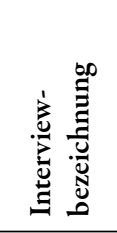 & 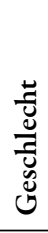 & 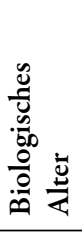 & 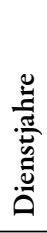 & 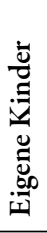 & 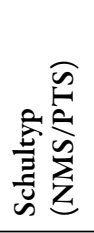 & 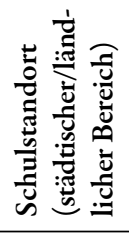 \\
\hline BA_01 & $\mathrm{w}$ & 24 & 3 & - & PTS & städt. B. \\
\hline BA_02 & $\mathrm{m}$ & 32 & 3 & - & PTS & ländl. B. \\
\hline BA_03 & $\mathrm{m}$ & 30 & 3 & - & PTS & städt. B. \\
\hline BA_04 & $\mathrm{w}$ & 25 & 1 & - & NMS & städt. B. \\
\hline BA_05 & $\mathrm{w}$ & 25 & 3 & - & NMS & ländl. B. \\
\hline BA_06 & $\mathrm{m}$ & 27 & 3 & 1 & PTS & ländl. B. \\
\hline BA_07 & $\mathrm{w}$ & 24 & 2 & - & PTS & ländl. B. \\
\hline
\end{tabular}




\begin{tabular}{|c|c|c|c|c|c|c|}
\hline 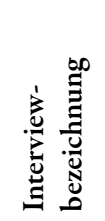 & 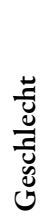 & 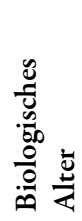 & 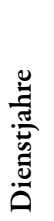 & 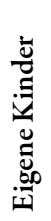 & 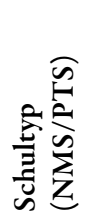 & 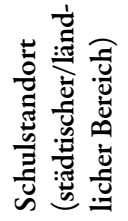 \\
\hline BA_08 & $\mathrm{w}$ & 24 & 3 & - & NMS & städt. B. \\
\hline BA_09 & $\mathrm{w}$ & 25 & 3 & - & NMS & städt. B. \\
\hline BA_10 & $\mathrm{w}$ & 23 & 2 & 1 & NMS & städt. B. \\
\hline BA_11 & $\mathrm{w}$ & 24 & 2 & - & PTS & ländl. B. \\
\hline BA_12 & $\mathrm{m}$ & 26 & 3 & - & PTS & städt. B. \\
\hline BA_13 & $\mathrm{w}$ & 22 & 2 & - & PTS & städt. B. \\
\hline BA_14 & $\mathrm{m}$ & 25 & 1 & - & PTS & städt. B. \\
\hline BA_15 & $\mathrm{w}$ & 24 & 3 & - & NMS & städt. B. \\
\hline BA_16 & $\mathrm{m}$ & 27 & 3 & - & PTS & städt. B. \\
\hline BA_17 & $\mathrm{w}$ & 25 & 2 & - & PTS & städt. B. \\
\hline BA_18 & $\mathrm{m}$ & 41 & 2 & 2 & PTS & städt. B. \\
\hline BA_19 & $\mathrm{m}$ & 26 & 3 & - & NMS & ländl. B. \\
\hline BA_20 & $\mathrm{m}$ & 32 & 3 & 1 & NMS & städt. B. \\
\hline BA_21 & $\mathrm{w}$ & 29 & 2 & - & NMS & städt. B. \\
\hline BA_22 & $\mathrm{w}$ & 23 & 1 & - & NMS & ländl. B. \\
\hline BA_23 & $\mathrm{w}$ & 23 & 1 & - & PTS & ländl. B. \\
\hline BA_24 & $\mathrm{w}$ & 26 & 1 & - & NMS & ländl. B. \\
\hline BA_25 & $\mathrm{w}$ & 24 & 1 & - & NMS & ländl. B. \\
\hline
\end{tabular}

Im folgenden Abschnitt werden die Kriterien des Samples BerufsanfängerInnen erläutert. Die Darstellungen erfolgen jeweils grafisch und deskriptiv.

\subsubsection{Kriterien}

\section{Dienstjahre}

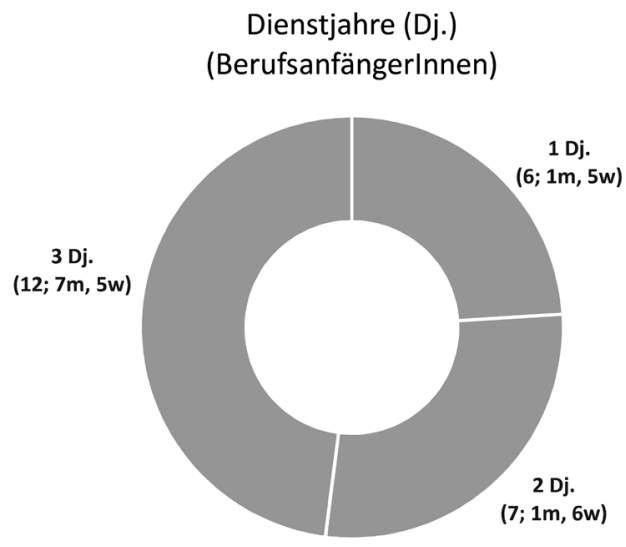

Abb. 37: Kriterium Dienstjahre; BA 
Aus dem 25-köpfigen BerufsanfängerInnen-Sample nahmen an den Interviews sechs BerufsanfängerInnen $(1 \mathrm{~m}, 5 \mathrm{w})$ im ersten Dienstjahr, sieben $(1 \mathrm{~m}, 6 \mathrm{w})$ im zweiten und zwölf BerufsanfängerInnen (7 m, $5 \mathrm{w}$ ) mit jeweils drei Dienstjahren teil (Abb. 37). Die Mehrheit der InterviewteilnehmerInnen mit einem und zwei Berufsjahren stellten die weiblichen Kolleginnen ( 5 von 6 bei 1 Dienstjahr und 6 von 7 bei 2 Dienstjahren), bei den TeilnehmerInnenzahlen von BerufsanfängerInnen im dritten Unterrichtsjahr war der Männeranteil leicht höher (7 m, $5 \mathrm{w})$.

Die Dienstjahre waren, wie bereits einleitend erwähnt, das zentrale Kriterium der Auswahl der BerufsanfängerInnen als InterviewpartnerInnen. Die vorliegende Untersuchung bezieht sich explizit auf BerufsanfängerInnen. Und nachdem in den ersten drei Berufsjahren „Überleben“ und „Entdecken“ die zentralen Motive (Kraler 2008b) der jungen Lehrkräfte sind, bevor dann eine Phase der Stabilisierung folgt (ebd.), wurde auf die Einhaltung dieses Zeitfensters von ein bis drei Jahren Berufserfahrung ausnahmslos geachtet. Die Einladung erging also ausschließlich an junge Lehrpersonen mit bis zur drei Jahren Berufserfahrung, wobei sich eher KollegInnen für das Interview bereit erklärten, die bereits länger im Dienst standen. Eine Erklärung dafür könnte sein, dass im dritten Unterrichtsjahr der Schwerpunkt bereits auf das „Entdecken“ fällt und die Neugier auf Forschungsergebnisse und Hintergründe zum Berufseinstieg größer ist als z.B. im ersten Berufsjahr, wo noch das „Überleben“ im Vordergrund steht und auch aus zeitressourcentechnischen Gründen die Bereitschaft für ein Interview nicht gegeben ist.

\section{Geschlecht und Schultyp}

\section{Berufsanfängerlnnen nach Geschlecht und Schultyp - Sample}

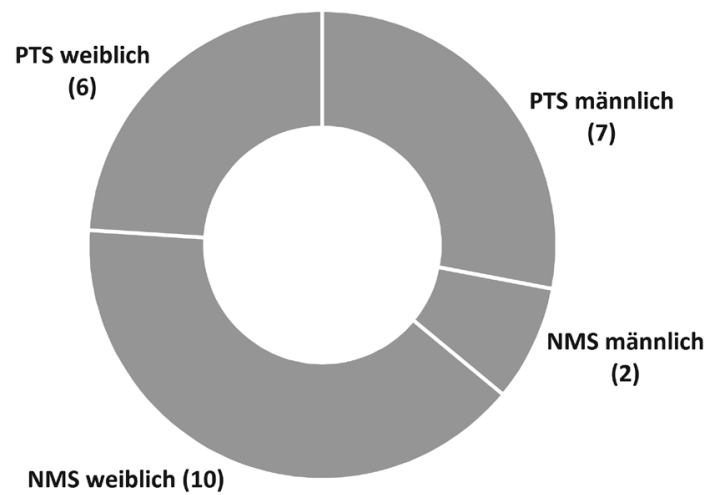

Abb. 38: Kriterien Geschlecht und Schultyp; BA

Insgesamt wurden 25 BerufsanfängerInnen befragt, 16 Frauen und neun Männer (Abb. 38). Das entspricht einem Frauenanteil von 64 Prozent. Zehn der weiblichen und zwei der männlichen Lehrpersonen unterrichteten zum Zeitpunkt der Untersuchung an einer Neuen Mittelschule, sechs Frauen und sieben Männer an einer Polytechnischen Schule. Der tirolweite Vergleich bzgl. Geschlecht und Schultyp stellt sich wie folgt dar (Abb. 39). 


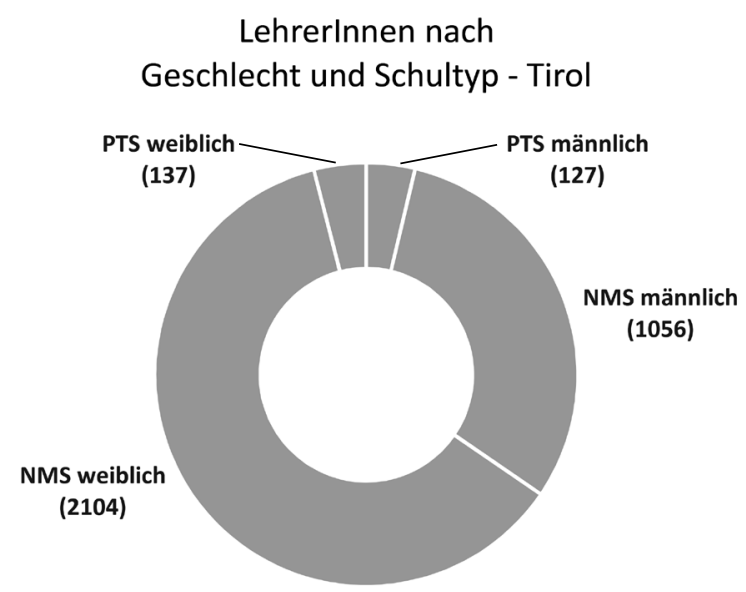

Abb. 39: Vergleichsverteilung nach Geschlecht und Schultyp; LehrerInnen; Quelle: Bildungsdirektion für Tirol, Stand: 20.02.2019

Tirolweit unterrichteten zum Zeitpunkt der Untersuchung 2241 Frauen (2104NNMS; 137 PTS) und 1183 Männer (1056 NMS; 127 PTS) an Neuen Mittelschulen und Polytechnischen Schulen. Von den weiblichen Lehrpersonen unterrichteten 2104 an einer Neuen Mittelschule und 137 an einer Polytechnischen Schule, 1056 Männer lehrten an einer Neuen Mittelschule und 127 an einer Polytechnischen Schule (Abb. 39). Das entspricht einem Frauenanteil (NMS+PTS) von rund 65 Prozent. 64 Prozent betrug der Frauenanteil im Sample der BerufsanfängerInnen (Abb. 38).

\section{Lage und Schultyp}

Etwas mehr als die Hälfte (15 von 25) der BerufsanfängerInnen unterrichteten im städtischen Bereich (7 NMS; 8 PTS), zehn LehrerInnen waren im ländlichen Bereich beschäftigt (5 NMS; 5 PTS). Die Verteilung zwischen den Schultypen Neue Mittelschule und Polytechnische Schule war ausgeglichen. Zwölf BerufsanfängerInnen unterrichteten an einer Neuen Mittelschule, 13 an einer Polytechnischen Schule (Abb. 40).

Schule nach Lage und Schultyp (BerufsanfängerInnen)

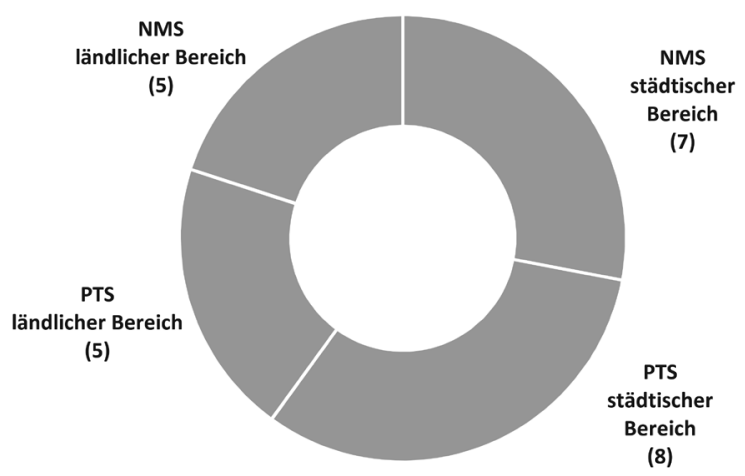

Abb. 40: Kriterien Lage und Schultyp; BA 
Biologisches Alter

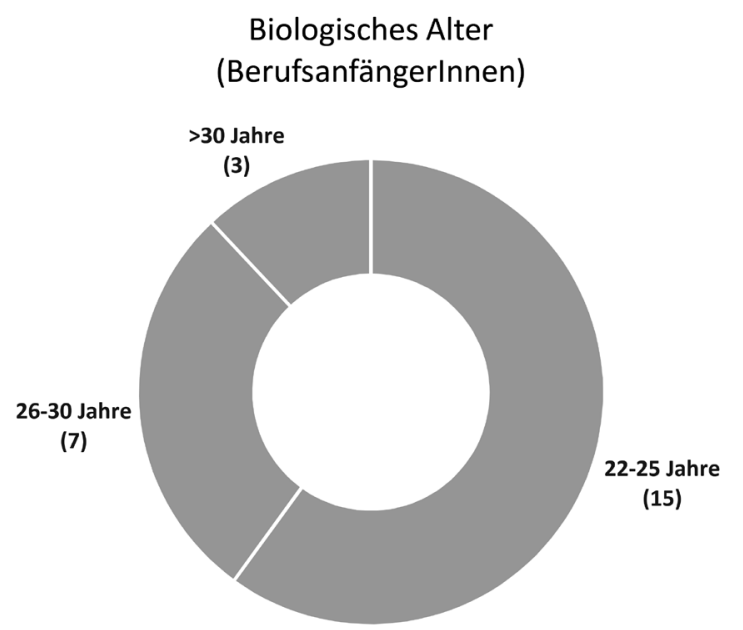

Abb. 41: Kriterium Biologisches Alter; BA

Das Sample der BerufsanfängerInnen bildete sich aus 15 Personen mit einem Alter von 22 bis 25 Jahren, sieben LehrerInnen waren 26 bis 30 Jahre alt und drei BerufsanfängerInnen hatten ein biologisches Alter von über 30 Jahren (Abb. 41).

\section{Eigene Kinder}

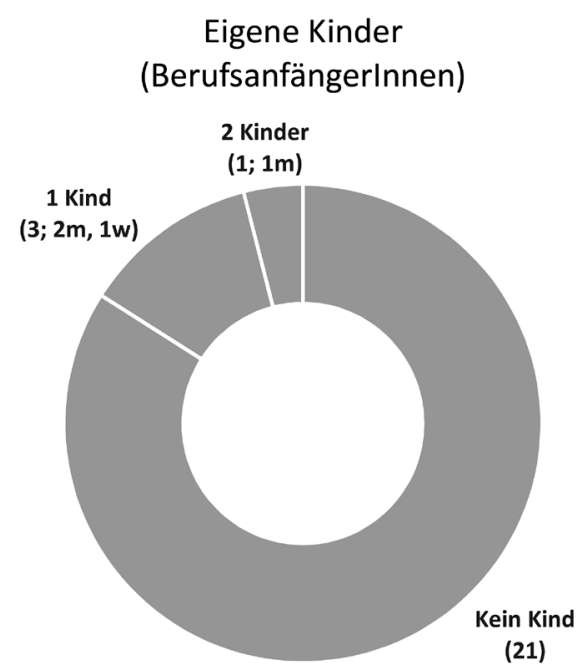

Abb. 42: Kriterium Eigene Kinder; BA 
Schließlich wurden im Zuge der biografischen Datenerhebung die eigenen Kinder der BerufsanfängerInnen erfasst (Abb. 42). Die Mehrheit (21 von 25) hatte zum Zeitpunkt der Befragung kein eigenes Kind, drei Befragte $(2 \mathrm{~m}, 1 \mathrm{w})$ hatten ein Kind und ein Interviewpartner $(\mathrm{m})$ hatte zwei eigene Kinder.

Im folgenden Abschnitt wird der Fokus auf die methodologischen Zugänge gelenkt. Die Methoden zur Datenerhebung werden ebenso beschrieben und deren Wahl begründet wie die Methoden der Datenauswertung und Analyseinterpretation. Abschließend erfolgt die Darstellung der methodologischen Grenzen der vorliegenden Arbeit. 


\section{Methodologie}

In Abschnitt III.2 Methodologie werden die Methoden zur Datenerhebung, -analyse und Konzeptualisierung beschrieben. Mit dem Fokus auf die charakteristischen Eigenschaften der jeweiligen Datenerhebungs-, -auswertungs- und -interpretationsmethode werden in diesem Abschnitt deren Anwendungen begründet. Der Forschungsansatz der vorliegenden Untersuchung lautet: Rekonstruktion von sozialer Wirklichkeit und Konzeptbildung durch Induktion. Insofern liegt der Fokus dieser Untersuchung auf einem qualitativen Forschungsparadigma.

Lamnek (2010, S. 106) stellt fest, dass die Basis für die Theorieentwicklung ${ }^{48}$ in der qualitativen Sozialforschung immer die soziale Realität sei und insofern davon ausgegangen werden kann, dass die so produzierten Theorien realistischer sind als etwa jene aus quantitativen Untersuchungen. An dieser Stelle ist noch einmal explizit zu erwähnen, dass das Ziel der vorliegenden Forschungsarbeit nicht die Generierung einer Theorie, sondern die Entwicklung eines Konzepts zur Umsetzung der Herausforderungen beim Berufseinstieg als Lehrperson darstellt (Abb. 43).

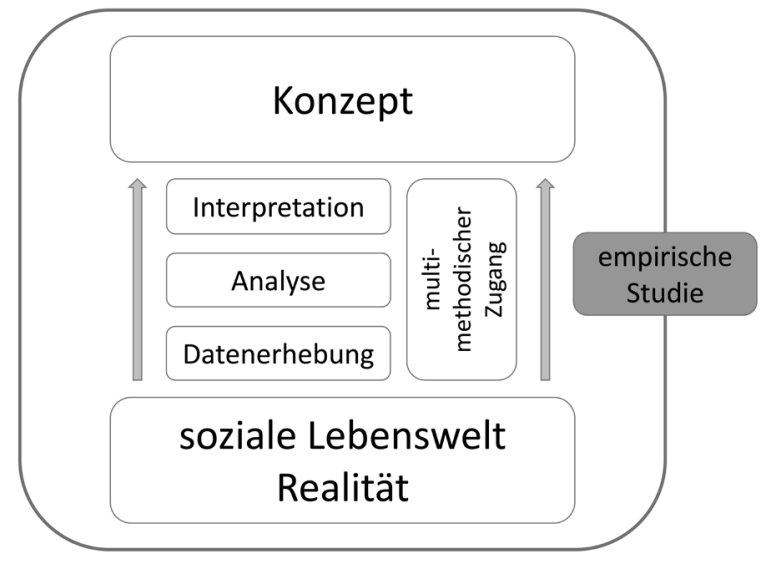

Abb. 43: Induktive Konzeptentwicklung mit multimethodischem Zugang; eigene Abbildung in Anlehnung an Lamnek und Krell (2010, S. 106)

Den Ausgangspunkt bildet die soziale Lebenswelt, die Realität (Abb. 43), das Ziel ist eine durch Induktion entwickelte Konzeptualisierung von Gelingensbedingungen und deren Umsetzungsmöglichkeiten. Die soziale Lebenswelt bzw. die Realität der BerufsanfängerInnen und SchulleiterInnen wird mithilfe leitfadengestützter Interviews erhoben. Die methodischen Schritte bilden sich aus Datenerhebung (Interview), Analyse (Kategorienbildung, Clusteranalyse) und Interpretation (Themen-Kategorien-Matrix, strukturierende qualitative Inhaltsanalyse) (Buber et al. 2000; Kuckartz 2016; Lamnek \& Krell 2010; Strauss \& Corbin 1996), es erfolgt ein multimethodischer Zugang. Als Ergebnis wird die Konzeptualisierung von Bedingungen bzw. Voraussetzungen für einen gelingenden Berufseinstieg, der sich positiv auf die weitere Entwicklung der Berufsbiografie auswirkt, angestrebt.

48 In der vorliegenden Arbeit wird die Entwicklung eines Konzeptes angestrebt. 
In diesem Sinne werden Stärken einzelner Methoden so eingesetzt, dass die Publikation „substanziell anspruchsvolle[r] und konsistente[r] Forschungsergebnisse " (Schründer-Lenzen 2010, S. 149) möglich wird. In den nächsten Abschnitten erfolgt die Beschreibung der einzelnen Verfahren und Methoden zur Datenerhebung und -auswertung.

\subsection{Datenerhebung: Leitfadeninterview, ExpertInneninterview, Postscript}

Die Datenerhebung für die vorliegende Untersuchung erfolgt im Sinne einer methodologischen Triangulation (Schründer-Lenzen 2010) (Abb. 44).

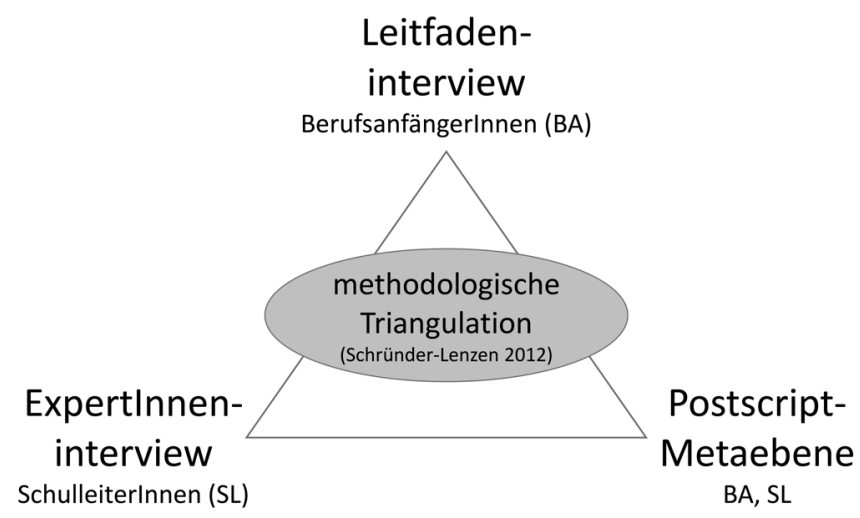

Abb. 44: Methodologische Triangulation zur Datenerhebung

Die Dreiteilung der Datenerhebung erfolgt durch Leitfadeninterviews mit den BerufsanfängerInnen, weiters mit einer Sonderform des Leitfadeninterviews, dem ExpertInneninterview mit den SchulleiterInnen als ExpertInnen und schließlich mit Texten auf Metaebene, den Postscripts. Schründer-Lenzen (ebd.) spricht in diesem Zusammenhang von der methodologischen Triangulation und beschreibt damit die Kombination unterschiedlicher Methoden bei der Datenerhebung.

In der qualitativen Forschung sucht man die Konstruktionen von Welt (Friebertshäuser \& Langer 2010) bzw. die soziale Lebenswelt (siehe auch Abb. 43) zu rekonstruieren. Die Entscheidung für die spezifische Interviewform des Leitfadeninterviews resultiert aus dem Interesse an internen Abläufen (ebd., S. 438), die in der konkreten Untersuchung innerhalb der Schule, innerhalb des Kollegiums, innerhalb des Klassenzimmers etc. vorzufinden sind. Leitfrageninterviews strukturieren die Befragung, begrenzen jedoch den Horizont möglicher Antworten. Sie setzen ein Vorverständnis zum Untersuchungsgegenstand des Forschers bzw. der Forscherin voraus, die sich u.a. aus den eigenen Kenntnissen des Feldes ableiten lassen (ebd., S. 439). Dieses Vorverständnis ist in Bezug auf die vorliegende Untersuchung durch die langjährige einschlägige Unterrichtspraxis gegeben.

Das ExpertInneninterview eignet sich zur Rekonstruktion komplexer Wissensbestände und wird z.B. im Bereich der Bildungsforschung häufig eingesetzt (Meuser \& Nagel 2010). Nicht nur die Rekonstruktion, ein Ziel der vorliegenden empirischen Untersuchung, ist der Anlass, ExpertInneninterviews durchzuführen. Auch die Identifikation von Wirkungszusammenhän- 
gen von Strukturvorgaben, von betrieblich-organisatorischen Kontextbedingungen sowie die Abschätzung zukünftiger Steuerungsaufgaben (ebd., S. 458) können mit dieser Sonderform des Leitfadeninterviews gewährleistet werden, da ExpertInnen ihr (Erfahrungs-)Wissen weitergeben. Den oben genannten Strukturvorgaben entspricht im schulischen Kontext z.B. die Bildung von Klassen- und Gruppenzusammensetzungen. Betrieblich-organisatorische Kontextbedingungen sind etwa im Stundenplanbau und in der Lehrfächerverteilung ${ }^{49} \mathrm{zu}_{\text {finden, }}$ die Abschätzung zukünftiger Steuerungsaufgaben kann in der Verteilung diverser Ämter, wie Schulleitungsstellvertretung, LerndesignerIn oder auch Berufsorientierungskoordination, gesehen werden. Die Entscheidungsgewalt in den genannten Bereichen liegt bei den ExpertInnen. Der Hinweis von Meuser und Nagel (2010, S. 464), dass sich der Feldzugang zu ExpertInnen vergleichsweise einfach gestaltet, hat sich in der vorliegenden Untersuchung bestätigt. Nach der Information über das Forschungsprojekt und der Darstellung der Intention, die Forschungsergebnisse zur Umsetzung von Gelingensbedingungen beim Berufseinstieg für BerufsanfängerInnen heranzuziehen, erklärte sich jede/r (!) der angeschriebenen SchulleiterInnen umgehend zur Teilnahme am Interview bereit. Die ExpertInnen sehen die Chance, durch ihre Teilnahme am Forschungsprojekt die von ihnen identifizierten Desiderate im Kontext der gesamten Forschungsthematik einer breiteren Öffentlichkeit sichtbar und damit lösbar zu machen.

Durch die Erfassung von Postscripts nach jedem Interview wurden Daten auf Metaebene gesammelt und ebenfalls analysiert. Die Analyse erfolgte auf der interpretativen und inhaltlichen Ebene. Interpretativ wird das, was im Interview selbst nicht zum Ausdruck kommt (Lamnek \& Krell 2010), in die Analyse mit einbezogen. Das sind Verhaltensweisen, Gesten etc. der InterviewpartnerInnen, aber auch Rahmenbedingungen wie Raumgestaltung, Umfeld etc. Inhaltlich sind Postscripts dann relevant, wenn eventuell Einzelheiten festgehalten werden, die vom Befragten erst nach dem eigentlichen Interview (ebd.) genannt werden. Dieser Fall trat im Laufe der vorliegenden Untersuchung regelmäßig ein. Im Sinne forschungsethischer Grundsätze haben die InterviewpartnerInnen auch in Bezug auf Metadaten das Recht auf Vertraulichkeit und Anonymität (Aeppli 2014; Flick 2007).

Die an die Transkription anschließende Datenanalyse nach der Grounded Theory (Strauss und Corbin 1996) und Clusteranalyse (Bortz und Döring 2006; Buber et al. 2000) wird im nächsten Abschnitt thematisiert.

\subsection{Datenanalyse: Grounded Theory und Clusteranalyse}

Die Datenanalyse und -interpretation (siehe auch Kap. III.2.3) erfolgt mittels eines multimethodischen Zugangs. Zur Analyse finden die Methoden Grounded Theory (Strauss \& Corbin 1996) und Clusterbildung (Bortz \& Döring 2006; Buber et al. 2000) Anwendung.

Das Paradigma, die Rekonstruktion der sozialen Wirklichkeit aus den Grunddaten zu generieren, wird durch den Ansatz der Grounded Theory verfolgt. Eine Variante, in der die Theorieerzeugung bzw. Konzeptualisierung in der vorliegenden Forschungsarbeit aus dem Material heraus im Zentrum steht, ist das Theoretical Coding nach Glasser und Strauss (Schmidt 2010). Diese Linie wird im Auswertungsprozess sukzessive verfolgt, bis schließlich das gesamte Datenmaterial in mehreren Kategorienebenen (siehe auch Kap. III.3.1) systematisiert analysiert vorliegt. Konkret bedeutet das, dass der Kodiervorgang mehrmals wiederholt wird. Dadurch

49 In der Lehrfächerverteilung wird in der Regel für den Zeitraum eines Schuljahrs festgelegt, welche Fächer in welchen Klassen von den Lehrpersonen unterrichtet werden. Diese Aufgabe liegt an Neuen Mittelschulen und Polytechnischen Schulen in der Kompetenz der Schulleitung. 
verändern sich Perspektiven, Kategorien werden neu- bzw. umbenannt oder ev. gestrichen. Dabei wird darauf hingesteuert, final eine Theorie bzw. ein Konzept zu erlangen, die bzw. das dem untersuchten Gegenstandsbereich gerecht wird (Strauss \& Corbin 1996). Auf die Erläuterung der verschiedenen Vorgänge des offenen, axialen und selektiven Kodierens (ebd., S. 43-165) wird an dieser Stelle bewusst verzichtet, sie käme einer Wiederholung von bereits mehrfach Publiziertem gleich (Breuer et al. 2018; Schmidt 2010; Strauss \& Corbin 1996).

Der multimethodische Zugang konstituiert sich in dieser Untersuchung insofern, als auf der Ebene der Hauptkategorien (siehe auch Kap. III.3.1), die nach dem Paradigma der Grounded Theory generiert werden, noch keine Theoriebildung bzw. Konzeptualisierung intendiert wird. Im Sinne der Multimethodik folgt auf die Kategorienbildung zur Konzeptentwicklung die Clusterbildung. Durch dieses Vorgehen wird eine thematische Verdichtung und Abstraktionsebene erreicht, die letztlich zur Theorie- bzw. Konzeptbildung führt (Schründer-Lenzen 2010). Dazu werden die Hauptkategorien auf der Inhaltsebene durchforstet, um sich überschneidende Themen bzw. Schlüsselstellen in verschiedenen Kategorien zu identifizieren und schließlich abstrahiert zu bündeln. Das Ziel der Clusteranalyse besteht in der Bündelung bzw. Zusammenfassung der lokalisierten Themen bzw. Schlüsselstellen zu Clustern (Clusterkategorienebene I [CK_I], Clusterkategorienebene II [CK_II]; siehe auch Kap. III.3.3 und III.3.4), wobei die Objektunterschiede innerhalb der Cluster möglichst klein und die Unterschiede zwischen den Clustern (Kap. III.3.3, CK_I_1 und CK_I_2) möglichst groß sein sollen (Bortz \& Döring 2006). Durch mehrmalige Verdichtungs- und Abstraktionsschleifen bildet sich in der Clusterkategorienebene II (Kap. III.3.4, CK_II_3) mit der Konzeptualisierung das Ergebnis der Untersuchung heraus.

\subsection{Dateninterpretation: Strukturierende qualitative Inhaltsanalyse, Bedingungsgefüge}

Die Interpretation der analysierten Ergebnisse erfolgt ebenfalls multimethodisch. Im zweistufigen, verdichtenden Prozess werden vorerst die bereits mithilfe der Grounded Theory generierten Kategorien $^{50}$ mit kategorienübergreifenden Themen in Anlehnung an Kuckartz (2016, S. 112) gekreuzt und in einer Themen-Kategorien-Matrix dargestellt. Der Begriff Thema (bzw. Themen) wird in dieser Arbeit im Zusammenhang mit Themen-Kategorien-Matrix explizit verwendet. Es handelt sich dabei um die Themen Administration, Systemwissen, Reflexion \& Feedback, Autorität in der Klasse, Kooperation mit SchülerInnen und Habitus (siehe auch Kap. III.4.1). Im Bewusstsein, dass die Inhaltsanalyse Bestandteil jeder soziologischen Forschung ist (Lamnek $\&$ Krell 2010), werden nach diesem Paradigma, konkret im Sinne der inhaltlich strukturierenden qualitativen Inhaltsanalyse (Kuckartz 2016), Schlüsselbegriffe identifiziert, die in mehreren Kategorien thematisch vorkommen. Zum Teil wird auf einer interpretativen höheren Abstraktionsstufe auch inhaltlich Verwandtes bzw. Kongruentes aufgenommen, nicht als Zitat, sondern in den eigenen Worten des Forschenden (Kuckartz 2016). Diese Stellen werden in der Untersuchung als Essenzen bezeichnet. Die Themen-Kategorien-Matrix dient als Ausgangspunkt für die spätere Interpretation auf der Ebene der Bedingungsgefüge. Durch den Schritt der systematischen thematischen Zusammenfassung in Form von Schlüsselbegriffen und Essenzen „wird das Material zum einen komprimiert, zum anderen pointiert und auf das für die Forschungsfrage wirklich Relevante reduziert“"(Kuckartz 2016, S. 111).

50 Konkret: Hauptkategorien (Kap. III.3.1) 
Anschließend an die thematische Verdichtung mit der Themen-Kategorien-Matrix als Ergebnis werden im Sinne einer Relevanzanalyse (Buber et al. 2000) Kausalitäten zwischen den Knotenpunkten (Hauptkategorien) und den Schlüsselbegriffen bzw. Essenzen als Kondensationspunkte identifiziert. Das Grundprinzip beim Analyse-Prozess lautet: „In interpreting the relevancy list, the main question is: What is important or relevant in the analyzed situation, to the research population?" (Buber et al. 2000) In der vorliegenden Untersuchung generieren sich aus den Originaltexten (Transkripte) bzw. aus den Interpretationen im Kontext zu den Originaltexten die Abhängigkeiten Voraussetzung, Ursache, Maßnahme, Konsequenz und Wirkung. Der Terminus Voraussetzung wird gewählt, wenn ein Phänomen vorliegen muss, damit ein anderes eintritt. Ursache unterstreicht im Vergleich dazu die Bedingtheit und die Aktivität, nicht bloßes Vorhandensein. Unter Maßnahme wird in diesem Kontext eine konkrete Handlungsweise zur Erfüllung einer Absicht verstanden. Schließlich kommen noch die Relevanzen Konsequenz und Wirkung vor, wobei die Konsequenz im Unterschied zur Wirkung die Aktivität im Sinne von aktiver Beitrag bzw. Reaktion impliziert. Eine Wirkung hingegen wird dann identifiziert, wenn bei passiver Betrachtung ein kausales Phänomen wahrgenommen werden kann.

Als grafische Grundlage zur Interpretation werden in der Darstellung der Ergebnisse Bedingungsgefüge (Kap. III.4.2) abgebildet, welche sich methodisch an den „causal network graphics“ (Buber et al. 2000) orientieren. Das Hauptgerüst bilden die Kontenpunkte, die den Hauptkategorien des ersten Analyseschritts entsprechen. Die Korrelationen und Kausalitäten werden in den Bedingungsgefügen als Linien (Korrelationen) und als durch Punkte fixierte Schlüsselworte bzw. Essenzen in rechteckigen Textfeldern (Kausalitäten bzw. Relevanzen) dargestellt. Ein zentrales Anliegen der wissenschaftlichen Tätigkeit (Rasch et al. 2014), und auch in der vorliegenden Untersuchung angestrebt, ist die Suche nach Zusammenhängen. Das Aufdecken von Kausalzusammenhängen kann Erklärungen für empirische Sachverhalte liefern. Letztlich ermöglicht die Kenntnis von Zusammenhängen, hier sind es vorwiegend durch Schlüsselbegriffe und Essenzen präzisierte Kausalitäten, Vorhersagen über künftige Ereignisse zu machen. Insofern spielen die Interpretationen der Ergebnisse, insbesondere die Erkenntnisse aufgrund der Bedingungsgefüge, im Abschnitt Zusammenführung und Integration (Kap. IV) eine zentrale Rolle. Gelingensbedingungen, die das Fundament der Forschungsfragen bilden, können daraus unmittelbar abgeleitet werden.

Um den ständigen Bezug zu den Grunddaten zu gewährleisten, womit sich der methodologische Kreis zur Grounded Theory schließt, werden auch an dieser Stelle zur Absicherung der Interpretationen SchulleiterInnen und BerufsanfängerInnen mit Originalaussagen zitiert.

\subsection{Methodologische Grenzen der Untersuchung}

Aufgrund des qualitativen Zugangs der vorliegenden Forschungsarbeit sind methodologische Grenzen gesetzt. Das interpretative Paradigma der Datenanalyse erlaubt, bezogen auf die Forschungsfragen, eine Rekonstruktion des Feldes in begrenztem Umfang. Diesem methodologischen Limit wurde mittels eines qualitativen multimethodischen Zugangs durch den Einsatz mehrerer Methoden (Grounded Theory, Clusteranalyse, Qualitative Inhaltsanalyse) und der daraus resultierenden, multimethodisch gestützten, Rekonstruktion entgegengewirkt. Jedoch stellt diese Herangehensweise keine Methodentriangulation dar, die Rekonstruktion unter Berücksichtigung qualitativer und quantitativer Forschungsparadigma bleibt eindimensional. Die Verfahrenstiefe ist begrenzt. Das Mittel der Wahl im Hinblick auf eine weitere, vertiefende Forschungstätigkeit zum Berufseinstieg von Lehrpersonen kann der Mixed-Methods-Ansatz sein 
(Aeppli 2014; Bortz \& Döring 2006; Friebertshäuser et al. 2010; Kuckartz 2016). Hier werden qualitative und quantitative Forschungszugänge systematisch und gezielt kombiniert bzw. integriert (Tashakkori \& Newmann 2010), um statistische und inhaltsanalytische Meta-Schlussfolgerungen in Bezug auf die Forschungsfragen zu erhalten. Die Güte der Rekonstruktion und der Grad der Generalisierbarkeit der Erkenntnisse steigt, wenngleich festgehalten werden muss, dass die Generalisierbarkeit der vorliegenden Erkenntnisse auch aus anderen, nicht methodologischen Gründen, begrenzt ist. Dazu wird im Abschnitt Grenzen der Arbeit (Kap. IV.3) Stellung genommen. 


\section{Analyse}

Im Folgenden wird die Analyse der Interviews der SchulleiterInnen (SL) und BerufsanfängerInnen (BA) wie in Methodologie (Kap. III.2) beschrieben, dargestellt. Die Analyse erfolgt mehrstufig, d.h. es wird für die Samples SchulleiterInnen und BerufsanfängerInnen der jeweilige Status quo der betreffenden Analyseebene beschrieben. Das sind alle Kategorien- und Subebenenkategorien (Strauss \& Corbin 1996) und die Clusterebenen I und II, welche letztlich die gegenstandsverankerte Konzeptualisierung darstellen.

\subsection{Haupt-, Sub-, Subsub- und Subsubsubkategorienbildung}

In einem ersten Kodierprozess der vorliegenden transkribierten Interviews nach dem Schema des Offenen Kodierens (ebd.) kann das Textmaterial auf der Ebene der Hauptkategorien in gröBere Einheiten aufgeschlüsselt werden, in Subkategorien erfolgt dies detaillierter und fallweise noch spezifischer auf den Ebenen der Subsub- bzw. Subsubsubkategorien. Zur Übersichtlichkeit und Einordnung der einzelnen Kategorien in die verschiedenen Kategorienebenen wird ein Kategorien-Nummerierungssystem aus einer Kombination von Buchstaben und Ziffern gewählt. Die Buchstabenkombinationen SL und BA geben darüber Auskunft, ob es sich um ein Interview mit einer Schulleiterin bzw. einem Schulleiter (SL) oder mit einer Berufsanfängerin bzw. einem Berufsanfänger (BA) handelt. Die Buchstabenkennung (A-F) bezeichnet die Hauptkategorie, die nachfolgenden, durch Unterstrich getrennten Ziffern benennen die Ebenen der Sub-, Subsub- und Subsubsubkategorien. Die Kategorienbezeichnung (BA_C_3_5_1) bezeichnet demnach die Subsubsubkategorie 1 in der Hauptkategorie C, generiert aus den Interviews mit BerufsanfängerInnen.

Das Kategoriensystem dieses ersten Kodierprozesses der Interviews mit SchulleiterInnen und BerufsanfängerInnen wird zur Wahrung der Übersicht im Abschnitt Kategoriensystem der Interviews mit SchulleiterInnen (Kap. III.3.1.2) als Aufzählung dargestellt. In der Beschreibung aller Kategorienebenen (Kap. III.3.1.4 und Kap. III.3.1.5) werden die jeweiligen Kategoriensysteme zusätzlich in Form von Vielwegbäumen (Ernst et al. 2016) grafisch dargestellt.

\subsubsection{Darstellungsformen der Kategoriensysteme}

Im Folgenden wird das gesamte Kategoriensystem (über SchulleiterInnen und BerufsanfängerInnen) hierarchisch abgebildet (Kap. III.3.1.2 und III.3.1.3). Hierbei ist der verbalen Bezeichnung der jeweiligen Kategorie (z.B. Fach- \& Methodenkompetenz) die numerische Kategorienbezeichnung (z.B.SL_A_1; siehe auch Beschreibung in Kap. III.3.1) vorangestellt. Damit wird sowohl die Hierarchie als auch die Nummerierungslogik berücksichtigt.

Die hierarchische Abbildung der vier Ebenen des Kategoriensystems folgt diesem Muster (Abb. 45):

(XX_X) Hauptkategorie

(XX_X_X) Subkategorie

$\left(X X \_X_{-} x_{-} x\right)$ Subsubkategorie

$\left(X x_{-}{ }_{-} x_{-} x_{-} x\right)$ Subsubsubkategorie

Abb. 45: Hierarchische Darstellung des Kategoriensystems; Aufzählung mit Nummerierungslogik 
Die Hauptkategorien werden in Schriftgröße 12, „fett“ formatiert, dargestellt (Abb. 45). Subkategorien werden wie alle nachfolgenden Kategorien jeweils tabulatorisch eingerückt in normaler Schriftstärke, ebenfalls in Schriftgröße 12, Subsubkategorien in Schriftstärke 10 abgebildet. Die hierarchisch unterste Subsubsubkategorienebene wird mit Schriftgröße 10 kursiv formatiert.

In der detaillierten Analyse der Interviews mit SchulleiterInnen und BerufsanfängerInnen wird jede Hauptkategorie inklusive aller vorhandenen Subkategorienebenen in Form von Vielwegbäumen (ebd.) dargestellt. Diese Darstellungsform hat gegenüber der Binärbaumdarstellung den Vorteil, dass ein Knoten (Ebene) beliebig viele Nachfolger haben kann. Die „familiären Verwandtschaften“ (ebd., S. 549), gemeint sind damit die inhaltlichen Beziehungen zwischen den Kategorienebenen, lassen sich in Baumdarstellungen nachvollziehbar abbilden und dienen aufgrund der grafischen Unterstützung zum besseren Verständnis der inhaltlichen Zusammenhänge bzw. Abgrenzungen zwischen den einzelnen Kategorien und Kategorienebenen. Das Hauptaugenmerk liegt auf den qualitativen Aussagen und Erkenntnissen, deshalb wird die bereits eingeführte Nummerierung in der Baumdarstellung jeweils als Suffix angegeben.

Die Abbildungen der Vielwegbäume in der Analyse der Interviews mit SchulleiterInnen (Kap. III.3.1.4) und BerufsanfängerInnen (Kap. III.3.1.5) folgen dem in Abbildung dargestellten Schema (Abb. 46).

Die hierarchisch oberste Hauptkategorienebene ist ganz links in einem grau hinterlegten, abgerundeten Rechteck abgebildet (Abb. 46). Alle nachfolgenden Kategorienebenen sind durch Verbindungslinien miteinander verbunden, um die inhaltliche Zusammengehörigkeit zu verdeutlichen.

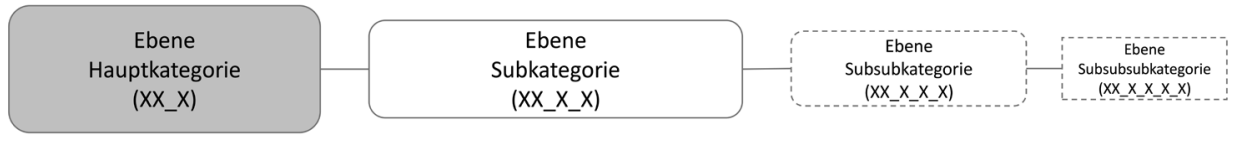

Abb. 46: Darstellung der Kategorien in Vielwegbäumen; Schema

Die abgerundeten Rechteckformen werden jeweils kleiner (v.l.n.r.), die hierarchisch untersten Kategorienebenen Subsub- und Subsubsubkategorie tragen strichlierte Umrandungen. Die Schriftgröße verkleinert sich mit der jeweils hierarchisch tiefer liegenden Kategorienebene.

\subsubsection{Kategoriensystem: SchulleiterInnen}

\section{(SL_A) Kompetenzen von BerufsanfängerInnen}

(SL_A_1) Fach- \& Methodenkompetenz

(SL_A_2) Positive Grundeinstellung \& Einsatzfreude

(SL_A_3) Selbst angeeignete Kompetenzen, Individualität

(SL_B Institutionelle Ausbildung aus der Sicht der SchulleiterInnen

(SL_B_1) Selektion

(SL_B_2) Anforderungen an die Ausbildung

(SL_B_3) Praktika

(SL_B_4) Ausbildung an PH/Universität 
(SL_C) Wahrnehmung der BerufsanfängerInnen durch die SchulleiterInnen

(SL_C_1) Einordnung der BA im Kollegium (SL_C_1_1) Definitionen der Bezeichnung „BerufsanfängerInnen“

(SL_C_2) Arbeitshaltung der BA

(SL_C_3) Geschlechtsspezifische Unterschiede zwischen BA

(SL_C_4) Assoziationen der SL mit dem Begriff BA

(SL_C_5) Herausforderungen an die BA

(SL_D) Unterstützung \& Begleitung der BerufsanfängerInnen im Schulalltag

(SL_D_1) Erwartungen von den BA an die SL

(SL_D_2) Strukturelle Hilfe \& Rahmenbedingungen

(SL_D_3) Mentoring

(SL_D_3_1) Vorbedingungen

(SL_D_3_2) KollegInnen als BegleiterInnen

(SL_D_3_3) SL als BegleiterInnen

(SL_D_3_4) Herausforderungen an die BA

Das Kategoriensystem aus der Analyse der SchulleiterInneninterviews besteht aus den vier Hauptkategorien SL_A bis SL_D. In allen Hauptkategorien bestehen Subkategorien, in den Subkategorien SL_C_1 und SL_D_3 kommen auch Subsubkategorien vor.

\subsubsection{Kategoriensystem: BerufsanfängerInnen}

(BA_A) Ersterfahrung der BerufsanfängerInnen beim Berufseinstieg

(BA_A_1) Herausforderungen an die BA

(BA_A_1_1) Schock bei unmittelbarem Berufseinstieg

(BA_A_1_2) Arbeitsüberlastung

(BA_A_1_3) „Keine Routine“ (In-vivo)

(BA_A_2) „Sprung ins kalte Wasser“ (In-vivo)

(BA_A_3) Eigene Rolle

(BA_A_3_1) Rollenfindung im System

(BA_A_3_2) Akzeptanz der BA durch die SchülerInnen

(BA_A_3_3) Worst Case: Scheitern

(BA_A_4) Lernen aus eigenen Erfahrungen

(BA_A_4_1) Selbstreflexion

(BA_A_4_2) Erste Routine

(BA_B) Mentoring aus der Sicht der BerufsanfängerInnen

(BA_B_1) Hilfe von SL

(BA_B_1_1) „Rund-um-Hilfe“ von SL

(BA_B_1_2) Fragen zulassen

(BA_B_1_3) Administrative Hilfe von SL

(BA_B_2) Hilfe von KollegInnen

(BA_B_2_1) „Rund-um-Hilfe“ von KollegInnen

(BA_B_2_2) Tipps \& Gespräche

(BA_B_2_3) Arbeitsmaterialien

(BA_B_2_4) Administrative Hilfe von KollegInnen

(BA_B_2_5) Konkrete Themen 
(BA_C) Institutionelle Ausbildung aus der Sicht der BerufsanfängerInnen

(BA_C_1) Organisation der Ausbildung

(BA_C_1_1) Bürokratie

(BA_C_1_2) Kommunikation

(BA_C_2) Selektion aus der Sicht der BA

(BA_C_2_1) Berufseignungsfeststellung

(BA_C_2_2) Notenwahrheit

(BA_C_3) Curriculum
(BA_C_3_1) DozentInnen
(BA_C_3_2) Fachausbildung
(BA_C_3_3) Fachdidaktik
(BA_C_3_4) Bildungswissenschaften
(BA_C_3_5) Praktika
(BA_C_3_5_1) Praxislehrperson
(BA_C_3_5_2) Nutzen
(BA_C_3_5_3) Praxis \& Realität
(BA_C_3_5_4) Meisterlebre

(BA_C_4) Desiderate \& Anwendbares Wissen

(BA_C_4_1) Administratives Wissen

(BA_C_4_2) Theorie-vs. Praxiswissen

(BA_D) Disziplin \& Classroom Management

(BA_D_1) Disziplin als Herausforderung beim Berufseinstieg

(BA_D_2) Disziplin \& Ausbildung

(BA_D_3) Umgang mit Disziplinproblemen

(BA_D_4) Disziplin ist kein Problem (BA_E) Kriterien für Berufszufriedenheit der BerufsanfängerInnen

(BA_E_1) Kooperation mit SchülerInnen und LehrerkollegInnen

(BA_E_2) Fach \& Fachkompetenz

(BA_E_3) Schultyp

(BA_F) Arbeitsbelastung \& Überforderung der BerufsanfängerInnen

(BA_F_1) Schulstrukturen \& Schulsystem

(BA_F_2) Supplierverpflichtung

Das Kategoriensystem aus der Analyse der BerufsanfängerInneninterviews besteht aus den sechs Hauptkategorien (BA_A) bis (BA_F). In allen Hauptkategorien bestehen Subkategorien, in den Subkategorien (BA_A_1), (BA_A_3), (BA_A 4), (BA_B_1), (BA_B_2), (BA_C_1), (BA_C_2), (BA_C_3) und (BA_C_4) kommen auch Subsubkategorien vor. Schließlich konnten in den Subsubkategorien (BA_C_3_5) auch noch Subsubsubkategorien gebildet werden.

Um dem Prinzip des Grounding und der Emergenz der theoretischen Strukturen (Breuer et al. 2018) Rechnung zu tragen, werden im Sinne der Transparenz anschließend alle Kategorienebenen (Haupt-, Sub-, Subsub- und Subsubsubkategorien) der Interviews mit SchulleiterInnen und BerufsanfängerInnen näher beschrieben und mit Originalzitaten der InterviewteilnehmerInnen belegt.

Die Quellenangaben zu den Originalzitaten berücksichtigen, ob es sich bei der interviewten Person um eine/n SchulleiterIn (SL) bzw. um eine/n BerufsanfängerIn (BA) handelt. Ferner 
werden die Nummer des Interviews (01-15 bei SchulleiterInnen und 01-25 bei BerufsanfängerInnen) und die Absatznummerierung der Textstelle angegeben. Die Zitation (SL_15: 56) steht somit für Absatz 56 im 15. eines SchulleiterInneninterviews.

\subsubsection{Interviews mit SchulleiterInnen}

Nach der Analyse der Interviews mit SchulleiterInnen bilden sich die Hauptkategorien Kompetenzen von BerufsanfängerInnen (SL_A), Institutionelle Ausbildung aus der Sicht der SL (SL_B), Wabrnehmung der BA durch die SL $\left(S L_{-} C\right)$ und Unterstützung \& Begleitung der BA im Schulalltag (SL_D) heraus (Abb. 47).

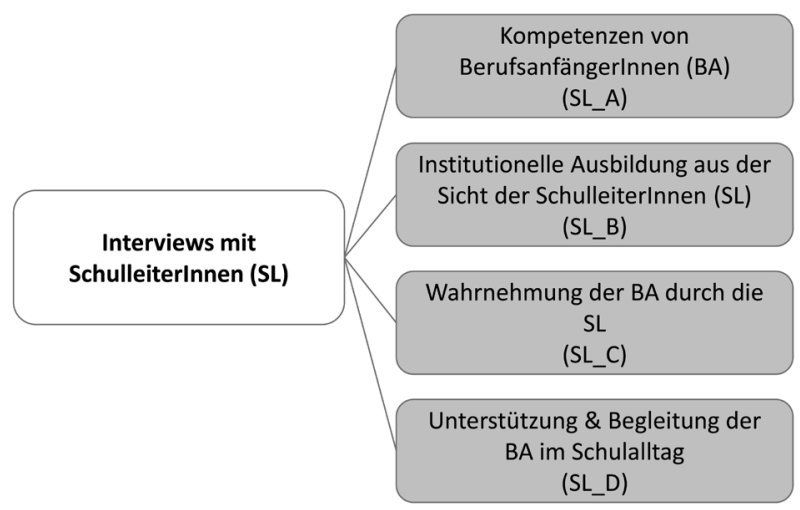

Abb. 47: Interviews mit SchulleiterInnen; Hauptkategorien (SL_A) - (SL_D)

Bevor im Folgenden die Haupt- und alle untergeordneten Subkategorien beschrieben werden, erfolgt eine tabellarische Übersicht zur Quantität von Subkategorien, Codes und SchulleiterInnen-Nennungen (Tab. 7).

Tab. 7: Interviews mit SchulleiterInnen - Häufigkeiten

\begin{tabular}{lcccc}
\hline Interviews mit SchulleiterInnen - Häufigkeiten \\
\hline Hauptkategorie & Subkategorie & Subsubkategorie & Codes & $\begin{array}{c}\text { n (verschiedene } \\
\text { SchulleiterInnen) }\end{array}$ \\
\hline SL_A & 3 & 0 & 40 & 11 \\
SL_B & 4 & 0 & 106 & 13 \\
SL_C & 5 & 1 & 112 & 14 \\
SL_D & 3 & 4 & 141 & 14 \\
\hline Summe & $\mathbf{1 5}$ & $\mathbf{5}$ & 759 & $15^{51}$ \\
\hline
\end{tabular}

Hauptkategorie (SL_A) Kompetenzen von BerufsanfängerInnen (Tab. 7) besteht aus drei Subkategorien mit insgesamt 40 Kodierungen (Codes), genannt von elf SchulleiterInnen. Haupt-

51 Aufgrund von Überschneidungen entspricht diese Größe nicht der nummerischen Summe von n in der Spalte oberhalb. 
kategorie (SL_B) Institutionelle Ausbildung aus der Sicht der SL besteht aus vier Subkategorien mit insgesamt 106 Kodierungen, genannt von 13 SchulleiterInnen. Hauptkategorie (SL_C) Wabrnehmung der BA durch die SL wird in fünf Subkategorien und in eine Subsubkategorie mit insgesamt 112 Kodierungen aufgeteilt, die von 14 SchulleiterInnen genannt werden. Hauptkategorie (SL_D) Unterstützung \& Begleitung der BA im Schulalltag besteht aus drei Sub- und vier Subsubkategorien mit insgesamt 141 Codes, welche von 14 SchulleiterInnen genannt werden. In den folgenden Abschnitten (Kap. III.3.1.4.1 - Kap. III.3.1.4.4) werden die jeweiligen Hauptmit allen untergeordneten Subkategorien beschrieben.

\subsubsection{Hauptkategorie Kompetenzen von BerufsanfängerInnen (SL_A)}

Die Hauptkategorie Kompetenzen von BA (SL_A) kann in die Subkategorien Fach-\& Methodenkompetenz (SL_A_1), Positive Grundeinstellung \& Einsatzfreude (SL_A_2) und Selbst angeeignete Kompetenzen, Individualität (SL_A_3), unterteilt werden (Abb. 48).

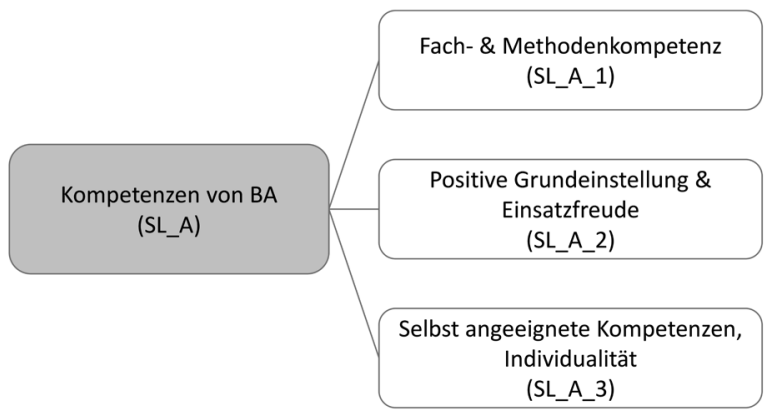

Abb. 48: Kompetenzen von BA; Haupt- und Subkategorien

Die Fach-und Methodenkompetenz (SL_A_l) der BerufsanfängerInnen wird von den SchulleiterInnen als vorhanden wahrgenommen. Ein Unterschied zwischen der Fach- und der Methodenkompetenz besteht insofern, als die Existenz der Fachkompetenz vorausgesetzt wird und über die größtenteils vorhandene Methodenkompetenz positive Überraschung herrscht. Umgekehrt formuliert, gehen die SchulleiterInnen eher von methodisch-didaktischen Desideraten aus als von fachlichen.

„Wo ich immer erstaunt bin, weil ich ja die Unterrichtsbeobachtungen machen muss, ist die extreme Vielfalt an Methodik und Didaktik. Also da bin ich immer wieder ganz stolz, wie vielfältig da unterrichtet wird, und nicht nur, wenn ich [in den Unterricht, Anm.] hinein gehe." (SL_10: 41)

Das sagt in diesem Zusammenhang ein/e SchulleiterIn. „[Die Fachkompetenz] setze ich voraus, wenn er für dieses Fach geprüft ist. Ich will ja das nicht anzweifeln“ (SL_12: 63), heißt es in einem weiteren Interview, wodurch bestätigt wird, dass das Vorhandensein der Fachkompetenz vorausgesetzt wird.

Mit dem folgenden Zitat wird die Subkategorie Positive Grundeinstellung \& Einsatzfreude (SL_A_2) und deren Auswirkung auf das LehrerInnenkollegium formuliert.

„Das ist wirklich eine Freude, so ein junges Blut tut der Schule wohl. In jede Schule gehörten ein, zwei Junglehrer hinein [...] Einfach auffrischen und sich gegenseitig austauschen.“ (SL_08: 44) 
Die Einsatzfreude der BerufsanfängerInnen zeigt sich einerseits in der Durchführung von diversen Schulprojekten, wo die SchulleiterInnen staunen, „welche Leistungen Junglehrer ${ }^{52}$ erbringen [... und] an welche Projekte sich Junglehrer herangewagt haben“ (SL_06: 56). Andererseits zeigt sich auch hier die positive Wirkung der BerufsanfängerInnen auf den gesamten Lehrkörper. Im folgenden Interviewausschnitt wird dieser Zusammenhang geschildert:

„Und in den Konferenzen entstehen oft interessante Diskussionen. Die Junglehrer haben oft andere Ideen oder sie haben andere Unterrichtsmethoden und sagen, das habe ich probiert'. Der Ältere hört das und kann das dann auch probieren, er wäre sonst nie auf die Idee gekommen, das zu probieren. Es gibt schon neue Impulse, also das schätze ich sehr.“ (D_08: 44)

Die/der SchulleiterIn betont zugleich, dass es sich um eine eigene Einschätzung handelt. Die Subkategorie Selbst angeeignete Kompetenzen, Individualität (SL_A_3) wird aus den Interviews mit den SchulleiterInnen gebildet, ohne im Sinne des Leitfadengesprächs danach gefragt zu haben, und ist somit als rein induktive Kategorie zu bewerten. Mit selbst angeeigneten Kompetenzen sind im Kontext der Interviewgespräche solche gemeint, die zum Beispiel in Vereinen, beim Ausüben von Hobbies, jedoch nicht im Rahmen der LehrerInnenausbildung angeeignet werden. In einem Interview wird dieser Sachverhalt folgendermaßen beschrieben:

„Vielleicht durch den eigenen Lebensstil, wenn ein Geographielehrer selber viel Reisen macht oder so. Dann kommt mir vor, ein Lehrer ist auch einer, der verschiedenste Interessen hat. Vereine, Gespräch.“ (SL_04: 49)

In Bezug auf die eigene Persönlichkeit macht es ein/e InterviewpartnerIn folgendermaßen deutlich: „Also ich merke, dass viel schon einmal Sache der Persönlichkeit ist. Dass das jetzt also nicht nur ein Produkt der Ausbildung ist. (lacht)“ (D_02: 62) Hier wird auch der Bezug zu „menschlichen Qualitäten“ (D_03: 69) hergestellt und der Bogen bis zur didaktischen Arbeit mit Kindern gespannt:

„Diese [menschlichen Qualitäten, Anm.] bringen sie [die BerufsanfängerInnen, Anm.] mit. Da spielen viele persönliche Sachen mit, das Temperament und so. ,Den Draht zum Kind', den spürst du einfach, wenn er da ist." (ebd.)

So ein/e SchulleiterIn, der die Empathie der BerufsanfängerInnen betont.

Insgesamt schätzen SchulleiterInnen die Kompetenzen der BerufsanfängerInnen als sehr hoch ein. Dies betrifft die Fach- und Medienkompetenz ebenso wie die Kompetenz, durch Motivation und Einsatzfreude das Schulklima positiv zu beeinflussen. Auch die durch die BerufsanfängerInnen selbst angeeigneten Kompetenzen werden von SchulleiterInnen in Bezug auf deren Individualität positiv bewertet.

\subsubsection{Hauptkategorie Institutionelle Ausbildung aus der Sicht der SchulleiterInnen (SL_B)} Die Hauptkategorie Institutionelle Ausbildung aus der Sicht der SchulleiterInnen (SL) (SL_B) steht nach der Analyse der Interviews mit SchulleiterInnen hierarchisch über den vier Subkategorien Selektion (SL_B_1), Anforderungen an die Ausbildung (SL_B_2), Praktika (SL_B_3) und Ausbildung an PH/UNI (SL_B_4) (Abb. 49).

Das Thema Selektion ( $S L_{-} B \_1$ ) wird beinahe von jedem/jeder zweiten SchulleiterIn (7 von 15) angesprochen, ohne dass im Interviewleitfaden explizit darauf eingegangen wird. Das unter-

52 SchulleiterInnen verwenden in den Interviews die Begriffe JunglehrerIn, BerufseinsteigerIn und BerufsanfängerIn weitgehend synonym. Ein expliziter Bezug zum biologischen Alter bei der Verwendung des Begriffs JunglehrerIn kann bei der Analyse der Interviews nicht hergestellt werden. 
streicht die Bedeutung dieser Thematik für SchulleiterInnen im Sinne einer induktiv gewonnenen Kategorie.

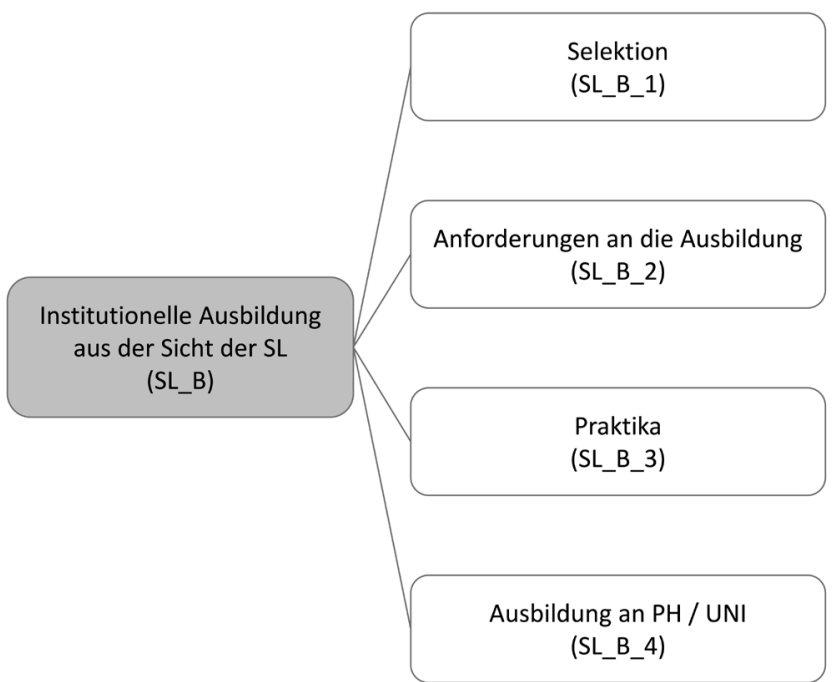

Abb. 49: Institutionelle Ausbildung aus der Sicht der SL; Haupt- und Subkategorien

Grundsätzlich unterscheiden die SchulleiterInnen zwischen messbaren und nicht-messbaren Faktoren zur Selektion, wobei der einhellige Tenor Selektion vor (z.B. durch Aufnahmetest) und während (z.B. mittels Notengebung) der LehrerInnenausbildung lautet.

„Es darf nicht jeder Lehrer werden können, der halt das Studium schafft, der die Prüfungen schafft. Es müssen die menschlichen Fähigkeiten, wie auch immer ( $2 \mathrm{~s}$ Nachdenkpause) und ich weiß, dass das schwierig ist, die müssen Priorität haben." (SL_03: 53)

Der/die SchulleiterIn deutet also auf die oben genannten nicht-messbaren Selektionskriterien hin. Ähnlich argumentiert ein/e weitere/r InterviewpartnerIn und meint, nachdem hin und wieder vom „Schülermaterial“ (SL_05: 45) gesprochen würde:

„[...] ob so ein Mensch mit so einer Menschenbildung Lehrer sein darf, also da gehört ausgemistet. Und da gehört sofort am Anfang ein Auswahlverfahren her, also das wäre einmal Grundsache." (SL_05: 50)

Ein anderes, laut SchulleiterInnen durch Selektion zu steuerndes Phänomen ist die Motivation zur Wahl des Lehramtsstudiums. SchulleiterInnen gehen davon aus, dass nicht für alle Lehramtsstudierenden die pädagogische Arbeit mit Kindern und Jugendlichen der Anlass zur Wahl dieses Studiums ist, wie die folgende Aussage zeigt:

„Wenn ich wüsste, wie man das objektiv feststellen könnte, aus welchen Gründen einer Lehrer werden möchte, dann könnte ich mir das schon vorstellen, einen Riegel vorzuschieben." (SL_13: 60)

Zusammenfassend kann gesagt werden, dass die SchulleiterInnen eine Selektion aufgrund der pädagogisch-didaktischen, im weiteren Sinne der bildungswissenschaftlichen Kompetenzen als notwendig empfinden. 
In der Subkategorie Anforderungen an die Ausbildung (SL_B_2) werden vorrangig pädagogische und administrative Kenntnisse, nicht etwa spezielle Kenntnisse bezogen auf das Unterrichtsfach, genannt. Das ist insofern interessant, als die Ausbildung (Bachelorstudium) in den Unterrichtsfächern mit 200 ECTS-AP (inkl. Fachdidaktik) tatsächlich den weitaus größeren Teil im Vergleich zu den Bildungswissenschaftlichen Grundlagen mit 40 ECTS-AP einnimmt (Leopold-Franzens-Universität Innsbruck 2016).

Bezogen auf die pädagogischen Kenntnisse sprechen die SchulleiterInnen von praxisnahem Classroom Management:

„[... manche Junglehrer nicht so ins offene Messer laufen lassen. Die [BerufsanfängerInnen, Anm.] kommen und sind mit dem Schulbetrieb, den ganzen Dingen nie konfrontiert worden. Auch von praktischen Abläufen, was sie einfach nicht gehört haben. Einfach zu theoretisch, klingt jetzt vielleicht ein bisschen blöd. Gut ausgebildet, aber manchmal ein bisschen an der Praxis vorbei." (SL_02: 50)

So beschreibt ein/e SchulleiterIn den typischen Berufseinstieg von BerufsanfängerInnen. Speziell disziplinäre Herausforderungen werden sehr oft thematisiert. Diese werden auch in $\mathrm{Her}$ ausforderungen an die BA (SL_C_5) weiter unten beschrieben. Ein/e SchulleiterIn sieht sich als VermittlerIn und dabei „[...] geht es nur um disziplinäre Sachen. Und das heißt für mich schon, dass das viel zu wenig gelehrt wird." (D_01: 36) Das Thema Disziplin spielt kategorienübergreifend eine zentrale Rolle. Bei den Befragungen der SchulleiterInnen und weitaus mehr noch in den Gesprächen mit den BerufsanfängerInnen (siehe auch Kategorienbeschreibung Classroom Management \& Disziplin (BA_D), Kap. III.3.1.5.4). Etwas weniger emotional wahrgenommen, jedoch häufig genannt werden die Themen rund um Amtsschriften, Reiserechnungen, C-Topf ${ }^{53}$, Struktur des Schuljahrs - insgesamt administrative Angelegenheiten im LehrerInnenberuf. Ein/e SchulleiterIn bringt es so auf den Punkt:

„Amtsschriften sind den Junglehrern weitgehend unbekannt und dass es bestimmte Termine im Schuljahr gibt, so wie halt ein Schuljahr strukturiert ist, ist auch nicht so bekannt.“ (SL_13: 19-20)

In der Subkategorie Praktika (SL_B_3) werden von den SchulleiterInnen drei Botschaften mitgegeben: Erstens sollte die Praxis verstärkt werden, zweitens müssten Realsituationen in den pädagogisch-praktischen Studien herbeigeführt werden und drittens sei die Wahrnehmung der SchulleiterInnen vorhanden, dass die schulpraktische Ausbildung wirkt. Ein/e SchulleiterInnen gibt in Bezug auf die Verstärkung der pädagogisch-praktischen Studienanteile Folgendes zu bedenken:

„Die Praxis ist sehr gut und das kann man noch verstärken, soweit es möglich ist. Dass jeder die Erfahrungen sammelt und dass er merkt ,Hoppla, da war ich zu wenig konsequent, da muss ich doch anders hinein gehen', dass er durch Erfahrungen lernt." (SL_12: 52)

Mit der Herbeiführung von Realsituationen werden im Wesentlichen die Praktikumsschulen bzw. -klassen und ganz konkrete Situationen im LehrerInnenalltag angesprochen. Ein/e SchulleiterIn meint:

„Bei manchen Klassen hat man einfach das Gefühl, dass die Junglehrer nicht entsprechend vorbereitet sind, dass sie sich manches nicht so vorstellen, wie die Praxis dann ist. [...] im zweiten Jahr ist die Sache dann schon wesentlich besser, heißt aber noch lange nicht, dass sie schon absolut fähig sind, damit umzugehen." (SL_02: 20)

$53 \operatorname{Im}$ C-Topf (Bereich C) des zur Zeit der Befragung geltenden alten Dienstrechts sind alle LehrerInnentätigkeiten enthalten, die über die Unterrichtserteilung und deren Vor- und Nachbereitung hinausgehen (Tiroler Landesregierung 2015). 
Auch der Umgang mit Disziplinproblemen wird an dieser Stelle wiederholt thematisiert, etwa in dieser Aussage:

„Dass manche Sachen besser gemacht gehören, das ist überall so, außer das Disziplinproblem. Also da glaube ich schon, dass sie da besser vorbereitet werden sollten, auf die realistische Situation der Schule.“ (SL_02: 54)

In Bezug auf die Vorbereitungsarbeit des Unterrichts sehen SchulleiterInnen die positive Auswirkung der Ausbildung. Ein/e Befragte/r meint dazu:

„Was sie [die BerufsanfängerInnen, Anm.] gut können, ist, wie sie mit den Vorbereitungen umgehen. Das klappt schon gut, auch schriftlich ist alles da und mit einem normalen Maß, also es ist nicht übertrieben. Also da merkt man, dass sie schon eine Ausbildung in der Praxis gehabt haben." (SL_09: 45)

Er/sie betont damit durchaus Positives in der LehrerInnenausbildung.

In der Subkategorie Ausbildung an PH/UNI (SL_B_4) wird über Methodik, Didaktik und die Fachausbildung bezogen auf das Unterrichtsfach bzw. die Unterrichtsfächer gesprochen. Die SchulleiterInnen sehen, wie bereits erwähnt, die Ausbildung an der Pädagogischen Hochschule und an der Universität durchwegs positiv. Die Statements in diese Richtung sind in quantitativer Hinsicht viel präsenter als negativ-kritische.

„Ich glaube, dass methodisch-didaktisch die Ausbildung nicht schlecht ist und dass sich in dem Zusammenhang ein neuer Lernbegriff durchgerungen hat. Da hat sich schon etwas getan.“ (SL_10: 44)

So meint ein/e SchulleiterIn und geht damit auf das in der Literatur diskutierte Paradigma des lehr- und lernseitigen Unterrichts (Christof und Schwarz 2013; Schratz et al. 2012) ein. Im Vergleich zur früheren, im Interview nicht näher deklarierten Ausbildung, sieht ein/e weitere/r Schulleiterin eine Qualitätssteigerung in der LehrerInnenausbildung:

„Was sich die jungen Lehrer methodisch-didaktisch manchmal antun, das ist schon phänomenal. Da haben sie einfach viele Beispiele und Werkzeuge mitbekommen, da merke ich einen markanten Sprung." (SL_10: 47).

Derselbe bzw. dieselbe SchulleiterIn erzählt in Bezug auf das verstärkt lernseitige Unterrichtskonzept weiter:

„[...] und den Trend, dass den Schülern mehr Aktivitäten zugelassen werden, merke ich seit ein paar Jahren deutlich. Also da muss ich der Ausbildung wirklich ein Kompliment machen, in der Richtung auf alle Fälle." (ebd.: 46).

In Bezug auf die Ausbildung im Unterrichtsfach ${ }^{54}$ werden die Aussagen in der Subkategorie Fach- \& Methodenkompetenz (SL_A_I) innerhalb der Hauptkategorie Kompetenzen von BerufsanfängerInnen ( $S L_{-} A$ ), wonach diese vorausgesetzt werden, bestätigt bzw. noch weitergeführt. Sie sind tatsächlich in hohem Maße vorhanden. „Ich glaube schon an die Ausbildung,

54 LehrerInnen der vorliegenden Untersuchung sprechen in den Interviews von Fachausbildung, wenn sie explizit das Studium des späteren Unterrichtsfachs meinen. Andernfalls wird die Terminologie Ausbildung in Methodik und (Fach-) Didaktik gewählt. Das Fach Bildungswissenschaften kommt im Schuljargon nicht vor bzw. wird dieses in Methodik und Didaktik subsumiert. Die Positionierung der Bildungswissenschaften bzw. die Akzeptanz dieser in der LehrerInnenausbildung hat im Denken vieler LehrerInnen und SchulleiterInnen noch wenig bis keinen Platz, das wurde interpretativ aus den Interviewgesprächen deutlich. Im Abschnitt Ansätze zur gelingenden Professionalisierung - Ausblick (Kap. V) wird auf die Triade Fachwissenschaft - Fachdidaktik - Bildungswissenschaft näher eingegangen. 
durch die Praxis und die Theorie, durch den Mix. Das finde ich schon gut. Also die Ausbildung ist okay“ (SL_12: 67), meint ein/e SchulleiterIn stellvertretend für viele.

Insgesamt kann festgestellt werden, dass SchulleiterInnen der Ausbildung bzw. den Ausbildungsinstitutionen gegenüber eine gute, wertschätzende Meinung vertreten. Desiderate orten sie beim Thema Selektion, die praktische Ausbildung könnte ihrer Meinung nach quantitativ ausgebaut werden. Die Vorbereitung der einsteigenden Lehrkräfte seitens der Ausbildung empfinden SchulleiterInnen insbesondere in Bezug auf neue Lehr- und Lernmethoden positiv.

\subsubsection{Hauptkategorie Wahrnehmung der BerufsanfängerInnen durch die SchulleiterInnen} $\left(S L_{-} C\right)$

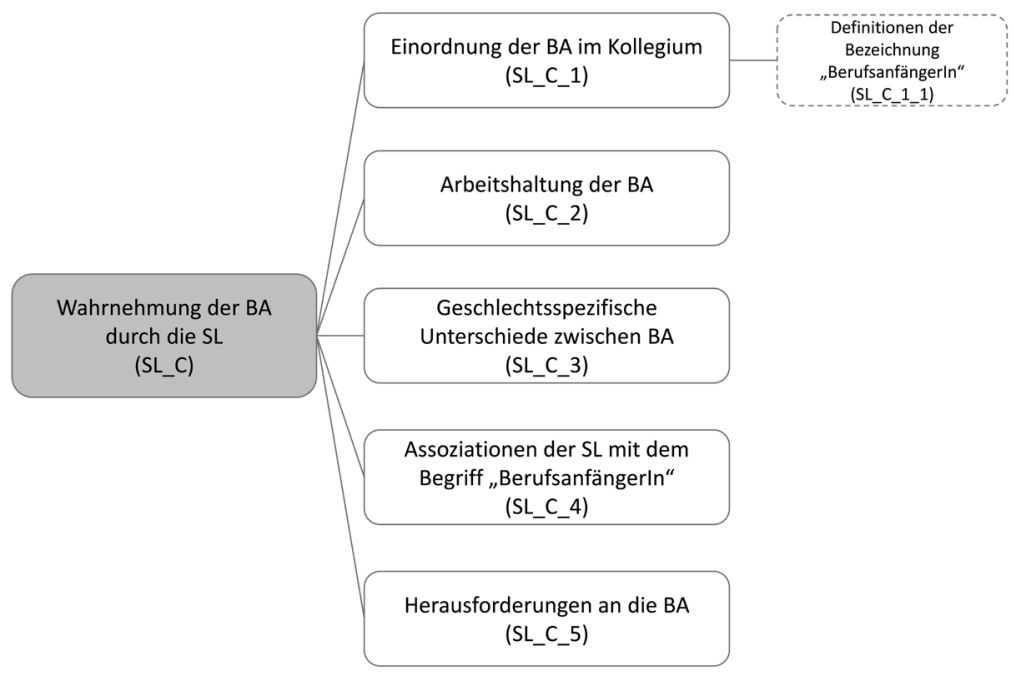

Abb. 50: Wahrnehmung der BA durch die SL; Haupt-, Sub- und Subsubkategorien

Die Hauptkategorie (Abb. 50). Wabrnehmung der BerufsanfängerInnen durch die SchulleiterInnen (SL_C) kann in die Subkategorien Einordnung der BA im Kollegium (SL_C_1), Arbeitshaltung der BA (SL_C_2), Geschlechtsspezifische Unterschiede zwischen BA (SL_C_3), Assoziationen der SL mit dem Begriff BerufsanfängerIn ( $S L_{-} C_{-} 4$ ) und Herausforderungen an die BA $\left(S L_{-} C_{-}\right.$5) unterteilt werden. Einordnung der BA im Kollegium (SL_C_1) wird in der Subsubkategorie Definitionen der Bezeichnung BerufsanfängerInnen ( $S L_{-} C_{-}{ }_{-}$1) weiter spezifiziert $\mathrm{Zu}$ Einordnung der BA im Kollegium ( $S L_{-} C_{-}$l) kann festgestellt werden, dass es laut Wahrnehmung der SchulleiterInnen für eine/n BerufsanfängerIn einen Unterschied ausmacht, in welchem Lehrkörper die junge Lehrkraft ihren Berufseinstieg absolviert. Folglich hängt es ganz wesentlich von der Altersstruktur des Kollegiums ab, wie lange sich der/die betreffende BerufsanfängerIn als JunglehrerIn fühlt. „Das Ganze hängt natürlich auch von der Struktur in einem Lehrkörper ab“ (SL_07: 13), sagt ein/e SchulleiterIn und präzisiert:

„Wenn ich einen sehr alten Lehrkörper habe, dann ist vielleicht die Definition ,Junglehrer ' noch länger - wenn ich einen sehr alten Lehrkörper habe und ein neuer Lehrer mit zehn Jahren Praxis ist dann im Vergleich zu den anderen halt auch noch ein Junglehrer." (SL_07: 13) 
Die Meinungen der SchulleiterInnen gehen auseinander, wenn es um die Bezeichnung JunglehrerIn im Zusammenhang mit dem biologischen Alter geht. Für die eine (größere) Gruppe bezieht sich die Bezeichnung JunglehrerIn auf das Berufsalter, auf die Dienstjahre im LehrerInnenberuf. „Das biologische Alter ist vielleicht nicht immer so ausschlaggebend“, meint ein/e SchulleiterIn, „weil wenn einer erst später einsteigt, der gehört dann auch zu den Junglehrern, der muss einfach die Erfahrung sammeln“ (SL_07: 15). „Im Prinzip sind für mich Junglehrer Lehrer, die das erste Mal bei mir an der Schule sind“ (D_13: 13), lautet eine weitere Eingrenzung des Begriffs JunglehrerIn. Die andere Gruppe der SchulleiterInnen vertritt die Auffassung: „Späteinsteiger bezeichnet man nicht mehr so gern als Junglehrer. Man begegnet ihm nicht mehr so als Junglehrer, das Alter spielt eine Rolle." (SL_08: 14) Für eine/n andere/n SchulleiterIn ist „[...] ein Junglehrer [...] im engeren Sinne [einer], der frisch kommt von der Ausbildung bis zu den ersten drei, vier Dienstjahren“" (SL_09: 13).

Das Dienstalter bzw. die absolvierten Dienstjahre der BerufsanfängerInnen spielen für die Subsubkategorie Definitionen ( $C_{-} 1_{-} 1$ ) eine zentrale Rolle. Sie sind das Kriterium, welches von den SchulleiterInnen zur Definition des Begriffs BerufsanfängerIn herangezogen wird. Die Spanne der Dienstjahre, die ein/e BerufsanfängerIn nach Ansicht der SchulleiterInnen braucht, um aus dem Status des Berufsanfängers/der Berufsanfängerin herauszutreten, variiert zwischen einem und sieben Dienstjahren. Dabei kristallisiert sich der Bereich von drei bis vier Dienstjahren als der am öftesten genannte heraus. Eine Aussage, die auch das biologische Alter berücksichtigt und die zusammenfassende Gesamtmeinung der SchulleiterInnen gut abbildet, lautet: „Die ersten drei Jahre, würde ich sagen, das ist ein Berufsanfänger. Egal, wie alt er ist." (SL_12: 13) In Bezug auf die Arbeitshaltung der BA (SL_C_2) äußern sich die SchulleiterInnen ausschließlich positiv. Zum einen nehmen sie Freude am LehrerIn-Sein wahr:

„Das Engagement bringen sie aus der Freude heraus, dass sie gerne Lehrer sind, mit. Aus dem heraus, dass da doch ein gewisser Berufungsaspekt dahinter ist. Dass man nicht Lehrer wegen der Ferien geworden ist, sondern weil man das machen möchte.“ (SL_14: 47)

Andererseits wird auch die Arbeitshaltung, also der aktive Beitrag der BerufsanfängerInnen, im Beruf thematisiert. Die Einsatzfreude dieser steht diesbezüglich an erster Stelle. Das folgende Interviewzitat steht stellvertretend für viele Wortmeldungen zum Thema Einsatzbereitschaft:

„Was sie [die BerufsanfängerInnen, Anm.] noch haben, also das kann man quer durch die Bank sagen, also totalen Einsatz. Also wirklich, sie wollen gut sein, etwas anderes habe ich noch nicht erlebt. Und sie wollen dir das zeigen, sie sind gute Lehrer und tun für die Kinder das Beste. Also der Einsatz ist sehr groß. Das muss ich sagen." (SL_03: 67)

Die Offenheit, im Sinne von bereit für Neues, wird ebenso thematisiert:

„Ich habe nur super Junglehrergehabt bisjetzt. Also sehr lernwillige und offene, die halt eine,Fragehaltung haben.BesteErfahrungen.[...] Die[BerufsanfängerInnen,Anm.] sindeinfachfroh,dasssieeinenJobhaben." (SL_11:15-17)

In Geschlechtsspezifische Unterschiede zwischen $B A$ (SL_C_3) lautet die übereinstimmende Grundmeinung, unabhängig davon, ob es sich um eine Schulleiterin oder um einen Schulleiter handelt: „Also ich würde sagen, da gibt es keine Unterschiede." (SL_13: 46) An dieser Stelle der Analyse wird deshalb von einer Grundmeinung gesprochen, da diese zwar mehrfach betont wird, aber trotzdem des Öfteren ein Phänomen auftaucht, wo das Geschlecht der jungen Lehrkraft eine Rolle spielt: die Emotion. „Frauen, das ist jetzt allgemein, Frauen sind emotiona- 
ler, nehmen alles viel persönlicher. Mir gegenüber und auch Eltern gegenüber“ (SL_03: 42), ist ein/e SchulleiterIn überzeugt. In einem weiteren Interview heißt es:

„In der Zusammenarbeit ist es mit den Männern teilweise einfacher, weil diese nicht jedes Wort so auf die Waagschale legen. Bei den Frauen wiederum, muss man ein bisschen aufpassen. Wenn du zu einem Mann sagst ,das hast du jetzt verbockt', dann steckt er es weg. Wenn du das zu einer Frau sagst, dann nimmt sie es persönlich." (SL_08: 33)

Ein Seitenthema in dieser Subkategorie ist die zahlenmäßige Unterrepräsentanz der männlichen Berufsanfänger. Ein/e SchulleiterIn bringt es auf die Frage nach geschlechtsspezifischen Unterschieden folgendermaßen zum Ausdruck: „Das ist jetzt natürlich eine gute Frage, weil die männlichen Junglehrer fast nicht mehr existent sind.“ (SL_02: 46) Und ein/e weitere/r SchulleiterIn verbreitet einen Hauch von Resignation:

„Zu viele Eltern sind geschieden, die Kinder wachsen schon nur mit der Mama auf, dann haben sie eine Oma, dann haben sie eine Kindergärtnerin, dann haben sie eine Volksschullehrerin und wenn sie jetzt in der Neuen Mittelschule oder im Gymnasium auch noch Lehrerinnen haben, es ist halt unnatürlich, sagen wir so. Aber das kann ich nicht beeinflussen." (SL_03: 47)

Die Reaktionen und Antworten auf die Frage nach den Assoziationen der SL mit dem Begriff BerufsanfängerIn (SL_C_4) fallen eindeutig positiv aus. Ein/e SchulleiterIn kommt ins Schwärmen:

„Mir fällt ein, dass sie [die BerufsanfängerInnen, Anm.] sehr engagiert sind, sehr fleißig sind und mit hundert Prozent bei der Sache. Motiviert, voll motiviert. Ich assoziiere positiv, nur positiv“" (SL_08:16).

Der Gewinn für die Schule, „da kommt ein frischer Wind mit den Jungen, das ist einfach so“ (SL_03: 76), wird ebenso assoziiert wie das Engagement, welches bereits an voriger Stelle erwähnt wurde:

„Also wenn ich an meine Junglehrer denke, wüsste ich nicht einen, der nur Dienst nach Vorschrift gemacht hat. Das taugt ihnen sogar, also für eine Institution da zu sein und Aufgaben zu übernehmen“ (SL_09: 48).

Die einzige negativ behaftete Assoziation mit BerufsanfängerInnen ist der realitätsfremde Berufseinstieg. „Sie [die BerufsanfängerInnen, Anm.] kommen mit vielen Illusionen“ (SL_09: 15), führt ein/e SchulleiterIn aus, um anschließend noch zu präzisieren: „[...] und dann ist das Problem, dass manche Berufseinsteiger mit einer idealisierten Vorstellung daher kommen." (ebd.: 27) $\mathrm{Er} /$ sie spielt auf die herausfordernde, später noch zu analysierende Aufgabe des Classroom Managements an.

In die Subkategorie Herausforderungen an die BA (SL_C_5) fallen jene Beobachtungen der SchulleiterInnen hinein, die Stolpersteine für den erfolgreichen Berufseinstieg von BerufsanfängerInnen darstellen, konkret in den Bereichen Classroom Management und SchülerInnenleistungen. Es handelt sich hier um „viele Fehler, die mit der Routine weggehen“ (SL_14: 17). Und „[... ] natürlich kommt das Fachliche dazu. Das bringt dann auch Unsicherheiten, vom Lehrbuch. Das Buch kennt er nicht, der Junglehrer“ (D_12: 39), berichten zwei SchulleiterInnen von ihren Beobachtungen. „Manche sind wieder zu streng in der Leistung“, erzählt der/die SchulleiterIn weiter. „Sie [die BerufsanfängerInnen, Anm.] verlieren den Blick dafür, was wir da für Schüler vor uns haben, vom Leistungsniveau her." (SL_14: 21) Ein/e weitere/r SchulleiterIn schildert die Herausforderung an die BerufsanfängerInnen, wenn deren Erwartungshaltung nicht erfüllt werden: 
„Und dann ist generell die Arbeitshaltung der Schüler manchmal ein Problem für Junglehrer, sie [die BerufsanfängerInnen, Anm.] kommen tiptop vorbereitet und erwarten sich im Prinzip das Gleiche von den Schülern. [...] Und damit müssen sie [die BerufsanfängerInnen, Anm.] fertig werden." (SL_08: 30)

Zusammenfassend kann festgestellt werden, dass SchulleiterInnen keine Hürden für BerufsanfängerInnen in Bezug auf die Integration im LehrerInnenkollegium feststellen, die Altersstruktur des Letzteren determiniert jedoch das Integrationsverhalten der neuen Lehrpersonen. In Bezug auf die Bezeichnung JunglehrerIn gehen die Meinungen der SchulleiterInnen auseinander. Für eine Gruppe hängt der Begriff mit dem biologischen Alter, für die anderen mit dem Dienstalter zusammen. Die größten Herausforderungen für BerufsanfängerInnen sehen SchulleiterInnen im realistischen Schuleintritt. Sie können teilweise nicht abschätzen, wie weit SchülerInnen fachlich und welche Verhaltensweisen im Kontext des Classroom Managements zu erwarten sind.

\subsubsection{Hauptkategorie Unterstützung und Begleitung der BerufsanfängerInnen im Schulalltag (SL_D)}

Die Hauptkategorie Unterstützung und Begleitung der BerufsanfängerInnen im Schulalltag (SL_D) ist quantitativ mit 141 Kodierungen und mit 14 von 15 bezugnehmenden SchulleiterInnen (siehe auch Tab. 7) die umfangreichste aller Kategorien und findet auch auf der qualitativen Ebene besondere Berücksichtigung (z.B. Induktionsphase). Sie steht hierarchisch über den Subkategorien Erwartungen von den $B A$ an die $S L$ (SL_D_1), Strukturelle Hilfe \& Rahmenbedingungen (SL_D_2) und Mentoring (SL_D_3). Die Subkategorie Mentoring (SL_D_3) wird zusätzlich in die Subsubkategorien Vorbedingungen ( $S L \_D \_3 \_1$ ), KollegInnen als BegleiterInnen ( $\left.S L \_D \_3 \_2\right)$, SL als BegleiterInnen (SL_D_3_3) und Herausforderungen an die BA (SL_D_3_4) unterteilt (Abb. 51).

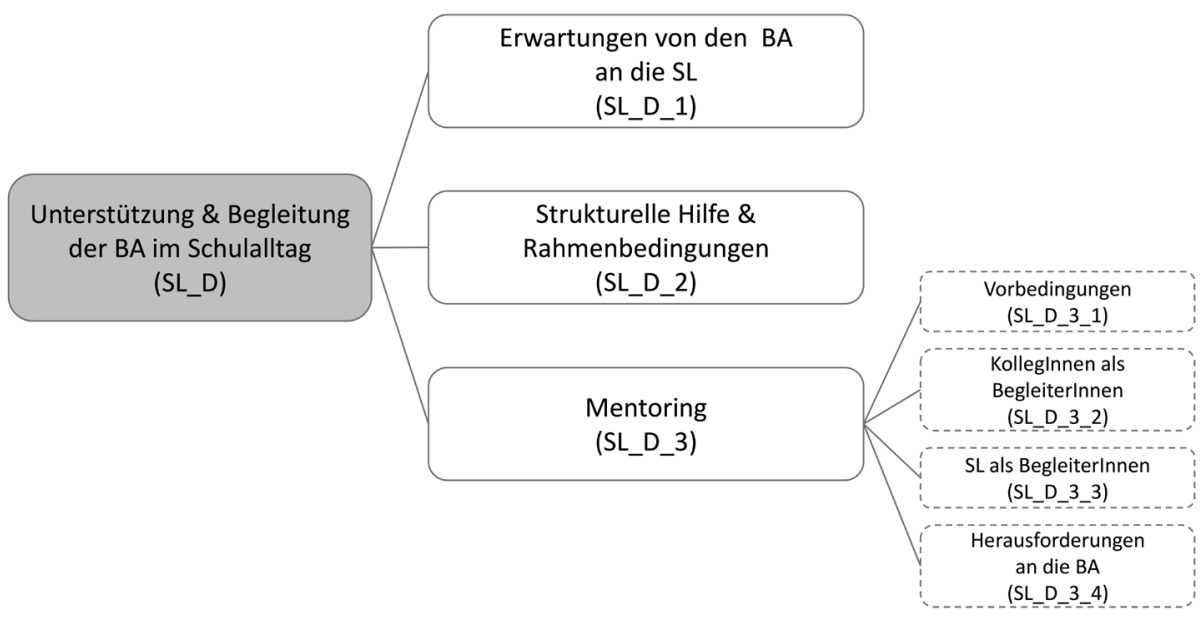

Abb. 51: Unterstützung \& Begleitung der BA im Schulalltag; Haupt-, Sub- und Subsubkategorien

An dieser Stelle wird noch einmal betont, dass es sich bei der Beschreibung der Subkategorie $E r$ wartungen von den $B A$ an die $S L\left(S L \_D \_1\right)$ wie bei allen vorangegangenen Kategorien um die Sicht der SchulleiterInnen handelt. Insbesondere für die Thematik Unterstützung \& Begleitung der BA im Schulalltag (SL_D) spielt der Fokus (BerufsanfängerInnen- oder SchulleiterInnenperspektive) eine essenzielle Rolle. 
Der Grundtenor der SchulleiterInnen lautet, dass die BerufsanfängerInnen „mit verhältnismäBig wenigen“ (SL_07: 20) Erwartungen kommen. Zum einen kann das damit erklärt werden, dass die BerufsanfängerInnen in erster Linie froh über ihre Anstellung sind und nicht sofort ihre Erwartungen in den Vordergrund stellen (siehe auch Kategorie Arbeitshaltung der BA $\left(S L \_C \_2\right)$, Kap. III.3.1.4.3), zum anderen damit, dass „das Kollegium vieles abnimmt“ (SL_10: 22). Die dritte Erklärung könnte sein, dass es sich eben um die Sicht der SchulleiterInnen handelt und diese Erkenntnisse auf Fremdwahrnehmung basieren.

Wenn die SchulleiterInnen von Erwartungen sprechen, dann nicht von fachlich-pädagogischen, sondern von organisatorischen. „Wo ist was an der Schule?" (SL_12: 37), formuliert ein/e SchulleiterIn und erklärt weiter: „Da ist es wichtig, dass man jemanden zuteilt, oder besser gesagt, der Junglehrer wird jemanden zugeteilt." (ebd.: 37)

In der Subkategorie Strukturelle Hilfe \& Rahmenbedingungen (SL_D_2) kommen ebenfalls organisatorische Dinge, aber auch das Thema Classroom Management zur Sprache:

„[...] also zuerst im Vorfeld musst du ihnen [den BerufsanfängerInnen, Anm.] einmal viel Information geben [...] und dann relativ genau zeigen, die verschiedenen Schulbücher und Unterrichtsmittel und überhaupt von den Gegebenheiten an der Schule, was so gemacht wird und möglich ist." (SL_04: 22)

Diese Aussage bezieht sich auf den Schultyp der Polytechnischen Schule ${ }^{55}$, die in der LehrerInnenausbildung kaum bzw. keine explizite Berücksichtigung findet. Für eine/n andere/n SchulleiterIn "geht [es] mehr um Verwaltungsgeschichten, [...] nicht [so sehr um] Pädagogik“"(SL_05: 15). Er/ sie relativiert unmittelbar: „[...] bei jedem Junglehrer ist das natürlich höchst unterschiedlich.“ (ebd.) Eine gute Möglichkeit zur Unterstützung der JunglehrerInnen beim Einstieg in das Berufsleben sehen die SchulleiterInnen in der Klassen- bzw. Gruppen- und auch in der Fächereinteilung. So spricht ein/e SchulleiterIn die disziplinären Schwierigkeiten an und sieht eine Lösung darin,

„dass man eine [disziplinär schwierige, Anm.] Gruppe diesem Junglehrer dann nicht mehr gibt. Ich glaube, das ist eine der wichtigsten Maßnahmen, die man überhaupt setzen kann." (SL_11:23)

Ein/e andere/r SchulleiterIn „bemüht [sich] immer, möglichst solche Fächer zu geben, wo sich der betreffende Lehrer darüber hinaus sieht“ (SL_13: 32). Ebenfalls in den Bereich der strukturellen Hilfe und Rahmenbedingungen fällt das Fachkoodinationssystem.

„Bei den Hauptfächern ist es ja ideal. Wir haben einen Koordinator, in der Neuen Mittelschule zumindest. Da wird er [der Berufsanfänger, Anm.] eh schon eingebunden, es ist auch in den ersten Tagen eine Koordinatorsitzung." (SL_12:41)

„Klare Richtlinien“ (SL_08: 28) an der Schule sind für SchulleiterInnen in diesem Zusammenhang eine sehr wichtige und wertvolle Maßnahme. Diese Klarheit bezieht sich auf zwei Phänomene im Schulbetrieb: auf die Notengebung und auf den Umgang mit Disziplinproblemen:

„Ob sie [die BerufsanfängerInnen, Anm.] bei der Notengebung alles richtig machen, da werde ich gefragt. Da haben wir auch in der Konferenz einmal ein Thema gemacht: Einheitliche Notengebung an der ganzen Schule." (ebd.: 27)

Zum Thema Disziplin lautet eine Devise:

55 Die Polytechnische Schule hat gemäß $\$ 28$ des Schulorganisationsgesetzes die Aufgabe, auf das weitere Leben und insbesondere auf das Berufsleben vorzubereiten und wurde im Jahre 1966 als Polytechnischer Lehrgang im österreichischen Schulsystem zur Möglichkeit der Absolvierung des verpflichtenden neunten Schuljahrs installiert (Bundesministerium für Bildung, Wissenschaft und Forschung 2020b; Jäger 2001). 
„Wenn jeder Lehrer im Lehrkörper eine unterschiedliche Linie verfolgt und jeder unterschiedliche Maßnahmen setzt, dann wird sich der Junglehrer sehr schwer tun. Wenn es aber gelingt, bei einem verhältnismäßig kleinen Lehrkörper einen Konsens zu finden, in welche Richtung das gehen kann, dann glaube ich, findet sich der Junglehrer wesentlich leichter zurecht." (SL_06: 30)

Der Stellenwert des Mentorings, abgebildet in der Subkategorie Mentoring (SL_D_3), wird durch die hohe Anzahl der Kodierungen sichtbar (15 von 15 SchulleiterInnen thematisieren Mentoring mit 103 Codes). Diese Subkategorie bildet sich aus den vier Subsubkategorien Vorbedingungen (SL_D_3_1), KollegInnen als BegleiterInnen (SL_D_3_2), SchulleiterInnen als BegleiterInnen (SL_D_3_3) und Herausforderungen (SL_D_3_4).

Die Subsubkategorie Vorbedingungen (SL_D_3_1) kann mit den Schlagworten Zeitangebot, Gespräche und Vertrauen zusammengefasst werden. Eine SchulleiterIn versucht, „sehr, sehr viel an der Schule zu sein, Vormittag und auch Nachmittag“ (SL_02: 26), um den BerufsanfängerInnen ständig helfend zur Seite stehen zu können. „Meine Tür steht jederzeit offen“ (SL_13: 23), lautet eine weitere Devise. „[...] dann kommen viele Fragen in allen Belangen. Pädagogisch, fachlich, was immer. [...] da ist immer die Tür offen“" (SL_05: 15), bringt ein/e weiter/e SchulleiterIn den Rundum-die-Uhr-Service zum Ausdruck. Mit der Aussage „[...] es geht um mentale und pädagogische Hilfen. Und da hilft oft schon das Gespräch“ (SL_04: 27) thematisiert ein/e SchulleiterIn die Bedeutung der Gespräche mit BerufsanfängerInnen. Eine Facette des Gesprächs ist das Feedback, welches von den SchulleiterInnen in den Interviews regelmäßig genannt wird.

„Da [nach dem Unterrichtsbesuch, Anm.] sage ich dann, was mir vorkommt, was gut gewesen ist und so weiter. Und das Feedback ist auch wichtig, weil da Vertrauen aufgebaut wird.“ (SL_03: 32)

„Sie [die BerufsanfängerInnen, Anm.] wollen wissen, ob das gut und recht ist [...] So ein bisschen Bestätigung brauchen sie, das kommt relativ oft vor" (SL_04: 36), teilt ein/e SchulleiterIn die diesbezüglichen Erfahrungen mit. Auch verpflichtende MitarbeiterInnengespräche werden von den SchulleiterInnen thematisiert. ,Ja, vielleicht könnte man irgendeine Art ,verpflichtende Gesprächsrunde` einführen“ (SL_01: 61), formuliert ein/e SchulleiterIn eine zukünftige Möglichkeit der Implementierung strukturierter Gespräche. Wie bereits einleitend zu dieser Subsubkategorie erwähnt, wird ein intaktes Vertrauensverhältnis für die gute Zusammenarbeit im Kollegium, aber auch für die Motivation und folglich für die weitere Entwicklung der jungen Lehrperson als entscheidender Faktor angesehen: „Wenn man arbeitet mit Leuten, man braucht ein gewisses Vertrauensverhältnis. [...] für die Motivation des Lehrers ist ein Vertrauensverhältnis, glaube ich, ganz gut.“ (SL_12: 59) Eine „Annahme [im Kollegium, Anm.] ohne Vorurteile“ (SL_03: 29) und eine „gewisse Vertrauensbasis“ (ebd.: 30) wird präzisierend als Schlüssel für den erfolgreichen Berufseinstieg von BerufsanfängerInnen genannt.

In den Gesprächen mit den SchulleiterInnen haben sich Unterschiede in der Wirksamkeit der Begleitung von BerufsanfängerInnen herauskristallisiert, je nachdem, ob es sich um KollegInnen als BegleiterInnen ( $\left.S L \_D \_3 \_2\right)$ oder um SchulleiterInnen als BegleiterInnen (SL_D_3_3) handelt. In der Subsubkategorie KollegInnen als BegleiterInnen (SL_D_3_2) wird deutlich, dass das Gespräch unter KollegInnen aus pragmatischen und hierarchischen Gründen leichter stattfindet als zwischen SchulleiterIn und BerufsanfängerIn: „Am ehesten“, schildert ein/e SchulleiterIn, „[funktioniert die Begleitung] mit Vorbildwirkung. Mit Teamteaching, mit Unterrichtsbesuchen." (SL_05: 19) Aus hierarchischer Sicht „ist der Direktor für Junglehrer eine Etage höher. Das heißt also, sobald er den Direktor braucht, vermittelt er das Gefühl, dass er nicht ganz genau weiß, was ihn erwartet" (SL_06: 28), gibt ein/e SchulleiterIn zu bedenken. Diese Hemm- 
schwelle, die Hilfe der Schulleitung in Anspruch zu nehmen, führt nach den Aussagen der SchulleiterInnen dazu, dass sich „die Junglehrer viel von den älteren Kollegen abschauen. Schon im Konferenzzimmer.“ (SL_08: 26) Hier wird deutlich, dass die Begleitung der BerufsanfängerInnen nicht erst im Klassenzimmer, sondern viel früher beginnt. In fachlicher und vorbereitungstechnischer Hinsicht werden BerufsanfängerInnen dadurch unterstützt, indem „wirklich ganze Vorbereitungsmappen weitergegeben [werden]“ (ebd.: 19), was die zeitaufwändige Arbeit der Stundenvorbereitung erleichtert.

In der Subsubkategorie SchulleiterInnen als BegleiterInnen (SL_D_3_3) kommen im Wesentlichen zwei Themen zur Sprache: Professionalisierung und Disziplin bzw. Disziplinprobleme. Im Bereich Professionalisierung werden einerseits pädagogisch-praktische Tipps für einen gelingenden Unterricht gegeben: „[...] der Junglehrer unterrichtet sehr lehrerzentriert, er lässt also wenig den Schülern über [...] und er hat dann die doppelte Arbeit." (SL_14: 19) Andererseits wird auch über Vorkommnisse außerhalb der Unterrichtes gesprochen, zum Beispiel über das Verhalten aufgebrachten Eltern gegenüber. Wenn ein Fehler passiert ist, kann man diesen „ruhig zugeben“ (SL_03: 40), lautet etwa ein Tipp, wobei sogleich relativiert wird:

„[...] oft sind es die Mütter, es kommen ja hauptsächlich die Mütter, die meinen, weil sie selber einmal in die Hauptschule gegangen sind, wissen sie über die Pädagogik Bescheid. Also da braucht er [der Berufsanfänger, Anm.] schon Unterstützung." (ebd.: 40)

Auch die Wissensaneignung auf der rechtlichen Ebene trägt laut SchulleiterInnen zur Professionalisierung der BerufsanfängerInnen bei. „Was bei den Jüngeren noch dazu kommt, sind dienstrechtliche Fragen, wo ich dann natürlich erster Ansprechpartner bin“ (SL_07: 22), weiß ein/e SchulleiterIn. Konkret sind das Fragen wie „Darf ich das?', gesetzliche Fragen, , Was könnte ich tun, dass mir das Amt das genehmigt?" und so weiter" (SL_05: 17).

Im Bereich Disziplin bzw. Disziplinprobleme sehen sich die SchulleiterInnen zuweilen als aktive HelferInnen:

„Also da bin ich teilweise einmal pro Woche mit in die Klasse gegangen, habe Schüler geholt und so weiter. Und das sind die [BerufsanfängerInnen, Anm.] froh um diese Unterstützung" (SL_03: 39).

Auch passiv, durch die bloße Anwesenheit an der Schule, unterstützen SchulleiterInnen die BerufsanfängerInnen, denn ein/e SchulleiterIn hat „auch schon Lehrer gehabt, bei denen [sie, die Schulleiterin, Anm.], wenn sie Nachmittagsunterricht gehabt haben, ungern aus dem Schulhaus gegangen [ist]“ (SL_13: 41). Die aktive Unterstützung der BerufsanfängerInnen bei Disziplinproblemen wird allerdings ambivalent diskutiert, „weil wenn man den Direktor sozusagen holt, dann ist man eigentlich schon verkauft“ (ebd.: 39). „Einen Schüler zum Direktor zu schicken ist ein Armutszeugnis“ (SL_14: 24f), wird ebenfalls dazu ganz pointiert formuliert.

Eine weitere Maßnahme ist die Zusammensetzung der verschiedenen Gruppen- und Klassenverbünde. Dies obliegt der Schulleitung der jeweiligen Schule und wird auch als unterstützendes Instrument zur Vermeidung oder Verminderung disziplinärer Probleme eingesetzt. „Wenn eine schwierige Schülerin aus ihrer gewohnten Umgebung heraus kommt, kann ich damit der Kollegin sicherlich helfen" (SL_07: 31), ist ein/e SchulleiterIn überzeugt.

Die Herausforderungen an die BA (SL_D_3_4) beim Mentoring bestehen laut Aussagen der SchulleiterInnen darin, dass die BerufsanfängerInnen ihre Rolle ohnehin selber definieren und finden müssen.

„Sowie er [der Berufsanfänger, Anm.] die Klasse betritt, ist er auf sich allein gestellt. Wie er sich der Klasse präsentiert und wie er von der Klasse angenommen wird, hängt in erste Linie von ihm selber ab“ (SL_06:24) 
Insofern „muss einfach jeder selber in der Klasse mit beiden Füßen drinnen stehen und einfach ,den Haufen' in den Griff bekommen“ (SL_07: 29), formuliert ein/e weitere/r SchulleiterIn. Außerdem ist eine Einmischung der Schulleitung ,immer mit Autorität verbunden [...], mit Gesichtsverlust [...], mit Zugeständnis von Schwäche“ (SL_06: 26) und damit wird eine Brücke zur Kategorie SchulleiterInnen als BegleiterInnen (SL_D_3_3) geschlagen. Daraus resultiert gewissermaßen, dass die SchulleiterInnen das „Hineingschaftln“56 (SL_09: 22) vermeiden und es am liebsten mögen, „wenn sich einer [ein Berufsanfänger, Anm.] selber zurecht findet, weil dann entwickelt er selber sein Profil“ (ebd.: 23).

In Bezug auf die Unterstützung und Begleitung der BerufsanfängerInnen durch die SchulleiterInnen kann zusammengefasst werden, dass BerufsanfängerInnen nicht aktiv mit Erwartungen an die SchulleiterInnen herantreten. Das schließt die erforderliche Unterstützung durch die Schulleitungen jedoch nicht aus. Im Bereich der Rahmenbedingungen, z.B. durch Schülergruppeneinteilungen und stundenplantechnischen Berücksichtigungen, werden BerufsanfängerInnen unterstützt. Einen großen Unterstützungsbedarf orten SchulleiterInnen im Bereich des Mentorings. Während sie selbst eher passiv durch die bloße Anwesenheit oder das Angebot zum Gespräch helfen, unterstützen die berufserfahreneren KollegInnen die BerufsanfängerInnen aktiv.

Nach der Darstellung der Kategorien, die sich aus der Analyse der SchulleiterInneninterviews gebildet haben, folgt nun die Beschreibung der Kategorien zu den Interviews mit BerufsanfängerInnen.

\subsubsection{Interviews mit BerufsanfängerInnen}

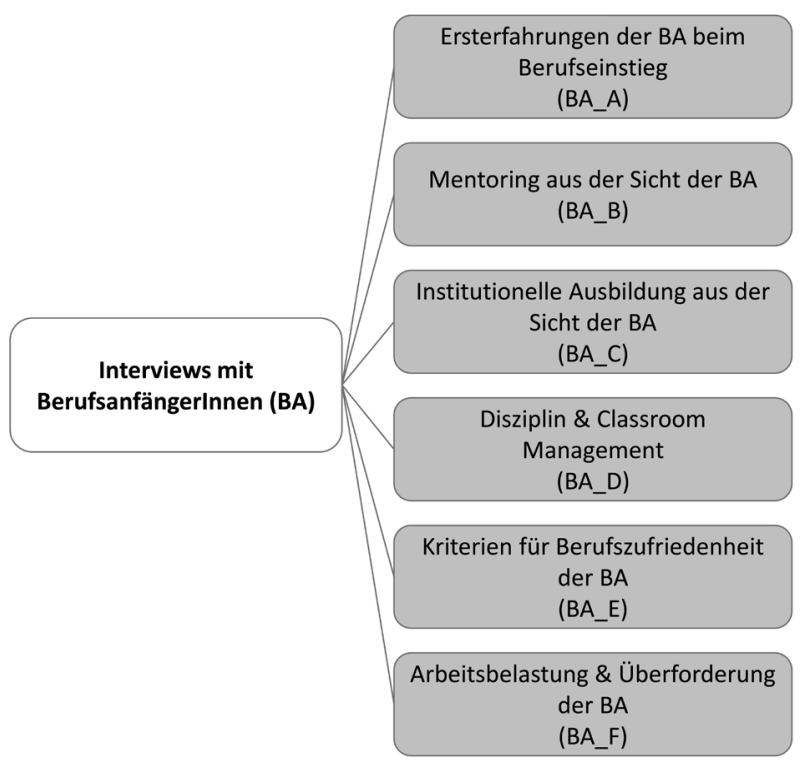

Abb. 52: Interviews mit BerufsanfängerInnen; Hauptkategorien (BA_A) - (BA_F)

Die Analyse der Interviews mit BerufsanfängerInnen liefert fünf Hauptkategorien. Diese sind Ersterfahrungen der BA beim Berufseinstieg (BA_A), Mentoring aus der Sicht der BA (BA_B), Institutionelle Ausbildung aus der Sicht $\operatorname{der} B A\left(B A_{-} C\right)$, Disziplin \& Classroom Management

56 Wichtigtuerei, unangemessenes Einmischen (Duden 2017). 
(BA_D), Kriterien für Berufszufriedenheit der BA (BA_E) und Arbeitsbelastung \& Überforderung der $B A$ (BA_F) (Abb. 52).

Vor der Beschreibung der Haupt- und untergeordneten Subkategorien (Kap. III.3.1.5.1Kap. III.3.1.5.6) wird in Tabelle 8 eine Übersicht zur Quantität von Subkategorien, Codes und BerufsanfängerInnen-Nennungen gegeben. Hauptkategorie (BA_A) Ersterfahrungen der BerufsanfängerInnen beim Berufseinstieg (Tab. 8) besteht aus vier Subkategorien und acht Subsubkategorien mit insgesamt 102 Kodierungen (Codes), welche von 19 BerufsanfängerInnen thematisiert werden (Tab. 8).

Tab. 8: Interviews mit BerufsanfängerInnen - Häufigkeiten

\begin{tabular}{lccccc}
\hline \multicolumn{7}{l}{ Interviews mit BerufsanfängerInnen - Häufigkeiten } \\
$\begin{array}{l}\text { Haupt- } \\
\text { kategorie }\end{array}$ & Subkategorie & $\begin{array}{l}\text { Subsub- } \\
\text { kategorie }\end{array}$ & $\begin{array}{l}\text { Subsub- } \\
\text { subkategorie }\end{array}$ & Codes & $\begin{array}{l}\text { n (verschiedene } \\
\text { BerufsanfängerInnen) }\end{array}$ \\
\hline BA_A & 4 & 8 & 0 & 102 & 19 \\
BA_B & 2 & 8 & 0 & 57 & 20 \\
BA_C & 4 & 11 & 4 & 454 & 25 \\
BA_D & 4 & 0 & 0 & 70 & 14 \\
BA_E & 3 & 0 & 0 & 68 & 18 \\
BA_F & 2 & 0 & 0 & 25 & 12 \\
\hline Summe & 19 & 27 & 4 & 776 & $25^{57}$ \\
\hline
\end{tabular}

Hauptkategorie (BA_B) Mentoring aus der Sicht der BA besteht aus zwei Subkategorien und acht Subsubkategorien mit insgesamt 57 Kodierungen, genannt von 20 BerufsanfängerInnen. Hauptkategorie (BA_C) Institutionelle Ausbildung aus der Sicht der BA beinhaltet vier Subkategorien, elf Subsubkategorien und vier Subsubsubkategorien mit insgesamt 112 Kodierungen. Diese werden von allen 25 BerufsanfängerInnen angesprochen. Hauptkategorie (BA_D) Disziplin \& Classroom Management besteht aus vier Subkategorien, 14 BerufsanfängerInnen tragen zu 70 Codes bei. Hauptkategorie (BA_E) Kriterien für Berufszufriedenheit der BA wird in drei Subkategorien unterteilt, 18 BerufsanfängerInnen thematisieren 68 Kodierungen und Hauptkategorie (BA_F) Arbeitsbelastung \& Überforderung der BA besteht aus zwei Subkategorien mit insgesamt 25 Codes unter Beteiligung von zwölf BerufsanfängerInnen (Tab. 6).

In den folgenden Abschnitten (Kap. III.3.1.5.1 - Kap. III.3.1.5.6) werden die jeweiligen Hauptmit allen untergeordneten Subkategorien beschrieben

3.1.5.1 Hauptkategorie Ersterfahrungen der BerufsanfängerInnen beim Berufseinstieg (BA_A) Die Hauptkategorie (Abb. 53) Ersterfahrungen der BerufsanfängerInnen beim Berufseinstieg (BA_A) wird in die Subkategorien Herausforderungen (BA_A_1), "Sprung ins kalte Wasser" (BA_A_2) (In-vivo $)^{58}$, Eigene Rolle (BA_A_3) und Lernen aus eigenen Erfahrungen (BA_A_4) unterteilt. Die Subkategorie Herausforderungen an die $B A\left(B A_{-} A_{-} 1\right)$ teilt sich in die Subsubkategorien Schock bei unmittelbarem Berufseinstieg (BA_A_1_1), Arbeitsüberlastung (BA_A_1_2) und „Keine Routine“(BA_A_1_3) (In-vivo).

57 Aufgrund von Überschneidungen entspricht diese Größe nicht der nummerischen Summe von n in der Spalte oberhalb.

58 In-vivo-Codes sind spezielle Redeweisen oder Bezeichnungen der Befragten, die in den Transkriptionen vorkommen und für das Feld charakteristische Phänomene darstellen. Deshalb können diese als Codebezeichnung wörtlich übernommen werden (Breuer et al. 2018; Kuckartz 2016). 


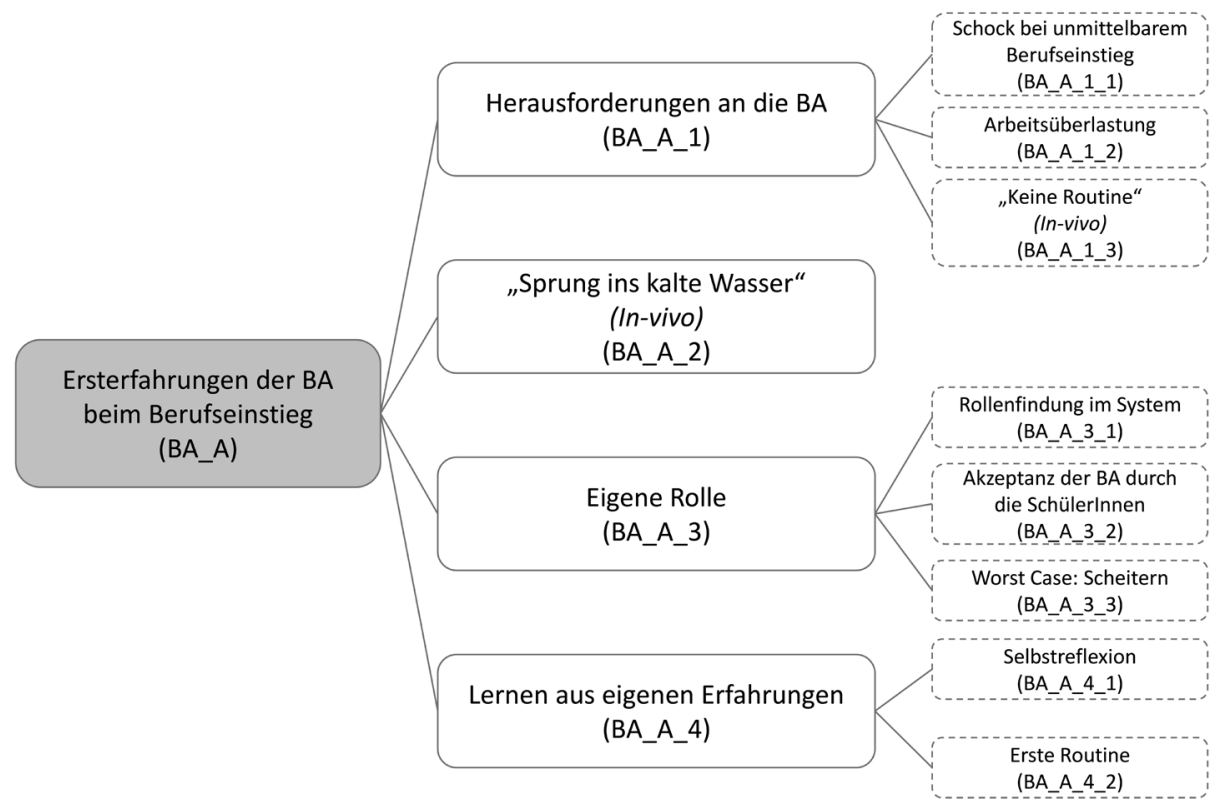

Abb. 53: Ersterfahrungen der BA beim Berufseinstieg; Haupt-, Sub- und Subsubkategorien

Die Subkategorie Eigene Rolle (BA_A_3) kann in die Subsubkategorien Rollenfindung im System (BA_A_3_1), Akzeptanz der BA durch die SchülerInnen (BA_A_3_2) und Worst Case: Scheitern (BA_A_3_3) differenziert werden. Schließlich werden aus der Subkategorie Lernen aus eigenen Erfahrungen ( $\left.B A \_A \_4\right)$ die Subsubkategorien Selbstreflexion (BA_A_4_1) und Erste Routine (BA_A_4_2) gebildet.

Die einzelnen Phänomene der genannten Subkategorie Herausforderungen an die BerufsanfängerInnen (BA_A_l) können mit den Subsubkategorien Schock bei unmittelbarem Berufseinstieg (BA_A_1_1), Arbeitsüberlastung (BA_A_1_2) und "Keine Routine“ (BA_A_1_3) (In-vivo) zusammengefasst werden (Abb. 53). „Also im ersten Jahr wollte ich es eigentlich wieder lassen. Weil ich mir gedacht habe, das ist einfach nicht mein Beruf“ (BA_15:21), beschreibt ein/e BerufsanfängerIn den Anfangsschock. Die große Unsicherheit bezüglich der richtigen Berufswahl wird auch mit der Aussage „[...] also ich war sehr lange im Zweifel, ist das jetzt der Beruf für mich oder nicht“" (BA_07: 71) ausgedrückt. Exemplarisch für den Schock beim unmittelbaren Berufseinstieg und den Umgang steht folgende Aussage:

„Ich habe keinen gekannt und dann habe ich mich schnell an das gewöhnen müssen und die Kollegen erst kennen lernen. Und dann, was am Anfang noch so viel war, es war so viel auf einmal. Dann kommt die Direktorin mit C-Topf und Zeug und Administration, das war einfach am Anfang irre viel, das zu bewältigen. Ja, so viele Eindrücke auf einmal und man hat halt mit so vielen Sachen gleichzeitig zu kämpfen gehabt oder halt, man hat das alles halt irgendwie schaffen müssen." (BA_17:25)

Der Unterschied zwischen dem Schock bei unmittelbarem Berufseinstieg (BA_A_1_1) und der Arbeitsüberlastung ( $\left.B A \_A \_1 \_2\right)$ besteht nach der Analyse der Interviews im Grad der Überforderung. So wird beim schockartigen Berufseinstieg die Überforderung bis hin zum möglichen 
Scheitern ${ }^{59}$ thematisiert, während die BerufsanfängerInnen die Arbeitsüberlastung zwar benennen, jedoch die Machbarkeit dieser Herausforderung meist zugleich artikulieren. „Du hast am Anfang sowieso viel zu tun, du brauchst ja dreimal so lang zum Hefte Korrigieren“ (BA_16: 87), sagt ein/e BerufsanfängerIn zum Arbeitsaufwand beim Berufseinstieg. Eine typische Beschreibung der Herausforderung zum Berufseinstieg inklusive Bewältigungsstrategie lautet folgendermaßen:

„Da ist man natürlich schon gleich einmal herangekommen, was man alles zu tun hat und es war ein Riesenberg. Aber den hat man abgearbeitet und es hat ganz gut geklappt. Zum Glück ist es in der Fächerverteilung auch letztes Jahr so ganz gut gegangen, dass ich eben die Unterstützung da gehabt habe und das dann ganz gut geschafft habe. Ich habe letztes Jahr noch nicht Mathematik unterrichtet als Schwerpunkt, sondern nur im Fachbereich Mathematik, so habe ich da ein bisschen weniger gehabt." (L_18:30)

In der Subsubkategorie „Keine Routine“ (BA_A_1_3) (In-vivo) schildert ein/e BerufsanfängerIn die Situation beim Berufseinstieg authentisch so:

„[...] und dann kommen halt einfach die ganzen Sachen dazu am Anfang, mit denen du nicht rechnest. Wo du noch keine Routine hast und nichts (3s) und nicht weißt, wie du in bestimmten Situationen reagieren sollst." (BA_16:31)

In diesem Fall wird „Keine Routine“ als In-vivo-Code übernommen, dessen Stärke in der schon erwähnten Authentizität und in der induktiven, sich aus dem Datenmaterial heraus generierenden Kategorienbildung liegt, was der Intention der Grounded Theory (Strauss und Corbin 1996) besonders nahe kommt.

Ein/e weitere/r Befragte/r hat die Desiderate in der Routine noch gut in Erinnerung: „Ich bin hineingeschmissen worden und habe oft nicht gewusst, wie und was ich tun soll vor der Klasse. Und da habe ich mir schon viel vermasselt." (BA_13: 76) Die Konsequenzen nach einem „vermasselten" Schulstart aufgrund der nicht vorhandenen Routine sind manchmal weitreichend. „Aber zumindest habe ich daraus gelernt und bin dann gleich von der Schule weggekommen“ (BA_19: 42), erzählt ein/e Befragte/r die persönliche Lösung dieses Problems.

Die Subkategorie „Sprung ins kalte Wasser“ (BA_A_2) (In-vivo) wird 21 Mal explizit genannt (bei $\mathrm{n}=25$ ). Da zumindest die Metapher des „kalten Wassers“ herangezogen wird, wird auch dieser Code als In-vivo-Code übernommen. Eine Schilderung dazu lautet folgendermaßen:

„Ich war gleich Klassenvorstand, ich hab Klassenbuch führen müssen, hab Schülerstammblätter schreiben müssen, ich hab wahnsinnig viel zu tun gehabt. Im Nachhinein war das nicht schlecht, weil es zum Lernen am besten war. Ich bin ins kalte Wasser geschmissen worden und hab dadurch viel gelernt. Ich hab zwar viel fragen müssen und so." (BA_11:28)

Ein/e andere/r BerufsanfängerIn spricht wörtlich vom „Sprung ins kalte Wasser“ (BA_14: 28) und „[ist] schon viel beim Nachdenken, beim Reflektieren“ (ebd.). Die Thematik des Reflektierens wird weiter unten in der Subkategorie $\left(B A_{-} A_{-} 4\right)$ Lernen aus eigenen Erfahrungen behandelt. Diese herausfordernde Ersterfahrung beim Berufseinstieg wird zwar mit der Konnotation einer Belastung erzählt, jedoch überwiegt am Ende das Positive, der Lerneffekt: „Ja, da weiß ich sehr viel, weil ich da ins kalte Wasser geworfen wurde“ (BA_18:27), wird etwa mitgeteilt. „Man steht da komplett alleine da, ich habe eine Klassenführung übernommen, das war schon ein Brocken muss ich sagen“" (BA_07: 35), sinniert ein/e BerufsanfängerIn, um sogleich etwas Positives

59 Scheitern als mögliches Szenario wird in der Kategorie (BA_A_3_3) Worst Case: Scheitern beschrieben. 
nachzulegen: „So viel Neues, also das Kollegium ist hundertprozentig hinter mir gestanden, aber auch die Eltern.“ (ebd.: 36)

Die Subkategorie Eigene Rolle (BA_A_3) kann in die Subsubkategorien Rollenfindung im System (BA_A_3_1), Akzeptanz der BA von den SchülerInnen (BA_A_3_2) und Worst Case: Scheitern (BA_3_3) unterteilt werden.

Den ersten Kontakt mit den LehrerkollegInnen auf dem Weg zur Rollenfindung im System (BA_A_3_1) schildert ein/e BerufsanfängerIn folgendermaßen:

„Man hat's als Junglehrer hin und wieder schwer (lacht). Wenn man als Junglehrer in das Lehrerteam hinein kommt, man ist halt doch der Neue, die anderen kennen sich schon und sind untereinander vertraut. Du kommst dann als Neuer dazu und sollst dich dann versuchen zu integrieren.“ (BA_11:23)

Als Präzisierung folgt: „[...] in etwas Bestehendes hineinkommen ist halt immer schwer.“ (ebd.: 24) Auf die konkretisierende Nachfrage kommt schließlich zur Antwort: „Weil es vielleicht so ein eingeschworenes Team war." (ebd.: 25) Einer/einem weiteren BerufsanfängerIn „[hat] also das ganze System [...] da einen kompletten Stress gemacht. Das ganze System einfach, ja.“ (BA_15:38) Einschneidende Erlebnisse schildern die BerufsanfängerInnen in Bezug auf die Eigene Rolle (BA_A_3), wenn die Subsubkategorie Akzeptanz der BA von den SchülerInnen (BA_A_3_2) thematisiert wird. Die Akzeptanz respektive die Nicht-Akzeptanz der BerufsanfängerInnen durch die SchülerInnen hängen laut den Aussagen der befragten BerufsanfängerInnen stark mit einem vorhergehenden LehrerInnenwechsel zusammen. So wird etwa in einem Interview erzählt:

„Ja, das war ja ein Drama, wie die alte Lehrerin gegangen ist und ich angefangen habe. Die (SchülerInnen, Anm.) haben ja geweint wegen der alten Lehrerin, das hat ja gedauert, bis sie mich akzeptiert haben als neue Lehreri“" (BA_04: 26)

Ein/e weitere/r BerufsanfängerIn führt aus: „[...] und dann merkst du zuerst einmal, die sind einen anderen Lehrer gewohnt und du machst es halt doch anders und da gibt es zuerst einmal Gegenwehr." (BA_16: 30) Die Herausforderung, bei der Übernahme einer Klasse als LehrerIn akzeptiert zu werden, wird neben dem Wechsel der Bezugsperson auch mit der Pubertät der SchülerInnen in Zusammenhang gebracht.

„Für mich war am Anfang das Problem, dass ich zwei dritte Klassen [Sek. I, Anm.] übernommen habe, die sind natürlich gerade mitten in der Pubertät. Da ist es dann verhaltenstechnisch schwierig für mich, mich da zu behaupten. [...] Also da tue ich mich recht schwer. Das Hauptproblem ist da, dass sie [die SchülerInnen, Anm.] mich als Nachfolger akzeptieren." (BA_10:21)

Weniger dramatisch, aber doch für die Relevanz der Bezugsperson bezeichnend, wird in einem letzten Zitat zu dieser Subsubkategorie festgehalten: „Für die Schüler war es komisch, weil sie eine andere Lehrerin gehabt haben und auf einmal stehe ich da." (BA_17:25)

In der Subsubkategorie Worst Case: Scheitern (BA_A_3_3) wird von den InterviewteilnehmerInnen die Thematik des Scheiterns im LehrerInnenberuf sowohl aus der Perspektive der Selbstwahrnehmung,als auch als Fremdwahrnehmung von Berufsanfänger-KollegInnen angesprochen. „Also ich hab's jetzt schon bei einem Lehrer mitbekommen, der bei uns [3 s Denkpause]. Ja es klingt schlimm, aber der ist einfach gescheitert“ (BA_01: 78), erzählt ein/e BerufsanfängerIn und präzisiert: 
„Der hat auch mit mir die Ausbildung gemacht und er hat mit uns auch immer schon seine eigene Art gehabt, also bei Referaten und so, dass ich mir gedacht habe ,wenn der mit Schülern so tut, dann glaube ich, dass sie [die SchülerInnen, Anm.] sich das ab einem gewissen Alter nicht mehr gefallen lassen." (ebd.)

Die Gedanken ans Aufhören, an einen Berufswechsel bzw. die Zweifel, ob die Berufswahlentscheidung die richtige ist, werden im Laufe der Interviewgespräche regelmäßig thematisiert. So hat sich ein/e InterviewpartnerIn „dann schon gedacht ,also wenn das immer so ist, dann weiß ich nicht, ob mir das gefällt auf Dauer?““ (BA_05: 36). Noch klarer formuliert ein/e weitere GesprächspartnerIn: „Letztes Jahr habe ich mir schon überlegt, ob ich nicht aufhören soll.“ (BA_13: 21) Und liefert einige Absätze später eine mögliche Erklärung.

„Sicher bin ich auch falsch hinein gegangen, einfach zu locker und nicht so streng wie ich hätte sein sollen. [...] ich habe zwischendrin Phasen gehabt, wo ich gesagt habe ,ich wechsle den Beruf, das schaff ich nicht mehr." (ebd.: 30-31)

Ein/e nächste/r BerufsanfängerIn formuliert die Überforderung sehr anschaulich und liefert auch gleich den Hilferuf nach:

„Die totale Überforderung. Das war ja auch der Punkt, wo ich gesagt habe, jetzt kann ich nicht mehr. Ich war mit dem überfordert, was ich zu tun hatte. Ich war mit dem überfordert, was ich nicht zu tun gehabt hätte, mir aber auferlegt worden ist. (2s) Ja. (3s) Aber ich war sicher überfordert, total. Und ich hab' das auch gesagt. Ich hab' zum Direktor gesagt, ich bin überfordert, ich brauche Hilfe irgendwo.“ (BA_15: 40-41)

Die erfahrenen Lehrpersonen werden in dieser Subsubkategorie als Stützen und Helfer ${ }^{60}$ erwähnt: „Die Kollegen waren mir letztes Jahr eine große Stütze. Wenn ich die nicht gehabt hätte, dann hätte ich es vielleicht hingeschmissen" (BA_17: 22), erinnert sich ein/e InterviewpartnerIn und spricht ebenso die Thematik des Scheiterns an.

In der Subkategorie Lernen aus eigenen Erfabrungen ( $B A_{-} A_{-} 4$ ) werden von den BerufsanfängerInnen erste reflexive Rückschlüsse formuliert. Die eigenen Erfahrungswerte werden analysiert und die Ergebnisse für das zukünftige LehrerInnenhandeln herangezogen. Folglich kann die Subkategorie Lernen aus eigenen Erfabrungen (BA_A_4) in die Subsubkategorien Selbstreflexion (BA_A_4_1) und Erste Routine (BA_A_4_2) unterteilt werden. So erzählt ein/e BerufsanfängerIn:

„[...] Erfahrungen habe ich viele gesammelt, da habe ich mir im ersten Jahr eigentlich ein dickes Fell zugelegt. Und das wahrscheinlich auch sein müssen. Also ich schaue da nicht negativ zurück." (BA_12:31)

Einblick in die eigene Reflexion zum Berufseinstieg gibt folgende Passage:

„Es sind ja schon fünf Monate. Ich bin beim Überlegen, was habe ich falsch gemacht, was muss ich nächstes Jahr anders machen' und so weiter und so fort." (BA_14: 28)

Ein/e weitere/r BerufsanfängerIn formuliert: „Ich hab immer versucht, und ich mach' das nach wie vor, mich zu reflektieren. Mich, meine Schüler, die Art und Weise, wie ich mit den Schülern umgehe." (BA_20: 30)

In Erste Routine (BA_A_4_2) äußert sich u.a. ein/e BerufsanfängerIn im dritten Unterrichtsjahr: „Man merkt schon, man ist routinierter, man kann mehr ausprobieren und man hat einen Vergleich zu den Jahren vorher." (BA_16: 24) „Ja, mir geht's jetzt ganz gut, muss ich sagen. Mir kommt vor, langsam kommt die Routine“ (BA_08: 21), erzählt ein/e BerufsanfängerIn explizit

60 Dieses Phänomen wird weiter unten u.a. in der Kategorie (BA_B) Mentoring aus der Sicht der BerufsanfängerInnen eingehend diskutiert. 
zur Routine. Den Routineprozess beschreibt diese/r BerufsanfängerIn folgendermaßen: „Ich sag' einmal so, es war immer eine Steigerung. Vom ersten Dienstjahr zum zweiten und dann vom zweiten zum dritten jetzt." (ebd.: 21)

Zusammenfassend kann festgestellt werden, dass sich die Ersterfahrungen der BerufsanfängerInnen ambivalent konstituieren. Beim unmittelbaren Berufseinstieg wird Schock, Arbeitsüberlastung, keine Routine bis hin zum Scheitern als Worst Case thematisiert. Den Sprung ins kalte Wasser formulieren beinahe alle Befragten, weshalb diese Metapher als In-vivo-Code übernommen wurde. Erfolge und Fortschritte in der Professionalisierung nehmen die BerufsanfängerInnen im Rahmen der Selbstwahrnehmung beim Lernen aus eigenen Erfahrungen, konkret durch die Selbstreflexion, wahr.

\subsubsection{Hauptkategorie Mentoring aus der Sicht der BerufsanfängerInnen (BA_B)}

In Abbildung 54 wird die Hauptkategorie Mentoring aus der Sicht der BerufsanfängerInnen $\left(B A \_B\right)$ dargestellt und subsummiert die Subkategorien Hilfe von $S L$ (BA_B_l) und Hilfe von KollegInnen (BA_B_2). Die Subkategorie Hilfe von SL (BA_B_1) kann in die Subsubkategorien "Rund-um"Hilfe von $S L$ (BA_B_1_1), Fragen zulassen (BA_B_1_2) und Administrative Hilfe von $S L$ (BA_B_1_3) differenziert werden. Die Subkategorie Hilfe von KollegInnen (BA_B_2) gliedert sich in die Subsubkategorien „Rund-um-Hilfe“von KollegInnen (BA_B_2_1), Tipps \& Gespräche (BA_B_2_2), Arbeitsmaterialien (BA_B_2_3), Administrative Hilfe von KollegInnen (BA_B_2_4) und Konkrete Themen (BA_B_2_5) (Abb. 54).

Für die BerufsanfängerInnen stellt es einen hohen Wert dar, wenn Hilfestellung gegeben wird, ohne dass explizit danach gefragt werden muss. Dies gilt für die Hilfestellung seitens der SchulleiterInnen gleichermaßen wie für die der LehrerkollegInnen. Deshalb werden die Subsubkategorien "Rund-um-Hilfe" von SL (BA_B_1_1) und „Rund-um-Hilfe" von KollegInnen (BA_B_2_1) in den beiden Subkategorien Hilfe von SchulleiterInnen (BA_B_1) und Hilfe von KollegInnen (BA_B_2) abgebildet. Der Unterschied zwischen diesen beiden Subsubkategorien besteht darin, dass in "Rund-um-Hilfe“von SL (BA_B_1_1) SchulleiterInnen und KollegInnen gemeinsam genannt werden, in der Kategorie „Rund um-Hilfe“ von KollegInnen (BA_B_2_1) wird jedoch explizit die Hilfestellung seitens der KollegInnen thematisiert.

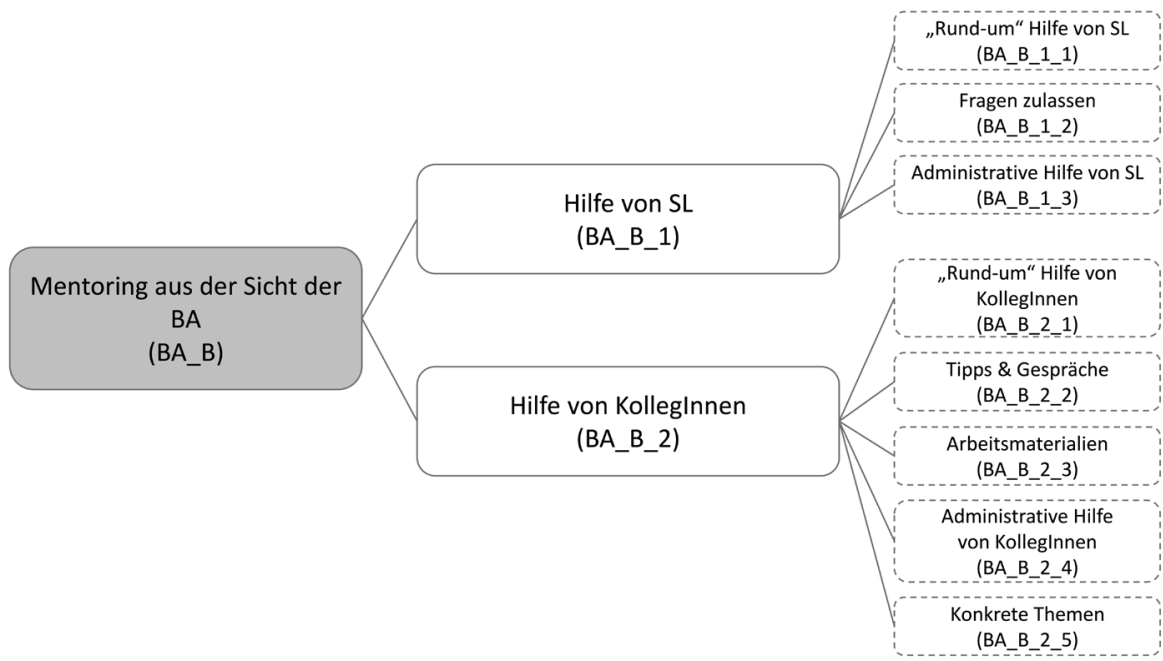

Abb. 54: Mentoring aus der Sicht der BA; Haupt-, Sub- und Subsubkategorien 
Insofern kann konstatiert werden, dass im Bereich des Mentorings aus der Sicht der BerufsanfängerInnen die SchulleiterInnen-Hilfe eine Teilmenge der KollegInnen-Hilfe darstellt. Deshalb werden an dieser Stelle die Subsubkategorien „Rund-um-Hilfe von SL (BA_B_1_1) und „Rund-um-Hilfe“ von KollegInnen (BA_B_2_1) direkt aufeinanderfolgend beschrieben. Anschließend wird wieder dem Prinzip der fortlaufenden Beschreibung jeder Subsubkategorie innerhalb einer Subkategorie nach Abbildung 54 Folge geleistet.

Eine stellvertretende Aussage für die Subsubkategorie „Rund-um-Hilfe “von SL (BA_B_1_1) beschreibt den „Rund-um“-Charakter und lautet folgendermaßen: „Wenn ich etwas brauche, kann ich fragen. Beim Direktor oder bei den Kollegen.“ (BA_14: 27) Die Aussagen „Sicher hat man Hilfe durch den Direktor und die älteren Kollegen“ (BA_03: 57) und „Mit den Kolleginnen und der Direktorin ist es ein perfektes System. [...] Kollegenmäßig und auch von der Direktorin hat man volle Unterstützung" (BA_18: 23) stellen ebenfalls die allgemeine Hilfe seitens der Schulleiterin bzw. des Schulleiters und der KollegInnen als gleichwertige MentoringPartnerInnen in den Vordergrund.

In „Rund-um-Hilfe“von KollegInnen (BA_B_2_1) wird nun explizit von KollegInnen als MentorInnen gesprochen: „Ja, von den Kollegen her passt es ganz gut. Da bekomme ich Unterstützung von allen Seiten, gerade weil ich die Dienstjüngste bin“ (BA_07: 23) und „[...] von den Kollegen her, muss ich sagen, die waren total hilfsbereit“ (BA_08: 30) lauten Aussagen von zwei BerufsanfängerInnen. Die „Rund-um-Hilfe“ bringt ein/e weitere/r BerufsanfängerIn folgendermaßen zum Ausdruck: „Sie [die KollegInnen, Anm.] sind mir von Anfang an überall entgegen gekommen, ich habe alles fragen können." (BA_20:25) Als ebenso hilfsbereit schildert ein/e BerufsanfängerIn ihre KollegInnen, diesmal ist die „Rund-um-Hilfe“ auf die Unabhängigkeit der Unterrichtsfächer bezogen. „Sie [die KollegInnen, Anm.] sind total freundlich und hilfsbereit, ich kann zu jedem hingehen. Ich habe bei allen Fächern, auch prüfungsfremden Fächern, Unterstützung.“ (BA_19:25)

In der Subsubkategorie Fragen zulassen (BA_B_1_2) in Hilfe von $S L$ (BA_B_1) wird thematisiert, dass sich die BerufsanfängerInnen im Gegensatz zur „Rund-um-Hilfe“ von $S L$ (BA_B_1_1) zum Teil auch nicht oder missverstanden fühlen, wenn sie mit Fragen an die SchulleiterInnen herantreten. „[...] man [hat] der Direktorin oft so blöde Fragen gestellt, weil man sich ja selber nicht auskennt. Und sie antwortet dann quasi so ,was fragst du denn so blöd?"“ (BA_11: 25), wird zum Beispiel erzählt. Ein/e weitere/r BerufsanfängerIn erinnert sich an eine Kontaktaufnahme mit dem/der SchulleiterIn mit einem scheinbar leicht lösbaren Anliegen und die darauf folgende Antwort: „[...] und du bekommst dann halt vom Direktor gesagt ,du hast ja die Matura. (lacht)“ (BA_15:37)

In der Subsubkategorie Administrative Hilfe von SL (BA_B_1_3) werden die SchulleiterInnen von den BerufsanfängerInnen als wesentliche Stütze in administrativen Belangen dargestellt. Hier wird als Begründung vorrangig das Desiderat der administrativen Ausbildung ${ }^{61}$ im Curriculum angesprochen. „[...] weil wir jetzt von dem ganzen Organisatorischen, also von der Schule her, nicht so viel gemacht haben. Allein diese C-Topf-Stunden ${ }^{62}$. Ich habe schon gewusst, dass es den C-Topf gibt, aber was das genau ist? (3s) Da habe ich dann Gott sei Dank die Hilfe des Direktors der Stammschule bekommen, der hat das gemanagt." (BA_12:34)

61 Vgl. hierzu die Analyse der Subsubkategorie (BA_C_4_I) Administratives Wissen (Kap. III.3.1.5.3).

62 Im Dienst- und Besoldungsrecht „Alt“ für Landesvertragslehrpersonen sind im Bereich C (C-Topf) sonstige lehramtliche Pflichten - neben der Unterrichtsvor- und -nachbereitung - zu erfüllen (Abteilung Bildung des Landes Tirol 2015). 
In der Subsubkategorie Tipps \& Gespräche (BA_B_2_2) innerhalb von Hilfe der KollegInnen (BA_B_2) wird angesprochen, dass KollegInnen, auch ohne explizit danach gefragt zu werden, Hilfestellung anbieten und so gewissermaßen prophylaktisch Fehlern vorbeugen. Ein/e BerufsanfängerIn erzählt:

„Ja schon einmal von vorne herein, man hat mir gleich erklärt, wie es abläuft. Grad vorweggenommen, was ich falsch machen könnte, wegen Aufsicht und sonstiges." (BA_07: 38)

Die Gesprächs- und Hilfsbereitschaft bringt ein/e InterviewpartnerIn folgendermaßen zum Ausdruck: „Ich kann ihn [[den Kollegen, Anm.]) am Tag fünfmal anrufen und es ist kein Problem." (BA_12: 26) Im folgenden Zitat wird geschildert, dass Mentoring manchmal auch Verstehen und Beruhigen bedeutet:

„Und wenn ich einmal ganz verzweifelt war, dann haben die Kollegen schon gesagt, nein, passt schon und das geht jedem so, denk dir nichts dabei. Sie haben mir gute Tipps gegeben und haben mir wahnsinnig geholfen." (BA_17: 28)

Ein/e weitere/r BerufsanfängerIn hat mit den KollegInnen „ganz viel geredet“ (BA_15: 45) und verdeutlicht, dass allein zuhören und Verständnis zeigen schon eine Hilfe darstellt: „[...] die haben mir geholfen, indem sie gesagt haben ,he, wir waren alle im ersten Jahr überfordert, das ist normal'“ (ebd.)

In Arbeitsmaterialien ( $B A \_B \_2 \_3$ ) betonen die BerufsanfängerInnen konkrete Hilfestellungen der KollegInnen in Form von Bereitstellung von Unterrichtsmaterialien und anderen Aufzeichnungen zum Unterricht. Ein/e BerufsanfängerIn erzählt:

„Also die Kollegen in der Schule sind sicher so, dass sie mich total unterstützen. Das ist zum Beispiel so, dass sie mir einfach Arbeitsblätter herlegen, ohne dass ich etwas sage und so.“ (BA_04: 23)

Ein/e weitere/r InterviewpartnerIn erinnert sich ebenfalls an diese Art von Hilfe: „[...] und sie [die KollegInnen, Anm.] haben mir auch geholfen mit Arbeitsblättern, so auf dieser Schiene." (BA_15:45) Auch im Bereich des Briefings in Bezug auf SchülerInnenverhalten wissen BerufsanfängerInnen die KollegInnen zu schätzen:

„Die Lehrerin, die vorher da war, ist auch noch gekommen und hat mir ihre Aufzeichnungen gegeben und gesagt ,bei dem und dem (Schüler, Anm.) musst du schauen, die und die und die (Schülerin, Anm.) ist so und so."(BA_05:34)

In der Subsubkategorie Administrative Hilfe der KollegInnen (BA_B_2_4) wird eindeutig kommuniziert, dass BerufsanfängerInnen auf die Hilfe der KollegInnen angewiesen sind. „Die ganzen Sachen [Administration, Anm.] habe ich erst durch die Kollegen bekommen [...]“ (BA_16:43), lautet exemplarisch eine Aussage dazu. „[... ] also schöner kann man es nicht haben“, schwärmt ein/e weitere/r BerufsanfängerIn, „dass man die Unterstützung jederzeit bekommt [...] und mir macht es Spaß, mit diesem Team zu arbeiten." (BA_18:24)

In der Subsubkategorie Konkrete Themen ( $\left.B A \_B \_2 \_5\right)$ werden auf Nachfrage beim Interview inhaltlich drei Bereiche angesprochen. Insofern handelt es sich um eine deduktive Subsubkategorie. Die BerufsanfängerInnen benötigen Mentoring im Bereich ihres Unterrichtsfachs bzw. ihrer Unterrichtsfächer, im „zwischenmenschlichen Umgang“ mit den SchülerInnen (BA_01: 49) und auf der bürokratischen Ebene. In Bezug auf die Unterrichtsfächer ,ist es fein, dass mehrere [KollegInnen, Anm.] dein Fach haben. Da kannst du dich ein bisschen austauschen“ (BA_08: 24), 
erzählt ein/e BerufsanfängerIn. Während einer/einem anderen „hin und wieder ein Kollege [hilft]“ (BA_12: 26). Sehr plakativ beschreibt ein/e InterviewpartnerIn den Berufseinstieg bzgl. bürokratischer Aufgaben: „Du kommst hinein [in die Schule, Anm.] und hast vom Tuten und Blasen keine Ahnung." (BA_10: 83)

Als Resümee wird festgehalten, dass BerufsanfängerInnen Hilfe von SchulleiterInnen und KollegInnen benötigen, angeboten bekommen und diese auch in Anspruch nehmen. Die „Rundum-Hilfe" unterscheidet sich insofern, als BerufsanfängerInnen die SchulleiterInnen aktiv darum bitten, die KollegInnen bieten diese an. Während im Bereich der Administrationstätigkeiten SchulleiterInnen und KollegInnen gleichermaßen hilfsbereit sind, reagieren SchulleiterInnen auf Fragen von BerufsanfängerInnen im Unterschied zu KollegInnen manchmal weniger verständnisvoll. Hilfe im Sinne des Mentorings bekommen BerufsanfängerInnen vorrangig von KollegInnen, was dem angestrebten Mentoring-Prozess im Rahmen der Induktionsphase nahe kommt (Raufelder und Ittel 2012; Schmid und Haasen 2011). Auch bei konkreten Themen, wie z.B. im Umgang mit verschiedenem Schülerverhalten, benötigen und erhalten BerufsanfängerInnen Hilfe im Sinne eines informellen Mentorings von erfahrenen KollegInnen.

3.1.5.3 Hauptkategorie Institutionelle Ausbildung aus der Sicht der BerufsanfängerInnen (BA_C) Die Hauptkategorie (Abb. 55) Institutionelle Ausbildung aus der Sicht der BerufsanfängerInnen $\left(B A_{-} C\right)$ wird in die Subkategorien Organisation der Ausbildung (BA_C_1), Selektion (BA_C_2), Curriculum (BA_C_3) und Desiderate \& Anwendbares Wissen (BA_C_4) unterteilt. Die Subkategorie Organisation der Ausbildung (BA_C_1) kann in die Subsubkategorien Bürokratie (BA_C_1_1) und Kommunikation ( $B A_{-} C_{-}{ }_{-}$2) differenziert werden, die Subkategorie Selektion (BA_C_2) in die Subsubkategorien Berufseignungsfeststellung (BA_C_2_1) und Notenwahrheit (BA_C_2_2). Subkategorie Curriculum (BA_C_3) wird in DozentInnen (BA_C_3_1), Fachausbildung (BA_C_3_2), Fachdidaktik (BA_C_3_3), Bildungswissenschaften (BA_C_3_4) und Praktika (BA_C_3_5) gegliedert.

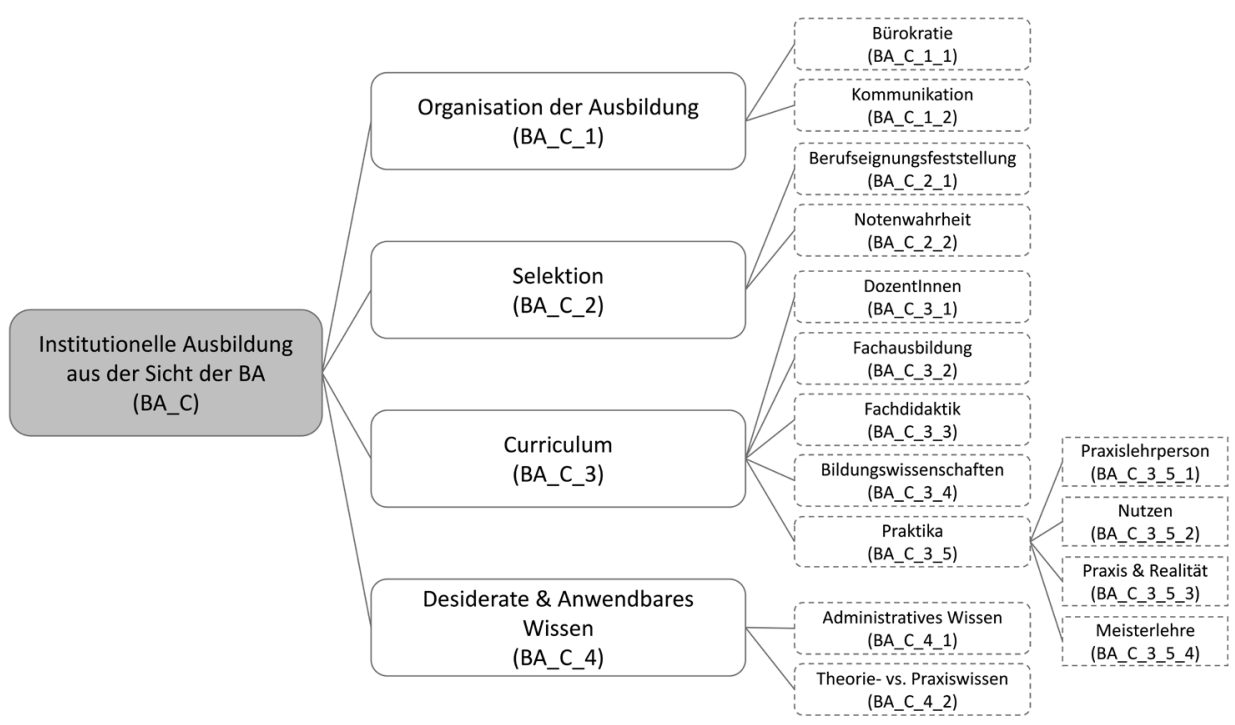

Abb. 55: Institutionelle Ausbildung aus der Sicht der BA; Haupt-, Sub-, Subsub- und Subsubsubkategorien 
Die Subsubkategorie Praktika (BA_C_3_5) können zusätzlich analysiert werden, und zwar in den Subsubsubkategorien Praxislehrperson (BA_C_3_5_1), Nutzen (BA_C_3_5_2), Praxis \& Realität (BA_C_3_5_3) und Meisterlehre (BA_C_3_5_4). Schließlich wird die Subkategorie Desiderate \& Anwendbares Wissen (BA_C_4) in die Subsubkategorien Administratives Wissen (BA_C_4_1) und Theorie-vs. Praxiswissen (BA_C_4_2) aufgeschlüsselt

Innerhalb der Subkategorie Organisation der Ausbildung ( $\left.B A_{-} C_{-} 1\right)$ werden von den BerufsanfängerInnen zwei Bereiche thematisiert: Bürokratie (BA_C_1_1) und Kommunikation (BA_C_1_2). „Die wahnsinnige Bürokratie, [...] bis man die einmal heraus gehabt hat, war's halt anstrengend" (BA_17: 54f), erinnert sich ein/e BerufsanfängerIn. Auch in Bezug auf Anerkennung von Vorstudien sehen sich die BerufsanfängerInnen bürokratischen Hürden ausgesetzt. „Ob das [Vorstudien, Anm.] jetzt an der Pädagogischen Hochschule in Klagenfurt passiert, ob das jetzt an der UNI Wien passiert, [...] dann muss das einheitlich anerkannt sein“ (BA_18: 50), meint ein/e weitere/r BerufsanfängerIn.

Ein Seitenthema innerhalb dieser Subsubkategorie Bürokratie (BA_C_1_1) ist die Planung des Curriculums. Die „zu vielen Stunden [...] war[en] eine der größten Hürden [...]“ (BA_14: 81), erinnert sich ein/e Befragte/r.

In Kommunikation ( $B A_{-} C_{-}{ }_{1}$ 2) erzählt ein/e BerufsanfängerIn vom Studienbeginn: „Ja für Einsteiger, wenn sich eh niemand auskennt, [...] ist niemand herum.“ (BA_10:75) „Also das [die Kommunikation, Anm.] war mühselig“ (BA_16: 67), erinnert sich ein/e weitere/r Befragte/r. Zum Thema Berufseignungsfeststellung in der Subsubkategorie Berufseignungsfeststellung zu Ausbildungsbeginn ( $\left.B A_{-} C_{-} 2 \_1\right)$ in der Subkategorie Selektion (BA_C_2) nimmt ein/e BerufsanfängerIn wie folgt Stellung: „Ich würde am Anfang jedenfalls einen Aufnahmetest machen.“ (BA_12: 39) „[...] weil das war erschreckend, wie viele [KommilitonInnen, Anm.] ich kennen gelernt habe, denen ich meine Kinder nicht anvertrauen würde“ (ebd.), wird noch präzisiert. „Und was mich am meisten gestört hat“, gibt ein/e andere/r BerufsanfängerIn zu Protokoll, „dass nicht wirklich herausgefiltert worden ist, wer ist jetzt geeignet und wer nicht.“ (BA_11: 54) Ein/e weitere/r Interviewte/r bring eine Berufseignungsfeststellung ins Spiel: „Dann müsste es irgendetwas geben, [...] ob jemand für den Beruf geeignet ist." (BA_16: 46) Eine des Öfteren kommunizierte Idee geht in Richtung einer längeren Studienbeobachtungs- bzw. -einstiegsphase. „[...] eher so, dass es vielleicht über eine Woche geht oder ein Probemonat. Wo man wirklich schaut, ,ist derjenige geeignet, in dieser Sparte zu arbeiten?'“' (BA_12: 39)

In der Subsubkategorie Notenwahrheit (BA_C_2_2) werden die Schilderungen der befragten Personen teilweise emotional. „Diese Sachen bewegen mich immer noch“ (BA_03: 87), heißt es etwa. Ein/e weitere/r BerufsanfängerIn wundert sich: „Und es kann ja auch nicht sein, dass ich im Gymnasium [...] mit acht Vierern die achte Klasse abschließe und dann [...] einen Notendurchschnitt von 1,8 habe.“ (BA_14: 74) In dieser Analysekategorie wird generell der Leistungsanspruch im Lehramtsstudium thematisiert.

„Das hat mich gestört“, erzählt ein/e BerufsanfängerIn, „dass da jeder hingehen kann. Egal ob mit Matura oder mit Studienberechtigungsprüfung. Wir haben einen dabei gehabt, der hatte vorher einen Beruf, den du überhaupt nicht mit dem Lehrerberuf in Verbindung bringst. Der ist jetzt fertig und ist jetzt Lehrer." (BA_12: 40)

In Bezug auf strengere Notengebung im Bereich der Bildungswissenschaften wird ein/e InterviewpartnerIn ganz deutlich:

„Dann das Notensystem in manchen Fächern. Es gibt nur Einser und Zweier. Dreier, wenn du einen Dreier hast, bist du eh schon die Oberflasche, unter Anführungszeichen." (BA_16: 45) 
Allerdings wird dieses Thema auch relativiert: „[...] es hat schon einige Professoren gegeben, die das [die Notengebung, Anm.] ernster genommen haben“ (BA_20: 42), erinnert sich ein/e Befragte/r. In der Subsubkategorie Dozentinnen (BA_C_3_1) innerhalb der Subkategorie Curriculum (BA_C_3) spielt die Vorbildwirkung eine zentrale Rolle. Neben kritischen Stimmen wie „[...] weil halt er kein Lehrer in dem Sinne ist, sondern halt ein Fachwissenschaftler“ (BA_16: 65) oder „Ja ich würde davon weggehen, dass man die Studenten viel arbeiten lässt und dass man einfach mehr bekommt, mehr Information“ (BA_08: 41) wird ebenso das Positive erwähnt: „Ich meine, es hat auch Professoren gegeben, die haben sich total ins Zeug gelegt [...].“ (BA_19: 81)

In Fachausbildung (BA_C_3_2) wird oft das praktikable, anwendbare Wissen erwähnt: „[...] wir [haben] viele Bücher vorgestellt bekommen, viele Lernbücher, was auch super gewesen ist“ (BA_13: 68), heißt es beispielsweise. Andererseits „haben wir [in der Fachwissenschaft] sinnlose Sachen gemacht, die man in der Schule nie anwenden kann" (BA_14: 36), erinnert sich ein/e Befragte/r. An anderer Stelle heißt es:

„Für mich persönlich hat es nichts gebracht, weil es zu hoch war (3 s Denkpause), für mein mathematisches Interesse. Da gibt es nichts, was ich für den Unterricht gebrauchen kann. Da müsste sich was ändern." (BA_16:51)

Im Gegensatz dazu meint wiederum ein/e andere/r Befragte/r:

„Also fachwissenschaftlich war es in meinen Augen okay. Da müssen einfach ein paar Exzesse gemacht werden, die man nicht umsetzen kann an der Schule. Dafür ist es eine Fachwissenschaft und nicht irgendwie ein Unterricht." (BA_18: 55)

Zur Fachkompetenz im eigenen Unterricht erinnert sich ein/e InterviewpartnerIn: „[...] in den Fächern, wo ich geprüft war, da habe ich die fachliche Sicherheit mitgebracht." (BA_16: 37) Ein häufig angesprochenes Thema, welches von den befragten BerufsanfängerInnen im Zusammenhang mit der Fachausbildung genannt wird, ist das große Feld der Leistungsbeurteilung. Die Zusammenstellung von Tests und Schularbeiten, die Erstellung von Notenschlüsseln, die Notengebung allgemein bis hin zu rechtlichen Vorgaben in Bezug auf die Leistungsbeurteilung werden hier genannt. Exemplarisch stelle ich eine Aussage einer jungen Lehrerin/eines jungen Lehrers im dritten Dienstjahr vor:

„Es ist auch Notengebung so ein Thema, das immer im Schwarzen liegt. Ich meine, ich bin jetzt im dritten Dienstjahr und weiß immer noch nicht, bin ich zu streng oder bin ich zu locker? Ich weiß nicht, hat man das nur am Anfang oder hat man das eh immer. Ich hab’s immer noch.“ (BA_15: 94)

In der Subsubkategorie Fachdidaktik (BA_C_3_3) sehen viele InterviewpartnerInnen die Herausforderung, diese „ein bisschen realistischer“ (BA_04: 68) zu gestalten, und sprechen damit die Orientierung am schulischen und nicht am universitären Unterricht an: „Die Ideen sind ja gut, aber es geht einfach realistisch gesehen schwer. Aber trotzdem finde ich gut, dass man es macht“ (ebd.: 69), fährt die/der Befragte fort und relativiert die anfängliche Kritik. „Von der Didaktik her, ja das stimmt, da nimmt man [...] sicher einiges unbewusst auf. Wenn man das nicht gehabt hätte, würde einem sicher was fehlen“ (BA_01: 60), erinnert sich ein/e BerufsanfängerIn.

Im Bereich der Bildungswissenschaften (BA_C_3_4) liegen die Erwartungen hoch, „weil da steckt ja die Essenz eigentlich drinnen“ (BA_20: 44). „Ich würde die ganzen Bildungswissenschaften [...] viel mehr auf den Schüler bezogen aufbauen“" (BA_11: 39), meint ein/e InterviewpartnerIn und lenkt das Thema in Richtung Anwendbarkeit im Unterricht. „Vielleicht unterschätzt man dieses Wissen auch?“, stellt ein/e BerufsanfängerIn den studentischen Zugang zu 
den Bildungswissenschaften in Frage und fährt fort: „Vielleicht ist man sich gar nicht bewusst, dass man es vorher nicht gewusst hat? Kann schon sein." (BA_02: 87)

Die Aussagen innerhalb der Subsubkategorie Praktika (BA_C_3_5) sind thematisch so weitreichend, dass eine nochmalige innere Gliederung auf der Ebene der Subsubsubkategorien ${ }^{63}$ notwendig ist. Die Subsubkategorie Praktika (BA_C_3_5) ist die einzige Kategorie in der vorliegenden Untersuchung, die bis zur Ebene der Subsubsubkategorien differenziert wird. Das kann als Hinweis für eine hohe thematische Relevanz gesehen werden.

Die Kernaussage in der Subsubsubkategorie Praxislehrperson (BA_C_3_5_1) lautet, dass der Erfolg der Studierenden in den Praktika auch von den beteiligten Praxislehrpersonen abhängt. Die Meinungen der BerufsanfängerInnen sind ambivalent. Ein/e InterviewpartnerIn hat „tolle Betreuungslehrer ${ }^{64}$ gehabt und [hat] die Qualität und den Standard der Praxis als recht hoch empfunden“ (BA_20: 49). Ein/e weitere/r BerufsanfängerIn erinnert sich, dass „die [Praxislehrpersonen, Anm.] einfach [sagen], was man besser machen kann, wie man mit den Schülern noch anders umgehen kann. Das [...] ist sehr gut, weil ein offenes Gespräch immer fördernd ist.“ (BA_18: 63) „Aber es gibt auch Betreuer“, fährt der/die GesprächspartnerIn fort, „die nicht für den Betreuerjob geschaffen sind und eigentlich nicht auf Studenten losgelassen werden sollten." (ebd.: 64) Eine weitere kritische Stimme meint: „Also die Auswahl der Betreuungslehrer müsste wirklich besser sein, dass kompetente Lehrer ausgewählt werden.“ (BA_16: 57) Die Herausforderung für Studierende in den Pädagogisch-praktischen Studien liege darin, „das umzusetzen, was sie ([die Praxislehrpersonen, Anm.] [wollen]“ (BA_01: 49), denn „das ist halt auch so subjektiv“" (B_07: 74), wird als Begründung angegeben.

In Nutzen (BA_C_3_5_2) wird artikuliert, dass „die Praxis [...] sicher etwas vom Wichtigsten“ (BA_04: 35) innerhalb der PädagogInnenausbildung darstellt. „In der Praxis merkt jeder Student: ,Ist das mein Beruf oder nicht? ‘“ (BA_06: 54), geht eine Erklärung in Richtung Berufsorientierung resp. -eignung. „[...] man sieht es von Anfang an, ob man hineinpasst oder nicht“ (BA_08: 51), ist ein/e weitere/r BerufsanfängerIn der gleichen Meinung. Bezüglich der Kompetenzsteigerung und Berufsvorbereitung weiß ein/e GesprächspartnerIn „[...] und natürlich [hilft] die Praxis, dass man da, das schätze ich also oft sehr, dass man irgendetwas schon in Vorbereitung hat." (BA_03: 45) Nicht zuletzt deshalb ,ist man in der Praxis immer top motiviert gewesen“ (BA_12: 74), lautet ein positives Abschlussstatement in diesem Bereich.

Die Subsubsubkategorie Praxis \& Realität (BA_C_3_5_3) wird kontrovers diskutiert. Einerseits sind BerufsanfängerInnen der Meinung, dass in den pädagogisch-praktischen Studien ein realer Blick ins Schulleben gewährt wird, auf der anderen Seite sind sich Befragte allerdings sicher, dass den PraktikantInnen die Schulrealität verwehrt bleibt. So erzählt ein/e BerufsanfängerIn:

„Da habe ich viel selbständig arbeiten können und eine Woche war meine Praxislehrerin krank, da war ich die meiste Zeit alleine. Das war nicht einfach, aber das war sehr interessant und hilfreich auch. Weil man sieht, was man so machen kann. Also es wäre jedenfalls wichtig, dass die Praxislehrer auch einmal hinaus aus der Klasse gehen und den Studenten allein lassen." (BA_08: 52)

Und sie/er liefert auch gleich ein mögliches Konzept für ein realitätsnahes Praktikum. Studierende sollten auch ohne Praxislehrperson unterrichten.

63 Vgl. zur Übersicht Kap. III.3.1.5.3 bzw. die Vielwegbaumgrafik (BA_C) Institutionelle Ausbildung aus der Sicht der BerufsanfängerInnen (Abb. 55).

64 Studierende und Lehrpersonen sprechen auch von Betreuungslehrpersonen oder Besuchsschullehrpersonen. Im Curriculum (Leopold-Franzens-Universität Innsbruck 2016) im Rahmen der pädagogisch-praktischen Studien und in der Kommunikation zwischen den Ausbildungsinstitutionen und den Praxisschulen (z.B. im Praktikumspass) wurde der Terminus Praxislehrperson (PLP) eingeführt. 
„Ich habe [...] den kompletten Stundenplan von meiner Lehrerin übernommen. [...] Jetzt habe ich da 21 Stunden gehabt, zwei Wochen lang. Das war ,zach' ${ }^{65}$, aber ich hab' gewusst, wie es abläuft." (BA_10:54-55)

So erzählt ein/e BerufsanfängerIn vom Praktikum und streicht damit den realistischen Praxisbezug hervor. „Also das ist so das Negativste, das ich da mitbekommen habe. Dass die Realität einfach fehlt, dass nie gesagt wird ,horch her, die Realität sieht anders aus “" (BA_15: 85), erinnert sich, ganz im Gegensatz zu den obigen Erzählungen, ein/e InterviewpartnerIn. An anderer Stelle heißt es: „Bei den Praxisstunden macht man halt immer eine Show, ein Thema einzuführen und da hat man einfach nicht mehr Zeit dafür.“ (BA_04: 70) Auch die Praxislehrpersonen selbst werden angesprochen, denn „die [Praxislehrpersonen, Anm.] machen für die Praxisstunden weiß Gott was für Sachen und der normale Unterricht läuft aber ganz anders ab. Das weiß ich und das sagen auch viele andere." (BA_10: 52) Abschließend zu Praxis \& Realität (BA_C_3_5_3) wird noch eine hilfreiche Maßnahme in Bezug auf die realistische Berufsvorbereitung erwähnt, die Absolvierung des Praktikums in sogenannten „schwierigen“ Klassen.

„Also das hat mir sicher am meisten gebracht [...]. Ich war in einer ganz schwierigen Klasse [...]. Ich habe mich jetzt nie vor einer Klasse gefürchtet und das habe ich sicher von der Praxis her." (BA_12: 35)

In der Subsubsubkategorie Meisterlehre (BA_C_3_5_4) thematisieren die BerufsanfängerInnen das Konzept der Meisterlehre, wonach „die Lehrerausbildung wie eine Lehre ablaufen würde“ (BA_15: 55). Ein/e Befragte/r ,würde das Ganze also auf die Praxis aufbauen und nicht auf die Theorie. Weil [...] das Ganze in der Praxis wesentlich einsichtiger war, als in der Theorie durchzukauen." (BA_11: 38) Darunter werden auch „Gespräche mit Lehrern“ (BA_17: 35) verstanden. „Also richtig offizielle Gespräche mit Lehrern während der Ausbildung“, wird noch weiter präzisiert, „dass man richtige Praxisberichte hört." (ebd.) Eine weitere Idee eines/einer Befragten zum Ausbildungsmodus ist: „Praxis und dann drei Monate Studium oder so." (BA_15: 57)

Die Subkategorie Desiderate \& Anwendbares Wissen (BA_C_4) in Institutionelle Ausbildung aus der Sicht der BA $\left(B A_{-} C\right)$ bildet sich aufgrund der Interviewfrage "Was hat dir/Ihnen beim Einstieg in den Lehrerberuf am meisten bzw. besonders gefehlt, was hättest du/hätten Sie noch gebraucht, was hätte dir/Ihnen den Berufseinstieg erleichtert?"zum einen als deduktive Kategorie (Desiderate). Zusätzlich wird das anwendbare, praktikable Wissen auch im Sinne der Unterscheidung zwischen Theorie- und Praxiswissen genannt und insofern als induktiver Teil (Anwendbares Wissen) in der vorliegenden Subkategorie gemeinsam mit den Desideraten abgebildet. Einen thematischen Schwerpunkt bildet die Subsubkategorie Administratives Wissen (BA_C_4_1). Hier werden laut den befragten BerufsanfängerInnen große Desiderate in der LehrerInnenausbildung wahrgenommen. „Also die bürokratischen Sachen [...], das haben wir nie gelernt und gemacht und so. Und dann war das halt einfach alles neu und alles auf einmal“ (BA_05: 37), wird beispielsweise erzählt. „Klassenbücher oder so“, konkretisiert ein/e InterviewpartnerIn, „sollte man auch einmal sehen während der Ausbildung. Das habe ich nämlich noch nie gesehen vorher, das wäre auch ganz interessant gewesen.“ (BA_08: 57) Ein/e GesprächspartnerIn relativiert und meint: „[...] gewisse Sachen kann die Ausbildung auch nicht übernehmen. Alles geht nicht. Aber es wäre schon ganz toll, wenn man das Organisatorische [...] hören würde." (BA_16: 86)

In der Subsubkategorie Theorie-vs. Praxiswissen (BA_C_4_2) äußern die Befragten, „dass eigentlich viel Theorie war und es oft wenig damit zu tun hatte, was in der Praxis wichtig ist“ (BA_07: 45). Die folgende Aussage steht stellvertretend für die durchgängige Meinung:

65 Mundart; zäh, schwierig 
„Ich glaube die Nähe zur Praxis wäre nicht schlecht, wenn sie mehr gemacht würde. Das heißt, die Fachwissenschaft hat schon ihren Stellenwert aber die praktische Seite muss im zeitlichen Plan noch mehr berücksichtigt werden." (BA_18: 72)

Bezüglich des Praxiswissens werden einerseits „die Arbeitsmaterialien [...] und viele didaktische Materialien“ (BA_09: 39) genannt, die beim Berufseinstieg „sehr weitergeholfen“ (ebd.) hätten. Andererseits werden ganz konkrete Maßnahmen zur Einhaltung der Disziplin in der Klasse genannt. Aufgrund der Emotionalität und der plakativen Schilderung eines scheinbaren Desiderats wird das folgende Zitat trotz unpassender Terminologie ausgewählt:

„Wie gehe ich mit einem Schüler um, der aufsteht und sagt: ,Leck' mich am Arsch. Was mache ich dann? Das lernt man nicht. Es wird eine Streichelpädagogik vermittelt, die eigentlich nicht geht, die einfach nicht möglich ist. [...] es heißt zwar immer praxisnahe Ausbildung, sie ist aber total praxisfremd“" (BA_14: 40f)

Als Zusammenfassung der größten (bezogen auf die Kategorienanzahl) Hauptkategorie Institutionelle Ausbildung aus der Sicht der BA (BA_C) wird rekapituliert, dass hier die BerufsanfängerInnen viele Wünsche bzw. negativ konnotierte Wortmeldungen abgeben. So sei die Bürokratie in der Ausbildungsinstitution Pädagogische Hochschule zu kompliziert und unflexibel, die Kommunikation zu wenig transparent. In Bezug auf Selektion vermissen die meisten BerufsanfängerInnen sowohl Einstiegsüberprüfungen vor als auch Notenwahrheit im Studium. Die Beurteilung der DozentInnen inklusive ihrer Fächer (Subkategorie Curriculum) fällt ambivalent aus, den Wert der Bildungswissenschaften erkennen laut den Aussagen der Befragten diese erst nach dem Studium bzw. im Laufe der ersten Berufsjahre. Einen großen Teil nimmt die Diskussion zu den Praktika ein. Ein Teil der BerufsanfängerInnen reüssiert aufgrund dieser, der andere Teil vermisst die Schulrealität in den Praktika. Zur Subkategorie Desiderate \& Anwendbares Wissen nennen die BerufsanfängerInnen einen Überhang des Theoriewissens auf Kosten des praktischen Wissens, Inhalte zur Administration als Lehrperson, z.B. der Umgang mit Klassenbüchern, vermissen BerufsanfängerInnen im Nachhinein gänzlich.

\subsubsection{Hauptkategorie Disziplin \& Classroom Management (BA_D)}

Die Hauptkategorie Disziplin \& Classroom Management (BA_D) wird in die Subkategorien Disziplin als Herausforderung beim Berufseinstieg (BA_D_1), Disziplin und Ausbildung (BA_D_2), Umgang mit Disziplinproblemen (BA_D_3) und Disziplin ist kein Problem (BA_D_4) unterteilt (Abb. 56).

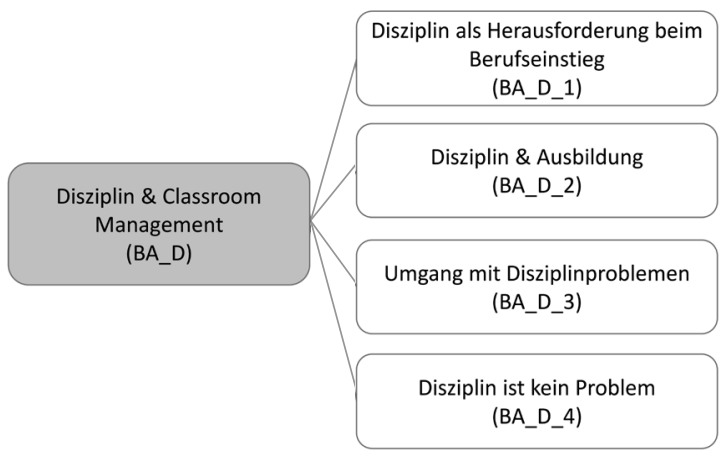

Abb. 56: Disziplin \& Classroom Management; Haupt- und Subkategorien 
In der Subkategorie Disziplin als Herausforderung beim Berufseinstieg $\left(B A_{-} D_{-} 1\right)$ ändert sich der Erzählcharakter der BerufsanfängerInnen im Vergleich zu den vorhergehenden Kategorien vom informativen in Richtung eines emotionalen. Dies ist insofern interessant, als die geschilderten Berufseinstiegserlebnisse disziplinärer Art teilweise zwei bis zweieinhalb Jahre zurückliegen. Trotzdem scheinen die Erinnerungen frisch und auch nachhaltig in Bezug auf jetziges Handeln zu sein. Die Schilderungen innerhalb dieser Subkategorie können in zwei inhaltliche Bereiche gegliedert werden. Zum einen wird der Umgang mit Disziplin beim Berufseinstieg als Herausforderung wahrgenommen, zum anderen bildet diese Thematik für die BerufsanfängerInnen ein Problem bzw. das Hauptproblem.

„Ich meine, mit den Schülern hat man immer wieder einmal einen Kampf“ (BA_13: 21), bringt ein/e BerufsanfängerIn die Herausforderung mit der Disziplin zum Ausdruck. An einer anderen Stelle wird nun bereits vom „Problem“ gesprochen:

„Die Probleme sind eigentlich disziplinärer Art, im Vergleich zu letztem Jahr, da hat man viele Sachen gar nicht sagen müssen. Heuer fällt ihnen [den SchülerInnen, Anm.] alles ein, sie rauchen im Klo, also das geht von A bis Z. Dass man sich oft denkt, das kann ja gar nicht wahr sein. (BA_01:28)

In vielen Gesprächen wird der Umgang mit disziplinären Auffälligkeiten, mit der Einforderung disziplinärer Standards bzw. mit Disziplin zum Hauptproblem beim Berufseinstieg erhoben. „Das Hauptproblem war eindeutig die Disziplin“ (BA_19: 41) oder „Die größten Probleme waren also eindeutig die disziplinären“ (BA_13: 41), lauten dazu zwei Aussagen von BerufsanfängerInnen.

In Disziplin \& Ausbildung (BA_D_2) werden Desiderate in der Ausbildung in Bezug auf den großen Themenbereich Disziplin im Unterricht bzw. in der Schule aufgezeigt. „Da habe ich, kommt mir vor, wenig Handhabe mitbekommen“ (BA_19: 41), erzählt ein/e BerufsanfängerIn. Etwas konkreter mit inhaltlichen Hinweisen schildert ein/e weitere/r InterviewpartnerIn die Disziplinthematik in der Ausbildung:

„Auf alle Fälle, ,wie gehe ich mit den Kindern um', also mehr das einbezogen, die Problemkinder, die laut sind, die stören., Was kann ich mit denen machen?‘. Also das ist etwas, das haben wir in der Ausbildung überhaupt nicht gelernt. Da war gar nichts, also bei mir zumindest nicht, und das hätte ich einige Male schon gebraucht." (BA_05: 46)

Der Umgang mit Disziplinproblemen ( $\left.B A \_D \_3\right)$ reicht von Abwarten und Akklimatisieren über Zurechtweisen bis hin zum Einsatz von Strafen, „weil das sind Sachen, die eigentlich schon wirken“ (BA_10: 63). Bezüglich Abwarten und Akklimatisieren meint ein/e BerufsanfängerIn im ersten Dienstjahr:

„Du musst zuerst einmal mit der ganzen Sache warm werden. Es ist etwas anderes, ob ich [...] die Praxis mache, wo ich im behüteten Umfeld bin. Da habe ich immer die Betreuungslehrer, die doch drauf schauen und die Schüler halten sich ja doch recht zurück. Und hier geht man halt hinein (in die Klasse, Anm.) und muss mit der Situation einmal klar kommen, $(2 \mathrm{~s})$ mit den Schülern. Man muss schauen, wie reagieren die Schüler auf einen, wenn man ganz allein in der Klasse steht und so weiter." (BA_20: 28-29)

Ein/e andere/r InterviewpartnerIn hat im zurechtweisenden Gespräch ein adäquates Mittel im Umgang mit disziplinären Herausforderungen gefunden: 
"Ja, ich tue sie [die SchülerInnen, Anm.] schon relativ viel zurechtweisen und sag ihnen viel, dass man Sachen nicht macht. Ich rede viel mit ihnen. Ich hole sie meistens nach der Stunde her und sag ihnen, dass die Sachen [Fehlverhalten, Anm.] einfach nicht gehen.“ (BA_01: 26)

Schließlich finden sich in der Subsubkategorie Disziplin ist kein Problem (BA_D_4) Stimmen, die „[...] disziplinär überhaupt keine Probleme [haben]“ (BA_03: 23). Einem/einer anderen BerufsanfängerIn ,geht es [mit den Schülern] hervorragend“ (BA_07: 24), während ein/e nächste/r Kollege/Kollegin etwas relativierend ,eigentlich nie gröbere Schwierigkeiten [hatte]" (BA_06: 37). Auffallend sind die verschiedenen Zugänge zu Disziplin bzw. die verschiedenen Kriterien für BerufsanfängerInnen, wonach sich disziplinierte SchülerInnen ausmachen lassen. Stellvertretend werden an dieser Stelle „brave und vor allem fleißige [Schüler]“ (BA_08: 22) genannt.

Die Hauptkategorie Disziplin \& Classroom Management kann zusammenfassend als emotionales Thema für BerufsanfängerInnen bezeichnet werden. Disziplin bzw. Disziplinierung wird als Herausforderung und Problem gesehen, insbesondere auch deshalb, da dieses Thema nach den Aussagen der Interviewten in der Ausbildung nicht adäquat behandelt wird. Der Umgang mit Disziplinproblemen reicht von Reden bis zum Extrem der Strafe. Ein kleiner Teil der Befragten gibt an, keine disziplinären Probleme im Unterricht zu haben.

\subsubsection{Hauptkategorie Kriterien für Berufszufriedenheit der BerufsanfängerInnen (BA_E)}

Die Hauptkategorie Kriterien für Berufszufriedenheit der BerufsanfängerInnen (BA_E) gliedert sich in die Subkategorien Kooperation mit SchülerInnen und LehrerkollegInnen (BA_E_1), Fach \& Fachkompetenz (BA_E_2) und Schultyp (BA_E_3) (Abb. 57).

In Kooperation mit SchülerInnen und LehrerkollegInnen (BA_E_1) werden die Themen Gruppengrößse bzw. Klassenzusammensetzung und zwischenmenschliche Beziehung angesprochen. Zur Berufszufriedenheit in Bezug auf die Gruppengröße erinnert sich ein/e BerufsanfängerIn:

„Ich habe ganz kleine Gruppen gehabt, winzige Gruppen mit zehn, zwölf Leuten. Das war total fein zum Anfangen, weil da hast du sie gleich einmal gekannt, erstens. Und zweitens habe ich sie recht gut im Griff gehabt, dadurch, dass sie so wenige waren." (BA_08: 29)

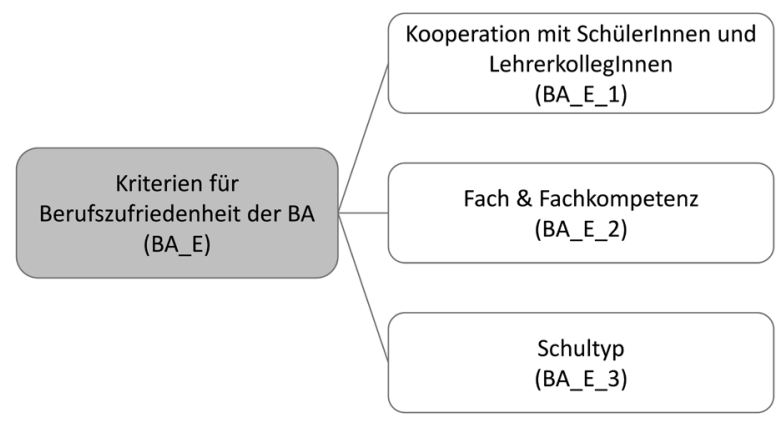

Abb. 57: Kriterien für Berufszufriedenheit der BerufsanfängerInnen; Haupt- und Subkategorien

Zur Förderung der zwischenmenschlichen Beziehungen ist ein/e InterviewpartnerIn „entweder auf sie [die SchülerInnen, Anm.] zugegangen oder [hat] sie an [sich] heran gelassen" (BA_01:37). 
Dies ist dann möglich, wenn „die bürokratischen Abläufe“ (ebd.) bereits bekannt sind und dadurch „mehr Zeit [bleibt, sich] mit diesen Dingen zu beschäftigen“ (ebd.). Auch das Einholen von SchülerInnenfeedback in Form von „persönliche[n] Gespräche[n]“ (BA_18:34) zum „Klima“ (ebd.) ergab für eine/n Befragte/n „eine angenehme Resonanz“ (ebd.) und „das war dann eigentlich dann [sic!] doch eine schöne Sache“ (ebd.).

Auch die zwischenmenschliche Beziehung zu den LehrerkollegInnen wird explizit erwähnt. Ein/e BerufsanfängerIn habe

„es hervorragend erwischt. Wir sind elf oder zwölf Lehrer und es ist nicht einer dabei, wo ich sagen müsste, er würde mich stören oder ich hätte irgendein Problem mit ihm. Also wirklich super.“ (BA_12: 23)

In der Subkategorie Fach \& Fachkompetenz (BA_E_2) wird deutlich, dass es zwischen der Intensität des Wohlfühlens der BerufsanfängerInnen beim Berufseinstieg und dem Fach bzw. der Fachkompetenz einen kausalen Zusammenhang gibt.

Für eine/n BerufsanfängerIn ist „der andere Fachunterricht mit Fachrechnen ${ }^{66}$ schon interessant zu unterrichten. [...] Dadurch, dass ich Mathematiklehrer bin“, lautet die Begründung (BA_02: 36). Im Pflichtschulbereich ist es gang und gäbe, dass LehrerInnen nicht nur ihre geprüften Fächer unterrichten. Insofern ist ein/e BerufsanfängerIn „sehr zufrieden“ (BA_09: 27) und berichtet weiter: „[...] ich habe genau meine Fächer, das passt perfekt eigentlich.“ (ebd.: 28) Ein/e weitere/r InterviewpartnerIn nimmt ebenso Bezug auf den Fächerkanon und erzählt: „Ja fachlich ist es bei mir so, dass ich extrem viele Fächer habe." (BA_15: 32) Das sei „teilweise nicht einfach“, denn „ich will halt mehr können oder mehr wissen wie das, was ich unterrichte“ (ebd.). In Bezug auf die Fachkompetenz meint ein/e BerufsanfängerIn, sie finde es schade, „dass man alle Fächer unterrichten muss und nur in zwei Fächern ausgebildet wird“ (BA_02: 60). So sei ein/e weitere/r BerufsanfängerIn zur fachlichen Vorbereitung „den ganzen Sommer gesessen“ (BA_03: 51). Und „[...] es ist eine große Herausforderung, weil es nicht meine Fächer sind“ (BA_07: 26), erzählt ein/e InterviewpartnerIn: „Einfach die Fülle der Fächer. Es macht mir zwar keine Probleme, aber es ist eine Herausforderung, es gibt viel zu tun.“ (ebd.)

In der Subkategorie Schultyp (BA_E_3) wird von den BerufsanfängerInnen explizit und ohne im Leitfadeninterview darauf hinzuführen, der Schultyp thematisiert. Konkret sind es in der vorliegenden Untersuchung ausschließlich BerufsanfängerInnen mit Erfahrungen an einer Polytechnischen Schule, die diesen Schultyp in Beziehung zu ihrer Berufszufriedenheit bringen:

„Von den Schülern her bin ich heuer total begeistert. Vielleicht liegt es auch daran, dass ich jetzt an einer Polytechnischen Schule bin. Ich weiß nicht, die Schüler sind einfach schon ein bisschen reifer, sie wissen, es geht um was. In der Neuen Mittelschule ist das noch ein bisschen anders." (BA_03: 22)

Konträr dazu liest sich die nachfolgende Aussage, die die Ambivalenz der Einstellung von BerufsanfängerInnen zur Polytechnischen Schule widerspiegelt. Entweder jemand ist davon absolut überzeugt oder genau das Gegenteil ist der Fall.

„Ich muss sagen, ich habe total brave Schüler heuer [an einer Neuen Mittelschule, Anm.], fleißig vor allem. Im Gegensatz zum Poly ${ }^{67}$ sind sie halt sehr motiviert. Das vergleiche ich halt. Und ich kann sie von der Ersten bis zur Vierten vielleicht begleiten. Und da wissen sie halt schon wie ich bin und was ich will und auch umgekehrt. Sie wissen halt schon viel mehr." (BA_08: 22)

66 An der Polytechnischen Schule (PTS) wird vorrangig in Fachgruppen mit jeweils eigenem Fächerkanon unterrichtet. 67 Abkürzung für Polytechnische Schule im Schuljargon. 
Zusammenfassend wird festgestellt, dass zwischenmenschliche Beziehungen (zu SchülerInnen und LehrerkollegInnen) für BerufsanfängerInnen einen großen Einfluss auf deren Berufszufriedenheit ausüben. Je wohler sich die Befragten mit den zu unterrichtenden Fächern fühlen, desto höher scheint auch die Berufszufriedenheit zu sein. Hier wird der Umstand, dass Lehrpersonen an Pflichtschulen auch nicht geprüfte Fächer unterrichten (müssen), als negativ diskutiert. Der Zusammenhang zwischen Schultyp und Berufszufriedenheit wird ambivalent dargestellt. BerufsanfängerInnen beider Schultypen sind ebenso zufrieden wie unzufrieden im Beruf.

\subsubsection{Hauptkategorie Arbeitsbelastung \& Überforderung der BerufsanfängerInnen (BA_F)}

Die Hauptkategorie Arbeitsbelastung \& Überforderung der BerufsanfängerInnen (BA_F) wird in die Subkategorien Schulstrukturen \& Schulsystem (BA_F_l) und Supplierverpflichtung (BA_F_2) unterteilt (Abb. 58).

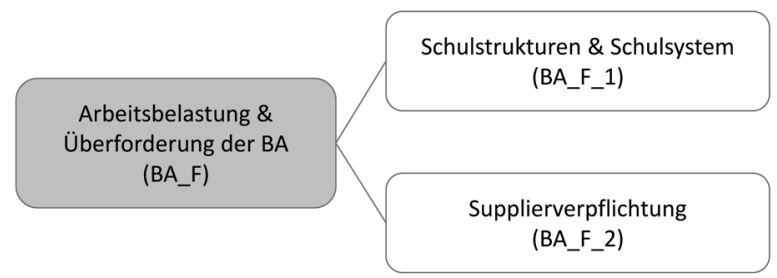

Abb. 58: Arbeitsbelastung und Überforderung der BerufsanfängerInnen; Haupt- und Subkategorien

In der Subkategorie Schulstrukturen \& Schulsystem (BA_F_1) werden Arbeitsbelastungen mit strukturellen, systemischen und dienstrechtlichen Ursachen thematisiert. So erinnert sich ein/e BerufsanfängerIn:

„Große Klassen gehabt. Zwei Fachmetallgruppen waren in der Klasse. Alles Jungs, kein einziges Mädel, alles Metaller. Eher heftige G'schicht. Das ist schon heftig zu unterrichten.“ (BA_02: 34)

Auch die Auswirkung der Arbeitsbelastung auf den Unterricht wird angesprochen:

„Auf die Schüler selber, glaube ich, habe ich gar nicht so viel eingehen können“, erzählt ein/e InterviewpartnerIn, „weil drum herum so viel neu war für mich. Der bürokratische Ablauf.' (BA_01:34)

Und in einem Nachsatz kommt nachdenklich: „Also ich weiß nicht, was die Schüler für ein Gefühl gehabt haben?" (ebd.)

In weiteren Gesprächen wird erzählt, „dass es so wahnsinnig viel auf einmal war“ (BA_17: 26) und dass im Schulsystem verschiedene Aufgaben neben dem Unterrichten eine Zusatzbelastung darstellen: „[...] dann Kustos, also Kustodiat für die Computer und lauter so Sachen. Das war natürlich schon ein bisschen schwer." (BA_03: 39)

„Ich war zwar nicht heillos überfordert, ich habe schon alles bewältigt, aber es war halt einfach stressig“ (BA_17: 27), schildert abschließend ein/e InterviewpartnerIn die Erinnerungen zum Berufseinstieg.

In der Subkategorie Supplierverpflichtung $\left(B A \_F \_2\right)$ werden einerseits die Arbeitsbelastung, andererseits die Unsicherheiten bzgl. der Verpflichtung zum Supplieren thematisiert. So schildert ein/e BerufsanfängerIn die Arbeitsbelastung: „Es war dann auch eine Zeit, wo ich viel zu viel supplieren habe müssen. Da waren es dann mehr als 21 Stunden, weil eine Lehrerin krank wurde. 
Da ist es mir dann natürlich weniger gut gegangen, weil das über Monate hinweg so gegangen ist. Und vier Deutschgruppen, das war einfach zu stressig." (BA_09: 33)

In Bezug auf die rechtlichen Unsicherheiten beim Supplieren stellt sich ein/e InterviewpartnerIn folgende Fragen:

„Welche Rechte und welche Pflichten habe ich? Was muss ich machen? [...] Was ist mit den Anstattstunden ${ }^{6}$, die ich ständig habe? [...] Ist das rechtlich erlaubt oder nicht?" (BA_15: 61f)

Hier wird als Resümee deutlich, dass BerufsanfängerInnen aufgrund der Schulstrukturen, dem System Schule allgemein und der Einsatzpraxis speziell mit Arbeitsbelastung bis hin zu Überforderung konfrontiert sind. Einerseits machen den jungen Lehrpersonen große Klassen zu schaffen, ebenso die Arbeitsbelastung durch Zusatzaufgaben, wie Kustodiatsbetreuung. Andererseits kommen rechtliche Aspekte bzw. Unsicherheiten zum Ausdruck. Neben den Heraus- bzw. Überforderungen durch pädagogisch-fachliche Phänomene beim Berufseinstieg sind die BerufsanfängerInnen durch die rechtlichen Unsicherheiten auch mit einer Form von Ohnmacht konfrontiert.

\subsection{Zusammenfassende Darstellung der Hauptkategorien}

Im folgenden Abschnitt werden die einzelnen Hauptkategorien inklusive ihrer Sub-, Subsubund Subsubsubkategorien inhaltlich verdichtet in der Terminologie der befragten Personen rekonstruiert (Aeppli 2014). Termini der InterviewteilnehmerInnen werden in der Rekapitulation kursiv abgebildet, ohne jedoch explizit als Zitat vermerkt zu werden, da ausnahmslos alle kursiv abgebildeten Termini in der Analyse der Interviews mit SchulleiterInnen (III.3.1.4) und BerufsanfängerInnen (III.3.1.5) erwähnt und zitiert sind. Am Beginn jeder Rekapitulation steht jeweils ein für die zusammengefasste Kategorie repräsentatives, manchmal pointiertes Zitat.

Das Ziel dieser inhaltlichen Rekapitulation ist zweierlei: Zum einen soll die dicht gefasste Zusammenfassung einen schnellen inhaltlichen Einblick in die Aussagen der SchulleiterInnen und BerufsanfängerInnen gewähren, zum anderen dienen die Kurzzusammenfassungen dazu, in weiterer Folge inhaltliche Überschneidungen zwischen den einzelnen Kategorien aufzuzeigen und im Sinne einer Clusterbildung eine nächsthöhere inhaltliche Abstraktionsebene zu erreichen.

\section{Kompetenzen von BerufsanfängerInnen (SL_A)}

„Wo ich immer wieder erstaunt bin [...] ist die extreme Vielfalt an Methodik und Didaktik." (SL_10: 41)

Die SchulleiterInnen nennen in dieser Kategorie primär die Fach- und Methodenkompetenzen der BerufsanfängerInnen, wobei die Fachkompetenzen als Ausbildungsinhalte im Rahmen des Curriculums vorausgesetzt werden und die Methodenkompetenzen mit positiver Überraschung erstaunt zur Kenntnis genommen werden.

Die BerufsanfängerInnen wagen sich an herausfordernde Schulprojekte heran und bringen damit für die SchulleiterInnen die Einsatzfreude zum Ausdruck, welche sich in weiterer Folge in Form neuer Impulse positiv auf den gesamten Lehrkörper auswirkt.

Die selbst angeeigneten Kompetenzen und die individuellen Stärken erwerben die BerufsanfängerInnen in Vereinen oder durch den eigenen Lebensstil und bringen so Persönlichkeit und menschliche Qualitäten mit, was den Drabt zum Kind ermöglicht.

68 IntegrationslehrerInnen werden zur Unterstützung im Unterricht eingesetzt. Wenn sie als Unterstützung abgezogen und als KlassenlehrerIn eingesetzt werden, spricht man von „Anstattstunden“ (Bundesministerium für Digitalisierung und Wirtschaftsstandort 1984). 


\section{Institutionelle Ausbildung aus der Sicht der SchulleiterInnen (SL_B)}

„Amtsschriften sind den Junglehrern weitgehend unbekannt.“ (SL_13: 19)

„Ich glaube, dass methodisch-didaktisch die Ausbildung nicht schlecht ist und dass sich in dem Zusammenhang ein neuer Lernbegriff durchgerungen hat." (SL_10: 44)

Die SchulleiterInnen favorisieren eine pädagogisch-didaktische Selektion am Anfang des Studiums, womit die menschlichen Fähigkeiten der Studierenden erfasst werden können, und gehen davon aus, dass nicht für alle Lehramtsstudierenden die pädagogische Arbeit mit Kindern und Jugendlichen das Kriterium für die Wahl dieses Studiums darstellt.

In Bezug auf die Anforderungen an die Ausbildungsinstitutionen nennen die SchulleiterInnen Desiderate im pädagogischen und administrativen Bereich. In Anspielung auf das Classroom Management seien die BerufsanfängerInnen zwar gut ausgebildet, aber manchmal an der Praxis vorbei. Die disziplinären Herausforderungen stellen ein Hauptthema dar und werden in den Augen der SchulleiterInnen viel zu wenig gelehrt. Schließlich weisen SchulleiterInnen in dieser Kategorie vehement auf die Ausbildungsdesiderate im administrativen Bereich hin und wissen, dass den BerufsanfängerInnen Amtsschriften weitgehend unbekannt sind.

In der Fremdwahrnehmung die SchulleiterInnen reüssieren die BerufsanfängerInnen durch die praktische Ausbildung, wobei realistische Situationen in der Schule zu bevorzugen sind und die Praxis noch verstärkt werden kann.

Den Ausbildungsinstitutionen wird vonseiten der SchulleiterInnen ein gutes Zeugnis ausgestellt. Sie sehen insgesamt eine Qualitätssteigerung in der LehrerInnenausbildung und denken, dass die BerufsanfängerInnen fachlich sehr gut vorbereitet sind.

\section{Wahrnehmung der BerufsanfängerInnen durch die SchulleiterInnen (SL_C)}

„Also wirklich, sie wollen gut sein. Etwas anderes habe ich noch nicht erlebt.“ (SL_03: 67)

SchulleiterInnen verbinden mit "JunglehrerIn“ eher im Dienst unerfahrene (bis ca. vier Dienstjahre) als biologisch junge Lehrpersonen und nehmen auch einen Zusammenhang zwischen der Altersstruktur des Lehrkörpers und der Zeit, wie lange sich ein/e BerufsanfängerIn als AnfängerIn fühlt, wahr. Einsatzfreude ist quer durch die Bank gegeben, ebenso Offenheit im Sinne von bereit für Neues. Geschlechtsspezifisch sehen die Schulleiterinnen und Schulleiter (explizit beide Geschlechter) zwischen Berufsanfängern und Berufsanfängerinnen bis auf die Emotionalität keine Unterschiede. SchulleiterInnen assoziieren mit BerufsanfängerIn „sehr engagiert“, „sehrfleißig“, aber auch „illusorisch"bzgl. idealisierter Vorstellungen im Unterricht.

Die größten Herausforderungen liegen im Bereich des Classroom Managements: Fehler im Umgang mit SchülerInnen und deren Arbeitshaltung, die mit der Routine weggehen.

\section{Unterstützung \& Begleitung der BA im Schulalltag (SL_D)}

„Meine Tür steht jederzeit offen.“ (SL_13:23)

Bezüglich Unterstützung und Begleitung kommen BerufsanfängerInnen mit wenigen Erwartungen, da diese einerseits frob über die Anstellung sind und weil die KollegInnen vieles abnehmen. Die wenigen Erwartungen liegen im Bereich der Schulorganisation und des Classroom Managements, eine strukturelle Hilfe bietet das FachkoordinatorInnensystem. Wichtig sind klare Richtlinien innerhalb der Schule in den Bereichen Notengebung und Disziplin.

Zum Mentoring liegt eine hohe Anzahl von Kodierungen vor, wesentlich sind Zeitangebot, Gespräche und gegenseitiges Vertrauen. In diesem Sinne sind die Türen immer offen. Gespräche unter 
KollegInnen finden aus pragmatischen (Pausen, Fensterstunden) und hierarchischen Gründen eher statt als Mentoring-Gespräche mit SchulleiterInnen. Die KollegInnen begleiten vorrangig durch Vorbildwirkung, die SchulleiterInnen punkten mit den Themen Professionalisierung, dienstrechtliche Fragen und Umgang mit Disziplinproblemen, wobei eine Einmischung der Schulleitung vor der Klasse mit Autoritätsverlust für den/die BerufsanfängerIn verbunden wird.

\section{Ersterfahrungen der BerufsanfängerInnen beim Berufseinstieg (BA_A)}

„Ich bin ins kalte Wasser geschmissen worden und hab' dadurch viel gelernt." (BA_11:28)

Die ersten Erfahrungen der BerufsanfängerInnen lassen sich zum Teil mit Zweifel und Schock umschreiben, wobei diese Erfahrungen aus einer Arbeitsüberlastung resultieren. Die Kombination aus keiner Routine und den vielen neuen, unbekannten Aufgaben macht den Einstieg nicht leichter, zumal auch das ganze System Stress macht. BerufsanfängerInnen berichten von einem Drama beim Berufseinstieg, bis man als LehrerIn akzeptiert wird. Der Worst Case ist das Scheitern. Die KollegInnen fungieren als Stützen und HelferInnen und nicht zuletzt werden die eigenen Erfahrungswerte selbstreflexiv analysiert. Aufgrund der ersten Routine ist eine Steigerung von Jahr zu Jahr erkennbar.

\section{Mentoring aus der Sicht der BerufsanfängerInnen (BA_B)}

„Du kommst hinein [in die Schule, Anm.] und hast vom Tuten und Blasen keine Ahnung.“ (BA_10: 83)

BerufsanfängerInnen benötigen und beanspruchen von SchulleiterInnen und KollegInnen Unterstützung. Diese ist dann besonders wertvoll, wenn nicht explizit danach gefragt werden muss. In überwiegendem Maße funktioniert dies perfekt, unabhängig davon, ob es sich um SchulleiterInnen oder KollegInnen bzw. um fachliche oder allgemeine Fragestellungen handelt. SchulleiterInnen unterstützen die BerufsanfängerInnen meist in administrativen Belangen, während KollegInnen oftmals durch Tipps prophylaktisch Fehlern vorbeugen. Zuhören und Verständnis zeigen sind ebenso hilfreich wie das Bereitstellen von Arbeitsmaterialien. Erst durch das Mentoring erhalten die BerufsanfängerInnen einen schulischen Überblick, insbesondere im zwischenmenschlichen Umgang mit den SchülerInnen und im Bereich der Bürokratie.

\section{Institutionelle Ausbildung aus der Sicht der BA (BA_C)}

„In der Praxis merkt jeder Student: ,Ist das mein Beruf oder nicht?““(BA_06: 54)

Neben der wahnsinnigen Bürokratie sind es vor allem die zu vielen Stunden, die den BerufsanfängerInnen negativ in Erinnerung sind. Zur Selektion würden die BerufsanfängerInnen einen Aufnahmetest anwenden, wo wirklich herausgefiltert wird, wer als LehrerIn geeignet ist und wer nicht. In der Ausbildung spielt das anwendungsorientierte Wissen eine zentrale Rolle, dies trägt zur fachlichen Sicherheit beim Berufseinstieg bei. Bezogen auf die Ausbildungsinhalte sind Leistungsfeststellung und -beurteilung dominant, sowohl in praktischer als auch in rechtlicher Hinsicht.

Die Erwartungen an die Bildungswissenschaften sind hoch, sie bilden die Essenz in der Ausbildung. Neben der Vorbildwirkung der DozentInnen tragen auch die Praxislehrpersonen zum Erfolg oder Misserfolg in der Ausbildung bei.

Die praktische Ausbildung ist etwas vom Wichtigsten in der Ausbildung. Leider fehlt zum Teil die Realität, denn Praxisstunden sind oft eine Show. Als Lösung dafür gelten Gespräche mit LehrerInnen in der Praxis, um richtige Praxisberichte zu hören.

Die größten Desiderate in der Ausbildung liegen laut den BerufsanfängerInnen im Bereich des administrativen Wissens. 


\section{Disziplin \& Classroom Management (BA_D)}

„Das Hauptproblem war eindeutig die Disziplin.“ (BA_19: 41)

In der Kategorie Disziplin \& Classroom Management thematisieren die BerufsanfängerInnen die Handhabe, wie sie mit SchülerInnen insbesondere bei Disziplinproblemen umgehen. Dieses Thema wird emotional diskutiert, es ist auch vom Kampf mit den SchülerInnen die Rede. Desiderate in der Ausbildung werden im engeren Sinne im Bereich des Themenbereichs Disziplin angesprochen, im Weiteren in Bezug auf das Classroom Management und die Frage: Wie gehe ich mit den Kindern um? Ein Lösungsansatz lautet, viel mit ihnen reden. Schließlich gibt es auch einzelne BerufsanfängerInnen, die beim Berufseinstieg keine gröberen Schwierigkeiten mit Classroom Management und Disziplin in der Klasse hatten.

\section{Kriterien für Berufszufriedenheit der BerufsanfängerInnen (BA_E)}

„Von den Schülern her bin ich heuer total begeistert." (BA_03: 22)

Ein wesentliches Kriterium für die Berufszufriedenheit der BerufsanfängerInnen ist die Qualität der Kooperation sowohl mit den SchülerInnen als auch mit den LehrerkollegInnen bzw. mit der Schulleitung. Wenn die bürokratischen Abläufe bekannt sind, bleibt mehr Zeit für SchülerInnenfeedback in Form von persönlichen Gesprächen und für die Pflege der zwischenmenschlichen Beziehungen zu den LehrerkollegInnen.

Wenn die BerufsanfängerInnen im Pflichtschulbereich ${ }^{69}$ die eigenen, studierten Fächer unterrichten und in einem Schultyp eingesetzt sind, wo sie sich mit der Altersstufe ${ }^{70}$ und den Intentionen ${ }^{71}$ der SchülerInnen identifizieren können, wird der Grad der Berufszufriedenheit ebenso erhöht.

\section{Arbeitsbelastung \& Überforderung der BerufsanfängerInnen (BA_F)}

\section{„Große Klassen [...] das ist schon heftig zu unterrichten.“ (BA_02: 34)}

In der Kategorie Arbeitsbelastung \& Überforderung nennen die BerufsanfängerInnen Kriterien mit strukturellen, systemischen und dienstrechtlichen Ursachen. Hier spielen Klassengrößen und Gruppenzusammensetzungen ebenso eine Rolle wie im System vorgesehene Zusatzaufgaben, z.B. die Betreuung eines Kustodiats. BerufsanfängerInnen fühlen sich dann zwar nicht heillos überfordert, es ist einfach stressig. Die an der Pflichtschule dienstrechtlich vorgeschriebene Supplierverpflichtung wirkt sich in zweifacher Hinsicht negativ aus, einerseits durch den erhöhten Mehraufwand als zusätzliche Arbeitsbelastung, andererseits durch rechtliche Unsicherheiten aufseiten der BerufsanfängerInnen als emotionale Zusatzbelastung.

Nach der Analyse der SchulleiterInnen- und BerufsanfängerInneninterviews, der Kategorisierung und inhaltlichen Beschreibung der Kategorien bzw. Kategorienebenen erfolgt im Anschluss eine inhaltliche Verknüpfung der verschiedenen Hauptkategorien über Schlüsselbegriffe. Über das Clustern (Bortz \& Döring 2006; Breuer et al. 2018; Buber et al. 2000) einzelner Hauptkategorien wird die nächst höhere Ebene zur Konzeptentwicklung erreicht: die Clusterkategorienebene I.

69 Sekundarstufe I: Neue Mittelschule (NMS), Polytechnische Schule (PTS)

70 NMS (Sek. I): 10-14 Jahre; PTS: 14-15 bzw. 16 Jahre (tlw. freiwilliges 10. Schuljahr)

71 Verschiedene Übergänge: Sek. I $\rightarrow$ Sek. II; Sek. I $\rightarrow$ PTS; PTS $\rightarrow$ duale Lehrausbildung (siehe auch Schultypen, Kap. III.1.3.1) 


\subsection{Clusterkategorienebene I}

Die Kategorien (SL_A) bis (SL_D) aus den Interviews mit SchulleiterInnen und (BA_A) bis (BA_F) aus den Interviews mit BerufsanfängerInnen hängen über Schlüsselbegriffe thematisch zusammen. Diese Schlüsselbegriffe stammen aus Zitaten der InterviewpartnerInnen und lassen eine Clusterbildung im Sinne einer empirischen Klassifikation zu. Im Gegensatz zur theoretischen (künstlichen oder logischen) Klassifikation sind bei der empirischen (natürlichen) Klassifikation ausschließlich empirisch angetroffenen Merkmalskombinationen relevant (Bortz \& Döring 2006). In der vorliegenden Arbeit sind die Schlüsselbegriffe Ausgangspunkt des Ordnungsversuchs. Die Herausforderung besteht nun darin, diese Schlüsselbegriffe zu identifizieren und sinn- und erkenntnisgewinnend zu verknüpfen. Buber, Kraler und Gadner schreiben u.a.: „To get the final grouping, the researcher chooses a parameter [...]. Damit wird die Initiativleistung des Forschers bestätigt (Buber et al. 2000).

In Abbildung 59 sind am rechten Bildrand thematische Verknüpfungen in Form von Klammern dargestellt. Die Clusterbildung, welche zu den hierarchisch höheren Clusterkategorienebene I führt, wird in Form von gebündelten Linien angezeigt. Auf diese Weise können Hauptkategorien über Schlüsselbegriffe verbunden und Cluster gebildet werden. Die Schlüsselbegriffe der Clusterkategorien werden in den Abschnitten Kap. III.3.3.1 und III.3.3.2 beschrieben. Kategorie (SL_A) kohäriert inhaltlich mit den Kategorien (SL_C), (BA_C) und (BA_D), Kategorie (SL_B) hängt mit (BA_C) und BA_E) zusammen. Kategorie (SL_D) kann über Schlüsselbegriffe mit (BA_B), (BA_D) und (BA_E) verknüpft werden, Hauptkategorie (BA_A) hängt mit (BA_F) zusammen. Schließlich gibt es noch inhaltliche Verknüpfungen über Schlüsselbegriffe zwischen (BA_B) und den Kategorien (BA_D) und (BA_E).

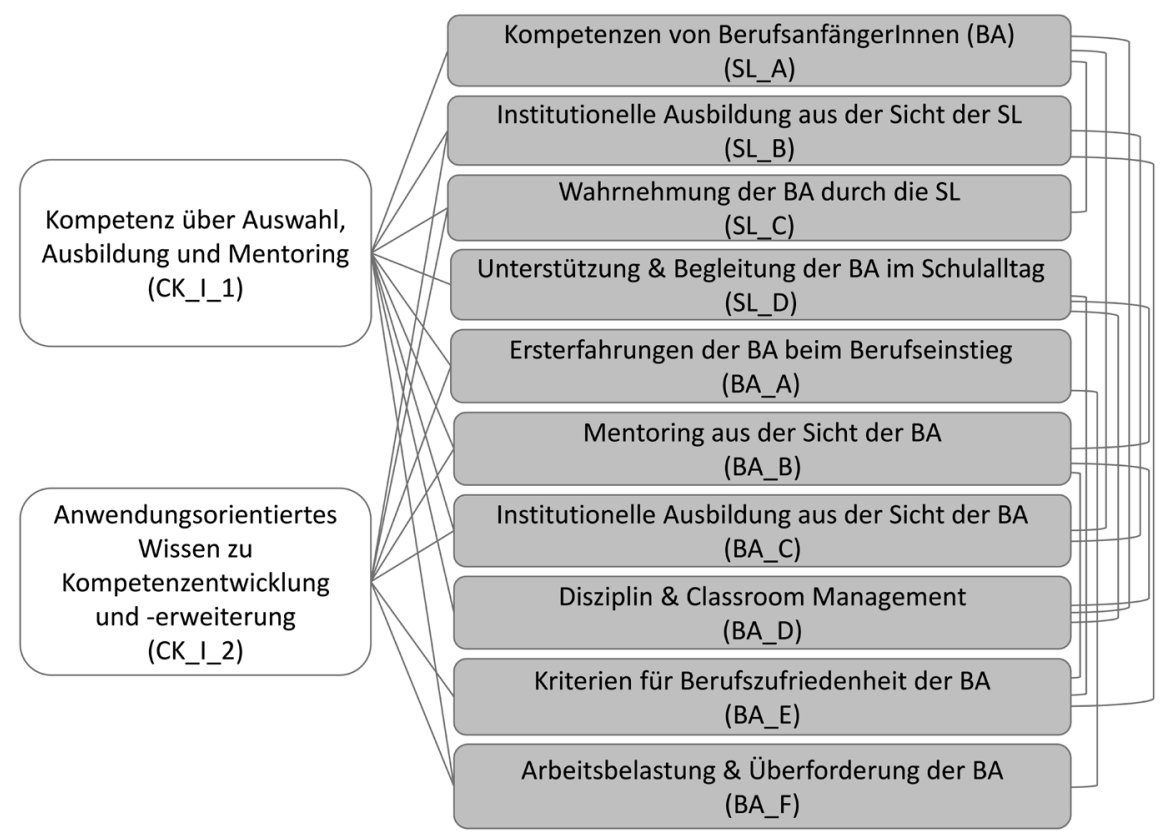

Abb. 59: Clusterkategorien 1 und 2 auf Clusterkategorienebene I (CK_I) 


\subsubsection{Schlüsselbegriffe und Clusterbildung: Clusterkategorie 1 (CK_I_1)}

Eine Reihe von Schlüsselbegriffen kommt wiederkehrend in verschiedenen Kategorien, sowohl von SchulleiterInnen als auch von BerufsanfängerInnen angesprochen, thematisch im Kontext Kompetenz vor (Tab. 9). Die Kompetenz hilft dem/der BerufsanfängerIn bei der Bewältigung des Berufseinstiegs und in Folge bei der Entwicklung der ersten Berufserfahrung. Maßgeblich ausschlaggebend für den Erwerb dieser Kompetenz sind drei Aspekte: die Selektion, die Ausbildung und die Begleitung.

Tab. 9: Kontext und Schlüsselbegriffe; Clusterkategorie 1

\begin{tabular}{ll}
\hline Clusterkategorie 1 (CK_I_1) & \\
\hline Kontext & Schlüsselbegriffe \\
\hline Kompetenzaspekte & Selektion vor und während der Ausbildung \\
Selektion, Ausbildung, Begleitung & Fach- und Methodenkompetenz \\
& Kooperation im Team \\
& Schulstruktur und -system \\
& Stress \\
\hline
\end{tabular}

Die mehrfach thematisierten Schlüsselbegriffe von SchulleiterInnen und BerufsanfängerInnen sind Selektion vor und während der Ausbildung, Fach- und Methodenkompetenz, Kooperation im Team, Schulstruktur, System und Systemstress (Tab. 9). Insofern streben BerufsanfängerInnen eine die Schlüsselbegriffe inkludierende Kompetenz an, welche gleichermaßen als Voraussetzung bzw. als Gelingensbedingung für einen erfolgreichen Berufseinstieg angesehen werden kann.

Dies führt zur Clusterkategorie 1 auf der analysehierarchisch nächst höheren Clusterkategorienebene I:

\section{Clusterkategorie 1: Kompetenz über Auswahl, Ausbildung und Mentoring (CK_I_1)}

(Abb. 59)

\subsubsection{Schlüsselbegriffe und Clusterbildung: Clusterkategorie 2 (CK_I_2)}

Die zweite Clusterkategorie (CK_I_2) emergiert ebenfalls aus dem Phänomen ${ }^{72}$ Kompetenz, hier (im Unterschied zu Clusterkategorie 1) im Zusammenhang von Anwendungsorientierung und Praktikabilität (Tab. 10).

Insofern überschneiden sich die beiden Clusterkategorien zwar terminologisch, jedoch nicht inhaltlich. Hiermit wird dem Prinzip der Clusteranalyse Rechnung getragen, wonach innerhalb eines Clusters die Objektunterschiede möglichst klein und zwischen den Clustern möglichst groß sein sollen (Bortz \& Döring 2006).

72 Dieser Begriff wird an dieser Stelle verwendet, um den empirisch gewonnenen Erkenntnissen Nachdruck zu verleihen. 
Tab. 10: Kontext und Schlüsselbegriffe; Clusterkategorie 2

\begin{tabular}{ll}
\hline Clusterkategorie 2 (CK_I_2) & \\
\hline Kontext & Schlüsselbegriffe \\
\hline Kompetenzaspekte & Methodenkompetenz \\
Anwendungsorientiertes Wissen & Illusion beim Berufseinstieg \\
Praktikabilität & Classroom Management \\
& Umgang mit Kindern \\
& Disziplin, Disziplinprobleme \\
& realistische Praxisausbildung \\
& Erwartungen an die bildungswissenschaftliche Ausbildung \\
\hline
\end{tabular}

In Clusterkategorie 2 thematisieren die SchulleiterInnen und BerufsanfängerInnen vor allem die praxisbezogene und praktikable (nicht praktische!) Ausbildung und deren Umsetzung im tatsächlichen LehrerInnendasein, insbesondere beim Berufseinstieg.

Die zugehörigen Schlüsselbegriffe lauten Methodenkompetenz, Illusion beim Berufseinstieg, Classroom Management, Umgang mit Kindern, Disziplin, Disziplinprobleme, realistische Praxisausbildung, Erwartungen an die bildungswissenschaftliche Ausbildung in Bezug auf die Vorbereitung auf das reale LehrerInnenberufsleben (Tab. 10).

Daraus wird Clusterkategorie 2 der Clusterkategorienebene I abgeleitet:

Clusterkategorie 2: Anwendungsorientiertes Wissen zu Kompetenzentwicklung und -erweiterung (CK_I_2) (Abb. 59)

Im folgenden Abschnitt wird der Abstraktions- und Verallgemeinerungsgrad erhöht. Aus den Clusterkategorien 1 und 2 auf Clusterkategorienebene I wird durch Integration Clusterkategorie 3 auf der in dieser Arbeit hierarchisch höchsten Analyseebene, Clusterkategorienebene II, gebildet.

\subsection{Konzeptualisierung: Clusterkategorie 3 (CK_II_3)}

Die höchste Hierarchieebene (Abb. 60) der vorliegenden Untersuchung, die Clusterkategorienebene II, bildet sich aus den beiden Clusterkategorien Kompetenz über Auswabl, Ausbildung und Mentoring (CK_I_I) und Anwendungsorientiertes Wissen zu Kompetenzentwicklung und -erweiterung (CK_I_2).

Die Clusterkategorien 1 und 2 hängen über die Hauptkategorien (SL_B), (SL_C), (BA_A), (BA_B), (BA_C) und (BA_F) zusammen. In diesen Hauptkategorien konnten inhaltliche Überschneidungen zu Selektion, Ausbildung, Mentoring und Anwendungsorientierung identifiziert werden. „The higher we go, the more general the summaries are“ (Buber et al. 2000) beschreibt den Abstraktionsprozess der Theorie- bzw. Konzeptfindung. Nach Subsumierung und Integration von Auswahl (Selektion), Ausbildung und Mentoring mit dem Anspruch, anwendungsorientiertes Wissen und Praktikabilität zu implementieren, folgt in der vorliegenden Untersuchung der Schluss, dass es sich thematisch um Professionalisierung im weitesten Sinne handelt (Abb. 60). 


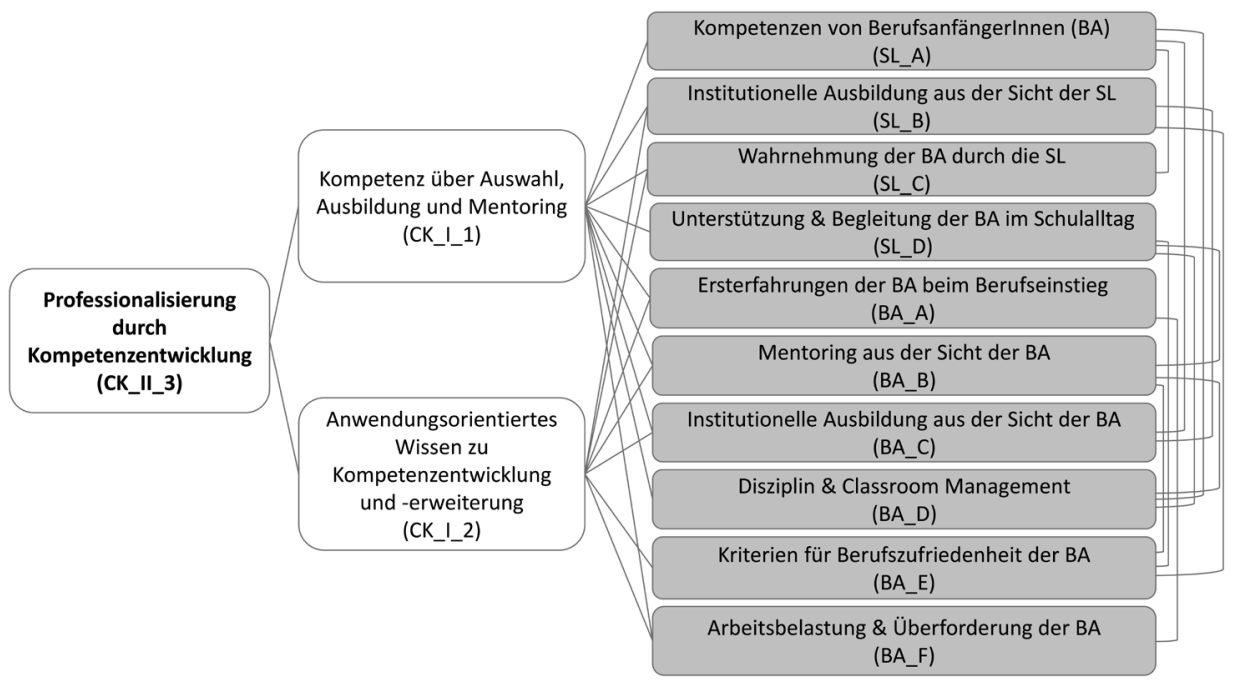

Abb. 60: Clusterkategorie 3 auf Clusterkategorienebene II (CK_II_3); Konzeptualisierung

Unter Berücksichtigung der vorliegenden Argumente lautet die finale Clusterkategorie 3 auf Clusterkategorienebene II:

Clusterkategorie 3: Professionalisierung durch Kompetenzentwicklung (CK_II_3) 


\section{Interpretation der analysierten Daten}

Die Interpretation erfolgt zweistufig. Zuerst werden nach dem Vorbild der inhaltlich strukturierenden qualitativen Inhaltsanalyse (Kuckartz 2016) Schlüsselbegriffe und Originalzitate bzw. deren abstrahierte Aussagen (Essenzen) aus den Interviewtexten identifiziert und als sogenannte Themen ${ }^{73}$ mit den Hauptkategorien aus der Analyse in einer Themen-Kategorien-Matrix dargestellt. Anschließend werden nach dem qualitativen Paradigma den Schlüsselbegriffen und Essenzen interpretativ Bedingungen ${ }^{74}$ bzw. Beziehungen zugeschrieben.

\subsection{Themen-Kategorien-Matrix}

Die Identifikation der Schlüsselbegriffe erfolgt einerseits nach dem quantitativen Paradigma mithilfe der Auswertungssoftware MAXQDA (Kuckartz 2016), indem nach Begriffen gesucht und deren Position und Quantität in den Texten festgestellt werden. Andererseits wird durch qualitatives Vorgehen nach Zitaten und deren Essenzen gesucht (Tab. 11).

Tab. 11: Themen-Kategorien-Matrix; eigene Darstellung nach Kuckartz (2016)

\begin{tabular}{|c|c|c|c|c|c|c|}
\hline & $\begin{array}{l}\text { Administra- } \\
\text { tion }\end{array}$ & Systemwissen & $\begin{array}{l}\text { Reflexion \& } \\
\text { Feedback }\end{array}$ & $\begin{array}{l}\text { Autorität in } \\
\text { der Klasse }\end{array}$ & $\begin{array}{l}\text { Kooperation } \\
\text { mit Schüler- } \\
\text { Innen }\end{array}$ & Habitus \\
\hline SL_A & & & & $\begin{array}{l}\text { geschlechts- } \\
\text { neutral, } \\
\text { Lebensstil }\end{array}$ & $\begin{array}{l}\text { Engagement, } \\
\text { Schulprojekte }\end{array}$ & \\
\hline SL_B & $\begin{array}{l}\text { Ausbildungs- } \\
\text { desiderat }\end{array}$ & Erwartungen & & & & \\
\hline SL_C & & & & $\begin{array}{l}\text { Einstellungs- } \\
\text { kriterium }\end{array}$ & $\begin{array}{l}\text { geschlechts- } \\
\text { neutral }\end{array}$ & $\begin{array}{l}\text { Illusion vs. } \\
\text { Realität }\end{array}$ \\
\hline SL_D & $\begin{array}{l}\text { Prophylaxe, } \\
\text { Sicherheit, } \\
\text { Ausgleich von } \\
\text { Ausbildungs- } \\
\text { desideraten }\end{array}$ & $\begin{array}{l}\text { Professionali- } \\
\text { sierung }\end{array}$ & $\begin{array}{l}\text { Zeit, } \\
\text { Sicherheit }\end{array}$ & & $\begin{array}{l}\text { Rahmenbe- } \\
\text { dingungen }\end{array}$ & $\begin{array}{l}\text { Entwick- } \\
\text { lungsprozess, } \\
\text { Orientierung }\end{array}$ \\
\hline BA_A & sicherer Start & $\begin{array}{l}\text { Einstiegswis- } \\
\text { sen }\end{array}$ & $\begin{array}{l}\text { Impulse, } \\
\text { Rückkoppe- } \\
\text { lung }\end{array}$ & $\begin{array}{l}\text { Akzeptanz, } \\
\text { Zweifel, } \\
\text { Schock, }\end{array}$ & $\begin{array}{l}\text { Akzeptanz, } \\
\text { Selbstrefle- } \\
\text { xion }\end{array}$ & $\begin{array}{l}\text { Zweifel, } \\
\text { Selbstrefle- } \\
\text { xion }\end{array}$ \\
\hline BA_B & $\begin{array}{l}\text { vorauseilende } \\
\text { Hilfe }\end{array}$ & $\begin{array}{l}\text { Tipps, } \\
\text { Gespräche, } \\
\text { Professionali- } \\
\text { sierung }\end{array}$ & $\begin{array}{l}\text { Professionali- } \\
\text { sierung, } \\
\text { Qualitäts- } \\
\text { zirkel }\end{array}$ & $\begin{array}{l}\text { Tipps, } \\
\text { Gespräche }\end{array}$ & $\begin{array}{l}\text { Tipps, } \\
\text { zwischen- } \\
\text { menschliche } \\
\text { Beziehungen }\end{array}$ & $\begin{array}{l}\text { Entwick- } \\
\text { lungs- und } \\
\text { Reifeprozess }\end{array}$ \\
\hline
\end{tabular}

73 Zur speziellen Verwendung des Begriffs Thema (bzw. Themen) in dieser Untersuchung siehe Abschnitt Strukturierende qualitative Inhaltsanalyse, Bedingungsgefüge (Kap. III.2.3).

74 Insofern werden die Abbildungen (Kap. III.4.2.1 - III.4.2.6) in der Interpretation als Bedingungsgefüge bezeichnet. 


\begin{tabular}{|c|c|c|c|c|c|c|}
\hline & $\begin{array}{l}\text { Administra- } \\
\text { tion }\end{array}$ & Systemwissen & $\begin{array}{l}\text { Reflexion \& } \\
\text { Feedback }\end{array}$ & $\begin{array}{l}\text { Autorität in } \\
\text { der Klasse }\end{array}$ & $\begin{array}{l}\text { Kooperation } \\
\text { mit Schüler- } \\
\text { Innen }\end{array}$ & Habitus \\
\hline BA_C & $\begin{array}{l}\text { großes Desi- } \\
\text { derat }\end{array}$ & $\begin{array}{l}\text { Verantwor- } \\
\text { tung, } \\
\text { Einstiegswissen }\end{array}$ & $\begin{array}{l}\text { Grundlage, } \\
\text { Know-how }\end{array}$ & Rüstzeug & $\begin{array}{l}\text { realistische } \\
\text { Praxis }\end{array}$ & $\begin{array}{l}\text { Perspektiven- } \\
\text { wechsel, } \\
\text { Fundament }\end{array}$ \\
\hline BA_D & & & & $\begin{array}{l}\text { Versagens- } \\
\text { ängste }\end{array}$ & $\begin{array}{l}\text { fehlende } \\
\text { Routine } \\
\text { Emotionalität }\end{array}$ & Sicherheit \\
\hline BA_E & Sicherheit & $\begin{array}{l}\text { Zeit für } \\
\text { pädagogisches } \\
\text { Handeln }\end{array}$ & Vertrauen & & $\begin{array}{l}\text { Engagement, } \\
\text { Schulprojekte }\end{array}$ & $\begin{array}{l}\text { Sicherheit, } \\
\text { Selbstrefle- } \\
\text { xion }\end{array}$ \\
\hline BA_F & & $\begin{array}{l}\text { Stress, } \\
\text { Überforde- } \\
\text { rung, } \\
\text { Unsicherheit }\end{array}$ & & & $\begin{array}{l}\text { Illusion, } \\
\text { fehlende } \\
\text { Routine }\end{array}$ & \\
\hline
\end{tabular}

Schließlich können in der Themen-Kategorien-Matrix (ebd.) über die gemeinsamen Schlüsselbegriffe die Vernetzungen der Themen (Kopfzeile) mit den Hauptkategorien (linke Spalte) dargestellt werden (Tab. 11).

Legende zu den Abkürzungen der Hauptkategorien in Tabelle 11:

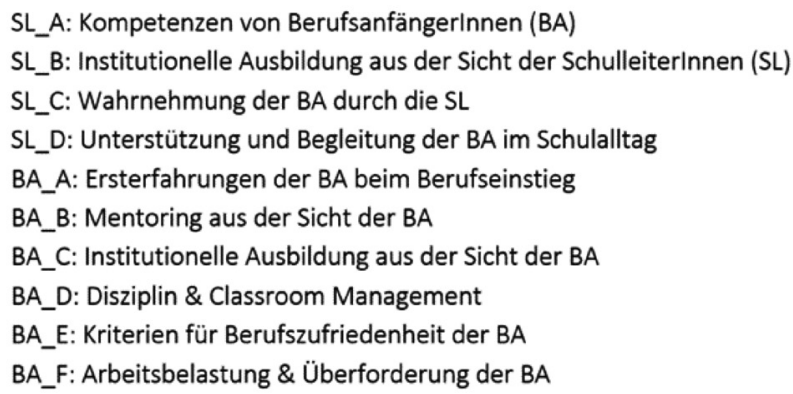

Abb. 61: Hauptkategorien; Legende

In Tabelle 11 wird dargestellt, dass sich auf der Ebene der Themen (Kopfzeile) die Begriffe Administration, Systemwissen, Reflexion \& Feedback, Autorität in der Klasse, Kooperation mit den SchülerInnen und als Metathema Habitus herauskristallisieren. In der linken Spalte sind die Hauptkategorien SL_A, SL_B etc. abgebildet. Die Positionen der Schlüsselbegriffe bzw. Essenzen einerseits und deren Häufigkeit andererseits zeigen die zu interpretierende Vernetzung der Themen mit den Hauptkategorien. Bezogen auf die Themen mit dem Blick auf die Ordinatenachse (Hauptkategorien) kann aus der Anzahl von vernetzenden Schlüsselbegriffen (Tab. 11) pro Thema der Grad der Vernetzung mit verschiedenen Hauptkategorien abgeleitet werden.

In der vorliegenden Untersuchung ist, wie in Abbildung 62 dargestellt, das Thema Administration mit sechs Hauptkategorien vernetzt. Das entspricht einem mittleren Vernetzungsgrad, die 
Werte reichen insgesamt von 5 bis 9. Das Thema Systemwissen hat eine mittlere Vernetzung mit sieben Hauptkategorien, Reflexion \& Feedback ist mit fünf Hauptkategorien vergleichsweise niedrig vernetzt. Das Thema Autorität in der Klasse ist bei mittlerem Vernetzungsgrad mit sechs Hauptkategorien vernetzt, das Thema Kooperation mit SchülerInnen zeigt eine hohe Vernetzung mit neun Hauptkategorien auf. Schließlich hat das Thema Habitus mit sieben Hauptkategorien einen mittleren Vernetzungsgrad in der vorliegenden Arbeit (Abb. 62).

\section{Vernetzungsgrad: Themen mit Hauptkategorien}

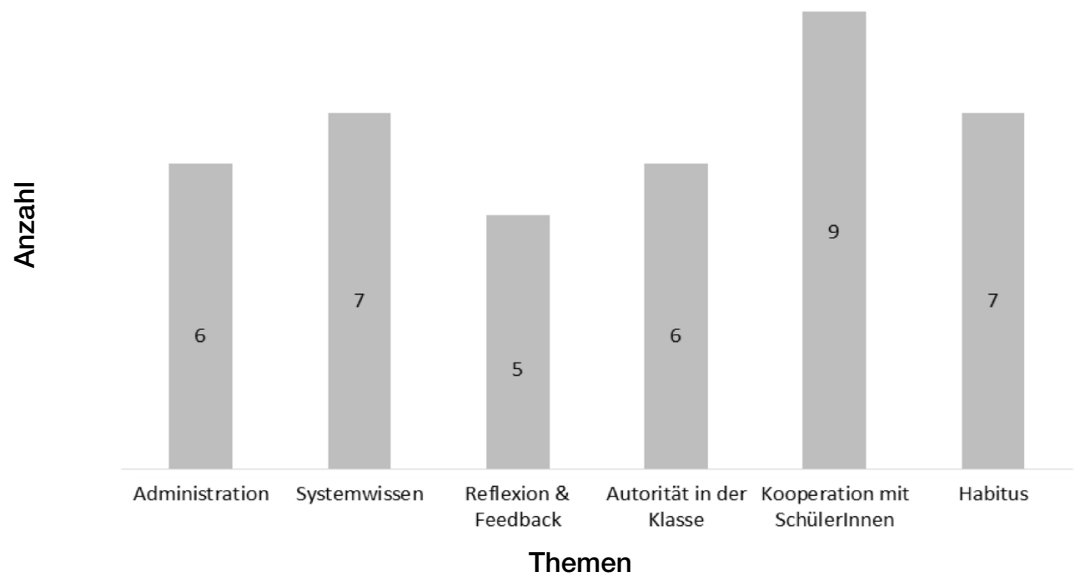

Abb. 62: Vernetzungsgrad nach Themen

\section{Vernetzungsgrad: Hauptkategorien mit Themen}

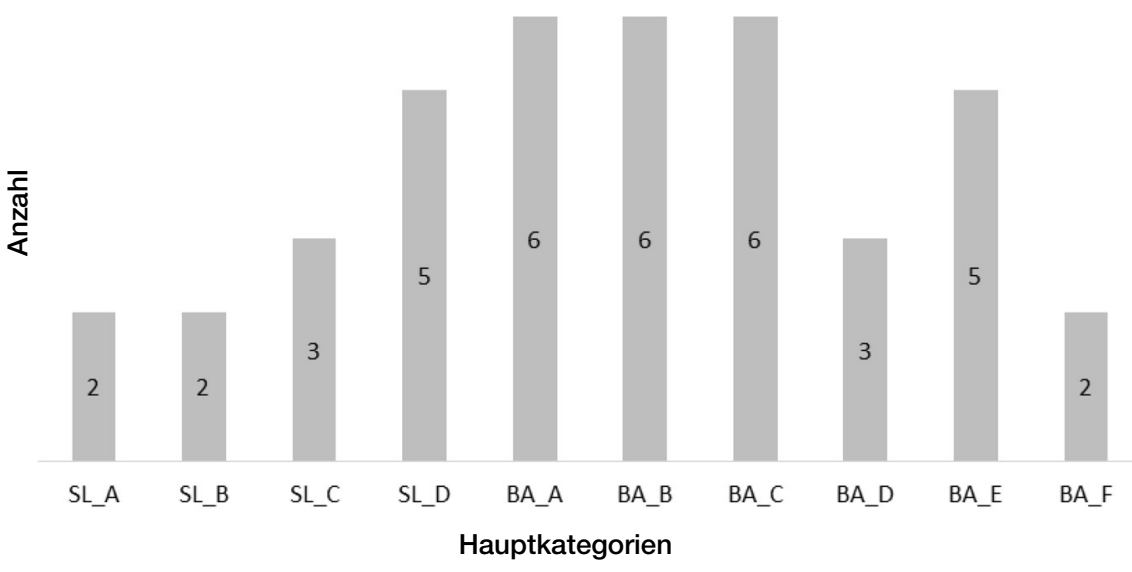

Abb. 63: Vernetzungsgrad nach Hauptkategorien (Legende s. Abb. 61)

In Bezug auf die Hauptkategorien mit Blickrichtung Abszisse (Themenachse) wird in Abbildung 63 dargestellt, mit wie vielen Themen die jeweilige Hauptkategorie vernetzt ist, daraus wird die Relevanz der jeweiligen Hauptkategorie abgeleitet. 
Die Vernetzungswerte der Hauptkategorien mit Themen reichen von zwei (z.B.SL_A) bis sechs (z.B. BA_A) (Abb. 63). In der vorliegenden Arbeit entspricht 2 einem (relativ) niedrigen und 6 einem (relativ) hohen Vernetzungsgrad. Die Hauptkategorien Kompetenzen von Berufsanfängerinnen aus der Sicht der SchulleiterInnen (SL_A), Institutionelle Ausbildung aus der Sicht der SchulleiterInnen (SL_B) und Arbeitsbelastung \& Überforderung (BA_F) weisen mit jeweils zwei Themen einen relativ niedrigen Vernetzungsgrad auf. Die Hauptkategorien Wahrnehmung der BerufsanfängerInnen durch die SchulleiterInnen (SL_C) und Disziplin \& Classroom Management (BA_D) mit jeweils drei Vernetzungen sowie Unterstützung und Begleitung der BerufsanfängerInnen im Schulalltag (SL_D) und Kriterien für Berufszufriedenheit der BerufsanfängerInnen (BA_E) mit jeweils fünf Vernetzungen sind mittelstark vernetzt. Die Hauptkategorien Ersterfahrungen der BerufsanfängerInnen beim Berufseinstieg (BA_A), Mentoring aus der Sicht der BerufsanfängerInnen (BA_B) und Institutionelle Ausbildung aus der Sicht der BerufsanfängerInnen $\left(B A_{-} C\right)$ sind mit jeweils sechs Vernetzungen in Bezug auf die vorliegende Untersuchung stark vernetzt.

Die Strukturierung des Datenmaterials in einer Matrix in der Form Hauptkategorie mal Thema ähnelt einer Datenmatrix in der quantitativen Forschung (Kuckartz 2016, S. 49). Im Unterschied dazu sind in der vorliegenden Forschungsarbeit nicht Korrelationskoeffizienten etc., sondern die bereits erläuterten qualitativ interpretierten und interpretierbaren Schlüsselbegriffe von besonderem Interesse. Insofern werden mit der Darstellung dieser Kategorien-Themen-Matrix (Tab. 11) und der inkludierten Vernetzungszusammenhänge in Form der Schlüsselbegriffe zwei Ziele verfolgt. Zum einen wird an dieser Stelle ganz im Unterschied zum Akt der Kategorien- und Konzeptbildung der Abstraktionsgrad verringert und im Sinne der Deduktion (bei vorhandenen Themen und Hauptkategorien) explizit nach konkreten Zusammenhängen (Vernetzungen) gesucht. Damit kann auch der Intention dieser Arbeit, der Darstellung von mikrodynamischen Zusammenhängen, nachgekommen werden. Zum anderen dient das in Tabelle 11 extrahierte Datenmaterial inkl. der qualitativen Vernetzungszusammenhänge als Grundlage für die im Anschluss dargestellten und interpretierten Bedingungsgefüge.

\subsection{Bedingungsgefüge}

Die Bedingungsgefüge werden zu den einzelnen Themen Habitus, Kooperation mit SchülerInnen, Autorität in der Klasse, Reflexion \& Feedback, Systemwissen und Administration erstellt. Inhaltlich verknüpfte Hauptkategorien werden über Schlüsselbegriffe und Essenzen miteinander in Beziehung gebracht. Den Schlüsselbegriffen und Essenzen (Tab. 11) können interpretativ Funktionen zugeschrieben werden, die die Abhängigkeiten bzw. Beziehungen der Hauptkategorien untereinander signalisieren. Es handelt sich hierbei um die Termini Voraussetzungen, Ursachen, Maßnahmen, Konsequenzen und Wirkungen. ${ }^{75}$ Die Interpretation und Darstellung der Bedingungsgefüge zu den einzelnen Themen ${ }^{76}$ erfolgt unter dem Gesichtspunkt Professionalisierung durch Kompetenzentwicklung, dem Ergebnis der Clusteranalyse bzw. der finalen Clusterkategorie 3 auf Clusterkategorienebene II (CK_II_3) bzw. der Ebene der Konzeptentwicklung. Dabei ist besonders zu erwähnen, dass das Thema Habitus ein Metathema darstellt. Dieses ist zusätzlich zur Clusterkategorie 3 (CK_II_3) auch mit allen anderen Themen inhaltlich verbunden (Abb. 64).

75 Die Beschreibung sowie die Intention der Verwendung der Termini Voraussetzung, Ursache etc. ist in Methodologie (Kap. III.2 bzw. III.2.3) näher ausgeführt.

76 Habitus, Kooperation mit SchülerInnen, Autorität in der Klasse, Reflexion \& Feedback, Systemwissen, Administration 


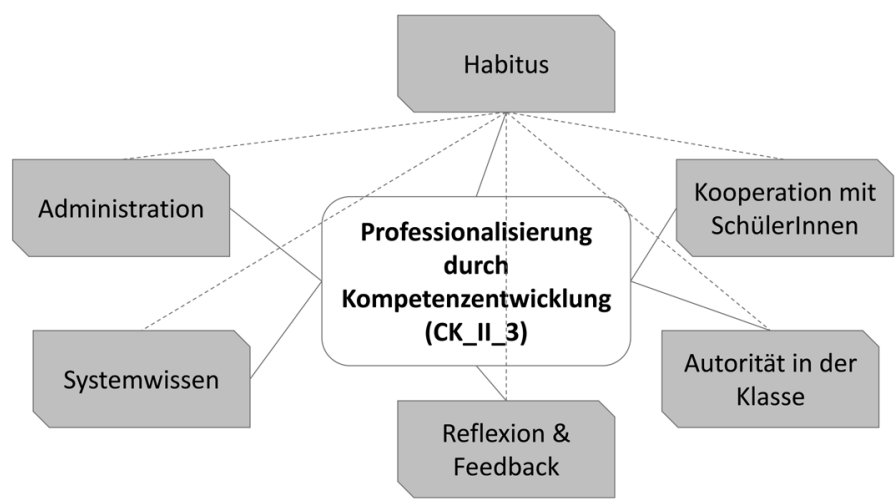

Abb. 64: Themen zur Interpretation: Übersicht und Zusammenhänge

Die Themen Habitus, Kooperation mit SchülerInnen, Autorität in der Klasse, Reflexion \& Feedback, Systemwissen und Administration sind über das Konzept der vorliegenden Arbeit, Professionalisierung durch Kompetenzentwicklung (CK_II_3), vernetzt. Habitus ist als Metathema überdies mit allen anderen Themen inhaltlich verbunden (Abb. 64).

\subsubsection{Vorbemerkungen zu Abbildungen und Tabellen der Bedingungsgefüge}

In den Abbildungen der Bedingungsgefüge (Kap. III.4.2.2 - Kap. III.4.2.7) stellen die Knotenpunkte jeweils jene Hauptkategorien dar, in denen die Themen Habitus, Kooperation mit SchülerInnen, Autorität in der Klasse, Reflexion \& Feedback, Systemwissen und Administration forschungstechnisch relevant angesprochen wurden. Als Grundlage dient die ThemenKategorien-Matrix (Kap. III.4.1, Tab. 11). Die Wirkungslinien symbolisieren die thematischen Überschneidungen in Form der bereits genannten Zusammenhänge Voraussetzungen, Ursachen, Maßnahmen, Konsequenzen und Wirkungen. In den verbalen Interpretationen werden die Termini kursiv formatiert, damit der schnelle und unmissverständliche Zusammenhang zu den Abbildungen und Tabellen der Bedingungsgefüge hergestellt werden kann. Die interpretierten Termini emergieren aus dem Kontext der Interviewanalyse und aus dem Forschervorwissen nach 26-jähriger einschlägiger Berufserfahrung.

Zur Visualisierung der Zusammenhänge von Hauptkategorien dienen Abbildungen (Abb. 65-Abb. 70), die thematische Einordnung verschiedener Termini erfolgt zusätzlich in Tabellen (Tab. 12 - Tab. 17). Die Begriffe in den rechteckigen Feldern auf den Wirkungslinien in den Abbildungen stellen die Schlüsselbegriffe bzw. Essenzen aus der strukturierenden qualitativen Inhaltsanalyse sowie interpretierte Termini dar. In den Tabellen werden die Bedeutungen der Termini zwischen den Knotenpunkten (Bedingung, Zusammenhang) sowie deren Position in der Abbildung dargestellt. Die Reihung der Termini innerhalb der Tabelle erfolgt alphabetisch.

\subsubsection{Bedingungsgefüge Administration}

Das Bedingungsgefüge Administration wird aus den Knotenpunkten (Hauptkategorien) Unterstützung \& Begleitung der BA im Schulalltag (SL_D), Ersterfahrungen der BA beim Berufseinstieg (BA_A), Mentoring aus der Sicht der BA (BA_B), Institutionelle Ausbildung aus der Sicht der BA (BA_C), Kriterien für Berufszufriedenheit der BA (BA_E) und Institutionelle Ausbildung aus der Sicht der SL (SL_B) gebildet (Abb. 65). 


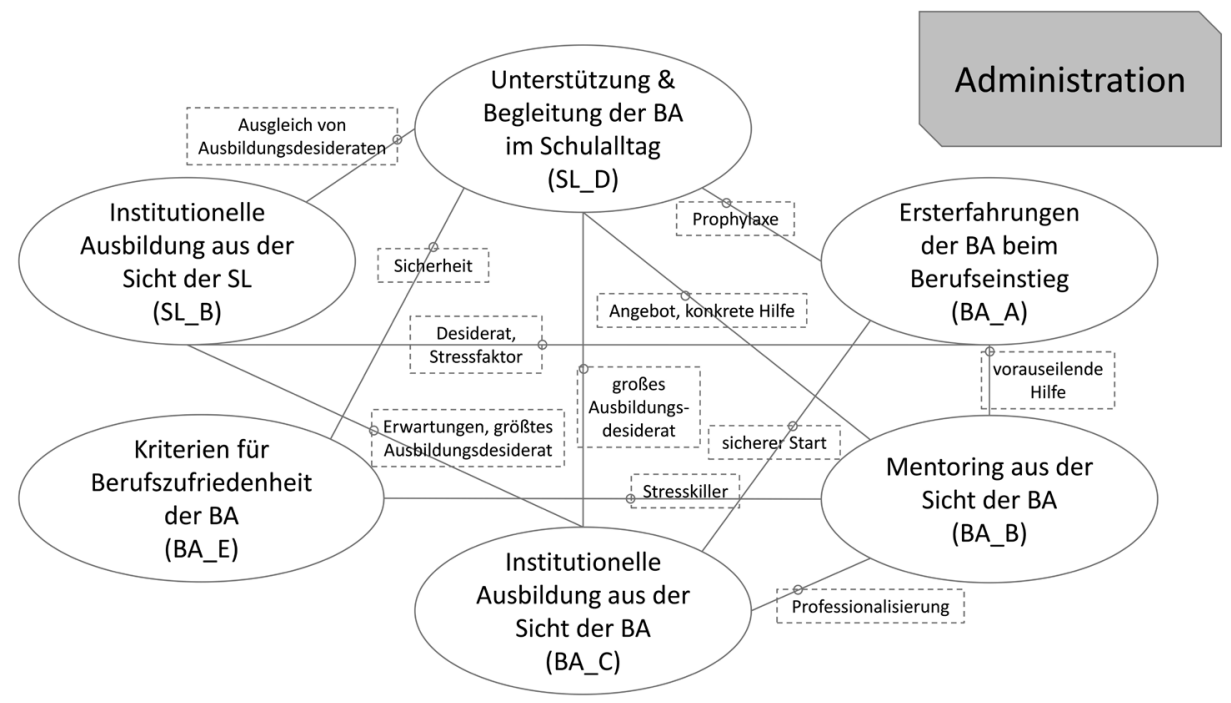

Abb. 65: Bedingungsgefüge Administration

Tab. 12: Bedingungsgefüge Administration; Übersicht

\begin{tabular}{lll}
\hline Terminus & $\begin{array}{l}\text { Zusammenhang } \\
\text { Voraussetzung, Ursache, Maßnah- } \\
\text { me, Konsequenz, Wirkung }\end{array}$ & \\
\hline Angebot, konkrete Hilfe & Maßnahme & BA_B - SL_D \\
Ausgleich von Ausbildungsdesideraten & Konsequenz & SL_B - SL_D \\
Desiderat, Stressfaktor & Ursache, Wirkung & BA_A - SL_B \\
Erwartungen, größtes Ausbildungsdesiderat & Ursache, Wirkung & BA_C - SL_B \\
großes Ausbildungsdesiderat & Ursache & BA_C -SL_D \\
Professionalisierung & Wirkung, Konsequenz & BA_B - BA_C \\
Prophylaxe & Maßnahme & BA_A-SL_D \\
sicherer Start & Wirkung & BA_A - BA_C \\
Sicherheit & Wirkung & BA_E - SL_D \\
Stresskiller & Bedingung & BA_B - BA_E \\
vorauseilende Hilfe & Maßnahme & BA_A - BA_B \\
\hline
\end{tabular}

Der Knotenpunkt Unterstützung und Begleitung der BA im Schulalltag (SL_D) spielt im Bedingungsgefüge Administration eine zentrale Rolle, was durch die hohe Vernetzung mit den anderen Knotenpunkten sichtbar wird. Bezogen auf die Ersterfahrungen der BA beim Berufseinstieg (BA_A) werden die SchulleiterInnen prophylaktisch tätig, um den BerufsanfängerInnen einen möglichst reibungslosen Schulstart zu ermöglichen. „Die organisatorische Seite natürlich [...]“ (SL_12:31) steht hier im Vordergrund. Die konkrete Hilfe in administrativen Belangen (Mentoring aus der Sicht der BA) wird durch die BerufsanfängerInnen, in diesem Fall Mentees, ${ }^{77}$ insbesondere 
von den SchulleiterInnen in Anspruch genommen: „Da habe ich dann Gott sei Dank die Hilfe des Direktors [...] bekommen." (BA_12:34) Was sich direkt auf die Berufszufriedenheit (Kriterien für Berufszufriedenheit der BA) in Form eines Stresskillers auswirkt. Ebenso gibt die Unterstützung \& Begleitung der BA im Schulalltag den BerufsanfängerInnen Sicherheit, „[...] also schöner kann man es nicht haben, dass man die Unterstützung jederzeit bekommt [...]“ (BA_18: 24), was wiederum positiv auf den Grad der Berufszufriedenheit wirkt. SchulleiterInnen sehen sich durchaus verpflichtet, für den Ausgleich von Ausbildungsdesideraten seitens der institutionellen Ausbildung zu sorgen. „Sie [die BerufsanfängerInnen, Anm.] erwarten einmal viel Vorinformation. Weil sie [...] keine Ahnung haben [...].“ (SL_04: 22) Dieses Desiderat bewirkt bei den BerufsanfängerInnen Stress (Desiderat, Stressfaktor), „[du weißt nicht], wie du in bestimmten Situationen reagieren sollst“ (BA_16: 31), und wird zusätzlich zur Unterstützung der SchulleiterInnen auch durch die vorauseilende Hilfe der KollegInnen abgefedert. „[...] sie [die KollegInnen, Anm.] haben mich aufgebaut. [...] Das war gewaltig" (BA_13: 87).

Zusammenfassend ist festzuhalten, dass der Bereich Administration aus der Sicht der BerufsanfängerInnen und der SchulleiterInnen das größte Ausbildungsdesiderat im Rahmen der LehrerInnenausbildung darstellt. Dies wirkt sich äußerst negativ auf den sicheren Start in das Berufsleben aus. Damit diesen Unsicherheiten, „[... ] ich weiß nicht wie ich sagen soll, aber da hat man es ganz schwierig“ (BA_01: 79), weitgehend entgegengewirkt werden kann, wird im Sinne der ganzheitlichen Professionalisierung in der Aus- und Weiterbildung in Form eines formellen MentoringProzesses (Induktionsphase) auch gezielt auf administrative Inhalte einzugehen sein.

\subsubsection{Bedingungsgefüge Systemwissen}

Das Bedingungsgefüge Systemwissen besteht aus den Knotenpunkten Institutionelle Ausbildung aus der Sicht der SL (SL_B), Unterstützung \& Begleitung der BA im Schulalltag (SL_D), Ersterfahrungen der BA beim Berufseinstieg (BA_A), Mentoring aus der Sicht der BA (BA_B), Institutionelle Ausbildung aus der Sicht der BA (BA_C), Kriterien für Berufszufriedenheit der BA (BA_E) und Arbeitsbelastung \& Überforderung der BA (BA_F) (Abb. 66).

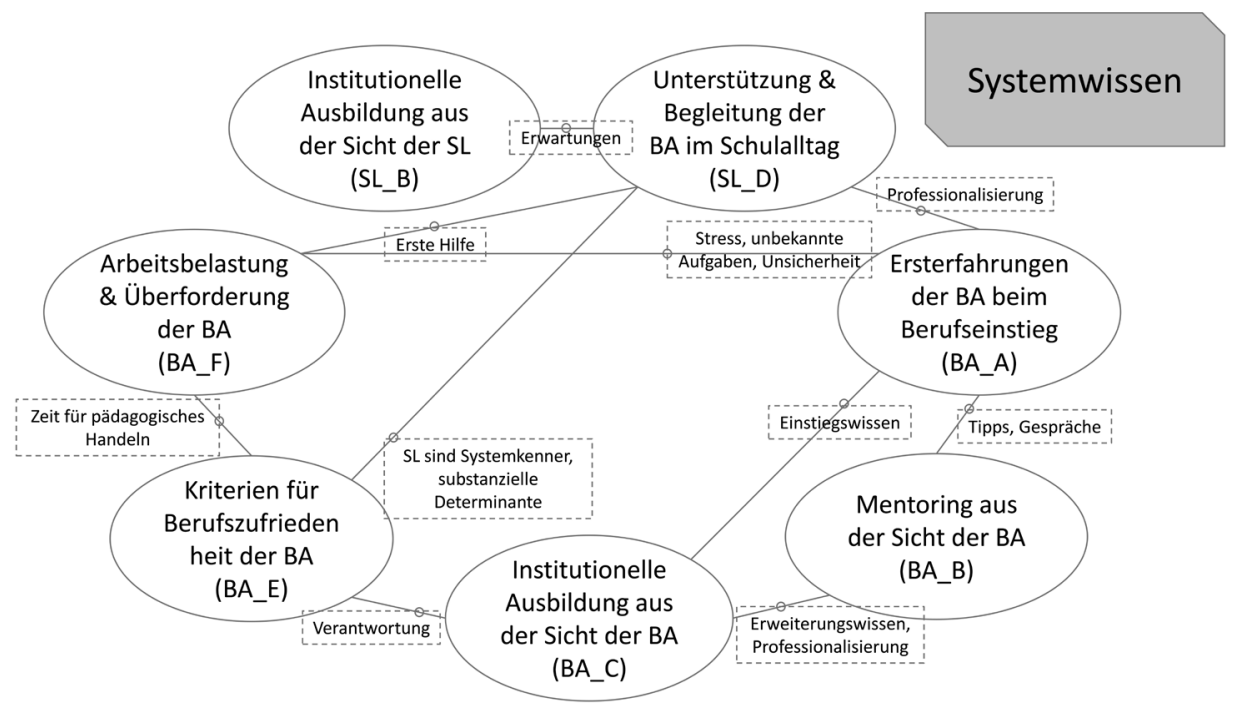

Abb. 66: Bedingungsgefüge Systemwissen 
Tab. 13: Bedingungsgefüge Systemwissen; Übersicht

\begin{tabular}{lll}
\hline Terminus & $\begin{array}{l}\text { Zusammenhang } \\
\text { Voraussetzung, Ursache, Maß- } \\
\text { nabme, Konsequenz, Wirkung }\end{array}$ & \\
\hline Einstiegswissen & Voraussetzung & BA_A - BA_C \\
Erste Hilfe & Maßnahme & BA_F - SL_D \\
Erwartungen & Voraussetzung & SL_B - SL_D \\
Erweiterungswissen, Professionalisierung & Konsequenz, Wirkung & BA_B - BA_C \\
Professionalisierung & Wirkung & BA_A - SL_D \\
SL sind Systemkenner, substanzielle Determinante & Voraussetzung, Wirkung & BA_E - SL_D \\
Stress, unbekannte Aufgaben, Unsicherheit & Ursache, Wirkung & BA_A - BA_F \\
Tipps, Gespräche & Maßnahme & BA_A - BA_B \\
Verantwortung & Voraussetzung & BA_C - BA_E \\
Zeit für pädagogisches Handeln & Wirkung, Voraussetzung & BA_E-BA_F \\
\hline
\end{tabular}

Die Vernetzung der einzelnen vorhandenen Knotenpunkte ist im Bedingungsgefüge Systemwissen sehr ausgeglichen. Einzig Institutionelle Ausbildung aus der Sicht der SL (SL_B) ist weniger stark eingebunden, was den Schluss zulässt, dass in der Ausbildung ein umfassendes Systemwissen aus der Sicht der SchulleiterInnen nicht weitergegeben werden kann. Die verschiedenen Systeme einzelner Schultypen und -standorte sind zu verschieden, um adäquat vermittelt werden zu können. Diese Informationen erhalten die BerufsanfängerInnen aus der Sicht der SchulleiterInnen weitgehend in den ersten Berufsjahren und die Erwartungen an die Ausbildung in dieser Hinsicht sind gering, denn „das [ist] ein großer Teil, der einfach an der PH und der UNI nicht vermittelt werden kann“ (SL_06: 44). Insbesondere im Schultyp der Polytechnischen Schule tragen die SchulleiterInnen wesentlich zur Professionalisierung in der ersten Zeit des Schuleinritts der BerufsanfängerInnen im Sinne von „die Struktur der PTS erklären“ (SL_13: 22) bei, denn das ist „für alle [BerufsanfängerInnen, Anm.] mehr oder weniger Neuland“ (ebd.). Die Institutionelle Ausbildung aus der Sicht der BA bietet in den schulpraktischen Studien neben dem fachlichen auch ein systemisches Einstiegswissen, denn da „sieht man halt den Schulalltag“ (BA_20: 51). Die Tipps und Gespräche haben im Mentoring aus der Sicht der BA neben den pädagogischen auch in systemischen Belangen einen hohen Stellenwert, denn „das sind Sachen, die man in der Ausbildung zu wenig hört“ (BA_07: 38), und tragen als Erweiterungswissen ganz wesentlich zur Professionalisierung der BerufsanfängerInnen bei. Die Unsicherheit, das „Unwissen“ der BerufsanfängerInnen über unbekannte Aufgaben führen zu Stress. So war „das erste Jahr war vom ganzen Drumherum schon schlimm“ (BA_12: 29) und trägt zur Arbeitsbelastung \& Überforderung bei. Besonders bei „rechtlicher Absicherung" (SL_13: 30) bieten SchulleiterInnen Erste Hilfe und sind als Systemkenner die Expertinnen bzw. Experten für die BerufsanfängerInnen. Diese Sicherheit stellt eine substanzielle Determinante für die BerufsanfängerInnen zur Berufszufriedenheit dar. Die Berufszufriedenheit der BA kann in der Gesamtschau auf das Systemwissen zusätzlich erhöht werden, indem die Institutionelle Ausbildung die Verantwortung zur Grundsteinlegung einer hohen Berufszufriedenheit wahrnimmt und die Vermittlung von Systemwissen nicht ignoriert. Der Grad der Arbeitsbelastung \& Überforderung soll minimiert werden, dadurch bleibt den Be- 
rufsanfängerInnen mehr Zeit für pädagogisches Handeln und um „ein sehr gutes Verhältnis zu den Schülern“ (BA_04: 26) aufbauen zu können.

Zusammenfassend wird festgehalten, dass Ausbildungsdesiderate im Systemwissen bei den BerufsanfängerInnen Stress auslösen. Den SchulleiterInnen und erfahrenen KollegInnen kommt in diesem Zusammenhang die Rolle der MentorInnen zu, einerseits durch Tipps das Wissen weiterzugeben, andererseits durch Gespräche die Berufszufriedenheit der jungen KollegInnen zu steigern und zur Professionalisierung beizutragen. Ressourcen der BerufsanfängerInnen, die durch Unwissen im System gebunden werden, fehlen bei der pädagogischen Arbeit mit SchülerInnen.

\subsubsection{Bedingungsgefüge Reflexion \& Feedback}

Das Bedingungsgefüge Reflexion \& Feedback wird aus den Knotenpunkten Unterstützung \& Begleitung der BA im Schulalltag (SL_D), Ersterfahrungen der BA beim Berufseinstieg (BA_A), Mentoring aus der Sicht der BA (BA_B), Institutionelle Ausbildung aus der Sicht der BA (BA_C) und Kriterien für Berufszufriedenheit der BA (BA_E) gebildet (Abb. 67).

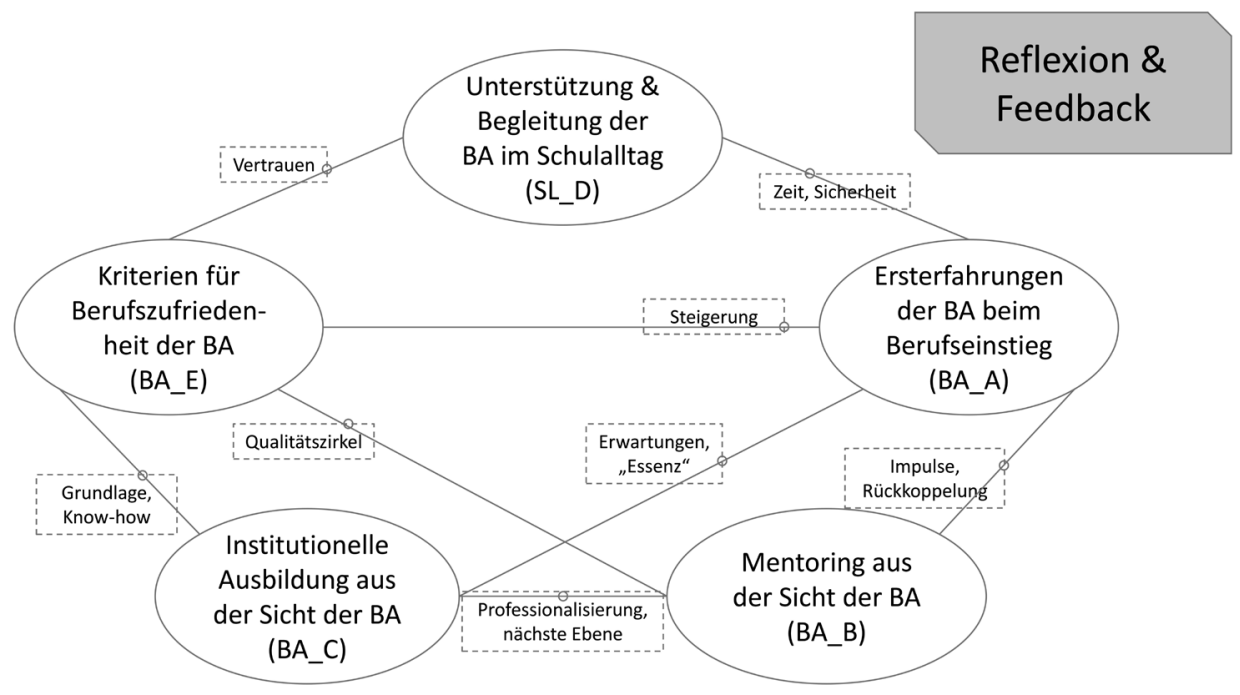

Abb. 67: Bedingungsgefüge Reflexion \& Feedback

Das Thema Reflexion \& Feedback wird eindeutig von den BerufsanfängerInnen initiiert (vier von fünf Knotenpunkten). Der Anteil der SchulleiterInnen besteht darin, die im Rahmen ihrer Möglichkeiten notwendigen Voraussetzungen zu schaffen. Einerseits geht es darum, den BerufsanfängerInnen die nötige Zeit und Sicherheit zu geben. „Wenn man ihnen [den BerufsanfängerInnen, Anm.] ein bisschen entgegen kommt und den Einstieg auf emotionaler Ebene erleichtert“ (SL_12: 24), wirkt sich das positiv auf den Berufseinstieg aus. 
Tab. 14: Bedingungsgefüge Reflexion \& Feedback; Übersicht

\begin{tabular}{lll}
\hline Terminus & $\begin{array}{l}\text { Zusammenhang } \\
\text { Voraussetzung, Ursache, Maßnah- } \\
\text { me, Konsequenz, Wirkung }\end{array}$ & \\
& Voraussetzung & \\
\hline Erwartungen, „Essenz“ & Voraussetzung & BA_A - BA_C \\
Grundlage, Know-how & Maßnahme & BA_C - BA_E \\
Impulse, Rückkoppelung & Wirkung & BA_A - BA_B \\
Professionalisierung, nächste Ebene & Maßnahme & BA_B-BA_C \\
Qualitätszirkel & Wirkung & BA_B-BA_E \\
Steigerung & Voraussetzung & BA_A-BA_E \\
Vertrauen & Voraussetzung & BA_E-SL_D \\
Zeit, Sicherheit & & BA_A - SL_D \\
\hline
\end{tabular}

Ebenso wirkt sich das Feedback der SchulleiterInnen für die BerufsanfängerInnen gewinnbringend aus, wenn auf einer Vertrauensbasis „Feedback als Hilfe“ (SL_03: 30) von einem „treuen Begleiter“ (ebd.) und nicht von einem „Aufsichtsorgan“ (SL_14: 26) gegeben wird. Nach den teilweise heftigen Ersterfahrungen („[Sprung] ins kalte Wasser“ (BA_18: 27)) sind die BerufsanfängerInnen „viel beim Nachdenken, beim Reflektieren“ (BA_14: 28) und bewirken damit eine Steigerung ihrer Berufszufriedenheit. Ebenso wirkt sich das Mentoring aus der Sicht der BA positiv auf die Berufszufriedenheit der BA aus, indem nach dem Muster eines Qualitätszirkels das Mentoring die Reflexion stets tiefer werden lässt. Die Institutionelle Ausbildung trägt über Umwege zur Berufszufriedenheit bei, indem durch das Know-how die Grundlage für einen adäquaten Reflexionsprozess und den Umgang mit Feedback gelegt wird. Weiters ist dies auch der Grundstein für die Reflexion und das Feedback im Mentoring-Prozess auf der Weiterbildungsebene, was schließlich im Sinne der Profession eine für LehrerInnen essenzielle Kompetenz darstellt. Die Erwartungen der BerufsanfängerInnen an die Institutionelle Ausbildung und an den Mentoring-Prozess sind bezogen auf Reflexion \& Feedback sehr hoch, zumal das Feedbackgeben ein zentraler Bestandteil der LehrerInnenaktivität darstellt. Allerdings wird dieser Themenkomplex ambivalent diskutiert. Für die BerufsanfängerInnen „steckt ja da die Essenz eigentlich drinnen“ (BA_20: 44), für zumindest eine/n SchulleiterIn ist die „Reflexionsfähigkeit von Lehrern ja gleich Null komma Null“ (SL_10: 37). Wenngleich diese letzte Aussage nicht als signifikant zu sehen ist, stellt sie doch die Notwendigkeit der Reflexions- und Feedbackkompetenz außer Frage. Eine Möglichkeit zur Erhöhung dieser Kompetenzen besteht in der Rückkoppelung zwischen BerufsanfängerInnen bereits nach ihren Ersterfahrungen und den MentorInnen sowie durch gezielte Impulse seitens der SchulleiterInnen und KollegInnen. In dieser Hinsicht „habe ich total viel bekommen, die [KollegInnen, Anm.] haben mich da sehr unterstützt“"(BA_08: 30) bestätigt ein/e BerufsanfängerIn.

Abschließend kann gesagt werden, dass Reflexion \& Feedback sehr stark im Zusammenhang mit Mentoring diskutiert wird. SchulleiterInnen und BerufsanfängerInnen sind sich der Relevanz des Feedbacks und Reflexionskompetenz bewusst. Diese Kompetenzen wirken sich u.a. auf die Vertrauensbasis, die Zufriedenheit und letztlich über den Qualitätsgewinn auf den Professionalisierungsprozess der BerufsanfängerInnen aus. Die Erwartungen der BerufsanfängerInnen in Bezug auf Feedback und Reflexion sind sehr hoch. 


\subsubsection{Bedingungsgefüge Autorität ${ }^{78}$ in der Klasse}

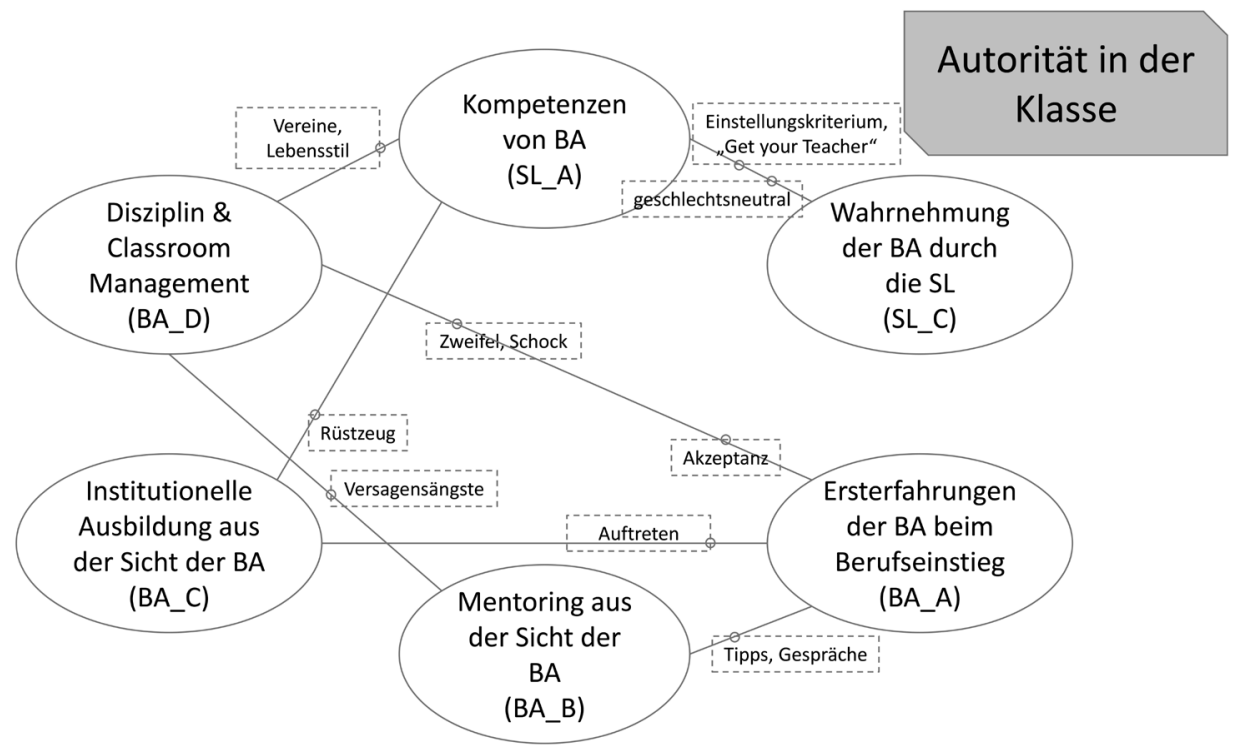

Abb. 68: Bedingungsgefüge Autorität in der Klasse

Das Bedingungsgefüge Autorität in der Klasse, dargestellt in Abbildung 68, wird durch die Knotenpunkte Kompetenzen von BA (SL_A), Wahrnehmung der BA durch die SL (SL_C), Ersterfahrungen der BA beim Berufseinstieg (BA_A), Mentoring aus der Sicht der BA (BA_B), Institutionelle Ausbildung aus der Sicht der BA (BA_C) und Disziplin \& Classroom Management (BA_D) gebildet.

Tab. 15: Bedingungsgefüge Autorität in der Klasse; Übersicht

\begin{tabular}{lll}
\hline Terminus & $\begin{array}{l}\text { Zusammenhang } \\
\text { Voraussetzung, Ursache, Maßnah- } \\
\text { me, Konsequenz, Wirkung }\end{array}$ & \\
\hline Akzeptanz & Voraussetzung, Wirkung & BA_A - BA_D \\
Auftreten & Voraussetzung, Wirkung & BA_A - BA_C \\
Einstellungskriterium, Get Your Teacher & Voraussetzung & SL_A - SL_C \\
geschlechtsneutral & Voraussetzung & SL_A - SL_C \\
Rüstzeug & Voraussetzung & BA_C - SL_A \\
Tipps, Gespräche & Maßnahme & BA_A - BA_B \\
Vereine, Lebensstil & Voraussetzung & BA_D -SL_A \\
Versagensängste & Wirkung & BA_B - BA_D \\
Zweifel, Schock & Wirkung & BA_A - BA_D \\
\hline
\end{tabular}

78 In dieser Diskussion wird der Terminus Autorität im Sinne von „einflussreiche, maßgebende Persönlichkeit von hohem [fachlichem] Ansehen" (Duden 2017) in der Klasse verstanden. 
Im Bedingungsgefüge Autorität in der Klasse wird die Autorität der Berufsanfängerin bzw. des Berufsanfängers im Zusammenhang mit dem Wirken in der Klasse, also dem Umgang mit SchülerInnen, thematisiert. Im Unterschied dazu, dies sei an dieser Stelle bereits klärend vorweggenommen, geht es im Bedingungsgefüge Habitus um das Erscheinungsbild (Riecke 2014), um das Auftreten der Lehrperson, welches nicht zwangsläufig im Kontext zur Arbeit mit SchülerInnen steht.

Die SchulleiterInnen nehmen bezüglich der Autorität von BerufsanfängerInnen in der Klasse ${ }^{79}$ keine geschlechtsspezifischen Unterschiede wahr. „Es ist nicht so, dass da bei uns ein Unterschied wäre“" (SL_01: 34), wird etwa bestätigt. Dies lässt den Schluss zu, dass das Geschlecht der jungen Lehrkraft in zweierlei Hinsicht keine unmittelbare Rolle spielen muss, erstens für den/die Betroffene/ $\mathrm{n}$ bei der Ausbildungsentscheidung und zweitens für die Schulleitung bei der Auswahl der Lehrkraft. Zukünftig werden SchulleiterInnen im Zuge der autonomen Schule mehr in den Auswahlprozess einbezogen und in diesem Zusammenhang kann die Autorität in der Klasse sehr wohl ein Einstellungskriterium darstellen. „Erwarten Sie von der neuen Lehrkraft Kompetenzen für Aufgaben, die über das Unterrichtsfach hinausgehen?“ (Bundesministerium für Bildung, Wissenschaft und Forschung 2018), lautet eine Grundfrage, die im LehrerInneneinstellungs-Programm Get-YourTeacher ${ }^{80}$ (ebd., S. 13) gestellt wird. Einen großen Vorteil haben BerufsanfängerInnen in Bezug auf die Autorität in der Klasse, wenn sie z.B. in Vereinen (SL_02: 63) die Persönlichkeit (ebd.: 62) bilden und auf einen anderen Werdegang bzw. Lebensstil (SL_01: 53) zurückblicken können. Trotzdem spielen im Bereich Disziplin \& Classroom Management Versagensängste eine große Rolle. „Die größten Probleme waren eindeutig die disziplinären“ (BA_13: 41), heißt es. Tipps und Gespräche im Rahmen des Mentoring-Prozesses können diesen Missstand ev. nicht beheben, aber mildern. Mit steigender Akzeptanz, „[...) ja das war ja ein Drama“ (BA_04: 26), der BerufsanfängerInnen durch die SchülerInnen weichen Schock und Zweifel, „[da] habe ich mir noch überlegt, ob ich nicht aufhören [zum Unterrichten, Anm.] soll“ (BA_13: 21), um schließlich behaupten zu können: „Aber jetzt inzwischen gefällt es mir gut." (BA_15: 23) Ein Schlüssel dafür ist das Zurechtrücken der Autoritätsverhältnisse innerhalb der Klasse.

Die fehlende Routine wirkt sich negativ auf das Auftreten der BerufsanfängerInnen in der Klasse aus, weil „du nicht weißt, wie du in bestimmten Situationen reagieren sollst“ (BA_16: 31). Insofern ist es umso wichtiger, den BerufsanfängerInnen im Rahmen der institutionellen Ausbildung das nötige Rüstzeug im Sinne von LehrerInnenkompetenzen mitzugeben, um sich so die Autorität in der Klasse zu holen. Mit der „extreme[n] Vielfalt an Methodik und Didaktik“ (SL_10: 41), die BerufsanfängerInnen bereits als Einstiegskompetenzen mitbringen, ist ein wichtiger Schritt in diese Richtung bereits getan.

Zusammenfassend wird festgehalten, dass SchulleiterInnen keine geschlechtsspezifischen Unterschiede bezüglich der Autorität von BerufsanfängerInnen in der Klasse wahrnehmen. Das Thema Classroom Management spielt für BerufsanfängerInnen eine zentrale Rolle, Versagensängste in Bezug auf die Autorität thematisiert. Mentoring bildet auch beim Thema Autorität einen zentralen Aspekt. So wird von den BerufsanfängerInnen erwartet, durch Tipps und Gespräche die fehlende Routine zumindest zu relativieren.

\subsubsection{Bedingungsgefüge Kooperation mit SchülerInnen}

Das Bedingungsgefüge Kooperation mit SchülerInnen ist das am dichtesten vernetzte Thema der vorliegenden Untersuchung. Die Knotenpunkte bilden Kompetenzen von BA (SL_A),

79 Diese Wahrnehmung gilt auch für andere Kriterien (z.B. Beliebtheit, Empathie etc.).

80 Bundesweite Software zur Unterstützung des LehrerInneneinstellungs-Prozesses 
Wahrnehmung der BA durch die SL (SL_C), Unterstützung \& Begleitung der BA im Schulalltag (SL_D), Ersterfahrungen der BA beim Berufseinstieg (BA_A), Mentoring aus der Sicht der BA (BA_B), Institutionelle Ausbildung aus der Sicht der BA (BA_C), Disziplin \& Classroom Management (BA_D), Kriterien für Berufszufriedenheit der BA (BA_E) und Arbeitsbelastung \& Überforderung der BA (BA_F) (Abb. 69).

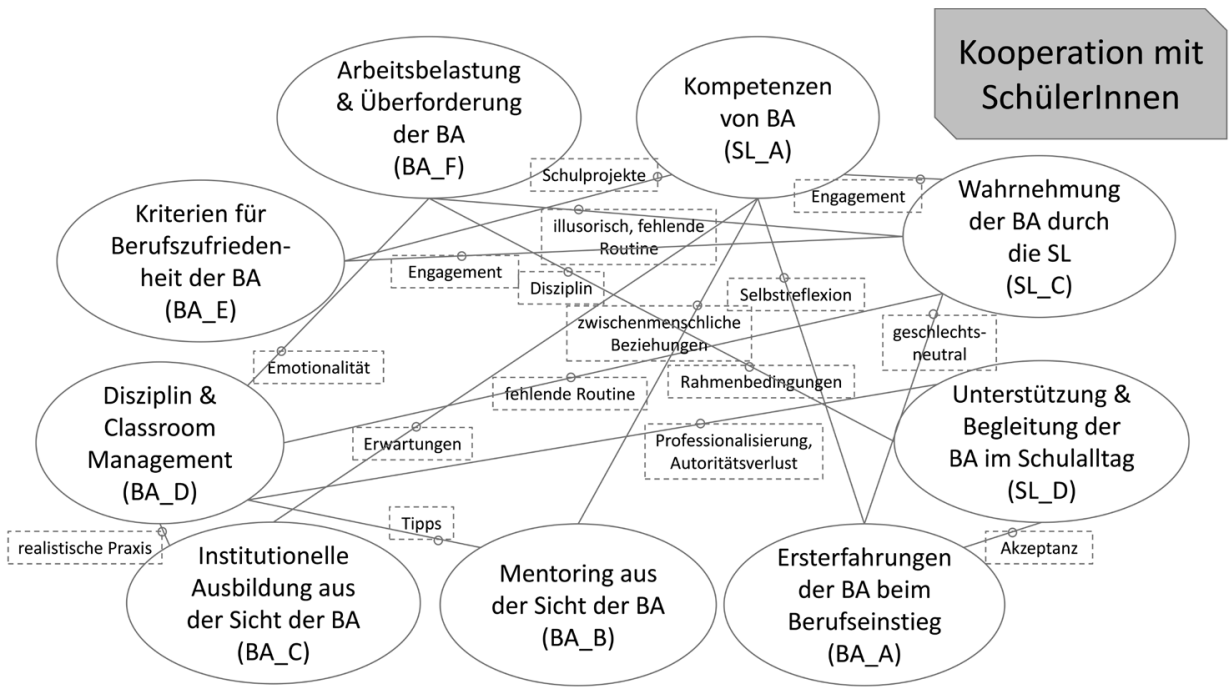

Abb. 69: Bedingungsgefüge Kooperation mit Schülerinnen und Schülern

Der große Themenbereich Kooperation mit SchülerInnen beinhaltet die Zusammenarbeit zwischen BerufsanfängerIn und SchülerIn im weitesten Sinne. Es sind damit die naheliegenden verschiedenen Unterrichtsmethoden bzw. kooperativen Lehr- und Lernformen, das gesamte Classroom Management inklusive des großen Themas Disziplin, aber auch Aktivitäten außerhalb des Unterrichts (Schulveranstaltungen) angesprochen. Die explizit positiv konnotierte Formulierung Kooperation mit SchülerInnen (im Vergleich zu Arbeit mit SchülerInnen) ist keine zufällige. Die zwischenmenschliche Beziehung zwischen BerufsanfängerIn und SchülerIn wird durchgehend thematisiert und als Prinzip im Sinne einer Voraussetzung für guten Unterricht vorangestellt. „Das Zwischenmenschliche ist so wahnsinnig wichtig, weil ich glaube, sonst kann man ihnen [den SchülerInnen, Anm.] das Fachliche gar nicht beibringen." (BA_01: 77). Die Aussage bringt beispielsweise den Grundwert dieses Zusammenhanges (Tab. 16) zum Ausdruck.

Tab. 16: Bedingungsgefüge Kooperation mit Schülerinnen und Schülern; Übersicht

\begin{tabular}{lll}
\hline Terminus & $\begin{array}{l}\text { Zusammenhang } \\
\text { Grundwert, Voraussetzung, Ursache, } \\
\text { Maßnahme, Konsequenz, Wirkung }\end{array}$ & \\
\hline Akzeptanz & Voraussetzung & BA_A - SL_D \\
Disziplin & Voraussetzung, Wirkung & BA_F - SL_D \\
Emotionalität & Wirkung & BA_D - BA_F
\end{tabular}




\begin{tabular}{lll}
\hline Terminus & $\begin{array}{l}\text { Zusammenhang } \\
\text { Grundwert, Voraussetzung, Ursache, } \\
\text { Maßnahme, Konsequenz, Wirkung }\end{array}$ & Position \\
\hline Engagement & Voraussetzung & BA_E - SL_C \\
Engagement & Grundwert & SL_A - SL_C \\
Erwartungen & Voraussetzung & BA_C - SL_A \\
fehlende Routine & Ursache & BA_D - SL_C \\
geschlechtsneutral & Grundwert & BA_A - SL_C \\
illusorisch, fehlende Routine & Ursache & BA_F-SL_C \\
Professionalisierung, Autoritätsverlust & Maßnahme, Wirkung & BA_D - SL_D \\
Rahmenbedingungen & Ursache & BA_F -SL_D \\
realistische Praxis & Voraussetzung, Maßnahme & BA_C - BA_D \\
Schulprojekte & Voraussetzung & BA_E - SL_A \\
Selbstreflexion & Maßnahme & BA_A - SL_A \\
Tipps & Maßnahme & BA_B - BA_D \\
zwischenmenschliche Beziehungen & Grundwert, Voraussetzung, Wirkung & BA_B -SL_A \\
\hline
\end{tabular}

Die zwischenmenschliche Beziehung wird von den BerufsanfängerInnen aber auch als Voraussetzung für kooperatives Arbeiten, ,[...] wenn du den richtigen Draht zu den Schülern findest [...] dann ist es eine super Sache“ (BA_12: 24), und als Wirkung nach guter Zusammenarbeit, „Im Laufe des Jahres habe ich dann [...] irgendwie eine andere Beziehung" (BA_01: 49), formuliert. Die Fähigkeit zur Pflege der zwischenmenschlichen Beziehungen wird den BerufsanfängerInnen von den SchulleiterInnen attestiert. „Sie haben ein gutes Gefühl für die Jugend [...] das gehört zu den Kompetenzen dazu" (SL_04: 46), und zugleich vertrauen die BerufsanfängerInnen auf „ein bisschen Hilfe [...] im zwischenmenschlichen Umgang“" (BA_01: 49) durch die KollegInnen als MentorInnen. Einen ebenso starken Zusammenhang in der Thematik Kooperation mit SchülerInnen stellt das Engagement der BerufsanfängerInnen dar. Einerseits kann dies als Grundwert interpretiert werden, „[...] die Junglehrer kommen normalerweise immer sehr enthusiastisch in die Schule“ (SL_01: 15), andererseits als positive Grundvoraussetzung für einen hohen Grad an Berufszufriedenheit. Dieses Engagement in Bezug auf Kooperationsbereitschaft mit SchülerInnen zeigt sich z.B. in der Durchführung von Schulprojekten. „Ich staune [...], an welche Projekte sich Junglehrer herangewagt haben [...], die zu überraschend positiven Ergebnissen gekommen sind.“ (SL_06: 56) „Ich tue gerne organisieren [und] mich mit den Schülern auseinandersetzen“ (BA_18: 29), zeigt auf der Seite der BerufsanfängerInnen die Berufszufriedenheit, die im weiteren Sinne mit der Kooperation mit SchülerInnen zusammenhängt. Das herausforderndste Thema innerhalb der Kooperation mit SchülerInnen ist der Umgang mit Disziplinproblemen. Folgende Aussage lässt viel Raum für Interpretation:

„Das Hauptproblem war eindeutig die Disziplin. Da habe ich, kommt mir vor, wenig Handhabe mitbekommen [in den Ausbildung, Anm.]. Auf das war ich nicht ganz vorbereitet." (BA_19: 41)

Hier kommt die Arbeitsbelastung bis hin zur Überforderung und zugleich ein Vorwurf an die Ausbildung zum Ausdruck. Die Erwartungen, die BerufsanfängerInnen in einem „Werkzeugkoffer“ (BA_02: 94), „das Werkszeug [sic!] dafür mitzugeben, wie er im Unterricht tun soll“ (ebd.: 48), werden in den Gesprächen regelmäßig thematisiert und als Voraussetzung für einen erfolgreichen Berufsstart im Sinne eines zufriedenstellenden Classroom Management gesehen. Neben den 
MentorInnentipps, „[...] da haben mir in dem Fall dann die Kollegen geholfen und gesagt ,also da musst du das und das probieren“" (BA_05: 47), können auch pädagogisch-praktische Studien in Realsituationen, „[...] weil da hast du wirklich etwas gesehen. Und da hast du auch mit schwierigen Klassen schon zu kämpfen gehabt" (BA_13: 75), den BerufsanfängerInnen hilfreiche Einblicke in erfolgreiches Classroom Management geben. Die feblende Routine aufseiten der BerufsanfängerInnen ist manchmal auch die Ursache dafür, dass sie „mit vielen Illusionen kommen“ (SL_09: 15) und dass die „,idealisierten Vorstellungen [...] nicht mit der Realität übereinstimmen“ (ebd.: 27). Dass nach der längst überwundenen schwarzen Pädagogik die Überforderung der BerufsanfängerInnen deren Emotionalität an eine Grenze bringt, wo „zu Altbewährtem [...], Strafen schreiben oder Nachsitzen lassen, was man theoretisch nicht tun darf [...], aber eigentlich schon [wirkt, zurückgegangen wird]“ (BA_10: 62-63), ist höchst bemerkenswert.

Zur Deeskalation solcher Situationen können die SchulleiterInnen präventiv durch die Schaffung geeigneter Rahmenbedingungen beitragen, durch die Auflösung ungünstiger Gruppen- oder Klassenkonstellationen, durch die Bildung geeigneter Teamteaching-Paare, durch die Installation von geeigneten Ruheräumen und ebenso durch das Engagement von BeratungslehrerInnen. Die SchulleiterInnen können „also alles ausschöpfen, was organisatorisch möglich ist“ (SL_08: 22), um das „Recht auf ungestörten Unterricht“ (SL_13: 42) bestmöglich zu gewährleisten.

Nach den ersten Erfahrungen im Classroom Management bzw. mit der Kooperation mit SchülerInnen erweitern die BerufsanfängerInnen ihre diesbezügliche Kompetenz vielfach durch regelmäßige Selbstreflexion: „Ich habe halt immer versucht, mich zu verbessern, zu reflektieren. [...] Aber ich habe immer noch das Gefühl, ich stehe ziemlich am Anfang." (BA_20: 30) An dieser Stelle ist zu erwähnen, dass sich die vielen Selbstreflexionsanteile im Curriculum zur LehrerInnenausbildung (Leopold-Franzens-Universität Innsbruck 2016) sehr positiv auf diese Kompetenzaneignung auswirken, auch wenn die Studierenden während ihrer Ausbildung den Wert der (Selbst-)Reflexion oftmals nicht erkennen.

Geschlechtsspezifisch sind in Bezug auf die Kooperation mit SchülerInnen bzw. den Umgang mit Herausforderungen keine signifikanten Unterschiede wahrnehmbar: „[...] ich könnte nicht sagen, dass Frauen mehr Probleme hätten oder Männer mehr Probleme hätten." (SL_04: 39) In Bezug auf die Empathiefähigkeit meint ein/e SchulleiterIn: „Den Draht zu finden mit den Schülern [...] das kann ein Mann in dem Sinne oft vielleicht gar nicht schaffen [...], das ist die Chance, die die Junglehrerinnen haben“" (SL_09: 40). „Frauen sind emotionaler, nehmen alles viel persönlicher" (SL_03: 42), erzählt ein/e weitere/r SchulleiterIn.

Abschließend kann gesagt werden, dass die SchulleiterInnen einen wesentlichen und verantwortungsvollen Part in der Professionalisierungsphase beim Berufseintritt in Bezug auf die Kooperation mit SchülerInnen einnehmen. Auf der einen Seite spielen für die positiven Ersterfahrungen der BerufsanfängerInnen Akzeptanz „und eine gewisse Vertrauensbasis“ (SL_03: 30) ein große Rolle. Andererseits beanspruchen die BerufsanfängerInnen die SchulleiterInnen „als Anker“ (SL_01: 31) und als „Amtsautorität“ (SL_13: 41). Die Kunst bzw. das professionelle Agieren der SchulleiterInnen besteht darin, die BerufsanfängerInnen genau im richtigen Maße zu unterstützen. Fällt die Unterstützung zu gering aus, steigt der Grad der Arbeitsbelastung und Überforderung der BerufsanfängerInnen bis hin zum Scheitern. „Wenn ich das so weiter mache, bin ich irgendwann am Ende, das halte ich nicht durch." (BA_03: 34) Agieren die SchulleiterInnen zu progressiv, ,ist es immer mit Autorität verbunden, das heißt, auch mit Gesichtsverlust verbunden, mit Zugeständnis von Schwäche“ (SL_06: 26). Die goldene Mitte kann in diesem Zusammenhang „nicht ,hineingschaftln" ${ }^{\text {(11 }}$, [...] vielleicht in eine Richtung lenken“ (SL_09: 22) sein. 


\subsubsection{Bedingungsgefüge Habitus: Metabedingungsgefüge}

Das Bedingungsgefüge Habitus wird durch die Knotenpunkte Wahrnehmung der BA durch die SL (SL_C), Unterstützung \& Begleitung der BA im Schulalltag (SL_D), Ersterfahrungen der BA beim Berufseinstieg (BA_A), Mentoring aus der Sicht der BA (BA_B), Institutionelle Ausbildung aus der Sicht der BA (BA_C), Disziplin \& Classroom Management (BA_D) und Kriterien für Berufszufriedenheit der BA (BA_E) gebildet (Abb. 70).

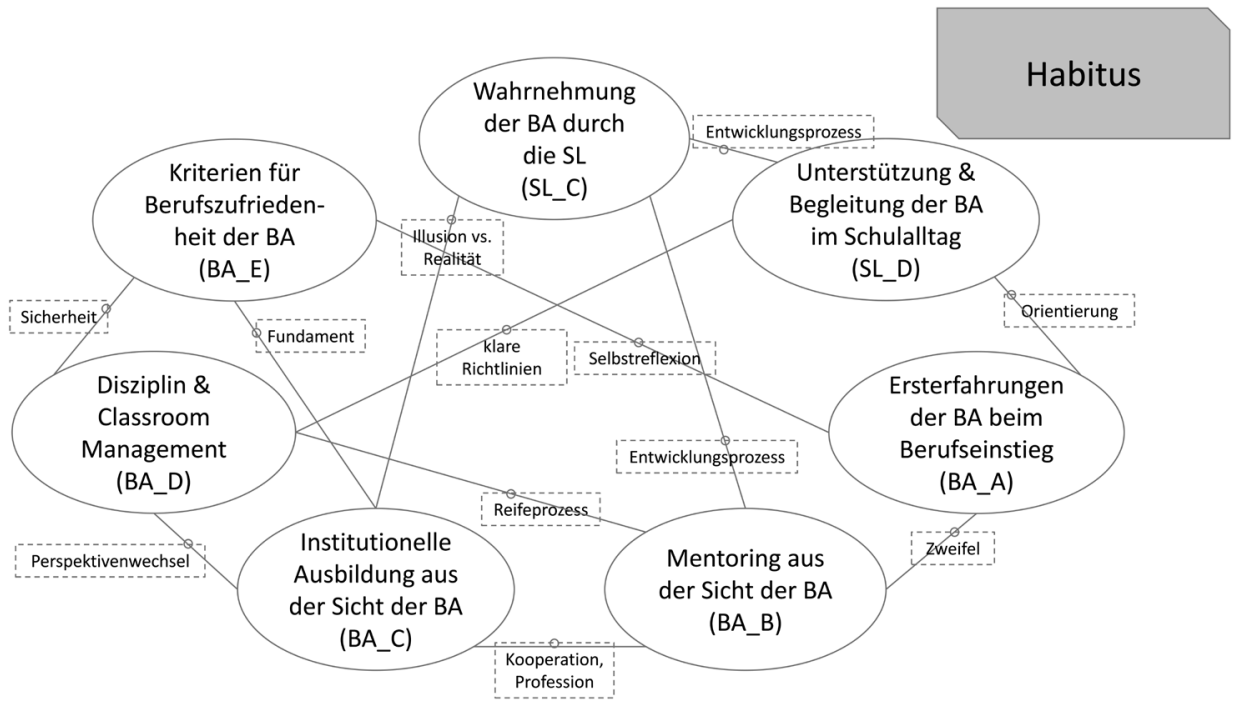

Abb. 70: Bedingungsgefüge Habitus

Innerhalb dieser Interpretation ist der Themenbereich Habitus komplexer als die vorhergehenden. In der Literatur wird auch von Lehrerhabitus bzw. Habitus von Lehrkräften (Paseka et al. 2018) gesprochen.

Tab. 17: Bedingungsgefüge Habitus; Übersicht

\begin{tabular}{lll}
\hline Terminus & $\begin{array}{l}\text { Zusammenhang } \\
\text { Grundwert, Voraussetzung, Ursache, } \\
\text { Maßnahme, Konsequenz, Wirkung }\end{array}$ & \\
\hline Entwicklungsprozess & Voraussetzung & BA_B - SL_C \\
Entwicklungsprozess & Voraussetzung & SL_C - SL_D \\
Fundament & Voraussetzung, Ursache & BA_C - BA_E \\
Illusion vs. Realität & Voraussetzung & BA_C - SL_C \\
klare Richtlinien & Voraussetzung, Maßnahme & BA_D - SL_D \\
Kooperation, Profession & Voraussetzung, Maßnahme, Wirkung & BA_B - BA_C \\
Orientierung & Voraussetzung, Konsequenz & BA_A - SL_D \\
Perspektivenwechsel, „Essenz“ & Voraussetzung, Konsequenz & BA_C - BA_D
\end{tabular}




\begin{tabular}{lll}
\hline Terminus & $\begin{array}{l}\text { Zusammenhang } \\
\text { Grundwert, Voraussetzung, Ursache, } \\
\text { Maßnahme, Konsequenz, Wirkung }\end{array}$ & \\
\hline Reifeprozess & Wirkung & BA_B - BA_D \\
Selbstreflexion & Ursache, Konsequenz & BA_A - BA_E \\
Sicherheit & Voraussetzung & BA_D - BA_E \\
Zweifel & Wirkung, Voraussetzung & BA_A - BA_B \\
\hline
\end{tabular}

In dieser Diskussion wird die Erscheinung, die Haltung, das Gehabe (Duden 2017) der BerufsanfängerInnen im Kontext Schule, z.B. Klasse, Kollegium, Umfeld (Elternkontakt, Kontakt nach außen etc.), Schulbehörde etc., ausgedrückt. Der Habitus subsummiert in der vorliegenden Interpretation auch die verschiedenen Rollenbilder der Lehrperson in unterschiedlichen Situationen und Settings (unter KollegInnen, beim Elternkontakt, mit Vorgesetzten etc.). Insofern ist hier Habitus ein Metathema, welches alle anderen Themenbereiche beeinflusst, ohne dort unbedingt explizit erwähnt zu sein. In den Grundlagen (Kap. II) der vorliegenden Arbeit wird die Habitualisierung als Lehrperson nach dem Habituskonzept Bourdieus eingehend diskutiert (Bourdieu 1996a, 2015a; Lenger et al. 2013; Nairz-Wirth 2011; Schumacher 2013). Die Erkenntnis, Habitus als Konzept von Professionalität zu verstehen, kann mit dem Konzept der Clusterkategorie 3 der vorliegenden Arbeit, Professionalisierung durch Kompetenzentwicklung zu erreichen, in Einklang gebracht werden.

Für die Entwicklung des Habitus sind neben den BerufsanfängerInnen selbst in hohem Maße die SchulleiterInnen, aber auch die KollegInnen bzw. die MentorInnen und nicht zuletzt die institutionelle Ausbildung beteiligt. Die BerufsanfängerInnen bekommen nach den ersten Erfahrungen vorrangig durch Selbstreflexion ein klares Bild darüber, „wie man eine klare Linie verfolgt“, und fallen zu Beginn der LehrerInnenlaufbahn nicht selten ,auf die Nase“ (BA_19: 72). Wird der Habitus gefestigt, steigen die gegenseitige „Akzeptanz“ und der „Respekt“ (BA_01:77), was sich unmittelbar auf die Berufszufriedenheit auswirkt. Bereits in der Ausbildung, vor allem im Rahmen der pädagogisch-praktischen Studien, wird ein Fundament zur Ausbildung des Habitus und damit zur höheren Berufszufriedenheit gelegt. „Was wirklich total wichtig ist, sind [sic!] die Praxisstunden“ (BA_06: 47), wird in diesem Zusammenhang erwähnt. Die Ausbildungsinstitutionen sind gefordert, den BerufsanfängerInnen die schulische Realität zu vermitteln, damit die Entwicklung des Habitus von Beginn an in die richtige Richtung verläuft. Hier sind ebenso die pädagogisch-praktischen Studien bzw. deren begleitende universitären Seminare maßgeblich gefordert.

SchulleiterInnen sehen sich oftmals als „ruhender Pol“ (SL_02: 36), aber auch „relativ strikt“ in der „Unterstützung“ (SL_03: 40), vor allem „wenn es mit Eltern oft einmal was gibt“ (SL_04: 26). Sie gelten für die BerufsanfängerInnen als Orientierungsmaßstab, insbesondere was den Habitus nach außen (z.B. in Elterngesprächen) betrifft. Im Unterschied dazu agieren die KollegInnen als MentorInnen kollegial auf hierarchisch gleicher Ebene, „wir haben die gleichen Probleme wie du“ (BA_15: 29), und sie fördern damit die Entwicklung des Habitus von BerufsanfängerInnen auf der Basis der Nachahmung.

Die Zweifel der BerufsanfängerInnen in den Ersterfahrungen beim Berufseinstieg, die teilweise der Theorie-Praxis-Schere geschuldet sind, „[... aber die Situationen treffen ja doch immer ganz anders ein“ (BA_09: 42), können andererseits auch als Voraussetzung dafür gesehen werden, dass die BerufsanfängerInnen aktiv das Gespräch mit den MentorInnen suchen und darüber hi- 
naus einen Mehrwert erfahren. Insgesamt beeinflusst der Habitus bzw. dessen Entwicklung das Classroom Management der jungen Lehrkraft. Die SchulleiterInnen bewirken eine Schärfung durch klare Richtlinien. „Der Junglehrer ist überhaupt froh, wenn es klare Richtlinien gibt." (SL_08: 28) In der Ausbildung ist es wesentlich, den Perspektivenwechsel der StudentInnen in die Richtung der Lehrperson einzuleiten und gegen Ende der Ausbildung zu vollziehen. Dies gibt den BerufsanfängerInnen die nötige Sicherheit beim Berufseinstieg und stellt ein unmittelbares Kriterium für die Berufszufriedenheit der BerufsanfängerInnen dar.

Abschließend kann gesagt werden, dass die KollegInnen als MentorInnen eine Schlüsselfunktion für die BerufsanfängerInnen bei der Ausbildung des Habitus einnehmen. Durch die Rückkoppelungsprozesse im Sinne von Fragen stellen \& Feedback bekommen wird der Reifeprozess der BerufsanfängerInnen unterstützt: „Die Unterstützung von den Kollegen hat mir sehr geholfen und habe ich auch gebraucht." (BA_07: 38) Dieser Feinschliff der jungen Lehrpersonen in Form des Mentorings sollte unbedingt in Abstimmung mit der bzw. aufbauend auf die Ausbildung erfolgen, in Form einer Kooperation. Dann kann der Anspruch an einen professionellen Habitus gestellt werden.

Im folgenden Abschnitt Zusammenführung und Integration (Kap. IV) werden die Erkenntnisse aus Grundlagen (Kap. II) und Empirischer Zugang (Kap. III) vereint und inhaltliche Zusammenhänge diskutiert. Mit der Formulierung von Conclusiones zu den Forschungsfragen schließt das Kapitel. 



\section{Zusammenführung und Integration}

Die Zusammenführung und Integration erfolgt in drei Abschnitten mit dem Ziel, den Konnex der theoretischen Konzepte und empirischen Erkenntnisse der vorliegenden Arbeit herzustellen. Die Fokusse dieses Teils stellen sich wie folgt dar:

In Vereinigung theoretischer und empirischer Erkenntnisse (Kap. IV.1) werden die empirisch gewonnenen Erkenntnisse in die theoretischen Konzepte zu Ungewissheit \& Kontingenz und LehrerInnenpersönlichkeit \& Habitus eingebettet.

In Erkenntnisse zu den Forschungsfragen: Conclusiones (Kap. IV.2) werden Antworten auf die Forschungsfragen gegeben, theorie- bzw. empiriebasierte Rückschlüsse gezogen und weiterführende Gedanken formuliert.

Grenzen der Arbeit (Kap. IV.3) zeigen Unschärfen in der Konzeptualisierung der vorliegenden Arbeit in Bezug auf die Rahmenbedingungen zur empirischen Forschung auf. 


\section{Vereinigung theoretischer und empirischer Erkenntnisse}

Die empirischen Erkenntnisse aus der Analyse (Kap. III.3) und der Interpretation der analysierten Daten (Kap. III.4) werden im Folgenden mit zwei theoretischen Konzepten, die in Bezug auf die Professionalisierung als LehrerIn vorkommen, verknüpft.

Die Themen-Kategorien-Matrix (Kap. III.4.1) und die Bedingungsgefüge (Kap. III.4.2) bilden die empirischen Erkenntnisse ab. Diese werden in Ungewissheit, Kontingenz (Kap. IV.1.1) mit theoretischen Konzepten von Ungewissheit und Kontingenz (Kap. II.1.1) und in LehrerInnenpersönlichkeit \& Habitus (Kap. IV.1.2) mit theoretischen Konzepten von LehrerInnenpersönlichkeit und Habitus (Kap. II.1.2) in Beziehung gebracht.

\subsection{Ungewissheit, Kontingenz}

Im Abschnitt Ungewissheit als Teil der pädagogischen Profession (Kap. II.1.1) wurde das Phänomen der Ungewissheit bzw. der Kontingenz aus folgenden Perspektiven beleuchtet: Zuerst wurde der Blick auf die Konstitution der Ungewissheit im pädagogischen Feld gerichtet (Kap. II.1.1.1), um anschließend den Umgang mit Ungewissheit als Krisenerfahrung zu thematisieren (Kap. II.1.1.2). Im Folgenden werden die theoretischen Erkenntnisse in Beziehung zu den empirischen Ergebnissen gesetzt und diskutiert.

Wenn Kontingenz als „Möglichkeit und gleichzeitige Nichtnotwendigkeit“ (Duden 2017) von Sachverhalten, Aussagen, Kooperationen, Interventionen etc. gesehen wird, ist eine Öffnung des Blicks bzw. eine Erhöhung des Abstraktionsgrads in der Einordnung der zwischenmenschlichen Verhaltensweisen notwendig. Kontingenz inkludiert die Erhöhung der Möglichkeiten bzw. der Interaktionsvariationen und gerade deshalb muss der Blick auch in Richtung Öffnung und Verallgemeinerung gerichtet werden. „Teachers cannot generalize skills they have not adequately learned“, stellt Scheeler (2008, S. 146) fest und ergänzt: „[...] they do not generalize newly acquired teaching techniques to real world settings." (ebd.) Nach den im Zuge dieser Forschungsarbeit durchgeführten Interviews und der Analyse der empirischen Daten kann unterstrichen werden, dass explizit die thematisierte Kontingenz bzw. Ungewissheit einen Großteil der von Scheeler erwähnten realen Umwelt im Sinne von realen Unterrichtssituationen ausmachen.

Im Anschluss werden thematische Zusammenhänge zwischen den im Grundlagenteil (Kap. II) diskutierten Kontingenzkonstitutionen und im Empirischen Zugang (Kap. III) analysierten Kontingenzvorkommen dargestellt und diskutiert. Als Grundlage für die empirischen Schwerpunkte dienen die in der Themen-Kategorien-Matrix (Tab. 11) bzw. in den Bedingungsgefügen (Kap. III.4.2) bereits dargestellten Themen Systemwissen, Reflexion \& Feedback, Autorität in der Klasse, Kooperation mit Schülerinnen und Schülern, Administration und Habitus. Sie stellen die Essenz der empirischen Erkenntnisse dar.

In Abbildung 71 sind die Themen mit der in Beziehung zu setzenden und theoretisch bereits diskutierten Materie Ungewissheit bzw. Kontingenz sowie die Schlüsselbegriffe abgebildet. Die den Themen beigefügten Begriffe bzw. Schlagworte sind Schlüsselbegriffe, die bereits in der Diskussion zu den Bedingungsgefügen vorkommen und entweder in-vivo von den InterviewpartnerInnen oder interpretativ im Zuge der Datenanalyse generiert wurden (Abb. 71).

Die in den Grundlagen thematisierten Phänomene Ungewissheit, Kontingenz und Unverfügbarkeit (Kap. II.1.1) können in allen empirisch ermittelten Themen, die mittels Bedingungsge- 
füge analysiert wurden, identifiziert werden, in Systemwissen, Reflexion \& Feedback, Autorität in der Klasse, Kooperation mit Schülerinnen und Schülern, Administration und Habitus.

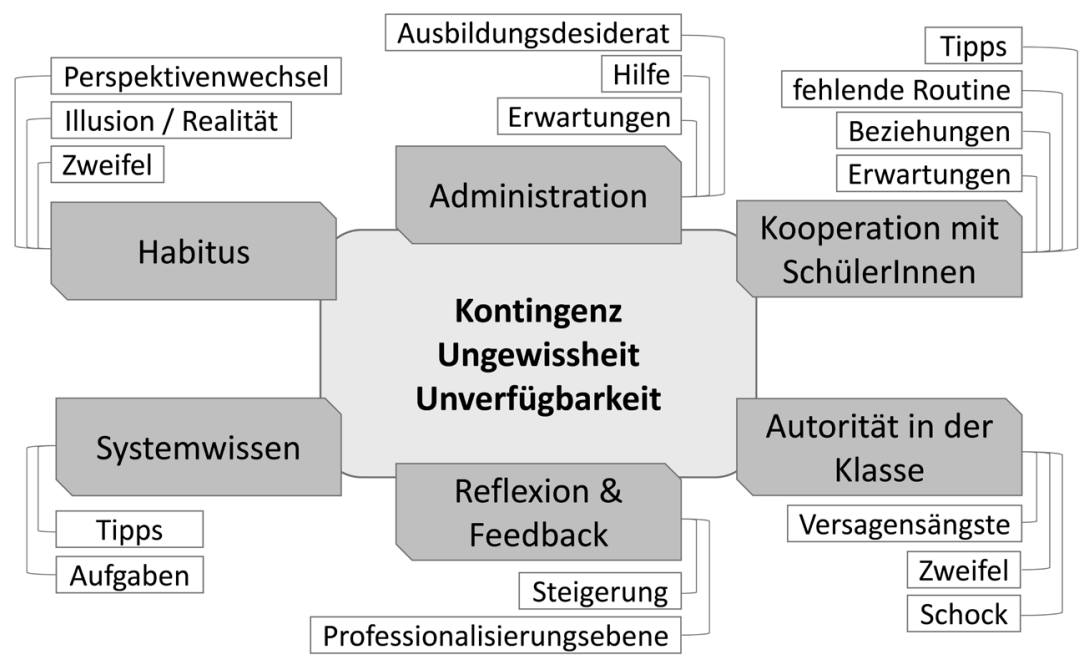

Abb. 71: Kontingenz, Ungewissheit, Unverfügbarkeit - Zusammenführung und Integration

Die Themen Systemwissen, Reflexion \& Feedback, Autorität in der Klasse, Kooperation mit SchülerInnen, Administration und Habitus, sowie die Schlüsselwörter in den rechteckigen Feldern (Abb. 71) werden zur Orientierung und Transparenz in der folgenden Diskussion kursiv dargestellt.

Die zum Teil nicht geklärten Aufgaben, besonders im Bereich Systemwissen, bewirken für Lehrpersonen im Allgemeinen und BerufsanfängerInnen im Speziellen Stress, letztlich wird der Grad der Ungewissheit erhöht. Dies trifft in der Gesamtheit der Herausforderungen betrachtet jedoch nicht nur das Systemwissen, sondern spielt z.B. auch in den Bereich des Unterrichts hinein, da Aufgaben im System mit den pädagogischen Aufgaben bzw. Erwartungen im Klassenzimmer teilweise eng verknüpft sind. Unterstützung erfahren die BerufsanfängerInnen von erfahrenen KollegInnen, DirektorInnen und nach Implementierung der Induktionsphase voraussichtlich vorrangig von MentorInnen. Die Hilfe reicht vom Schließen der Ungewissheit (Kosinár 2018), indem z.B. MentorInnen mit Tipps helfend zur Seite stehen, bis zum allgemeinen Umgang mit Kontingenz- bzw. Krisenerfahrungen (Connor et al. 2012).

In Bezug auf Reflexion \& Feedback ist die Erwartungshaltung aufseiten der BerufsanfängerInnen sehr hoch, gerade was den Umgang mit Ungewissheit, Kontingenz und Unverfügbarkeit betrifft. Da Reflexion \& Feedback auf mehreren Ebenen stattfindet (z.B. Lehrperson-SchülerIn, Lehrperson-SchulleiterIn, BerufsanfängerIn-MentorIn), soll nach den empirischen und analytischen Erkenntnissen der vorliegenden Arbeit dieser Möglichkeit des Umgangs mit Kontingenz ein besonderes Augenmerk geschenkt werden. Dass durch den professionellen Umgang mit Ungewissheit, welcher durch Reflexion \& Feedback initiiert werden kann, die Berufszufriedenheit steigt, ist nicht als positiver Nebeneffekt, sondern als wesentliche Determinante für den weiteren berufsbiografischen Weg der BerufsanfängerInnen zu verstehen. Interessant erscheint in diesem Zusammenhang auch das Phänomen der doppelten Kontingenz (Kärtner 2015; Luh- 
mann 2018), welches in den Reflexions- und Feedbackgesprächen auf der Metaebene bzw. auf der Ebene zweiter Ordnung auftreten kann. Letztlich führt Reflexion \& Feedback in Bezug auf Ungewissheit und Kontingenz zu einer Steigerung in der Professionalisierung. Die Chance für die BerufsanfängerInnen, sich auf die nächste Professionsebene zu heben, wird u.a. dann signifikant erhöht, wenn die Bereitschaft der BerufsanfängerInnen besteht, sich mit der Ungewissheit auseinanderzusetzen (Keller-Schneider 2018). Dies kann z.B. in Form von Reflexion \& Feedback initiiert und etabliert werden.

Das Thema Autorität in der Klasse weist eine sehr hohe Korrelation zum Phänomen der Ungewissheit bzw. Kontingenz auf. Die BerufsanfängerInnen nennen diesbezüglich in erster Linie Versagensängste und, in weniger stark ausgeprägter Form, Zweifel. Der Schock beim Berufseinstieg, in der Literatur und auch in der vorliegenden Untersuchung als Praxisschock thematisiert, wird als nahezu erwartbares Übel angesehen, dem im Sinne der theoretischen Aufarbeitung der Kontingenzthematik am besten mit Offenheit begegnet wird. Die Nicht-Standardisierbarkeit (Helsper 2001) von Handlungen verschiedener Akteure wird hier ebenso ins Treffen geführt wie die strukturelle Unhintergehbarkeit (Dietrich 2018) von Krisenerfahrungen. Trotz dieser theoretischen Erklärungsmuster stellt sich die Frage, ob angesichts der pädagogisch-praktischen Studien im Laufe des Lehramtsstudiums das Schockerlebnis für BerufsanfängerInnen so ohne Weiteres hingenommen werden soll, oder ob es Praktikumssettings gäbe, die noch realitätsnaher als die bisherigen sind und insofern den Praxisschock verringern bzw. bestenfalls verhindern können. Auch in Bezug auf die bereits erwähnten Versagensängste der BerufsanfängerInnen liefert der in der Literatur diskutierte Umgang mit Kontingenz bzw. mit Krisen teilweise keine befriedigende Aussicht. Wie erklärt man einer Berufsanfängerin bzw. einem Berufsanfänger, ohnehin von Zweifel und Ängsten geplagt, dass der offensive Umgang mit Kontingenz eine aktive Kriseninitiierung (Combe et al. 2018) sein kann. Hier ist, so meine Vermutung, ein Punkt erreicht, wo sich theoretische Erkenntnisse und praktische Erfahrungen nicht treffen.

Die Kooperation mit Schülerinnen und Schülern umfasst den Bereich des Classroom Managements und die wesentliche Facette der Beziehungen bzw. Beziehungsebene zwischen Lehrperson und SchülerIn (Hattie 2015). Gerade BerufsanfängerInnen können aufgrund der fehlenden Routine, welche aus den Interviews als noch markantere In-vivo-Codierung „Keine Routine“ hervorgeht, den Abstand zu den SchülerInnen oftmals schwer oder nicht einschätzen, zumal sich die SchülerInnen zum Teil genau in derselben Situation befinden. Hier liegt ein typischer Fall der doppelten Kontingenz (Combe et al. 2018; Luhmann 2018) vor, wonach zwei Akteure mit Kontingenzpotenzial aufeinandertreffen.

Die SchulleiterInnen erwarten sich von den BerufsanfängerInnen in Bezug auf die Kompetenz der Kooperation mit SchülerInnen erste Ansätze und formulieren zugleich ein Desiderat in der Ausbildung. Auch hier zeigt sich doppelte Kontingenz und die komplexen, systemischen Variationen in den BerufsanfängerIn-SchülerIn-SchulleiterIn-Begegnungen lassen sich in laborähnlichen universitären Studiensituationen nicht durchspielen, geschweige denn erlernen. Auch die praxisbezogenen Seminare der pädagogisch-praktischen Studien können in Bezug auf Alltagspraxis als Laborveranstaltungen interpretiert werden. Letztlich hoffen BerufsanfängerInnen auch hier auf Tipps und Tricks seitens der älteren KollegInnen, MentorInnen und SchulleiterInnen, wenngleich die Mentoring-Ansätze eher die Unterstützung und Entwicklung (Wang \& Odell 2007) im Gegensatz zum Präsentieren von Lösungen präferieren. Die sensible Phase des Berufseinstiegs (Kraler et al. 2017) manifestiert sich besonders im Kooperationsverhalten. Umso mehr wird nicht erst in dieser Phase, sondern bereits in der Ausbildung die Anerkennung von Ungewissheit (Keller-Schneider 2018) zu thematisieren sein. 
Ausbildungsdesiderate in der Administration im LehrerInnenberuf erzeugen große Unsicherheiten, die sich in Kontingenzerfahrungen niederschlagen können. Ähnlich wie beim Systemwissen sind administrative sehr eng mit den pädagogischen LehrerInnentätigkeiten verbunden, sodass sich Unsicherheiten auf der einen Seite (Administration) als Ungewissheiten auf der anderen (zwischenmenschlich und pädagogisch) konstituieren können. In diesem Zusammenhang ist der Fokus eher auf das Kooperationsverhältnis zwischen BerufsanfängerIn und Schulleitung als auf jenes zwischen BerufsanfängerIn und SchülerIn zu richten. SchulleiterInnen versuchen dieses Ausbildungsdesiderat und die resultierenden Kontingenzerfahrungen durch vorauseilende Hilfeleistungen auszugleichen. Schrittesser (2011) spricht in diesem Zusammenhang vom prophylaktischen Handeln, Oevermann (2017) vom implizit Therapeutischen als einer pädagogischen Funktion. Erwartungsgemäß werden diese Erste-Hilfe-Maßnahmen nach der Implementierung der Induktionsphase zukünftig auch MentorInnen übernehmen, wenngleich die ursprüngliche Intention des MentorInnenhandelns nicht Erste Hilfe sondern die Unterstützung in der persönlichen Entwicklung (Raufelder \& Ittel 2012) ist. Zur Minderung des Spannungsfelds zwischen den Erwartungen der Schulleitungen, KollegInnen etc. und dem Ausbildungsdesiderat, in dem sich die BerufsanfängerInnen befinden und das für Ungewissheit und Krisenerfahrungen mitverantwortlich ist, können die Ausbildungsinstitutionen beitragen. Neben theoretischen, wissenschaftlichen und pädagogisch-praktischen Lehrinhalten darf auf die administrativ-praktischen Inhalte im Curriculum nicht vergessen werden. Die Erwartungen an die Ausbildungsinstitutionen seitens der BerufsanfängerInnen und der SchulleiterInnen werden diesbezüglich eindeutig formuliert und in der Beantwortung der Forschungsfrage 3 thematisiert (Kap. IV.2.3).

Das Thema Habitus wird aus der Analyse der empirischen Daten interpretativ generiert. Der Vollständigkeit halber sei erwähnt, dass sich die Thematik der Habitualisierung in Verbindung mit LehrerInnenpersönlichkeit bzw. deren Genese durch die gesamte vorliegende Arbeit durchzieht und insofern als Metathema zu betrachten ist.

Der Perspektivenwechsel von den Studierenden zur Lehrperson fällt den BerufsanfängerInnen schwer. Daran ändern auch die pädagogisch-praktischen Studien wenig, zumal die Rolle der Praktikantin bzw. des Praktikanten ohnehin eine andere ist als jene der Lehrperson resp. der Berufsanfängerin bzw. des Berufsanfängers. Die Ungewissheit aufgrund der Handlungen, Interaktionen etc. von AkteurInnen in unterschiedlichen Rollen bestätigt Keller-Schneider (2018). Wenn verschiedene Rollen (Studierende bzw. Studierender, PraktikantIn, BerufsanfängerIn bzw. Lehrperson in den ersten Dienstjahren) in zeitnahen Abständen bzw. in ineinandergreifenden Phasen eingenommen werden, ist die Ungewissheit bzw. die Kontingenz inkl. der einhergehenden Krisenerfahrung vermutlich umso größer.

In engem Zusammenhang damit steht die Diskrepanz zwischen den Illusionen und den Realitätserfahrungen der BerufsanfängerInnen. Die Differenzen zwischen dem Erwarteten (Illusion) und dem Tatsächlichen (Realität) führen zu Kontingenz- und Krisenerfahrungen, Ausführungen der BerufsanfängerInnen und SchulleiterInnen bestätigen dies ausdrücklich. Trotzdem oder gerade deshalb treffen BerufsanfängerInnen Entscheidungen (Combe 2018) und insofern können Krisenerfahrungen allgemein (Combe \& Paseka 2012) und Diskrepanzen zwischen Illusion und Realität im Speziellen als Chance zur Entscheidungsfindung gesehen werden. Zweifel werden in den Befragungen der BerufsanfängerInnen oft thematisiert und stehen in den Gesprächen mit Schock, Überforderung und Krise im Zusammenhang. Hier orte ich - ebenso wie beim bereits diskutierten Thema Autorität in der Klasse - eine in der Theorie gefundene, jedoch wenig bis nicht praktikable Lösung: die Initiierung der Krise. Im Falle des Zweifels scheint der lösungsorientierte, durch MentorInnen begleitete Ansatz der Stärkung der Persönlichkeit der bessere zu sein. 


\subsection{LehrerInnenpersönlichkeit \& Habitus}

In LehrerInnenpersönlichkeit und Habitus im Kontext der Professionalisierung (Kap. II.1.2) wurde einleitend das Habituskonzept nach Bourdieu (Kap. II.2.2.1) vorgestellt. Im Anschluss erfolgte die ausführliche Darstellung und Diskussion des Habitusmodells (Kap. II.1.2.2). Abschließend wurden in Anwendung des Habituskonzepts nach Bourdieu auf die LehrerInnenprofessionalisierung (Kap. II.1.2.3) Querverbindungen zwischen dem theoretischen Konzept und der LehrerInnenbildung hergestellt. Im vorliegenden Abschnitt werden theoretische Erkenntnisse in Beziehung zu den empirischen Befunden gebracht und diskutiert.

Auch wenn bzw. gerade weil Kramer und Pallesen (2019, S. 15) betonen, dass in Bezug auf den LehrerInnenhabitus und seine empirische Erschließung „noch längst nicht auf ein fertiges und unstrittiges Programm zurückgegriffen werden kann, sondern noch immer Pionierarbeit zu leisten ist“, wird anschließend die Verbindung von LehrerInnenpersönlichkeit und Habitus sowie deren Determiniertheit in Bezug auf die in der Analyse identifizierten Themen rekonstruiert. Die in der Bourdieu'schen Habitusdiskussion dominanten Konstitutionen Feld, Kapital und Macht stehen im Zentrum des Interesses, wobei ein Hauptanliegen die Diskussion über die Verwendung des Begriffs des LehrerInnenhabitus im Verhältnis von Habitus und Feld in Form des Bildungswesens allgemein, der jeweiligen Schulform, der Einzelschule etc. (ebd., S. 13) unter Berücksichtigung des Berufseinstiegs in den LehrerInnenberuf darstellt.

In Abbildung 72 sind die Themen Systemwissen, Reflexion \& Feedback, Autorität in der Klasse, Kooperation mit Schülerinnen und Schülern, Administration und Habitus mit der in Beziehung zu setzenden und theoretisch bereits diskutierten Materie LehrerInnenpersönlichkeit \& Habitus sowie die Schlüsselbegriffe abgebildet. Diese Themen wurden in der Themen-Kategorien-Matrix (Tab. 11) bzw. in den Bedingungsgefügen (Kap. III.4.2) bereits diskutiert und stellen die wesentlichen empirischen Erkenntnisse in Form von relevanten Themen der vorliegenden Arbeit dar. Die den Themen beigefügten Begriffe bzw. Schlagworte sind Schlüsselbegriffe, die bereits in der Diskussion zu den Bedingungsgefügen vorkommen und entweder in-vivo von den InterviewpartnerInnen oder interpretativ im Zuge der Datenanalyse generiert wurden (Abb. 72).

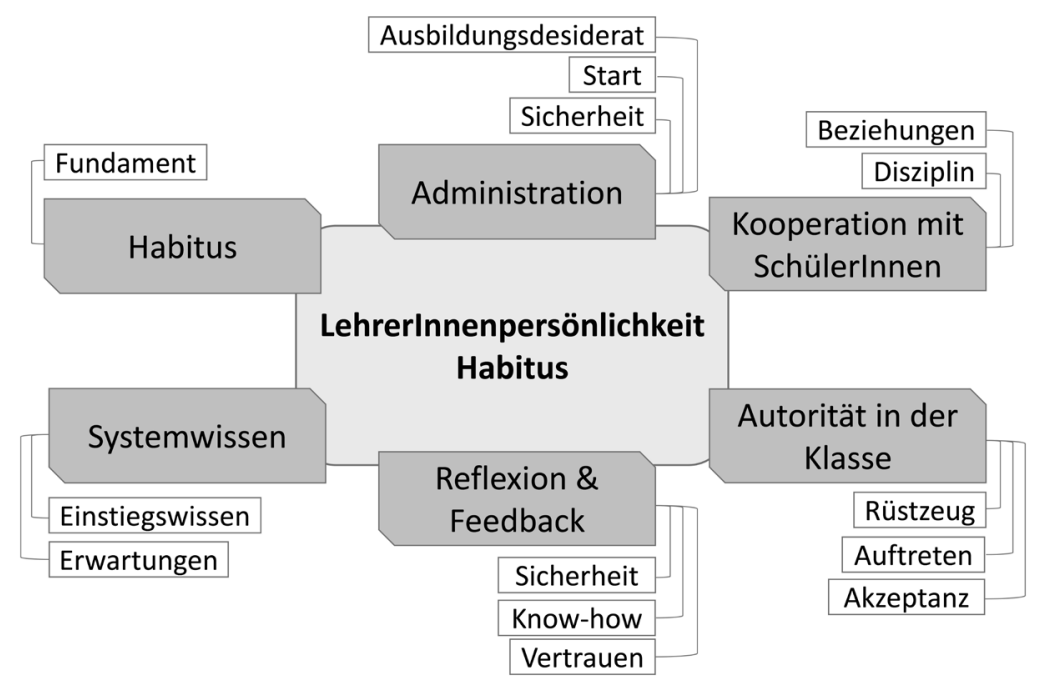

Abb. 72: LehrerInnenpersönlichkeit \& Habitus - Zusammenführung und Integration 
Die in den Grundlagen diskutierten Konstitutionen von LehrerInnenpersönlichkeit und Habitus werden mit allen in der Themen-Kategorien-Matrix (Tab. 11) bzw. in den Bedingungsgefügen interpretierten Themen (Kap. III.4.2) im vorliegenden Kapitel zueinander in Beziehung gesetzt und diskutiert. Die Themen und in den rechteckigen Feldern dargestellten Schlüsselwörter werden zur Orientierung und Transparenz der Diskussion im folgenden Text kursiv dargestellt.

Der thematische Komplex Systemwissen kann aus zwei Perspektiven betrachtet werden. Einmal bildet die abstrakte, gewissermaßen anonyme Organisation das System, zum anderen besteht das System aus einzelnen, konkreten AkteurInnen. Das (Schul-)System beginnt de facto beim (konkreten) LehrerInnenkollegium und geht bis in die höchste bildungspolitische (anonyme) Ebene im Bildungsministerium. Wenn die gesellschaftspolitische Dimension von Schule und Ausbildung mitgedacht wird, greift das System praktisch in alle gesellschaftlichen Lebensbereiche ein. Dementsprechend gehen die Erwartungen von und an Lehrpersonen in viele Richtungen. In dieser Forschungsarbeit beschränkt sich der Blick auf BerufsanfängerInnen und Lehrpersonen in den ersten Dienstjahren. Dies macht insofern einen Unterschied, als die Phase der Habitualisierung, der Inkorporierung und letztlich der Herausbildung der LehrerInnenpersönlichkeit (Bourdieu 2011; Helsper 2018; Lenger et al. 2013) berufsbiografisch differenziert zu betrachten ist (Terhart 2001). Die Erwartungen seitens der BerufsanfängerInnen sind, bezogen auf das Systemwissen, auf die schulinternen Unterstützungssysteme gerichtet, auf die SchulleiterInnen, Kolleginnen und zukünftig auch auf die MentorInnen. Im Verlauf von Ausbildung und Berufsausübung konstituiert sich im Zusammenspiel von Feld (System) und Habitus ein feldspezifischer Teilhabitus (Helsper 2018) und in der Auseinandersetzung mit den Erwartungen wird dieser kontinuierlich reproduziert und transformiert (Liegmann et al. 2018).

BerufsanfängerInnen benötigen für einen gelingenden Berufseinstieg ein Mindestmaß an Einstiegswissen nicht nur in fachlich-pädagogischen, sondern auch in systemrelevanten Bereichen. Desiderate auf dieser Ebene schwächen die BerufsanfängerInnen bzw. verbrauchen Ressourcen, die besser eingesetzt werden könnten. Wenn das System als Feld im Sinne der Bourdieu'schen Habitusdiskussion interpretiert wird, kann die Professionalisierung nur über die Vernetzung von Habitus und Feld (Nairz-Wirth 2011), also Habitus der BerufsanfängerInnen und System, erfolgen. Auch die Idee der Inkorporierung in Bezug auf die Habitualisierung (Bourdieu 2015a; Lenger et al. 2013) kann letztlich nur bei vorhandenen Erfahrungen, Wissensbeständen etc. Fuß fassen, sodass einerseits dem System selbst (LehrerInnenkollegium, SchulleiterInnen), andererseits nicht minder den Aus- und Fortbildungsinstitutionen (Universität, Pädagogische Hochschule) eine enorme Verantwortung zukommt. In den formellen Mentoring-Prozessen und im Rahmen der inhaltlichen und strukturellen Anpassungen der Aus- und Fortbildungscurricula wird insofern nicht nur das fachlich-pädagogische Paradigma zu berücksichtigen sein, sondern auch jenes des Systems Schule.

Das Thema Reflexion \& Feedback konstituiert sich insbesondere basierend auf den Aussagen der BerufsanfängerInnen. Eine Begründung dafür kann sein, dass die BerufsanfängerInnen in ihrer Ausbildung als Studierende mit Reflexion und Feedback vertraut gemacht werden, stellen diese Themen doch einen wesentlichen Teil des Curriculums der LehrerInnenausbildung dar. Zusätzlich nutzen BerufsanfängerInnen offensichtlich die Kraft des Feldes, um sich im Umfeld ihres Wirkens zu stärken bzw. über den Habitus zu professionalisieren (Dietrich 2018). An dieser Stelle wird die Kernidee des Habitus als Konzept der Professionalität deutlich. Gerade in Reflexion und Feedback kann die Triade des Wahrnehmens, Denken und Handelns (Bourdieu 2015a) (siehe auch Abb. 15, Kap. II.1.2.3.2) zur Professionalisierung der BerufsanfängerInnen beitragen. Wenn davon ausgegangen wird, dass das Feld in dynamischer Relation zum Habitus 
(Bourdieu 1996b) steht, liegt diese Korrelation nahe, vorausgesetzt, das nötige Vertrauen ist im Feld vorhanden. Neben persönlichen Charakteristika der AkteurInnen (hier: SchulleiterInnen, KollegInnen, MentorInnen, BerufsanfängerInnen) ist für die Positionierung Letzterer im Feld und deren individuelle Habitualisierung (Nairz-Wirth 2011) das Know-how ein ganz wesentlicher Faktor. Bourdieu (1996a) spricht in diesem Zusammenhang von Machtstrukturen, NairzWirth (2011) versteht das Kapital als Machtmittel und je nach Ausstattungsgrad der BerufsanfängerInnen mit dem Kapital Know-how positionieren sich diese unterschiedlich im Feld. Die Vertrauensbasis kann hier zweifach interpretiert werden, entweder als Ursache für die Wahl der Positionierung der Berufsanfängerin bzw. des Berufsanfängers oder als Wirkung, indem sich Vertrauen nach der Positionierung im Feld bildet oder nicht. Insofern sind die Aus- und Fortbildungsinstitutionen angehalten, den BerufsanfängerInnen die nötige Sicherheit mitzugeben, um sich im Feld bestmöglich positionieren zu können.

Die Verwobenheit des Feldes (Bourdieu 1996a) erklärt die Relevanz des gegenseitigen Vertrauens, die noch zu verbessernde Aus- und Fortbildung der BerufsanfängerInnen (Nairz-Wirth 2011) deutet den Handlungsbedarf an.

In der Diskussion zum Thema Autorität in der Klasse ist, insbesondere wenn der Fokus auf die LehrerInnenpersönlichkeit bzw. auf den Habitualisierungsprozess gerichtet ist, noch einmal darauf hinzuweisen, dass in der vorliegenden Arbeit der Terminus Autorität im Sinne von „einflussreiche, maßgebende Persönlichkeit von hohem Ansehen“ (Duden 2017) verstanden wird. Insofern ist dieses Thema sehr entscheidend. Einerseits für den erfolgreichen Berufseinstieg von BerufsanfängerInnen, andererseits für die weitere Entwicklung der Berufsbiografie von Lehrpersonen. BerufsanfängerInnen erwarten sich, von der Ausbildung ein Rüstzeug zur Verfügung gestellt zu bekommen, ähnlich einem Werkzeugkoffer, der alle möglichen Tools für bestimmte Situationen enthält. Auch wenn in diversen Ratgebern solche Werkzeugkoffer für den Berufseinstieg als Lehrperson angeboten werden (Schaper 2017), sind es letztlich „nur“ Tipps und Tricks, die den Einstieg in den LehrerInnenberuf erleichtern. Aufgrund der Nicht-Standardisierbarkeit des pädagogischen Handelns (Helsper 2001) gibt es diesen Werkzeugkoffer nicht. Insofern existiert auch der universell gute Lehrer (Bromme \& Haag 2008) nicht und umso herausfordernder ist die Suche nach der idealen LehrerInnenpersönlichkeit (Terhart 2007). Das Auftreten der BerufsanfängerInnen, insbesondere der erste Auftritt, ist laut den Aussagen der SchulleiterInnen maßgeblich für die weitere Akzeptanz der Lehrperson im Klassenzimmer und im Feld Schule generell. Insofern spielen wieder die Bourdieu'schen Kategorien Feld, Kapital und Macht eine entscheidende Rolle. Die Kapitalausstattung (Nairz-Wirth 2011) der BerufsanfängerInnen zum Berufseinstieg ist eine relevante Größe zur ersten Positionierung im Feld und in Bezug auf die weitere individuelle Habitualisierung (ebd.). Die Interaktion zwischen Subjekt und Objekt (Bourdieu 2015a) ist Teil der Inkorporierung im Feld (Bourdieu 2015b; Schumacher 2013), die sich ihrerseits als individuelle Habitualisierung (Nairz-Wirth 2011) konstituiert.

Die Thematik Kooperation mit SchülerInnen wird aus dem Blickwinkel der LehrerInnenpersönlichkeit von den BerufsanfängerInnen und Schulleitern ähnlich diskutiert wie das Thema Autorität in der Klasse. Die fehlende Handhabe im Umgang mit dem Hauptproblem Disziplin, was den bereits angesprochenen Werkzeugkoffer in den Vordergrund stellt, sind Argumente für den belastenden Berufseinstieg. Interessant erscheint in diesem Zusammenhang die Erkenntnis, dass die individuelle Habitualisierung strukturierend und feldgestaltend (Nairz-Wirth 2011) wirkt. Scheinbar ist für die Kooperation im Feld, also im vorliegenden Fall für die Kooperation mit SchülerInnen, mehr notwendig als - im übertragenen Sinne - anwendbares Handwerkzeug, 
nämlich LehrerInnenpersönlichkeit durch Habitualisierung. Auch die vielfach thematisierte, für pädagogisches Arbeiten vorausgesetzte Beziehungsebene (Hattie 2015) hängt eng mit der Kooperation im schulischen Feld zusammen. SchulleiterInnen sehen die Bereitschaft der BerufsanfängerInnen, Beziehungsebenen zu gestalten, als mitgebrachte Kompetenz, entweder seitens der Ausbildung oder aus privaten Ressourcen kommend, z.B. durch die Mitwirkung in Vereinen. Nairz-Wirth (2011) spricht in diesem Zusammenhang von der Anerkennung des Status als Expertin bzw. als Experten, was wiederum Ausgangspunkt der schon thematisierten Inkorporierung und der daraus folgenden weiteren persönlichen Habitualisierung sein kann. Die Annahme, dass insofern zumindest bezogen auf die Beziehungsfähigkeit im Feld Schule eine längerfristige Professionalisierung und der Übergang in einen professionellen Habitus (Hericks et al. 2018) eingeleitet werden kann, ist durchaus vielversprechend.

Wie bereits in Vereinigung theoretischer und empirischer Erkenntnisse (Kap. IV.1) diskutiert, ist ebenso in Bezug auf LehrerInnenpersönlichkeit und Habitus das Thema Administration durch eine Schlüsselaussage relevant: das Ausbildungsdesiderat. Dadurch, dass BerufsanfängerInnen mit diesem Wissensrückstand ${ }^{82}$ in ihr Berufsleben starten, fehlt es ihnen an Sicherheit. Dieser Umstand beschränkt sich nicht auf die administrativen Tätigkeiten, denn durch Überschneidungen von Pädagogischem und Administrativem wirkt sich das Ausbildungsdesiderat Administration auch auf das bereits diskutierte Auftreten vor der Klasse, im Kollegium etc. aus und hat schließlich eine negative Wirkung in Bezug auf die Positionierung der BerufsanfängerInnen als LehrerInnenpersönlichkeit. Das Feld und letztlich das Kapital als Machtinstrument (Bourdieu 1996b; Nairz-Wirth 2011) spielen in dieser Diskussion wiederholt eine Rolle, in diesem Fall als fehlendes Kapital. In Umkehrung der Erkenntnisse über den habitualisierenden Vorgang der Inkorporierung (Bourdieu 2015a; Schumacher 2013) kann abgeleitet werden, dass dieser Vorgang bei fehlenden Voraussetzungen eben nicht oder nur schleppend in Gang gesetzt wird und insofern auch die gewünschte individuelle Habitualisierung bzw. die Ausbildung der LehrerInnenpersönlichkeit darunter leiden. Sich auf die schöpferische Intuition zu verlassen, wie sie Combe und Paseka (2012) in Bezug auf die Ungewissheit in Betracht ziehen, oder die schöpferische Dimension der Habitualisierung (Bourdieu 2011) in Anspruch zu nehmen, scheint in Bezug auf das klare, strukturierte Thema der Administration nicht zielführend zu sein. Die Aus- und Fortbildungsinstitutionen sind hier gefordert, den BerufsanfängerInnen den Start in das Berufsleben zu erleichtern. Die Herausforderung besteht darin, administrative Tätigkeiten in Unterrichtphasen ${ }^{83}$ so einzuplanen und zu gestalten, dass dieses Handlungswissen beim Berufseinstieg tatsächlich nutzbar gemacht werden kann (Whitehead 2011, S. 43). Im Rahmen des formellen Mentoring-Prozesses während der Induktionsphase bietet sich die Gelegenheit, BerufsanfängerInnen im Dienst in ihren aktiven administrativen Tätigkeiten zu unterstützen.

Das Thema Habitus markiert den Abschluss der in diesem Kapitel diskutierten Beziehungen zwischen den theoretischen und den empirischen Erkenntnissen. Mit diesem Thema, insofern ist es kein Zufall, dass es am Ende dieser Diskussion steht, schließt sich der Kreis. Letztlich, so bestätigen die vorliegenden Erkenntnisse, geht es um Habitus, um Habitualisierung, um die Bildung der LehrerInnenpersönlichkeit. Das Bedingungsgefüge Habitus (Kap. III.4.2.7) ist ein

82 In dieser Diskussion ist es irrelevant, ob es sich um einen Wissens-, Erfahrungs- oder etwa einen Kompetenzrückstand handelt. Es wird die Faktizität des Ausbildungsdesiderats thematisiert. Insofern wird der allgemeine Begriff des Wissens verwendet.

83 Studierende sind in administrative Tätigkeiten im Rahmen der pädagogisch-praktischen Studien nicht zwingend eingebunden. 
Metabedingungsgefüge, das Thema Habitus ein Metathema, Habitus als Konzept der Professionalität stellt die Kernidee der Grundlagen (Kap. II) dar.

Einerseits ist es illusorisch, ein Set von Eigenschaften und Verhaltensweisen für die perfekte LehrerInnenpersönlichkeit zu nennen (Terhart 2007). Das wäre zu trivial, zu kurz gedacht. Andererseits existiert der Ansatz, die Qualität einer Lehrperson und deren Professionalität als berufsbiografisches Entwicklungsproblem zu sehen (ebd.). Ich bevorzuge in diesem Zusammenhang die Terminologie der Entwicklungsherausforderung, entscheiden sich die BerufsanfängerInnen doch aus freien Stücken für diesen Beruf, mit der guten Absicht, sich beruflich weiter-, zum Besseren zu entwickeln, um den SchülerInnen gute Lehrpersonen sein zu können. Dieser Trend geht aus den vielen Befragungen eindeutig hervor, im Sinne der Selbstwahrnehmung durch die BerufsanfängerInnen und, eventuell noch aussagekräftiger, bestätigt durch die Fremdwahrnehmung der SchulleiterInnen. Die Phänomene der Freude, Neugierde und Spannung lassen die Problembehaftung entschieden im Schatten stehen. Mit dem Fokus auf die Entwicklung der BerufsanfängerInnen zur LehrerInnenpersönlichkeit werden die Professionalisierung und die Anbahnung eines wissenschaftlich-reflexiven Habitus (Hericks et al. 2018) impliziert. Über die Triade des professionellen Wahrnehmens, Denkens und Handelns (siehe auch Abb. 14 und 15, Kap. II.1.2.3.2) wird die sukzessive Habitualisierung zum individuellen Habitus durch wissenschaftlich-reflexive Kompetenzen (Helsper 2018; Hericks et al. 2018; Kraler 2008b) zur professionellen Habitualisierung mit dem Idealziel des professionellen LehrerInnenhabitus erweitert. Der Grundstein, das Fundament dafür wird bereits in der Ausbildung gelegt. Um die mikrodynamischen, auf die einzelne Berufsanfängerin bzw. den einzelnen Berufsanfänger bezogenen Habitualisierungs- bzw. Professionalisierungsschritte im Übergang zu begünstigen, braucht es ein Vor- und ein Nachher. Das hat das Wesen des Übergangs an sich. Die Phase der Inkorporierung setzt Integration und Resonanz voraus (Bourdieu 2015b; Lenger et al. 2013; Rosa 2018). Auch dafür benötigen BerufsanfängerInnen im Speziellen und Lehrpersonen im Allgemeinen Zeit (siehe auch Konstitutionen des Feldes LehrerInnenbildung, Abb. 12, Kap. II.1.2.3.1), womit der Übergang eben zur Phase und nicht zum Zeitpunkt wird. Insofern gilt auch hier: Zur Professionalisierung der BerufsanfängerInnen bedarf es der Aus- und Fortbildung und des scharfen Blicks auf die Inhalte der Curricula bzgl. der habitualisierungsfördernden Inhalte. Noch konkreter kann konstatiert werden, dass LehrerInnenpersönlichkeitsbildung bzw. Habitualisierung bzw. Professionalisierung vor dem Berufseinstieg in der Ausbildung, während des Berufseinstiegs im Übergang bzw. in der Induktionsphase und nach dem unmittelbaren Übergang, und begleitet durch die Fortbildung, stattfindet.

Nach der zusammenführenden Diskussion der Erkenntnisse aus den Grundlagen und des empirischen Zugangs folgt im Anschluss die Darstellung der Conclusiones bzgl. der in Abschnitt III.1.1 formulierten, dieser Arbeit zugrunde liegenden Forschungsfragen. 


\section{Erkenntnisse zu den Forschungsfragen: Conclusiones}

Im vorliegenden Kapitel werden Antworten auf die in der empirischen Arbeit gestellten Forschungsfragen (Kap. III.1.1) gegeben. Die formulierten Schlussfolgerungen können unter dem Aspekt der Professionalisierung durch Kompetenzentwicklung subsumiert werden. Insofern kann dieser Aspekt, der zugleich die Clusterkategorie 3 (CK_II_3) (Kap. III.3.4) bzw. die Konzeptualisierung der empirischen Erkenntnisse darstellt, als Metaconclusio betrachtet werden.

\subsection{Conclusio Forschungsfrage 1}

Die dieser Forschungsarbeit zugrunde liegende Forschungsfrage 1 lautet:

Welche Inhalte brauchen Lehramtsstudierende während der Ausbildung und welche Hilfen benötigen BerufsanfängerInnen während der ersten Berufsjahre, um den Berufseinstieg für sich und die Personen ihres unmittelbaren Arbeitsumfelds (SchülerInnen, Eltern, KollegInnen, Vorgesetzte) erfolgreich absolvieren zu können?

Die Beantwortung dieser Forschungsfrage gestaltet sich insofern zweiteilig, als hier zuerst die Phasen kurz vor dem Berufseinstieg während der LehrerInnenausbildung und anschließend jene der ersten Berufsjahre betrachtet werden.

Für beide Phasen werden die folgenden Erkenntnisse herangezogen: Die durch den multimethodisch Zugang durchgeführte Konzeptualisierung, wonach die Professionalisierung durch Kompetenzentwicklung (Kap. III.3.4) als der Schlüssel für einen gelingenden Berufseinstieg anzusehen ist, und die in der Empirie identifizierten Themen Administration, Systemwissen, Reflexion \& Feedback, Autorität in der Klasse, Kooperation mit SchülerInnen sowie Habitus (Kap. III.4.1).

In der folgenden Tabelle werden konkrete Hilfen bzw. Inhalte zu den jeweiligen Themen mit den Foki auf die Phasen der Ausbildung und der ersten Berufsausübung dargestellt (Tab. 18) und erläutert.

Tab. 18: Konkrete Hilfen in der Ausbildungs- und Berufseinstiegsphase

\begin{tabular}{|c|c|c|}
\hline Thema Phase & $\begin{array}{l}\text { Ausbildungsphase } \\
\text { Lehramtsstudium }\end{array}$ & $\begin{array}{l}\text { Berufseinstiegsphase } \\
\text { Mentoring, Fortbildung }\end{array}$ \\
\hline Administration & $\begin{array}{l}\text { Kennenlernen von Software, Über- } \\
\text { blickswissen zu administrativen } \\
\text { Tätigkeiten }\end{array}$ & $\begin{array}{l}\text { Konkrete Unterstützung im Umgang } \\
\text { mit Administrationssoftware, Betreu- } \\
\text { ung durch MentorIn und/oder Kolle- } \\
\text { gium, rechtliches Hintergrundwissen } \\
\text { durch Schulleitung }\end{array}$ \\
\hline Systemwissen & $\begin{array}{l}\text { Kennenlernen der Organigramme der } \\
\text { Institutionen Bildungsdirektion und } \\
\text { Bildungsministerium, Exkursionen in } \\
\text { höheren Semestern zum persönlichen } \\
\text { Kennenlernen der Leitungspersonen, } \\
\text { Überblickswissen zur Schulorganisation } \\
\text { (SGA, Jahreskalender etc.) }\end{array}$ & $\begin{array}{l}\text { Einführungskonferenzen für Berufsan- } \\
\text { fängerInnen, Übernahme der Einfüh- } \\
\text { rungsverantwortung durch MentorIn } \\
\text { und/oder Kollegium }\end{array}$ \\
\hline
\end{tabular}




\begin{tabular}{|c|c|c|}
\hline Thema Phase & $\begin{array}{l}\text { Ausbildungsphase } \\
\text { Lehramtsstudium }\end{array}$ & $\begin{array}{l}\text { Berufseinstiegsphase } \\
\text { Mentoring, Fortbildung }\end{array}$ \\
\hline $\begin{array}{l}\text { Reflexion \& } \\
\text { Feedback }\end{array}$ & $\begin{array}{l}\text { Reflexion \& Feedback aus den Perspek- } \\
\text { tiven SchülerIn - LehrerIn; Initiierung } \\
\text { des Perspektivenwechsels }\end{array}$ & $\begin{array}{l}\text { Kollegiale Beratung in formellen Set- } \\
\text { tings (z.B. Professionelle Lerngemein- } \\
\text { schaft), Mentoring, aktives Feedback } \\
\text { über das Mentoring hinaus }\end{array}$ \\
\hline $\begin{array}{l}\text { Autorität in der } \\
\text { Klasse }\end{array}$ & $\begin{array}{l}\text { Reale Unterrichtssituationen ohne Pra- } \\
\text { xislehrperson in den pädagogisch-prak- } \\
\text { tischen Studien, Pädagogisch-praktische } \\
\text { Studien als Proberaum }\end{array}$ & $\begin{array}{l}\text { Persönlichkeitsbildung im Mento- } \\
\text { ring, Feedback durch Hospitationen, } \\
\text { Schulleitungsfeedback als Unterstüt- } \\
\text { zungsmaßnahme (nicht als Kontrolle } \\
\text { und Begutachtung), zusätzlich zur } \\
\text { Induktionsphase Angebot von Fortbil- } \\
\text { dungsveranstaltungen }\end{array}$ \\
\hline $\begin{array}{l}\text { Kooperation mit } \\
\text { Schülerinnen und } \\
\text { Schülern }\end{array}$ & $\begin{array}{l}\text { Pädagogisch-praktische Studien mit } \\
\text { Schwerpunkt Beziehungsarbeit }\end{array}$ & $\begin{array}{l}\text { Hospitationen bei MentorIn und/oder } \\
\text { KollegInnen, Kollegiale Beratung in for- } \\
\text { mellen Settings, Kollegiales Feedback, } \\
\text { Mentoring, Zusätzlich zur Induktions- } \\
\text { phase Angebot von Fortbildungsveran- } \\
\text { staltungen }\end{array}$ \\
\hline Habitus & $\begin{array}{l}\text { Inhaltlicher theoretischer Schwerpunkt } \\
\text { zur Habitualisierung und LehrerIn- } \\
\text { nenpersönlichkeit in den universitären } \\
\text { Seminaren }\end{array}$ & $\begin{array}{l}\text { Zusätzlich zur Induktionsphase } \\
\text { Theorie-Praxis-Verknüpfung in Fortbil- } \\
\text { dungsveranstaltungen }\end{array}$ \\
\hline
\end{tabular}

Das Thema Administration (Tab. 18) ist eher für die BerufsanfängerInnen als für die SchulleiterInnen eine brennendes, zumal die neuen Lehrpersonen das Gefühl haben, völlig ohne administratives Vorwissen ihre Berufslaufbahn zu starten. Dabei ist anzumerken, dass diese Einstiegshürde mit wenig Mitteln und Aufwand erheblich zu erleichtern ist, indem bereits im Rahmen der LehrerInnenausbildung entsprechende Software exemplarisch vorgestellt und mittels Testzugängen ausprobiert wird. Als Schulverwaltungssoftware ist in Österreich das Programm Sokrates im Einsatz und wird für alle möglichen administrativen Vorgänge an der Schule verwendet, von der Einschreibung der SchülerInnen über die Kommunikation mit den Erziehungsberechtigten bis zum Zeugnisdruck am Jahresende. Durch die Vernetzung der Ausbildungsinstitutionen mit der jeweiligen Bildungsdirektion ist es ein Formalakt, dieses Desiderat zu beheben. Zusätzlich zum konkreten Softwareeinstieg benötigen die Studierenden ein Überblickswissen über die beim Berufseinstieg anfallenden administrativen Tätigkeiten. Studierende haben oftmals keine Vorstellung darüber, dass LehrerIn-Sein viel mehr ist, als vor der Klasse zu stehen und zu unterrichten. Außerdem ist die pädagogische Überforderung (Stichwort: Praxisschock) zum Berufseinstieg ohnehin gegeben und kann durch die Entlastung aufgrund administrativen Vorwissens, welches während des Studiums angeeignet wird, eingedämmt werden.

In der Phase des Berufseinstiegs bzw. in den ersten Berufsjahren sind BerufsanfängerInnen auf die Unterstützung Ihrer MentorInnen, KollegInnen und SchulleiterInnen angewiesen. Neue Lehrpersonen fühlen sich insbesondere in Bezug auf administrative Tätigkeiten allein gelassen. Somit wird die Einstiegshürde signifikant erhöht. Neben konkreten Hinweisen im Umgang mit der Schulverwaltungssoftware oder zu Terminplanungen anfallender Administrationstätigkeiten, was z.B. im Mentoring oder in der Rolle der Berufsanfängerin bzw. des Berufsanfängers als Co-Klassenvorstand einfach zu bewerkstelligen ist, sind rechtliche Hinweise relevant. Diese 
betreffen z.B. zeitgerechte Mitteilungen an die Eltern bzgl. Leistungsabfall etc. und werden idealerweise durch die Schulleitung selbst gegeben.

Ähnlich wie bei Administration herrschen beim Systemwissen laut den BerufsanfängerInnen und deren SchulleiterInnen die größten Ausbildungsdesiderate innerhalb der vorliegenden Themenpalette. Während des Studiums kann den Studierenden ein erster (theoretischer) Einblick in das Schulsystem gewährt werden, indem Organigramme der schulbezogenen Institutionen, z.B. der Bildungsdirektion, des Bildungsministeriums, in den universitären Vorlesungen oder Seminaren tiefgehend thematisiert werden. Ein praktischer Zugang kann für höhersemestrige Studierende die Durchführung von Exkursionen in die Institutionen sein, mit dem zusätzlichen Vorteil, (Leitungs-)Personen im Gespräch kennenzulernen. Ein Blick ins Schulsystem auf der Mikroebene bringt den Studierenden in Bezug auf die Erfassung der Gesamtheit ihres angestrebten Berufs eine Horizonterweiterung. Schulgemeinschaftsausschuss, Klassenparlament, schulischer Jahreskalender sind Themen, mit denen sie später beim Berufseinstieg unweigerlich konfrontiert werden.

Die BerufsanfängerInnen können beim Berufseinstieg durch die persönliche Betreuung im Mentoring-Programm und zusätzlich durch Einführungskonferenzen ins System einsteigen. Auch hier wird das Gefühl des Allein-gelassen-Werdens thematisiert, welches durch eine verbindliche persönliche Einführungsverantwortung, z.B. durch die Mentorin bzw. den Mentor, gelindert werden kann. Der Schulleitung kommt eine Delegierungsverantwortung zu, die allerdings in vielen Fällen nicht wahrgenommen wird.

Mit Reflexion \& Feedback sprechen BerufsanfängerInnen und SchulleiterInnen ein Thema an, das im bildungswissenschaftlichen Curriculum der PädagogInnenbildung NEU bereits sehr stark verankert ist. Es geht darum, das eigene Tun zu reflektieren, anderen Feedback zu geben und von anderen Feedback anzunehmen. Dies geschieht idealerweise aus den Perspektiven der Studierenden selbst, aber auch nach einem initiierten kognitiven Perspektivenwechsel aus der Sicht der SchülerInnen und, noch wesentlicher, aus der Sicht angehender Lehrpersonen. Diese Perspektivenwechsel tragen letztlich zur LehrerInnenpersönlichkeitsbildung und Habitualisierung bei, indem Inkorporierungsvorgänge bereits in diesem Stadium beginnen können.

Die Chance, das eigene LehrerInnenhandeln zu reflektieren, Feedback zu geben und anzunehmen, besteht für BerufsanfängerInnen im ersten Dienstjahr im Rahmen der Induktionsphase durch den formellen Mentoring-Prozess. Hier liegt die Verantwortung nicht nur auf der Seite der BerufsanfängerInnen, sondern ebenso bei den MentorInnen und den SchulleiterInnen, die letztlich den Rahmen durch eine adäquate Lehrfächerverteilung, einen passenden Stundenplan etc. vorgeben. Eine weitere Möglichkeit zur Professionalisierung der BerufsanfängerInnen durch Reflexion \& Feedback besteht durch kollegiale Beratung in Form des strukturierten Settings Professionelle Lerngemeinschaft. Bonsen und Rolff (2006) zeigen signifikante Zusammenhänge zwischen der Durchführung Professioneller Lerngemeinschaften und der beruflichen Zufriedenheit von Lehrpersonen auf, die sich, ebenfalls signifikant nachweisbar, in qualitativ steigenden SchülerInnenleistungen niederschlagen. Die Berufszufriedenheit ist neben der Relevanz für BerufsanfängerInnen eine wesentliche Determinante für die Entwicklung der weiteren Berufsbiogra-fie der Lehrperson.

Das Thema Autorität in der Klasse ist jenes, wo BerufsanfängerInnen und zum Teil auch SchulleiterInnen nach dem Werkzeugkoffer mit den für alle möglichen Interaktions-Situationen passenden Tools fragen. Dass es diesen Koffer nicht gibt, wurde u.a. in der Diskussion zur Ungewissheit und Kontingenz mit den dazugehörenden Krisenerfahrungen thematisiert, wenngleich Rogers (2013) diesbezüglich teilweise rezeptartige Hinweise gibt. Eine Chance für Studierende 
besteht darin, Realsituationen in den pädagogisch-praktischen Studien herzustellen, indem z.B. Praxislehrpersonen vermehrt Verantwortung an die Studierenden abgeben. Ein weiterer Ansatz ist, die pädagogisch-praktischen Studien weniger seminar- bzw. leistungsbezogen mit Benotungspriorität zu sehen, sondern die Praktika der Studierenden in einer Art Proberaum stattfinden zu lassen. Handlungsvariationen und „Fehler“ sind dann explizit erwünscht, um die Bandbreite der Autoritätserfahrungen zu erweitern. Der Umgang mit Kontingenzerfahrungen sowie das Induzieren von Krisen wurden eingehend thematisiert. Wo kann das besser geübt und ausprobiert werden als im Proberaum der pädagogisch-praktischen Studien?

In der Berufseinstiegsphase können die MentorInnen entscheidend für Gelingensmomente für BerufsanfängerInnen sein. Diese artikulieren die Beanspruchung der Hilfe von erfahrenen KollegInnen explizit. Durch Hospitationen und gezieltes Feedback können MentorInnen wesentlich zur Persönlichkeitsbildung unerfahrener KollegInnen beitragen, dies betrifft ebenso den Ansatz der Hilfe zur Selbsthilfe. Die hierarchisch höhere Schulleitungsebene kann ebenfalls durch Feedback hilfreich agieren, da in diesem Fall aufgrund der anderen Perspektive eine neue wertvolle Dimension hinzukommt. In Bezug auf Hospitationen durch die SchulleiterInnen ist laut deren eigenen Aussagen allerdings Sensibilität gefragt. Diese können, wenn sie progressiv im Sinne eines Autoritätsakts durchgeführt werden, von den BerufsanfängerInnen als Misstrauen bzw. Kontrolle und von den SchülerInnen als Schwäche der BerufsanfängerInnen interpretiert werden. Beides kommt aufgrund der empirischen Erkenntnisse vor, beides dient der Entwicklung der natürlichen Autorität von BerufsanfängerInnen nicht. Neben den in der Induktionsphase verpflichtenden Seminaren für die VertragslehrerInnen in der Induktionsphase ${ }^{84}$ gilt es, generell in der Fortbildung für LehrerInnen auf Seminarangebote mit Inhalten zum LehrerInnenhandeln zu achten.

Auch in Bezug auf Kooperation mit Schülerinnen und Schülern findet in erster Linie Learningby-doing statt. In der LehrerInnenausbildung können im Rahmen der pädagogisch-praktischen Studien und den begleitenden universitären Seminaren kooperative Situationen theoretisch aufbereitet, durchgeführt bzw. geübt, reflektiert und im Sinne des Spiralprinzips auf qualitativ höherer Ebenen weiter behandelt werden. Operative Momente in der Auseinandersetzung mit SchülerInnen, KollegInnen etc. können am besten durch praktisches Tun vermittelt bzw. erarbeitet werden. Bei einer übermäßigen oder gar ausschließlich theoretischen Befassung fehlt den Studierenden der Konnex, was wiederum Unverständnis bewirkt.

In der Phase des Berufseinstiegs ist das kollegiale Feedback der MentorInnen und KollegInnen ebenso wichtig und von den BerufsanfängerInnen erwünscht wie jenes der SchulleiterInnen. Auch in Bezug auf Kooperation mit Schülerinnen und Schülern können formelle Settings wie die bereits erwähnten Professionellen Lerngemeinschaften (Bonsen \& Rolff 2006) sehr hilfreich sein. Die Implementierung dieser idealerweise regelmäßigen Treffen von LehrerInnen ist allerdings insofern eine Herausforderung, als einerseits deren physische Anwesenheit in der unterrichtsfreien Zeit und andererseits ein Know-how zur Durchführung der Professionellen Lerngemeinschaften notwendig ist. Das Engagement externer Berater kann sowohl die Verbindlichkeit zur Teilnahme als auch die Ergebnisqualität dieses Lernsettings positiv beeinflussen. Seminare im Rahmen der Induktionsphase und weiterführende Fortbildungsveranstaltungen sind selbstverständlich, wie auch schon bei vorangegangenen Themen, seitens der Ausbildungsinstitutionen zu forcieren.

Die theoretische Einführung des Themas Habitus im Sinne der Bildung der LehrerInnenpersönlichkeit bzw. des Vorgangs der Habitualisierung und des Setzens erster Kondensationspunkte zur Inkorporierung muss im Rahmen der universitären Lehrveranstaltungen erfolgen. Selbst wenn die Habitualisierung ein Leben lang stattfindet, beginnt die Formung des LehrerInnen-

84 Eine Vertragslehrperson in der Induktionsphase wird im Schuljargon als VLIP bezeichnet. 
habitus mit der Ausbildung und vorerst auf unbewusster Ebene. Die Ausbildungsinstitutionen haben die Chance und die Pflicht, Lehramtsstudierende bewusst auf diese berufsbiografische Entwicklung vorzubereiten und diese durch Reflexionen, Feedback etc. in der LehrerInnenpersönlichkeitsbildungs- und Habitualisierungsphase zu begleiten.

Auch in den Seminaren zur Induktionsphase beim Berufseinstieg und in der weiterführenden Fortbildung besteht die Chance, den professionellen Habitus der LehrerInnen in Form einer durchaus theoretischen Auseinandersetzung mit dem Thema der Habitualisierung zu stärken und weiterzuentwickeln. Professionalisierung hat nicht nur mit Besserwerden im Umgang mit täglichen, eventuell banalen und trotzdem durchaus herausfordernden Tätigkeiten zu tun, sondern bezieht sich auch oder gerade auf theoretische Inhalte, die mit praktischen Erfahrungen verglichen, verknüpft oder in Widerspruch gesetzt werden. Dieser Auftrag liegt bei den Aus- und Fortbildungsinstitutionen, sodass im Sinne der LehrerInnenprofession die Entwicklung von der Meisterlehre zur professionellen Lehre mit wissenschaftlich-reflexivem Habitus weiter vorangetrieben werden kann.

\subsection{Conclusio Forschungsfrage 2}

Forschungsfrage 2 lautet wie folgt:

Wie wirkt sich die initiale Berufserfahrung auf den unmittelbaren weiteren beruflichen Verlauf (bis drei Jahre) der BerufsanfängerInnen aus?

Im folgenden Abschnitt werden jene in den Bedingungsgefügen (Kap. III.4.2) diskutierten Schlüsselbegriffe und Essenzen herausgegriffen, die auf eine Determinierung der weiteren Berufsausübung bzw. der kurzfristigen Berufsbiografie bis drei Jahre schließen lassen. Nach der Sortierung der Schlüsselbegriffe werden die Wirkungen vorerst nach den Kriterien positiv, ambivalent und negativ unterschieden (Abb. 73), anschließend erfolgt die Beschreibung.

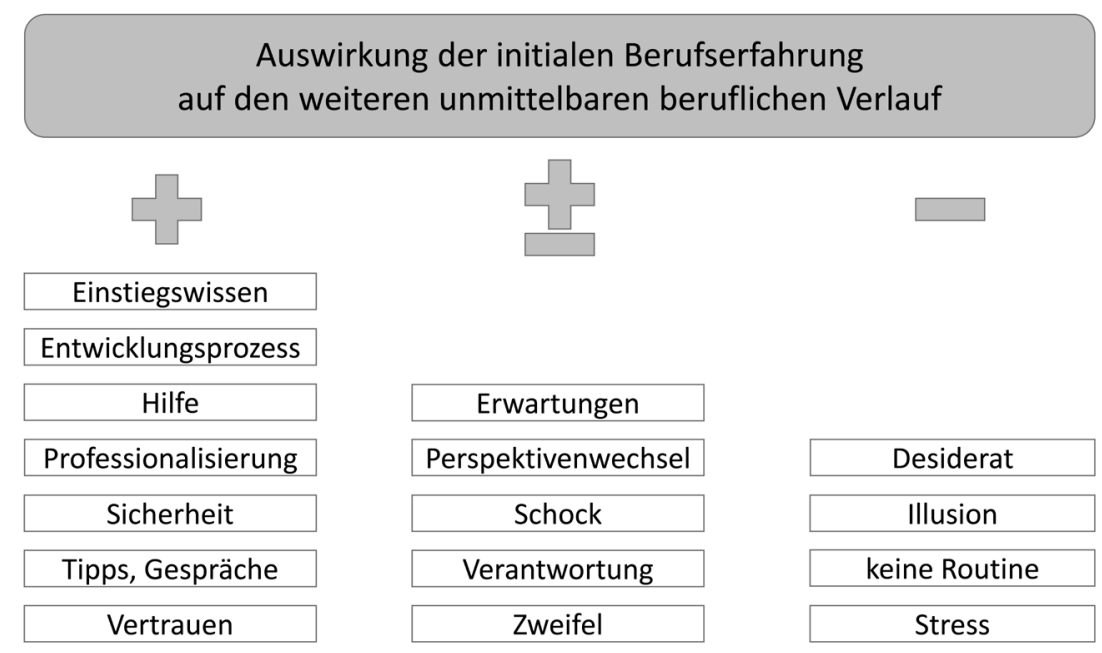

Abb. 73: Schlüsselbegriffe und Auswirkungen der initialen Berufserfahrungen 
Zunächst kann festgestellt werden, dass in der vorliegenden Untersuchung für positive Determiniertheiten mehr Schlüsselbegriffe zu identifizieren sind als für negative. Daraus kann abgeleitet werden, dass das Stimmungsbild des Status quo, erzeugt durch die BerufsanfängerInnen und SchulleiterInnen, und die zu erwartenden Auswirkungen auf die nächsten Berufsjahre eher positiv besetzt sind.

$\mathrm{Zu}$ den positiv konnotierten Schlüsselbegriffen in Bezug auf die Auswirkungen der initialen Berufserfahrung auf den weiteren unmittelbaren beruflichen Verlauf gehören Einstiegswissen, Entwicklungsprozess, Hilfe, Professionalisierung, Sicherheit, Tipps, Gespräche und Vertrauen. Ambivalente Begriffe sind Erwartungen, Perspektivenwechsel, Schock, Verantwortung und Zweifel. Die kleinste Gruppe der negativ konnotierten Schlüsselbegriffe besteht aus Desiderat, Illusion, keine Routine und Stress (Abb. 73).

Zur besseren Nachvollziehbarkeit und Transparenz werden die Schlüsselbegriffe der Abbildung 73 in der folgenden Diskussion kursiv dargestellt.

\section{Positive Wirkungen}

Positiv determiniert wird der weitere berufliche Verlauf durch vorhandenes Einstiegswissen. Dieses bezieht sich auf mehrere Bereiche des LehrerInnenberufs, beginnt bei administrativen und endet bei pädagogischen Belangen. Die positive Wirkung zeigt sich dadurch, dass Lehrpersonen die ihnen zur Verfügung stehenden Ressourcen für sie bestmöglich verteilen können, ohne vom Gefühl der Überforderung übereilt zu werden. Das wiederum gibt den ein bis drei Jahre im Dienst stehenden LehrerInnen das - eventuell subjektive - Gefühl der Sicherheit und ebenso des Vertrauens, eine Art positive Spirale wird in Gang gesetzt. Aber auch das Vertrauen, das die SchulleiterInnen den Lehrpersonen entgegenbringen, wirkt positiv. Die Berufszufriedenheit steigt signifikant, was sich in weiterer Folge auch auf andere Bereiche des LehrerInnenalltags auswirkt. Die Hilfe wird von den BerufsanfängerInnen oft thematisiert. Hier kann zwischen vorauseilender, nicht eingeforderter Hilfe und jener auf Nachfrage unterschieden werden. Ebenso können die Hilfemaßnahmen in unstrukturierte bzw. informelle (Tipps zwischendurch) und strukturierte bzw. formelle Settings (Reflexion und Feedbackgespräche im Rahmen des Mentoring-Prozesses) eingeteilt werden. Für beide Fälle gilt, dass BerufsanfängerInnen auf diese Hilfe angewiesen und ebenso dankbar dafür sind. In den Berufsjahren zwei und drei reduzieren sich die „Hilferufe“ signifikant, dies wird durch die Selbstwahrnehmung der betroffenen LehrerInnen ebenso bestätigt wie von den SchulleiterInnen aufgrund deren Fremdwahrnehmung. Unter den konkreten Hilfemaßnahmen verstehen die betroffenen Lehrpersonen nicht etwa das "Zeigen, wie es geht“, sondern viel mehr Tipps und Gespräche. Dies entspricht mit Einschränkung dem Ansatz des Mentoring-Prozesses, wo die Stärkung der Persönlichkeit und das Prinzip der Hilfe zur Selbsthilfe im Vordergrund stehen. Gerade durch die Gespräche, in erster Linie sind es formelle Mentoring-Gespräche, werden Entwicklungsprozesse in Gang gesetzt, die besonders von den SchulleiterInnen wahrgenommen und in den Gesprächen zur empirischen Untersuchung erwähnt werden. Letztlich befördern die Entwicklungsprozesse die Professionalisierung und Habitualisierung der noch am Beginn der Berufslaufbahn stehenden Lehrpersonen. Die möglicherweise wesentlichste Auswirkung sind Inkorporierungsvorgänge durch erste positive Berufserfahrungen und die initiale Entwicklung eines wissenschaftlich-reflexiven Habitus mit der Triade des professionellen Wahrnehmens, Denkens und Handelns.

\section{Ambivalente Wirkungen}

Ein Schlüsselbegriff, der zu ambivalenten Wirkungen bei den befragten Lehrpersonen führt, ist der Perspektivenwechsel. Dieser wird bereits in der Ausbildung initiiert, indem in den pädagogisch-praktischen Studien die Rolle der Lehrperson einzunehmen versucht wird. Tatsächlich 
unterscheidet sich jedoch die Rolle der Lehrperson von jener der Praktikantin bzw. des Praktikanten maßgeblich. Die Herausforderungen des Perspektivenwechsels werden nicht nur von BerufsanfängerInnen im ersten Dienstjahr, sondern auch von Lehrpersonen im zweiten und dritten Dienstjahr thematisiert. Einerseits ist vom guten Gefühl, endlich Lehrperson zu sein, die Rede und andererseits wird die Herausforderung des Wechsels von der bzw. dem Studierenden zur Lehrperson genannt. Letztlich ist es eine Herausforderung der Habitualisierung. Der SchülerInnenhabitus wirkt nach und dessen inkorporierte und mit dem Feld verknüpften Erfahrungen stehen teilweise im Widerspruch zum sich jetzt ausbildenden LehrerInnenhabitus mit ganz anderen Kapital- und Machtgefügen im selben Feld Schule, um in der Terminologie Bourdieus zu bleiben.

Auch die Erwartungen und die damit verbundene Verantwortung wirken ambivalent. Die Lehrpersonen stehen nach ihrer Ausbildung endlich im Berufsleben und sind nun fachlich und dienstrechtlich autorisiert, SchülerInnen zu unterrichten, was ein Gefühl des Stolzes mit sich bringt. Zugleich bewirken Erwartungen und Verantwortung auch Erfolgsdruck im Kollegium, gegenüber der Schulleitung, den SchülerInnen, Eltern etc. Dieser Erfolgsdruck, insbesondere wenn diesem nicht oder nicht genügend Stand gehalten werden kann, führt zu Zweifel, sogar von Gedanken über Berufsabbruch ist in den Interviews die Rede. Der Schock bzw. Praxisschock, eine Auswirkung der Überforderung in den ersten Berufsjahren, kann Verhaltensweisen in zwei Richtungen hervorrufen. Im negativen Fall führt er zu Lähmung, Stagnation bzw. zu unproduktivem LehrerInnenverhalten, im positiven fühlen sich Lehrpersonen angefeuert. Diese sprechen in diesem Zusammenhang vom Durchstarten.

Zusammengefasst kann zu ambivalenten Wirkungen konstatiert werden, dass dieses Thema mit Ungewissheit, Kontingenz und Unverfügbarkeit bzw. mit krisenhaften Erfahrungen in Zusammenhang steht. Der Umgang mit Krisen ist Ausdruck der Ambivalenz des LehrerInnenwirkens in den ersten Berufsjahren.

\section{Negative Wirkungen}

Explizit negative Wirkungen können aus den empirischen Erkenntnissen lediglich mit einigen wenigen Schlüsselbegriffen konnotiert werden. An erster Stelle sind hier, und diese Positionierung entspricht auch dem Erkenntnisbild der Befragung, Desiderate in der Ausbildung zu nennen. Ärger und Überforderung sind in diesem Zusammenhang die Wirkungen - und laut den Äußerungen der Lehrpersonen völlig unnötig. So sind häufig Desiderate von einfach in die Ausbildung zu integrierenden Inhalten die Ursache für Überforderung und unnötigen Stress, der sich dann aufgrund der fehlenden Zeitressourcen in die pädagogische Arbeit überträgt. Die Rede ist von den bereits diskutierten Themen Administration und Systemwissen. Die fehlende bzw. nicht vorhandene Routine führt ebenso zu Stressverhalten von Lehrpersonen in den ersten Dienstjahren. Vom nächtelangen Unterrichtsplanen ist in diesem Zusammenhang die Rede. Dass viele Berufsanfängerinnen mit Illusionen in den Beruf starten und die ersten Berufsjahre benötigen, um ein reales Berufsbild zu erhalten, wirkt insofern, als die Lehrpersonen nicht sofort zielgerichtete pädagogische Arbeit leisten können. Zu viel Aufmerksamkeit muss für das Zurechtrücken des „verschobenen“ LehrerInnenbilds aufgewendet werden. Möglicherweise trägt diese berufsbiografische Phase wesentlich zum Perspektivenwechsel von der bzw. vom Studierenden zur Lehrperson bei. Damit einher geht der noch zu geringe Grad der Habitualisierung als Lehrperson, insbesondere wenn der Studierenden- oder gar SchülerInnen-Habitus zu präsent sind. Insofern ist die Absolvierung des Lehramtsstudiums in Mindestzeit kein Vorteil per se. BerufsanfängerInnen, die an einer achtjährigen Schulform maturieren und ohne Zivilbzw. Präsenzdienst das Studium starten, haben wenig Zeit für den in Bezug auf die Illusionen 
wesentlichen Perspektivenwechsel und die Habitualisierung mit der Inkorporierungsphase zur initialen Ausbildung der LehrerInnenpersönlichkeit.

\subsection{Conclusio Forschungsfrage 3}

Forschungsfrage 3 lautet folgendermaßen:

Welche Anforderungen ergeben sich aus den gewonnenen Erkenntnissen an ein Curriculum für das Lehramtsstudium und an die Induktionsphase für BerufsanfängerInnen?

Die gewonnenen empirischen Erkenntnisse und jene aus den Diskussionen in den Grundlagen gelten nur zum Teil für das Curriculum der LehrerInnenausbildung und für die Induktionsphase mit dem begleitenden, informellen Mentoring-Prozess gleichermaßen. Partiell sind sie differenziert zu betrachten. In Bezug auf Forschungsfrage 3 wurden vier Themenfelder identifiziert, die im Folgenden diskutiert werden.

\section{Habitualisierung und Professionalisierung auf allen Systemebenen}

Für einen gelingenden Berufseinstieg ist die Rollenübernahme als LehrerIn Voraussetzung. Das geht sowohl aus Grundlagen (Kap. II) als auch aus Empirischer Zugang (Kap. III) hervor. In erster Linie sind es in einem ersten Schritt die Studierenden, deren Habitus sich bildet und deren Professionalitätsgrad sich entwickelt. In weiterer Folge professionalisieren sich die BerufsanfängerInnen in der Induktionsphase, ähnlich dem Spiralprinzip (Brunner 2009) mit sich wiederholenden Inhalten auf der jeweils nächst höheren Ebene. Dass die Implementierung von theoretischen und praktischen Inhalten im Curriculum zur LehrerInnenausbildung (Vorlesungen, Seminare, pädagogisch-praktische Studien) und in der Induktionsphase (Mentoring, Hospitationen, Feedback, eigene Unterrichtspraxis) zur Habitualisierung und Bildung der professionellen LehrerInnenpersönlichkeit führt, wurde eingehend diskutiert. Eine Ebene darf jedoch nicht vergessen werden. Auch für die AusbildnerInnen (Dozierende, Praxislehrpersonen, MentorInnen) gelten seit der Einführung der PädagogInnenbildung NEU andere Voraussetzungen. Alle AbsolventInnen des Lehramtsstudiums sind zukünftig AkademikerInnen, der Professionalitätsanspruch ist gewissermaßen implementiert und alle daran Beteiligten sind dahingehend gefordert. Das gilt verstärkt im Bereich des Mentorings. Die BerufsanfängerInnen befinden sich nicht mehr in Ausbildung, sie sind aus dienstrechtlicher Sicht Lehrpersonen im ersten Dienstjahr und treten den MentorInnen als KollegInnen gegenüber. Der Professionalitätsanspruch existiert auf beiden Seiten und vor allem in der abschließenden Phase der Begutachtung bzw. Bewertung wird die Profession der MentorInnen auf die Probe gestellt. Sie arbeiten mit KollegInnen zusammen und helfen dabei, deren Persönlichkeiten zu stärken und die Habitualisierung positiv zu beeinflussen. Zugleich sind sie in die Entscheidung, ob die Kollegin bzw. der Kollege im nächsten Jahr eine weitere Anstellung als Lehrperson bekommt, über die Erstellung eines Gutachtens zumindest beratend eingebunden. Hier können kollegiale Beratungen sowohl unter Dozierenden, Praxislehrpersonen und MentorInnen als auch in den Hochschullehrgängen zur Qualifizierung als MentorIn an der kontinuierlichen Entwicklung des eigenen Habitus und der eigenen Professionalisierung unter Einbeziehung curricularer Inhalte der Aus- und Fortbildung wertvoll sein.

\section{Vernetzung aller Beteiligten}

In Bezug auf die viel diskutierte Theorie-Praxis-Schere (Helmke 2007; Helsper 2001; Schneider \& Haas 2019; Terhart 2001, 2007) bietet sich neben der curricular-inhaltlichen auch eine opera- 
tional-personelle Vernetzung an. Insbesondere Studierende der Ausbildung fühlen sich zwischen den universitären Lehrveranstaltungen und den pädagogisch-praktischen Studien an den (Praxis-) Schulen nicht genügend vernetzt. Dies führt zu Verunsicherungen und letztlich zu inhaltlichen Defiziten. Insofern kann ein Ziel sein, die in Modulen abgebildeten Lehrveranstaltungen (Praktika und begleitende universitäre Seminare) kohärenter zu gestalten. Die handelnden Personen sprechen sich in Bezug auf gegenseitige Erwartungen auf inhaltlicher, administrativer etc. Ebene konkret ab (Abb. 74).

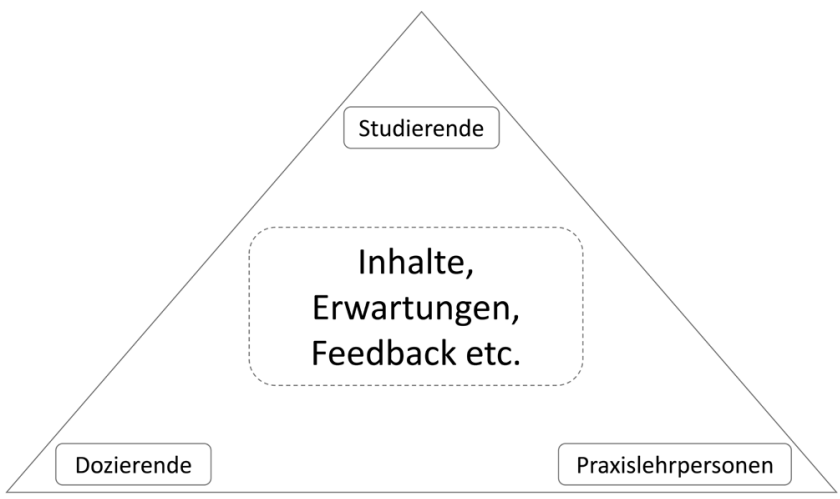

Abb. 74: Beziehungsdreieck im Ausbildungscurriculum

Durch physische Treffen der im Beziehungsdreieck beteiligten AkteurInnen Studierende, Dozierende und Praxislehrpersonen (Abb. 74) können durch gegenseitiges Feedback Barrieren abgebaut und Missverständnisse im Sinne Oevermanns (2017) prophylaktisch behandelt und vermieden werden. Dies betrifft die Induktionsphase auf Fortbildungsebene gleichermaßen. Das Beziehungsdreieck in der Induktionsphase bzw. im Fortbildungscurriculum wird von Mentees, MentorInnen und SchulleiterInnen oder anderen schulischen AkteurInnen gebildet (Abb. 75).

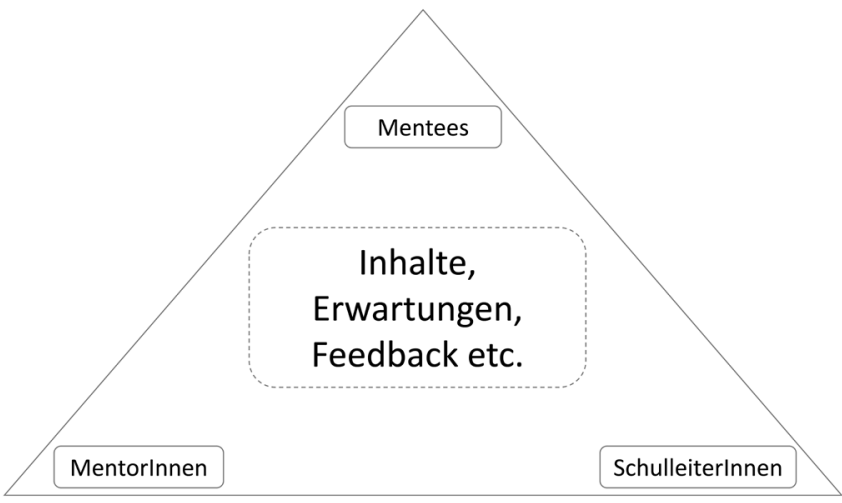

Abb. 75: Beziehungsdreieck in der Induktionsphase 
Mentees, MentorInnen und SchulleiterInnen befinden sich im Beziehungsdreieck (Abb. 75) und kommunizieren über Inhalte, Administration (Berücksichtigungen im Stundenplan) etc. Die Vernetzung der handelnden Personen kann zur positiven Beeinflussung der Feedbackkultur und letztlich zur Verbesserung der Professionalisierung aller AkteurInnen beitragen. Der Inkorporierungsprozess im Rahmen der Triade professionelles Wahrnehmen, Denken und Handeln, der Interaktion und Resonanz voraussetzt, ist ein immer wiederkehrender, nie abgeschlossener (Bourdieu 1996a, 2015b; Rosa 2018) (siehe auch Abb. 12 und 13, Kap. II.1.2.3.2). Kollegiale Beratungen, z.B. in Form von formellen Professionellen Lerngruppen (Bonsen \& Rolff 2006), erhöhen die Chance auf kontinuierliche Weiterentwicklung und Erweiterung des Blickwinkels. Regelmäßige Vernetzungstreffen in Form der erwähnten strukturierten Settings können zwischen MentorInnen, Mentees, SchulleiterInnen in jeweils hierarchisch homogenen oder gemischten Gruppen stattfinden. Der Nutzen dieser Professionellen Lerngemeinschaften ist wissenschaftlich nachgewiesen und beschränkt sich nicht auf die unmittelbar teilnehmenden Personen. Im Endeffekt sind die SchülerInnen die Profiteure. Sie sind diejenigen, denen Erkenntnisse in Theorie und Empirie letztlich zugutekommen.

\section{Mut zur Selektion}

Eine weitere Conclusio in Bezug auf die Anforderungen an die Curricula und in weiterer Folge auf den Umgang mit zu handhabenden Konsequenzen geht in eine andere als bisher kommunizierte Richtung, die Selektion. Insbesondere SchulleiterInnen vermissen, dass im Lehramtsstudium im Rahmen des Curriculums Selektionsprozesse nach deren Wahrnehmung nicht vorgesehen sind. Hier ist von Einstiegsüberprüfungen und Aufnahmetests gleichermaßen die Rede wie von Schwellenprüfungen im Laufe des Studiums an definierten Positionen im Curriculum. Aber auch BerufsanfängerInnen vermissen insbesondere zu Studienbeginn und während des Studiums im Rahmen der pädagogisch-praktischen Studien, wenn sich Studierende als nicht geeignet erweisen, Selektionsprozesse.

Die einhellige Meinung der SchulleiterInnen und BerufsanfängerInnen lautet dahingehend, dass es für SchülerInnen und das Schulsystem insgesamt verständlicherweise besser ist, wenn nicht geeignete Studierende durch Selektion den LehrerInnenberuf nicht ergreifen können. Ebenso wird begründet, dass selbst für die betreffenden Studierenden eine frühzeitige Selektion auf den berufsbiografischen Lebenslauf bezogen ein Vorteil sein kann.

Auch im Rahmen des Mentoring-Prozesses sind durch die abschließenden Vorgänge des Begutachtens und Bewertens durch die MentorInnen und in letzter Konsequenz durch die SchulleiterInnen Möglichkeiten der Selektion vorgesehen. Argumentieren SchulleiterInnen und BerufsanfängerInnen eine frühe, zeitgerechte Selektion mit dem weiteren berufsbiografischen Verlauf der Studierenden, kann die Devise zur Selektion im Mentoring-Prozess „besser spät als nie“ lauten. Wissenschaftlich-reflexive Habitualisierung, Professionalisierung und Inkorporierung bedingen ausbaufähige Fundamente, deren Konstitution, wenn sie in der Induktionsphase noch nicht vorhanden sind, äußerst fragwürdig erscheint. Hier stellt sich die Frage, ob die AkteurInnen (Dozierende, Praxislehrpersonen, MentorInnen, SchulleiterInnen) bei Bedarf eben nicht den Weg des geringsten Widerstandes wählen sollten, sondern sich im Sinne der Verantwortung den SchülerInnen, den Eltern, der LehrerInnenprofession und letztlich der Gesellschaft gegenüber bei begründetem Bedarf für den durchaus unpopulären Weg der Selektion entscheiden müssen.

\section{Anpassung der curricularen Inhalte und Ausbildungssettings}

„Pedanten spotten über Bildung, welche nützlich ist. Aber wenn Bildung nicht nützlich ist, was ist sie dann?", fragt Whitehead (2011, S. 40) und spricht damit ein Thema an, welches zu den 
primären Anliegen der SchulleiterInnen, BerufsanfängerInnen und Lehrpersonen in den ersten Dienstjahren zählt. Das im Curriculum bewährte und thematisierte Professionswissen wird dabei nicht in Frage gestellt. Vielmehr geht es um zusätzliches nutzbares Einstiegswissen, um die Erleichterung der ersten Hürden beim Berufseinstieg und darum, idealerweise den berüchtigten Praxisschock zu verhindern. Die bereits thematisierten Ausbildungsdesiderate in den Bereichen Administration und Systemwissen stellen vergleichsweise triviale Inhalte dar und können in ein Curriculum ohne immensen vorherigen Forschungsaufwand übernommen werden. Durch Vernetzung der Ausbildungsinstitutionen mit den Schulen (SchulleiterInnen, Lehrpersonen) können Desiderate, Wünsche und, wie in der vorliegenden Forschungsarbeit, Lösungen identifiziert werden. Werden die Ergebnisse den zuständigen Behörden (Bundesministerium, Bildungsdirektionen) weitergegeben, können Curricula angepasst werden. Die Theorie-Praxis-Schere wird enger, der "Sprung ins kalte Wasser“ (In-vivo-Code) wird für die BerufsanfängerInnen verkraftbar, im Idealfall entfällt er. Letztlich kann der Wegfall der Einstiegsüberforderung, ausgelöst auch durch Desiderate in administrativen und schulsystemischen Belangen, für BerufsanfängerInnen den Weg für Inkorporierung, Habitualisierung und letztlich Professionalisierung frei machen. Die Bildung der LehrerInnenpersönlichkeit wird dadurch ebenso lanciert wie die Offenheit für Kontingenz und pädagogische Krisenerfahrungen.

In Bezug auf das curriculare Ausbildungssetting der pädagogisch-praktischen Studien wird aufgrund der vorliegenden Erkenntnisse darauf hingewiesen, dass dieses zwar praktisch orientiert stattfindet, trotzdem aber laborähnlichen Charakter besitzt. Die Studierenden vermissen teilweise das verbindliche, eigenständige Agieren-Dürfen und realitätsnahe Unterrichtssituationen, welche z.B. durch die ständige Anwesenheit der Praxislehrpersonen eher gehemmt werden. Das kann eine Erklärung dafür sein, dass die BerufsanfängerInnen nach den im Rahmen der LehrerInnenausbildung absolvierten Praktika trotzdem vom Sprung ins kalte Wasser sprechen. Pädagogisch-praktische Studiensettings mit mehr Verantwortungsübernahme durch die Studierenden und zurückhaltender Präsenz der Praxislehrpersonen, dafür umso detailliertere Reflexions- und Feedbackgespräche nach den Unterrichtsstunden bzw. Praktika können einen Ansatz zur Verminderung der Berufseinstiegshürden und damit eine Gelingensbedingung für einen positiven Berufsstart darstellen. 


\section{Grenzen der Arbeit}

Neben der begrenzt durchführbaren Rekonstruktion des Feldes aufgrund methodologischer Grenzen (siehe Kap. III.2.4), ist die Generalisierbarkeit der Ergebnisse der vorliegenden Arbeit eingeschränkt. Dieser Umstand ist sowohl dem Sample als auch den geografischen Bedingungen geschuldet. Das Sample bilden ausschließlich AkteurInnen (SchulleiterInnen und BerufsanfängerInnen) aus der Sekundarstufe 1, womit die Generalisierbarkeit der empirischen Erkenntnisse auf die gesamte Bildungslandschaft nicht gegeben ist. Insofern wird in Interpretation der analysierten Daten (Kap. III.4) als eine Konzeptualisierung und nicht als Theoriebildung vorgenommen. Das Sample wurde deshalb aus SchulleiterInnen und BerufsanfängerInnen gebildet, damit Erzählungen und Erkenntnisse der InterviewteilnehmerInnen aus den jeweiligen Perspektiven der Selbst- und Fremdwahrnehmung berücksichtigt werden. Trotzdem, das stellt eine weitere Grenze der Generalisierbarkeit der Erkenntnisse dar, basieren die Interviewdaten einerseits auf Selbsteinschätzung und sind andererseits subjektiv interpretierbar, da Sprache und Kommunikation per se vieldeutig sind (Aeppli 2014, S. 191).

$\mathrm{Zu}$ den geografischen Bedingungen ist anzumerken, dass die tirolweite Befragung bei Betrachtung auf nationaler und internationaler Ebene letztlich einen ländlichen Raum abbildet, auch wenn Lehrpersonen z.B. aus urbanen Lebensbereichen wie Innsbruck und Kufstein interviewt wurden. In Sample (Kap. III.1.3) wird deshalb nicht zwischen Stadt und Land, sondern zwischen städtischem und ländlichem Bereich unterschieden. Auch die relativ homogene Bevölkerungsschicht (z.B. in Bezug auf Migration, soziale Herkunft, Lebensstandards etc.) ist, wie die Beschränkung auf den ländlichen Raum, eine limitierende Determinante bzgl. Generalisierbarkeit der empirischen Erkenntnisse. Dies gilt für internationale Vergleiche ebenso wie für nationale, da selbst innerhalb von Österreich z.B. in Wien völlig andere Voraussetzungen in Bezug auf die Homogenität der Bevölkerungsschicht gelten wie in Tirol, wo die vorliegende Untersuchung durchgeführt wurde. 


\section{Ansätze zur gelingenden Professionalisierung - Ausblick}

In der Einleitung (Kap. I) wurde Bezug auf die verschiedenen Ansätze der LehrerInnenprofessionalisierung genommen, auf den strukturtheoretischen Bestimmungsansatz mit vielen antinomischen Strukturen in der Ausübung der LehrerInnenrolle, auf den kompetenztheoretischen Bestimmungsansatz, der die Aufgabenbeschreibungen, Kompetenzbereiche und Wissensdimensionen des LehrerInnenberufs definiert, und schließlich auf den berufsbiografischen Bestimmungsansatz, der den allmählichen Kompetenzaufbau im Laufe der Berufsjahre thematisiert (Terhart 2011). In dieser Forschungsarbeit treten diese Ansätze in unterschiedlichen Zusammenhängen zutage, seien es strukturtheoretische Herausforderungen in Bezug auf Antinomie mit daraus resultierenden Kontingenz- bzw. Krisenerfahrungen oder kompetenztheoretisch positionierte Entwicklungen, wo es z.B. um Classroom Management oder den Umgang mit schulsystemischen Herausforderungen geht. Schließlich spielt in der vorliegenden Arbeit auch oder gerade der berufsbiografische Bestimmungsansatz eine wesentliche Rolle. So fällt in diesen Bereich die Bildung der LehrerInnenpersönlichkeit und die Thematik der Habitualisierung als Lehrperson.

Kraler (2008b, S. 7) sieht Professionalität als „berufsbiografische Entwicklungsaufgabe“ und legt den Fokus hierbei insbesondere auf die BerufsanfängerInnen, deren Überforderungen beim Berufseinstieg meist unterschätzt, durch Einführungsprogramme, wie den Mentoring-Prozess, jedoch wirkungsvoll und nachhaltig unterstützt werden kann. Insofern sind nicht nur die inhaltlichen Dimensionen der verschiedenen, oben genannten Bestimmungsansätze von Bedeutung, sondern auch die zeitliche Dimension von der Ausbildung über den Berufseintritt bis zu den ersten Berufsjahren.

Die Erkenntnisse der vorliegenden Arbeit zeigen, dass der Schlüssel zum gelingenden Berufseinstieg und zur anschließenden Entwicklung zur erfolgreichen Lehrperson in der ganzheitlichen Professionalisierung liegt. In den Clusterkategorien zur Konzeptualisierung von Gelingensbedingungen für einen erfolgreichen Berufseinstieg und eine positive berufsbiografische Entwicklung werden Kompetenzen im Rahmen der Ausbildung und des Mentorings sowie anwendungsorientiertes Wissen bzgl. Kompetenzen identifiziert (Clusterkategorien 1 und 2). Auf höchster Konzeptebene steht die Professionalisierung durch Kompetenzentwicklung (Clusterkategorie 3) als prioritäre Erkenntnis.

Diese Kompetenzentwicklung ist nicht, wie aufgrund der Terminologie angenommen werden könnte, auf den kompetenztheoretischen Bestimmungsansatz ausgelegt. Aus den empirischen Erkenntnissen geht eindeutig hervor, dass die Kompetenzentwicklung erstens aus der jeweiligen Perspektive des strukturtheoretischen und kompetenztheoretischen Ansatzes zu betrachten ist. Zweitens stellt die jeweilige Phase (Ausbildung, Berufseinstieg, erste Berufsjahre) im Sinne des berufsbiografischen Ansatzes eine im Moment der Betrachtung besondere Bedeutung für die nachhaltige Entwicklung der Lehrperson dar. Letztlich führt die integrative, sich im stetigen triadischen Prozess „professionelles Wahrnehmen, Denken und Handeln“ befindliche Habitua- 
lisierung zur ganzheitlichen Professionalisierung. Habitus kann als Konzept der Professionalität verstanden werden.

Im Folgenden werden zwei Ansätze vorgestellt, die die Idee der ganzheitlichen Professionalisierung von (angehenden) Lehrpersonen verfolgen. Unabhängig davon, ob sich diese in der Phase der Ausbildung, des Übergangs oder in den ersten Berufsjahren befinden. Zugleich betone ich, dass die Darstellung der folgenden Konzepte Kontingenz- bzw. Unverfügbarkeitsfenster öffnet und die Neugierde auf weitere, detaillierte Beforschung dieser konkreten Themen weckt. 


\section{Spiralförmig in kleinen Schritten zur Professionalität: Delta P}

Diverse Modelle (Bromme 1992; Hubermann 1991; Messner \& Reusser 2000) zur Bildung der LehrerInnenpersönlichkeit, zu berufsbiografischer Entwicklung etc. zeigen, dass der Prozess der Habitualisierung bzw. der Professionalisierung als Lehrperson einen lang andauernden, wenn nicht einen lebenslangen Entwicklungsprozess darstellt. Die empirischen Erkenntnisse der vorliegenden Arbeit machen deutlich, dass eben diese Erkenntnis bei den Studierenden in der Phase der Ausbildung sowie bei den BerufsanfängerInnen in der Phase des Berufseinstiegs bzw. in den ersten Berufsjahren Stress auslösen. Im Wissen, noch nicht bzw. noch nicht genügend professionalisiert zu sein, wird bereits von BerufsanfängerInnen der professionelle Habitus erwartet. Im Wissen, dass die Phase der Professionalisierung eine sehr lange ist, wird den Studierenden eventuell suggeriert, mit Abschluss des Studiums als „Profis“ in den Beruf zu starten. Die Erwartung des professionellen Handelns und die Aussicht auf den professionellen Berufseinstieg sind unrealistisch und irreführend, in gewisser Weise für die Studierenden und BerufsanfängerInnen deprimierend. Der Praxisschock und das Phänomen der Konstanzer Wanne (Müller-Fohrbrodt et al. 1978) scheinen als logische Konsequenz unausweichlich auf die BerufsanfängerInnen hereinzubrechen. Im Kompetenzstufenmodell nach Gordon und Burch (Oerter et al. 2002) und in der Erweiterung dieses Modells durch Kraler (2008b), welches an dieser Stelle bezugnehmend auf das Konzept Professionalisierung durch Kompetenzentwicklung (Clusterkategorie 3, Kap. III.3.4) stellvertretend für die bereits genannten Entwicklungsmodelle ausgewählt wird, durchlaufen Auszubildende und bereits im Beruf stehende Personen mehrere Stufen mit unterschiedlichen Kompetenzniveaus (Abb. 76).

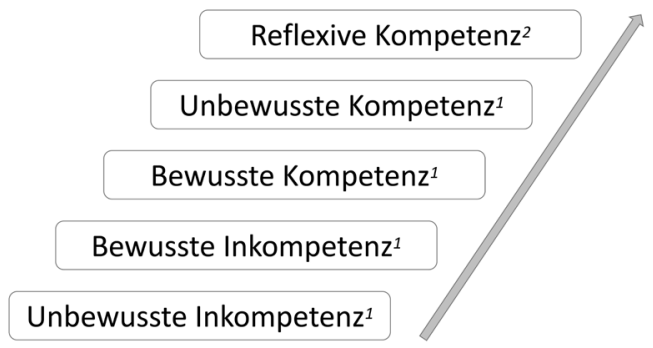

Abb. 76: Kompetenzstufenmodell nach Gordon \& Burch $^{(1)}$ und Kraler ${ }^{(2)}$

Wie in Abbildung 76 dargestellt, entwickeln sich Personen von der Inkompetenz zur Kompetenz und in einer Pendelbewegung vom Unbewussten zum Bewussten. So werden die Stadien unbewusste Inkompetenz, bewusste Inkompetenz, bewusste Kompetenz und unbewusste Kompetenz (Oerter et al. 2002) durchlaufen. Zum Abschluss wird die höchste Stufe, die reflexive Kompetenz (Kraler 2008b), auf der bewussten Ebene erreicht.

Die Studierenden kommen in der Phase der unbewussten Inkompetenz naiv und ohne professionsspezifische theoretische und praktische Grundlagen in die Ausbildung, Handlungen erfolgen intuitiv falsch. Auf der Stufe der bewussten Inkompetenz werden professionsspezifische Defizite zwar wahrgenommen, sie können jedoch noch nicht analytisch erfasst werden. In der Phase der bewussten Kompetenz können berufliche Herausforderungen zuverlässig, jedoch 
nicht automatisiert behandelt werden, während auf der Stufe der unbewussten Kompetenz diese Herausforderungen z.B. aufgrund der Handlungsroutine ohne überlegen zu müssen gelöst werden. Schließlich wird in der Phase der reflexiven Kompetenz das Handeln „problembezogen selektiv kritisch hinterfragt, verfeinert, adaptiert und weiterentwickelt“ (Kraler 2008b, S. 6). Im Habitualisierungsprozess nach Bourdieu (2015b) (siehe auch Abb. 15; Kap. II.1.2.3.2) befinden sich die AkteurInnen auf dieser reflexiven Kompetenzstufe im Besitz der wissenschaftlichreflexiven Kompetenz (Helsper 2018; Hericks et al. 2018; Kraler 2008b). Die professionelle Habitualisierung findet aufgrund der Triade professionelles Wahrnehmen - professionelles Denken - professionelles Handeln statt.

Wird nun die Aufmerksamkeit in der Aus- und Fortbildung, z.B. im begleitenden MentoringProzess, sofort auf das große Ganze gelenkt, im obigen Beispiel auf das Stadium der reflexiven Kompetenz, können Überforderung, Deprimierung etc. der Aus- und Fortzubildenden stattfinden. Zwei Ansatzunkte zur Deeskalation dieser Phänomene werden festgestellt. Zum einen kann im Sinne eines Spiralcurriculums (Bruner 2009), wo Inhalte immer wieder auf jeweils höheren Niveaus bzw. Kompetenzstufen behandelt werden, der Druck des sofortigen, fehlerfreien und professionellen Handelns von den AkteurInnen genommen werden. Bruner drückt die Idee des „spiral curriculum“ folgendermaßen aus: „Let the topics be developed and redeveloped in later grades“" (ebd., S. 54).

Ein zweiter möglicher Ansatz lautet, die Studierenden im Lehramtsstudium und den BerufsanfängerInnen in der Induktionsphase bzw. in den ersten Berufsjahren in kleinen Schritten zur Professionalität, ich nenne es Delta $\mathrm{P}(\Delta \mathrm{P})^{85}$, zu begleiten. Bourdieu $(2011, \mathrm{~S}$. 156) verurteilt jene Personen, „die es gewohnt sind, in den Begriffen Alles oder Nichts zu denken“. Er drückt damit den kontinuierlich-integrativen Zugang zu Wissen bzw. Kompetenz in kleinen Schritten ebenso aus wie die Verantwortung der handelnden Personen. Die kleinen Schritte der Professionalisierung, $\Delta \mathrm{P}$, halten das Kontinuum der Professionalisierungstriade wahrnehmen - denken - handeln (Bourdieu 2015b) über die stetige Inkorporierung (ebd.) am Leben und bringen Studierende sowie Lehrpersonen auf höhere Kompetenzstufen (Kraler 2008a; Oerter et al. 2002). Mit professionellem LehrerInnenhabitus wird zum Ziel, zur Vision navigiert, zur professionellen Lehrperson mit beliebig vielen Handlungsschemata für beliebig viele (Unterrichts-) Situationen.

Diese große Verantwortung liegt u.a. bei den Dozierenden, Praxislehrpersonen, MentorInnen und SchulleiterInnen. Sie sind es, die den Blick auf konkrete, überschau- und realisierbare Kompetenz- und letztlich Professionalitätszuwächse lenken können. Reflexion, Feedback und Unterstützung in der persönlichen Entwicklung wurden in der vorliegenden Arbeit ausführlich diskutiert und können die passenden Werkzeuge dafür sein. 


\section{Ganzheitliche Professionalisierung in der Ausbildung}

Dass die professionelle Habitualisierung als Lehrperson eine lange Zeit in Anspruch nimmt, sofern sie überhaupt abgeschlossen werden kann, wurde ausführlich diskutiert. Umso wichtiger erscheint es, in der Aus- und Fortbildung die Fühler in viele Richtungen auszustrecken, um den Lehrpersonen ein möglichst umfassendes Professionalitätsbild zugänglich zu machen. Für Blömeke (2003) setzt sich eine umfassende professionelle Kompetenz aus der Trias Wissen, Handlungsroutinen und Ethos zusammen. Wissen beschränkt sich dabei nicht auf das isolierte Fachwissen, sondern inkludiert auch dessen Vermittlung und ist in diesem Sinne eher fachdidaktisch als fachwissenschaftlich organisiert. Handlungsroutinen zeichnen sich dadurch aus, dass Situationen schneller wahrgenommen und Handlungsentscheidungen schneller und besser getroffen werden können. Das Berufsethos wirkt im gesamten Berufsalltag und ist von Verantwortung und Engagement gekennzeichnet (Schönknecht 2005). Kraler (2012a) identifiziert die kulturelle Dimension einer umfassenden und, um in meiner Terminologie zu bleiben, ganzheitlichen Professionalisierung und sieht kulturelle Tradierungs- und Entwicklungsprozesse auf der Makro-, Meso- und Mikroebene. Die Makroebene beschreibt das Bildungs- und gesellschaftspolitische System, die Mesoebene die Institutionen (Schule, Aus- und Fortbildungsinstitutionen, Bildungsdirektion etc.) und die Mikroebene ist als personale Ebene, jene der einzelnen Lehrperson, Schulleitung, Schulaufsichtsperson etc., zu verstehen. Als Ziel der LehrerInnenausbildung formuliert Kraler (2012b, S. 66) „die Vermittlung solider inhaltlicher Kenntnisse in den Fächern und die Einführung in innovative Zugänge zu pädagogischem und fachdidaktischem Wissen“, was einerseits die große, ganzheitliche Bandbreite der zu vermittelnden Kompetenzen und des zu thematisierenden Wissens zum Ausdruck bringt und andererseits den Beginn eines langen Professionalisierungsprozesses andeutet.

Die Idee der ganzheitlichen Professionalisierung besteht darin, im Wissen, dass in der Ausbildung Professionalisierungsprozesse lediglich initiiert werden und in der Fortbildung zwar weiterentwickelt, aber nach den Erkenntnissen der vorliegenden Arbeit nicht abgeschlossen werden, die Curricula der jeweiligen Disziplinen Fachausbildung, fachdidaktische Ausbildung und bildungswissenschaftliche Grundlagen in ihrer Ganzheitlichkeit zu betrachten. Darüber hinaus ist zu berücksichtigen, die pädagogisch-praktischen Studien aufgrund der Positionierungen im Spiralcurriculum (Leopold-Franzens-Universität Innsbruck 2016) aufeinander aufbauend zu konzipieren. Das Prinzip der kleinen Professionalisierungsschritte $\Delta \mathrm{P}$ (Kap. V.1) hilft den Studierenden und diese sind in den begleitenden Lehrveranstaltungen durch Dozierende und Praxislehrpersonen dahingehend zu betreuen.

Lehramtsstudierende absolvieren im Rahmen ihrer Ausbildung Fach- und Fachdidaktikstudien sowie Studien zu bildungswissenschaftlichen Grundlagen und pädagogisch-praktische Studien (Abb. 77). Die pädagogisch-praktischen Studien umrahmen die Curricula und stellen den praktischen Bezug zur theoretischen Ausbildung im Sinne der Schließung der Theorie-Praxis-Schere her. 


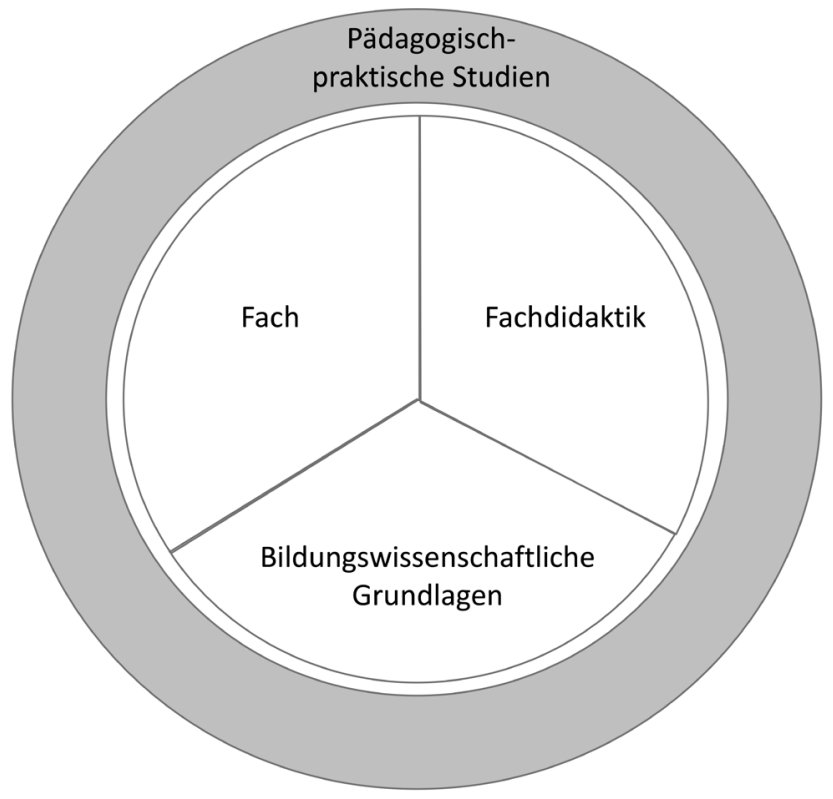

Abb. 77: Ganzheitliche Professionalisierung

„Such knowledge should be linked organically with the practical activities of teaching and a constant interrelation between theory and practice should be envisaged", stellt Ostinelli (2009, S. 305) fest und bestätigt die empirischen Erkenntnisse bezüglich der notwendigen Integration von Theorie und Praxis. Auch Conway (2012, S. 334) unterstreicht die Relevanz der pädagogisch-praktischen Inhalte: „A focus an the pracitcal“ lautet die Devise. Das Prinzip der ganzheitlichen Professionalisierung kann dann funktionieren, wenn sich die AkteurInnen der einzelnen Curricula (Fach, Fachdidaktik, Bildungswissenschaftliche Grundlagen, pädagogisch-praktische Studien) auf der inhaltlichen und personellen Ebene vernetzen, um den Studierenden und BerufsanfängerInnen fachliche, fachdidaktische und pädagogische Inhalte aus verschiedenen Perspektiven näher zu bringen und zu erschließen. Die Perspektive der pädagogisch-praktischen Studien wird idealerweise in alle anderen Teilstudien (Fach, Fachdidaktik, Bildungswissenschaft) integriert.

In Abbildung 78 wird das Wissensnetz dargestellt, welches die verschiedenen AusbildungsakteurInnen bilden. Studierende haben die Chance, aus je verschiedenen Blickwinkeln Ihrer AusbildnerInnen letztlich eine ganzheitliche Entwicklung zu erleben.

Die AusbildungsakteurInnen ihrer jeweiligen Fachdisziplin (Fachdidaktik, Bildungswissenschaft, Fach, pädagogisch-praktische Studien) bilden ein personelles und über die Curricula determiniertes Wissens- und Kompetenznetz. Die Studierenden sind in diesem (Wissens-)Netz geborgen und können sich bei ganzheitlicher Betrachtung der Ausbildung, bei Vernetzung der AkteurInnen über Inhalte, ganzheitlich professionalisieren (Abb. 78). 


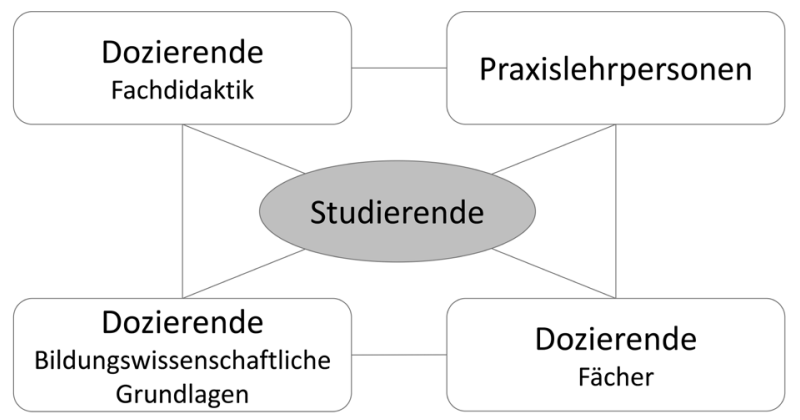

Abb. 78: Studierende in der Vernetzung der AusbildungsakteurInnen und Curricula

Die AkteurInnen tragen in diesem Sinne über die Inhalte der Curricula zur Professionalisierung der Studierenden bei.

Diese Arbeit schließt mit einem Gedanken Terharts (2013), wonach insbesondere selbstkritische und reflektierende Rückwendung auf das eigene Handeln einen zentralen Motor für die Weiterentwicklung von Professionalität darstellt. Das kann ein guter Leitgedanke für die am Professionalisierungsprozess beteiligten AkteurInnen sein. Durch das kritische Hinterfragen und Reflektieren des eigenen Handelns können Freiräume für Vernetzung und Kooperation, letztlich für ganzheitliche Professionalisierung, geschaffen werden. 



\section{Verzeichnisse}

\section{Literaturverzeichnis}

Abteilung Bildung des Landes Tirol. (2015). Das neue Dienst- und Besoldungsrecht für Landesvertragslehrpersonen. Kurzinformation. https://www.tirol.gv.at/fileadmin/themen/bildung/bildung/downloads/2015/Formulare/ Kurzinformation_Dienstrecht.pdf.

Aeppli, J. (2014). Empirisches wissenschaftliches Arbeiten. Ein Studienbuch für die Bildungswissenschaften (UTB, 4201: Pädagogik, 3., überarb. Aufl.). Bad Heilbrunn: UTB; Klinkhardt.

Artmann, M. \& Herzmann, P. (2018). Studienprojekte im Praxissemester. Forschungsfragen zwischen Erfahrungsbasierung und fachlichen Forschungslogiken. In M. Artmann, M. Berendonck, P. Herzmann \& A. Liegmann (Hrsg.), Professionalisierung in Praxisphasen der Lehrerbildung. Qualitative Forschung aus Bildungswissenschaft und Fachdidaktik (Studien zur Professionsforschung und Lehrerbildung, S. 56-73). Bad Heilbrunn: Verlag Julius Klinkhardt.

Atkinson, W. (2011). From sociological fictions to social fictions: some Bourdieusian reflections on the concepts of 'institutional habitus' and 'family habitus'. British Journal of Sociology of Education 32(3), 331-347.

Barlösius, E. (2011). Pierre Bourdieu (2. Aufl.). Frankfurt am Main: Campus Verlag.

Bennewitz, H. (2012). Der Blick auf Lehrer/-innen. In H. d. Boer (Hrsg.), Beobachtung in der Schule - Beobachten lernen (Lehrbuch, S. 203-214). Wiesbaden: Springer VS.

Blömeke, S. (2003, Oktober). Professionelles Lehrerhandeln. Kriterien und aktuelle empirische Erkenntnisse aus der Unterrichtsforschung, Vortrag an der Universität Passau.

Boelhauve, U. (2005). Forschendes Lernen - Perspektiven für erziehungswissenschaftliche Praxisstudien. In A.H. Hilligus \& H.-D. Rinkens (Hrsg.), Zentren für Lehrerbildung - neue Wege im Bereich der Praxisphasen. 24. Bundestagung der Leiterinnen und Leiter der Schulpraktischen Studien im Mai 2004 (Paderborner Beiträge zur Unterrichtsforschung und Lehrerbildung, Bd. 10, S. 103-126). Münster: Lit-Verl.

Boer, H. d. (2012a). Beobachtung und Professionalisierung. In H. d. Boer (Hrsg.), Beobachtung in der Schule - Beobachten lernen (Lehrbuch, S. 301-311). Wiesbaden: Springer VS.

Boer, H. d. (2012b). Der Blick auf sich selbst. In H. d. Boer (Hrsg.), Beobachtung in der Schule - Beobachten lernen (Lehrbuch, S. 215-226). Wiesbaden: Springer VS.

Bohnsack, R. (2017). Praxeologische Wissenssoziologie. Opladen: Barbara Budrich, UTB.

Bönsch, M. (2006). Das didaktische Dreieck als Grundmodell. In M. Bönsch (Hrsg.), Allgemeine Didaktik. Ein Handbuch zur Wissenschaft vom Unterricht (1. Aufl., S. 149-150). Kohlhammer Verlag.

Bonsen, M. \& Rolff, H.-G. (2006). Professionelle Lerngemeinschaften von Lehrerinnen und Lehrern. Zeitschrift für Pädagogik 52(2), 167-184.

Bortz, J. \& Döring, N. (2006). Forschungsmethoden und Evaluation. Für Human- und Sozialwissenschaftler; mit 87 Tabellen (Springer-Lehrbuch Bachelor, Master, 4., überarb. Aufl., [Nachdr.]. Heidelberg: Springer-Medizin-Verl.

Bourdieu, P. (1982). Die feinen Unterschiede. Kritik der gesellschaftlichen Urteilskraft. Frankfurt am Main: Suhrkamp.

Bourdieu, P. (1996a). Die Logik der Felder. In P. Bourdieu \& L. Wacquant (Hrsg.), Reflexive Anthropologie. Frankfurt am Main: Suhrkamp.

Bourdieu, P. (1996b). Die Praxis der reflexiven Anthropologie. In P. Bourdieu \& L. Wacquant (Hrsg.), Reflexive Anthropologie (S. 251-294). Frankfurt am Main: Suhrkamp.

Bourdieu, P. (1998a). Praktische Vernunft. Zur Theorie des Handelns. Frankfurt am Main: Suhrkamp.

Bourdieu, P. (1998b). Vom Gebrauch der Wissenschaft. Für eine klinische Soziologie des wissenschaftlichen Feldes. Konstanz: UVK Verl.-Ges.

Bourdieu, P. (2011). Rede und Antwort (Edition Suhrkamp, 3. Aufl.). Frankfurt am Main: Suhrkamp.

Bourdieu, P. (2015a). Entwurf einer Theorie der Praxis. Auf der ethnologischen Grundlage der kabylischen Gesellschaft (Suhrkamp-Taschenbuch Wissenschaft, Bd. 291, 4. Auflage). Frankfurt am Main: Suhrkamp.

Bourdieu, P. (2015b). Sozialer Sinn. Kritik der theoretischen Vernunft (Suhrkamp-Taschenbuch Wissenschaft, Bd. 1066, 9. Auflage). Frankfurt am Main: Suhrkamp.

Bourdieu, P. \& Wacquant, L. (Hrsg.). (1996). Reflexive Anthropologie. Frankfurt am Main: Suhrkamp. 
Brandhorst, A., Goerigk, P., Schöning, A. \& Dempki, C. (2018). Zwischen Forschung und Praxis - Das Praxissemester aus der Perspektive von Lehrenden der Fachdidaktiken. In M. Artmann, M. Berendonck, P. Herzmann \& A. Liegmann (Hrsg.), Professionalisierung in Praxisphasen der Lehrerbildung. Qualitative Forschung aus Bildungswissenschaft und Fachdidaktik (Studien zur Professionsforschung und Lehrerbildung, S. 93-110). Bad Heilbrunn: Verlag Julius Klinkhardt.

Brennecke, B., Pfaff, N., Schrader, T.-B. \& Tervooren, A. (2018). Das Praxissemester als Erfahrungsraum Forschenden Lernens? Ambivalenzen in der Aneignung qualitativer Forschungsmethoden. In M. Artmann, M. Berendonck, P. Herzmann \& A. Liegmann (Hrsg.), Professionalisierung in Praxisphasen der Lehrerbildung. Qualitative Forschung aus Bildungswissenschaft und Fachdidaktik (Studien zur Professionsforschung und Lehrerbildung, S. 38-55). Bad Heilbrunn: Verlag Julius Klinkhardt.

Breuer, F., Muckel, P., Dieris, B. \& Allmers, A. (2018). Reflexive Grounded Theory. Eine Einführung für die Forschungspraxis (3., vollständig überarbeitete und erweiterte Auflage). Wiesbaden: Springer.

Bromme, R. (1992). Der Lehrer als Experte. Bern: Huber.

Bromme, R. \& Haag, L. (2008). Forschung zur Lehrerpersönlichkeit. In W. Helsper \& J. Böhme (Hrsg.), Handbuch der Schulforschung (2., durchgesehene und erw. Aufl., S. 803-819). Wiesbaden: VS Verlag für Sozialwissenschaften/ GWV Fachverlage, Wiesbaden.

Bruner, J.S. (2009). The Process of Education. Cambridge: Harvard University Press.

Buber, R., Kraler, C. \& Gadner J. (2000). How GABEK and WinRelan support Qualitative Research. In R. Buber \& J. Zelger (Hrsg.), GABEK II. Zur qualitativen Forschung - on qualitative research (S. 111-139). Innsbruck: StudienVerl.

Buhren, C.G. \& Rolff, H.-G. (2011). Personalmanagement für die Schule. Ein Handbuch für Schulleitung und Kollegium (Beltz Pädagogik, Bd. 2, Neu ausgestattete Sonderausg). Weinheim: Beltz.

Bundeskanzleramt. (1986). Bundesgesetz über die Ordnung von Unterricht und Erziehung in den im Schulorganisationsgesetz geregelten Schulen (Schulunterrichtsgesetz - SchUG).

Bundeskanzleramt. (2013). Bundesgesetzblatt Nr. 211/2013, Teil I. BGBl. I Nr. 213/2013.

Bundeskanzleramt. (2018). Landeslehrer-Dienstrechtsgesetz. LDG 1984. In Rechtsinformationssystem des Bundes RIS.

Bundesministerium für Bildung, Wissenschaft und Forschung. (1962). Gesamte Rechtsvorschrift für Schulorganisationsgesetz. SchOG (BGBl. Nr. 242/1962).

Bundesministerium für Bildung, Wissenschaft und Forschung. (2014). Empfehlungen des BMBWF zu Mentoring und Induktion. Dienstrechtsnovelle 2013, 539 a VBG.

Bundesministerium für Bildung, Wissenschaft und Forschung. (2018). Schritt für Schritt zur neuen Lebrkraft. Ein anwendungsorientierter Leitfaden für Schulleitungen. Wien: Digitales Druckzentrum Renngasse.

Bundesministerium für Bildung, Wissenschaft und Forschung. (2020a). Neue Mittelschule. https://www.bmbwf.gv.at/ Themen/schule/schulsystem/sa/nms.html (Zugriff: 07022020).

Bundesministerium für Bildung, Wissenschaft und Forschung. (2020b). Polytechnische Schule. https://www.bmbwf. gvat/Themen/schule/schulsystem/sa/pts.html (Zugriff: 07022020).

Bundesministerium für Digitalisierung und Wirtschaftsstandort. (1984). Gesamte Rechtsvorschrift für LandeslehrerDienstrechtsgesetz. LDG 1984. https://www.ris.bka.gv.at/GeltendeFassung.wxe?Abfrage=Bundesnormen\&Gesetzesnummer $=10008549$. Zugegriffen: 17 . Oktober 2019 .

Christof, E. \& Schwarz, J. (Hrsg.). (2013). Lernseits des Geschehens. Über das Verhältnis von Lernen, Lehren und Leiten. Innsbruck: StudienVerlag.

Combe, A. (2018). Ungewissheit als Risiko der Anschlussverfehlung. Verstehen als Gespräch. Eine differenztheoretische Konzeptualisierung von Unterricht. In A. Paseka, M. Keller-Schneider \& A. Combe (Hrsg.), Ungewissheit als Herausforderung für pädagogisches Handeln (S. 81-102). Wiesbaden: Springer VS.

Combe, A. \& Paseka, A. (2012). Und sie bewegt sich doch? Gedanken zu Brückenschlägen in der aktuellen Professionsund Kompetenzdebatte. Zeitschrift für Bildungsforschung 2(2), 91-108.

Combe, A., Paseka, A. \& Keller-Schneider, M. (2018). Ungewissheitsdynamiken des Lehrerhandelns. Kontingenzzumutung - Kontingenzbelastung - Kontingenzfreude - Kontingenzbewusstsein. In A. Paseka, M. Keller-Schneider \& A. Combe (Hrsg.), Ungewissheit als Herausforderung für pädagogisches Handeln (S. 53-79). Wiesbaden: Springer VS.

Connor, L., Borradori, G., Brennan, M., Doane, M.A., Fletcher, A., Geimer, P., Kury, G., Ledbury, M., Rose, B., Spalding, F. \& Spring, C. (2012). Notes from the Field: Contingency. The Art Bulletin 94(3), 344-361.

Conway, C.M. (2012). Ten Years Later_Teachers Reflectt on „Perceptions of Beginning Teachers, Their Mentors, and Administrator Regarding Preservice Music Teacher Preparation“.Journal of Research in Music Education 60 (3), 324-338.

David, T. (2000). Programs in practice: Teacher mentoring - benefits all around. Kappa Delta Pi Record 36(3), $134-136$.

Diaz-Bone, R. (2010). Kulturwelt, Diskurs und Lebensstil: Eine diskurstheoretische Erweiterung der Bourdieuschen Distinktionstheorie. Wiesbaden: VS Verlag für Sozialwissenschaften. 
Dietrich, F. (2018). Professionalisierung als Krisenbearbeitung. In A. Paseka, M. Keller-Schneider \& A. Combe (Hrsg.), Ungewissheit als Herausforderung für pädagogisches Handeln (S. 277-297). Wiesbaden: Springer VS.

DuBois, D.L., Hollowa, B., Valtentine, J.C. \& Cooper, H. (2002). Effectiveness of Mentoring Programs für Youth: A Meta-Analytic Review. American Journal of Community Psychology 30(2), 157-197.

Duden. (2017). Die deutsche Rechtschreibung. Auf der Grundlage der aktuellen amtlichen Rechtschreibregeln in zwölf Bänden; das Standardwerk zur deutschen Sprache; Band 1. (2015). Duden. In zwölf Bänden; das Standardwerk zur deutschen Sprache.

Ernst, H., Schmidt, J. \& Beneken, G.H. (2016). Grundkurs Informatik. Grundlagen und Konzepte für die erfolgreiche IT-Praxis - Eine umfassende, praxisorientierte Einführung (6. Auflage 2016). Wiesbaden: Springer Vieweg.

Evans, L. (2002). What Is Teacher Development? Oxford Review of Education 28(1), 123-137.

Evans, L. (2008). Professionalism, Professionality and the Development of Education Professionals. British Journal of Educational Studies 56(1), 20-38.

Flick, U. (1995). Qualitative Forschung. Reinbek bei Hamburg: Rowohlt-Taschenbuch-Verl.

Flick, U. (2007). Qualitative Sozialforschung. Eine Einführung (Rowohlts Enzyklopädie, Bd. 55694, Vollst. überarb. und erw. Neuausg. 2007, 4. Aufl.). Reinbek bei Hamburg: Rowohlt-Taschenbuch-Verl.

Friebertshäuser, B., Boller, H. \& Richter, S. (Hrsg.). (2010). Handbuch qualitative Forschungsmethoden in der Erziehungswissenschaft (Juventa-Handbuch, 3., vollst. überarb. Aufl., (Neuausg.)). Weinheim: Juventa-Verl.

Friebertshäuser, B. \& Langer, A. (2010). Interviewformen und Interviewpraxis. In B. Friebertshäuser, H. Boller \& S. Richter (Hrsg.), Handbuch qualitative Forschungsmethoden in der Erziehungswissenschaft (Juventa-Handbuch, 3., vollst. überarb. Aufl., (Neuausg.), S. 437-455). Weinheim: Juventa-Verl.

Fuchs-Heinritz, W. \& König, A. (2014). Pierre Bourdieu. Eine Einführung (UTB, Bd. 2649, 3., überarb. Aufl.). Konstanz: UVK-Verl.-Ges.

Führer, F.-M. \& Heller, V. (2018). Reflektieren als interaktive Praktik in Unterrichtsbesprechungen zwischen Mentoren und Deutsch-Studierenden im Praxissemester. In M. Artmann, M. Berendonck, P. Herzmann \& A. Liegmann (Hrsg.), Professionalisierung in Praxisphasen der Lehrerbildung. Qualitative Forschung aus Bildungswissenschaft und Fachdidaktik (Studien zur Professionsforschung und Lehrerbildung, S. 113-130). Bad Heilbrunn: Verlag Julius Klinkhardt.

Gadamer, H.-G. (1990). Wabrheit und Methode (Gesammelte Werke, Bd. 1). Tübingen: Mohr.

Greiner, U. (2010). Induktionsphase. Ergänzende Expertise zu LehrerInnenbildung NEU. Die Zukunft der pädagogischen Berufe. Im Auftrag des bmukk und des BMWF (S. 9-15), Wien.

Greiner, U. (2019). Neue Lehrerinnen und Lehrer: Einführung und Mentoring. Kommentar. In J. Schmich \& U. Itzlinger-Bruneforth (Hrsg.), TALIS 2018. Rahmenbedingungen des schulischen Lehrens und Lernens aus Sicht von Lehrkräften und Schulleitungen im internationalen Vergleich (Bd. 1, S. 80-82). Graz: Leykam Buchverlagsgesellschaft.

Gruschka, A. (2018). Ungewissheit, der innere Feind für unterrichtliches Handeln. In A. Paseka, M. Keller-Schneider \& A. Combe (Hrsg.), Ungewissheit als Herausforderung für pädagogisches Handeln (S. 15-29). Wiesbaden: Springer VS.

Haas, E. \& Schneider, K. (2018). Schulpraktikum I im Lehramtsstudium der Sekundarstufe Allgemeinbildung: Erkenntnisse aus der Sicht von Studierenden. In C. Fridrich, G. Mayer-Frühwirt, R. Potzmann, W. Greller \& R. Petz (Hrsg.), Forschungsperspektiven 10 (S. 47-64). Wien: Lit Verlag GmbH \& Co. KG.

Hanuscin, D.L. \& Lee, M.H. (2008). Perspectives: Mentoring New Teachers. Science and Children 45 (9), 56-57.

Hattie, J. (2015). Lernen sichtbar machen (3., erweiterte Auflage mit Index und Glossar). Baltmannsweiler: Schneider Verlag Hohengehren.

Helmke, A. (2007). Was wissen wir über guten Unterricht? Wissenschaftliche Erkenntnisse zur Unterrichtsforschung und Konsequenzen für die Unerrichtsentwicklung, Landau/Pfalz.

Helmke, A. (2012). Unterrichtsqualität und Lehrerprofessionalität. Diagnose, Evaluation und Verbesserung des Unterrichts: [Orientierungsband] (Unterricht verbessern - Schule entwickeln, 4., aktualisierte Aufl.). Seelze: Kallmeyer in Verbindung mit Klett.

Helsper, W. (2001). Praxis und Reflexion. Die Notwendigkeit einer „doppelten Professionalisierung“ des Lehrers. Journal für LehrerInnenbildung 1 (3), 7-15.

Helsper, W. (Hrsg.). (2005). Ungewissheit. Pädagogische Felder im Modernisierungsprozess (2. Aufl.). Weilerswist: Velbrück Wiss.

Helsper, W. (2018). Lehrerhabitus. Lehrer zwischen Herkunft, Milieu und Profession. In A. Paseka, M. Keller-Schneider \& A. Combe (Hrsg.), Ungewissheit als Herausforderung für pädagogisches Handeln (S. 105-140). Wiesbaden: Springer VS.

Helsper, W. \& Böhme, J. (Hrsg.). (2008). Handbuch der Schulforschung (2., durchgesehene und erw. Aufl.). Wiesbaden: VS Verlag für Sozialwissenschaften/GWV Fachverlage, Wiesbaden. 
Hericks, U., Meister, N. \& Meseth, W. (2018). Professionalisierung durch Perspektivenwechsel? Lehramtsstudierende zwischen schulischer und universitärer Praxis. In M. Artmann, M. Berendonck, P. Herzmann \& A. Liegmann (Hrsg.), Professionalisierung in Praxisphasen der Lehrerbildung. Qualitative Forschung aus Bildungswissenschaft und Fachdidaktik (Studien zur Professionsforschung und Lehrerbildung, 255-270). Bad Heilbrunn: Verlag Julius Klinkhardt.

Hericks, U. 1. (2006). Professionalisierung als Entwicklungsaufgabe. Rekonstruktionen zur Berufseingangsphase von Lehrerinnen und Lehrern (Studien zur Bildungsgangforschung, Bd. 8, 1. Aufl). Wiesbaden: VS Verl. für Sozialwiss.

Herzig, B. \& Grafe, S. (2005). Lernen im Praktikum als Entwicklung einer Reflexionskultur. In A.H. Hilligus \& H.-D. Rinkens (Hrsg.), Zentren für Lebrerbildung - neue Wege im Bereich der Praxisphasen. 24. Bundestagung der Leiterinnen und Leiter der Schulpraktischen Studien im Mai 2004 (Paderborner Beiträge zur Unterrichtsforschung und Lehrerbildung, Bd. 10, S. 149-170). Münster: Lit-Verl.

Herzmann, P. \& Liegmann, A. (2018). Studienprojekte im Praxissemester. Eine Heuristik von forschungsvorhaben im Kontext Forschenden Lernens. In M. Artmann, M. Berendonck, P. Herzmann \& A. Liegmann (Hrsg.), Professionalisierung in Praxisphasen der Lehrerbildung. Qualitative Forschung aus Bildungswissenschaft und Fachdidaktik (Studien zur Professionsforschung und Lehrerbildung, S. 74-92). Bad Heilbrunn: Verlag Julius Klinkhardt.

Herzog, S. (2014). Über den Berufseinstieg hinaus: Berufsbiografien von Lehrerinnen und Lehrern im Blickfeld der Forschung. In E. Terhart, H. Bennewitz \& M. Rothland (Hrsg.), Handbuch der Forschung zum Lebrerberuf (S. 408432). Münster, Westf: Waxmann.

Höller, I., Toferer, B. \& Winter, S. (2019). Neue Lehrerinnen und Lehrer: Einführung und Mentoring. In J. Schmich \& U. Itzlinger-Bruneforth (Hrsg.), TALIS 2018. Rabmenbedingungen des schulischen Lehrens und Lernens aus Sicht von Lehrkräften und Schulleitungen im internationalen Vergleich (Bd. 1, S. 71-82). Graz: Leykam Buchverlagsgesellschaft.

Holloway, J. (2001). The benefits of mentoring. Educational leadership 58(8), 85-86.

Horster, L. (2001). Die Einarbeitung neuer Lehrkräfte als Beginn der Personalentwicklung. In H. Buchen (Hrsg.), Personalfübrung und Schulentwicklung. Stuttgart.

Hubermann, M. (1991). Der berufliche Lebenszyklus von Lehrern: Ergebnisse einer empirischen Untersuchung. In E. Terhart (Hrsg.), Unterrichten als Beruf(S. 249-267). Köln, Wien: Bölau-Verlag.

Idel, T.-S. \& Kahlau, J. (2018). Professionalisierung in Studien-Praxis-Projekten. Beziehungsgestaltung und Rollenkonstruktion in gemeinsamen Entwicklungsvorhaben von Studierenden und Lehrkräften. In M. Artmann, M. Berendonck, P. Herzmann \& A. Liegmann (Hrsg.), Professionalisierung in Praxisphasen der Lehrerbildung. Qualitative Forschung aus Bildungswissenschaft und Fachdidaktik (Studien zur Professionsforschung und Lehrerbildung, S. 238254). Bad Heilbrunn: Verlag Julius Klinkhardt.

Ingersol, R.M. \& Strong, M. (2011). The impact of induction and mentoring programs for beginning teachers. Review of Educational Research 81 (2), 201-233.

Ittel, A. \& Raufelder, D. (2009). Mentoring in der Schule: Professionelle Praxis und Qualitätssicherung. In H. Stöger, A. Ziegler \& D. Schimke (Hrsg.), Mentoring: Theoretische Hintergründe, empirische Befunde und praktische Anwendungen (1. Auflage, S. 193-206). Lengerich: Pabst Science Publishers.

Jäger, P. (2001). Die Österreichische Polytechnische Schule im Wandel. Schulpädagogische Perspektiven (1.). Wien.

Kärtner, J. (2015). Das Problem der doppelten Kontingenz als Ausgangsproblem des Sozialen und der soziologischen Theorie. Vorschlag zu einer Systematisierung der soziologischen Systemtheorie Niklas Luhmanns. Zeitschrift für theoretische Soziologie 4(1), 60-88.

Keller-Schneider, M. (2018). „Es genügt nicht mehr, einfach zu unterrichten“. Den Umgang mit Ungewissheit als Herausforderung annehmen. In A. Paseka, M. Keller-Schneider \& A. Combe (Hrsg.), Ungewissheit als Herausforderung für pädagogisches Handeln (S. 231-254). Wiesbaden: Springer VS.

Keller-Schneider, M. \& Hericks, U. (2014). Forschungen zum Berufseinstieg. Übergang von der Ausbildung in den Beruf. In E. Terhart, H. Bennewitz \& M. Rothland (Hrsg.), Handbuch der Forschung zum Lehrerberuf (386-407). Münster, Westf: Waxmann.

Koballa, T., Bradbury, L. \& Deaton, C.M. (2008). Realize Your Mentoring Success: Conceptions of mentoring help shape interactions between new science teachers and their mentors. The Science Teacher 75 (5), 43-47.

Kosinár, J. (2018). Konstruktionen von Professionalität und Ungewissheitserfahrungen im Referendariat. In A. Paseka, M. Keller-Schneider \& A. Combe (Hrsg.), Ungewissheit als Herausforderung für pädagogisches Handeln (S. 255275). Wiesbaden: Springer VS.

Kraler, C. (2008a). Kompetenzorientierte Lehrerbildung und ihre Wirkung. Überlegungen zur Nachhaltigkeit in Forschung und Praxis. In C. Kraler \& M. Schratz (Hrsg.), Wissen erwerben, Kompetenzen entwickeln. Modelle zur kompetenzorientierten Lehrerbildung (S. 151-180). Münster: Waxmann. 
Kraler, C. (2008b). Professionalisierung in der Berufseingangsphase - Berufsbiographie und Kompetenzentwicklung. Entwicklungsaufgaben der ersten Berufsjahre und Unterstützungsmöglichkeiten. SchulVerwaltung spezial 2008(1), 4-7. https://www.uibk.ac.at/ils/mitarbeiter/christian-kraler/papers/kraler_spez08-01.pdf. Zugegriffen: 21. Oktober 2018 .

Kraler, C. (Hrsg.). (2012a). Kulturen der Lehrerbildung. Professionalisierung eines Berufsstands im Wandel. Münster: Waxmann.

Kraler, C. (2012b). Selbstähnlichkeiten in der Lehrer/innenbildung. In C. Kraler (Hrsg.), Kulturen der Lehrerbildung. Professionalisierung eines Berufsstands im Wandel (S. 41-72). Münster: Waxmann.

Kraler, C., Dittrich, A.-K. \& MacKay-Falls, F. (2017). Ausbildungsreform als Chance für einen Musterwechsel im Verständnis pädagogisch-psychologischen Professionswissens. Zeitschrift für Bildungsforschung 7(3), $193-207$. doi: $10.1007 / \mathrm{s} 35834-017-0198-\mathrm{z}$

Kraler, C. \& Menges, R. (2007). Fallvignetten in der Lehrerbildungsforschung. In C. Kraler \& M. Schratz (Hrsg.), Ausbildungsqualität und Kompetenz im Lehrerberuf (Österreichische Beiträge zur Bildungsforschung, Bd. 4, S. 56-77). Wien: Lit-Verl.

Kraler, C. \& Reich, K. (2019). Kontext, Genese und Entwicklung des Lehramtsstudiums im Verbund LehrerInnenbildung West. In M.-L. Braunsteiner \& C. Spiel (Hrsg.), PädagogInnenbildung (S. 251-264). Heiligenkreuz: BeBe.

Kraler, C., Reich, K. \& Fügenschuh, B. (2017). PädagogInnenbildung Neu im Verbund LehrerInnenbildung West. Eine Standortbestimmung zu Gelingensbedingungen und Herausforderungen. Zeitschrift für Hochschulrecht 16, 79-86.

Kramer, R.-T. \& Pallesen, H. (2019). Theoretisch-konzeptionelle und empirische Herausforderungen des Lehrerhabitus. Eine Einleitung. In R.-T. Kramer \& H. Pallesen (Hrsg.), Lehrerhabitus. Theoretische und empirische Beiträge zu einer Praxeologie des Lehrerberufs (Studien zur Professionsforschung und Lehrerbildung, S. 9-23).

Kuckartz, U. (2016). Qualitative Inhaltsanalyse. Methoden, Praxis, Computerunterstützung (Grundlagentexte Methoden, 3., durchgesehene Aufl.). Weinheim, Bergstr: Beltz Juventa.

Lamnek, S. \& Krell, C. (2010). Qualitative Sozialforschung. Lehrbuch; [Online-Materialien (Grundlagen Psychologie, 5., überarb. Aufl.). Weinheim: Beltz.

Lenger, A., Schneickert, C. \& Schumacher, F. (Hrsg.). (2013). Pierre Bourdieus Konzeption des Habitus. Grundlagen, Zugänge, Forschungsperspektiven. Wiesbaden: Springer VS.

Leonhard, T. \& Lüthi, K. (2018). Auf Linie gebracht? Adressierungen und Normen der Anerkennbarkeit im Praktikum. In M. Artmann, M. Berendonck, P. Herzmann \& A. Liegmann (Hrsg.), Professionalisierung in Praxisphasen der Lehrerbildung. Qualitative Forschung aus Bildungswissenschaft und Fachdidaktik (Studien zur Professionsforschung und Lehrerbildung, S. 183-200). Bad Heilbrunn: Verlag Julius Klinkhardt.

Leopold-Franzens-Universität Innsbruck. (2016). Curriculum für das Bachelorstudium Lehramt Sekundarstufe (Allgemeinbildung). https://www.uibk.ac.at/fakultaeten-servicestelle/pruefungsreferate/gesamtfassung/ba-lehramtsekundarstufe_stand-01.10.2016.pdf.

Liegmann, A., Artmann, M., Berendonck, M. \& Herzmann, P. (2018). Diskurse zu Professionalisierung in Praxisphasen der Lehrerbildung. Eine Einleitung in den Sammelband. In M. Artmann, M. Berendonck, P. Herzmann \& A. Liegmann (Hrsg.), Professionalisierung in Praxisphasen der Lehrerbildung. Qualitative Forschung aus Bildungswissenschaft und Fachdidaktik (Studien zur Professionsforschung und Lehrerbildung, S. 7-18). Bad Heilbrunn: Verlag Julius Klinkhardt.

Luhmann, N. (2015). Gesellschaft der Gesellschaft (Suhrkamp-Taschenbuch Wissenschaft, Bd. 1360, 1. Aufl., [9. Nachdr.]. Frankfurt am Main: Suhrkamp.

Luhmann, N. (2018). Soziale Systeme. Grundriß einer allgemeinen Theorie (Suhrkamp-Taschenbuch Wissenschaft, Bd. 666, 17. Auflage). Frankfurt am Main: Suhrkamp.

Messner, H. \& Reusser, K. (2000). Die berufliche Entwicklung von Lehrpersonen als lebenslanger Prozess. Beiträge zur Lehrerbildung 18(2), 157-171.

Meuser, M. \& Nagel, U. (2009). Experteninterview und der Wandel der Wissensproduktion. In A. Bogner, B. Littig \& W. Menz (Hrsg.), Experteninterviews. Theorien, Methoden, Anwendungsfelder (3., grundlegend überarbeitete Auflage, S. 35-60). Wiesbaden: VS Verlag für Sozialwissenschaften.

Meuser, M. \& Nagel, U. (2010). Experteninterviews - wissenssoziologische Voraussetzungen und methodische Durchführung. In B. Friebertshäuser, H. Boller \& S. Richter (Hrsg.), Handbuch qualitative Forschungsmethoden in der Erziehungswissenschaft (Juventa-Handbuch, 3., vollst. überarb. Aufl., (Neuausg.), S. 457-471). Weinheim: JuventaVerl.

Molnar, A., Lindquist, B. \& Grau, U. (2013). Verhaltensprobleme in der Schule. Lösungsstrategien für die Praxis (10., durchges. Aufl.). Dortmund: Borgmann.

Müller-Fohrbrodt, G., Cloetta, B. \& Dann, H.D. (1978). Der Praxisschock bei jungen Lehrern. Formen, Ursachen, Folgerungen. Stuttgart: Klett. 
Nairz-Wirth, E. (2011). Professionalisierung nach Pierre Bourdieu. In M. Schratz (Hrsg.), Pädagogische Professionalität: quer denken - umdenken - neu denken. Impulse für next practice im Lehrerberuf (1. Aufl., S. 163-186).

Niggli, A. (2005). Unterrichtsbesprechungen im Mentoring (Pädagogik bei Sauerländer Praxis, 1. Aufl.). Oberentfelden: Sauerländer.

Odell, S.J. \& Huling, L. (2000). Quality mentoring for novice teachers. Joint publication. Washington.

Oerter, R., Montada, L. \& Oerter-Montada. (2002). Entwicklungspsychologie. Lehrbuch (5., vollst. überarb. Aufl.). Weinheim: Beltz PVU.

Oevermann, U. (2017). Theoretische Skizze einer revidierten Theorie professionalisierten Handelns. In A. Combe \& W. Helsper (Hrsg.), Pädagogische Professionalität. Untersuchungen zum Typus pädagogischen Handelns (SuhrkampTaschenbuch Wissenschaft, Bd. 1230, 9. Auflage 2017, S. 70-182). Frankfurt am Main: Suhrkamp.

Ostinelli, G. (2009). Teacher Education in Italy, Germany, England, Sweden and Finland. European Journal of Education 44(2), 291-308.

Pädagogische Hochschule Tirol. (2019). Curricula der Hochschullehrgänge an der PHT. https://ph-tirol.ac.at/de/ content/curricula-der-hochschullehrgaenge-der-pht. Zugegriffen: 3. Oktober 2019.

Paseka, A. (2011). Transformationen - Brüche - Entgrenzungen. Personal Mastery als Suchbewegung. In M. Schratz (Hrsg.), Pädagogische Professionalität: quer denken - umdenken - neu denken. Impulse für next practice im Lehrerbe$\operatorname{ruf}(1$. Aufl., 123-164).

Paseka, A., Keller-Schneider, M. \& Combe, A. (Hrsg.). (2018). Ungewissheit als Herausforderungfürpädagogisches Handeln. Wiesbaden: Springer VS.

Paseka, A., Schratz, M. \& Schrittesser, I. (2011). Professionstheoretische Grundlagen und thematische Annäherung. In M. Schratz (Hrsg.), Pädagogische Professionalität: quer denken - umdenken - neu denken. Impulse für next practice im Lehrerberuf (1. Aufl., S. 8-45).

Patry Jean-Luc. (2014). Theoretische Grundlagen des Theorie-Praxis-Problems in der Lehrer/innenbildung. In K.-H. Arnold, A. Gröschner \& T. Hascher (Hrsg.), Schulpraktika in der Lehrerbildung Pedagogical field experiences in teacher education. Theoretische Grundlagen, Konzeptionen, Prozesse und Effekte Theoretical foundations, programmes, processes, and effects (S. 29-44). Münster, Westf: Waxmann.

Poel, K. t. \& Heinrich, M. (2018). Empathiefähigkeit und Professionalisierungsprozesse im Praxissemester. Eine Fallrekonstruktion im qualitativen Längsschnitt. In M. Artmann, M. Berendonck, P. Herzmann \& A. Liegmann (Hrsg.), Professionalisierung in Praxisphasen der Lehrerbildung. Qualitative Forschung aus Bildungswissenschaft und Fachdidaktik (Studien zur Professionsforschung und Lehrerbildung, S. 221-237). Bad Heilbrunn: Verlag Julius Klinkhardt.

Pollmanns, M., Kabel, S., Leser, C. \& Kminek, H. (2018). Krisen der Professionalisierung. Wie sich Studierende in Schulpraktischen Studien forschungsbezogenen Typs der schulischen Praxis zuwenden. In M. Artmann, M. Berendonck, P. Herzmann \& A. Liegmann (Hrsg.), Professionalisierung in Praxisphasen der Lehrerbildung. Qualitative Forschung aus Bildungswissenschaft und Fachdidaktik (Studien zur Professionsforschung und Lehrerbildung, S. 2137). Bad Heilbrunn: Verlag Julius Klinkhardt.

Rädiker, S. \& Kuckartz, U. (2019). Analyse qualitativer Daten mit MAXQDA. Text, Audio und Video. Wiesbaden: Springer Fachmedien Wiesbaden.

Rasch, B., Friese, M., Hofmann, W. \& Naumann, E. (2014). Quantitative Methoden 1. Einführung in die Statistik für Psychologen und Sozialwissenschaftler (Springer-Lehrbuch, 4., überarb. Aufl.). Berlin: Springer.

Raufelder, D. \& Ittel, A. (2012). Mentoring in der Schule: ein Überblick; theoretische und praktische Implikationen für Lehrer/-innen und Schüler/-innen im internationalen Vergleich. Diskurs Kindheits- und Jugendforschung 7(2), 147-160.

Reay, D. (2004). „It's All Becoming a Habitus“: Beyond the Habitual Use of Habitus in Educational Research. British Journal of Sociology of Education 25 (4), 431-444.

Reh, S. (2012). Beobachten und aufmerksames Wahrnehmen. In H. d. Boer (Hrsg.), Beobachtung in der Schule - Beobachten lernen (Lehrbuch, S. 3-25). Wiesbaden: Springer VS.

Resta, V., Huling, L., White, S. \& Matschek, D. (1997). A year to grow on. Journal of Staff Development 18(1), 43-45.

Riecke, J. (2014). Duden, das Herkunftswörterbuch. Etymologie der deutschen Sprache (Der Duden in zwölf Bänden, Bd. 7, 5., neu bearbeitete Auflage). Berlin: Dudenverlag.

Rogers, B. (2013). Classroom Management. Das Praxisbuch (Pädagogik Praxis). Weinheim: Beltz.

Rosa, H. (2018). Unverfügbarkeit (Unruhe bewahren). Wien: Residenz Verlag.

Rosemann, I. \& Bonnet, A. (2018). „Oder wäre doch was anderes sinnvoller?“ - Mentoringgespräche über Englischunterricht als Professionalisierungsgelegenheiten an der Schnittstelle zwischen Studium und Schulpraxis. In M. Artmann, M. Berendonck, P. Herzmann \& A. Liegmann (Hrsg.), Professionalisierung in Praxisphasen der Lehrerbildung. Qualitative Forschung aus Bildungswissenschaft und Fachdidaktik (Studien zur Professionsforschung und Lehrerbildung, S. 131-148). Bad Heilbrunn: Verlag Julius Klinkhardt. 
Sayer, J. (2006). European Perspectives of Teacher Education and Training. Comparative Education 42 (1), 63-75.

Schaper, C. (Hrsg.). (2017). Werkzeugkoffer Pädagogisches Handeln. Ein Handbuch für den Start in den Lehreralltag (1. Aufl.). Göttingen: Vandenhoeck Ruprecht.

Scheeler, M.C. (2008). Generalizing Effective Teaching Skills: The Missing Link in Teacher Preparation. Journal of Behavioral Education 17(2), 145-159.

Schlömerkemper, J. (2010). Konzepte pädagogischer Forschung. Eine Einführung in Hermeneutik und Empirie (UTB Erziehungswissenschaft, Pädagogik, Bd. 3273, 1. Aufl.). Bad Heilbrunn: Klinkhardt.

Schmich, J. \& Itzlinger-Bruneforth, U. (Hrsg.). (2019). TALIS 2018. Rahmenbedingungen des schulischen Lehrens und Lernens aus Sicht von Lehrkräften und Schulleitungen im internationalen Vergleich (Bd. 1). Graz: Leykam Buchverlagsgesellschaft.

Schmid, B. \& Haasen, N. (2011). Einführung in das systemische Mentoring (Compact, 1. Aufl.). Heidelberg: Carl-Auer-Verl.

Schmidt, C. (2010). Auswertungstechniken für Leitfadeninterviews. In B. Friebertshäuser, H. Boller \& S. Richter (Hrsg.), Handbuch qualitative Forschungsmethoden in der Erziehungswissenschaft (Juventa-Handbuch, 3., vollst. überarb. Aufl., (Neuausg.), S. 473-486). Weinheim: Juventa-Verl.

Schneider, K. \& Haas, E. (2019). Schulische Lerngelegenheiten als ein Ort das Aufbau Pädagogischen Wissens. Eine Analyse der Emotion von Studierenden im Schulpraktikum I in der Lehrer/innenbildung West. Erziehung und Unterricht $169(9-10), 825-832$.

Schober, B., Finsterwald, M., Wagner, P. \& Spiel, C. (2009). Lebenslanges Lernen als Herausforderung der Wissensgesellschaft: Die Schule als Ort der Förderung von Bildungsmotivation und selbstreguliertem Lernen. In W. Specht (Hrsg.), Nationaler Bildungsbericht. Band 2: Fokussierte Analysen bildungspolitischer Schwerpunktthemen (Bd. 2, S. 121-139). Graz: Leykam Buchverlagsgesellschaft.

Schönknecht, G. (2005). Die Entwicklung der Innovationskompetenz von LehrerInnen aus (berufs-)biographischer Perspektive, bwpat.de.

Schratz, M. (2011). Professionalität und Professionalisierung von Lehrerinnen und Lehrern in internationaler Perspektive. In M. Schratz (Hrsg.), Pädagogische Professionalität: quer denken - umdenken - neu denken. Impulse für next practice im Lehrerberuf (1. Aufl., S. 46-94).

Schratz, M., Schwarz, J.F., Westfall-Greiter, T. \& Rumpf, H. (2012). Lernen als bildende Erfahrung. Vignetten in der Praxisforschung (Erfolgreich im Lehrberuf, Bd. 8). Innsbruck: StudienVerlag.

Schratz, M., Wiesner, C., Kemethofer, D., George, A.C., Rauscher, E., Krenn, S. \& Huber, G. (2015). Schulleitung im Wandel: Anforderungen an eine ergebnisorientierte Führungskultur. In M. Bruneforth, F. Eder, K. Krainer, C. Schreiner, A. Seel \& C. Spiel (Hrsg.), Nationaler Bildungsbericht Österreich. Band 2: Fokussierte Analysen bildungspolitischer Schwerpunktthemen (Bd. 2, S. 221-262). Wien: Leykam.

Schratz, M. 1., Schrittesser, I., Forthuber, P., Pahr, G., Paseka, A. \& Seel, A. (2008). Domänen von Lehrer/innen/professionalität. Rahmen einer kompetenzorientierten Lehrer/innen/bildung. In C. Kraler \& M. Schratz (Hrsg.), Wissen erwerben, Kompetenzen entwickeln. Modelle zur kompetenzorientierten Lehrerbildung (S. 123-137). Münster: Waxmann.

Schrittesser, I. (2011). Professionelle Kompetenzen: Systematische und empirische Annäherungen. In M. Schratz (Hrsg.), Pädagogische Professionalität: quer denken - umdenken - neu denken. Impulse für next practice im Lehrerberuf (1. Aufl., S. 95-122).

Schründer-Lenzen, A. (2010). Triangulation - ein Konzept zur Qualitätssicherung von Forschung. In B. Friebertshäuser, H. Boller \& S. Richter (Hrsg.), Handbuch qualitative Forschungsmethoden in der Erziehungswissenschaft (Juventa-Handbuch, 3., vollst. überarb. Aufl., (Neuausg.), S. 149-158). Weinheim: Juventa-Verl.

Schumacher, F. (2013). Norbert Elias' „sozialer Habitus“ als Vorläufer des Bourdieu’schen Habitus? Eine vergleichende Analyse. In A. Lenger, C. Schneickert \& F. Schumacher (Hrsg.), Pierre Bourdieus Konzeption des Habitus. Grundlagen, Zugänge, Forschungsperspektiven (S. 131-145). Wiesbaden: Springer VS.

Steinke, L.J. \& Putnam, A.R. (2011). Mentoring Teachers in Technology Education: Analyzing the Need. The Journal of Technology Studies 37(1/2), 41-49.

Stichweh, R. (1992). Professionalisierung, Ausdifferenzierung von Funktionssystemen, Inklusion. Betrachtungen aus systemtheoretischer Sicht. In B. Dewe (Hrsg.), Erziehen als Profession (S. 36-48). Opladen.

Stichweh, R. (2017). Professionen in einer funktional differenzierten Gesellschaft. In A. Combe \& W. Helsper (Hrsg.), Pädagogische Professionalität. Untersuchungen zum Typuspädagogischen Handelns (Suhrkamp-Taschenbuch Wissenschaft, Bd. 1230, 9. Auflage 2017, S. 49-69). Frankfurt am Main: Suhrkamp.

Strauss, A.L. \& Corbin, J.M. (1996). Grounded theory. Grundlagen qualitativer Sozialforschung. Weinheim: Beltz, PsychologieVerlagsUnion.

Tashakkori, A. \& Newmann, I. (2010). Mixed methods. In P. Peterson, E. Baker \& B. McGaw (Hrsg.), International Encyclopedia of Education (3. Aufl., S. 514-520). Oxford: Elsevier.

Terhart, E. (2001). Lehrerberuf und Lehrerbildung. Forschungsbefunde, Problemanalysen, Reformkonzepte (Beltz Pädagogik). Weinheim: Beltz. 
Terhart, E. (2007). Was wissen wir über gute Lehrer? Ergebnisse aus der empirischen Lehrerforschung. Friedrich Jahresheft, 20-24.

Terhart, E. (2011). Lehrerberuf und Professionalität: Gewandeltes Begriffsverständnis - neue Herausforderungen. Zeitschrift für Pädagogik (Beiheft) 57, 202-224.

Terhart, E. (2013). Erziehungswissenschaft und Lehrerbildung (Waxmann Studium). Münster: Waxmann.

Terhart, E., Bennewitz, H. \& Rothland, M. (Hrsg.). (2014). Handbuch der Forschung zum Lehrerberuf. Münster, Westf: Waxmann.

Tippelt, R. (Hrsg.). (2010). Handbuch Bildungsforschung (3., durchges. Aufl.). Wiesbaden: VS, Verl. für Sozialwiss.

Tiroler Landesregierung. (2015). Das neue Dienst- und Besoldungsrecht für Landesvertragslehrpersonen: Zugriff: 08.09.2018.

Wang, J. \& Odell, S.J. (2007). An alternative conception of mentor-novice relationships: Learning to teach in reformminded ways as a context. Teaching and Teacher Education. Teacher and Teacher Education 23 (4), 473-489.

Weischer, C. (2007). Sozialforschung (UTB Soziologie, Bd. 2924). Konstanz: UVK Verl.-Ges.

Whitehead, A.N. (2011). Die Ziele von Erziehung und Bildung. Und andere Essays (Suhrkamp Taschenbücher Wissenschaft, Bd. 2015, 1., Deutsche Erstausgabe). Berlin: Suhrkamp.

Wilfert, C. \& Thünemann, H. (2018). Subjektive Theorien Geschichtsstudierender vor und nach dem Praxissemester. Zum Verhältnis geschichtsdidaktischen Theorie- und unterrichtspraktischen Erfahrungswissens. In M. Artmann, M. Berendonck, P. Herzmann \& A. Liegmann (Hrsg.), Professionalisierung in Praxisphasen der Lehrerbildung. Qualitative Forschung aus Bildungswissenschaft und Fachdidaktik (Studien zur Professionsforschung und Lehrerbildung, S. 203-220). Bad Heilbrunn: Verlag Julius Klinkhardt.

Ziegler, A. (2009). Mentoring: Konzeptionelle Grundlagen und Wirksamkeitsanalyse. In H. Stöger, A. Ziegler \& D. Schimke (Hrsg.), Mentoring: Theoretische Hintergründe, empirische Befunde und praktische Anwendungen (1. Auflage, S. 7-29). Lengerich: Pabst Science Publishers.

Zorn, S.K. (2018). Begleitung Studierender als kooperativer Prozess. Das Bilanz- und Perspektivengespräch im Praxissemester. In M. Artmann, M. Berendonck, P. Herzmann \& A. Liegmann (Hrsg.), Professionalisierung in Praxisphasen der Lehrerbildung. Qualitative Forschung aus Bildungswissenschaft und Fachdidaktik (Studien zur Professionsforschung und Lehrerbildung, S. 271-289). Bad Heilbrunn: Verlag Julius Klinkhardt. 


\section{Abbildungsverzeichnis}

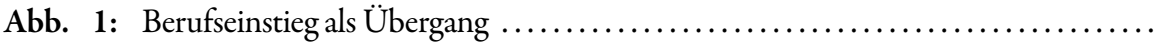

Abb. 2: Erscheinungsformen von Kontingenz; eigene Abbildung nach Combe et al. $(2018$, S. 54)

Abb. 3: Fragen zur Ungewissheit; eigene Abbildung nach Gruschka (2018, S. 18) ...... 23

Abb. 4: Umgang mit Ungewissheit; eigene Abbildung nach Kosinár (2018) .......... 26

Abb. 5: Subjekt und Objekt interagieren und resonieren im Feld ................. 31

Abb. 6: Trias von Resonanz (Rosa 2018) und Habitualisierung (Bourdieu 1996) ....... 33

Abb. 7: Inkorporierung im Habitualisierungsprozess ........................ 34

Abb. 8: Habitualisierungsschema nach Bourdieu (1982) ..................... 35

Abb. 9: Habitus als holistisches Konzept (Bourdieu 1982) ................... 35

Abb. 10: Generierung beliebig vieler Handlungsschemata ....................... 36

Abb. 11: Wechselwirkung zwischen den Feldern im schulischen Kontext ............ 37

Abb. 12: Konstitution des Feldes LehrerInnenbildung ....................... 38

Abb. 13: Subjekt und Objekt in den Feldern Ausbildung, Berufspraxis und

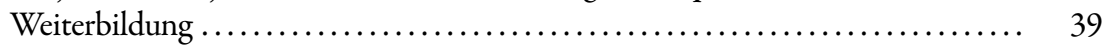

Abb. 14: Sukzessive Habitualisierung über Inkorporierung und Lernprozesse . .......... 40

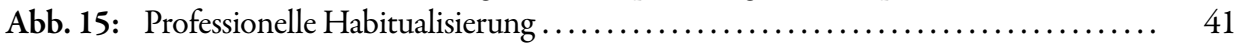

Abb. 16: Strukturanalogien allgemeiner und pädagogischer Professionen; eigene Abbildung nach Schrittesser (2011) ........................ 46

Abb. 17: Professionalisierung in den Praxisphasen; eigene Abbildung nach Liegmann et al. (2018) ..................................... 50

Abb. 18: Triaden der Habitualisierung und Professionalisierung in den Praxisphasen ..... 51

Abb. 19: Professionalisierung durch Forschendes Lernen; eigene Abbildung nach

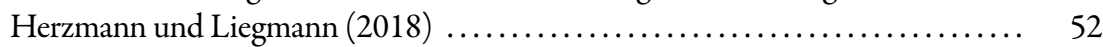

Abb. 20: Gesichtspunkte zum Forschenden Lernen; eigene Abbildung nach Boelhauve (2005) .........................................

Abb. 21: Professionalisierung durch reflexives Lernen; eigene Abbildung nach

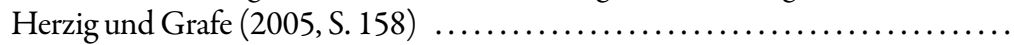

Abb. 22: Mögliche Kooperationsvarianten der Akteure zwischen den

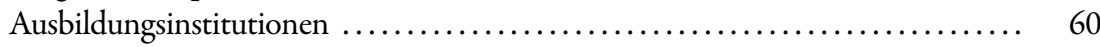

Abb. 23: Professionalisierung der Mentees und MentorInnen .................. 68

Abb. 24: Mentoring-Ansätze; eigene Abbildung nach Wang und Odell (2007) ......... 73

Abb. 25: Drei-Wege-Modell; eigene Abbildung nach Niggli (2005) .................. 74

Abb. 26: Lerntriade; eigene Abbildung nach Ziegler (2009) ..................... 74

Abb. 27: Effektives Lernen beim Mentoring; eigenen Abbildung nach Ziegler (2009) .... 75

Abb. 28: Hauptziel (HZ) und Nebenziele (NZ) beim Mentoring; eigene Abbildung

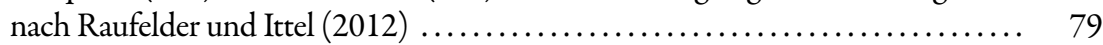

Abb. 29: Pädagogische Professionalisierung auf mikrodynamischer Ebene $\ldots \ldots \ldots \ldots \ldots . \quad 82$

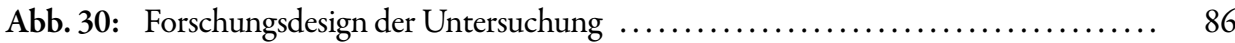

Abb. 31: Kriterien Geschlecht und Schultyp; SL ......................... 93

Abb. 32: Vergleichsverteilung nach Geschlecht und Schultyp; SL, Quelle: Bildungsdirektion für Tirol, Stand: $20.02 .2019 \ldots \ldots \ldots \ldots \ldots \ldots \ldots \ldots \ldots . \quad 93$

Abb. 33: Kriterium Dienstzeit; SL .................................... 94

Abb. 34: Kriterium Unterrichtstätigkeit; SL $\ldots \ldots \ldots \ldots \ldots \ldots \ldots \ldots \ldots \ldots \ldots \ldots . \quad 94$ 
Abb. 35: Kriterium Größe des Kollegiums; SL ........................... 95

Abb. 36: Kriterien Lage und Schultyp; SL . ............................ 95

Abb. 37: Kriterium Dienstjahre; BA ................................ 97

Abb. 38: Kriterien Geschlecht und Schultyp; BA .......................... 98

Abb. 39: Vergleichsverteilung nach Geschlecht und Schultyp; LehrerInnen;

Quelle: Bildungsdirektion für Tirol, Stand: 20.02.2019 ............... 99

Abb. 40: Kriterien Lage und Schultyp; BA ................................. 99

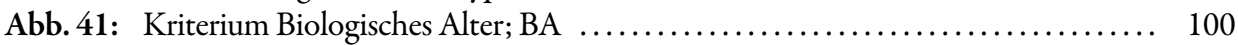

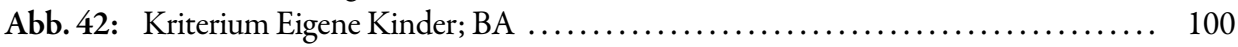

Abb. 43: Induktive Konzeptentwicklung mit multimethodischem Zugang; eigene Abbildung in Anlehnung an Lamnek und Krell (2010, S. 106) .............. 102

Abb. 44: Methodologische Triangulation zur Datenerhebung ................... 103

Abb. 45: Hierarchische Darstellung des Kategoriensystems; Aufzählung mit Nummerierungslogik .................................. 108

Abb. 46: Darstellung der Kategorien in Vielwegbäumen; Schema .................. 109

Abb. 47: Interviews mit SchulleiterInnen; Hauptkategorien (SL_A) - (SL_D) ........ 112

Abb. 48: Kompetenzen von BA; Haupt- und Subkategorien ................... 113

Abb. 49: Institutionelle Ausbildung aus der Sicht der SL; Haupt- und Subkategorien ..... 115

Abb. 50: Wahrnehmung der BA durch die SL; Haupt-, Sub- und Subsubkategorien ....... 118

Abb. 51: Unterstützung \& Begleitung der BA im Schulalltag; Haupt-, Sub- und

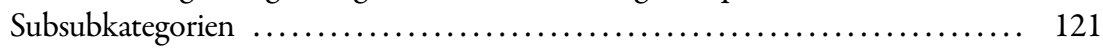

Abb. 52: Interviews mit BerufsanfängerInnen; Hauptkategorien (BA_A) - (BA_F) ..... 125

Abb. 53: Ersterfahrungen der BA beim Berufseinstieg; Haupt-, Sub- und

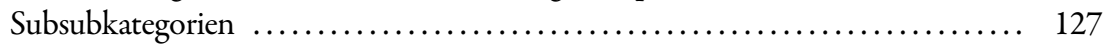

Abb. 54: Mentoring aus der Sicht der BA; Haupt-, Sub- und Subsubkategorien ......... 131

Abb. 55: Institutionelle Ausbildung aus der Sicht der BA; Haupt-, Sub-, Subsub- und

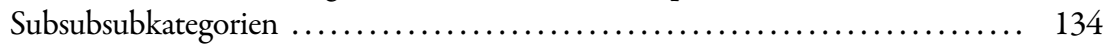

Abb. 56: Disziplin \& Classroom Management; Haupt- und Subkategorien ............ 139

Abb. 57: Kriterien für Berufszufriedenheit der BerufsanfängerInnen; Haupt- und Subkategorien ............................................

Abb. 58: Arbeitsbelastung und Überforderung der BerufsanfängerInnen; Haupt-

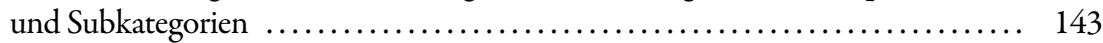

Abb. 59: Clusterkategorien 1 und 2 auf Clusterkategorienebene I (CK_I) . .......... 148

Abb.60: Clusterkategorie 3 auf Clusterkategorienebene II (CK_II_3);

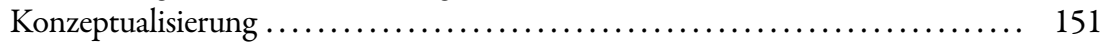

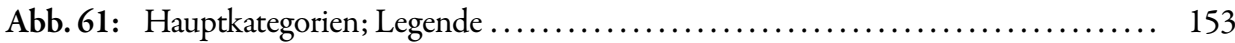

Abb. 62: Vernetzungsgrad nach Themen ............................... 154

Abb. 63: Vernetzungsgrad nach Hauptkategorien (Legende s. Abb. 61) ............. 154

Abb. 64: Themen zur Interpretation: Übersicht und Zusammenhänge ............. 156

Abb. 65: Bedingungsgefüge Administration ............................ 157

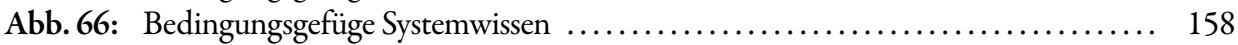

Abb. 67: Bedingungsgefüge Reflexion \& Feedback ........................ 160

Abb. 68: Bedingungsgefüge Autorität in der Klasse .......................... 162

Abb. 69: Bedingungsgefüge Kooperation mit Schülerinnen und Schülern ............ 164

Abb. 70: Bedingungsgefüge Habitus ............................... 167 
Abb. 71: Kontingenz, Ungewissheit, Unverfügbarkeit - Zusammenführung

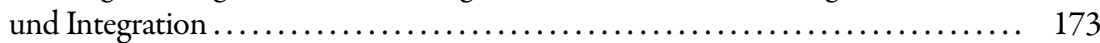

Abb. 72: LehrerInnenpersönlichkeit \& Habitus - Zusammenführung und Integration .... 176

Abb. 73: Schlüsselbegriffe und Auswirkungen der initialen Berufserfahrungen ......... 185

Abb. 74: Beziehungsdreieck im Ausbildungscurriculum ...................... 189

Abb. 75: Beziehungsdreieck in der Induktionsphase ........................ 189

Abb. 76: Kompetenzstufenmodell nach Gordon, Burch \& Kraler .................. 195

Abb. 77: Ganzheitliche Professionalisierung ............................. 198

Abb. 78: Studierende in der Vernetzung der AusbildungsakteurInnen und Curricula ..... 199

Abb. 79: Bibliografische Datenerhebung - BerufsanfängerInnen ................ 213

Abb. 80: Leitfaden - BerufsanfängerInneninterview ........................ 214

Abb. 81: Bibliografische Datenerhebung-SchulleiterInnen .................... 215

Abb. 82: Leitfaden - SchulleiterInneninterview ........................... 216

\section{Tabellenverzeichnis}

Tab. 1: Professionelles Wahrnehmen, Denken und Handeln ................. 41

Tab. 2: Relevanz von Mentoring; Quelle: Schmich und Itzlinger-Bruneforth

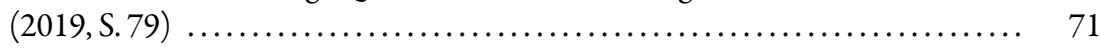

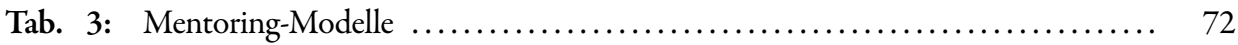

Tab. 4: Institutionelle Rahmenbedingungen für Mentoring in der LehrerInnenbildung

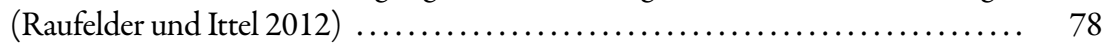

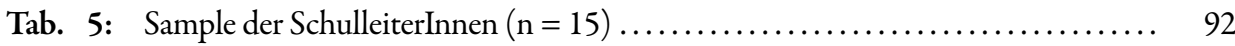

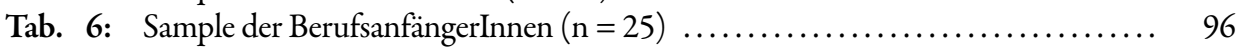

Tab. 7: Interviews mit SchulleiterInnen - Häufigkeiten .................... 112

Tab. 8: Interviews mit BerufsanfängerInnen - Häufigkeiten ................... 126

Tab. 9: Kontext und Schlüsselbegriffe; Clusterkategorie $1 \ldots \ldots \ldots \ldots \ldots \ldots \ldots \ldots \ldots$

Tab. 10: Kontext und Schlüsselbegriffe; Clusterkategorie $2 \ldots \ldots \ldots \ldots \ldots \ldots \ldots \ldots \ldots . \ldots \ldots$

Tab. 11: Themen-Kategorien-Matrix; eigene Darstellung nach Kuckartz (2016) ........ 152

Tab. 12: Bedingungsgefüge Administration; Übersicht ..................... 157

Tab. 13: Bedingungsgefüge Systemwissen; Übersicht $\ldots \ldots \ldots \ldots \ldots \ldots \ldots \ldots \ldots \ldots \ldots$

Tab. 14: Bedingungsgefüge Reflexion \& Feedback; Übersicht .................. 161

Tab. 15: Bedingungsgefüge Autorität in der Klasse; Übersicht ................... 162

Tab. 16: Bedingungsgefüge Kooperation mit Schülerinnen und Schülern; Übersicht ..... 164

Tab. 17: Bedingungsgefüge Habitus; Übersicht .......................... 167

Tab. 18: Konkrete Hilfen in der Ausbildungs- und Berufseinstiegsphase ............ 181 


\section{Abkürzungsverzeichnis}

$\begin{array}{ll}\text { AHS } & \text { Allgemeinbildende höhere Schule } \\ \text { BA } & \text { BerufsanfängerIn, BerufsanfängerInnen } \\ \text { BL } & \text { BetreuungslehrerIn, BetreuungslehrerInnen } \\ \text { BMHS } & \text { Berufsbildende mittlere und höhere Schule } \\ \text { HTL } & \text { Höhere Technische Lehranstalt } \\ \text { MS } & \text { Mittelschule } \\ \text { NMS } & \text { Neue Mittelschule } \\ \text { PFL } & \text { Projekt Forschendes Lernen } \\ \text { PLP } & \text { Praxislehrperson, Praxislehrpersonen } \\ \text { PTS } & \text { Polytechnische Schule } \\ \text { QDA } & \text { Qualitative Datenanalyse } \\ \text { SGA } & \text { Schulgemeinschaftsausschuss }{ }^{86} \\ \text { SL } & \text { SchulleiterIn, SchulleiterInnen } \\ \text { Sek. I } & \text { Sekundarstufe 1 } \\ \text { Sek. II } & \text { Sekundarstufe 2 } \\ \text { VLIP } & \text { Vertragslehrperson in der Induktionsphase }\end{array}$

86 Der Schulgemeinschaftsausschuss setzt sich aus Schüler-, Eltern- und LehrervertreterInnen zusammen. Die Schulleitung hat den Vorsitz ohne Stimmrecht (Bundesministerium für Bildung, Wissenschaft und Forschung 1962). 


\section{Anhang}

\section{Interview:}

Ort: Zeit:

Geschlecht: W/m

Alter:

Geschwister:

Eigene Kinder:

Schule/Matura:

Vorherige Berufsausbildung:

Vorherigen Beruf ausgeübt: ja nein

Beruf der Eltern: Vater:

Mutter:

Ausbildungsinstitution:

Geprüfte Fächer:

Dzt. Unterrichtsfächer:

U-Jahre: 123

Schule: NMS PTS

Region: Land Stadt

Tätigkeiten: KV

FB-Leiterln

Kustos

sonstiges

Vorwiegender Unterricht:

Klasse

Fach-Gruppe

Teamteaching Nachhilfe

Abb. 79: Bibliografische Datenerhebung - BerufsanfängerInnen 
Wie geht es Ihnen jetzt gerade mit der Arbeit als Lehrerln?

Versuchen Sie sich bitte in Ihre erste Zeit als LehrerIn zurückzuversetzen. Wie war es, als Sie mit dem Unterrichten begonnen haben?

Versuchen Sei sich in die Ausbildungszeit zurückzuversetzen.

Was, bezogen auf die Ausbildung, war für Sie in der ersten Zeit als Lehrerln hilfreich?

Angenommen, Sie säßen an einer Schaltstelle und hätten die Möglichkeit, die Lehrerlnnenausbildung mitzugestalten.

Was hätten Sie in der Ausbildung noch gebraucht, was würden Sie noch hineinbringen bzw. weglassen?

Gibt es etwas, was Ihnen an der Lehrerlnnenausbildung besonders gut gefällt bzw. was Sie besonders stört?

Was hat Ihnen beim Einstieg in den LehrerInnenberuf am meisten bzw. besonders gefehlt, was hätten Sie noch gebraucht, was hätte Ihnen den Berufseinstieg erleichtert?

Möchten Sie noch etwas sagen oder ergänzen?

Was waren Ihre Beweggründe bzw. was hat Sie motiviert, an dieser Befragung teilzunehmen? 


\section{SchulleiterInneninterview $\mathrm{Nr}$.}

Ort:

Datum / Zeit:

Geschlecht:

Lehrerln seit

Schulleiterln seit

Eigene Unterrichtstätigkeit: ja/nein

Dzt. Unterrichtsfächer:

Schule:

Stadt / Land

Schulgröße / Anzahl der Klassen:

Kollegium / Anzahl der Lehrerlnnen:

Abb. 81: Bibliografische Datenerhebung - SchulleiterInnen 
Was sind für Sie „BerufsanfängerInnen“ (in Folge: BA)? Wie lange ist ein Lehrer ein $B A$, wie würden Sie diesen Begriff definieren?

Wenn Sie als SchulleiterIn an BA denken, was fällt Ihnen dazu ein, was assoziieren Sie damit?

Welche Unterstützung erwarten sich Ihrer Meinung nach BA von Ihnen als Schulleiterln bzw. als erfahrenem( $r$ ) Lehrerln? ( $\rightarrow$ Erwartung!)

Welche Unterstützung können Sie als Schulleiterln bzw. als erfahrene(r) Lehrerln BA geben? ( $\rightarrow$ Erfüllung!)

Denken Sie bitte an „Ihre" BA seit Sie Schulleiterln sind:

Was sind die häufigsten Anliegen, mit denen BA zu Ihnen kommen bzw. gekommen sint.

Macht es einen Unterschied, ob es sich um eine Frau oder einen Mann handelt?

oder: Ist es ein Unterschied, ob es eine junge Lehrerin oder ein junger Lehrer ist?

(vgl. Assoziationen, Erwartungen, Unterstützung, häufige Anliegen)

Nehmen wir an, Sie säßen an einer Schaltstelle bzgl. der

Lehrerlnnenausbildung und Sie könnten Ihre langjährige Berufserfahrung als Lehrerln bzw. Schulleiterln einbringen:

Was würden Sie an der LehrerInnenausbildung bzw. an den

Ausbildungsinstitutionen verändern, wenn Sie an den Berufseinstieg der LehrerInnen denken?

Zu den Kompetenzen der BA:

Welche Kompetenzen bringen BA in den Beruf mit?

Wo bzw. wie haben sie sich diese Kompetenzen Ihrer Meinung nach angeeignet?

Möchten Sie noch etwas anmerken oder ergänzen?

Abb. 82: Leitfaden - SchulleiterInneninterview 
Im vorliegenden Buch werden Gelingensbedingungen für einen erfolgreichen Berufseinstieg als Lehrperson rekonstruiert. Der Übergang mit den pädagogisch-praktischen Studien als Vorbereitung und der Induktionsphase als folgende und erste berufliche Professionalisierungsphase wird fokussiert, wobei der begleitende MentoringProzess besondere Berücksichtigung findet. Das Habituskonzept (Bourdieu) mit der Trias wahrnehmen - denken - handeln bildet mit der Integration der Resonanz-Diskussion (Rosa) und dem KontingenzPhänomen die theoretische Grundlage bzw. die Kernidee der Rekonstruktion: Habitus als Konzept der Professionalität. Die Datengrundlage der empirischen Arbeit besteht aus Interviews mit 25 BerufsanfängerInnen und 15 SchulleiterInnen, die multimethodisch nach der Grounded Theory, der qualitativen Inhaltsanalyse und einer Clusteranalyse qualitativ untersucht werden. Die Arbeit schließt mit dem Ausblick auf konkrete Umsetzungsmöglichkeiten der gewonnenen Erkenntnisse in Curricula für LehrerInnenaus- und -fortbildung.

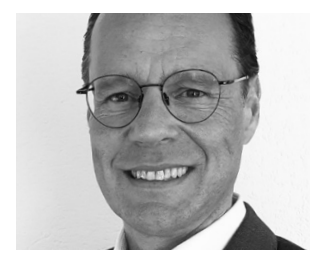

\section{Der Autor}

Klaus Schneider, Jahrgang 1969, 23 Jahre Lehrer in der Sekundarstufe, Studium der Pädagogik, Promotion in Schulpädagogik. Seit 2015 berufliches Engagement an der Pädagogischen Hochschule Tirol, Hochschuldozent für Bildungswissenschaftliche Grundlagen - Lehramt Sekundarstufe Allgemeinbildung, Verantwortung des Hochschullehrganges Mentoring - Berufseinstieg professionell begleiten. 